OLIVER JOHANNES EBNETH

\title{
INTERNATIONALISIERUNG UND \\ UNTERNEHMENSERFOLG BÖRSENNOTIERTER \\ BRAUKONZERNE
}

Göttingen, im Mai 2006 


\section{VORWORT}

$\mathrm{Zu}$ Beginn dieser Arbeit danke ich ganz herzlich meinem Doktorvater und akademischen Lehrer, Herrn Univ.-Prof. Dr. Ludwig Theuvsen für die Überlassung dieses Themas und die inhaltliche Betreuung meiner Dissertation. Während dieser Zeit ermöglichte mir Ludwig Theuvsen nicht nur meine zahlreichen Auslandsprojekte und Konferenzreisen, sondern stand mir stets in freundschaftlicher Weise mit Rat und Tat zur Seite.

Gut drei Jahre Doktorarbeit bedeuten viel ,Blut, Schweiß und Tränen', doch gab es auch zahlreiche unvergessliche Stunden und Erlebnisse, die ich nicht missen möchte. Mein Dank gilt deshalb allen Mitarbeitern des Instituts für Agrarökonomie sowie meinen Freunden und Kollegen vom Lehrstuhl für Betriebswirtschaftslehre des Agribusiness.

Besonderer Dank gilt auch Herrn Marc Koster, Director Corporate Business Development bei Heineken International, der mir eine zweimalige Projektmitarbeit im Heineken Headoffice in Amsterdam ermöglichte. Auch danke ich Berend Odink, mit dem ich viele Stunden bei Bilanzanalyse und Unternehmensbewertung internationaler Braukonzerne verbrachte.

Diese Arbeit widme ich meinen Eltern, die mir das Studium ermöglichten, eine glückliche Kindheit und Jugend in Ostbayern schenkten und stets alles gaben was in ihrer Kraft lag, kurz: ihre ganz Liebe. 
Abstract

EINLEITUNG

Internationalisierung und Unternehmenserfolg in der Weltbraubranche

\section{KAPITEL I}

Der Weltbiermarkt: Globalisierung einer Branche

I.1 Globalization of the Brewing Industry. Trends, Perspectives and Strategies

I.2 Main West European Beer Markets at a Glance 54

I.3 Review of the Central \& Eastern European Beer Markets 64

$\begin{array}{lll}\text { I.4 Review of the Chinese Brewing Sector } & 73\end{array}$

I.5 Recent Developments in Southern American Beer Markets 81

I.6 Overview over Northern American Beer Markets 90

\section{KAPITEL II}

Internationalisierung führender Braukonzerne

II.1 Exploring Globalization in the Brewing Industry. Internationalization Strategies of World-wide Leading Brewers

II.2 Konzentrationstempo. Internationalisierung und Erfolg europäischer Braukonzerne

\section{KAPITEL III}

Internationalisierung und Unternehmenserfolg:

Diskussion und Operationalisierung verschiedener Messkonzepte

III.1 Accelerating Growth Opportunities.

Profit Pools in the World Brewing Industry

III. 2 Growth and Profit Opportunities.

Analyzing the World Brewing Industry

III.3 Does Internationalization Pay Off?

Accounting-Based Evaluation of M\&As of European Brewers

III.4 Large Mergers and Acquisitions of European Brewing Groups.

Event Study Evidence on Value Creation

III.5 Multinationality and Financial Performance.

Findings from a Set of Listed Brewing Groups

III.6 The Relationship between Business Internationalization and

Financial Performance of Listed Brewing Groups

III.7 Performance and Internationalization. A Comparison of

Capital Market-Based and Accounting-Based Analysis 
KAPITEL IV

Exkurs: Internationalisierung anderer Subsektoren des Agribusiness

IV.1 Internationalization of Cooperatives in the Agribusiness.

Concepts of Measurement and their Application 304

IV.2 Internationalisierung europäischer Molkereigenossenschaften 327

IV.3 Internationalization and Corporate Success.

Empirical Evidence from the European Dairy Sector 338

FAZIT

Entwicklung und Ausblick einer sich konsolidierenden Branche

ANHANG

Publikationen und Konferenzteilnahmen

Lebenslauf

Erklärungen 


\section{ABSTRACT}

This thesis strives to analyze the major developments and trends in the world beer industry over the last decade that transformed it from the local market it was into a global one. This development arose from the fact that larger brewers faced low prospects for volume growth in developed markets leading them to seek growth either via acquisition of other brewers, by aggressive participation in developing markets or both. Thus, in the brewing industry of recent years, acquisitions have been the growing trend, providing the opportunity for firms to markedly enhance their degree of internationalization and their market share through diverse one-off deals. Recent spectacular acquisitions in the brewing industry have highlighted the apparent urgency of the major brewers to build scale and improve their strategic positioning. But, according to various studies on other industries, most cross-border deals did not meet shareholders' expectations. Assessing the corporate success and the financial performance of the latest Mergers \& Acquisitions in the brewing sector, therefore, deserves more attention.

Where methodology is concerned, this thesis sheds some empirical light on the relationship between degree of internationalization and financial performance for a sample of the largest stock-listed brewing groups around the globe. This investigation is innovative in two ways. First, as most studies in economics and international management focus on the financial service and manufacturing industries, there is hardly any literature at all about internationalizing agribusiness, food processing or consumer goods companies. Second, this thesis does not confine itself to common accounting-based procedures as have the studies by the bulk of previous scholars; instead, it will expand scientific knowledge to include capital-market-oriented measurements. The use of event study methodology is discussed as a potentially important and useful way to assess the dynamic effects of mergers and acquisitions on the brewing scene. The event study data employed in the empirical analysis are daily stock returns for the brewing groups involved in 34 Mergers \& Acquisitions between March 2000 and July 2005. The accounting-based examination employs financial data provided in the 18 largest brewing groups' annual reports for the eight financial years from 1998 to 2005. 
The outcomes of both methodologies adopted in this thesis are in line with the existing literature on internationalization-performance studies in the finance and economic literature. Indeed, there are some brewers whose Mergers \& Acquisitions transactions did not meet shareholder's expectations. But at the same time, many acquisitions have created significant value for some of the fastest growing brewers in the sample. Furthermore, there seems to be a clear link between the speed of external growth and a significant boost in brewer's profitability. Brewing groups show substantial improvement in both their profitability ratios following this period of intensive external growth. Regarding the decomposition of the performance indicators, this work is new and adds details to knowledge about the internationalizationperformance relationship. Furthermore, it discusses the development of the brewers' market capitalization, which is of paramount importance and represents the core element of a brewing group's sustained corporate success. The comparison of capital-market-based analysis with accounting-based measures provides evidence supporting the belief that these two methods should not be seen as alternative ways to evaluate performance, but as complementary ones.

A central managerial implication looks apparent as brewing executives face a new paradigm: They have to grow faster than the market in terms of market capitalization and enterprise valuation, whether to become a global company or to remain one. Otherwise, they run the risk of losing their independence and being acquired by more successful brewing groups. Heineken, SABMiller and InBev in particular have proven how effective M\&A transactions can contribute to satisfying this new paradigm.

In the last chapter of this thesis, the investigation is amended by going beyond the brewing industry. Like brewing groups, cooperatives in agribusiness are increasingly confronted with the challenges of internationalizing food markets. This development has also affected meat and dairy cooperatives, which are being forced to become more competitive by joining forces through international mergers and acquisitions. The mode and amount of the cooperatives' international activities is determined and their financial performance assessed by applying balance sheet analysis. The investigation ends with a discussion of why German cooperatives are noticeably less internationalized and show weaker financial performance than their European competitors. The managerial implications are obvious: German cooperatives in the meat and dairy industries have to solve their financial problems and redesign their outdated corporate governance mechanisms by professionalizing their management structures. 


\section{EINLEITUNG}

INTERNATIONALISIERUNG UND UNTERNEHMENSERFOLG IN DER WELTBRAUBRANCHE 


\title{
INTERNATIONALISIERUNG UND UNTERNEHMENSERFOLG IN DER WELTBRAUBRANCHE
}

\author{
,Es gibt nichts Praktischeres als eine gute Theorie'
}

Immanuel Kant

Seit Ende der 1990er Jahre hat das Phänomen der Globalisierung der Wirtschaftswelt zunehmend auch eine Branche ergriffen, die in der wirtschaftswissenschaftlichen Literatur bisher kaum Beachtung fand: Die Brauindustrie. Diese verzeichnete im globalen Kontext in den vergangenen Jahren ein kaum vorherzusehendes Wachstum. Parallel dazu haben sich weltweit große multinational tätige börsennotierte Braukonzerne herausgebildet, die durch zahlreiche M\&A-Transaktionen als Treiber hinter diesem Internationalisierungsprozess standen. Dabei entstanden global tätige Brauunternehmen mit Umsätzen von teilweise über 10 Milliarden Euro und einem Netzwerk weltweit gestreuter Tochtergesellschaften. Der starke Anstieg von Fusionen und Akquisitionen der agierenden Unternehmen führte in der letzten Dekade zu teils deutlicher Erhöhung der Konzentrationsrate innerhalb der Branche und zu einer beschleunigten Konsolidierung verschiedener Teilmärkte.

Praktisch alle in der Literatur existierenden Internationalisierungstheorien unterstellen implizit, dass Entscheidungen in Unternehmen stets mit dem Ziel der Gewinnmaximierung getroffen werden. Fusionen und Akquisitionen waren in den vergangenen Jahren vermehrt Gegenstand intensiver Forschungsbemühungen. Vor allem in jüngerer Zeit drängt sich allerdings zunehmend die Frage auf, ob die Strategie intensiver geographischer Diversifikation und der Aufbau von operativen Tochtergesellschaften im Ausland tatsächlich dazu dienen, Marktunvollkommenheiten auszunutzen, und damit den Erfolg der Unternehmen zu steigern (Glaum, 1996). Wie sich diese beschleunigte Globalisierung auf die Gesamtbranche sowie deren größte handelnde Unternehmen ausgewirkt hat wird in der vorliegenden Dissertation untersucht. Zentraler Untersuchungsgegenstand der vorliegenden Arbeit sind deshalb die Bestimmungsgründe und die Erfolgswirkung der Internationalisierung von börsennotierten Braukonzernen. 
$\mathrm{Zu}$ Beginn der vorliegenden kumulativen Dissertation soll ein ausführliches einleitendes Kapitel einen grundsätzlichen Überblick über die zu behandelnde Thematik geben. Nachdem die Zielsetzung dieser Dissertation einleitend knapp umrissen wird, werden nachfolgend die hauptsächlich in dieser wissenschaftlichen Arbeit zur Anwendung kommenden wirtschaftswissenschaftlichen Methodiken und Fragestellungen behandelt. Dabei wird jeweils auch auf die zu behandelnde Branche eingegangen und die theoretischen Konstrukte anhand von Praxisbeispielen verdeutlicht. Diese Erklärungsansätze sind im Überblick:

1) Internationalisierung und Multinationale Unternehmen

2) Messung von Internationalisierungsgraden

3) Fusionen \& Akquisitionen

4) Internationalisierung und Unternehmenserfolg

Es wird dabei auf die jeweiligen Schwerpunktkapitel verwiesen und nicht zu sehr in die Tiefe gegangen um nicht den einzelnen Beiträgen der vorliegenden Dissertation vorzugreifen. Anschließend daran folgt eine kurze Präsentation der vier Hauptkapitel der Arbeit. 


\section{ZIELSETZUNG}

Die grundsätzliche Relevanz dieser Arbeit für die bestehende Forschung als auch deren Bedeutung für die zu behandelnde Branche besteht darin, dass mit der vorliegenden Dissertation in mehrfacher Hinsicht wissenschaftliches 'Neuland' betreten wird. Zum einen sind die Konzepte zur Messung von Internationalisierung und deren Auswirkung auf unternehmerische Performance sowohl in der internationalen als auch der deutschsprachigen wirtschaftswissenschaftlichen Literatur erst im Laufe der 1990er Jahre verstärkt entwickelt worden. Zum anderen finden sich zum Stichwort ,Internationalisierung und Unternehmenserfolg' in den einschlägigen Fachzeitschriften des internationalen Agribusiness kaum Beiträge, da sich die bestehende Forschung nahezu ausschließlich auf die Finanzdienstleistungsbranche sowie die verarbeitende Industrie bezieht. Die Untersuchung zu den Auswirkungen steigender Internationalisierungsgrade führender Braukonzerne auf deren Unternehmenserfolg stellt also ein Novum in der Forschung zum Strategischen Internationalen Management in der Ernährungswirtschaft dar und soll auch Rückschlüsse auf den Konzentrations- bzw. Globalisierungsgrad der betrachteten Branche erlauben.

Voran gehen soll dem empirischen Teil ein umfassender Branchenüberblick über den Weltbiermarkt, um die grundsätzliche Frage beantworten zu können, ob es sich bei der Brauwirtschaft um eine globale Branche handelt oder nicht. Dabei wird auch versucht, anhand einer Konkurrentenanalyse die Herausbildung sowie die Verflechtungen und Rivalitätsbeziehungen zwischen den weltweit führenden Braukonzernen aufzuzeigen.

Den Hauptteil der Arbeit bildet ein explorativ-empirischer Teil über das bisherige Internationalisierungsverhalten bzw. die verfolgten Internationalisierungsstrategien sowie den daraus resultierenden unternehmerischen Erfolges führender börsennotierter Braukonzerne. Methodisch erfolgt dies mittels Bilanzanalyse und Kapitalmarkt orientierten Ereignisstudien. Dabei wird auch der Einfluss der internationalen Brauunternehmen auf die zunehmende Konsolidierung des Weltbiermarktes analysiert. Aus den Ergebnissen der empirischen Untersuchung werden Handlungsempfehlungen für das Management in der Brauindustrie abgeleitet.

In einem abschließenden Kapitel (IV) wird die behandelte Thematik ergänzend auf andere Subsektoren des Agribusiness angewandt: den genossenschaftlichen Schlacht- und Molkereisektor. Diese beiden Branchen zeigten in der jüngeren Vergangenheit ähnliche Entwicklungstendenzen wie die Brauwirtschaft, wobei gerade die deutschen Unternehmen vor zahlreichen Herausforderungen stehen, um zukünftig im internationalen Wettbewerb bestehen zu können. 


\section{1) INTERNATIONALISIERUNG UND MULTINATIONALE UNTERNEHMEN}

\section{Begriff der Internationalisierung}

Vor dem Hintergrund unzureichender Wachstumsraten auf den heimischen Märkten, zunehmender internationaler Konkurrenz, verkürzter Produktlebenszyklen und stetig steigender Forschungs- \& Entwicklungskosten haben Unternehmen ihr internationales Engagement deutlich forciert und in den letzten Jahren zu einer verschärften Dynamik und Intensität des internationalen Wettbewerbs beigetragen (Scholl, 1989; Marx, 1998). Eine gewisse Konzeptlosigkeit herrscht unverändert hinsichtlich der spezifischen Bedeutungsinhalte von 'Globalisierung', insbesondere in Abgrenzung zur 'Internationalisierung' als der Zunahme grenzüberschreitender ökonomischer Verflechtungen. Eine einheitliche Definition der Begriffe hat sich in der Literatur bis heute nicht gebildet und oftmals werden sie auch synonym verwandt, oder 'Globalisierung' wird lediglich als Steigerung von 'Internationalisierung' verstanden. Internationalisierung auf der betrieblichen Ebene bezeichnet die strategische Entscheidung eines Unternehmens, in allen relevanten Ländern der Erde präsent und ökonomisch tätig zu sein (Germann et al, 1996; Steger und Kummer, 1996; Glaum und Lindemann, 2002). Abgesehen von der Verwendung sehr unterschiedlicher Begriffe werden im Zusammenhang mit der Globalisierung der Wirtschaftsaktivitäten auch verschiedenste empirisch-analytische Verfahren für quantitative Beschreibungen verwandt. Globalisierung wird zum einen als eine intensivierte Form weltwirtschaftlicher Verflechtungen angesehen. Zum anderen wird sie als Ausdruck einer weltweiten Betätigung von Unternehmen - im geographischen Sinne - betrachtet. Die erste Beschreibung betont also die Intensität des Globalisierungsprozesses, die zweite dessen geographische Verbreitung (Schwab, 1997).

\section{Strategische Planung von Internationalisierungsschritten}

Angesichts steigender Globalisierung des Wettbewerbs in zahlreichen Branchen durch weltweit tätige Konkurrenzunternehmen sowie die umfängliche internationale Einbindung zahlreicher Unternehmen gestaltet sich die internationale Tätigkeit von Unternehmen zunehmend risikoreicher (Scholl, 1989). Für international tätige Unternehmen implizieren die im Vergleich zu früheren Perioden erhöhte Unsicherheit und Komplexität sowie der beschleunigte Wandel eine Abkehr vom 'Steady State' und die Notwendigkeit einer systematischen und planvollen Entwicklung geeigneter Internationalisierungsstrategien (Macharzina, 1984). Voraussetzung für eine dauerhafte Verbesserung der internationalen Wettbewerbsposition ist eine 
umfassende strategische Planung unter Berïcksichtigung externer Umfeldanforderungen und unternehmensinterner Potentiale. Im Rahmen einer internationalen Strategieentwicklung muss eine dezidierte Auseinandersetzung mit den grundlegenden Fragestellungen der Wettbewerbsprofilierung, der Marktexpansion, der Marktselektion, des Markteintritts und der Marktbearbeitung erfolgen (Steinmann, 1989; Marx, 1998; Keegan, 1999). Diese Ausgestaltung und inhaltliche Konkretisierung von Internationalisierungsstrategien im weiteren bzw. Markteintrittsstrategien im engeren Sinne ist Gegenstand zahlreicher Arbeiten innerhalb des Forschungsfeldes des Internationalen Strategischen Managements.

\section{Triebfedern der Globalisierung}

Als Treiber der zunehmenden Globalisierung werden meist politische, technologische, ökonomische (Markt bezogene), und interne Faktoren genannt (Yip, 1992; Bamberger und Wrona, 1997). Gerade im Weltbiermarkt wird die zunehmende Globalisierung der Branche gerade von diesen internen Kräften vorangetrieben, die sich aus dem strategischen, expansiven Verhalten der relevanten Unternehmen ergeben. Auch hat insbesondere der hohe Wettbewerbsdruck in den vergangenen Jahren viele Unternehmen veranlasst, sich stärker auf jene Geschäftsfelder zu konzentrieren, in denen sie besondere Stärken besitzen und Kundenbedürfnisse in hohem Maße erfüllen können. Als weitere Triebfeder zunehmender Globalisierung lässt sich die Notwendigkeit einer weltweiten Marktpräsenz aufgrund kritischer Größenerfordernisse in zahlreichen Märkten nennen. Hohe Investitionserfordernisse für Produktneuentwicklungen bei gleichzeitiger Verkürzung der Produktlebenszyklen fordern die Realisierung von weltweiten Skaleneffekten. Angesichts der hohen Wettbewerbsdynamik erscheint es häufig unmöglich, die kritische Größe durch internes Wachstum zu erreichen. Viele Unternehmen sehen sich aus diesem Grund zu Akquisitionen (oder Desinvestitionen) gezwungen (Glaum et al, 2003). Gerade vor dem Hintergrund zunehmender Globalisierung kommt neben internen Wachstumsstrategien insbesondere der Rolle der unternehmerischen Akquisitionspolitik als Wachstumsmotor eine zentrale Rolle zu. Allerdings sind im Kontext der Weltbraubranche in jüngerer Zeit auch vermehrt flexiblere Zusammenschlussformen wie Joint Ventures und strategische Allianzen an Bedeutung gewonnen, die bei veränderten Rahmenbedingungen schneller wieder gelöst werden können. 


\section{Multinationale Unternehmen in der Weltbraubranche}

Um ein internationales Unternehmen von einem nationalen abzugrenzen wurden von zahlreichen Autoren quantitative Kriterien zur theoretischen Betrachtungen des internationalen Unternehmens vorgeschlagen. Vorherrschende Sichtweisen gehen davon aus, dass es jeweils unterschiedliche, d. h. alternative Archetypen des international tätigen Unternehmens gibt. In der Literatur zum Internationalen Management existieren zwei häufig zitierte Typologisierungs-Vorschläge: das EPRG-Schema von Howard Perlmutter und das Konzept von Bartlett/Ghoshal. Zusammenfassend kann die Typologie Perlmutters als primär an der Einstellung des Managements orientiert und das Konzept von Bartlett/Ghoshal als durch eine primär an strategischen Anforderungen ausgerichtete Typologie beschrieben werden (Heenan und Perlmutter, 1979; Bartlett und Ghoshal, 1989).

Vor dem Hintergrund dieser Erklärungsansätze multinational tätiger Unternehmen können die börsennotierten (v. a. europäischen) Konzerne innerhalb der Weltbraubranche am ehesten als polyzentrisch operierende Unternehmen charakterisiert werden. D. h., die zahlreichen Unterschiede zwischen Mutter- und Gastland, wie z. B. eine unterschiedliche Kultur, werden akzeptiert und folglich das Management in Tochtergesellschaften bspw. mit lokalen Mitarbeitern besetzt, denen man die Kompetenz zuschreibt, am besten im lokalen Markt agieren zu können. Das Unternehmen wird hier als ein Gebilde aus mehr oder weniger selbstständigen Einheiten angesehen, die am Ende des Geschäftsjahres ihren Gewinn abliefern. Der von den Braukonzernen verfolgte Strategietyp lässt sich teils durch den Typ des ,multinationalen Unternehmens' nach Bartlett und Ghoshal am besten definieren. Dieses wird durch ein Portfolio nationaler Einheiten, die strategisch weitgehend unabhängig voneinander agieren, charakterisiert. Die einzelnen Niederlassungen oder Tochtergesellschaften treten quasi als einheimische Aktoren am Markt auf und verfügen über relativ große Autonomie. Führende europäische Braukonzerne wie InBev, SABMiller oder Heineken, die sich durch eine lange Historie sich internationalisierender Geschäftstätigkeit auszeichnen, können allerdings auch schon als ,transnationale Unternehmen' bezeichnet werden. Sie vereinen die Lokalisierungsvorteile durch länderspezifische Markenportfolios, können aber v. a. durch ihr weltweites Distributionsnetz Vorteile bei der Vermarktung ihrer ,global flagship brands' nutzen. 


\section{Internationalisierung der Brauwirtschaft}

Durch die vermehrten externen Wachstumsschritte hat sich gerade in der Weltbraubranche das Konzentrations- und Konsolidierungstempo in den vergangenen Jahren deutlich erhöht. Der Grad der absoluten Konzentration erfolgt anhand der Berechnung der Konzentrationsrate (CR). Im Jahr 2005 hatte der weltgrößte Braukonzern (InBev) einen Marktanteil von 14,1\% im Vergleich zu 8,9\% im Jahr 1998 (Anheuser-Busch). Der CR3 (CR5) erhöhte sich von $16,9 \%(22,6 \%)$ in 1998 auf 35,2 \% (47,2\%) in 2005. Vor allem zu Beginn des neuen Jahrtausends hat sich dieses Konzentrationstempo nochmals deutlich verschärft. Innerhalb der letzten sechs Jahre stieg der CR10 durch vermehrte M\&A-Tätigkeiten um fast 30\% auf inzwischen 62,5\%. In der Brauwirtschaft ist ein Trend zu beobachten, den auch andere Industrien im Zuge der Globalisierung vollzogen haben: Der Trend zur Konzentration auf das Kerngeschäft. So verkaufte der französische Molkereikonzern Danone im März 2000 seine Brauereisparte Kronenbourg an den britischen Brauer Scottish\&Newcastle (S\&N). Philipp Morris wiederum veräußerte seine Beteiligung am US-Brauer Miller im Jahr 2002 an South African Breweries. Weiterhin lässt sich beobachten, dass sich die zehn größten Braukonzerne weltweit fast ausschließlich auf die Bierproduktion beschränken. Das Engagement im Soft Drinks-, Spirituosen- und Weinsegment ist sehr begrenzt. Lediglich unter den Top 20-Unternehmen finden sich Misch-Getränkekonzerne wie Diageo, Foster's, Lion Nathan oder Asahi.

Das Phänomen Globalisierung auf die einzelbetriebliche Ebene von Braukonzernen bezogen, bedeutet, dass die Unternehmen entscheiden, ihre Geschäftstätigkeit auf eine internationale Basis auszuweiten, um in allen relevanten Ländern der Erde präsent und ökonomisch tätig zu sein. Beispiel dafür sind die europäischen Braukonzerne Heineken und InBev. So nimmt Heineken für sich in Anspruch, mit seiner gleichnamigen Biermarke in (fast) allen Ländern in der Welt vertreten zu sein (Heineken, 2006). Diese zunehmende globale Verflechtung erfolgt in überwiegendem Maße in Form von Fusionen und Akquisitionen, doch gibt es zwischen den führenden Unternehmen auch Beispiele für Joint Ventures und strategische Allianzen: In der Weltbrauwirtschaft sind zum einen die Vertriebskooperation zwischen dem US-Konzern Anheuser-Busch und dem niederländischen Brauer Grolsch über die Distribution der gleichnamigen Marke in den USA zu nennen. Der australische Getränke- und Bierkonzern Foster's wiederum geht bei seiner Auslandsexpansion primär den Weg über Kooperationen mit lokalen Partnern. Den russischen Markt etwa erschließt das Unternehmen seit 2004 mit Hilfe von Baltika, einem 50/50-Joint Venture zwischen Carlsberg und S\&N. In den USA arbeitet Foster's mit der Molson Coors Brewing Company (MCBC) zusammen, in Großbritan- 
nien mit S\&N und in der Türkei mit Efes (Dengel und Rössing, 2005). Heineken wiederum vertreibt Anheuser-Busch's Marken in West- und Osteuropa; beide Unternehmen haben kürzlich gar gemeinschaftlich für die Akquisition der chinesischen Fujian Sedrin geboten (Anonymus, 2005a).

Im Gegensatz zu den sich schnell internationalisierenden führenden Brauunternehmen kam es im weltweiten Kontext der einzelnen Biermärkte bisher allerdings kaum zu Homogenisierungen. So beträgt beispielsweise der Anteil der zehn größten Biermarken im Jahr 2005 nach wie vor lediglich etwa 19,5\% an der gesamten Weltbierproduktion. Auch zeichnet sich der größte Braukonzern InBev durch ein Markenportfolio von etwa 200 nationalen und regionalen Biermarken aus (siehe dazu auch Kapitel I.1).

Trotz des geschilderten hohen Konzentrationstempos und der zunehmenden Internationalisierung der Weltbraubranche kann Theodor Levitts These aus dem Jahr 1983, im Zuge der Globalisierung komme es zu einer weltweiten Vereinheitlichung der Präferenzen der Konsumenten und Unternehmen müssten infolgedessen globale Standardisierungsstrategien verfolgen, als zu vereinfachend abgelehnt werden (Glaum et al, 2003). Wie auch die weitaus meisten Konsumgütermärkte weist die Braubranche im internationalen Kontext weiterhin eine Vielzahl regionalspezifischer Differenzierungen auf. So wird die Etablierung internationaler Marken wie etwa Heineken (Heineken), Beck's (InBev), Stella Artois (InBev) oder Carlsberg (Carlsberg) von den Big Playern eher als Ausnahme gesehen; zumeist geht es um das Vorantreiben regionaler Marken. Somit kann im Kontext der Weltbraubranche teilweise auch der These Michael Porters widersprochen werden, dass sich der Trend zur zunehmenden Homogenisierung der internationalen Produktanforderungen fortsetzen werde und sich ursprünglich länderspezifische Konsumgüterbranchen in einem verstärkten Globalisierungsprozess befänden (Porter, 1995). 


\section{2) MESSUNG VON INTERNATIONALISIERUNGSGRADEN}

\section{Definition von Internationalisierungsgrad}

Um sich mit Konkurrenten der eigenen Branche zu vergleichen oder die Veränderung der Intensität ihrer eigenen Internationalisierung im Laufe der Zeit zu analysieren und daraus Konsequenzen für die Unternehmensstrategie abzuleiten, ist es für Braukonzerne zweckmäBig, den eigenen Internationalisierungsgrad messen und beurteilen zu können (Kornmeier und Müller, 2002). Der allgemein anerkannte Ansatz des Internationalisierungsgrades wird von Schmidt (1989) wie folgt definiert: „Unter Internationalisierungsgrad versteht man das Ausmaß der wirtschaftlichen Verbundenheit eines Unternehmens, einer Branche oder einer ganzen Volkswirtschaft mit dem Ausland. Die Auslandsaktivitäten werden dabei in Relation zu den das Inland betreffenden oder weltweiten Aktivitäten gesetzt.“ Die in dieser Definition genannte Relation der Auslandsaktivitäten zu den Gesamtaktivitäten wird als Auslandsquote bezeichnet. Als 'Ausland' ist hierbei alles das definiert, was nicht Heimatland ist (IettoGillies, 1998; Kutschker und Schmid, 2004). Die Analyse des Internationalisierungsgrades bezweckt, Wechselwirkungen zwischen Aktivitäten im Inland und im Ausland zu erkennen, um auf Basis entsprechender theoretischer Konzepte die zukünftige Gestaltung der Aktivitäten im Interesse der Zielerreichung zu verbessern (Schmidt, 1989).

\section{Eindimensionale Messkonzepte}

Bei der Messung von Internationalisierungsgraden kann man zwischen ein- und mehrdimensionalen Konzepten unterscheiden. Eindimensionale Messkonzepte ermitteln den Internationalisierungsgrad lediglich über Indikatoren einer Dimension und gehen davon aus, dass die Internationalisierung anhand eines einzigen Merkmals bzw. einer Dimension ausreichend dargestellt werden kann (Friedrichs, 1980). Dazu werden aus allgemeinen Merkmalen der Unternehmenstätigkeit diverse Kennzahlen der Internationalisierung entwickelt und dabei zwischen Bestands- und Bewegungsgrößen unterschieden ${ }^{1}$ (Kornmeier und Müller, 2002). Bei der Berechnung des Internationalisierungsgrades werden Relativzahlen gebildet, die die Bedeutung des Auslands für eine Wirtschaftseinheit darstellen, ohne dabei die räumliche Ver-

\footnotetext{
${ }^{1}$ Bestandsgrößen wären z. B. die Anzahl der Länder mit Niederlassungen, Tochtergesellschaften, Export, Lizenzproduktion etc., im Ausland vorhandenes Anlage-, Umlaufvermögen, Aktionäre im Ausland, Ausländer im Top- Management etc.
} 
teilung zu beachten. Für die Bildung dieser Indikatoren gibt es zwei grundsätzliche Möglichkeiten: Die Bildung einer Auslandsquote oder einer Auslandsrelation:

$$
\text { Auslandsquote }=\frac{\text { Auslandsaktivität }}{\text { Gesamtaktivität }}, \text { bzw. Auslandsrelation }=\frac{\text { Auslandsaktivität }}{\text { Inlandsaktivität }} \text {. }
$$

So kann z. B. der Auslandsumsatz bzw. -gewinn am Gesamtumsatz bzw. -gewinn relativiert werden, um die Auslandsquote zu errechnen. Da verschiedene Größen für die Berechnung des Internationalisierungsgrades herangezogen werden können, gibt es folglich nicht nur eine Kennzahl für die Internationalisierung eines Unternehmens. Die zu verwendende Größe sollte daher problemadäquat auf das Ziel der betreffenden Untersuchung zugeschnitten werden ${ }^{2}$ (Schüle, 1992; Glaum, 1996; Schwab, 1997). Der Vorteil der eindimensionalen Messkonzepte liegt in ihrer Einfachheit, da die Ergebnisse eindeutig, leicht interpretierbar, schnell zu berechnen und die erforderlichen Daten relativ leicht zu generieren sind. Nicht berücksichtigt wird dabei allerdings die Tatsache, dass die jeweiligen Auslandsquoten keine Aussagen über die Anzahl der einbezogenen Länder, deren geographisch-kulturelle Streuung und Konzentration erlauben. Weiterhin ist problematisch, dass sich die Form der Internationalisierung eines Unternehmens im Zeitverlauf ändern kann und in Dimensionen vorstößt, die durch die bisher verwendeten Indikatoren nicht berücksichtigt wurden. Der gesamte Umfang des Internationalisierungsprozesses wird mittels der eindimensionalen Messkonzepte deshalb nicht vollständig erfasst (Germann et al, 1999; Fisch and Oesterle, 2003).

\section{Mehrdimensionale Messkonzepte}

Aufgrund der soeben beschriebenen Nachteile eindimensionaler Messkonzepte ist es unumgänglich, sich der Kombination von Indikatoren bzw. der Kategorisierung unterschiedlicher Dimensionen durch mehrdimensionale Konzepte in Form von Indizes zu bedienen, um Internationalisierung umfassender abbilden zu können. Mehrdimensionale Messkonzepte berücksichtigen zum einen meist die geographische Streuung internationaler Unternehmenstätigkeiten, zum anderen fließen oftmals auch die kulturellen Unterschiede dieser Aktivitäten in den Internationalisierungsgrad ein. Es kann somit ein kompletteres Abbild verschiedener Merk-

\footnotetext{
${ }^{2}$ In der vorliegenden Untersuchung wurde beispielsweise die Zahl der Direktinvestitionen empfangenden Länder auf diejenigen beschränkt, die auch im Kontext der Weltbraubranche eine relevante Rolle spielen. Siehe dazu die Kapitel III.5, III.6 und III.7.
} 
male gemäß der zuvor aufgestellten Internationalisierungsdefinition gemessen werden (Germann et al, 1999; Fisch und Oesterle, 2000). Die wichtigsten dieser Konzepte sind der Ansatz von Sullivan (1994), der Transnationality Index der UNCTAD (1995), der Network Spread Index von Ietto-Gillies (1998), das multidimensionale Kennzahlensystem von Germann, Raab und Setzer (1999) und das Messkonzept von Fisch und Oesterle (2003).

Zur Validität dieser Messkonzepte lässt sich sagen, dass durch die Kombination von Einzelindikatoren Mehrdeutigkeiten in der Interpretation eliminiert und Abhängigkeitsbeziehungen zwischen den einzelnen Elementen erfasst werden sollen. Wichtig ist, dass die quantitativen Variablen eine sachlich sinnvolle Beziehung zueinander aufweisen, sich gegenseitig ergänzen, erläutern und Ziel gerichtet auf die betreffende Untersuchung bzw. Definition zugeschnitten werden. Die Indizes stellen damit verdichtete Größen dar, die essentielle Zusammenhänge deutlich machen sollen. Durch die Messgröße eines Indexes soll bei empirischen Untersuchungen dann ersichtlich werden, zu welchem Grad die Merkmale und Kriterien der zuvor erarbeiteten Definition der Internationalisierung erfüllt sind (Van Koolwijk und Wieken-Mayser, 1976; Glaum, 1996; Germann et al, 1999). Ziel dieser Messkonzepte ist es unter anderem, ein Urteil über das Globalisierungsprofil eines Untersuchungsobjekts (bspw. der zehn weltgrößten Braukonzerne) im Vergleich zu anderen fällen zu können, womit es möglich wird, ein differenziertes Bild der Globalisierung zu zeichnen. Dadurch können auch Aussagen darüber getroffen werden, ob eine Branche in allen Bereichen (Faktoreinsatz, Produktion, Absatz) global verflochten ist, oder sich ihre Auslandsaktivitäten auf bestimmte Bereiche oder Regionen bzw. Länder beschränken.

Ausführlicher werden diese ein- und mehrdimensionalen Messkonzepte in den Kapiteln IV.2 und IV.3 dieser Arbeit beschrieben.

\section{Stufenmodell der Internationalisierung}

Internationalisierungsschritte von Unternehmen lassen sich auch durch deskriptive Stufenmodelle beschreiben. Meissner und Gerber (1980) vertreten die Auffassung, dass die Internationalisierungsaktivitäten sich als ein in mehreren Stufen verlaufender Prozess darstellen, der sich nach Intensität der Kapital- und Managementleistung im Stamm- und Gastland von Exporten, über Lizenzvergaben, Franchising, Joint Venture, Auslandsniederlassungen, (Produktions-) Betrieben bis hin zu Tochtergesellschaften vollzieht. Mit einem zunehmenden Internationalisierungsgrad sinkt die Kapital- und Managementleistung im Stammland und steigt die im Gastland. So ist beim Export die Bindung der Leistungen an das Stammland sehr hoch und 
an das Gastland sehr gering. Bei einer selbständigen Tochtergesellschaft im Gastland hingegen ist dies genau umgekehrt der Fall (Meissner und Gerber, 1980; Swoboda, 2002). So kommen je nach Branche, Land, und Unternehmensgröße verschiedenen Internationalisierungsstrategien unterschiedliche Bedeutungen zu. Beschränkt sich der überwiegende Teil der deutschen Brauwirtschaft bisher auf direkten oder indirekten Export, so haben Fusionen \& Akquisitionen im weltweiten Kontext als zentrale Markteintritts- und Marktbearbeitungsstrategie eine besondere Bedeutung für die Internationalisierung von Braukonzernen gewonnen.

\section{Internationalisierungsgrade in der Weltbrauindustrie}

Beispielhaft aus der Braubranche sei hier der weltgrößte Braukonzern InBev genannt. Durch forcierte Direktinvestitionen im Ausland konnte InBev zwischen 1998 und 2005 die Gesamtzahl seiner ausländischen (Minderheits- und Mehrheits-) Beteiligungen von 91 auf 322 fast vervierfachen. Konkreter lässt sich diese internationale Verflechtung an der Anzahl der Länder belegen, in denen die Unternehmen Tochtergesellschaften besitzen. So unterhält InBev Mehrheits- und Minderheitsbeteiligungen in 77 Ländern weltweit, Heineken in 65 Ländern (InBev, 2006). Ein weiterer Indikator für die zunehmende Internationalisierung führender Braukonzerne ist der Auslandsanteil am Gesamtumsatz. Für die weitgehend global agierenden europäischen Brauunternehmen beläuft sich dieser inzwischen auf 97,2\% (InBev), 95,3\% (Carlsberg), 95,1\% (Heineken) und 86,1\% (SABMiller). Dies veranschaulicht die steigende Bedeutung, die den Auslandsmärkten der führenden Brauer zukommt. Das Auslandsengagement der deutschen Brauer beschränkt sich dagegen bisher fast ausschließlich auf reine Exporttätigkeit. Zwar konnte seit Ende der 90er Jahre die Exportquote der deutschen Brauwirtschaft von gut $7 \%$ auf $13 \%$ gesteigert werden, doch betreiben bis auf wenige Ausnahmen keine deutschen Braugruppen ausländische Tochtergesellschaften. Gründe dafür sind die - im internationalen Kontext - zu geringe Unternehmensgröße sowie die daraus (indirekt) resultierende mangelnde Kapitalkraft. 


\section{3) FUSIONEN \& AKQUISITIONEN}

Geschäftswachstum kann auf dem Wege des internen (organischen) und des externen Wachstums erzielt werden. Internes Wachstum liegt vor, wenn ein Unternehmen aus eigener Kraft in die Entwicklung neuer Produkte oder Märkte bzw. in eine intensivere Durchdringung seiner bisherigen Märkte investiert. Externes Wachstum besteht demgegenüber in dem Erwerb bereits bestehender Unternehmen. Im Gegensatz zur langsamen und kontinuierlichen Entwicklung durch internes Wachstum bewirken Übernahmen eine sprunghafte Veränderung von Struktur und Größe eines Unternehmens (Glaum und Lindemann, 2002).

\section{Vorteile von Fusionen und Akquisitionen}

Die Vorteile von Akquisitionen können wie folgt beschrieben werden: Zum einen ermöglichen sie - je nach Zielsetzung -, komplementäre Ressourcen zu erwerben, um damit economies of scope zu erzielen, oder auch ähnliche Ressourcen zu erwerben, wodurch die Realisierung von economies of scale möglich wird. Diese Verbund- und Größenvorteile könnten prinzipiell auch durch Neugründung von Auslandsgesellschaften erreicht werden, doch lässt das Markt- und Wettbewerbsumfeld in vielen Fällen einen solchen langsamen generischen Aufbau nicht zu. Man spricht dann von economies of speed (Kutschker und Schmid, 2004). Der entscheidende Vorteil externen Wachstums ist der Faktor 'Zeit'. Gerade in dynamisch wachsenden Teilmärkten der Weltbraubranche kann dieser Zeitvorteil entscheidend sein und externe Wachstumsschritte über M\&A-Transaktionen erscheinen aus Unternehmenssicht unumgänglich, um einen schnellen Markteintritt und eine zügige Marktdurchdringung zu gewährleisten (Barney, 2002; Kutschker und Schmid, 2004). Externes Wachstum eignet sich allerdings auch als Mittel der Restrukturierung in reifen und schrumpfenden Märkten. Durch Akquisitionen verringert sich die Zahl der Anbieter, es können Überkapazitäten abgebaut und die typischerweise sehr hohe Wettbewerbsintensität bestimmter Branchen reduziert werden (Glaum et al, 2003). Akquisitionen werden allerdings auch in einem defensiven langfristigen Sinne getätigt, um die eigene Unabhängigkeit zu sichern. Dies gilt primär für weitgehend konsolidierte Branchen wie beispielsweise der Automobilindustrie, wo sich im Laufe der Jahre weltweit einige große Anbieter formiert haben (Kutschker und Schmid, 2004).

Interessant ist auch die Tatsache, dass es zu einer vergleichsweise schnellen Rückzahlung der geflossenen Investitionen kommen kann. Dies liegt daran, dass schon im ersten Finanzjahr Cash Flows in erheblichem Umfang generiert werden, was bei Neugründungen von Tochter- 
gesellschaften nicht in gleichem Umfang der Fall ist (Gerpott, 1993). Strategisch besonders wichtig ist der Vorteil der existierenden Humankapitalbasis bei Markteintritten durch Akquisition bestehender Unternehmen. Weiterhin bieten Akquisitionen die Möglichkeit, auf bestehende Strategien, Strukturen und Prozesse oder auch erfolgreiche Produkte und Marken von Akquisitionsobjekten zugreifen zu können. Akquisitionen stellen oftmals die einzige wirkliche Möglichkeit dar, in einen bestimmten Markt einzutreten. Dies gilt vor allem für Märkte, die durch eine geringe Marktkonzentration und eine hohe Wettbewerbsintensität der Marktteilnehmer gekennzeichnet sind (Stein, 1992). Wird ein einheimisches Unternehmen übernommen, so wird das in den einheimischen Markt neu eintretende Unternehmen eher als lokales Unternehmen wahrgenommen. Das akquirierende Unternehmen kann auf diese Weise das Image des bisher nationalen Unternehmens nutzen.

Im Folgenden sollen deshalb die im Kontext von Braukonzernen wichtigen Vorteile externer Wachstumsschritte über M\&As näher analysiert werden. 


\section{M\&As in der Weltbrauindustrie}

Betrachtet man die Entwicklung der Weltbraubranche über die letzten Jahre, fällt auf, dass gerade die economies of speed von entscheidender Bedeutung sind, um in strategisch wichtige Märkte einzutreten ${ }^{3}$. Beispiele aus der Weltbraubranche sind die zahlreichen teils spektakulären M\&A-Transaktionen europäischer Braukonzerne, die im Laufe der letzten fünf Jahre v. a. die dynamisch wachsenden - sich noch durch einen relativ niedrigen Pro-Kopf-Konsum von Bier auszeichnenden - osteuropäischen, asiatischen und südamerikanischen Märkte konsolidiert haben. Vor allem der ehemals aus mittelständischen belgischen Privatbrauereien zu Anfang der 1990er Jahre hervorgegangene Braukonzern InBev hat diese Strategie über das vergangene Jahrzehnt mit großem Erfolg verwirklicht. Das Unternehmenswachstum des mittlerweile weltgrößten Braukonzerns erscheint vor diesem Hintergrund besonders beeindruckend. In Bezug auf das Produktionsvolumen wuchs InBev mit 223 Millionen Hektolitern in 2005 gegenüber 17 Millionen Hektolitern in 1994 um 1170\%. Der Unternehmensumsatz hat sich im gleichen Zeitraum von 1,2 Milliarden Euro auf 11,7 Milliarden Euro fast verzehnfacht.

Dass Akquisitionen oftmals die einzige wirkliche Möglichkeit darstellen, in einen bestimmten Markt einzutreten, zeigt das Beispiel des deutschen Biermarktes, der über Jahrzehnte bedingt durch seine atomisierte Marktstruktur und das deutsche Reinheitsgebot als uninteressant für internationale Braukonzerne galt. Lange Jahre lag der Anteil ausländischer Importbiere am deutschen Markt bei unter 3\%. Doch dann erfolgte innerhalb kurzer Zeit eine schrittweise Konsolidierung der deutschen Brauwirtschaft durch die Markteintritte der europäischen Braukonzerne Heineken, Interbrew und Carlsberg. So erwarben diese Unternehmen durch zahlreiche Akquisitionen größerer deutscher Brauer im Laufe von drei Jahren für etwa 4,2 Milliarden Euro einen kumulierten Marktanteil von etwa 35\% am deutschen Biermarkt.

\footnotetext{
${ }^{3}$ Dies lässt sich am Beispiel des US-Biermarktes veranschaulichen. Lange Jahre der volumenmäßig größte Markt (seit 2003 ist dies China), sind die USA immer noch der profitabelste Biermarkt weltweit. Während der niederländische Braukonzern Heineken über den Export seiner gleichnamigen internationalen Premium-Marke seit fast 100 Jahren in den USA tätig ist und inzwischen etwa 8 Millionen Hektoliter absetzt, ging der südafrikanische Brauer South African Breweries (SAB) dagegen den Weg des externen Unternehmenswachstums durch Akquisition, indem er im Mai 2002 für etwa 6,2 Milliarden Euro die Miller Brewing Company erwarb. Durch die Fusion zu SABMiller gelang es den Südafrikanern auf einen Schlag zur Nummer 2 der USA mit einem Bierabsatz von 45 Millionen Hektolitern und einen daraus resultierenden Marktanteil von etwa $18 \%$ zu werden.
} 
Des Weiteren verringerte sich durch Akquisitionen ausländischer Braukonzerne im deutschen Biermarkt die Zahl der Anbieter, es konnten teils Überkapazitäten abgebaut und die hohe Wettbewerbsintensität etwas reduziert werden. Durch die zahlreichen Akquisitionen der globalen Brauunternehmen hat sich auch der Konzentrationsgrad in der deutschen Braubranche in den letzten Jahren auf einen CR5 von inzwischen etwa 50\% deutlich erhöht. Durch zahlreiche Restrukturierungen, Preiserhöhungen aber auch Neuinvestitionen konnte angeführt von den großen teils neu entstandenen Braugruppen zudem die Profitabilität im deutschen Biermarkt deutlich verbessert werden. Lag die EBIT-Marge 2001 noch bei etwa einem Euro pro Hektoliter so konnte sie bis zum Jahr 2005 auf etwa sechs Euro/hl erhöht werden (Anonymus, 2005b).

Als Beispiel für die schnelle Rückzahlung der geflossenen Investitionen lässt sich erneut InBev anführen. Der weltgrößte Braukonzern entstand Anfang 2004 durch eine Fusion der belgischen Interbrew mit dem brasilianischen Marktführer AmBev. Durch eine komplizierte Transaktion durch Kapitalerhöhung und Aktientausch gelang es Interbrew, AmBev zu akquirieren. Durch den bisher wertmäßig größten M\&A-Deal in der Weltbraubranche erreichte Interbrew eine marktbeherrschende Stellung im strategisch wichtigen, weltweit viertgrößten und schnell wachsenden Biermarkt Brasilien. Darüber hinaus war AmBev das zweitprofitabelste Unternehmen der Branche und mit einer EBITDA-Marge von 38,5\% in 2004 weltweit eines der ertragreichsten Unternehmen überhaupt. Schon im ersten Finanzjahr (2004) der Fusion trug AmBev mit 1,26 Milliarden Euro etwa 35\% zum Gesamt-EBITDA bei, im ersten voll konsolidierten Finanzbericht 2005 waren es mit etwa 1,23 Milliarden Euro 39\% des Konzern-EBITDAs. Der operative Cash Flow des AmBev-Segments belief sich 2005 auf ca. 1,18 Milliarden Euro (InBev, 2006).

Ein Beispiel für die Möglichkeit, durch Akquisitionen auf erfolgreiche Produkte und Marken von Übernahmeobjekten zugreifen zu können ist die Akquisition des führenden deutschen Exportbrauers Beck's durch die belgische Interbrew im Jahre 2001. Interbrew, das bis dato durch eine Vielzahl nationaler Marken gekennzeichnet war, erwarb dadurch eine weltweit schon relativ breit etablierte Marke und führt ,Beck's' seither als ,global flagship brand'. Auch hat die Übernahme der Bremer Exportbrauerei Beck's dem Ansehen der gleichnamigen Marke nicht geschadet hat. Vielmehr wuchs ,Beck's' im In- und Ausland seither im hohen einstelligen Prozentbereich, wohingegen der übliche deutsche Biermarkt jährlich um etwa $1 \%$ schrumpft. 


\section{4) INTERNATIONALISIERUNG UND UNTERNEHMENSERFOLG}

\section{Problemrelevanz}

Unternehmen der Braubranche sehen sich einem zunehmend starken Druck der Kapitalmarktteilnehmer ausgesetzt, mit Hilfe von Akquisitionen ihre internationale Präsenz auszubauen und ihre Positionierung in den auf Umsatz- bzw. Produktionszahlen basierenden Branchenranglisten zu verbessern ${ }^{4}$. Andererseits ist beobachtbar, dass M\&As nur selten zu signifikanten Wertsteigerungen bei den handelnden Unternehmen führen. Gewinner bei Akquisitionen sind in aller Regel die (Alt-)Aktionäre des aufgekauften Unternehmens. Die Kurse der kaufenden Unternehmen erleiden demgegenüber häufig Einbußen. Nach Bekannt werden eines Bieterwettstreits um die Akquisition des kolumbianischen Brauers Bavaria im Januar 2005 stieg bspw. der Aktienkurs von Bavaria bis zur Übernahme durch SABMiller im Juli desselben Jahres um fast $80 \%$. Auf der anderen Seite sank - wenn auch nur für kurze Zeit - der Aktienkurs des niederländischen Braukonzerns Heineken nach Veröffentlichung seines Kaufgebotes für die österreichische Braugruppe BBAG um gut 13\%, da nach Analystenmeinung der Akquisitionspreis zu hoch gewesen sei.

Aufgrund der eingangs beschriebenen Konzentrations- und Konsolidierungstendenzen in der Weltwirtschaft im Allgemeinen und der Brauindustrie im Speziellen stellt sich die Frage nach der Erfolgswirkung eines forcierten internationalen Engagements und eines deutlich intensivierten externen Wachstums. Unternehmenserfolg im Sinne von langfristigen, nachhaltigen Wertsteigerungen kann somit nur durch langfristiges, nachhaltiges Umsatzwachstum erreicht werden. Wird allerdings Umsatzwachstum auf Kosten der Kapitalrendite erzielt, wird das Unternehmen zum Wertvernichter (Glaum et al, 2003). Braukonzerne müssen deshalb auf beiderlei abzielen: Einerseits schneller als der Markt (bzw. die Vergleichsgruppe oder 'peer group') zu wachsen, andererseits aber auch ständig ihre Profitabilität zu steigern.

\footnotetext{
${ }^{4}$ In der Braubranche basieren diese Rankings nach wie vor nicht auf Umsatzzahlen, sondern auf Produktionsvolumina. Dies macht einerseits Sinn, da somit auch nur die hauptsächlich im Biergeschäft tätigen Unternehmen erfasst werden. Diageo verzeichnet bspw. die höchste Marktkapitalisierung der Branche, ist aber v. a. im Spirituosenmarkt tätig und durch sein beschränktes Engagement im Biergeschäft weltweit nur auf Position 16. Andererseits kommt es bei einer rein produktionsbezogenen Sicht zu anderweitigen Verwerfungen. Die chinesische Tsingtao, die Nummer 9 im Weltbiermarkt, kommt mittlerweile auf eine Jahresproduktion von etwa $36 \mathrm{mhl}$, erlöst damit allerdings nur 747 Millionen $€$. Asahi hingegen (Japan, Nummer 12) erlöst mit gut 32mhl den achtfachen Umsatz von Tsingtao.
} 


\section{Methoden zur Messung unternehmerischen Erfolges}

In wissenschaftlichen Studien werden vor allem zwei wesentliche Methoden in breiter Form angewandt, um unternehmerischen Erfolg zu messen: Auswertung von Jahresabschlusskennzahlen und Kapitalmarkt orientierte Ereignisstudien ('event studies'). Von zentraler Bedeutung für den Aufbau der empirischen Untersuchung ist - neben der Messung zunehmender internationaler Geschäftstätigkeit - die Operationalisierung des Begriffes 'Unternehmenserfolg'. Die korrekte Ermittlung des Erfolges eines Unternehmens ist eine der zentralen betriebswirtschaftlichen Fragestellungen. Dem bilanziellen, kalkulatorischen und ökonomischen Gewinn steht das Kapitalmarkt orientierte Konzept des 'Shareholder Values' gegenüber, der sich aus ausgeschütteten Dividenden sowie aus Kursgewinnen zusammensetzt (Glaum, 1996).

\section{Bilanz orientierte Methodik}

Die Bilanz- oder besser Jahresabschlussanalyse ist ein systematisches Verfahren der Ausschöpfung und Verarbeitung des Informationspotentials von Bilanz sowie Gewinn- und Verlustrechnung und Cash Flow-Statement mit dem Ziel, Einsichten in die wirtschaftliche Lage und die Zukunftsaussichten eines Unternehmens zu erlangen. Als Bilanz orientierte Erfolgsgrößen gelten allgemeine erfolgswirtschaftliche Kennzahlen wie die Gesamt-, Eigenkapitalund Umsatzrentabilität, sowie finanzwirtschaftliche Kennzahlen wie die Eigenkapitalquote und der Verschuldungsgrad. Im Zuge der Internationalisierung der Rechnungslegung erlangen die im anglo-amerikanischen Raum gebräuchlichen Cashflow-orientierten EBIT-Kennzahlen zunehmende Bedeutung. Im Rahmen der Unternehmenswert gerichteten Unternehmensführung tritt jüngst v. a. die EBITDA-Größe in den Vordergrund. Beide Kennzahlen haben den Vorteil, eine Vergleichbarkeit verschiedener Unternehmen bei unterschiedlicher Kapitalstruktur und zwischen verschiedenen Rechtsformen und Perioden zu ermöglichen (Born, 2001; Küting und Weber, 2004).

Ziel der Bilanzanalyse ist es nicht, endgültige Urteile zu fällen, sondern mögliche Fehlentwicklungen und Auffälligkeiten zu erkennen sowie Entwicklungstendenzen und Prognosen zukünftigen unternehmerischen Erfolges aufzuzeigen (Born, 2001; Gräfer, 2001). Durch Aufbereitung des Datenmaterials mittels Kennzahlenbildung können Unternehmen eingeschätzt werden, wobei möglichst ähnliche Unternehmen derselben Branche betrachtet werden, um einen zusätzlichen Beurteilungsmaßstab zu erhalten und dadurch den Informationswert der Bilanzanalyse zu steigern. 
Prinzipiell wurden in der Vergangenheit zwar mittels empirischer Bilanzanalyse vielfach Performancestudien zur Bewertung singulärer unternehmenspolitischer Ereignisse wie Fusionen und Akquisitionen durchgeführt, doch verliert die Bilanzanalyse an Aussagekraft und Validität, da sich Profitabilitätskennzahlen (EBITDA-Marge, Umsatzrendite etc.) vor und nach Unternehmensübernahmen nicht allein auf diese zurückführen lassen (Glaum und Lindemann, 2002; Beitel und Schiereck, 2003). Zur allgemeinen Messung von Profitabilität eignet sich die empirische Bilanzforschung wiederum besser als Kapitalmarkt orientierte Methoden, da sie den gesamten Finanzerfolg eines Unternehmens im Zeitablauf messbar macht.

\section{Kapitalmarkt orientierte Methodik}

Ende der 1960er Jahre wurde eine neue Richtung der empirischen Kapitalmarktforschung kreiert, die als ,event studies' bezeichnet wurde. Nur Ereignisstudien erlauben die direkte Messung der Veränderung des Shareholder Values. In einer Ereignisstudie wird die Anpassung der Aktienkurse auf die Information eines bestimmten Ereignisses untersucht. Im Rahmen Kapitalmarkt orientierter Analysen werden Aktionärsreaktionen auf das spezifische Ereignis einer Unternehmensfusion oder -akquisition betrachtet und analysiert (Röder, 1999). Eine wesentliche Voraussetzung zur Durchführung einer event study ist demnach eine möglichst exakte Datierung des jeweiligen Ereignisses. Oft ist dieser genaue Ankündigungszeitpunkt nur näherungsweise zu bestimmen. Am besten lässt sich dies wohl durch das Datum definieren, an dem die Information erstmals in der Finanzpresse publiziert und somit allen Marktteilnehmern zugänglich gemacht wird (Gross, 2004). Zur Identifikation der erstmaligen Bekanntgabe der 34 in der vorliegenden Arbeit untersuchten M\&A-Transaktionen wurde eine Literaturrecherche der gängigen Finanzzeitungen durchgeführt (u. a. Handelsblatt, Wall Street Journal (online-Ausgabe), Financial Times Europe/Deutschland). Die Kapitalmarkt orientierte event study-Methodik basiert auf der Vorstellung, dass die Marktkapitalisierung eines börsennotierten Unternehmens dessen tatsächlichen Unternehmenswert widerspiegelt. Eine Kursveränderung aufgrund einer M\&A-Transaktion drückt die Bewertung aller Kapitalmarktteilnehmer aus, welche die veränderte Unternehmenssituation in Folge der Transaktion neu einschätzen. Den Maßstab zur Erfolgsmessung bildet die so genannte 'abnormale Rendite'. Sie wird als Differenz der tatsächlich beobachteten Rendite der betreffenden Aktie und der theoretisch erwarteten, mittels eines Gleichgewichtsmodells geschätzten 'normalen' Rendite über den Ereigniszeitraum errechnet. Eine positive abnormale Rendite bedeutet, dass die Kapitalmarktteilnehmer als Folge der Transaktion - in der vorliegenden Untersuchung transnati- 
onale M\&As - eine Steigerung der zukünftigen Cash Flows des Unternehmens erwarten (Glaum und Lindemann, 2002). Eine negative Kapitalmarktreaktion kann meist auf zu hohe Kaufpreise zurückgeführt werden. Zwar wird an event studies vor allem die zentrale Annahme informationseffizienter Märkte kritisiert, doch können Ereignisstudien trotzdem als die theoretisch am besten fundierte und in der Literatur am weitesten verbreitete Methode zur Beurteilung des Erfolges von Fusionen und Akquisitionen gelten.

Zentrale These der vorliegenden Arbeit ist nun, dass es durch vermehrte M\&A Transaktionen führender Braukonzerne zu einer messbaren Erhöhung der jeweiligen Internationalisierungsgrade kommt und sich diese auch in steigendem Unternehmenserfolg ausdrücken. Diesen zu messen kann allerdings nicht durch jeweils eine der beiden Methodiken allein in allen Dimensionen erfolgen. Ereignisstudien eignen sich hervorragend zur Greifbar Machung singulärer Phänomene wie der Auswirkung einer Akquisition auf den Aktienkurs eines Unternehmens, die Bilanzanalyse stellt wiederum ein profundes Instrument dar, gesamtunternehmerischen Erfolg im Zeitablauf zu messen, der durchaus durch intensive M\&A-Tätigkeit signifikant beeinflusst sein kann.

\section{Internationalisierung und Unternehmenserfolg in der Brauwirtschaft}

Die Fragestellung nach der Messung und Quantifizierung eines möglichen Zusammenhangs zwischen steigender internationaler Geschäftstätigkeit und daraus resultierendem - positiven oder negativen - unternehmerischen Erfolges bildet den zentralen thematischen Schwerpunkt der vorliegenden Arbeit. Deshalb soll an dieser Stelle keinen Ergebnissen vorweg gegriffen werden. In den Kapiteln III. 3 bis III.7 wird diese Fragestellung empirisch untersucht. 


\section{LITERATUR}

Anonymus (2005a). Anheuser-Busch und Heineken arbeiten zusammen, in: www.lz-net.de, 02.11.2005.

Anonymus (2005b). Paulaner will weiter zukaufen, in: www.lz-net.de, 22.09.2005.

Bamberger, I. und Wrona, T. (1997). Globalisierungsbetroffenheit und Anpassungsstrategien von Klein- und Mittelunternehmen, in: Zeitschrift für Betriebswirtschaft (ZfB), Band 67, Heft 7, S. 713-735.

Barney, J. B. (2002): Gaining and Sustaining Competitive Advantage, New Jersey: Pearson Education, Inc.

Bartlett, C. A. und Ghoshal, S. (1989): Managing Across Borders. The Transnational Solution, Boston.

Beitel, P. und Schiereck, D. (2003). Zum Erfolg von Akquisitionen und Zusammenschlüssen von Banken. Eine Bestandaufnahme der empirischen Forschung, in:Bank-Archiv: Zeitschrift für das gesamte Bank- und Börsenwesen, 2003 (51): 501-515.

Born, K. (2001). Bilanzanalyse international. Deutsche und ausländische Jahresabschlüsse lesen und beurteilen, 2. Auflage, Stuttgart.

Dengel, B. und Rössing, S. (2005). Braukonzerne drängen in neue Märkte, in: www.ftd.de, 13.09.2005.

Fisch, J. H. und Oesterle, M.-J. (2000). Globalisation can be Measured: Unveiling Tales of Mystery and Globalisation With a New Integrative and Metric Measurement Concept, Paper Submitted to the 26th Annual Conference of the European International Business Academy, Maastricht.

Fisch, J. H. und Oesterle, M.-J. (2003). Exploring the Globalization of German MNCs with the Complex Spread and Diversity Measure, in: Schmalenbach Business Review, 55(2003): 2-21.

Friedrichs, J. (1980). Methoden empirischer Sozialforschung, 14. Auflage, Opladen.

Germann, H. et al (1996). Globalisierung der Wirtschaft: Begriff, Bereiche, Indikatoren, in: Steger, U. (Hrsg.), Globalisierung der Wirtschaft: Konsequenzen, für Arbeit, Technik und Umwelt, Berlin: 18-55.

Germann, H. et al (1999). Messung der Globalisierung: ein Paradoxon, in: Steger, U. (Ed.), Facetten der Globalisierung: ökonomische, soziale und politische Aspekte, Berlin etc.: $1-25$.

Gerpott, T. J. (1993). Integrationsgestaltung und Erfolg von Unternehmensakquisitionen. Schäffer-Poeschel, Stuttgart.

Glaum, M. (1996). Internationalisierung und Unternehmenserfolg, Wiesbaden.

Glaum, M. und Lindemann, J. (2002). Externes Wachstum: Theoretische Überlegungen und empirische Befunde, in: Glaum et al. (Hrsg.), Wachstumsstrategien internationaler Unternehmungen. Internes vs. externes Unternehmenswachstum, Stuttgart: 269-299.

Gräfer, H. (2001). Bilanzanalyse, 8. Auflage, Berlin.

Glaum, M. et al (2003). Wachstumsstrategien internationaler Unternehmungen: 10 Thesen, in: Zeitschrift für betriebswirtschaftliche Forschung 55(2003): 823-846.

Gross, S. (2004). Branchenspezifischer Erfolg vertikaler und horizontaler Des/Integrationsentscheidungen. Eine empirische Ereignisstudie von Kapitalmarktbewertungen US-amerikanischer und europäischer Unternehmensvereinigungen und veräußerungen. Berlin.

Heenan, D. A. und Perlmutter, H. V. (1979). Multinational Organization Development, Reading et al.

Heineken (2006). Heineken N.V. Annual Report 2005, Amsterdam. 
Ietto-Gillies, G. (1998). Different Conceptual Frameworks for the Assessment of the Degree of Internationalization: An Empirical Analysis of Various Indices for the Top 100 Transnational Corporations, in: Transnational Corporations, 7(1998): 17-39.

InBev (2006). InBev Annual Report 2005, Brussels.

Keegan, W. J. (1999). Global Marketing Management, 6. Auflage, Prentice Hall, Englewood Cliffs.

Kornmeier, M. und Müller, S. (2002). Strategisches Internationales Management, München.

Küting, K.-H. und Weber, C.-P. (2004). Die Bilanzanalyse. Lehrbuch zur Beurteilung von Einzel- und Konzernabschlüssen, 7. Auflage, Stuttgart.

Kutschker, M. und Schmid, S. (2004). Internationales Management, München.

Macharzina, K., (Hrsg.) (1984). Diskontinuitätsmanagement, Berlin.

Marx, T. (1998). Internationale Marketingstrategien in der deutschen Brauwirtschaft, Frankfurt am Main u.a.

Meissner, H. G. und Gerber, S. (1980). Die Auslandsinvestition als Entscheidungsproblem, in: BFuP, 3(1980): 217-228.

Porter, M. E. (1995). Wettbewerbsstrategie, 8. Auflage, Frankfurt am Main, New York.

Röder, K. (1999). Kurswirkungen von Meldungen deutscher Aktiengesellschaften, Köln.

Schmidt, R. (1989). Internationalisierungsgrad, in: Macharzina, K. und Welge, M. (Hrsg.), Handwörterbuch Export und internationale Unternehmen, Stuttgart, S. 964-973.

Scholl, R. (1989). Internationalisierungsstrategien, in: Macharzina, K. und Welge, M. (Hrsg.), Handwörterbuch Export und internationale Unternehmen, Stuttgart: 983-1001.

Schüle, F. M. (1992). Diversifikation und Unternehmenserfolg: eine Analyse empirischer Forschungsergebnisse, Wiesbaden.

Schwab, J. A. (1997). Begriff und Messung der Globalisierung. Ein Vorschlag zur Integration von Intensitäts- und Streuungskonzepten in der empirischen Globalisierungsanalyse, Mainz.

Steger, U. und Kummer, C. (1996). Einleitende Zusammenfassung: Globalisierung verstehen und gestalten, in: Steger, U. (Hrsg.), Globalisierung der Wirtschaft: Konsequenzen für Arbeit, Technik und Umwelt, Berlin: 3-17.

Stein, I. (1992). Motive für internationale Unternehmensakquisitionen, Wiesbaden.

Steinmann, H. (1989). Internationalisierung der mittelständischen Unternehmungen, in: Macharzina, K. und Welge, M. (Hrsg.), Handwörterbuch Export und internationale Unternehmen, Stuttgart, S. 1508-1520.

Sullivan, D. (1994). Measuring the Degree of Internationalization of a Firm, in: Journal of International Business Studies, 25(1994): 325-342.

Swoboda, B. (2002). Dynamische Prozesse der Internationalisierung. Managementtheoretische und empirische Perspektiven des unternehmerischen Wandels, Wiesbaden.

Van Koolwijk, J. und Wieken-Mayser, M. (1976). Techniken empirischer Sozialforschung, München.

Yip, G. S. (1992). Total Global Strategy. Managing for Worldwide Competitive Advantage, Englewood Cliffs. 
Im Folgenden werden die einzelnen Themenblöcke der Dissertation zusammenfassend vorgestellt und thematisch voneinander abgegrenzt.

\section{KAPITEL I}

\section{Der Weltbiermarkt: Globalisierung einer Branche}

Kapitel I gibt einleitend einen Eindruck von der Bedeutung des Phänomens der Globalisierung innerhalb der Braubranche anhand von jüngeren Entwicklungen der bedeutendsten regionalen Weltbiermärkte. Es wird dabei stets auf spezifische Entwicklungen und Tendenzen in den jeweiligen Märkten eingegangen. Dieses erste Kapitel erlaubt somit einen Einblick in verschiedenste Einzelmärkte der globalen Brauindustrie und vermittelt damit einen Überblick über die zu analysierende Branche und deren führende Unternehmen. Das erste Unterkapitel I.1 führt dabei in die zu behandelnde Thematik ein und stellt verschiedene Trends (Konsum, Weltbierproduktion, größte Einzelmärkte, Marktwachstum, Konzentration und Konsolidierung, Markenführung, wichtigste Akteure etc.) und Strategien der handelnden Unternehmen der Weltbraubranche dar. Die weiteren Unterkapitel weisen alle eine ähnliche Strukturierung auf und befassen sich mit den wichtigsten Weltbiermärkten: Diese sind Westeuropa, Zentral\& Osteuropa, China, Südamerika und Nordamerika. Kumuliert repräsentieren die betrachteten Regionen etwa 94\% des Gesamtvolumens des Weltbiermarktes. Neben Produktion bezogenen Daten werden in Kapitel I der Dissertation vor allem auch auf wesentliche Profitabilität bezogene Unterschiede weltweiter Biermärkte herausgearbeitet. So gibt es hinsichtlich der EBITDA-Margen, die in verschiedenen Biermärkten erzielt werden können, erhebliche Differenzen. Die Margen reichen dabei von etwa $1 € / \mathrm{hl}$ (China), $4 € / \mathrm{hl}$ (Deutschland), $6 € / \mathrm{hl}$ (Russland) bis zu 26€/hl (Japan), 31€/hl (Kanada) und 32€/hl (Irland, Australien). 


\section{KAPITEL II}

\section{Internationalisierung führender Braukonzerne}

Dass es sich bei der Brauwirtschaft im weltweiten Kontext mittlerweile um eine von multinationalen Unternehmen geprägte Branche handelt, verdeutlichen die zahlreichen M\&ATransaktionen, die in zunehmendem Maße die Konzentration innerhalb der Weltbraubranche bestimmen. Vor allem die führenden europäischen Braukonzerne stellen dabei die Triebfedern zunehmender Konsolidierung der Braubranche dar. Diese so genannte 'peer group' umfasst InBev (Belgien/Brasilien), Heineken (Niederlande), SABMiller (UK/Südafrika), Carlsberg (Dänemark) und Scottish\&Newcastle (UK). Diese Unternehmen sind als die wesentlichen Player und Treiber des Internationalisierungsprozesses der Branche anzusehen und haben in den letzten fünf Jahren etwa 70 Mrd. $€$ in Fusionen und Akquisitionen investiert. Interessant ist ebenfalls das Wachstum - gemessen am Nettoumsatz - der einzelnen Unternehmen im Zeitablauf. Gerade die peer group der europäischen Braukonzerne wuchs im Vergleich zu den übrigen Brauunternehmen mit großem Abstand am stärksten und verzeichnete ein Umsatzwachstum von durchschnittlich 103\% (1998-2005); Hauptanteil daran hatte der nunmehr weltgrößte Braukonzern InBev mit 224\%. Es folgen mit erheblichem Abstand die Braugruppen aus Entwicklungs- und Schwellenländern mit durchschnittlich $42 \%$ sowie die nordamerikanischen Brauer mit 35\% Wachstum im Betrachtungszeitraum. Im Durchschnitt kaum Umsatzwachstum konnten die Braukonzerne aus dem japanisch/pazifischen Raum mit lediglich $2 \%$ verzeichnen. Daran wird wiederum deutlich, dass v. a. die fünf europäischen Braugruppen durch ihr aggressives externes Wachstum der vergangenen Jahre als Haupttreiber hinter der zunehmenden Konsolidierung der Weltbraubranche zu sehen sind.

Inhaltlich stellt Kapitel II eine Überleitung von Kapitel I auf Kapitel III dar. In ersterem werden Entwicklungstendenzen innerhalb der Weltbraubranche anhand verschiedener regionaler Biermärkte veranschaulicht. Kapitel II stellt deshalb einleitend (II.1) das zu behandelnde Untersuchungsobjekt ,internationale Braukonzerne' vor und diskutiert abschließend (II.2) bereits überleitend den Zusammenhang Internationalisierung-Unternehmenserfolg. 


\section{KAPITEL III}

\section{Internationalisierung und Unternehmenserfolg: Diskussion und Operationalisie- rung verschiedener Messkonzepte}

Kapitel III stellt dann den methodischen Schwerpunkt sowie den inhaltlichen Hauptteil der vorliegenden Arbeit dar. In diesem zentralen empirisch-explorativen Abschnitt wird der $\mathrm{Zu}-$ sammenhang zwischen steigender internationaler Geschäftstätigkeit weltweit führender Braukonzerne und dem daraus resultierenden Unternehmenserfolg ergründet. Dies erfolgt mittels zweier zentraler methodischer Vorgehensweisen: Empirischer Bilanzforschung und empirischer Kapitalmarktforschung.

Ein Blick auf wesentliche operative und erfolgswirtschaftliche Kennzahlen der weltweit führenden Braukonzerne verdeutlicht Unterschiede in Größe und finanzieller Performance der Unternehmen. Mit einem Gesamtvolumen von 1,02 Mrd. Hektolitern repräsentieren die insgesamt 18 untersuchten Braukonzerne etwa 66\% der gesamten Weltbierproduktion. Die kumulierte Marktkapitalisierung der 18 Brauunternehmen betrug 2004 mehr als 130 Mrd. $€$, der kumulierte Umsatz lag bei über 80 Mrd. $€$, der Gesamt-EBITDA bei knapp 18 Mrd. $€$ und der Gesamt-EBIT bei etwa 12 Mrd. €. Die erfolgswirtschaftlichen Kennzahlen der EBITDAbzw. EBIT-Marge zeigen eine deutliche Schwankungsbreite. Die Werte rangieren zwischen einer fast 40-prozentigen EBITDA-Marge für den kolumbianische Brauer Bavaria und etwa 10\% für das japanische Unternehmen Sapporo. Weiterhin wurde die event study-Methodik zur Analyse von insgesamt 34 Fusionen und Akquisitionen in der Weltbraubranche angewandt. Für diese 34 M\&As ergaben sich im Ereigniszeitraum von 21 Tagen im Durchschnitt negative kumulierte Überrenditen von $-0,50 \%$ und positive abnormale Renditen von $0,48 \%$ am Ankündigungstag. Diese Ergebnisse entsprechen den Befunden der meisten event studies.

Einleitend befassen sich die ersten drei Unterkapitel mit Facetten und Fragestellungen hinsichtlich Profitabilität weltweiter Biermärkte und Unterschieden hinsichtlich finanzieller Performance der agierenden Brauunternehmen. Die Kapitel III.4 bis III.7 stellen dann den Kern der vorliegenden Dissertation dar und diskutieren anhand einer event study (III.4) und bilanzieller Untersuchungen (III.5 und III.6) den Zusammenhang InternationalisierungUnternehmenserfolg. Das abschließende Unterkapitel III.7 vergleicht - in der Internationalisierungsprozessforschung bisher erstmalig - Bilanz orientierte und Kapitalmarkt orientierte Herangehensweisen anhand eines Datensamples der größten nordamerikanischen und europäischen Braukonzerne. 


\section{KAPITEL IV}

\section{Exkurs: Internationalisierung anderer Subsektoren des Agribusiness}

Viele genossenschaftliche Unternehmen der Agrar- und Ernährungswirtschaft sind in den letzten Jahren aus verschiedenen Gründen - geringes Marktwachstum, Wandel der Verbrauchergewohnheiten, Konzentration im Handel usw. - unter verstärkten wirtschaftlichen Druck geraten. Im letzten Kapitel dieser Arbeit werden deshalb der genossenschaftliche Schlachtund v. a. der Molkereisektor als größte Subsektoren der deutschen Ernährungsindustrie betrachtet. Bemerkenswert ist dabei, dass es hinsichtlich der Entwicklung deutscher Unternehmen im Vergleich mit den europäischen Wettbewerbern deutliche Parallelen zur Brauindustrie gibt. Wie auch in der deutschen Brauwirtschaft herrschen in der Schlacht- und Molkereibranche relativ kleine Betriebsgrößen und verhältnismäßig schwach konsolidierte Branchenstrukturen vor. Internationales Engagement erfolgt bisher fast ausschließlich über die Form direkten oder indirekten Exports. Genossenschaften aus anderen EU-Staaten haben dagegen ihre Aktivitäten bereits sehr viel stärker internationalisiert und sich insofern einen großen Vorsprung vor ihren deutschen Konkurrenten erarbeiten können.

Kapitel IV zeigt an ausgewählten Beispielen, wie groß die Unterschiede im Bereich der Internationalisierung (IV.1) aber auch hinsichtlich wesentlicher unternehmerischer Kennzahlen (IV.2 und IV.3) zwischen deutschen und ausländischen Schlacht- und Molkereigenossenschaften aktuell sind und diskutiert mögliche Ursachen dieser Entwicklung. Zusammenfassend ist festzuhalten, dass die deutschen Genossenschaften in der Vergangenheit nur bedingt in der Lage waren, dem internationalen Wettbewerb standzuhalten und im Zuge der Globalisierung der Märkte eine aktive Rolle zu spielen. Wogegen die deutsche Schlacht- und Zerlegebranche inzwischen zu etwa 50\% von ausländischen Konzernen kontrolliert wird, gibt es in der genossenschaftlichen Molkereiwirtschaft durchaus noch Hoffnung auf die Entwicklung schlagkräftiger nationaler Unternehmen, die damit langfristig nicht nur mit den führenden europäischen Genossenschaftsmolkereien, sondern auch mit den internationalen Molkereikonzernen wie Danone, Nestlé und Unilever konkurrieren könnten. 


\section{FAZIT}

\section{Entwicklung und Ausblick einer sich konsolidierenden Branche}

Die vorliegende Analyse zeigt auf, wo in der Weltbraubranche wie viel von welchen Unternehmen erwirtschaftet wird und welche gewaltigen Differenzen es nach wie vor hinsichtlich der Ertragslage unterschiedlicher Braukonzerne in verschiedenen Teilmärkten gibt. Es scheint klar, dass weitere Konzentrationsprozesse in vielen heute noch unprofitablen Märkten unumgänglich sein werden. Methodisch konnte die Erkenntnis belegt werden, dass Bilanz orientierte und Kapitalmarkt orientierte Bewertungen steigender Internationalisierung nicht konkurrierend sondern als komplementäre Herangehensweisen verwandt werden sollten.

Das abschließende Fazit gibt Ausblicke auf mögliche künftige Entwicklungen innerhalb der Weltbraubranche und deren Hauptakteuren. Abzuwarten bleibt, wann es erneut zu einer Fusion zwischen den ,Big Playern' in der Branche kommt. Spekuliert wird bspw. seit langem über eine mögliche Fusion von SABMiller mit InBev, Heineken mit Anheuser-Busch oder auch eine Allianz von Carlsberg mit Scottish\&Newcastle. Auch könnte es zu einer Fusion oder strategischen Allianz mit den großen Spirituosen- und Getränkekonzernen kommen. Fest scheint zu stehen, dass sich die Konzentrationswelle innerhalb der Weltbraubranche in der näheren Zukunft wohl mit unverminderter Geschwindigkeit fortsetzen wird.

Limitationen, Erweiterungen und Implikationen für die weitere Forschung auf dem Gebiet des Internationalen Managements im Speziellen und der empirischen Bilanz- und Kapitalmarktforschung im Agri-Food-Business im Besonderen schließen die vorliegende Arbeit. 
KAPITEL I

DER WELTBIERMARKT: GLOBALISIERUNG EINER BRANCHE 


\section{KAPITEL I.1}

\section{Globalization of the Brewing Industry \\ Trends, Perspectives and Strategies}

\section{Oliver Ebneth and Ludwig Theuvsen}

This paper has been presented at the $V^{\text {th }}$ International PENSA Conference, $27^{\text {th }}-30^{\text {th }}$ of July 2005, Ribeirão Preto (Brazil).

Published in a shorter version in:

Brewing and Beverage Industry International 2(2005): 10-15. 


\title{
Globalization of the Brewing Industry Trends, Perspectives and Strategies
}

\begin{abstract}
Despite its long heritage as a local industry, the brewing sector is becoming a global market governed by cross-border takeovers and company growth through acquisitions. This development has arisen from the fact that larger brewers faced low prospects for volume growth in developed markets, which has led them to seek growth either through acquisition of other brewers or through aggressive participation in developing markets - or both. Thus, consolidation and acquisitions have been the growing trend in recent years as the major brewers, like Heineken and InBev (former Interbrew), pursue growth strategies outside their own backyards.
\end{abstract}

\section{KEYWORDS}

Globalization, Brewing Industry, Trends, Perspectives, Strategies.

\section{INTRODUCTION}

Although globalization is a general trend in many industries, the brewing industry has long lagged behind and remained very fragmented. Globalization in the beer industry has proceeded at a much slower pace than in many related businesses. Still, five years ago management consultants feared that "Even today's most global brewers are relatively small and vulnerable to takeovers, perhaps by other packaged goods companies aiming to extend their food brands or distribution" (Lewis, 2001; Kaplan, 2003). Larger brewers are faced with low prospects for volume growth in developed markets, which leads them to seek growth either through acquisition of other brewers or through aggressive participation in developing markets - or both. Thus, faced with saturated home markets, in recent years pioneer enterprises, like Heineken and InBev, have started internationalizing their activities in order to continue growing and expanding (Benson-Armer et al, 1999; Todd, 2004). This paper strives to illustrate the major developments and trends in the world beer industry over the last decade which have transformed it from the local market it was into a global one. First, a general overview about trends in consumption, production and profitability is given. Then we describe the dis- 
tinct features of certain beer markets around the globe. Not only do we focus on size and trends in the beer markets, but we also pay particular attention to the largest players in the world beer industry. In the conclusion, the consolidation and concentration within the industry are highlighted by analyzing developments and trends among the peer group of world-wide leading brewers.

\section{GLOBAL INDUSTRY OVERVIEW}

\subsection{Consumption}

Generally, global beer consumption appears to be increasing. Since 1989 the trend line (see figure 1) has picked up from just below $2 \%$ per annum to about $2.3 \%$ per annum. This positive trend is being driven mainly by the increasing importance of faster growing emerging markets such as China and Russia (Blythman et al, 2003; Canadean, 2004; Joh.Barth\&Sohn, 2004).

Figure 1: Annual Global Beer Consumption Growth (1989-2004).

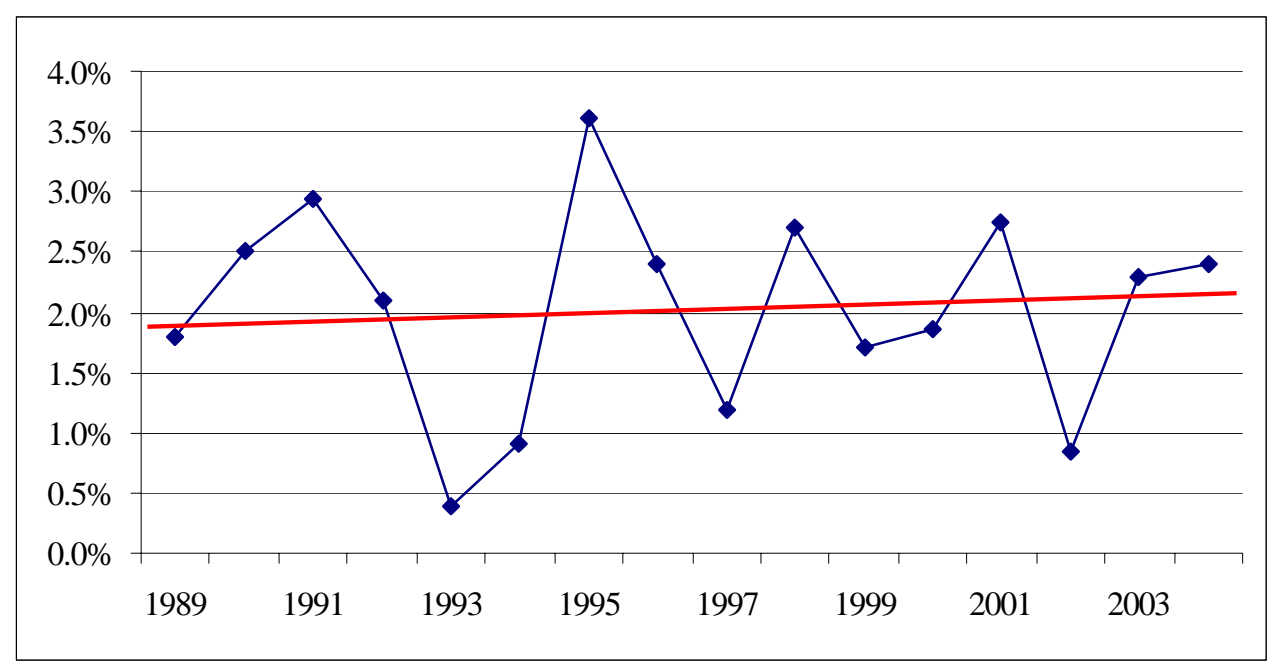

Source: Canadean, Blythman et al, 2004.

The markets with the highest per capita consumption (PCC) as well as the market growth of important and fast growing markets are highlighted in the following figure. Generally, the trend for the leaders in PCC has been downward, which is consistent with the trends in mature markets. Unsurprisingly, the highest growth rates for beer are seen in countries with the lowest per capita consumption. 
Figure 2: Per Capita Consumption and Market Growth.

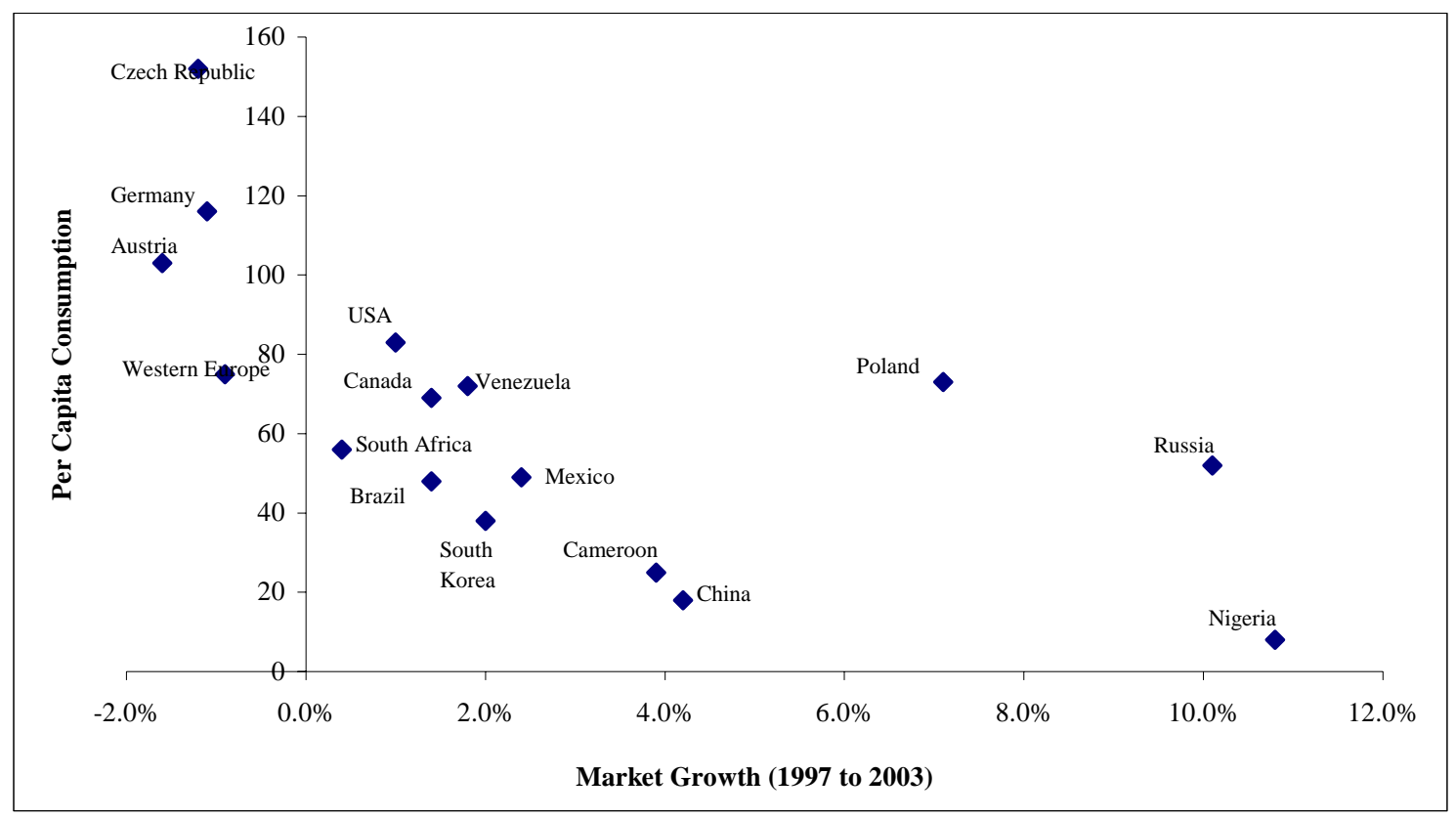

Source: Canadean, Bleakly et al, 2004.

Ongoing volume growth is likely to be seen in the emerging markets because of the headroom in terms of consumption per capita, but more importantly GDP and demographics are both key drivers of beer consumption (Bleakley et al, 2004). Since 1992, Eastern Europe has achieved the highest growth in PCC at $4.2 \%$ p.a., rising from 30 liters to 45 liters in just ten years. Asia-Pacific has also achieved good PCC growth (3.1\% p.a.) but from a much lower base. Africa, North America and Western Europe all showed declines in PCC - the latter two off their very high bases, consistent with their predominantly mature markets.

The following figure breaks the consumption growth down on a regional basis. 
Figure 3: Regional Per Capita Consumption.

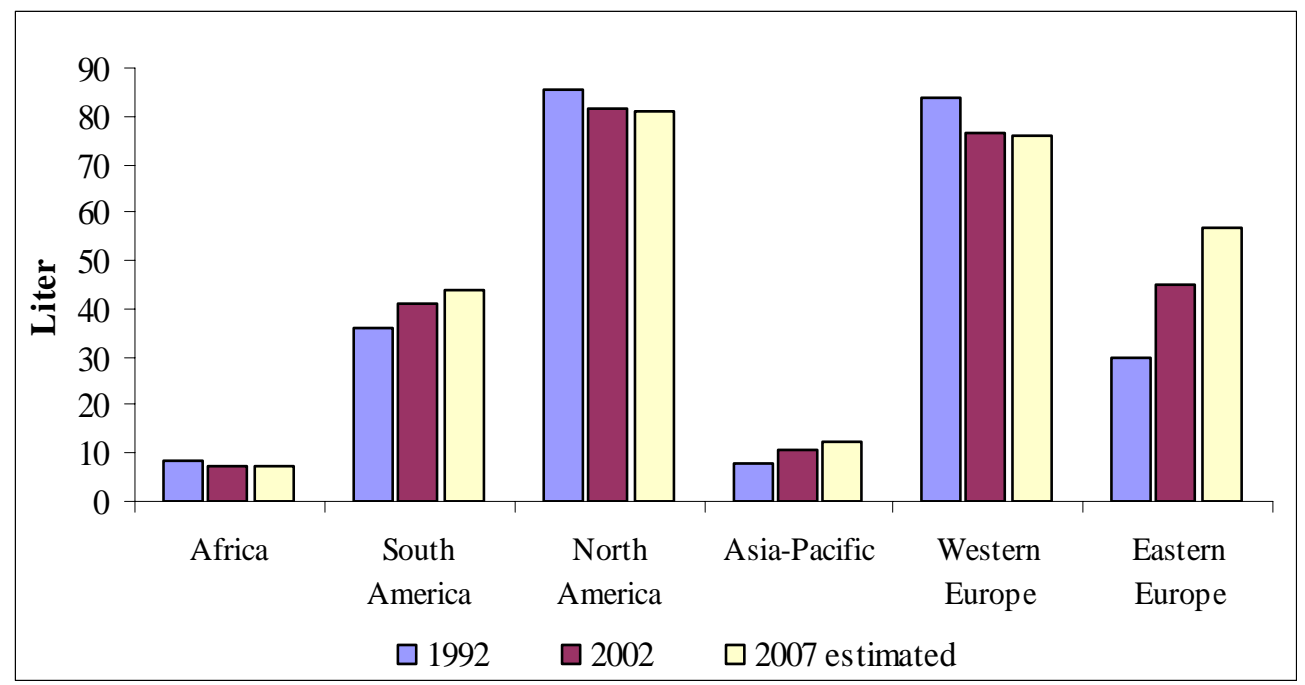

Source: The Barth Report, Canadean, Merrill Lynch estimates.

\subsection{Production}

Over the last 15 years the world beer market has experienced an average annual growth rate of about $2 \%$, reaching a production volume of $1,455 \mathrm{mhl}$ (million hectoliters) (compared to $1,076 \mathrm{mhl}$ in 1988). The following figure shows the growth in world beer production compared to the production growth of the top ten leading brewers. In the decade from 1988 to 1998 the Cr10 rose moderately from $32.4 \%$ to $37.6 \%$, but over the last five years this development has accelerated strongly. The Cr10 increased by $20 \%$ and is now $57.6 \%$. This reflects the above-average growth of the ten largest brewing companies over the past five years.

Figure 4: World Beer Production - Top Ten Brewers.

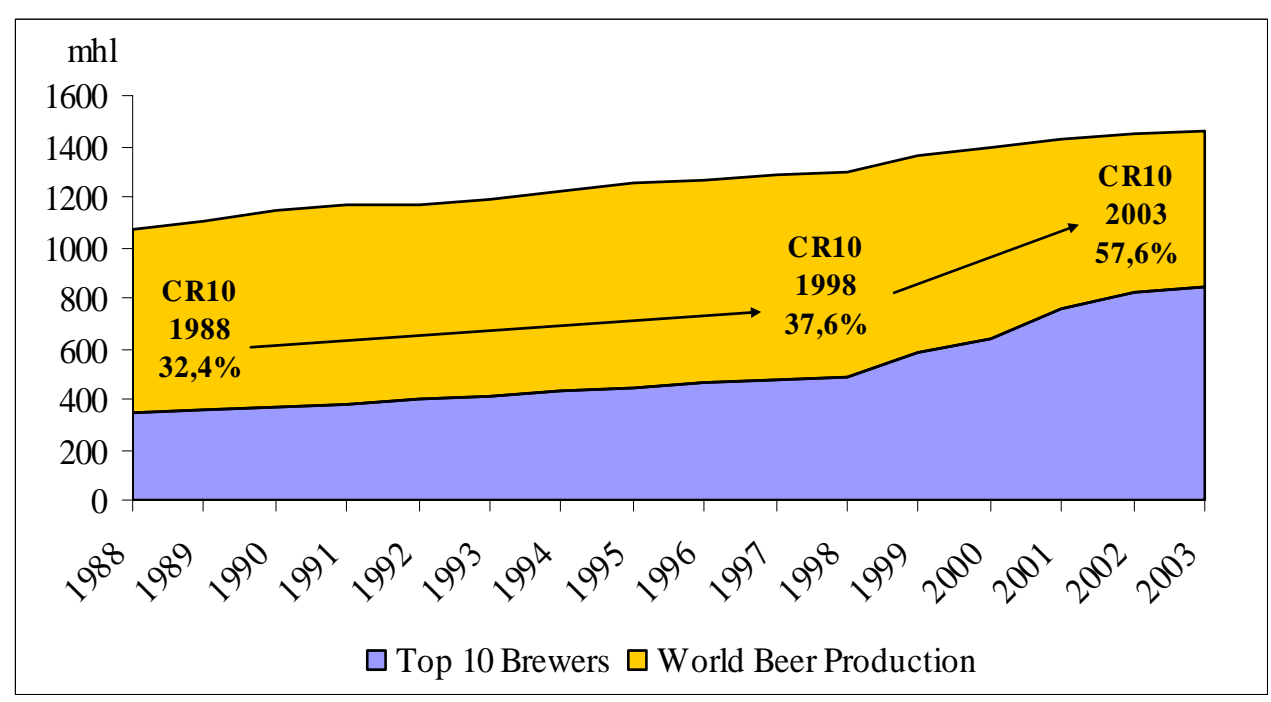




\subsection{Profitability}

Another interesting question regarding the world beer market is profitability. The most lucrative markets in the world are shown in the following table, ranked by EBITA.

Table 1: Top Ten Most Profitable Beer Markets - 2003.

\begin{tabular}{lrrrrrr}
\hline Country & $\begin{array}{r}\text { Volume } \\
(\mathbf{m h l})\end{array}$ & $\begin{array}{r}\text { Sales } \\
\text { (USDm) }\end{array}$ & $\begin{array}{r}\text { Sales/hl } \\
\text { (USD) }\end{array}$ & $\begin{array}{r}\text { EBITA } \\
\text { (USDm) }\end{array}$ & $\begin{array}{r}\text { EBITA/hl } \\
\text { (USDm) }\end{array}$ & $\begin{array}{r}\text { EBITA } \\
\text { Margin \% }\end{array}$ \\
\hline USA & 241 & 25347 & 105 & 3194 & 13 & $12.6 \%$ \\
UK & 59 & 9757 & 165 & 937 & 16 & $9.6 \%$ \\
Brazil & 81 & 2583 & 32 & 594 & 7 & $23.0 \%$ \\
Germany & 98 & 9218 & 94 & 593 & 6 & $6.4 \%$ \\
Canada & 21 & 3808 & 178 & 586 & 27 & $15.4 \%$ \\
Japan & 66 & 8750 & 132 & 542 & 8 & $6.2 \%$ \\
Mexico & 52 & 3998 & 76 & 524 & 10 & $13.1 \%$ \\
Australia & 17 & 3144 & 183 & 503 & 29 & $16.0 \%$ \\
Spain & 32 & 3796 & 120 & 444 & 14 & $11.7 \%$ \\
Russia & 75 & 2980 & 40 & 432 & 6 & $14.5 \%$ \\
\hline Top 10 & $\mathbf{7 4 3}$ & $\mathbf{7 3 3 8 1}$ & $\mathbf{9 9}$ & $\mathbf{8 3 5 0}$ & $\mathbf{1 1}$ & $\mathbf{1 1 . 4 \%}$ \\
\hline Share & $51.5 \%$ & $68.5 \%$ & & $68.8 \%$ & & $\varnothing$ \\
\hline World & $\mathbf{1 4 4 4}$ & $\mathbf{1 0 7 1 5 8}$ & $\varnothing 74$ & $\mathbf{1 2 1 2 4}$ & $\varnothing 8$ & $\varnothing 11.3 \%$ \\
\hline
\end{tabular}

Source: The Barth Report, Winston et al, 2004.

The US is clearly the most significant sales and profit pool in the global beer industry, generating about $24 \%$ of global sales and $27 \%$ of the global EBITA pool. The UK is the second largest profit pool, followed by the declining Japanese market, Brazil, Germany and Canada. Overall, these top ten markets generated $52 \%$ of global volumes, $69 \%$ of global revenues and $69 \%$ of global profits at $\$ 99 / \mathrm{hl}$ revenues and $11.4 \%$ EBITA margin. This means that the rest of the world generated revenues of $\$ 48 / \mathrm{hl}$ at a margin of $11.2 \%$.

\subsection{Consolidation and Competition Issues}

Over the last few years the consolidation process has clearly started to take off, as figure 4 illustrated. Just recently the industry has seen two big deals, again enhancing the concentration significantly. Belgian Interbrew and Brazilian AmBev announced their merger creating the world's leading brewing group, InBev, with a global market share of $14 \%$. The merger between Canadian Molson and US Coors created a new number seven in the world marketplace and represents another regional roll-up (Bevan and Greenberg, 2004; Koster, 2004).

Generally, the more highly concentrated a market, the more profitable it is. Figure 5 illus- 
trates the relationship between the Hirschmann-Herfindahl-Index (HHI) 1 and the EBITA2 margins. It shows two things: firstly, that there seems to be a correlation between concentration and profitability, and, secondly, those under-consolidated markets where future opportunities to consolidate might exist amongst the biggest beer markets in the world.

\section{Figure 5: HHI versus EBITA Margin.}

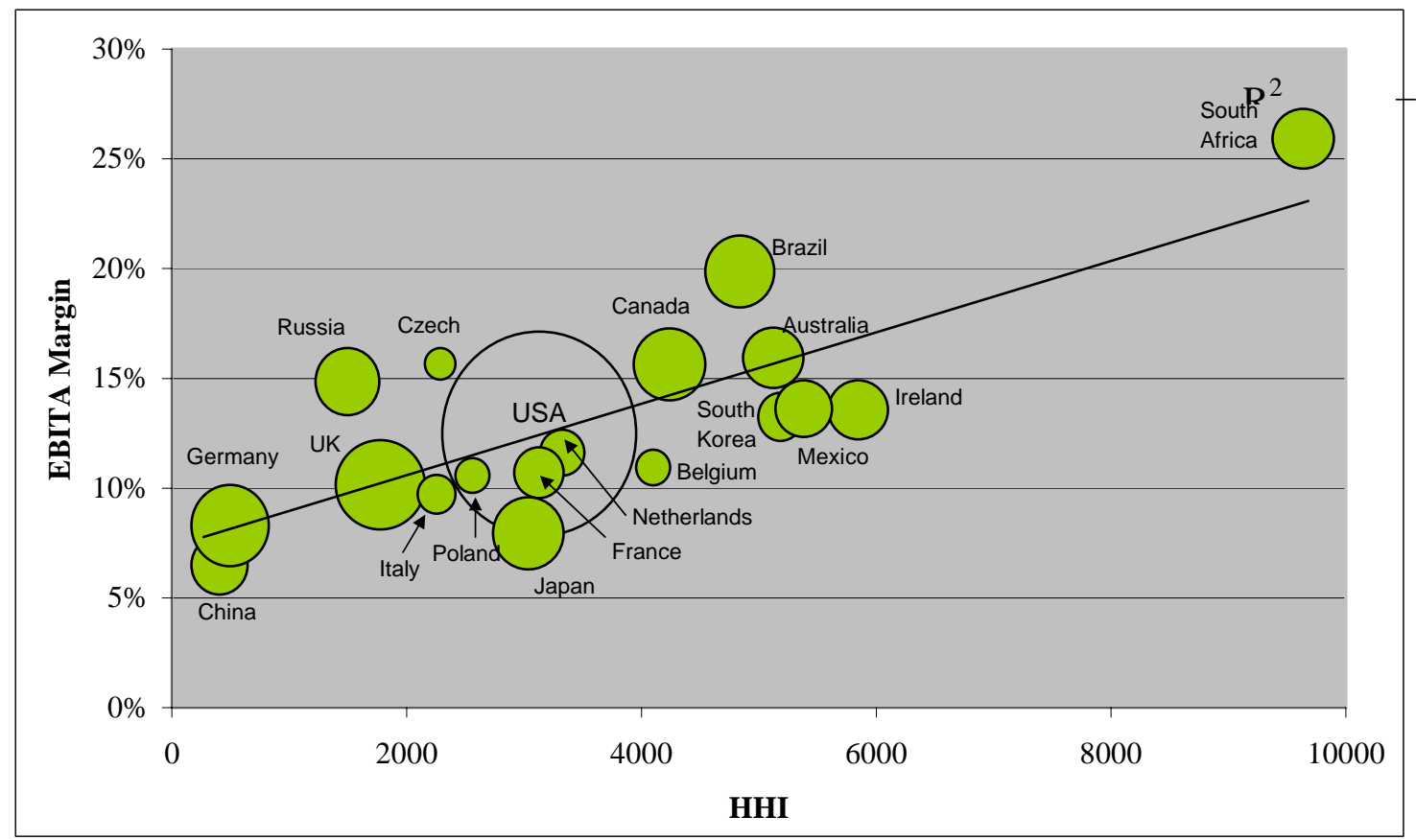

Source: Company data, Canadean, Merrill Lynch.

This figure builds up a fairly accurate picture of the sales and profit pools within the world brewing industry in 2003. The correlation between the HHI and the EBITA margin of a country market is $82 \%$. This is using a sample of the world's twenty largest brewing profit pools, which collectively account for $88 \%$ of the global EBITA pool (Winston et al, 2004).

So, what markets does this analysis suggest are ripe for further consolidation? The markets with the lowest HHI scores are China and Germany, followed by Russia, the UK, Italy, Spain and Poland. At the other end of the scale is South Africa, where SABMiller has a $98 \%$ market share and an EBITA margin of $25 \%$. But in recent months there have been some major deals

${ }^{1}$ The HHI is a specific measurement of market concentration, that is, of the extent to which a small number of firms account for a large proportion of output. The HHI is used as one possible indicator of market power or competition among firms. It measures market concentration by adding the squares of the market shares of all firms in the industry (Buntinas and Funk, 2005).

${ }^{2}$ EBITA = Earnings Before Interest, Taxes and Amortization. 
in Germany and China, and further acquisitions in these markets are very likely to proceed in the future.

\section{Regional ANALysis, OpPORTUNities AND TRAding TrENDS}

Breaking down the global consumption trends into regions highlights some strong disparities. The next figure illustrates historical growth by region over the last decade.

Figure 6: Percentage of the World Beer Production by Region (1994-2004).

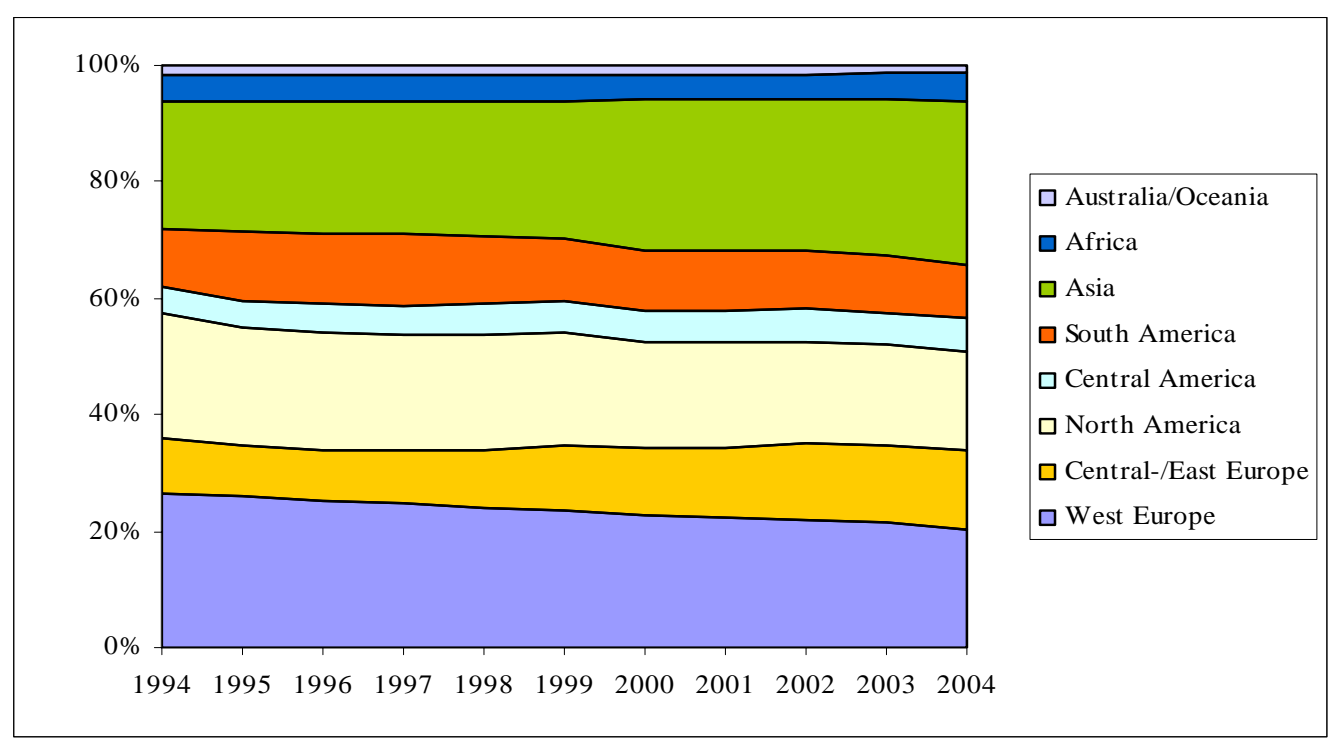

Source: The Barth Report.

Within the 2\% annual global growth from 1994 to 2004, Asia-Pacific was the fastest growing region at $4.5 \%$ p.a., whilst Western Europe was the worst performer, declining at 0.6\% p.a. Herewith, Asia-Pacific contributed 55\% of the additional $275 \mathrm{mhl}$ of beer consumption, followed by Eastern Europe at $32 \%$ and Central/South America at 16\%. Therefore, these trends indicate the growing importance of emerging markets to fuel global and company volume growth. Breaking down the regional development to single markets, the following figure gives an overview of the leading volume drivers.Germany and Japan, as the two most rapidly declining markets, are also included. The top ten markets grew at $2.6 \%$ p.a. compared to a global growth of 2\% (Blythman et al, 2003). These markets contributed 96\% to the incremental global growth since 1992, with China contributing 50\%, Russia 14\% and Brazil 10\%. This can be seen in figure 7 , which shows the ten markets with the highest growth in beer consumption over the past eleven years. 
Figure 7: Cumulative Growth in the Beer Consumption (1992-2003).

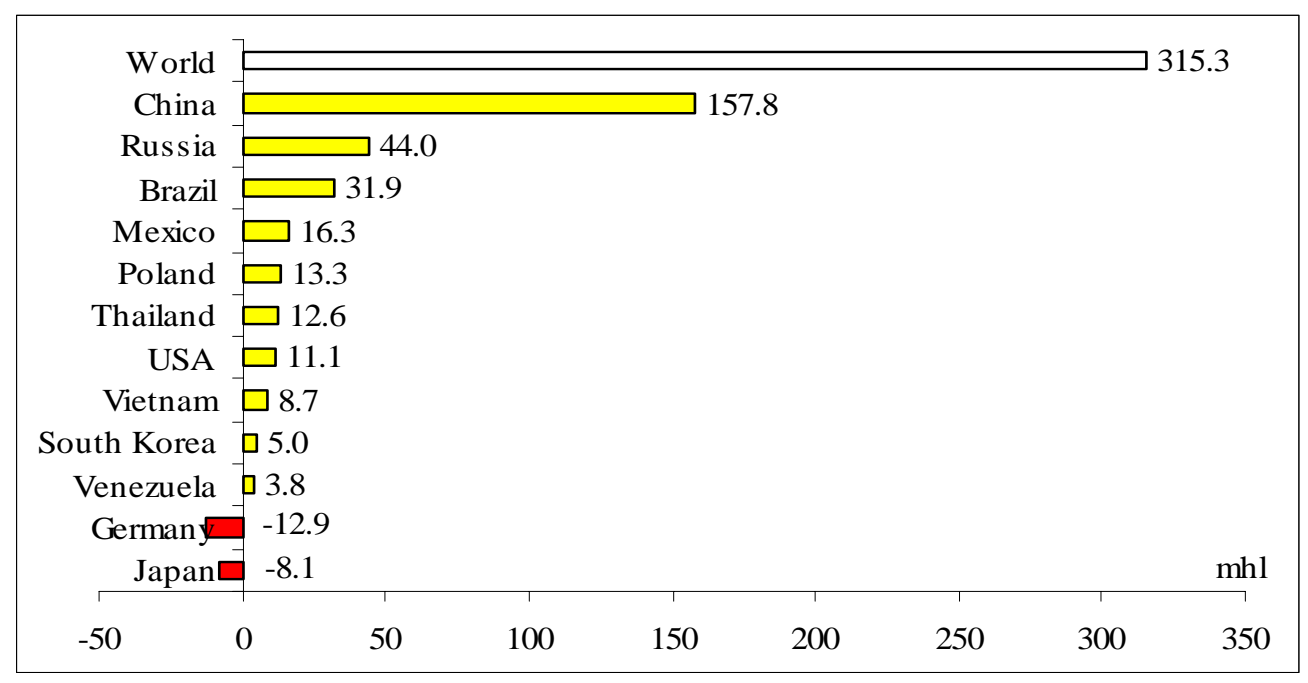

Source: Canadean, The Barth Report.

Emerging beer markets are growing at higher rates than more developed markets. To our mind this is likely to continue given the headroom that exists in term of per capita consumption and favorable underlying demographics and economic growth (Bleakley et al, 2004).

Figure 8: Growth in Emerging Markets versus Developed Markets.

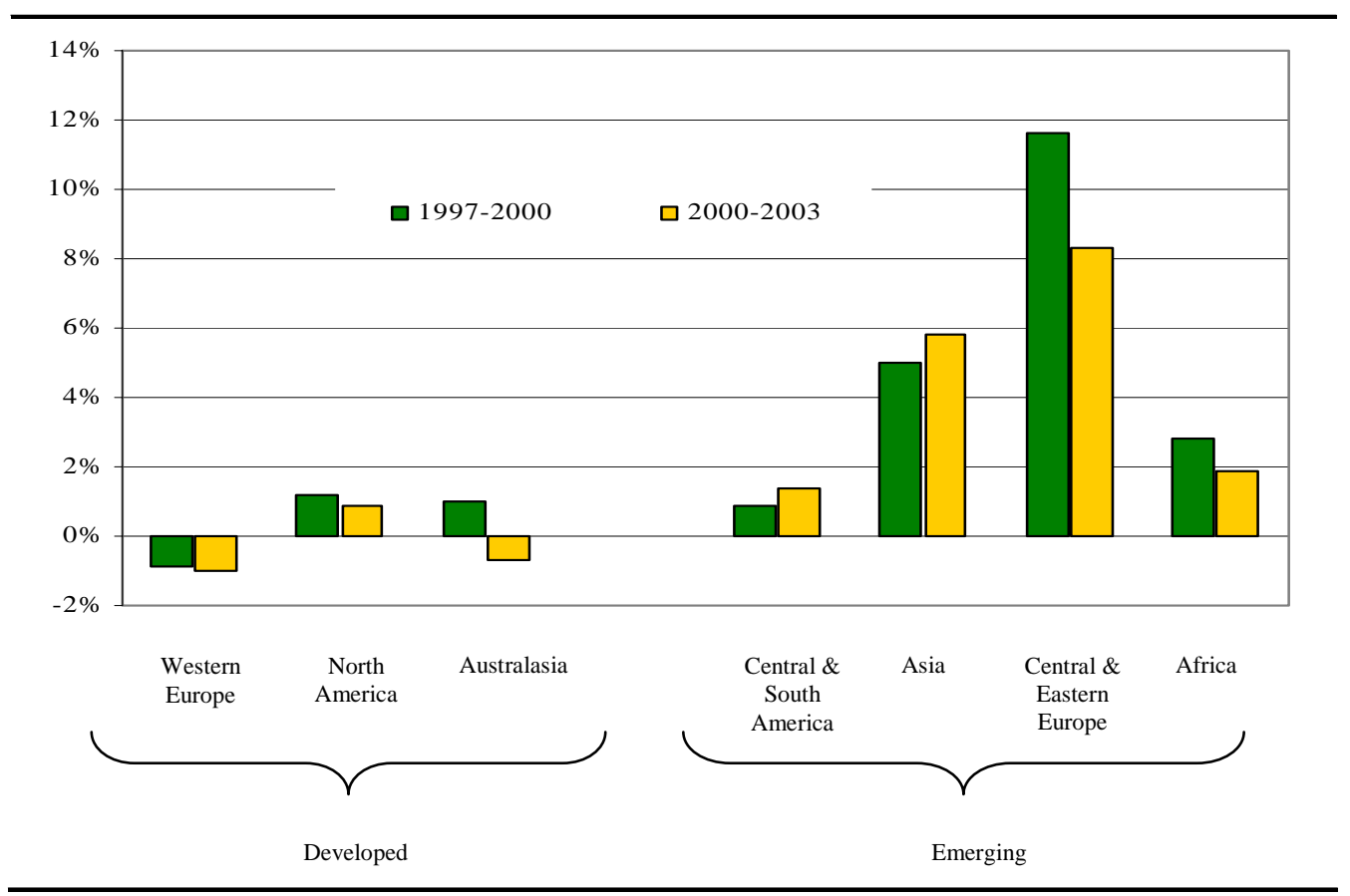

Source: Canadean, Bleakley et al, 2004. 
Emerging markets are seeking strong momentum in terms of beer consumption growth. Developed markets like Europe and Australia are in decline. The US offers some growth prospects. However, the growth being seen in emerging markets offers much more upside. But higher growth rates for the emerging beer markets are founded on these markets coming from much lower rates of per capita consumption (see also figure 1). Indeed, Asia, Latin America and Eastern Europe are all well placed to offer higher levels of growth than is the case for developed markets on these core drivers.

\section{Branding in the Global Brewing Industry}

Apart from gaining market share and expanding in emerging markets there is another step that can be taken to enhance brewers' global competitiveness and allow them to grab further share of the global profit pool: to create a truly international beer brand. When fighting for market share in the brewing industry, strategies centered around branding are of paramount importance. The principle importance of branding is its ability to add value caused to economies of scale in $R \& D$, production, marketing and distribution (Vrontis, 1998). Against the background of internationalizing consumer goods markets, leading branded companies are developing more and more global "mega-brands" due to synergetic effects in marketing spending (Meffert and Bolz, 1998; Jain, 2003).

But, in this regard, the collective success has been limited so far. The world's biggest brewer, InBev, for example, has more than 200 beer brands in its portfolio, which are all competing for a limited Advertising\&Promotion budget. There are signs that some brewers are increasing marketing focus on the core, drive brands, but the industry is still some way from being as focused as Diageo or Coca-Cola are on their priority brands. The only beer brand that really can be seen as an international one is Heineken. The following table highlights the leading ten brands in the world and their growth from 1998 to 2003. 
Table 2: Top 10 Beer Brands.

\begin{tabular}{lrrrrr}
\hline Brand & Owner & $\begin{array}{r}\text { Volume } \\
(\mathbf{m h l}) \mathbf{1 9 9 8}\end{array}$ & $\begin{array}{r}\text { Volume } \\
(\mathbf{m h l}) \mathbf{2 0 0 3}\end{array}$ & $\begin{array}{r}\text { CAGR* } \\
\mathbf{1 9 9 8 - 2 0 0 3}\end{array}$ & $\begin{array}{r}\text { Market } \\
\text { Share 2003 }\end{array}$ \\
\hline Budweiser & Anheuser-Busch & 53.9 & 49.3 & $-1.8 \%$ & $3.4 \%$ \\
Bud (range) & Anheuser-Busch & 33.4 & 46.6 & $\mathbf{6 . 9 \%}$ & $3.2 \%$ \\
Skol & AmBev & 16.0 & 31.2 & $\mathbf{1 4 . 3 \%}$ & $2.1 \%$ \\
Corona & Modelo & 21.1 & 27.9 & $\mathbf{5 . 7 \%}$ & $1.9 \%$ \\
Heineken & Heineken & 19.4 & 22.4 & $2.9 \%$ & $1.5 \%$ \\
Coors Light & Coors & 18.6 & 21.4 & $2.8 \%$ & $1.5 \%$ \\
Super Dry & Asahi & 23.6 & 19.5 & $-3.7 \%$ & $1.3 \%$ \\
Miller Lite & SABMiller & 19.2 & 19.0 & $-0.2 \%$ & $1.3 \%$ \\
Brahma Chopp & AmBev & 22.6 & 16.7 & $-5.9 \%$ & $1.1 \%$ \\
Busch (range) & Anheuser-Busch & 16.9 & 16.0 & $-1.1 \%$ & $1.1 \%$ \\
\hline Top 10 & & $\mathbf{2 4 4 . 7}$ & $\mathbf{2 7 0 . 0}$ & $\mathbf{2 . 0 \%}$ & \\
World Volumes & & 1301.1 & 1455.0 & $2.3 \%$ & \\
Top 10 Share & & $\mathbf{1 8 . 8 \%}$ & $\mathbf{1 8 . 6 \%}$ & & \\
\hline
\end{tabular}

*CAGR = Cumulative Average Growth Rate.

Source: Plato World Brewer Factfile, The Barth Report.

Thus the top ten brands in 2003 held just a 19\% market share of global beer volumes. This creates a significant opportunity for the major international brewers - as long as they continue to redirect resources behind their drive, premium international brands and continue to acquire incremental distribution and capacity within new markets, both mature and emerging.

\section{Global Positioning of Leading International Brewers}

\subsection{Market Shares}

So far in this paper we have dealt with developments in the different beer markets around the globe. Now we will turn our attention to some of the trends and developments within the peer group of the world's leading players in the beer industry. During recent years pioneer enterprises like Heineken and InBev, in response to saturated home markets, have started internationalizing their activities in order to continue growing and expanding (Todd, 2004). According to various studies aggregate volume of the world's top 10 brewers (see figure 9) has grown at more than four times the pace of total industry volume since the mid-1990s. 
Figure 9: Top Ten Global Brewers (1998-2003)

Top Global Brewers 1998

Share of Production Volume $(1,301 \mathrm{mhl})$

$37.6 \%$ Market Share

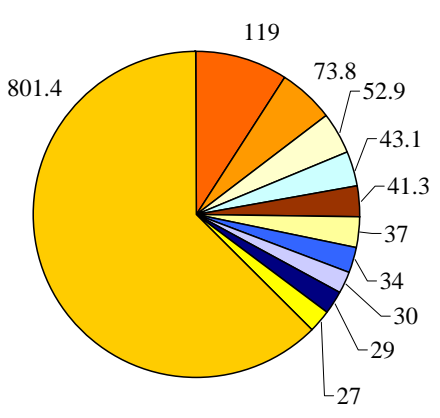

Top Global Brewers 2003

Share of Production Volume $(1,455 \mathrm{mhl})$ $\mathbf{5 7 . 6} \%$ Market Share

$\square$ Anheuser-Busch
$\square$ Heineken
$\square$ Miller
$\square$ SAB
$\square$ Brahma
$\square$ Interbrew
$\square$ Carlsberg
$\square$ Modelo
$\square$ Kirin
$\square$ Foster's
$\square$ Others

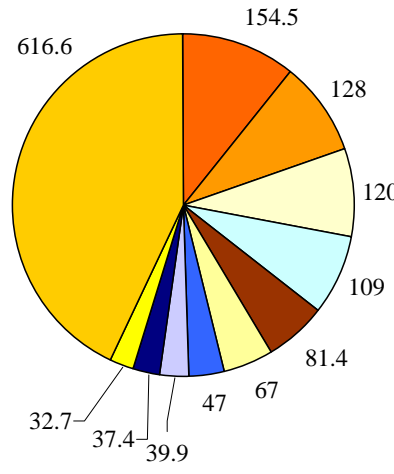

$\square$ Anheuser-Busch
$\square$ SABMiller
$\square$ Interbrew
$\square$ Heineken
$\square$ Carlsberg
$\square$ Ambev
$\square$ Scottish\&Newcastle
$\square$ Grupo Modelo
$\square$ Coors
$\square$ Kirin
$\square$ Others

Source: Company data.

Thus, internationalization in the beer industry is now moving at a much higher speed. Within only a couple of years formerly medium-sized InBev has become the world's number one brewer by aggressively expanding and acquiring foreign competitors.

The following table shows the above-average growth of the five largest brewing companies over the past five years. During the same period of time the Cr5 has risen strongly compared to the preceding five years.

Table 3: Ranking of the Leading Brewers and Concentration Ratio

\begin{tabular}{|c|c|c|c|c|c|c|c|c|c|c|c|}
\hline \multicolumn{6}{|c|}{ Ranking of the world's biggest brewers } & \multicolumn{6}{|c|}{$\%$ of the world beer production } \\
\hline \multirow{2}{*}{$\begin{array}{l}\text { Rank } \\
2004\end{array}$} & \multirow[t]{2}{*}{ mhl } & \multirow{2}{*}{$\begin{array}{l}\text { Rank } \\
1999\end{array}$} & \multirow[t]{2}{*}{ mhl } & \multirow{2}{*}{$\begin{array}{l}\text { Rank } \\
1994\end{array}$} & \multirow{2}{*}{ mhl } & \multicolumn{2}{|c|}{$\begin{array}{c}2004 \\
\mathbf{1 , 4 7 5} \mathbf{~ m h l}\end{array}$} & \multicolumn{2}{|c|}{$\begin{array}{c}1999 \\
\mathbf{1 , 3 6 5} \mathbf{~ m h l}\end{array}$} & \multicolumn{2}{|c|}{$\begin{array}{c}1994 \\
\mathbf{1 , 2 2 2} \mathbf{~ m h l}\end{array}$} \\
\hline & & & & & & $\%$ & $\mathbf{C r}$ & $\%$ & $\mathbf{C r}$ & $\%$ & $\mathrm{Cr}$ \\
\hline InBev & 162 & $\begin{array}{l}\text { Anheuser- } \\
\text { Busch }\end{array}$ & 123 & $\begin{array}{l}\text { Anheuser- } \\
\text { Busch }\end{array}$ & 104 & 11.0 & $\begin{array}{c}\mathrm{Cr} 1 \\
\mathbf{1 1 . 0}\end{array}$ & 9.0 & $\begin{array}{l}\mathrm{Cr} 1 \\
\mathbf{9 . 0}\end{array}$ & 8.5 & $\begin{array}{l}\mathrm{Cr} 1 \\
\mathbf{8 . 5}\end{array}$ \\
\hline $\begin{array}{l}\text { AnheuserB } \\
\text { usch }\end{array}$ & 155 & Heineken & 91 & Heineken & 61 & 10.5 & $\begin{array}{l}\mathrm{Cr} 2 \\
21.5\end{array}$ & 6.7 & $\begin{array}{l}\mathrm{Cr} 2 \\
\mathbf{1 5 . 7}\end{array}$ & 5.0 & $\begin{array}{l}\mathrm{Cr} 2 \\
13.5\end{array}$ \\
\hline SABMiller & 144 & Interbrew & 56 & Miller & 54 & 9.8 & $\begin{array}{l}\mathrm{Cr} 3 \\
\mathbf{3 1 . 3}\end{array}$ & 4.1 & $\begin{array}{l}\mathrm{Cr} 3 \\
19.8\end{array}$ & 4.4 & $\begin{array}{l}\mathrm{Cr} 3 \\
17.9\end{array}$ \\
\hline Heineken & 122 & Miller & 54 & Kirin & 36 & 8.3 & $\begin{array}{l}\mathrm{Cr} 4 \\
\mathbf{4 0 . 6}\end{array}$ & 4.0 & $\begin{array}{l}\mathrm{Cr} 4 \\
23.8\end{array}$ & 2.9 & $\begin{array}{l}\mathrm{Cr} 4 \\
20.8\end{array}$ \\
\hline Carlsberg & 92 & AmBev & 43 & Foster's & 33 & 6.2 & $\begin{array}{l}\mathrm{Cr} 5 \\
46.8\end{array}$ & 3.2 & $\begin{array}{l}\mathrm{Cr} 5 \\
27\end{array}$ & 2.7 & $\begin{array}{l}\mathrm{Cr} 5 \\
23.5\end{array}$ \\
\hline
\end{tabular}

Source: Data compiled from annual reports. 


\subsection{Domestic versus International}

Interestingly, however, the global market does not show the real picture. The next table breaks down each of the major brewers' volumes into domestic and international. Although Anheuser-Busch was the world's biggest brewer until 2003, only 22\% of its volumes are international. That compares to over $90 \%$ for Carlsberg, InBev and Heineken and over $80 \%$ for SABMiller (Bleakley et al, 2004).

Table 4: Top Ten Brewers - Domestic versus International Volumes - 2003.

\begin{tabular}{lrrrrr}
\hline Brewer & Total (mhl) & $\begin{array}{r}\text { Domestic } \\
(\mathbf{m h l})\end{array}$ & $\begin{array}{r}\text { Domestic } \\
\text { Share }\end{array}$ & $\begin{array}{r}\text { International } \\
(\mathbf{m h l})\end{array}$ & $\begin{array}{r}\text { International } \\
\text { Share }\end{array}$ \\
\hline Anheuser-Busch & 154.5 & 123.3 & $79.8 \%$ & 31.2 & $20.2 \%$ \\
SABMiller & 128.0 & 22.0 & $17.2 \%$ & 106.0 & $\mathbf{8 2 . 8 \%}$ \\
Interbrew & 120.0 & 5.7 & $4.8 \%$ & 114.3 & $\mathbf{9 5 . 3 \%}$ \\
Heineken & 109.0 & 6.6 & $6.1 \%$ & 102.4 & $\mathbf{9 3 . 9 \%}$ \\
Carlsberg & 81.4 & 3.8 & $4.7 \%$ & 77.6 & $\mathbf{9 5 . 3 \%}$ \\
AmBev & 67.0 & 58.7 & $87.6 \%$ & 8.3 & $12.4 \%$ \\
Scottisch\&Newcastle & 48.2 & 15.0 & $31.1 \%$ & 33.2 & $\mathbf{6 8 . 9 \%}$ \\
Grupo Modelo & 39.9 & 29.5 & $73.9 \%$ & 10.4 & $26.1 \%$ \\
Coors & 37.4 & 26.1 & $69.8 \%$ & 11.3 & $30.2 \%$ \\
Kirin & 32.7 & 20.4 & $62.4 \%$ & 12.3 & $37.6 \%$ \\
\hline Total Top 10 & 818.1 & 311.1 & $\varnothing 38.0 \%$ & 507.0 & $\emptyset 62.0 \%$ \\
\hline
\end{tabular}

Source: Data compiled from annual reports.

As mentioned in section 3 above, growth in world beer production is driven mainly by several emerging markets. Tapping into this emerging market growth is the key to value upside for the brewers. The players most exposed to emerging markets are SABMiller and InBev as seen in figure 10. However, the flip side here is that a higher level of exposure to emerging markets brings risks due to higher political, economic and liquidity problems. 
Figure 10: Developed versus Emerging Market Volumes - 2003.

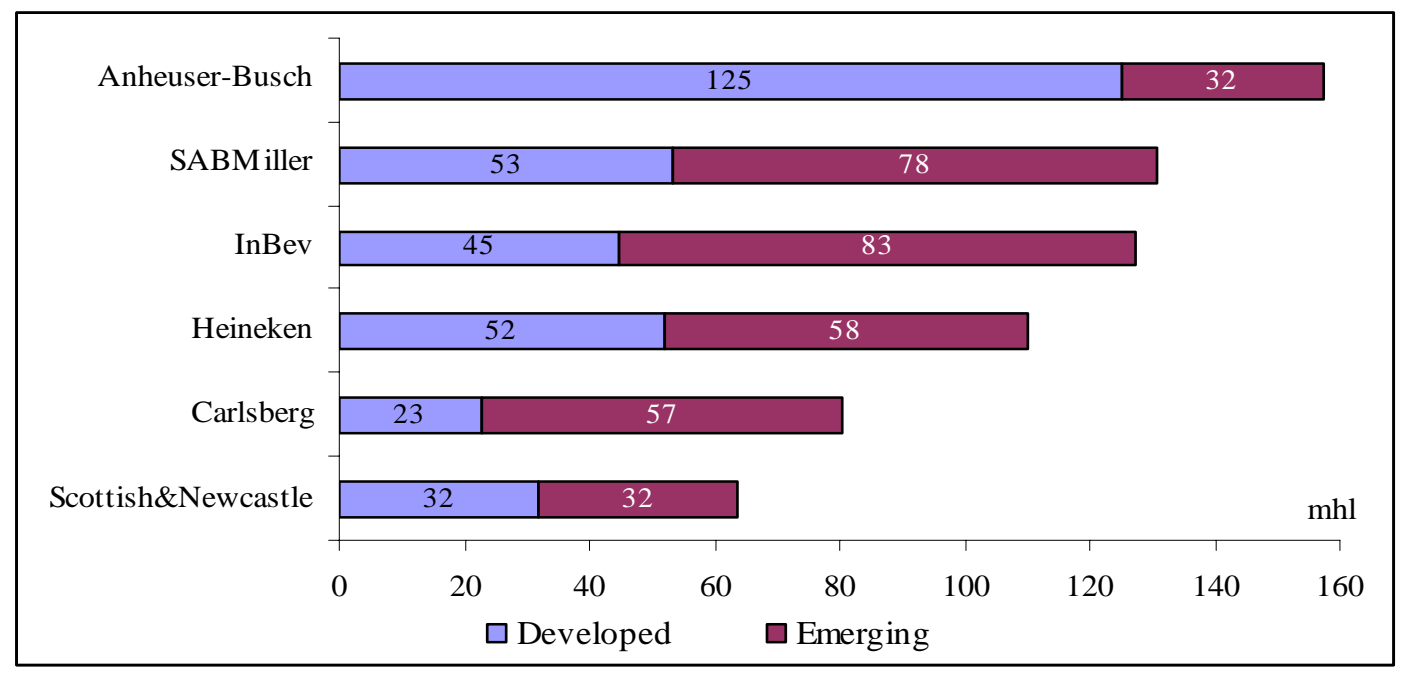

\subsection{Country Positioning}

In this chapter, we can show how well placed the major brewers are to the major profit pools and growth markets within global brewing. The next table highlights the market shares owned by the leading international brewers in the ten largest profit pools in the world (see again table 1). In all but two of the ten largest beer markets, Japan and Australia, the major brewers have a significant presence. In five of those markets the major brewers lead the market and in three of them, they have the number 1 and 2 positions. This creates a significant barrier to entry for other competitors. Only Anheuser-Busch has a higher weighted exposure to these profit pools. InBev now has five top five positions, in the UK, Brazil, Germany, Canada and Russia.

Table 5: Critical Mass Market Positions - Sorted by Profit - USDm.

\begin{tabular}{lrrrrrrr}
\hline Country & $\begin{array}{r}\mathbf{2 0 0 3} \\
\text { EBITA }\end{array}$ & $\begin{array}{r}\text { Anheuser- } \\
\text { Busch }\end{array}$ & SABMiller & InBev & Heineken & Carlsberg & $\begin{array}{r}\text { Scottish\& } \\
\text { Newcastle }\end{array}$ \\
\hline USA & 3194 & $\mathbf{5 0 \%}$ & $19 \%$ & $2 \%$ & $3 \%$ & $0 \%$ & $0 \%$ \\
UK & 937 & $3 \%$ & $0 \%$ & $16 \%$ & $1 \%$ & $14 \%$ & $\mathbf{2 7 \%}$ \\
Brazil & 594 & $0 \%$ & $0 \%$ & $\mathbf{6 6 \%}$ & $0 \%$ & $0 \%$ & $0 \%$ \\
Germany & 593 & $0 \%$ & $0 \%$ & $11 \%$ & $5 \%$ & $6 \%$ & $0 \%$ \\
Canada & 586 & $0 \%$ & $0 \%$ & $\mathbf{4 4 \%}$ & $0 \%$ & $0 \%$ & $0 \%$ \\
Japan & 542 & $0 \%$ & $0 \%$ & $0 \%$ & $0 \%$ & $0 \%$ & $0 \%$ \\
Mexico & 524 & $\mathbf{5 6 \%}$ & $0 \%$ & $0 \%$ & $0 \%$ & $0 \%$ & $0 \%$ \\
Australia & 503 & $0 \%$ & $0 \%$ & $0 \%$ & $0 \%$ & $0 \%$ & $0 \%$ \\
Spain & 444 & $0 \%$ & $3 \%$ & $0 \%$ & $\mathbf{3 3 \%}$ & $0 \%$ & $0 \%$ \\
Russia & 432 & $0 \%$ & $2 \%$ & $14 \%$ & $5 \%$ & $\mathbf{3 3 \%}$ & $\mathbf{3 3 \%}$ \\
\hline Critical Mass & & $\mathbf{2}$ & $\mathbf{0}$ & $\mathbf{2}$ & $\mathbf{1}$ & $\mathbf{1}$ & $\mathbf{2}$ \\
\hline
\end{tabular}

Source: Company data, The Barth Report, Winston et al, 2004.

Furthermore, the major brewers are also exposed to the fastest growing beer markets in the 
world (see table 6). Interestingly, the smallest brewers within this peer group - Carlsberg and S\&N - seem to have the best exposure to the fastest growing major markets in global brewing (Winston et al, 2004). But at closer inspection the markets with critical mass are as yet only Russia, Ukraine and Kazakhstan.

Table 6: Critical Mass Market Positions - Sorted by Volume Growth 2003 to 2008.

\begin{tabular}{lrrrrrrr}
\hline Country & $\begin{array}{r}\text { Volume } \\
\text { CAGR* }\end{array}$ & $\begin{array}{r}\text { Anheuser- } \\
\text { Busch }\end{array}$ & SABMiller & InBev & Heineken & Carlsberg & $\begin{array}{r}\text { Scottish\& } \\
\text { Newcastle }\end{array}$ \\
\hline Vietnam & $9.2 \%$ & $0 \%$ & $0 \%$ & $0 \%$ & $13 \%$ & $9 \%$ & $0 \%$ \\
Ukraine & $6.9 \%$ & $0 \%$ & $0 \%$ & $\mathbf{3 4 \%}$ & $0 \%$ & $\mathbf{2 2 \%}$ & $\mathbf{2 2 \%}$ \\
Russia & $5.6 \%$ & $0 \%$ & $0 \%$ & $14 \%$ & $5 \%$ & $\mathbf{3 3 \%}$ & $\mathbf{3 3 \%}$ \\
Uzbekistan & $5.4 \%$ & $0 \%$ & $0 \%$ & $0 \%$ & $0 \%$ & $0 \%$ & $0 \%$ \\
Kazakhsten & $5.4 \%$ & $0 \%$ & $0 \%$ & $0 \%$ & $8 \%$ & $\mathbf{2 1 \%}$ & $\mathbf{2 1 \%}$ \\
India & $5.0 \%$ & $0 \%$ & $\mathbf{3 5 \%}$ & $0 \%$ & $0 \%$ & $0 \%$ & $\mathbf{3 5 \%}$ \\
Thailand & $4.7 \%$ & $0 \%$ & $0 \%$ & $0 \%$ & $8 \%$ & $0 \%$ & $0 \%$ \\
Romania & $3.9 \%$ & $0 \%$ & $18 \%$ & $16 \%$ & $\mathbf{3 3 \%}$ & $6 \%$ & $0 \%$ \\
China & $3.9 \%$ & $2 \%$ & $9 \%$ & $9 \%$ & $0 \%$ & $0 \%$ & $0 \%$ \\
Nigeria & $3.6 \%$ & $0 \%$ & $0 \%$ & $0 \%$ & $\mathbf{5 2 \%}$ & $0 \%$ & $0 \%$ \\
\hline Critical Mass & & $\mathbf{0}$ & $\mathbf{1}$ & $\mathbf{1}$ & $\mathbf{2}$ & $\mathbf{3}$ & $\mathbf{4}$ \\
\hline
\end{tabular}

Source: Company data, The Barth Report, * Merrill Lynch estimates.

Thus, the major brewers are well positioned amongst the most profitable beer markets and are making inroads into the fastest growing markets, too, allowing them to take a significant portion of the incremental profit pool (Blythman et al, 2003).

It seams to be clear that a balance between emerging market positions for topline growth and developed market positions for profit and cash is best to maximize value in this industry.

\subsection{Major Merger and Acquisitions}

As figure 4 has shown, the concentration ratio amid the brewing industry has grown significantly over the last five years. This is mainly due to cross-border acquisitions, which have been the growing trend in recent years. This phenomenon has several reasons. First, acquisitions give the opportunity to enhance remarkably and quickly a firm's degree of internationalization and its market share through diverse one-off deals (Agrawal et al, 1992; Jensen, 1992; Müller-Stewens, 2000; Müller-Stewens et al, 2002). Second, economies of scale and synergies can be realized earlier through external rather than internal growth. Furthermore it may be possible to increase revenues in certain markets due to lack of competition. This motive assumes that the company will get rid of a major competitor and thus increase its power to set prices. Recent spectacular acquisitions in the brewing industry have highlighted the apparent urgency of the major European brewers to build scale and improve their strategic 
positioning. Apart from Anheuser-Busch, which is hardly internationalized, we consider five brewers as serious contestants in the global arena: Heineken, InBev, SABMiller, Carlsberg and Scottish\&Newcastle (Hoojimaijers, 2003). In the course of the last years, leading brewers and European groups in particular spent more than $€ 53 \mathrm{bn}$ in diverse cross-national M\&A deals. The largest transactions have been the SAB-Miller merger in 2002, the merger between Interbrew and AmBev in March 2004 - creating InBev, the largest brewing group around the globe, and just recently SABMiller's spectacular acquisition of Colombian brewer Bavaria. Figure 11 illustrates some of these high value-scaled cross-border deals recently transacted by the world-wide leading brewing groups.

Figure 11: Transaction Values of Recent Major Acquisitions.

\begin{tabular}{|c|c|c|c|c|}
\hline $\begin{array}{c}\text { Date } \\
\text { Announced }\end{array}$ & Acquiror & Target & $\%$ Acquired & $\begin{array}{r}\text { Transaction } \\
\text { Value (€m) }\end{array}$ \\
\hline 19-Jul-05 & SABMiller & Bavaria & $100 \%$ & 6,473 \\
\hline 18-Jul-05 & InBev & Tinkoff & $100 \%$ & 167 \\
\hline 03-Jan-05 & InBev & SunInterbrew & $100 \%$ & 260 \\
\hline 16-Sep-04 & SABMiller & Lion Nathan China & $100 \%$ & 119 \\
\hline 12-Aug-04 & Interbrew & SunInterbrew & $91 \%$ & 1,212 \\
\hline 22-Jul-04 & Coors & Molson & $50 \%$ & 3,200 \\
\hline 15-Jun-04 & Anheuser-Busch & Harbin & $29 \%$ & 640 \\
\hline 03-Mar-04 & Interbrew & Ambev & $58 \%$ & 16,341 \\
\hline 19-Feb-04 & Carlsberg & Orkla & $40 \%$ & 7,054 \\
\hline 20-Jan-04 & Carlsberg & Holsten & $100 \%$ & 437 \\
\hline 07-Jan-04 & Interbrew & Oriental Brewery & $45 \%$ & 1,547 \\
\hline 18-Sep-03 & Interbrew & Spaten & $100 \%$ & 533 \\
\hline 11-Sep-03 & Interbrew & Apatinska Pivara & $100 \%$ & 262 \\
\hline 08-Sep-03 & Interbrew & Lion & $50 \%$ & 114 \\
\hline 13-Мау-03 & $\mathbf{S} \& \mathbf{N}$ & Centralcer & $51 \%$ & 828 \\
\hline 13-Маy-03 & SABMiller & Peroni & $60 \%$ & 563 \\
\hline 02-May-03 & Heineken & BBAG & $100 \%$ & 1,899 \\
\hline 28-Apr-03 & S\&N & Bulmer & $100 \%$ & 398 \\
\hline 14-Jan-03 & Heineken & $\mathrm{CCU}$ & $31 \%$ & 1,525 \\
\hline 15-Nov-02 & Interbrew & Gilde & $100 \%$ & 575 \\
\hline 12-Sep-02 & Heineken & Al Ahram & $100 \%$ & 273 \\
\hline 05-Jun-02 & Heineken & Karlsberg & $45 \%$ & 135 \\
\hline 30-Мау-02 & SAB & Miller & $100 \%$ & 6,194 \\
\hline 18-Mar-02 & Heineken & Molson Brazil & $20 \%$ & 250 \\
\hline \multirow[t]{2}{*}{ 14-Feb-02 } & S\&N & Hartwall & $100 \%$ & 2,273 \\
\hline & & & & 53,272 \\
\hline
\end{tabular}




\section{OUTLOOK}

In the course of the last decade, the brewing industry has evolved from an industry that concentrated on domestic markets to one that views itself as part of a global market (Karrenbrock, 1990). The consolidation process picked up pace in recent years, and there are some clear signs that this process will continue to gather speed. One reason is that the biggest international brewing groups are well capitalized and all are seeking further external growth. Second, there has never been a more opportune time to try and establish a stronger position in the emerging markets or attempt to find synergistic deals in their home territories (Bevan and Greenberg, 2004). To ensure the appropriate balance between developed and emerging markets (see figure 10) the next priority for both InBev and SABMiller would most likely be to extend their positions in Western Europe or North America. In contrast, there is pressure for Anheuser-Busch and Heineken to extend their emerging market positions. For example, at present Heineken's position in China is weak, and, although the market remains relatively fragmented, there are hardly opportunities left to take a sizeable step forward in a single transaction.

In conclusion, future developments within the beer industry will be displaying many of the characteristics of recent years - with intense consolidation activity being set against the background of relatively demanding trading conditions in Western markets and relatively buoyant trends in emerging ones. We have shown that the peer groups' benefit can only be maximized if the brewers are well positioned to tap into both the largest profit pools, where the impact of mix improvements is that much greater, and have critical mass in the best growth markets. 


\section{REFERENCES}

Agrawal, A. et al (1992). The Post-Merger Performance of Acquiring Firms: A Re-

Examination of an Anomaly, in: The Journal of Finance 47(1992): 1605-1621.

Benson-Armer, R. et al (1999). Global Beer: What's on Tap? The McKinsey Quarterly 1999, No. 1.

Bevan, N. and Greenberg, M. (2004). Global Brewing - The Global Pitcher 2004 - Volume V, Deutsche Bank Report, London.

Bleakley, M. et al (2004). European Brewers - Going for Growth, Credit Suisse First Boston Equity Research.

Blythman, M. et al (2003). Pan European Brewing Review - 2003, Merrill Lynch, London.

Buntinas, M. and Funk, G. M. (2005). Statistics for the Sciences, Belmont.

Canadean (2004). Global Beer Report. Annual Report - Cycle 2004, Basingstoke.

Hoojimaijers, M. (2003). European Breweries. Medium-Sized Brewery Targets in Europe. Julius Baer European Brokerage, Amsterdam.

Jain, S. C. (2003). Handbook of Research in International Marketing, Cheltenham.

Jensen, M. C. (1992). Market for Corporate Control, in: Newman, P. et al. (Eds.), The New Palgrave Dictionary of Money and Finance, London: 657-665.

Joh.Barth\&Sohn (2004). The Barth Report. Hops 2003/04, Nuremberg.

Kaplan, A. (2003). Global Beer: Tapping into Growth, in: Beverage World 122(2003): 24-28.

Karrenbrock, J. D. (1990). The Internationalization of the Beer Brewing Industry, in: Review - Federal Reserve Bank of St. Louis, 72(1990): 3-19.

Koster, P. (2004). Global Beer Industry Report 2004, FBS Bankiers, Amsterdam.

Lewis, C. (2001). The Future of British Brewing: Strategies for Survival, in: Strategic Change, 10(2001): 151-161.

Meffert, H. and Bolz, J. (1998). Internationales Marketing-Management, Third Edition, Stuttgart etc.

Müller-Stewens, G. (2000). Akquisitionen und der Markt für Unternehmenskontrolle: Entwicklungstendenzen und Erfolgsfaktoren, in: Picot, A. (Ed.), Management von Akquisitionen: Akquisitionsplanung und Integrationsmanagement, Stuttgart: 41-61.

Müller-Stewens, G. et al (2002). Stand und Entwicklungstendenzen von Cross-BorderAkquisitionen, in: Krystek, U. and Zur, E. (Eds.) Handbuch Internationalisierung: 141-169.

Todd, H. (2004). Global Beer, in: Beverage World 123(2004): 39-41.

Vrontis, D. (1998). Strategic Assessement: The Importance of Branding in the European Beer Market, in: British Food Journal 100(1998): 76-84.

Winston, C. et al (2004). Brand New World. Marketing and Brands in the Global Beer Industry, Merrill Lynch, London. 


\section{KAPITEL I.2}

\section{Main West European Beer Markets at a Glance}

\section{Oliver Ebneth}

This article will be published in:

Brewing and Beverage Industry International 4(2006). 


\section{Main West European Beer Markets at a Glance}

This article strives to illustrate the major developments and trends in the West European beer industry over recent years. First, a general overview about trends in consumption, production, importing/exporting and M\&A activities is given. Then the distinct features of certain beer markets that play an important role within West Europe are described in detail. Not only do we focus on size and trends in the beer markets, but we also pay particular attention to the largest players in the European beer industry.

\section{Growth Prospects for Further Consumption are Likely to Slow Down}

The Western European beer market totals 317 million hectoliters (mhl) in 2004. Generally, global beer consumption quickened over the last decade but the trend line for West Europe is slightly declining by $0.3 \%$ per annum since the mid-1990s. But this negative trend is being driven mainly by the still dominating but remarkably decreasing German market which is waning volume by $1 \%$ each year. Figure 1 illustrates both the total consumption as well as the per capita consumption in all West European markets.

Figure 1: West European Consumption and Per Capita Consumption.

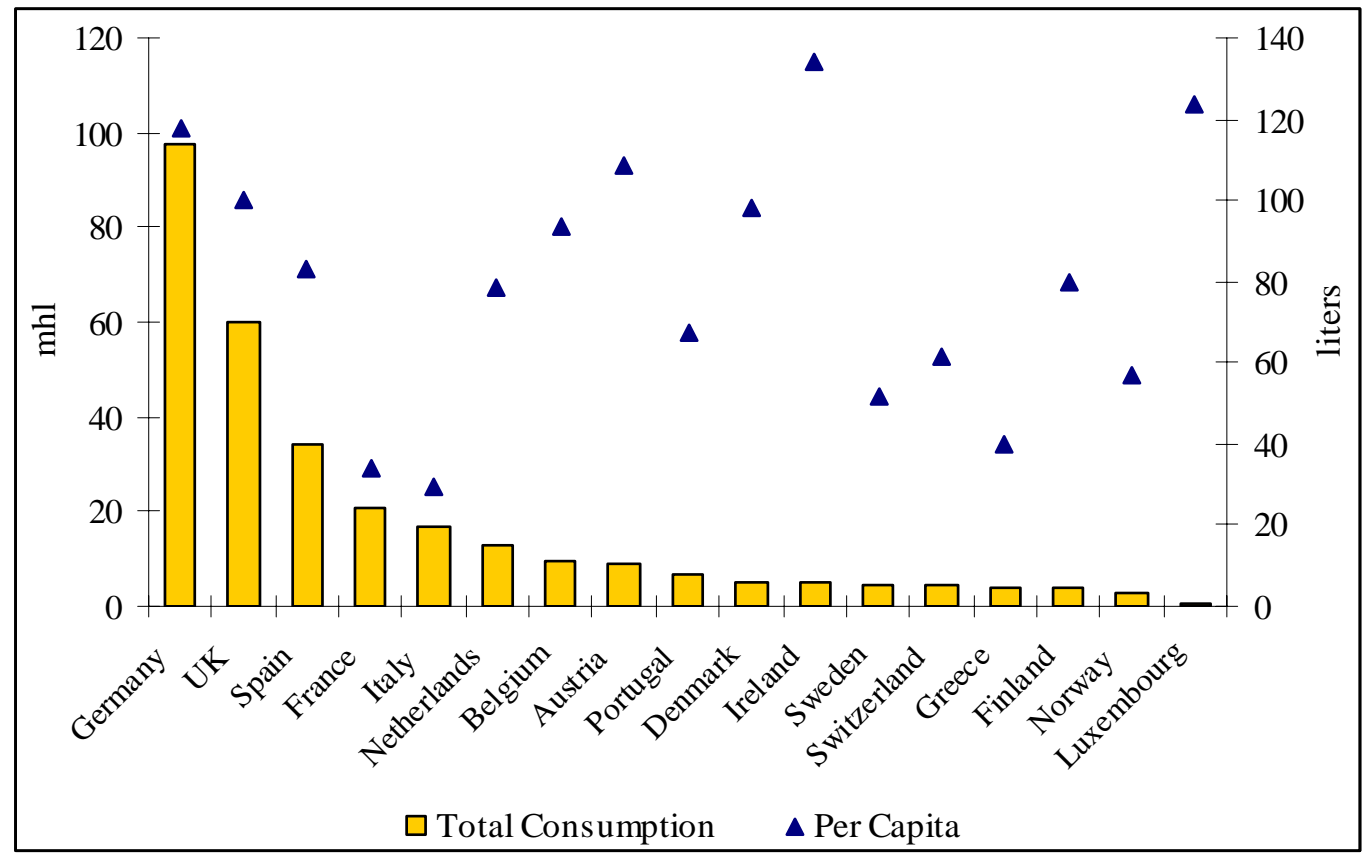

Source: (Canadean, 2004; Joh.Barth\&Sohn, 2005; Plato, 2005). 
Indeed the Western European matured market is stagnating or even shrinking since more than a decade. But still it is well ahead of the rest of the world and accounts for $20 \%$ of the world beer production. The picture in 2004 was again mixed, but in general many markets have shown a correction versus the hot summer driven growth in 2003. Markets have seen more normal conditions and declined by $0.5 \%$. Germany and the UK were both lower and France as well as the Netherlands were sharply down due to less favourable weather. The climate also saw the Italian market fall, although Spain was higher. Scandinavia was mixed, with Finland benefiting from a duty reduction and Norway also higher. Many industry insiders expect to see further short-term fluctuations, but the overall outlook is for continued stability, with a slight downward tendency, mainly influenced by a negative long-term outlook in UK and Germany. This outlook is emphasized by the development in the past, underlined by figure 2 which shows the changing importance of the ten largest beer markets within Western Europe.

Figure 2: Concentration of West European Beer Markets.

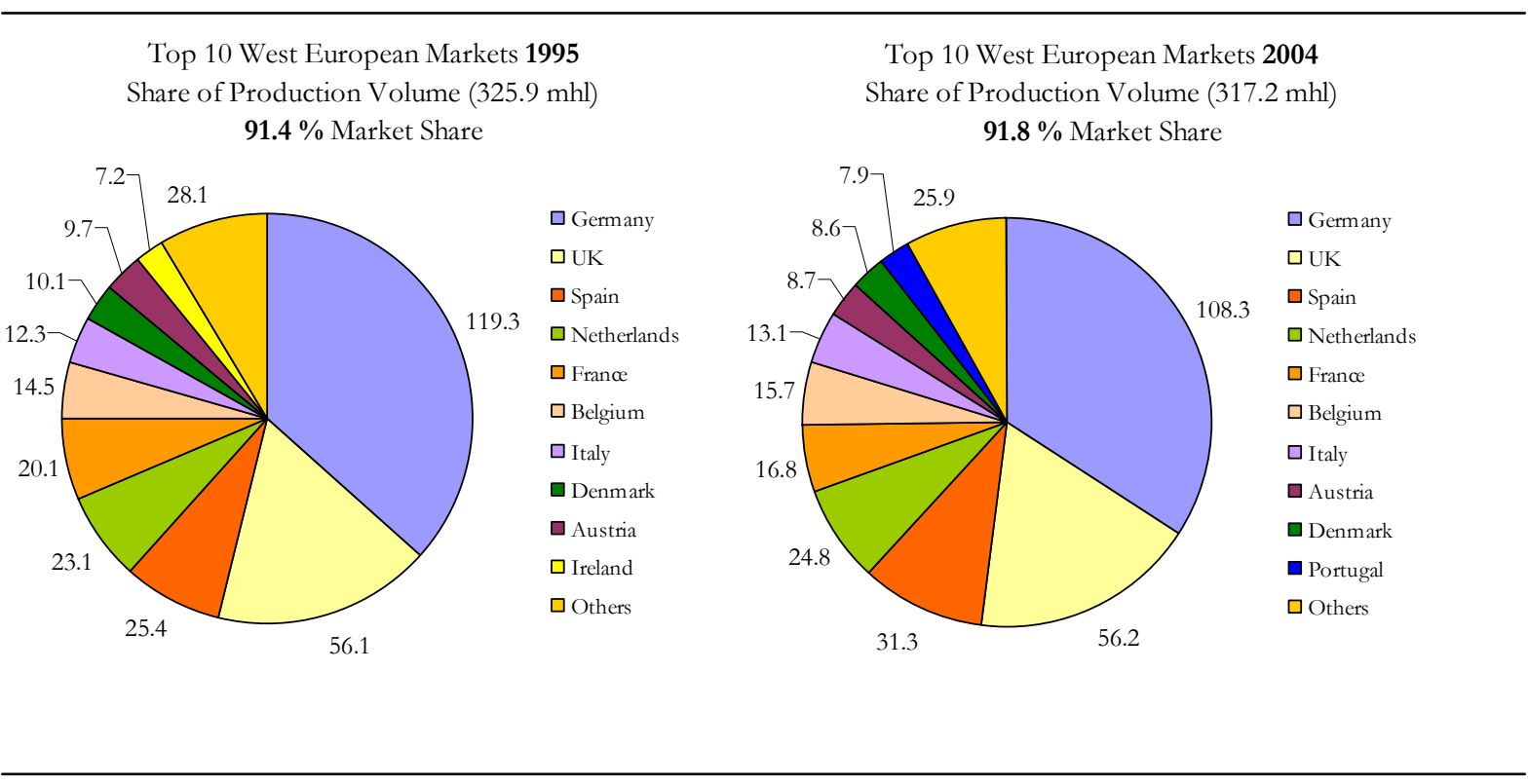

Source: (Plato, 2005).

Despite the continued picture of stagnation, the medium-term outlook for total beer consumption in West Europe is certainly not all bleak. The 1990s were in general a very difficult economic environment, in which beer nevertheless proved comparative resilient in that there was no evidence of sustained decline. Many countries in Southern Europe and Scandinavia still have comparatively low levels of per capita consumption suggesting further growth potential. 
The sustained phase of industry consolidation now seems to be drawing to a close, leaving fitter and more competitive companies dominating many markets.

\section{Patterns of Foreign Trade and M\&A Activities}

Exports reached a new high in 2004, passing $50 \mathrm{mhl}$ (according to Plato Logic Ltd.), or over $16 \%$ of total production. Over $50 \%$ of exports are supplied from Germany and the Netherlands. Recent growth is mainly attributable to inter-company movements and own-lable shipments. Imports reflected this, also reaching a new high of $32 \mathrm{mhl}$, equivalent to almost $11 \%$ of consumption. About $50 \%$ of imports are received by UK, Italy and Spain.

Since the end-1990s in particular, the level of corporate activity has continued to be significant. However while there were major deals announced internationally, 2004 was a comparatively quiet year for M\&A activity within West Europe. West Europe's Big 4 of Heineken, InBev, S\&N and Carlsberg now have a clear lead in the region (see figure 3), with combined volume of $142 \mathrm{mhl}$, being almost $50 \%$ of the market. Heineken remains West Europe's largest brewer, with $44 \mathrm{mhl}$ being 15\% market share. The Heineken brand is also market leader with $11 \mathrm{mhl}$ sales. Mainly as a result of their major investments in the UK and Germany, InBev has risen from $7^{\text {th }}$ place in 1994 to $2^{\text {nd }}$ in 2004. S\&N is now number 3 in West Europe, with total sales of $31 \mathrm{mhl}$ being $11 \%$ market share followed by Carlsberg with $28 \mathrm{mhl}$.

Figure 3: Main Western European Brewers.

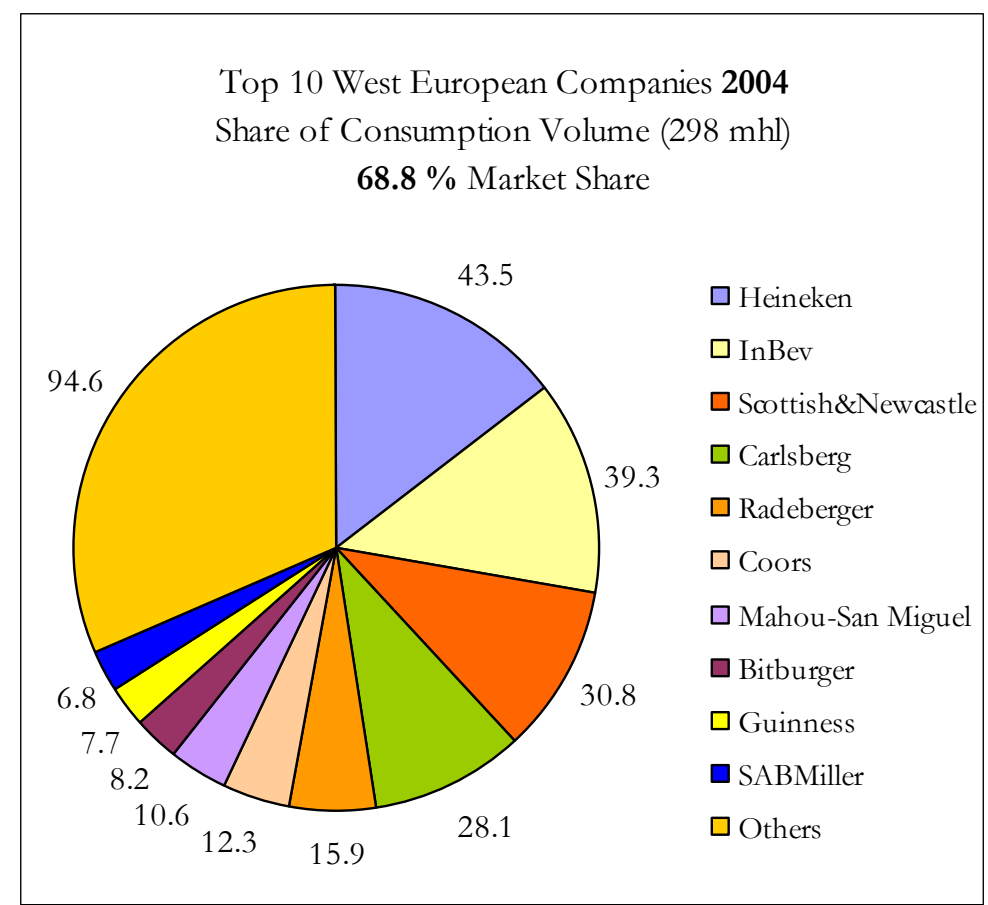

Source: (Plato, 2005). 


\section{Branding and Retailing in West European Beer Markets}

Given the exceptional weather enjoyed through the key summer months in Europe in 2004, volume comparisons this year were always going to be challenging. However, the brewers are not just complaining about the weather in Europe. More specifically a continued weak consumer environment is resulting in a lack of recovery in volumes through the higher margin on-trade and also a growth in demand for private label in the off-trade. The growth in own label brands in part reflects the rising influence of hard discount chains within the retail sector - which has an obvious connection with continued weak consumer confidence. Following the slight recovery seen in the end-1990s, the beer market in West Europe resumed a negative trend over the last years, but was a little higher in 2003. But in general, where the hot weather could be seen to provide a stimulus to the beer market, it was the off-trade that benefited more. With the critical exception of Germany, canned beer sales rose in nearly all markets. Apart from gaining market share and expanding in emerging markets there is another step that can be taken to enhance brewers' global competitiveness and allow them to grab further share of the global profit pool: to create a truly international beer brand. When fighting for market share in the brewing industry, strategies centered around branding are of paramount importance. Figure 7 illustrates movements within West Europe's leading brands in the period from 1999 to 2004.

Figure 4: Main Brands in West European Beer Markets.

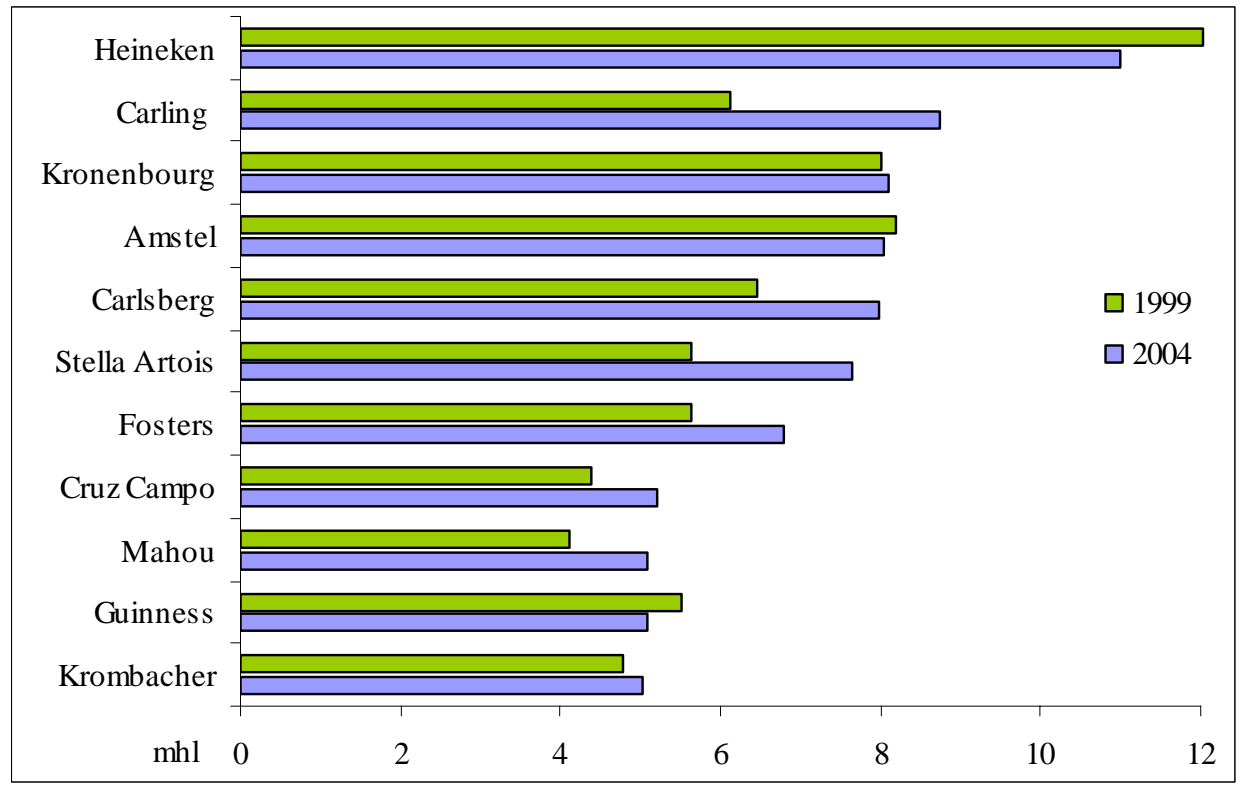

Source: (Plato, 2005). 


\section{Main Markets in Detail}

Breaking down the regional development of Western Europe to single markets, the following table gives an overview of the leading European key markets, ranked by production volume. As figure 2 has painted, the largest six markets account for approximately $80 \%$ of the total West Europe's volume. Table 1 highlights total consumption, per capita consumption, imports and exports as well as the respective market leaders. In the successional section, these main markets are described in-depth (see: Deutsche Bank Industry Research, DrKW, Plato).

Table 1: Main West European Beer Markets - Key Data.

\begin{tabular}{|c|c|c|c|c|c|c|c|c|c|c|c|c|}
\hline & \multicolumn{2}{|c|}{ Germany } & \multicolumn{2}{|c|}{ UK } & \multicolumn{2}{|c|}{ Spain } & \multicolumn{2}{|c|}{ Netherlands } & \multicolumn{2}{|c|}{ France } & \multicolumn{2}{|c|}{ Belgium } \\
\hline & 2000 & 2004 & 2000 & 2004 & 2000 & 2004 & 2000 & 2004 & 2000 & 2004 & 2000 & 2004 \\
\hline Beer consumption (mhl) & 103,056 & 96,500 & 58,550 & 59,100 & 29,153 & 34,981 & 13,227 & 12,550 & 20,837 & 20,040 & 10,064 & 9,560 \\
\hline Per capita consumption (1) & 126 & 118 & 99 & 99 & 73 & 88 & 83 & 78 & 35 & 35 & 98 & 92 \\
\hline Beer imports $(000 \mathrm{hl})$ & 3,574 & 2,740 & 7,455 & 8,800 & 3,373 & 3,802 & 1,043 & 2,000 & 4,881 & 4,900 & 801 & 917 \\
\hline Beer exports $(000 \mathrm{hl})$ & 10,947 & 13,500 & 3,637 & 3,920 & 633 & 884 & 12,888 & 13,000 & 2,340 & 2,220 & 5,474 & 6,771 \\
\hline Main brewers & Radeberger & $12 \%$ & S\&N & $27 \%$ & Heineken & $33 \%$ & Heineken & $49 \%$ & S\&N & $39 \%$ & $\mathrm{InBev}$ & $56 \%$ \\
\hline \multirow[t]{3}{*}{ Market share (\%) } & InBev & $10 \%$ & Coors & $20 \%$ & Mahou & $29 \%$ & InBev & $16 \%$ & Heineken & $36 \%$ & Alken-M. & $15 \%$ \\
\hline & Carlsberg & $8 \%$ & InBev & $20 \%$ & Damm & $18 \%$ & Grolsch & $13 \%$ & InBev & $9 \%$ & Palm & $6 \%$ \\
\hline & Top 5 & $37 \%$ & Top 5 & $83 \%$ & Top 5 & $81 \%$ & Top 5 & $94 \%$ & Top 5 & $89 \%$ & Top 5 & $95 \%$ \\
\hline
\end{tabular}

Source: (Bevan and Greenberg, 2005; Plato, 2005).

\section{Germany}

Germany - as the largest beer market in Europe with the second highest per capita consumption - continues to experience a steady decline in consumption with 2004 volumes down around $2 \%$. This on-going decline reflects a weak consumer environment and also the negative impact of a deposit scheme which was introduced in 2003 on cans and non-returnable bottles. This has led to a collapse in demand. Having seen a number of the major multiple retail chains de-list cans and one-way packaging because of the complexity of the scheme, the brewers are offering PET-bottled beer with the retail chain's logo stamped on the bottle for identification purposes. Additionally, many German brewers are seeking to offload a portion of their unwanted canned beer into Central European markets. Despite the tough trading outlook a number of the leading European brewers have moved to expand their presence within Germany. In January 2005, Heineken acquired through its 50/50 joint-venture BHI $90.7 \%$ of the shares of Würzburger Hofbräu AG. Heineken's position in Germany had already been strengthened substantially by two acquisitions in 2004. The most significant deal in 2004 was the $€ 1.1$ bn acquisition of Holsten by Carlsberg and Bitburger. Then in October the acquisition 
by InBev of two German brewing businesses, Dinkelacker-Schwaben and Spaten received competition approval. The deals cement InBev's position as market leader in Germany with a $13 \%$ market share. With no forthcoming change in the deposit laws anticipated, continued weak consumer confidence and challenging summer weather comparisons, total beer volumes probably will decline modestly in 2005 again. M\&A activity is expected to continue as the above-mentioned pressures are only likely to accelerate the consolidation process in one of the few remaining fragmented major European beer markets (Holland et al, 2005).

\section{UK}

The UK beer market was broadly stable in 2004 at $56 \mathrm{mhl}$ - which was a creditable performance given the tough summer weather related comparisons from 2003. However, the market continued to see a shift in consumption away from the on-trade (-2\% in 2004) and towards the off-trade (+4\% in 2004), reflected by a persistent fall in the retail price of beer within the multiple grocers. This shift in consumption and the growing power of both tenanted operators and managed pub companies is resulting in the beer industry becoming increasingly dominated by a small number of 'megabrands'. In 2004 the top 10 beer brands accounted for c.65\% of industry volumes compared to $42 \%$ in $1995 . \mathrm{S} \& \mathrm{~N}$ is the market leader in the UK with $27 \%$ market share - it also enjoys a $66 \%$ share of the cider category. InBev's business is concentrated on Stella Artois - the no.1 UK premium lager. It has recently been losing share as it seeks to improve the price positioning of its brand - particularly in the off-trade. Coors business is predominantly focused on Carling (no.1 standard lager) and Grolsch. Its profitability has been volatile since Coors acquired the business three years ago - but the Carling brand continues to gain share. Carlsberg UK is the no.4 player and is typically forced to compete on price. It has been forced to aggressively restructure its business to maintain profitability as the dynamics of the industry have deteriorated. UK volumes in 2005 are likely to be down at least $2 \%$ with S\&N continuing to gain share.

\section{Spain}

Within a West European beer market that has been struggling to display any positive growth, the Spanish market has been one of the few exceptions (+3\% in 2004). However, this growth was skewed towards the off-trade - with cultural changes and a relatively weak economic situation contributing to a decline in on-trade volumes. Heineken is market leader with just over 30\% market share. Its Spanish business continues to dominate Southern Spain following 
the acquisition of Cruzcampo. The business continues to enjoy strong profit growth and Heineken is investing over $€ 200 \mathrm{~m}$ in building a new state of the art $4.5 \mathrm{mhl}$ brewery in Seville. The no.2 brewer continues to be Mahou-San Miguel which dominates the central region and the North West. The future of Mahou-San Miguel continues to attract speculation - as it would be one of the few remaining large-scale family brewers within Western Europe. InBev has sold its $12 \%$ stake in the no.3 brewer, Damm for $€ 176 \mathrm{~m}$. Damm dominates the North East of Spain and again its future remains the subject of speculation. Although the Spanish beer industry continues to grow its development has not been aided by a number of government interventions (i. e. increase of the legal drinking age to 18 years, restrictions on advertisements, implementation of a $10 \%$ increase in excise duties). Aided by favorable summer weather and a growth in tourism flows the Spanish beer market maintained its solid growth trend during 2005 (Holland et al, 2005). Although per capita consumption is heading towards European average levels it is estimated that tourists account for c.20 liters per head of the annual consumption. Spain is therefore one of the most attractive markets in Western Europe and, as such, it seems to be sure that brewers like InBev, SABMiller, MolsonCoors would be keen to gain an exposure via Mahou-San Miguel or possibly Damm.

\section{Netherlands}

The Dutch beer market is saturated (-2\% in 2004) and competition is intense. However, this only exacerbated more structural problems. Firstly, there was the competition from other drinks, with beer losing ground in terms of image. A second factor was the continuing economic recession, and the third, the changing demographic composition of the Dutch population in terms of ageing. Price wars within the Dutch food retail industry have been intense, as the full service operators have had to respond to the growth of hard discounters by slashing prices on a wide number of branded goods. Beer has been one of the categories impacted. The market leader, Heineken, insists that it has not funded any of the additional discounts introduced by the retail chains but it is clearly worrying to see the price of its flagship brand cut aggressively. Heineken is the number one player in the Dutch beer market with a c.50\% market share. Against a falling market, Heineken's domestic sales slipped from $6.0 \mathrm{mhl}$ to 5.9 mhl. The slight loss of market share was due to Heineken's relatively strong position in the on-trade, where sales fell more sharply than in the rest of the beer market. InBev, Bavaria and Grolsch are the other big names in the Dutch beer market each holding 13-16\% shares respectively (Bevan and Greenberg, 2005). Heineken, InBev and Grolsch have all made moves re- 
cently to reduce costs from their Dutch brewing operations - whether it be brewery closures or more general efficiency gains. Cost reduction is likely to remain essential to driving any profit growth from the Dutch industry in the near-term as the prospects for a revival in beer consumption remains bleak.

\section{France}

The combination of a weak consumer environment and mediocre summer weather conspired to put downward pressure on consumption in the French beer market (-5\% in 2004). The pressure on the two leading French brewers, Kronenbourg (S\&N) and Heineken was compounded by the corresponding growth in private label volumes - which caused the big 2 to lose market share. Although the long-term volume trend for beer in France has been poor the industry has been able to continue growing value due to continued positive pricing environment (dominance of two brewers, fragmented on-trade, existence of 'Loi Galland' within the off-trade which has prevented beer being used as a loss-leader to build customer traffic). But there is no question that this pricing strategy has created the opportunity for the growth in private label as duty is also very low in France and so the bulk of retail prices are driven by the brewer and retailer not the government. However, the uncertainty remains on whether the price reductions will be funded by the retailer or the brewer (Bevan and Greenberg, 2005). S\&N is the market leader in France with a 39\% market share. Its two leading brands (Kronenbourg and 1664) are numbers one and three, and together comprise one-third of the French market, followed by Heineken with a $36 \%$ market share. It was a mixed bag for S\&N with Kronenbourg brand volumes falling by $6.4 \%$, Grimbergen volumes moving up by $13 \%, 1664$ volumes declining at a slower rate than the market by $3.5 \%$, and Foster's volumes marginally down by $1.6 \%$. Heineken France continued to focus on its leading premium brands. In the first half of 2005 the French beer market declined a further $4 \%$ - with the branded operators losing further share to private label.

\section{Belgium}

The year 2004 was also difficult for the highly competitive Belgium beer market (-4 to 5\%). Despite the dormant domestic consumption, Belgium remains the $12^{\text {th }}$ largest in Europe with annual consumption of around $9.6 \mathrm{mhl}$. InBev dominates the market with a $56 \%$ market share; Alken Maes (S\&N) follows with $15 \%$; the remaining $29 \%$ of the market is largely comprised of small family-owned brewers specialising in dark beer and specialty brands that are syn- 
onymous with Belgium. InBev's leading brand is Jupiler, accounting for just over one-third of all beer sold. InBev's other sizeable domestic brand is Stella Artois, which accounts for around a 7\% market share. Maes Pils and Grimbergen are Alken-Maes' key brands; Maes Pils accounts for approximately $10 \%$ of the national market share and therefore more than $60 \%$ of Alken-Maes' sales. Maes Pils volumes fell 4.5\%, in line with mainstream beer trends, but there was continued strong growth in the premium brand Grimbergen, where volumes grew $6 \%$. Both InBev and Alken-Maes are on a path of restructuring to provide stronger operational focus and efficiency. In Belgium, beer markets' performance in 2005 is likely to be relatively subdued as the outlook for consumer confidence remains depressed. For the two leading brewers, the focus remains on value growth although the growth of the discount chains may pose some threat to continued positive off-trade pricing (Bevan and Greenberg, 2005). However, despite these modest domestic consumption trends, national production is expected to grow reflecting the growing global appeal of speciality beers.

\section{REFERENCES}

Bevan, N. and Greenberg, M. (2005). Global Brewing - The Global Pitcher 2005 - Volume VI, Deutsche Bank Report, London.

Canadean (2004). Global Beer Report. Annual Report - 2004 Cycle, Daneshill.

Holland, A. et al (2005). Beverages Quarterly: Q3 2005, Dresdner Kleinwort Wasserstein Equity Research, London.

Joh.Barth\&Sohn (2005). The Barth Report. Hops 2004/05, Nuremberg.

Plato (2005). Country Draughts: West Europe, Plato Logic Limited, London. 
KAPITEL I.3

Review of the Central \& Eastern European Beer Markets

Oliver Ebneth

This article will be published in: Brewing and Beverage Industry International 3(2006). 


\section{Review of the Central \& Eastern European Beer Markets}

Our analysis of the beer markets of Central \& Eastern Europe shows that this region offers Europe's five major brewers strong potential and an attractive investment profile, with five key drivers:

- Favourable volume trends, notably in Russia, Ukraine, Poland, Turkey and Romania, where consumption of beer is rising strongly.

- Opportunities to improve margins, through synergies with local brewers acquired in recent years by rationalising and upgrading production and distribution facilities.

- Mix improvements, which should be driven by developing brand franchises. Brands in most markets remain fragmented. Moreover, we expect volume growth for domestic premium beers to outpace that for the standard and the economy segments in the medium to long term. The major brewers should also benefit from a change in the distribution channel mix, as the higher-margin "on-trade" is still in its development phase.

- We estimate that InBev, SABMiller, Heineken, Carlsberg and Scottish \& Newcastle have an aggregate market share in Central \& Eastern Europe of around 60\%. Further consolidation is expected to continue in some markets, notably Russia and Romania, where local brewers still have a large market share.

- While we are confident about the dynamic growth prospects for beer consumption in Central $\&$ Eastern Europe over the next years, we recognise that demographic factors suggest that growth beyond this period is likely to slow significantly.

\section{Growth prospects for further consumption are likely to slow down}

In the context of modest volume growth, at best, in their domestic beer markets, Europe's major brewers are pursuing opportunities in higher-growth areas. Over the last decade AsiaPacific and Central \& Eastern Europe offered the most appealing growth for the beer consumption per capita offering a compound annual growth rate (CAGR) of $6.5 \%$ (see figure 1). We estimate this growth rate to slow down a bit but remains on a comparatively high level. Thus Central \& Eastern Europe will still outperform the projected feeble growth of $0.6 \%$ and $0.7 \%$ respectively for Western Europe and North America. 
Figure 1: C\&E European Consumption and Per Capita Consumption.

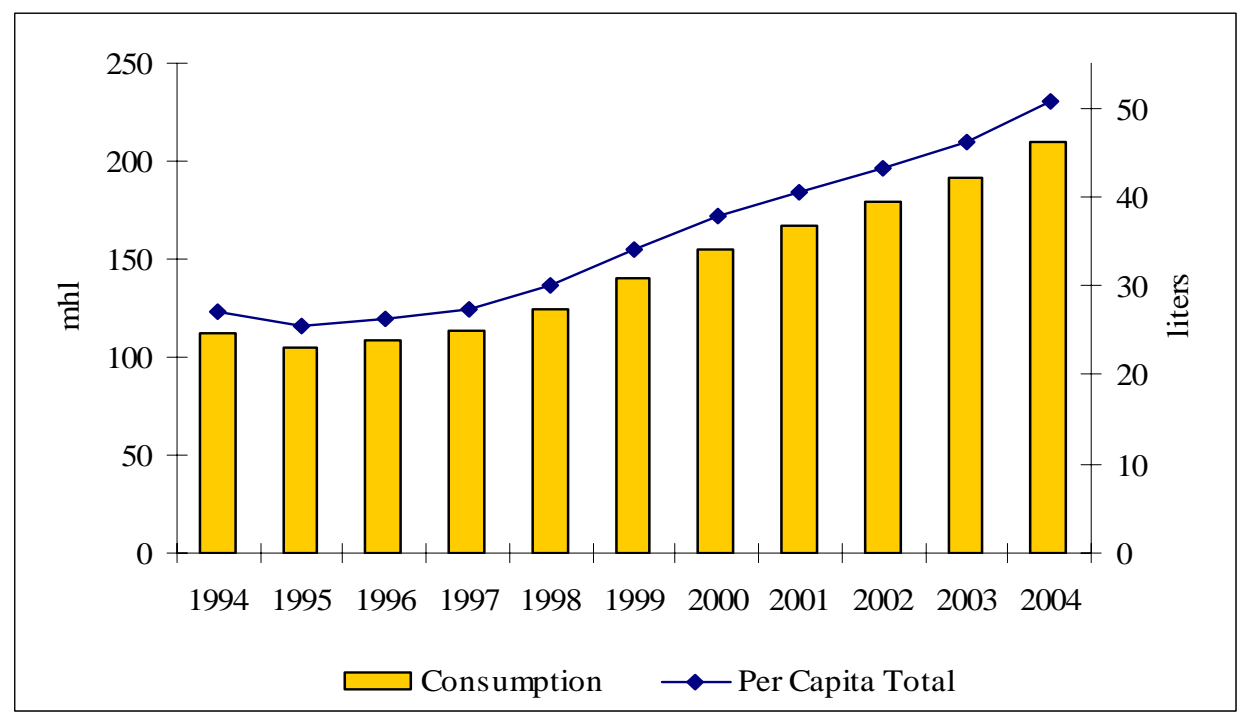

Source: (Canadean, 2004; Joh.Barth\&Sohn, 2005; Plato, 2005).

Beer consumption in Central \& Eastern Europe amounted to 210 million hectoliters (mhl) in 2004, representing $13.5 \%$ of world consumption and compared with intake of $320 \mathrm{~m} \mathrm{hl}$ in Western Europe. Russia is the region's largest market, accounting for $85.2 \mathrm{~m} \mathrm{hl}$ or $41 \%$ of regional beer consumption in 2004, followed by Poland (27.7 mhl), the Czech Republic (18.7 mhl) and Ukraine (17.3 mhl). Figure 2 highlights the total growth in beer volumes by market in the decade from 1994 until now.

Figure 2: Volume Growth of Central \& Eastern European Beer Markets.

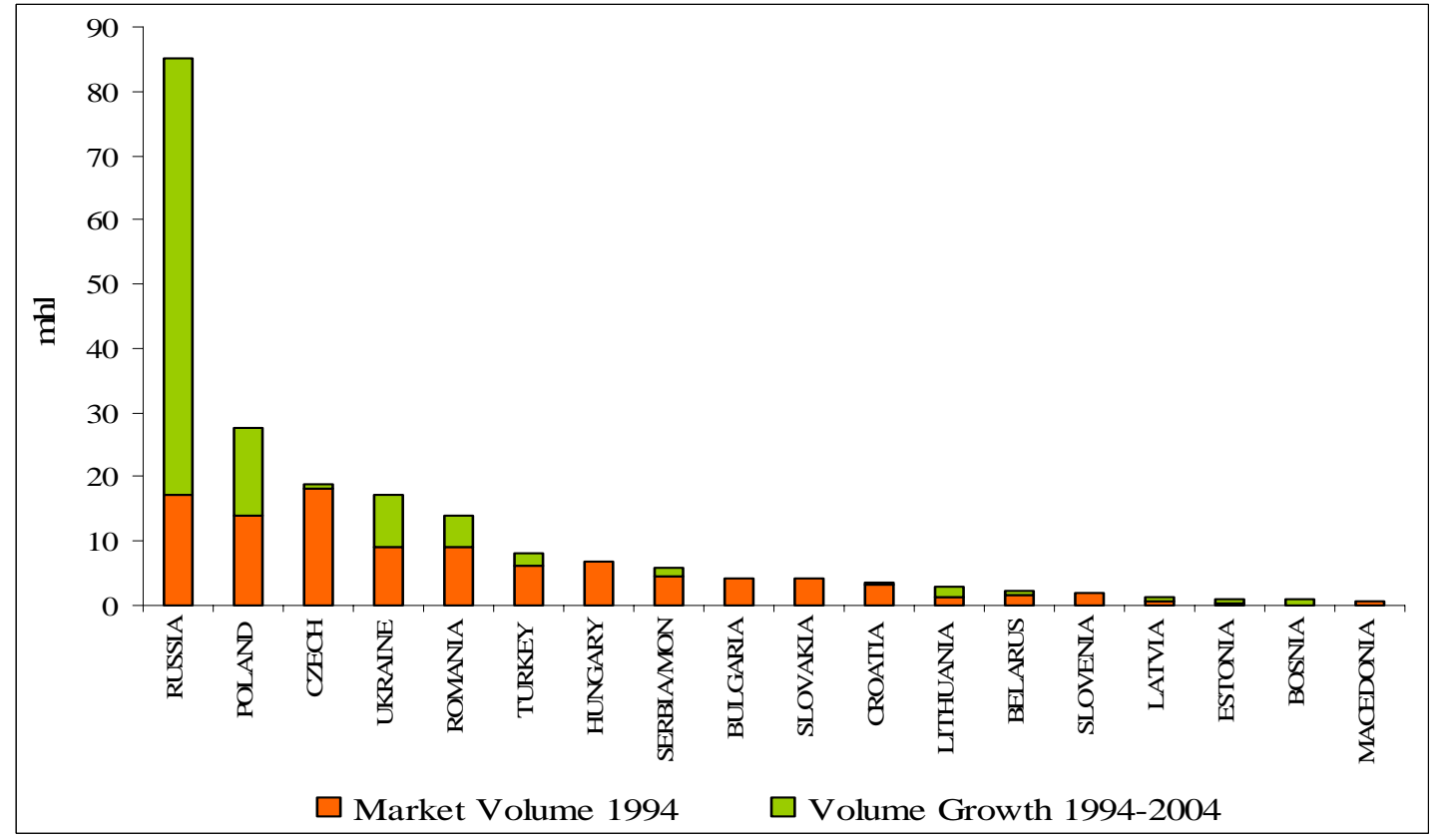

Source: Barth Reports (1995 -2005). 
As figure 2 shows, Russia represents by far the largest beer market within Central \& Eastern Europe. Russia has seen the strongest growth in terms of absolute volume increase as well as regarding the CAGR. In the decade between 1994 and 2004 the Russian beer market has risen from 17.3 to $85.2 \mathrm{mhl}$, which was a boost of $67.9 \mathrm{mhl}$ in total (CAGR 17.3\%). This has been followed by Poland (+13.7 mhl; CAGR 7.0\%), Ukraine (+8.2 mhl; CAGR 6.7\%), Romania (+4.9 mhl; CAGR 4.4\%) and Turkey (+2.2 mhl; CAGR 3.2\%). These five countries together represent $93 \%$ of the total $103 \mathrm{mhl}$ increase in beer volumes for Central \& Eastern Europe in the last decade. We expect Russia's beer market to see the strongest growth in beer consumption in absolute terms also over the next years. Table 1 illustrates some key figures of these most important C\&E European markets.

Table 1: Main Central \& Eastern European Beer Markets - Key Data.

\begin{tabular}{|c|c|c|c|c|c|c|c|c|c|c|c|c|}
\hline & \multicolumn{2}{|c|}{ Russia } & \multicolumn{2}{|c|}{ Poland } & \multicolumn{2}{|c|}{$\begin{array}{c}\text { Czech } \\
\text { Republic }\end{array}$} & \multicolumn{2}{|c|}{ Ukraine } & \multicolumn{2}{|c|}{ Romania } & \multicolumn{2}{|c|}{ Turkey } \\
\hline & 1998 & 2004 & 1998 & 2004 & 1998 & 2004 & 1998 & 2004 & 1998 & 2004 & 1998 & 2004 \\
\hline Beer consumption (mhl) & 31.3 & 85.2 & 21.0 & 27.7 & 16.9 & 18.8 & 6.9 & 17.3 & 9.8 & 14.0 & 5.9 & 8.2 \\
\hline Per capita consumption (1) & 21 & 58 & 54 & 74 & 164 & 165 & 14 & 37 & 44 & 49 & 9 & 12 \\
\hline Beer consumption (USDm) & 4,298 & 12,357 & 2,830 & 4,547 & 1,328 & 2,202 & 886 & 1,658 & 1,138 & 896 & 2,335 & 3,489 \\
\hline Value per hl (USDm) & 137.3 & 148.3 & 134.9 & 158.9 & 78.6 & 130.3 & 128.0 & 94.0 & 116.0 & 81.0 & 393.0 & 414.0 \\
\hline Main brewers & $\mathrm{BBH}$ & $34.8 \%$ & Heineken & $37.3 \%$ & SABMiller & $47.3 \%$ & InBev & $31.2 \%$ & Heineken & $33.1 \%$ & Efes & $77.0 \%$ \\
\hline \multirow[t]{3}{*}{ Market share (\%) } & InBev & $14.8 \%$ & SABMiller & $31.9 \%$ & InBev & $14.7 \%$ & Obolon & $26.3 \%$ & SABMiller & $15.8 \%$ & Carlsberg & $22.3 \%$ \\
\hline & Efes & $4.5 \%$ & Carlsberg & $13.4 \%$ & Praszké & $12.1 \%$ & $\mathrm{BBH}$ & $18.3 \%$ & InBev & $7.8 \%$ & Tekel & $0.7 \%$ \\
\hline & Top 5 & $62.1 \%$ & Top 5 & $89.7 \%$ & Top 5 & $83.8 \%$ & Top 5 & $91.2 \%$ & Top 5 & $79.1 \%$ & Top 5 & $100.0 \%$ \\
\hline
\end{tabular}

Source: (Faes et al, 2004; Plato, 2005).

Beer consumption in Central \& Eastern Europe is expected to continue to rise beyond 2008, but at a more moderate pace than in the coming four years due to demographic trends. In every Central \& Eastern European country, the population of core drinking age (15 to 34 years old) is projected to decline gradually over the period 2004-2050, according to the OECD. In most countries, the forecast rate of decline in the core drinking age is between $1 \%$ and $2 \%$ p.a., reflecting a projected decline in the total population of $0.2-1 \%$ annually. Within Central \& Eastern Europe, brokers (like BNP Paribas, Merrill Lynch) forecast the highest volume growth for the beer markets of Eastern Europe (Russia, Ukraine, Belarus), of $6.8 \%$ p.a. over 2004-2008. For Central Europe an annual growth of 3.3\% is projected, with divergences between the higher-growth (emerging) and lower-growth (mature) countries. We divide Central Europe's beer markets as follows:

A group of mature Central European markets, for which volume growth for beer is expected to grow by $2.3 \%$ p.a. over 2004-2008. In these markets, GDP per head is higher than in 
the region's emerging countries, ranging from USD5,000 to USD9,000 (according to the OECD). The beer markets are also the region's most competitive, with well developed modern retail infrastructures and aggressive pricing, and in which private labels have started to appear. In this group we include the Czech Republic, Poland, Hungary, Slovakia and Slovenia (Winston et al, 2005).

A group of emerging Central European markets, for which brokers project a 2004-2008 CAGR in beer volumes of $4.7 \%$. GDP per head in most of these countries is lower, at between $\$ 3,000$ US and \$5,000 US. Their economies are recovering slowly, and their retail trades are still geared towards independent food stores and kiosks. In this cluster we include the Baltic States, Bosnia, Bulgaria, Macedonia, Romania, Turkey, Serbia-Montenegro and Croatia.

\section{Branding and Marketing in Central \& Eastern European Beer Markets}

By improving the quality of beer available in Central \& Eastern Europe, the international brewers have increased many consumers' preference for beer over other alcoholic beverages. These efforts have been assisted in some countries by fiscal policies implemented by governments to encourage a switch from high-alcohol content spirits to lower-alcohol content beers for health reasons. This shift in consumer tastes is exemplified in Poland, where spirits accounted for $58 \%$ of all alcohol consumed in 1995 but beer has now become the main alcoholic drink (52\%), ahead of spirits (31\%) and wine (17\%).

One development discernible across Central \& Eastern Europe is the international Brewers' moves to convert promising local beer brands into national brands. Developing local brands on a national scale is also in evidence among the region's smaller domestic brewers. The premium beer segment is small in most Central \& Eastern European markets, accounting for an average $9 \%$ of the region's beer consumption in 2004, compared with nearly $15 \%$ on a global basis. Premium beers are still a niche product in Central \& Eastern Europe, as their prices are out of reach for most consumers. In the more mature Western European beer markets, brewers are focusing on improving the price/mix in these markets to compensate for negligible growth in volumes (Faes et al, 2004).

In those Central \& Eastern European markets with robust economies and consumption of beer geared towards the economy segment, growth in volumes tends to be driven by standard and premium lagers (e.g. Hungary, Russia and Ukraine). Conversely, in countries with weaker and/or more volatile economies, such as Romania and Slovakia, private labels and economy lager sell best. For the region overall, French investment bank BNP Paribas fore- 
casts a 2004-2008 volume CAGR for premium beers of $7.7 \%$, compared with $5 \%$ for the standard segment and $2.4 \%$ for economy. As Table 2 highlights, we expect this growth in premium beers to be driven by Eastern Europe $(+16.7 \%)$. For Central Europe, we forecast volume growth for the premium segment of $1.6 \%$, versus $2.9 \%$ and $1.7 \%$ for standard and economy respectively (Winston et al, 2004).

Table 2: Regional Growth Rates in Beer Consumption by Segment.

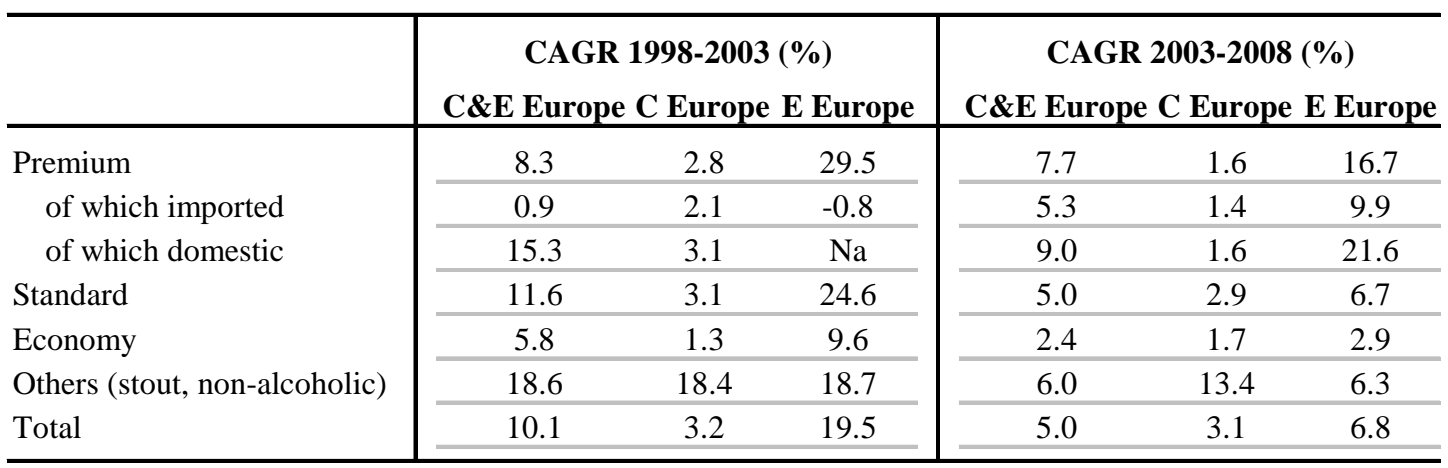

Source: (Faes et al, 2004).

\section{There is still scope for consolidation}

Central \& Eastern Europe's attractive growth profile for beer has prompted the international brewers to pursue market share gains aggressively in the region and as a result the industry has become increasingly competitive. Additionally to this competition is over-capacity of supply and price differentials between markets (encouraging cross-border sales). For example, surplus capacity in the canned beer industry in Germany is now exported to Hungary and Austria, and could also pose a threat to Poland's beer industry (although we have yet to see signs of this). Hungary also sells cheap beer imported from Slovakia. We believe these competitive pressures, along with rapid growth in the region of the major international retail chains, will keep price inflation down during the next years. The improvement in mix stemming from new products, the development of the on-trade and rising disposable incomes, is likely to be favourable. However, in most countries affordability continues to be an issue. As a result, the international brewers active in the region are rationalising their production facilities and improving distribution in order to boost profitability. Further consolidation between brewers and wholesalers, with brewers taking on some wholesale activities, should also help boost margins. 
Most Central \& Eastern European beer markets have consolidated considerably in recent years, as the international brewers have actively acquired local firms. However, we believe some countries still offer scope for the international brewers to reinforce their operations further through link-ups with local players.

While expansion should continue to be driven by volume growth from the companies' existing portfolios, some markets still offer opportunities growth from acquisitions. Figure 3 shows the top three brewers' aggregate market shares in each country. Turkey, Macedonia, Slovenia, Hungary and Estonia are now fully consolidated, with the three largest brewers' combined market shares exceeding 90\%. Other highly concentrated markets are (in descending order) Lithuania, Latvia, Croatia, Slovakia, Bosnia, Poland, Ukraine and the Czech Republic. In these markets, the three largest brewers between them control over $70 \%$ of sales volumes.

Figure 3: Concentration of Central \& Eastern European Beer Markets.

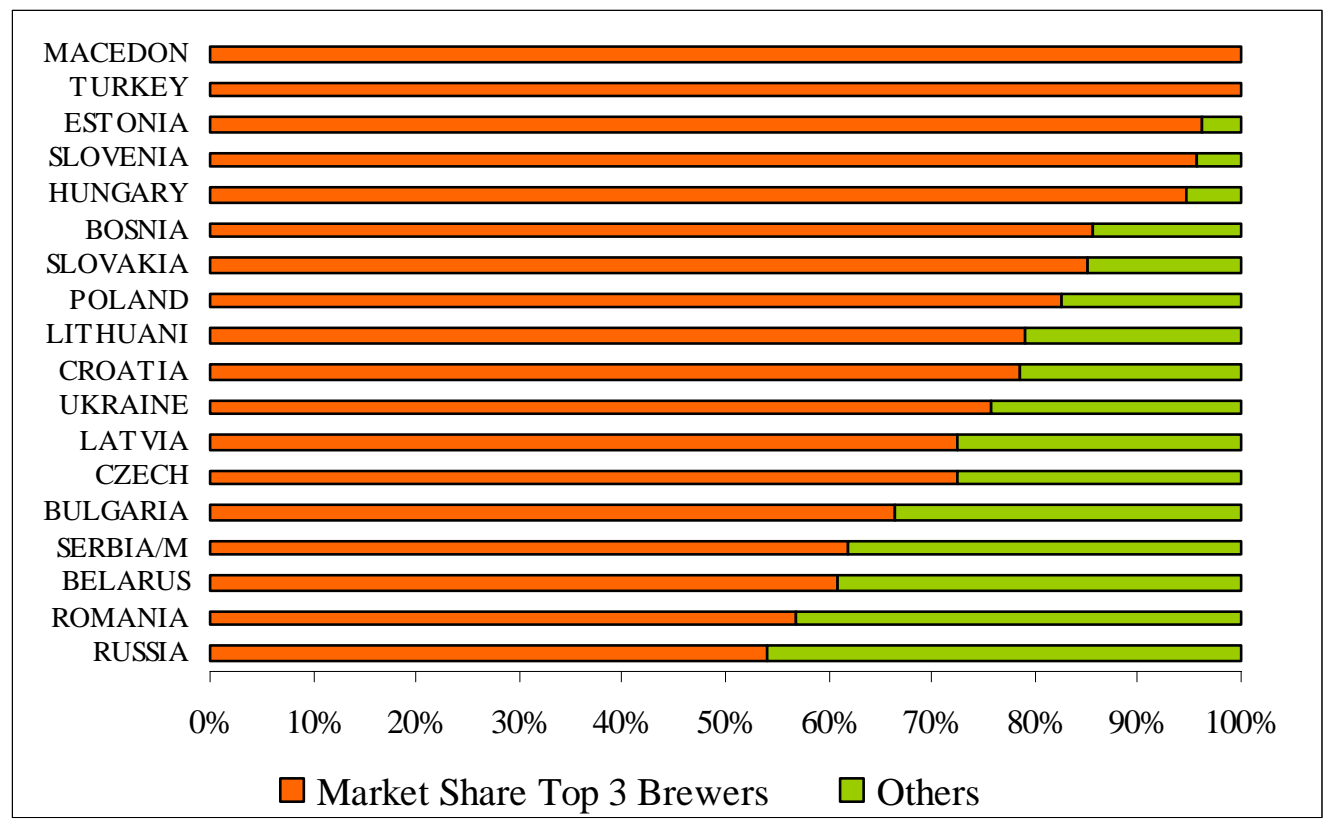

Source: (Plato, 2005).

The markets of Belarus, Bulgaria, Serbia-Montenegro, Romania and Russia are less consolidated. We expect most attention in this respect to be focused on Russia. In Russia, InBev, Heineken and SABMiller have all expressed a potential interest in the assets of the two largest remaining local players, Ochakovo and Krasny Vostok. Other potential targets could include Obolon and Sarmat (Ukraine), Akmaya (Romania), Budvar and Moravskoles (Czech Republic) and Topvar (Slovakia). Additionally, Bulgaria still has a few medium-sized independent 
brewers, such as Bulbrew, Zlaten, Mel and FIN Metal. In Bosnia-Herzegovina, Sarajevsja Pivaraand might join forces with an international brewer. The international brewers are not present in Slovenia, and their potential entry is likely to depend chiefly on whether the local player, Lasko, which controls $98 \%$ of the market directly and indirectly, wishes to remain independent.

The international brewers were quick to take up the opportunities to expand into Central \& Eastern Europe opened up by economic liberalisation. Since the 1990s, Heineken, InBev, SABMiller, Scottish \& Newcastle and Carlsberg have participated actively in developing the region's beer industry, both organically and through acquisitions. These five largest brewers present in Central \& Eastern Europe nowadays have an aggregate market share of $60.8 \%$. InBev is the largest brewer in Central \& Eastern Europe overall, with a market share in 2004 of $15.4 \%$, reflecting mainly its strong lead in Ukraine and Croatia and number two position in Russia. Within Central Europe, the company ranks third with an $11.9 \%$ share, as it is currently absent from the key market of Poland. Heineken, SABMiller and Carlsberg each have market shares in Central \& Eastern Europe of close to 14\%. Carlsberg's and S\&N's positions relate mainly to their 50\% stake in $\mathrm{BBH}$, market leader in Russia and the Baltic States and number three in Ukraine. Heineken is strong in Poland, Romania and Bulgaria, and recently enhanced its Russian market share to 15\% through diverse M\&As (Baikal, Taranov). SABMiller operates mainly in Poland and the Czech Republic.

\section{Outlook}

Further growth in Central \& Eastern Europe should help boost growth in margins and earnings for all five brewers to varying degrees. We expect InBev to benefit the most in terms of margin and earnings growth (assisted by volume growth, mix improvements and the reorganisation of production and distribution). Heineken, InBev, SABMiller and Carlsberg all have local expertise and international management teams in the region, and therefore look well positioned to take up further acquisition opportunities. In most of their operating countries, they now have national sales and distribution coverage that would enable them to extract synergies from further potential acquisitions. 


\section{REFERENCES}

Canadean (2004). Global Beer Report. Annual Report - Cycle 2004, Basingstoke.

Faes, N. et al (2004). Beverages. Central \& Eastern Europe in Focus. Exane BNP Paribas

Equity Research, Paris.

Joh.Barth\&Sohn (2005). The Barth Report. Hops 2004/05, Nuremberg.

Plato (2005). World Datasheets 2005, Plato Logic Limited, London.

Winston, C. et al (2005). European Beverages Valuations. The Sweet Sixteen - Standardising Valuations Across the Beverages Sector, Merrill Lynch, London.

Winston, C. et al (2004). Brand New World. Marketing and Brands in the Global Beer Industry, Merrill Lynch, London. 


\section{KAPITEL I.4}

\section{Review of the Chinese Brewing Sector}

\section{Oliver Ebneth}

Published in:

Brewing and Beverage Industry International 2(2006): 10-13.

This article will be published in:

Brewing and Beverage Industry China 2(2006). 


\section{Review of the Chinese Brewing Sector}

Although beer as a product has been consumed in China for many years, increased income levels have supported faster growth in consumption relative to the developed world. And combined with the increased exposure of the Chinese consumer to international brands, it seems only logical that the large beer producers worldwide have viewed China as an attractive growth market. Consequently, a broad push in establishing production footholds in the region has been made. In this article, we examine the current position of beer producers in China, and their capabilities to generate sustainable growth in profits. Surprisingly, the large international brewers as well as leading domestic brewers are not more profitable than smaller companies which act on a regional level.

Companies in the brewing sector have been investing heavily in recent years in the hope of long-term returns. But, we think their outlook is problematic, particularly for breweries, although a small proportion of brewers may eventually prosper once the sector reaches a more consolidated phase. We believe China's beer market is made up of hundreds and even thousands of small captive markets: small cities, districts, individual eateries, and countless entertainment venues. Distance, inefficient distribution systems, exclusive distribution arrangements, and, most importantly, very low beer prices have combined to erect high barriers around each small captive market. However, despite these high protective walls, the individual breweries that control these captive markets know well enough that they still have no pricing power. The walls would fall, as soon as the breweries were to raise beer prices. Besides, as an imported consumer item, beer has a very short history in China. To the vast majority of Chinese consumers, the history is as short as two decades. Beer is viewed as a soft substitute for traditional liquor for less masculine consumers. Beer's extremely low and falling prices have not helped its image and branding. Under this scenario, we see beer producer's margins in China coming under pressure over the coming period.

\section{The Largest and Fastest Growing Beer Market in the World}

China is now the largest beer market in the world - having recently overtaken the US (see figure 1). The market is now in excess of 290 million hectoliters (mhl) per annum (pa), and the growth rate over the past ten years has averaged around the 8\% level (Compound Annual Growth Rate (CAGR) 1994-2004: 7.6\%), which is well in excess of the majority of developed 
world growth rates. On around $290 \mathrm{mhl}$ of beer currently consumed in China, 6-8\% per annum growth represents approximately an additional $20 \mathrm{mhl}$ consumed pa (see figure 2). We believe similar growth over the next decade is possible, if not probable. This would mean China will remain the fastest-growing major beer market globally.

Figure 1: Top 10 Beer Markets 1994-2004.

Top 10 Beer Markets 1994

Share of Production Volume (1,214 mhl) $\mathbf{6 5 . 0} \%$ Market Share

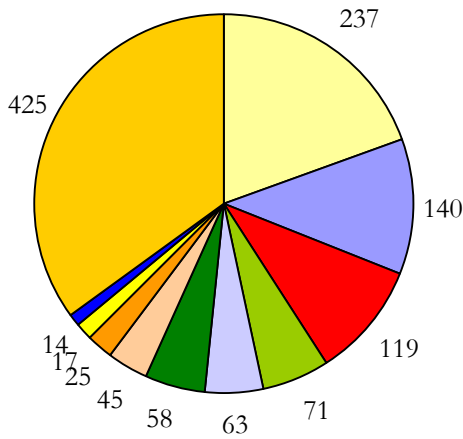

口USA

$\square$ China

$\square$ Germany

$\square$ Japan

$\square$ Brazil

$\square$ U.K.

$\square$ Mexico

$\square$ Spain

$\square$ Russia

Poland

$\square$ Rest of the World
Top 10 Beer Markets 2004

Share of Production Volume (1,552 mhl) $67.8 \%$ Market Share

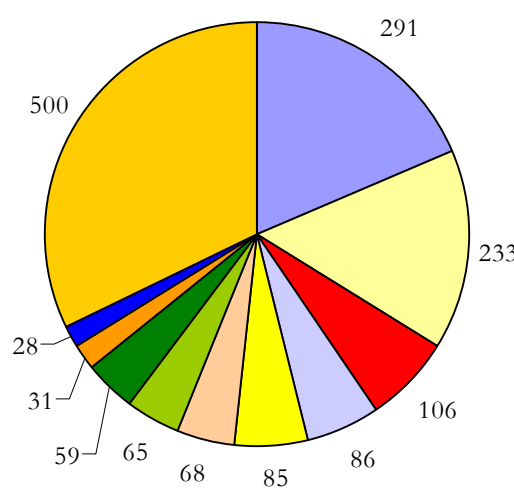

Source: (Joh.Barth\&Sohn, 1995; Joh.Barth\&Sohn, 2005).

Figure 2: Total Chinese Beer Consumption and Annual Growth Rates 1994-2004.

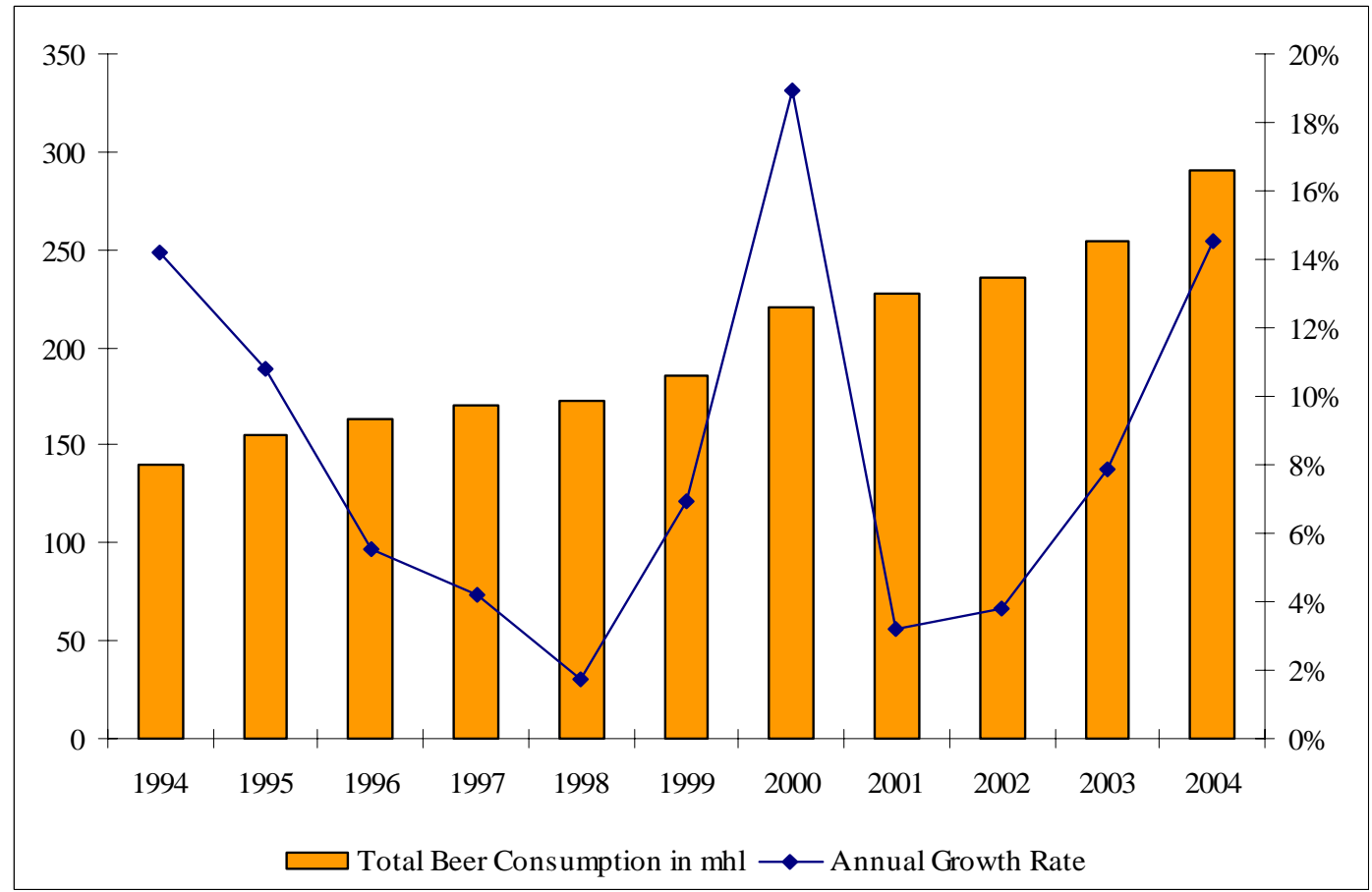

Source: Barth Reports (1995-2005). 
China's per-capita beer consumption of 22 liters in 2004 was lower than the global average and lower than consumption in some other Asian countries and regions: Korea (44.5 liters), Japan (42), Hong Kong (29), Singapore (24), and Taiwan (24). However, we believe the above data understates the true per-capita consumption in Chinese cities, for two reasons. First, most people in China had not tasted beer until around 20 years ago. Rice wines and liquors are much more deeply rooted in Chinese culture. Second, about $70 \%$ of China's population still lives in the countryside, despite two decades of urbanisation. These rural inhabitants are part of the denominator for the per-capita calculation, but their beer consumption will always likely be negligible because of low income and a lack of refrigeration. In other words, China's per-capita beer consumption is already high in cities. Thus, in most parts of China, beer consumption has reached a mature level and is unlikely to increase considerably. Therefore, consumption growth may have to come from poorer, rural regions.

\section{Consolidation Issues and Profitability}

Over the last decade, China emerged into the fastest-growing major beer market globally. As a result, China will continue to attract multinational brewers. Over 30 multinational brewers have already had operations in China. We believe their presence will grow as other markets stagnate. China may well remain a small portion of the multinationals' global operations. However, these overseas companies, despite being generally Return on Equity (ROE)conscious, will have a strong desire to maintain their China operations as strategic investments, even though these operations may make a loss or little profit according to some investment banks (Zhong and Wong, 2005).

We observe that few sectors in China today attract more media and investor attention than the brewery and retail sectors. This is because of the growing numbers of high-profile merger and acquisition (M\&A) deals that are emerging. Just recently Belgian brewing giant InBev obviously won the bidder's rivalry for the Chinese Fujian Sedrin Brewery. InBev acquired a majority at Fujian for approximately $\$ 750$ million US, according to the "New York Times". InBev competed against the Dutch Heineken NV, the US brewery Anheuser-Busch and the Chinese Yanjing Brewery. Table 1 lists the eight leading brewers ranked by their market share in 2004. 
Table 1: Top Eight Brewers in China (2003 market share).

\begin{tabular}{lc}
\hline Tsingtao (10\% owned by Anheuser-Busch) & $12.9 \%$ \\
CRB (SABMiller/CRE) & $11.5 \%$ \\
Beijing Yanjing & $10.5 \%$ \\
InBev (including KK and Lion) & $5.4 \%$ \\
Harbin (fully acquired by Anheuser-Busch in 2004) & $4.6 \%$ \\
Zhujiang (InBev has a 24\% stake) & $3.4 \%$ \\
Chongqing (Scottish has a 20\% stake) & $2.6 \%$ \\
CSI/Asahi & $2.2 \%$ \\
\hline
\end{tabular}

Source: (Zhong and Wong, 2005), author's calculations.

Total profit in China's brewery sector is low, despite sales volumes that have exceeded those in the US. The top three brewers in China-accounting for 35\% of the beer market-made a combined profit of solely about $\$ 100 \mathrm{~m}$ US in 2004, equivalent to 5\% of Anheuser-Busch's 2004 profit and 14\% of Heineken's. Beer prices in China are lower than in other countries about half of those in Western Europe, a third of those in the US and only a quarter of those being paid in Japan. Note that the costs of producing the beer - for raw materials and machinery - are fairly similar across these markets.

The size of a brewery does not equate to its profitability (see table 2). This is partly because so far, there is no financial incentive or reward for sector consolidation. Those breweries that have consolidated generally have relatively little to show for their efforts of the past six to seven years. Increasing managerial pressures are likely to more than offset the limited synergy from cost savings and brand recognition, in our view.

In theory, foreign entry should help accelerate the sector's consolidation. But, in reality, it may make consolidation more difficult. This is because multinationals' investments in China are relatively small for these large companies, so they can afford to wait for a recovery. While they wait, the sector will likely remain fragmented. Capacity utilization is currently around $65 \%$, resulting in producers consistently discounting (in order to gain market share).

In China, there is little evidence in favour of large breweries. We can look at them from two angles. First, the industry's three leaders - Tsingtao, CRB (a joint venture between China Resources and SABMiller), and Yanjing - have lower profitability than smaller players, such as Harbin and Kingway, despite the industry leaders having widely recognised brands (Zhong and Wong, 2005). Second, the three leaders still derive most of their profits, or $40 \%$ in Yan- 
jing's case, from their home markets, even five to six years after embarking on an acquisition drive.

Table 2: Six Leading Brewers’ Financial Performance 2002-2004.

\begin{tabular}{|c|c|c|c|c|c|c|c|c|c|c|c|c|c|c|c|c|c|c|}
\hline & \multicolumn{3}{|c|}{ Tsingtao } & \multicolumn{3}{|c|}{ Yanjing } & \multicolumn{3}{|c|}{ Kingway } & \multicolumn{3}{|c|}{ Harbin } & \multicolumn{3}{|c|}{ CRB } & \multicolumn{3}{|c|}{ Chongqing } \\
\hline & 2002 & 2003 & 2004 & 2002 & 2003 & 2004 & 2002 & 2003 & 2004 & 2002 & 2003 & 2004 & 2002 & 2003 & 2004 & 2002 & 2003 & 2004 \\
\hline Sales Volume (mhl) & 29.9 & 32.6 & 37.6 & 20.9 & 22.3 & 27.8 & 2.2 & 2.7 & 3.2 & 9.0 & 11.5 & 13.1 & 23.4 & 25.3 & 28.8 & 3.8 & 4.6 & 5.8 \\
\hline Gross Profit Margin & $31.2 \%$ & $31.8 \%$ & $31.4 \%$ & $25.1 \%$ & $27.7 \%$ & $28.5 \%$ & $40.7 \%$ & $45.5 \%$ & $48.8 \%$ & $42.4 \%$ & $43.6 \%$ & $41.2 \%$ & $\mathrm{Na}$ & $\mathrm{Na}$ & $\mathrm{Na}$ & $36.6 \%$ & $37.3 \%$ & $34.6 \%$ \\
\hline Return on Equity & $7.5 \%$ & $7.8 \%$ & $7.9 \%$ & $5.3 \%$ & $6.1 \%$ & $7.4 \%$ & $6.6 \%$ & $8.6 \%$ & $9.0 \%$ & $19.2 \%$ & $12.3 \%$ & $15.9 \%$ & $\mathrm{Na}$ & $\mathrm{Na}$ & $\mathrm{Na}$ & $7.6 \%$ & $8.7 \%$ & $9.3 \%$ \\
\hline EBIT Margin & $6.7 \%$ & $6.9 \%$ & $7.9 \%$ & $9.0 \%$ & $10.0 \%$ & $10.5 \%$ & $17.7 \%$ & $20.7 \%$ & $21.3 \%$ & $18.8 \%$ & $13.5 \%$ & $8.0 \%$ & $9.3 \%$ & $10.1 \%$ & $9.3 \%$ & $10.8 \%$ & $11.6 \%$ & $10.4 \%$ \\
\hline Net Profit Margin & $3.3 \%$ & $3.4 \%$ & $3.4 \%$ & $7.3 \%$ & $7.1 \%$ & $6.2 \%$ & $12.7 \%$ & $15.3 \%$ & $17.6 \%$ & $9.8 \%$ & $8.2 \%$ & $1.8 \%$ & $\mathrm{Na}$ & $2.9 \%$ & $\mathrm{Na}$ & $5.7 \%$ & $5.9 \%$ & $5.6 \%$ \\
\hline
\end{tabular}

Source: (Errington et al, 2004; Zhong and Wong, 2005), author's calculations.

Profitability in China's brewery sector is hard to ascertain, as only a few large breweries are publicly listed and have audited accounts. However, government data puts the sector's total profits at only $€ 260 \mathrm{~m}$ and net profit margin at just $4 \%$ in 2002 . The numbers are broadly consistent with those of the three largest breweries. Tsingtao, Yanjing, and CRB produced combined net profit of less than $€ 85 \mathrm{~m}$ in 2003 and their combined market share was about $35 \%$. One of the most successful foreign breweries in China is SABMiller. However, even after 12 years, we estimate its ROE from its China operations is still only 5-6\% (CRB made roughly $€ 24 \mathrm{~m}$ net profit in 2004 for an investment of about $€ 437 \mathrm{~m}$ ). At present, CRB is still expanding, acquiring and building new breweries (Zhong and Wong, 2005).

We believe Chinese breweries are stuck in a kind of poverty trap. Consumers generally disregard breweries' advertising and branding. Meanwhile, overcapacity in the brewery sector exerts downward pressure on beer prices. Most breweries cannot afford to advertise, given the low beer prices and therefore low gross margins. This factor, coupled with insufficient advertising, means consumers will continue to view beer as a non-branded product. Overcapacity must moderate if beer prices are to rise. But, it is very difficult to reach the goal of higher beer prices, for a number of reasons (Errington et al, 2004).

First of all, while the sector has a low ROE of roughly 4-5\%, it is by no means in dire straits. China's breweries are generally optimistic about their outlook. Breweries have underperformed the rest of the economy (where ROE is about 7\%), but the stock market continues to value breweries highly and there is no shortage of capital for the sector. Incumbents are unlikely to quit the sector voluntarily. Many breweries are state-owned enterprises, so they have high tolerances for prolonged low returns and even accounting losses, as long as cash 
flows are positive. Second, foreign and local breweries will likely keep adding new capacity faster than demand growth, which we estimate will be at a 6-8\% CAGR in the next decade.

\section{Branding and Retailing}

One reason for the lack of profitability in the Chinese beer market reflects the fact, that branding in China's brewery sector is still significantly underdeveloped. Breweries' advertising budgets in China are much lower than those of traditional liquor producers because breweries have much lower profit margins. It is disappointing for breweries that China's consumers treat beer as a non-branded product and that there is very little space for advertising and brand building. According to some brokers (UBS, Merrill Lynch Equity Research), consumers simply buy local beers for their freshness and because they are cheaper, having travelled a shorter distance than beers brewed elsewhere. Some observers argue the 'buy local' tendency is due to unique local tastes, but this observation remains uncertain.

Most major breweries in China control a large number of beer brands, as a result of acquisitions in the past six to seven years. For example, Tsingtao still controls around 50 brands, having phased out around 40 weaker brands. Yanjing now manages about 15 brands, and China Resources Breweries (CRB) around 30. Tsingtao, Yanjing, and CRB, along with other larger breweries that have acquired smaller brands, have been trying to push their main brands in the rest of the country and phase out acquired brands to increase average selling prices. But, the process has been very slow (Zhong and Wong, 2005).

As branding appears not to be important in China's brewery sector, we study how beer is sold. Our research shows that the distribution system has further cemented the fragmented beer production system. In most small cities and rural areas, there are usually one and at most two brands - the local brands. This is not only because of distance, but also because of local political intervention. In recent years, official media have exposed strong-arm tactics employed by local governments and local breweries to exclude beer brewed in other regions (Errington et al, 2004).

China's brewery sector is already far more consolidated than the retail sector. The top three breweries account for $35 \%$ of the country's beer market by volume (see table 1), and a far greater percentage by value. In their respective core markets, their actual market share is often as much as $70-90 \%$. However, in our view they still have no pricing power. We believe the consolidation of the Chinese retail sector will pick up the pace. This means the brewery sector 
will likely lose some of its bargaining power to large retailers in the process. In the past few years, breweries' biggest distributors (eateries) have been reasonably stable, and breweries have had stable relationships with them, but their established relationships with retailers are facing challenges due to retail sector consolidation. Retailers' growing size can become a rising industry entry barrier, but, for breweries, size might not help create a meaningful entry barrier.

\section{Outlook}

China is the largest and the fastest growing beer market in the world. However, from a manufacturer's perspective, it is also probably the toughest to be successful. Competition is fierce, and infrastructure needed to support manufacturing (raw materials, distribution, retailing) is under developed. Many of the companies we analyzed with exposure to China recognize that risks to earnings over the coming period are emerging. Most have strategies in place where they believe they will be insulated (move to premium products, increase productivity within their operations, be more vertically integrated). Whether the companies are successful in offsetting input price pressures and beer price deflation remains to be seen.

\section{REFERENCES}

Errington, D. et al (2004). Chinese Beer Industry. Supply - A Beer Producer's 'Achilles Heel'. Merrill Lynch, London.

Joh.Barth\&Sohn (1995). The Barth Report. Hops 1994/95, Nuremberg. Joh.Barth\&Sohn (2005). The Barth Report. Hops 2004/05, Nuremberg.

Zhong, J. and Wong, J. (2005). China Beer Sector Review. UBS Investment Research, Hong Kong. 


\section{KAPITEL I.5}

\section{Recent Developments in Southern American Beer Markets}

\section{Oliver Ebneth}

This article will be published in:

- Brewing and Beverage Industry Espagñol 3(2006),

- Brewing and Beverage Industry International 5(2006). 


\section{Recent Developments in Southern American Beer Markets}

In this article we shed light on some trends and developments in Southern American beer markets. We not only give an overview about consumption, main players and branding in these markets but we analyze some main Latin American beer markets more detailed. The outlook for South America appears similarly attractive. Per capita volume has grown remarkably by $1.6 \%$ each year over the last decade and is unlikely to loose the pace in the near future due to favorable demographics. Volume growth should be led by Chile, Argentina, Guatemala, Costa Rica and Brazil. Channel-of-trade is set to have no impact; geographic mix to be slightly negative, but in-market price and mix have increased revenues by $2.5 \%$ in 2004 and should rise by $2.7 \%$ in 2005, led by Ecuador, Puerto Rico, Paraguay, Bolivia, Panama, Peru and Venezuela. This has generated overall revenue growth of $4.4 \%$ in 2004 and will generate $4.1 \%$ in 2005 (see also DrKW Global Beer Report).

\section{Consumption Growth in Southern American Beer Markets}

The world beer market totals 1.55 billion hectoliter in 2004, increasing at a compound annual growth rate (CAGR) of $2.4 \%$ over the last decade. This positive trend is being driven mainly by the increasing importance of faster growing emerging markets such as China and Russia. Matured markets like the US and particularly West European markets stagnate or even shrink since more than a decade. Southern American markets stuck in the middle between the fast rising Eastern European and Asian markets and the saturated Western markets. Altogether these Southern American markets account for $10.3 \%$ of the world beer production. Figure 1 illustrates the developments of the six largest Southern American markets which account for 94\% of the total Latin American beer consumption. Glancing at figure 1 in detail shows some differences between these leading Latin American beer markets. Whereas, some have exposed sound growth rates over the last decade (Brazil: $+27.9 \%$, Venezuela: $+24.1 \%$, Argentina: $+19.0 \%$ ), others were more or less stagnating (Chile: $5.2 \%$ ), some markets even shrunk outstandingly in turn (Colombia: $-14.2 \%$, Peru: $-19.8 \%$ ). The overall CAGR of the South American beer market was $2.1 \%$ in the course of the last decade. 
Figure 1: Development of Consumption in Southern Americas Main Markets.

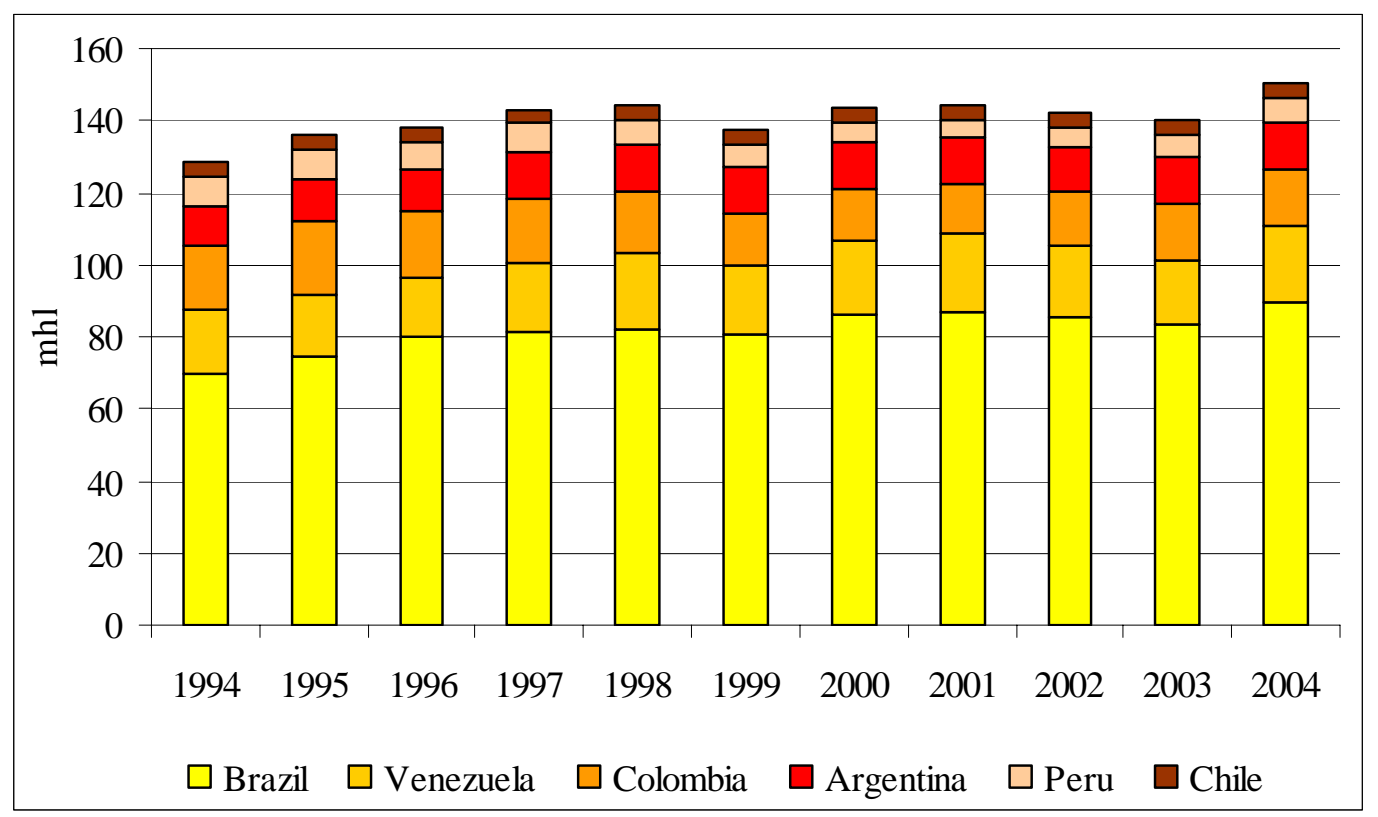

Source: (Joh.Barth\&Sohn, 1995; Joh.Barth\&Sohn, 2005; Plato, 2005a).

The following figure detects some large variations in terms of volume and per capita consumption within the Southern American beer market which is dominated by Brazil, the fourth-largest beer market world-wide, reaching a production volume of more than 89 million hectolitres (mhl) in 2004.

Figure 2: Southern American Consumption and Per Capita Consumption.

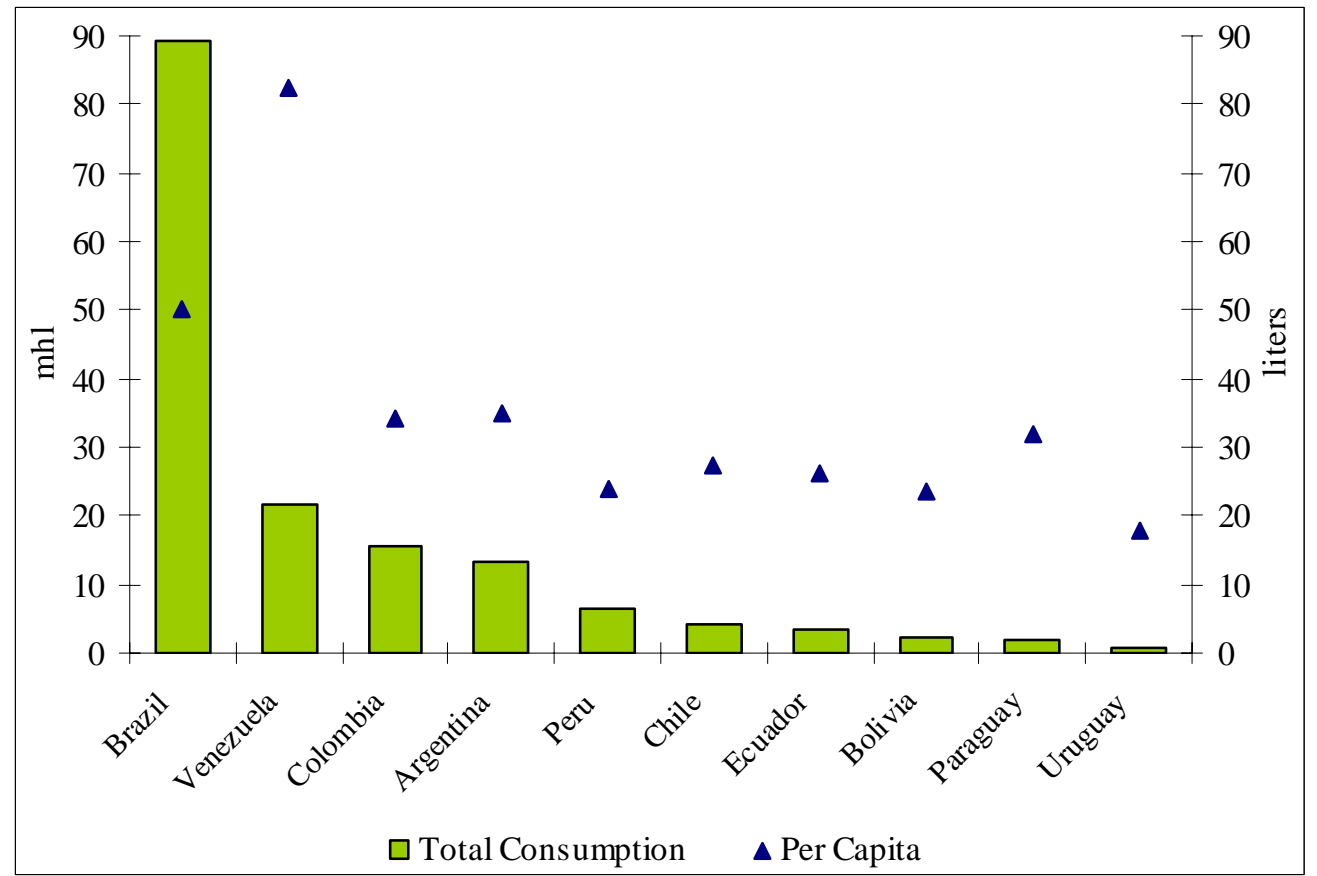

Source: (Plato, 2005a). 
The Brazilian market is followed by far by Venezuela $(21.5 \mathrm{mhl})$, Colombia (15.4mhl) and Argentina $(13.5 \mathrm{mhl})$ and a third group which consists of Peru $(6.5 \mathrm{mhl})$, Chile $(4.3 \mathrm{mhl})$ and Ecuador (3.5mhl). The remaining seven Latin American countries hardly play a significant role in terms of volume. We can see that some markets already have reached a quite high level of per capita consumption that does not much lie behind developed and matured markets in the Western hemisphere (average consumption ca. 70-80 liters per year). Brazil and Venezuela do not seem to offer much space for future growth in per capita consumption. Venezuela in particular has attained a saturated level of per capita consumption and is even decreasing since a couple of years (see also table 1). The remaining eight markets in figure 2 have all about the same per capita consumption that ranges between approximately 20 and 35 liters. These markets still have enough potential for further growth in beer consumption.

Interestingly in investigating the South American beer market is the dominance of big brands, in opposite to the whole world beer market, which is characterized by a fragmented brand structure. In 2004, the leading ten beer brands around the globe just account for $18.6 \%$ of the total world beer production, whereas South Americans biggest brands Antarctica (9.0mhl), Polar (11.1 mhl), Schincariol (11.4mhl), Brahma (19.7mhl) and Skol (31.2mhl) represent $51.8 \%$ of Latin America's beer volume.

Apart from production volumes and markets, analyzing the Latin American beer market demands glancing at the leading players within the South American brewing scene.

Figure 3: Southern American Largest Brewers - Volumes and Operating Statistics.
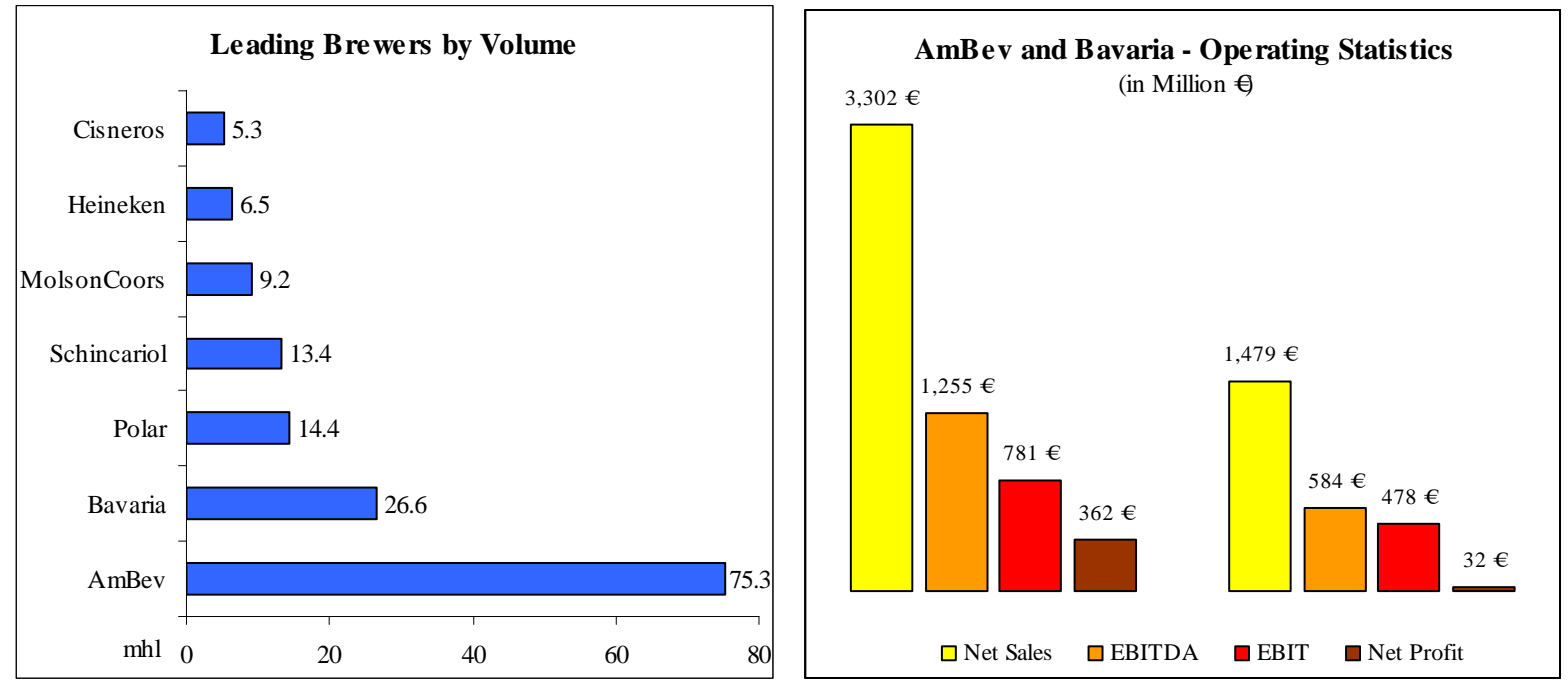

Source: Company publications, author's calculations, (Plato, 2005a). 
Looking at the right section of the above figure gives some information of outstanding importance if one analyzes the world brewing industry. Comparing AmBev's and Bavaria's financial performance data with the world-wide leading stock-listed brewing groups reveals that these brewers both are well ahead of the rest of their industry's peer group. The authors of this article have carefully examined in-depth the balance sheets and profit\&loss accounts of the 22 largest brewers around the globe. The average EBITDA-margin for the financial year 2004 has been $22.7 \%$; at the same time the EBIT-margin (= return on sales) has been $15.3 \%$. AmBev (38.0\% and 23.6\%) and Bavaria (39.5\% and 32.3\%) outperform these figures by far. With an EBIT-margin of $32.3 \%$, Bavaria more than doubles the industry's average $(15.3 \%)$. These extraordinary financial ratios can mainly be explained by the enormous domestic market shares of AmBev and Bavaria. Bavaria e.g., holds a 99.6\% market share in Colombia and Peru, respectively; AmBev controls about $68 \%$ of the huge Brazilian beer market. But not even these excellent performance figures could prevent AmBev and Bavaria from being acquired. None of them could hold up its independence, and have been bought by European brewing giants consecutively; the first deal has been Interbrew's merger with AmBev in March 2004, creating the world's largest brewer in terms of production volume, the second was SABMiller's spectacular acquisition of Bavaria in July 2005 for approximately $6.5 € \mathrm{bn}$.

\section{Main Markets in Detail}

Breaking down the regional development of South America to single markets, the following table gives an overview of the largest Latin American markets, ranked by production volume in 2004. As figure 1 has highlighted, these leading six markets account for approximately 94\% of the total South America's volume. Table 1 highlights total consumption, per capita consumption, imports and exports as well as the respective three market leaders. In the following section, these main markets are described in-depth (see also: Deutsche Bank Industry Research 'Global Brewing 2005', Plato Logic Limited). 
Table 1: Main South American Beer Markets - Key Data.

\begin{tabular}{|c|c|c|c|c|c|c|c|c|c|c|c|c|}
\hline & \multicolumn{2}{|c|}{ Brazil } & \multicolumn{2}{|c|}{ Venezuela } & \multicolumn{2}{|c|}{ Colombia } & \multicolumn{2}{|c|}{ Argentina } & \multicolumn{2}{|c|}{ Peru } & \multicolumn{2}{|c|}{ Chile } \\
\hline & 2000 & 2004 & 2000 & 2004 & 2000 & 2004 & 2000 & 2004 & 2000 & 2004 & 2000 & 2004 \\
\hline Per capita consumption (1) & 51.4 & 50.3 & 87.2 & 82.5 & 33.6 & 34.2 & 34.7 & 35.0 & 22.0 & 23.9 & 26.5 & 27.2 \\
\hline Beer imports $(000 \mathrm{hl})$ & 40 & 20 & 18 & 30 & 138 & 209 & 95 & 69 & 12 & 23 & 62 & 79 \\
\hline Main brewers & $\mathrm{AmBev}$ & $68 \%$ & Polar & $67 \%$ & Bavaria & $100 \%$ & Quilmes & $77 \%$ & Bavaria & $100 \%$ & $\mathrm{CCU}$ & $90 \%$ \\
\hline \multirow[t]{3}{*}{ Market share (\%) } & Schincariol & $12 \%$ & Regional & $19 \%$ & - & - & $\mathrm{CCU}$ & $15 \%$ & - & - & Quilmes & $9 \%$ \\
\hline & Molson & $10 \%$ & AmBev & $13 \%$ & - & - & Warsteiner & $7 \%$ & - & - & - & - \\
\hline & Top 5 & $98 \%$ & Top 5 & $100 \%$ & Top 5 & $100 \%$ & Top 5 & $100 \%$ & Top 5 & $100 \%$ & Top 5 & $100 \%$ \\
\hline
\end{tabular}

Source: (Bevan and Greenberg, 2005; Plato, 2005b; Plato, 2005a).

\section{Brazil}

Brazil is the world's fourth largest beer market $(89 \mathrm{mhl}$ in 2004) and is still growing by a CAGR of $2.5 \%$. However, per capita beer consumption had been fairly stable since 1995 and therefore still offers some space for further growth to reach the level of other large markets and. The market structure has evolved dramatically over the past five years. First, the merger between Brahma and Antarctica in March 2000 resulted in the creation of AmBev, which nowadays controls $68 \%$ of the beer market and $40 \%$ of the total beverage market. In 2001, Canadian brewer Molson bought the Bavaria brand from AmBev and acquired Brazil's second largest brewer Kaiser in May 2002 (14\% market share) from the Coca-Cola Company. Privately-owned brewer Schincariol could increase its market share by nearly to $12 \%$ by yearend 2004, becoming the new number two in Brazil. In contrast, Kaiser (Molson) has experienced increasing difficulties and its most recent market share has been below $10 \%$. Throughout 2005, industry volumes have been growing at a high-single digit pace as a result of excellent weather and an improving consumption environment (Bevan and Greenberg, 2005). The bulk of beer sales in Brazil (70\%) are through the on-premise segment in $600 \mathrm{ml}$ returnable glass bottles. Historically, the trend has been for joint beer and soft drink distribution, as there are no alcohol licence requirements for the majority of the points of sale. Uncertainty currently exists over the future ownership of Kaiser and Schincariol and it is possible both assets will change hands during 2006. Probable acquirer could be in each case Heineken or SABMiller. 


\section{Chile}

Although CCU continues to dominate the Chilean market with 90\&, the entrance of Quilmes ten years ago prompted heavy promotional activity, which benefited the domestic beer market. Between 1998 and 2002 overall volume growth and per capita consumption remained flat as a result of depressed consumption spending in Chile and the reduction of the price of wine in the domestic market. However, the 2004-2005-period has seen a steady increase in beer volumes as a result of a recovery in domestic consumption and poor grape harvests that have resulted in an increase in wine prices, the main substitute for beer in Chile. Despite CCU's high market share, the Quilmes/AmBev agreement presents the likelihood of a more aggressive pricing environment in Chile once AmBev takes full control of Quilmes in 2009, owing to the emergence of a much stronger No. 2 player. In 2004, around $70 \%$ of beer sales were packaged in returnable glass bottles (Bevan and Greenberg, 2005). In contrast, onpremise beer consumption is fairly low. According to Deutsche Bank Industry Research, the recovery of beer consumption in Chile will continue to be a function of this country's solid economic recovery, which begun to materialize in 2004, and the price of substitute alcoholic beverages like wine.

\section{Argentina}

Beer consumption in Argentina grew considerably in the early 1990s, reflecting the favorable economic environment and a shift towards low-alcoholic beverages in this typically winedrinking country. The economic slowdown that prevailed in the end-1990s caused beer consumption to remain relatively stagnant during that period. The main players in Argentina are Quilmes (77\%) and CCU (15\%). In May 2002, AmBev and Quilmes announced an agreement to integrate their operations. This transaction has had a remarkable effect on the market structure. Quilmes has now regained its clear leadership and is implementing new commercial strategies that are likely to contribute to the expansion of per capita consumption. The combined market share of AmBev and Quilmes is ca. 80\%. After the closing of the AmBev-Quilmes deal in 2003, Heineken sold its stake in Quilmes' to AmBev and transferred the brewing rights to the Heineken brand in Argentina to CCU. Most beer sales in Argentina are conducted through independent distributors (over $80 \%$ in 2004), which usually carry a company's products on an exclusive basis within a defined area. 


\section{Colombia}

Based on Plato statistics, total beer consumption in 2004 was ca. 15.4mhl - equivalent to a per capita consumption of 34.2 liter. However, if we go back to the mid-1990s, total beer consumption in Colombia was $20.4 \mathrm{mhl}$ and per capita consumption was 53 liters. This sharp fall in consumption reflected the late-1990s economic crisis, but it would suggest that culturally there is nothing to preclude per capita consumption from rising back towards its historical 45-50 liter level. Canadean and Deutsche Bank Research expect the Colombian beer market to grow volumes by $2 \%$ per annum over the next five years. Colombia offers a very attractive beer market with relatively low per capita consumption, heavy market concentration (Bavaria 99.6\%), high prices and stable macroeconomic environment. While Bavaria was clearly the quickest way for SABMiller to establish a dominant position in Colombia, there are other ways to enter this attractive market (Bevan and Greenberg, 2005). AmBev could replicate the successful entry model it has established in other countries by acquiring Pepsi franchises and combining them with greenfield beer operations. The dynamics of the industry are likely to intensify in favor of consumption with the entrance of SAB Miller in the management of Bavaria.

\section{Peru}

Peru's beer market size was $6.5 \mathrm{mhl}$ at year-end 2004, coming almost entirely from Backus (owned and controlled by Bavaria: 99.6\% market share). One of the attractive features of the Peruvian beer market is its low per capita consumption of only 23.9 liters versus its peak of 34 liters in the mid-1990s. The market in Peru has been steadily recovering after a tremendous decline of $7.8 \mathrm{mhl}$ in 1997 to $5.3 \mathrm{mhl}$ in 2001. In February 2003, AmBev announced its decision to enter the Peruvian market through investing US\$38mn in a greenfield project to build a brewing production facility. A few months later, AmBev acquired assets from Peru's main Pepsi bottler for US\$32mn, providing it with the Pepsi franchise for Lima and Northern Peru. AmBev quickly upgraded its soft drinks distribution system in Peru by introducing AmBev sales tools to its direct distribution system in Lima and direct distribution in key areas in the north of the country. Given its already strong distribution presence on the soft drinks side, brokers like Deutsche Bank Industry Research believe AmBev will be able to quickly garner market share in Peru. 


\section{Venezuela}

Venezuela is a very competitive market with a relatively high consumption per capita $(82.5$ liter) on by the end of 2004. Polar is the leading player in the market with a $67 \%$ market share, followed by Regional (19\%) and AmBev (13\%). Both brewers have taken market share away from Polar in the last five years. AmBev entered the Venezuelan market in 1994 through the acquisition of Cervecera Nacional and produces and commercializes its flagship brand Brahma and Brahma Light in Venezuela on a nationwide scale. AmBev's superior revenue management tools and exchange of best practices should secure a long-term market share of at least $25 \%$ by 2010 . Beer consumption in Venezuela dropped by $20 \%$ in 2001 to 2003 due to the harsh economic and political crisis the country endured during this period. Despite a rapid recovery in 2004 and 2005, beer consumption per capita is still far from the high levels achieved in 2000 and 2001 of 87 and 90 liters. The market is expected to continue with its upturn trend- rising close to $4 \%$ in the next five years (Bevan and Greenberg, 2005). However, faster growth rates in order to reach its very high consumption levels will depend on the country's sustained economic recovery, which at this point is not guaranteed given its high political risk.

\section{REFERENCES}

Bevan, N. and Greenberg, M. (2005). Global Brewing - The Global Pitcher 2005 - Volume VI, Deutsche Bank Report, London.

Joh.Barth\&Sohn (1995). The Barth Report. Hops 1994/95, Nuremberg.

Joh.Barth\&Sohn (2005). The Barth Report. Hops 2004/05, Nuremberg. Plato (2005a). South America Beer Report, Plato Logic Limited, London.

Plato (2005b). World Brewer Factfile: International Groups - Datasheets \& Consolidations, Plato Logic Limited, London. 
KAPITEL I.6

Overview over Northern American Beer Markets

\section{Oliver Ebneth}

This article will be published in:

Brewing and Beverage Industry International 6(2006). 


\section{Overview over Northern American Beer Markets}

With a production volume of $324 \mathrm{mhl}$ and world market share of $20.9 \%$ respectively, Northern America still is the second-largest beer market world-wide, behind Asia (28.4\%) and in front of Western Europe (20.6\%). In this expose we describe in detail this Northern American market which consists of the United States, Canada and Mexico. The latter two markets will be presented in two short paragraphs as the main focus of this article is to emphasize on the US beer market. Hereby, we stress on consumption trends, recent market developments and market segmentation, patterns of foreign trade and pay attention to the competitive scene in particular. The outlook for North America appears quite mixed. On the one hand side, per capita volume in the Mexican market has grown remarkably each year over the last decade and is unlikely to loose the pace in the near future due to favorable demographics. The Canadian market showed minimal growth in 2005, reflecting a stagnating but stable market size since more than a decade. On the other side, the US American shift towards a surge in demand for low carbohydrate products had a material impact on the beer industry. From an overall perspective the impact has been negative - shifting alcohol consumption away from beer and towards areas with lower carbohydrates ('carbs') e.g. spirits and wine. However, the trend has also created a series of winners and losers within the US beer market.

\section{Canada}

The Canadian beer market is characterized by a relatively steady consumption volume of ca. $23 \mathrm{mhl}$. Similarly, the per capita consumption was stable over the last decade at ca. 68 liter per annum (see figure 1). Indeed, Canada is the world's $13^{\text {th }}$ largest beer market in terms of production volume, but in terms of EBITDA generated by the brewing companies, Canada represents the sixth most profitable beer market with an estimated EBITDA-pool of approximately US\$680mn. Profit per hectoliter is guessed to amount to more than US\$30. The growing demand for value brands is causing some challenges for the two dominant operators - Labatt (InBev) and Molson (mergered with Coors in 2004 creating the world's number six Molson Coors Brewing Company). The growth of the value category has threatened to destabilize the positive pricing environment which has characterized the Canadian market for a number of years. 
Figure 1: Consumption and Per Capita Consumption - Canada.

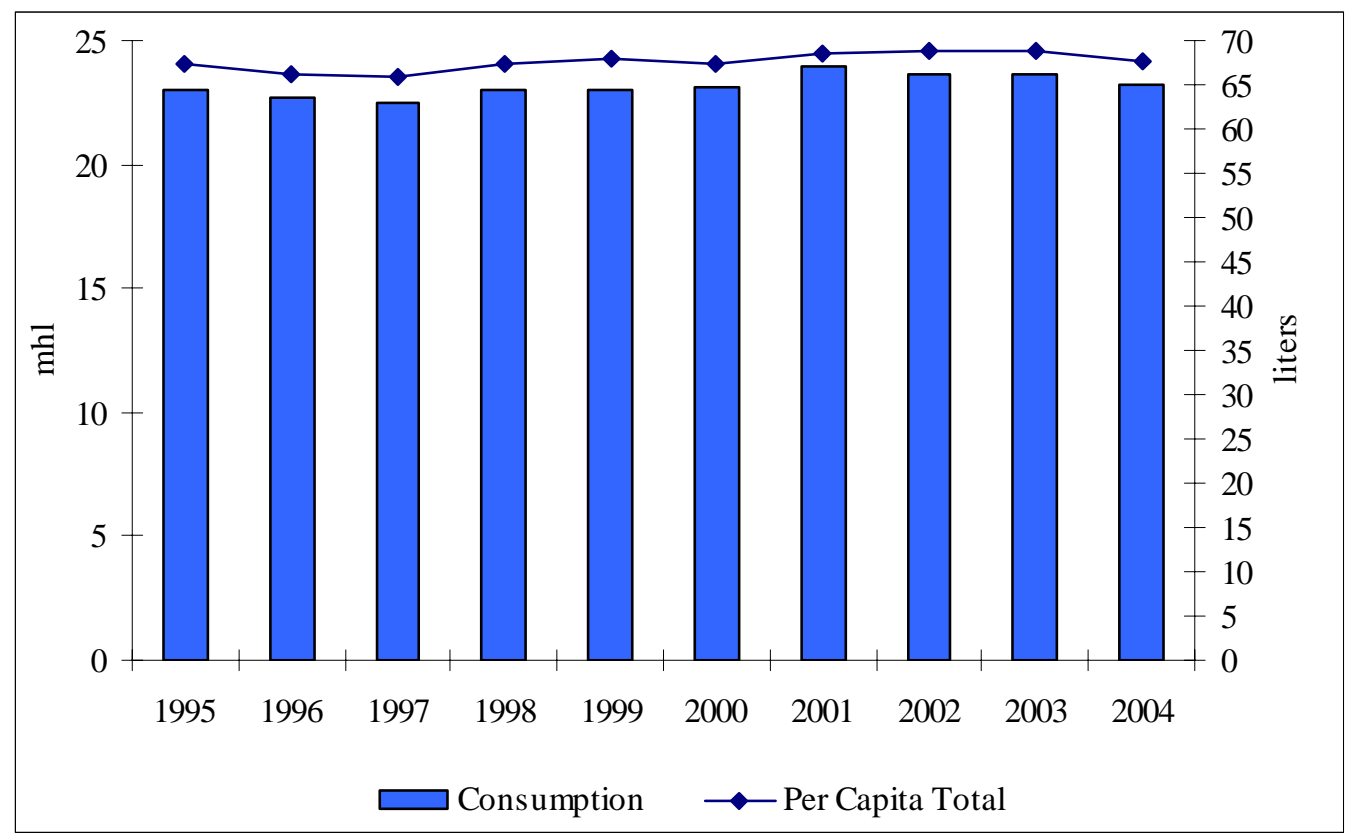

Source: (Joh.Barth\&Sohn, 1995; Joh.Barth\&Sohn, 2005; Plato, 2005).

The import category currently represents around $10 \%$ of the market dominated by two brands, Mexican Corona and European premium lager brand Heineken, both imported by Molson. Labatt has enjoyed success with Budweiser - which is brewed domestically - such that it now ranks as Labatt's largest brand. In Canada, we can detect the same trend like in the USA, towards 'low carb beer'. Consequently, Molson is enjoying positive momentum with Coors Lite. Labatt, originally a subsidiary of the Belgian-based Interbrew, is now a part of InBev, following Interbrew's merger with AmBev of Brazil in March 2004. This has resulted in an aggressive drive to reduce costs - including the closure of two breweries. In January 2005 Molson announced its 'merger of equals' with US-based brewer Coors to create the world's sixth-largest brewer by volume (see also Deutsche Bank Industry Strategy 'Global Brewing $\left.2005^{\prime}\right)$.

\section{Mexico}

Mexico is now the sixth largest beer market in the world with per capita consumption of 52.5 liters and a total consumption volume of $68.5 \mathrm{mhl}$. Like Canada and the USA, Mexico is one of the most profitable beer markets in the world. In terms of profitability, the cumulated Mexican EBITDA-pool is estimated to US\$945mn at year-end 2004. Thus, Mexico stands for the third largest profit pool in the world beer industry, behind the USA and Japan, followed 
by Brazil and the UK. The profit per hectoliter amounts to around US\$18. Figure 2 shows the sound and sustained consumption growth within the Mexican beer industry.

Figure 2: Consumption and Per Capita Consumption - Mexico.

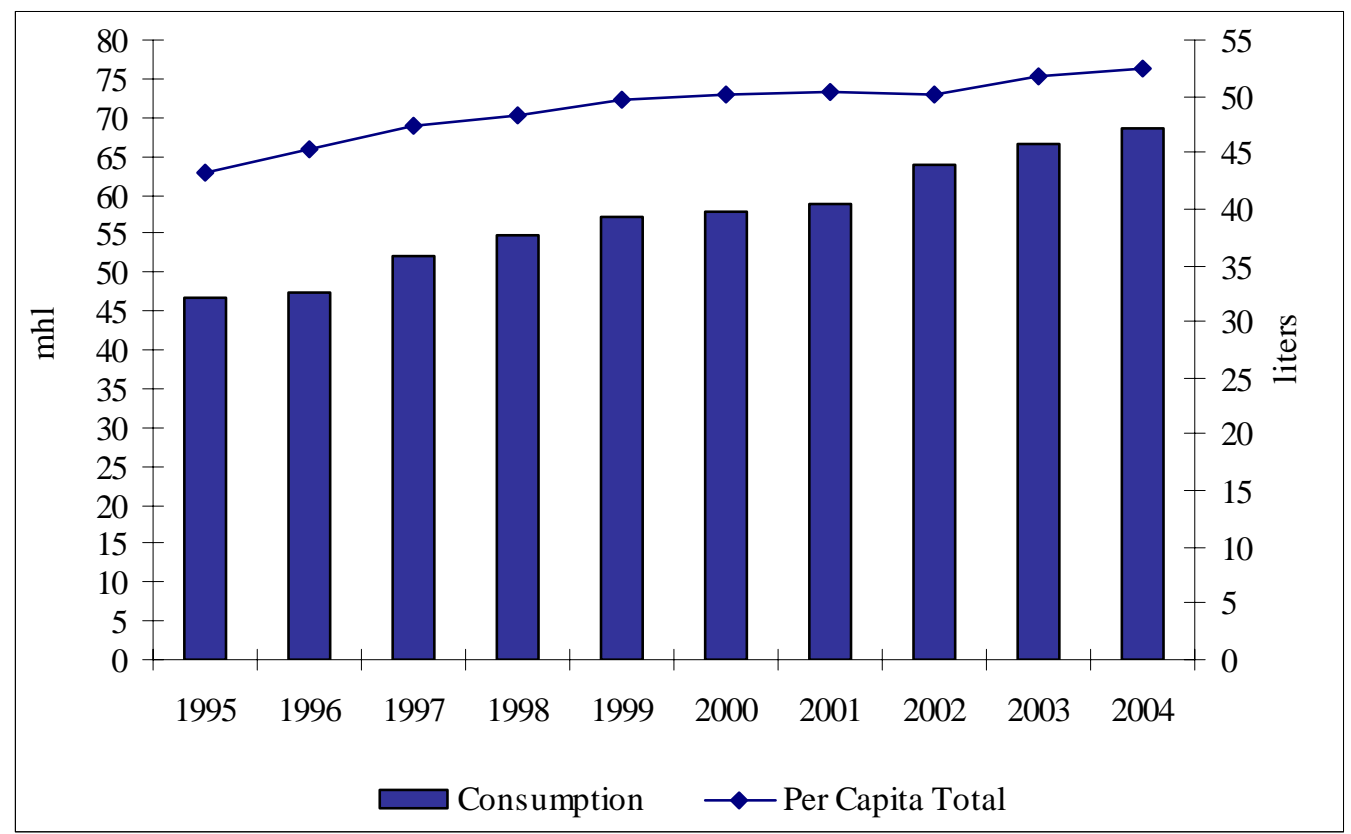

Source: Plato Logic Limited, Barth Report 1995-2005.

The Mexican beer industry is a relatively stable duopoly. Since the mid-1980s, the two domestic brewers Femsa and Grupo Modelo have dominated this market. In May 2004 Femsa loosened its ties with InBev, which held a 30\% stake in Femsa's beer division, and afterwards established an exporting partnership with Heineken USA and a licence to import and market Coors light in Mexico. Anheuser Busch has 50.2\% economic interest in the main subsidiary of Grupo Modelo. The off-premise channel serves around $80 \%$ of total Mexican beer demand. Approximately $70 \%$ of the sales volume is sold in returnable bottles. After seven years of consistent real price increases (1995- 2002) to recover gross margin, the industry reached a pricing plateau. Various brokers expect pricing to be in line with inflation at best, otherwise volume expansion will be limited (Bevan and Greenberg, 2005; Yule et al, 2005).

Before describing the US beer market in-depth we throw a glance away from single market, volume and consumption issues and towards the main player and their role in the respective North American markets. As table 1 illustrates, there are four main stock-listed brewing groups that play an important role in the North American brewing scene. For many, years, US-based Anheuser Busch (A-B) has been the largest brewer by production volume. But over 
recent years, A-B was outrun first by Belgium-based InBev, few years later by its main AU competitor SABMiller. Indeed, in terms of net sales, A-B still is by far the largest brewing group around the globe. Table 1 highlights some operating statistics about US-based brewers A-B and Coors, Canadian brewer Molson and the Mexican-based Grupo Modelo. A-B and Grupo Modelo (50.2\%-owned by A-B), are some of the most profitable in the whole world brewing industry, offering excellent profitability ratios. The EBITDA-margin of Anheuser Busch (Modelo respectively) was 28.8\% (34.0\%) and the Return on Sales (ROS) was 22.5\% (29.3\%). Both brewers, but Grupo Modelo in particular are well ahead of their peer group of leading publicly listed brewing groups. Molson, but especially Coors, perform much more feebly, showing EBITDA-margins of $22.8 \%$ and $14.1 \%$ and a ROS of $20.3 \%$ and $7.9 \%$.

Table 1: Northern America’s Largest Brewers - Operating Statistics 2004.

\begin{tabular}{|c|c|c|c|c|c|c|c|}
\hline & Country of & $\begin{array}{c}\text { Volume } \\
\text { (mhl) }\end{array}$ & $\begin{array}{c}\text { Market } \\
\text { Cap (€m) } \\
\end{array}$ & $\begin{array}{c}\text { Net Sales } \\
(€ \mathrm{~m}) \\
\end{array}$ & $\begin{array}{c}\text { EBITDA } \\
(€ \mathrm{~m}) \\
\end{array}$ & $\begin{array}{c}\text { EBIT } \\
(€ m) \\
\end{array}$ & $\begin{array}{c}\text { Net Profit } \\
(€ \mathbf{m}) \\
\end{array}$ \\
\hline Company & Incorporation & 2004 & Sep 05 & 2004 & 2004 & 2004 & 2004 \\
\hline Anheuser Busch & USA & 144.2 & 27,874 & 12,006 & 3,468 & 2,724 & 1,830 \\
\hline Coors & USA & 38.4 & 4,392 & 3,462 & 489 & 275 & 166 \\
\hline Molson & Canada & 21.0 & 2,905 & 1,589 & 362 & 322 & 149 \\
\hline Modelo & Mexico & 41.9 & 8,579 & 3,186 & 1,084 & 935 & 440 \\
\hline
\end{tabular}

Source: Company publications, author's calculations.

One main reason for Anheuser Busch's and Modelo's corporate success is their extreme domestic market shares. The Mexican market leader Grupo Modelo e.g. can base its business upon a comfortable 56\% market share in the fast growing Mexican beer market, which nowadays is the number six world-wide. As we can see from table 2, all three North American markets are highly dominated by few main players. The Mexican market is divided by a Duopoly, consisting of Femsa and Modelo, the Canadian market can be characterized by e Oligopoly, dominated by Molson (44\% market share) and Labatt (42\% market share). Whereas Canada and Mexico are completely consolidated, the US market still offers some space for smaller competitors, but the CR10 already is beyond $95 \%$. 
Table 2: Main North American Beer Markets - Key Data.

\begin{tabular}{|c|c|c|c|c|c|c|}
\hline & \multicolumn{2}{|c|}{ USA } & \multicolumn{2}{|c|}{ Canada } & \multicolumn{2}{|c|}{ Mexico } \\
\hline & 2000 & 2004 & 2000 & 2004 & 2000 & 2004 \\
\hline Beer consumption (mhl) & 232,500 & 233,300 & 23,074 & 23,130 & 57,812 & 68,482 \\
\hline Per capita consumption (1) & 83.0 & 79.0 & 67.0 & 69.0 & 50.9 & 51.8 \\
\hline Beer imports (000 hl) & 23,477 & 29,150 & 1,199 & 1,677 & 218 & 567 \\
\hline Beer exports $(000 \mathrm{hl})$ & 2,497 & 2,245 & 3,936 & 4,267 & 10,278 & 14,473 \\
\hline Main brewers & Anheuser-B & $50 \%$ & Molson & $44 \%$ & Modelo & $56 \%$ \\
\hline \multirow[t]{3}{*}{ Market share (\%) } & Miller & $18 \%$ & Labatt & $42 \%$ & Femsa & $43 \%$ \\
\hline & Coors & $10 \%$ & Sleeman & $5 \%$ & - & - \\
\hline & Top 5 & $82 \%$ & Top 5 & $98 \%$ & Top 5 & $99 \%$ \\
\hline
\end{tabular}

Source: (Bevan and Greenberg, 2005; Plato, 2005).

\section{USA}

The USA are now the second largest beer market world-wide, overtaken by China in 2003 but still offering a consumption of $233 \mathrm{mhl}$. This amount is based on both, a relatively high and stable per capita consumption and a slightly increasing population. Thus, the outlook for the longer-term is likely to be stagnation rather than any sustained movement up or down. Figure 3 highlights recent developments of both, the US per capita consumption as well as the total amount of national beer consumption. 
Figure 3: Consumption and Per Capita Consumption - United States.

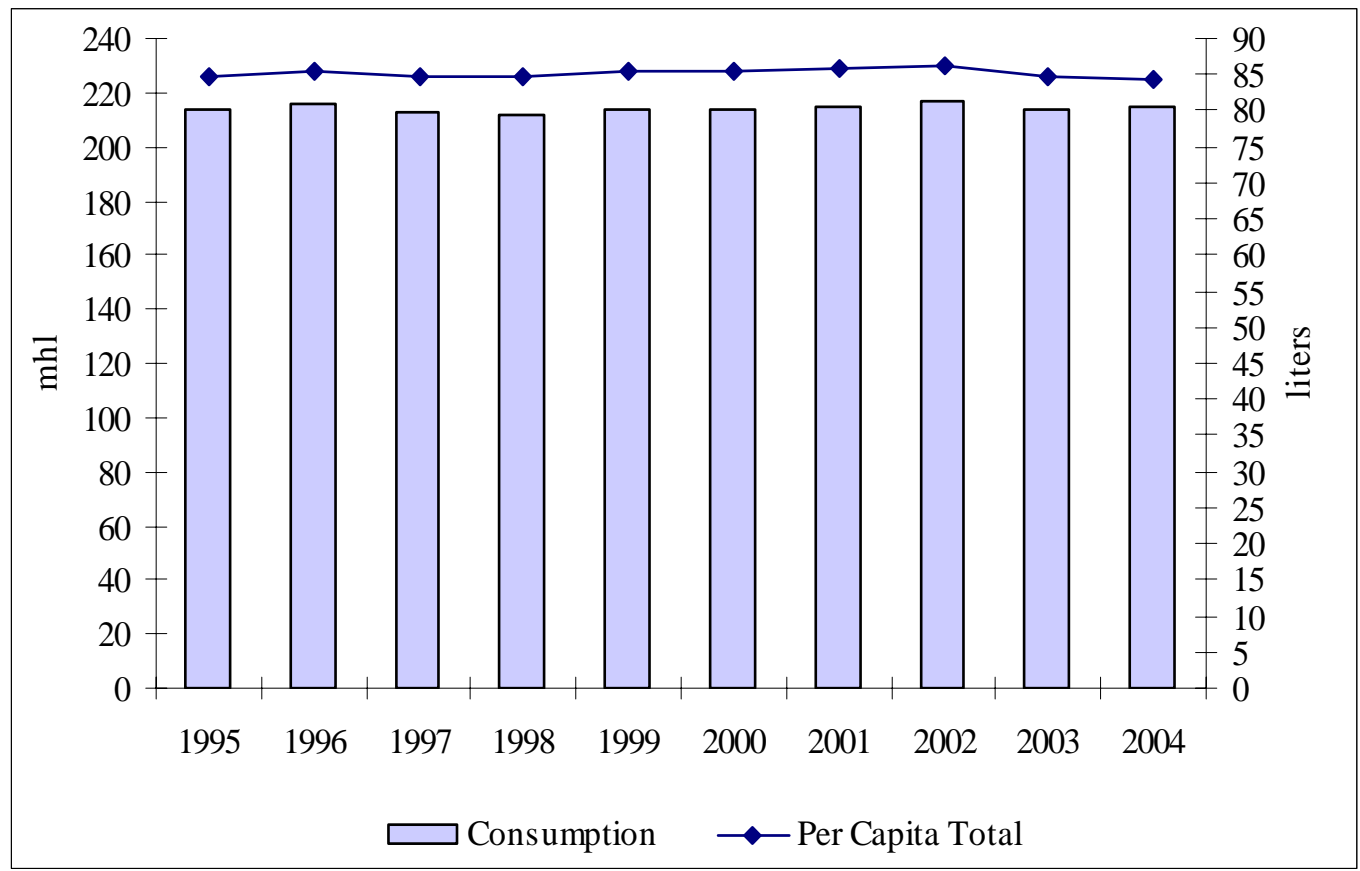

Source: Plato Logic Limited, Barth Report 1995-2005.

Total consumer spend on beer exceeded US\$60bn in 2004 and the total US profit-pool (expressed in EBITDA) is estimated at more than US\$5bn. Thus, the USA are still by far the most profitable beer market world-wide. Our survey about the US beer market is mainly based on Plato Logic Limited (see 'USA Beer Market 2005').

\section{Exports and Imports in the US Beer Market}

Confronted with a saturated domestic market, US brewers seek to develop their export activities over recent years. In 2004, 2.24mhl were exported, although this is well down on the mid-1990s peak of 6.7mhl. Major markets are Mexico, Japan and China. Indeed, imports have long been a niche in the US beer market but in the course of the last years, foreign brewing groups could foster their import businesses, reaching a new peak of $29.15 \mathrm{mhl}$ in 2004. Thus, the US market is still the largest import market world-wide. This import sector can be divided into three major categories: First, international premium lagers, led by European brewing groups such as Heineken and European specialties (e.g. stout and ale); Second, ice beers and regular brands from Canada; Third, Mexican beers, especially Corona. 


\section{Competition and Branding in the US Brewing Scene}

About $80 \%$ of the US market is accounted by a combination of mainstream beers and 'light' beers. Light beers (e.g. Michelob Ultra), which take around $50 \%$ of demand, are the largest category since the end-1990s and continue to take share. The US beer industry is polarized between large national stock-listed brewing groups and so called 'microbreweries'. The 'big three' consist of the world's number three, Anheuser Busch, Miller (part of SABMiller) and Coors. South African Breweries' acquisition of Miller in May 2002 for approximately €6.2bn, was the biggest deal, so far seen in the world brewing scene. Since then, Miller's share has begun to recover, but it is likely that SAB's strategy will be to operate as a strong and firm No. 2. Another large transaction has been the merger between Molson and Coors in 2004 (ca. $€ 3.2 \mathrm{bn}$ ), creating Molson Coors Brewing Company (MCBC). MCBC is a comparatively distant No. 3 with $10 \%$ market share, but with more than $26 \mathrm{mhl}$ in the USA it is still one of the world's leading brewing groups. The other medium-sized brewers have been struggling in recent years. At the end-1990s, Heileman and Stroh both have withdrawn from brewing business. Pabst remains the No. 4 with approximately $8.8 \mathrm{mhl}$, followed by Heineken (USA) and Labatt (InBev). The last domestic brewer with significant production volume is the Boston Beer Company (1.5mhl). Despite the importance of the leading large brewing groups, with the 'top three' accounting for $83 \%$ of the total market, the emergence of the microbreweries is a key dynamic. Growth has been astonishingly - admittedly from a quite low level - although volumes remain modest and have hardly grown since the mid-1990s. There are some 1,400 microbreweries currently in operation, and all tend to produce a wide range of specialties, often based on European traditions and recipes. The success of these microbreweries has not harmed the larger groups. It has rather encouraged diversification into more profitable areas both through new products and the creation of a price umbrella (Bevan and Greenberg, 2005; Plato, 2005). 
Figure 4 underlines the ranking of the leading US American brewers as well as the largest brands in the US brewing scene. The ranking of national brands reflects the overall competitive environment. Anheuser Busch's main brand 'Budweiser' has been the clear market leader in the USA for many years, recently overtaken by 'Bud Light'. Bud Light nowadays is A-B's growth brand, capturing market share from 'Miller Lite', the nation No. 3, but Miller also has notable mainstream volume with 'Miller Genuine Draft' and 'High Life'.

However, the clearest winner from the emergence of the low carb craze has been Miller. Miller's fortune in having the lowest carb light beer brand (Miller Lite) has served to transform the growth prospects for its flagship brand and in some senses the prospects for the entire business. The revival in Miller Lite has certainly served to dampen the growth rate of its two main rivals, Bud Light and Coors Light. But the main losers from the low carb growth have been the European importers - particularly Heineken. From a carbohydrate perspective the darker European brands are clearly at a disadvantage to lighter beers, such as the domestic light brands or the Mexican brands and this has certainly been part of the reason why the European import category has struggled to return to growth (Bevan and Greenberg, 2004; Bevan and Greenberg, 2005).

Figure 4: Largest Brewers and Leading Brands in the United States - 2004.
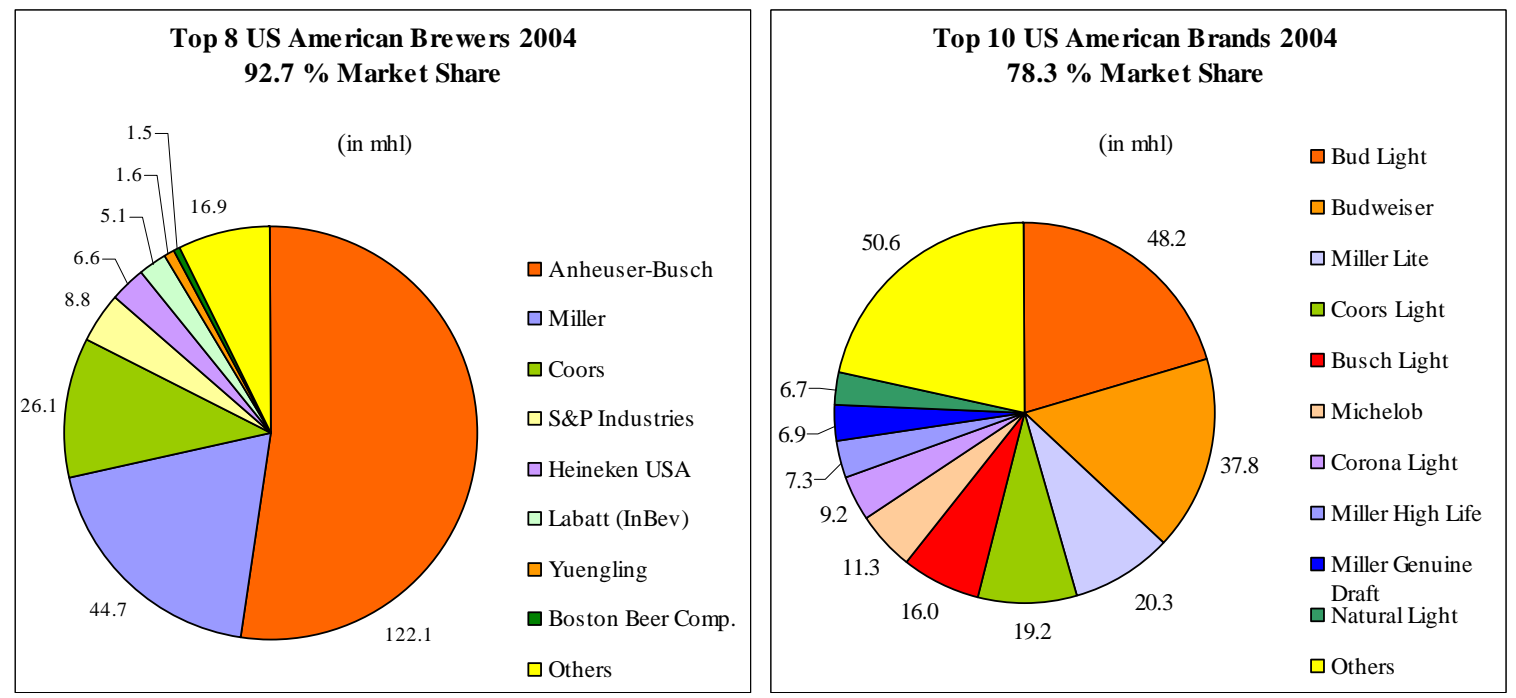

Source: Plato Logic Limited, Barth Report 2004/05.

Imported beers still are dominated by Heineken and Corona, but Corona (especially Corona Light) overtook Heineken as the leading import brand at the end-1990s. Facing this low carb wave, Heineken has just recently introduced its Heineken Light into the US market, spending an extraordinary marketing expenditure of $€ 100 \mathrm{mn}$ in 2005 . In fact, Heineken's US sales 
contribute only ca. $6 \%$ to its total sales but represent $20 \%$ of Heineken's profits, reflecting the paramount importance of the profitable US importing market.

Foster's and Miller have formed a new joint-venture to distribute the Foster's brand in the USA, which continues to be brewed by Molson. The Labatt USA business reached $5.1 \mathrm{mhl}$ in 2004 (see figure 4), including now the InBev export business (Beck's imported brands). Femsa and InBev have unwound their business in the USA in 2004; subsequently, Femsa have announced a new distribution agreement with Heineken USA to become the exclusive importer, marketer and distributor of the Femsa beer portfolio in the USA.

\section{REFERENCES}

Bevan, N. and Greenberg, M. (2004). Global Brewing - The Global Pitcher 2004 - Volume V, Deutsche Bank Report, London.

Bevan, N. and Greenberg, M. (2005). Global Brewing - The Global Pitcher 2005 - Volume VI, Deutsche Bank Report, London.

Joh.Barth\&Sohn (1995). The Barth Report. Hops 1994/95, Nuremberg.

Joh.Barth\&Sohn (2005). The Barth Report. Hops 2004/05, Nuremberg.

Plato (2005). Country Draughts: USA Beer Market, Plato Logic Limited, London.

Yule, M. et al (2005). Beer Appreciation: Episode 1. Morgan Stanley Research, London. 


\section{KAPITEL II}

INTERNATIONALISIERUNG FÜHRENDER BRAUKONZERNE ALS TRIEBFEDER ZUNEHMENDER KONSOLIDIERUNG DER BRAUBRANCHE 


\section{KAPITEL II.1}

\section{Exploring Globalization in the Brewing Industry Internationalization Strategies of World-wide Leading Brewers}

Ludwig Theuvsen and Oliver Ebneth

Poster presented at the $14^{\text {th }}$ Annual World Food and Agribusiness Forum, $12^{\text {th }}-15^{\text {th }}$ of June 2005, Montreux. 


\section{Exploring Globalization in the Brewing Industry Internationalization Strategies of World-wide Leading Brewers}

\section{Problem Statement and Objectives}

Although globalization is a general trend in many industries, the brewing industry has long been lagging behind and has remained very fragmented. It is sobering to learn that beer is one of the least global consumer goods. Globalization in the beer industry has proceeded at a much slower pace than in many related businesses. But during recent years pioneer enterprises like Heineken or Interbrew have started internationalizing their activities in order to continue growing and expanding in view of saturated home markets. The leading brewing groups thereby follow different paths in their internationalization strategies, which are to be examined and illustrated in this paper by considering some ways of measuring the degree of internationalization as well as its applicability to the brewing industry.

Consolidation led by major international brewers and volume growth in developing markets are undoubtedly the most important recent trends in the global beer industry. According to various studies aggregate volume of the world's top 10 brewers has grown at more than four times the pace of total industry volume since the mid-1990s. But larger brewers are faced with low prospects for volume growth in developed markets leading them to seek growth either via acquisition of other brewers, by aggressive participation in developing markets or both. Nevertheless, globalization in the brewing sector is developing moderately compared to other branches of industry (Kaplan, 2003). In the soft drinks sector, 50\% of the world market is held by the top 5 players, compared to 10 companies in the beer industry and the largest brewer (Anheuser-Busch) derives $90 \%$ of its sales from its home market (Lewis, 2001). But this situation has started to change dramatically. Internationalization in the beer industry is now moving at a much higher speed. Within only two years Interbrew has become Germany's number one brewer. Internationalization in the brewing sector, therefore, deserves more scientific attention. Figure 1 illustrates the market shares of the world's largest brewing groups. 
Figure 1: Top Ten Global Brewers - 2002.

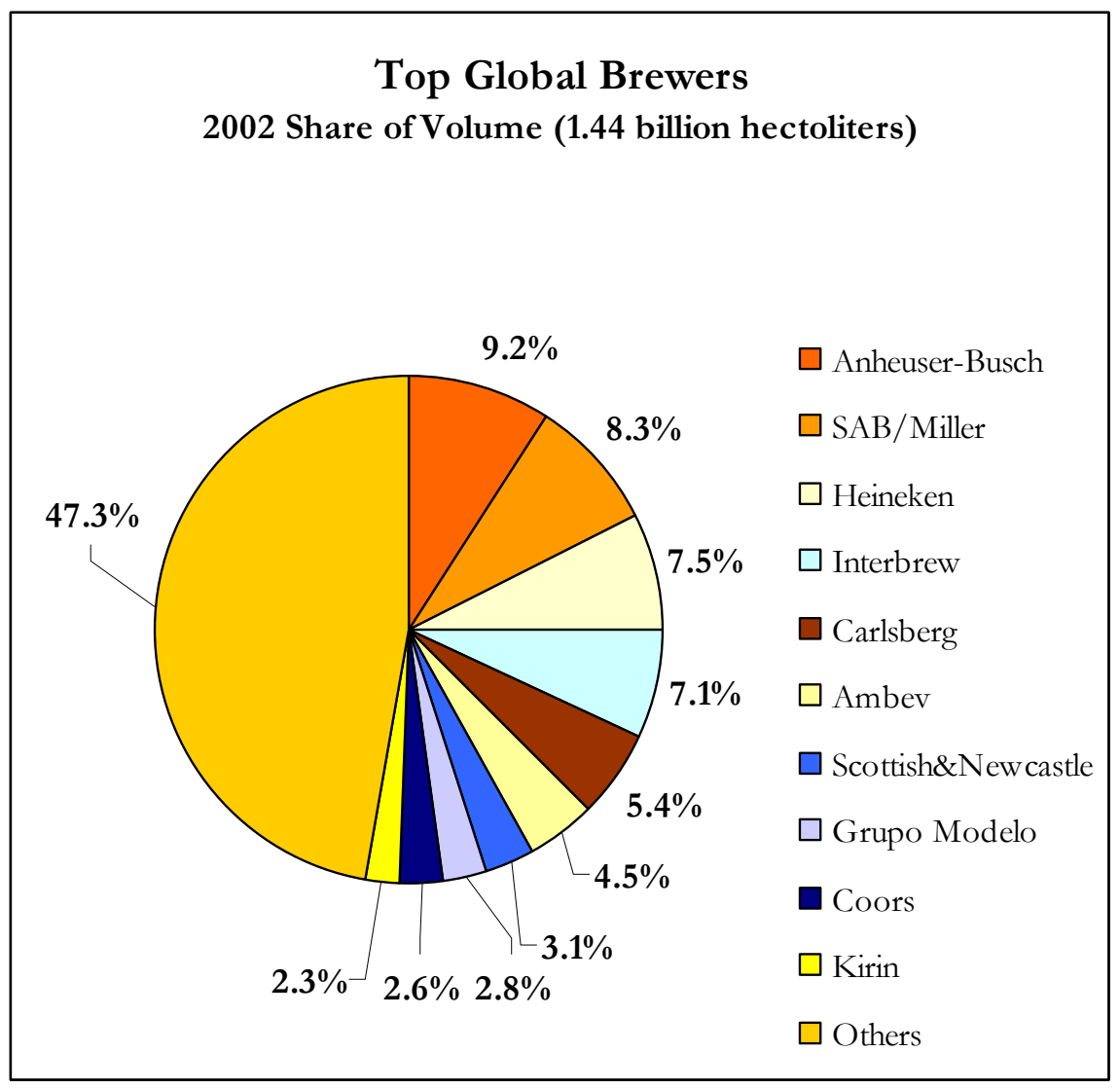

Source: Data compiled from annual reports.

But apart from simply measuring concentration degrees of an industry, this paper attempts to determine the degree of internationalization of single brewing companies by utilizing mathematical models. By applying traditional and advanced methods further conclusions are to be drawn on the different internationalization strategies used by the leading brewing groups. The central question here is as follows: Which is the degree of globalization within the regarded industry and what does this insinuate for developing a global competitive advantage in the brewing industry?

\section{Procedures}

The most common ways of measuring the degree of internationalization are quite simple, for example calculating:

- the ratio of foreign sales to total sales or the share of foreign assets to total assets (Reeb et al., 1998),

- the ratio of foreign pre-tax income to total pre-tax income (Chen et al., 1997), 
- the number of countries with foreign operations and the shares of foreign employees, profits, value added or shareholders.

Advantages of these unidimensional approaches are the availability of information compiled from databases with open access. Also foreign ratios are clear indicators of international activities and they are easy to handle in mathematical operations. But this simplicity implicates an obvious disadvantage: Such measurements capture only one single dimension of internationalization (Fisch and Oesterle, 2003).

In contrast multidimensional measures try to cover a larger and more representative range of the construct 'internationalization' but the challenge is to derive compact conclusions from a multiple set of numbers. Sullivan (1994) developed an innovative approach which can be viewed as the first multidimensional measuring concept including three dimensions of internationalization. Ramaswamy and Kroeck (1996) argue that internationalization is more complex than envisioned by this index, and it is suggested that further refinement of the construct is necessary before constructing indices. Another measure, the UNCTAD transnationality index (TNI), which weighs the percentages of foreign assets, sales and employees, suffers from corresponding deficiencies. Ietto-Gillies (1998) presented the so called 'Transnational Activity Spread Index' (TASI) which stands for a synthesis of two factors of internationalization (the volume of foreign activities and the number of countries it is spread over). But Fisch and Oesterle criticize that an arbitrarily weighted summation cannot be satisfactory because the elements represent different dimensions of internationalization. Germann et al. (1999) keep the percentage of foreign revenues and employees and a categorization of the international spread separately in so called 'bundles' of internationalization indicators. The idea of separate treatment is easy to justify but does not solve the problem. The 'Complex Spread and Diversity Measure' is probably the most fastidious concept in terms of mathematics, rejudged by Fisch and Oesterle (2003). But instead of mixing the volume of foreign activities and the number of countries in a multiplication they capture them in a joint measure of spread operating with Lorenz curve and Gini coefficient.

The examination of all annual reports of the leading brewing companies led to the conclusion that data was first available for the TNI and thus for accounting the TASI. 


\section{Results}

First findings show clear differences between the leading brewing companies regarding the degree of internationalization apart from individual market shares. Taking a closer look at the different brewers shows clearly, that there is still no sign of genuinely global beer brands for example. Thus the top 10 global brewers pursue quite different internationalization strategies, which shall be treated and demonstrated scientifically by the results of this research project.

\section{Conclusions}

Our empirical study proves that consolidation in the brewing sector is far from being concluded and the internationalization process has just begun to pick up speed. Compared to other branches, the beer brewing industry differs in the area of mergers, irrespective of how they were executed. Further research on MNCs in the agribusiness in general and in the brewing sector in detail should focus on the question whether full globalization would be economically desirable. More work should be done on evaluating the efficiency of various strategies and considering the structural consequences of different internationalization strategies.

\section{REFERENCES}

Chen, C. J. P. et al. (1997): An Investigation of the Relationship between International Activi ties and Capital Structure, in: Journal of International Business Studies, 1997(28): 563-577.

Fisch, J. H. and Oesterle, M.-J. (2003): Exploring the Globalization of German MNCs with the Complex Spread and Diversity Measure, in: Albers, S. et al. (ed.) Schmalenbach Business Review 2003(55): 2-21.

Germann, H. et al. (1999): Messung der Globalisierung: ein Paradoxon, in: Steger, U. (ed.), Facetten der Globalisierung: ökonomische, soziale und politische Aspekte, Berlin: 125 .

Ietto-Gillies, G. (1998): Different Conceptual Frameworks for the Assessment of the Degree of Internationalization: an Empirical Analysis of Various Indices for the Top 100 Transnational Corporations, in: Transnational Corporations, 1998(7): 17-39.

Kaplan, A. (2003): Global Beer: Tapping into Growth, in: Beverage World (122): 24-28.

Lewis, C. (2001): The Future of British Brewing: Strategies for Survival, in: Strategic Change (10): 151-161.

Ramaswamy, K. and Kroeck, G. (1996): Measuring the Degree of Internationalization of a Firm: A Comment, in: Journal of International Business Studies 1996(27): 167-177.

Reeb, D. et al. (1998): Systemic Risk of the Multinational Corporation, in: Journal of Interna tional Business Studies, 1998(29): 263-279.

Sullivan, D. (1994): Measuring the Degree of Internationalization of a Firm, in: Journal of International Business Studies, 1994(2): 325-342. 


\title{
Exploring Globalization in the Brewing Industry - Internationalization Strategies of World-wide Leadling Brewers -
}

\author{
Oliver Ebneth ${ }^{1}$ and Ludwig Theuvsen ${ }^{2}$
}

Problem Statement and Objectives

Although globalization is a general trend in many industries, the brewing industry has long been lagging behind and has remained very fragmented. It is sobering to leam that beer is one of the smallest global consumer goods. Globalization in the beer industry has proceeded at a much slower pace than in many similar industries. But during recen years pioneer enterprises like Heineken and Interbrew have started internationalizing ther activities in order to continue growing and expanding in view of satumted home markets. The leading brewing groups therby follow. their intemationalization strategies, which are to be examined an lllustrated in this paper by measuring the degree of internationalization (DOI), its applicability to the brewing industry as well as the companies' potential of further internationalization.

\section{Measuring the Degree of Intemationalization}

Apart from simply measuring concentration degrees of an industry it is possible to determine the degree of intemationalization of single brewing companies by applying traditional (unidimensional) and advanced (multidimensional) methods. This provides an empirical basis for further conclusions on the different intemationalization strategies of the leading brewing groups.

Unidimensional approaches

of measuring the degree of internationalization

$\ddot{y}$ the ratio of foreign sales to total sales or the share of foreign assets to total assets,

$\ddot{y}$ the ratio of foreign pretax income to total pre-tax income,

$\ddot{y}$ the number of countries with foreign perations and the shares of foreig employees, profits or shareholders.

Advantages of these unidimensional approaches are the availability of information compiled from databases with open access. Also foreign ratios are clear indicators of intem forion activities and they are easy to handle in mathematical operations.
In contrast multidimensional measures try to cover a larger and more representative range of the construct 'intemationalization':

ÿ Sullivan (1994) develops an innovative approach which can be viewed as the first multidimensional measuring concept including three dimensions of internationalization. According to Sullivan these attributes are performanoe (what goes on overseas), structural (what resources overseas) and attitudinal (top management's int orientation)

$\ddot{y}$ the UNCTAD transnationality index (TN I) is multidimensional and is calculated as the average of three ratios. Each of these elements provides scope for a unidimensional index of its own. The UNCTAD index is an average of the three as follows: $\mathrm{TNi}=[\mathrm{A} i+\mathrm{Si}+\mathrm{Ei}]: 3$, with: $\mathrm{Ai}=$ Foreign Assets Index, $\mathrm{Si}=$ Foreign Sales Index, $\mathrm{Ei}=$ Foreign Employment Index, y Ietto-G illies (1998) presented the so called 'Transnational Activity Spread Index' (TA SI) which stands for a synthesis of two factors of intemationalization (the volume of foreign activities as it is also computed for the T N $\mathrm{i}$ and the number of countries it is spread over),

$\ddot{y}$ the 'Complex Spread and Diversity Measure' Fisch and 0 esterle (2003) is probably the mathematically most advanced concept. They capture the volume of foreim activities and the number of countries in ajoint measure of spread operating with Lorenz curve and Gini coefficient.

Consolidation led by major international brewers and volume growth in developing markets are undoubtedly the most important recent trends in the global beer industry. According to various studies aggregate volume of the word's top 10 brewers has grown at more than four times the pace of total industry volume since the mid-1990s.
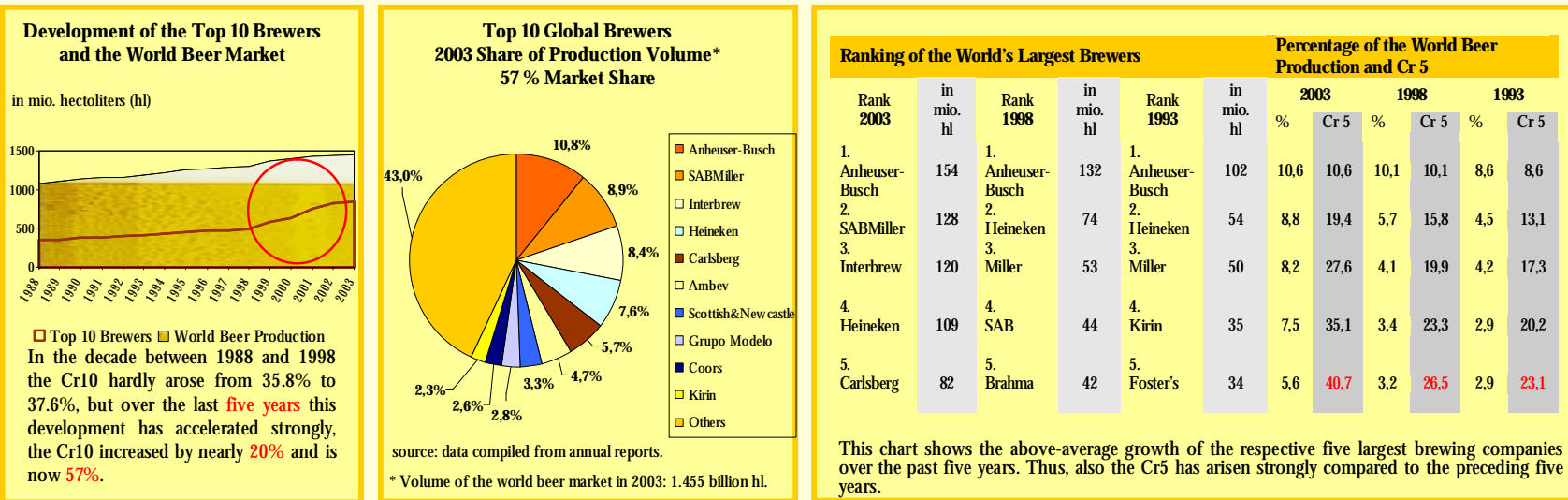

This chart shows the above-average growth of the respective five largest brewing companies over the past five years. Thus, also the $\mathrm{Cr} 5$ has arisen strongly compared to the preceding five years.

Our empirical study shows clear differences between the leading brewing companies regarding the degree of internationalization apart from individual market shares. Furthermore first findings prove that consolidation in the brewing sector is far from ending and the internationalization process has just begun to pick up speed.

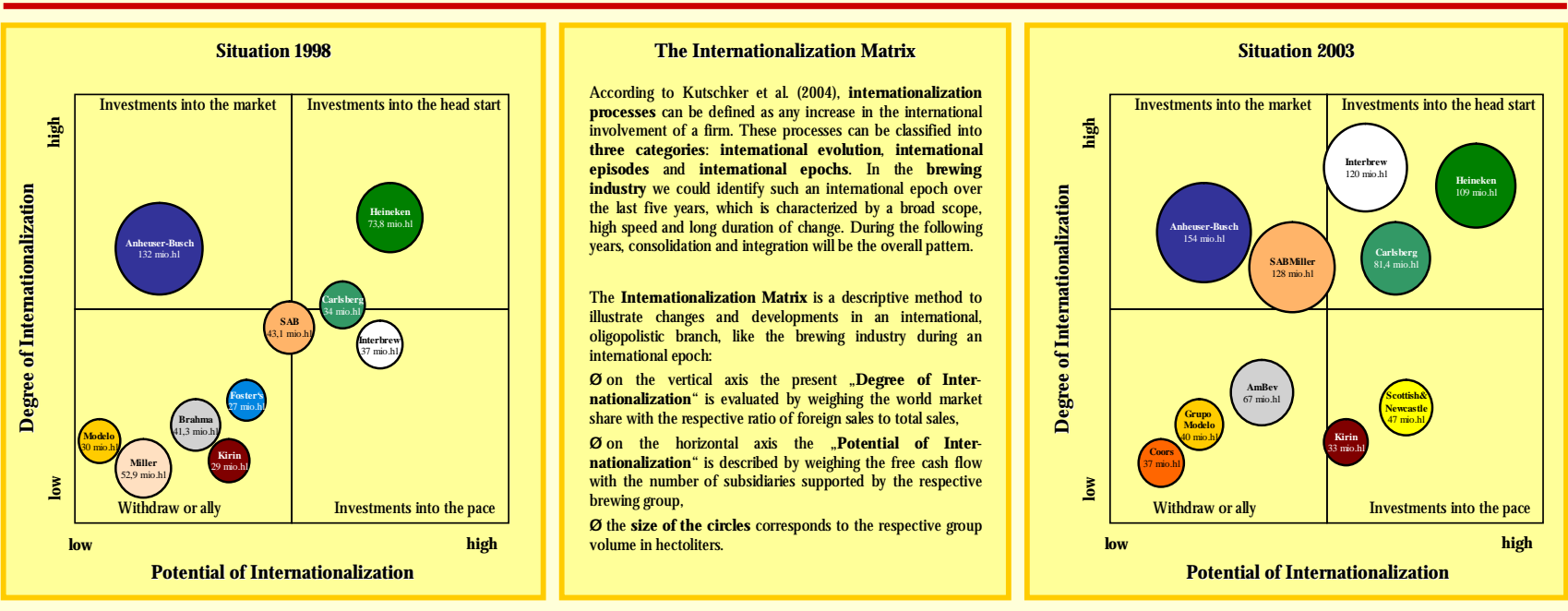

\section{Future Research}

Further research on MNCs in agribusiness in general and in the brewing sector in detail should focus on the question whether full globalization is economically desirable. More work should be done on evaluating the efficiency of various strategies and considering the structural consequences of different internationalization strategies. In addition we will empirically prove the link between entrepreneurial success and the effects of a rising degree of internationalization respectively periods of intensified intemationalization based on key balance-sheet data and key capital-market data. Finally statistically significant differences between internationalization strategies of various firms are revealed. This provides a starting point for the analysis of other industries. 
KAPITEL II.2

\author{
Konzentrationstempo \\ Internationalisierung und Erfolg europäischer Braukonzerne
}

Oliver Ebneth

Erschienen in: Brauindustrie 9(2005): 44-49. 


\section{Konzentrationstempo \\ Internationalisierung und Erfolg europäischer Braukonzerne}

Seit einigen Jahren ist bekanntlich ja auch der deutsche Biermarkt keine Insel der Glückseligen mehr, da internationale Brauriesen trotz Reinheitsgebot und einzigartiger Bierkultur in den deutschen Markt eintraten und diesen innerhalb weniger Jahre zumindest teilweise konsolidierten. Fasst man alle Beteiligungen, Joint Ventures und Tochtergesellschaften zusammen, kontrollieren die europäischen Konzerne inzwischen wohl gut $40 \%$ der heimischen Produktion. Heinekens Einstieg über Schörghuber und Karlsberg vor mittlerweile vier Jahren folgten weitere spektakuläre Übernahmen wie Interbrews Einkaufstour quer durch die Republik, von Diebels, Beck's bis Spaten-Löwenbräu. Die dänische Carlsberg übernahm im Januar 2004 Holsten und nur noch die RB-Brauholding (Radeberger-Gruppe) hält die deutsche Fahne hoch. Folgende Abbildung gibt einen Eindruck davon, wie stark die Konsolidierung und Konzentration der Weltbraubranche in den vergangenen Jahren vonstatten ging.

Abbildung 1: Entwicklung der weltweit führenden Braukonzerne 1998-2004.

Top 10 Braukonzerne 1998 Anteil an der Weltbierproduktion (1.301 Mio. hl) $37,6 \%$ Marktanteil

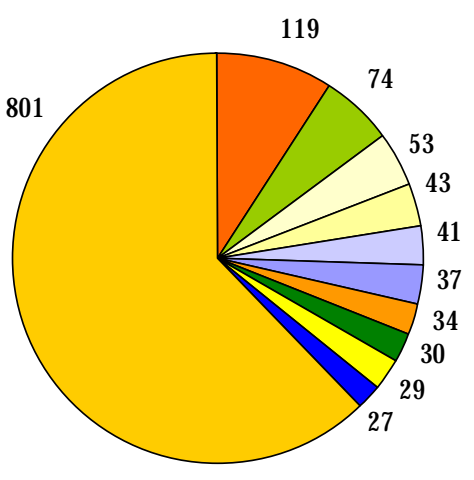

$\square$ Anheuser-Busch $\square$ Heineken $\square$ Miller $\square \mathrm{SAB}$

$\square$ Brahma

$\square$ Interbrew

$\square$ Carlsberg

- G rupo Modelo

$\square \mathrm{Kinin}$

- Foster's

$\square$ Rest
Top 10 Braukonzerne 2004

Anteil an der Weltbierproduktion (1.552 Mio. hl)

59,2 \% Marktanteil

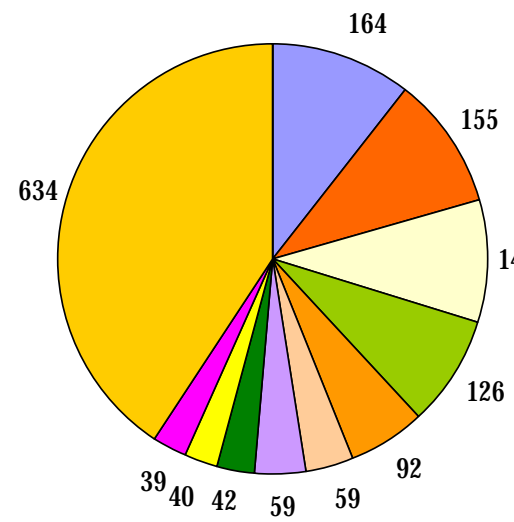

InBev

$\square$ Anheuser-Busch

$\square$ SABMiller

$\square$ Heineken

$\square$ Carlsberg

$144 \square$ MolsonCoors

$\square$ Soottish\&Newcastle

$\square$ Grupo Modelo

$\square \mathrm{K}$ inin

$\square$ Tsingtao

$\square$ Others

$\square 2004$

Quelle: Unternehmensdaten, (Joh.Barth\&Sohn, 1999; Joh.Barth\&Sohn, 2005).

Doch jenseits von den bekannten Transaktionen und den Ausstoßzahlen der führenden Braukonzerne ist das Wissen um Hintergründe und Erfolg der weltweiten Bierleader recht begrenzt. Vor den periodisch erscheinenden Hektoliter-Rankings, die auf eindrucksvolle Weise 
das schier unaufhaltsame Wachstum der Branchenriesen vor Augen führen wird wohl kaum ein heimischer Brauer unbeeindruckt sein. Doch über diese reinen Produktionszahlen, die zudem in fast allen Branchenblättern divergieren, ist wenig bekannt über Kennzahlen, Erfolg und internationale Tätigkeit vieler Braukonzerne. In diesem Artikel sollen deshalb die für den deutschen Markt besonders wichtigen europäischen Bierleader analysiert werden. Diese europäische Führungsgruppe bestimmt auch weltweit - abgesehen von den beiden nordamerikanischen Gruppen Anheuser-Busch und der neuen CoorsMolson - weitestgehend alleine die Internationalisierung der Braubranche. In den vergangenen fünf Jahren investierten alleine die europäischen Brauriesen an die 70 Milliarden $€$ in internationale Akquisitionen, Joint Ventures und Fusionen.

Hatten die Top 10 Braukonzerne noch 1998 lediglich einen Anteil von gut 37\% an der weltweiten Bierproduktion, so stieg dieser Anteil innerhalb nur weniger Jahre auf $62 \%$ an und dies angesichts einer gleichzeitigen Erhöhung der Weltbiererzeugung um 13,4\%. Dabei hat sich zwar im Wesentlichen die Zusammensetzung der führenden Braukonzerne nicht geändert, doch gab es doch einige Verschiebungen und Positionsänderungen im Konzert der Top 10. War Heineken Jahrelang die weltweite Nummer 2 und die unangefochtene Nummer 1 unter den europäischen Gruppen, mussten sich die Niederländer jüngst verglichen zu ihren Hauptwettbewerbern mit relativ moderatem Wachstum zufrieden geben. SABs spektakuläre Fusion mit dem US-amerikanischen Brauer Miller ließ 2001 auf einen Schlag einen Konzern entstehen, der v.a. in vielen Schwellen- und Wachstumsmärkten stark und erfolgreich vertreten ist. Carlsberg konnte v.a. durch die Expansion der Baltic Beverages Holding (BBH), eines 50/50Joint Ventures mit S\&N, in Osteuropa und Russland starkes Wachstum verzeichnen. Dadurch gelang es auch Scottish\&Newcastle in die Riege der führenden Konzerne vorstoßen. Relativ stabil blieben die Positionen der japanischen Kirin und der mexikanischen Grupo Modelo. Mit Tsingtao war 2004 erstmals ein Brauer aus China unter den Top 10 vertreten und angesichts des boomenden chinesischen Riesenmarktes wird sich Tsingtaos Position sicherlich schnell verbessern. Führte Anheuser-Busch über lange Jahre und Jahrzehnte die Liga der weltgrößten Brauer an, wurden die Amerikaner im vergangenen Jahr zum ersten Mal vom Thron gestoßen. Die neue Nummer 1 in der Weltbraubranche ist die belgisch-brasilianische InBev. Umso erstaunlicher ist dies, hält man sich die rasante Entwicklung der Belgier einmal vor Augen. 1994 produzierte der Zusammenschluss ehemaliger mittelständischer belgischer Familienbrauer gerade einmal 14 Millionen Hektoliter, zehn Jahre später wurde diese Zahl auf inzwischen über 160 Millionen hl verzwölffacht. Zusammen mit der durch die Fusion mit 
AmBev erworbenen soft-drink-Sparte verfügt InBev inzwischen über einen Gesamtgetränkeausstoß von 233,5 Millionen Hektolitern! Doch jenseits dieser produktionsorientierten Zahlen muss der Blick auch über die berühmte Hektoliterfixierung hinausgehen. Sind doch in der Weltbraubranche, genauso wie in der noch viel ärger gebeutelten deutschen Braubranche nicht die reine Produktion, sondern der daraus resultierende Umsatz und in einem weiteren Schritt der aus dem Umsatz generierte Gewinn die entscheidenden Kennzahlen, nach denen Unternehmen viel aussagekräftiger gerankt werden sollten. Deshalb werden im Folgenden anhand einiger bilanzanalytischer Kennzahlen Stärken und Schwächen, sowie Trends und Entwicklungen unter den fünf europäischen Braukonzernen aufgezeigt. Abbildung 2 stellt die fünf Brauer anhand von Umsatz und EBITDA (Ergebnis vor Zinsen, Steuern, Tilgung und Abschreibungen) sowie ihres jeweiligen Unternehmenswertes dar. Letzterer berechnet sich aus Marktkapitalisierung, Minderheitsbeteiligungen und Nettoschulden.

Abbildung 2: Unternehmenswert und Bilanzkennzahlen - Top 5 Braukonzerne.

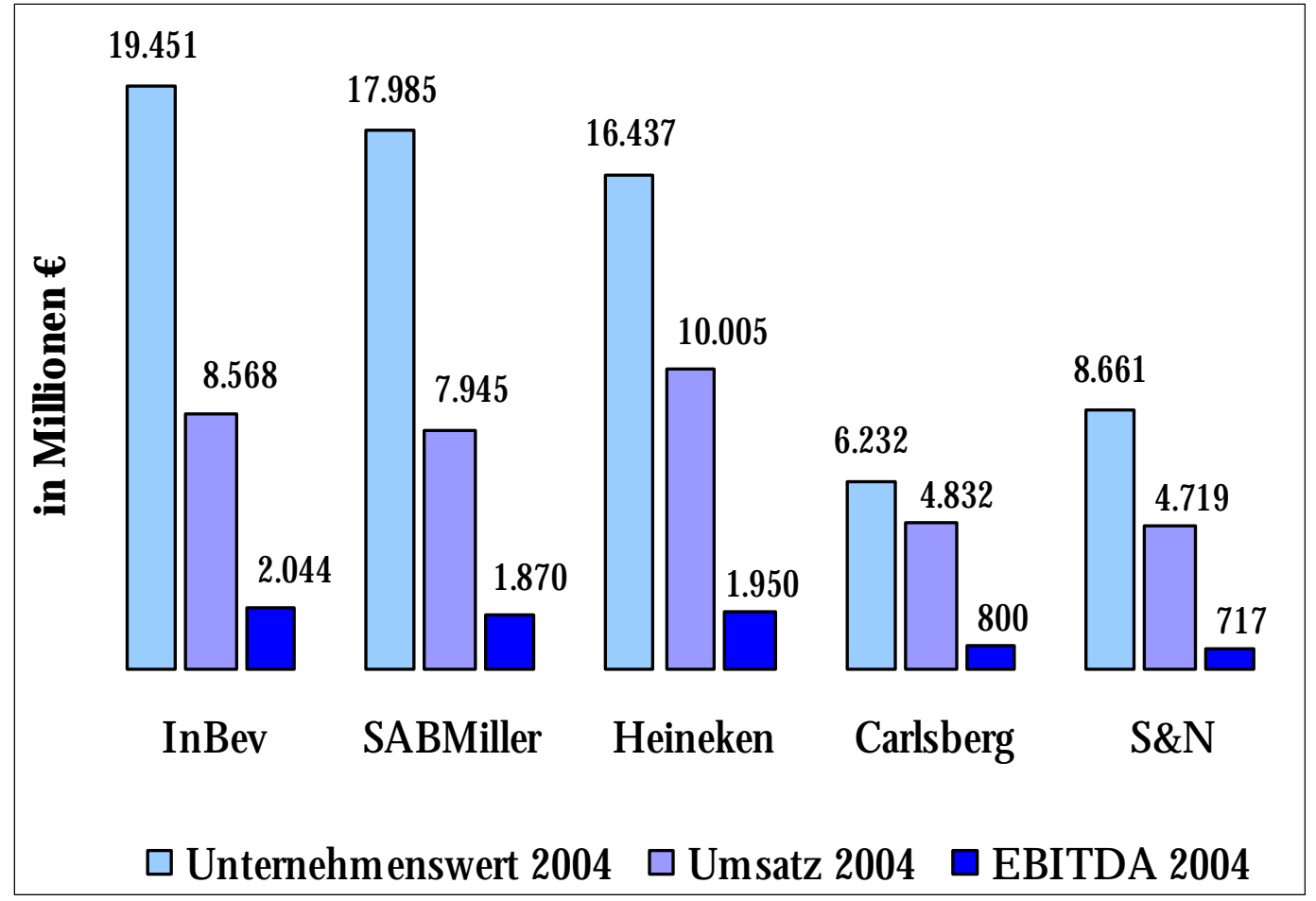

Quelle: Unternehmensdaten, eigene Berechnungen.

Das Ranking in dieser Abbildung erfolgt aufgrund der Relation von Umsatz zu EBITDA. Dabei kommt InBev auf eine EBITDA-Marge von 23,9\%, Sabmiller auf 23.6\% gefolgt von Heineken mit $19.1 \%$ und fast gleichauf Carlsberg und S\&N mit jeweils 16,5\% und 16,3\%. Beim 
Unternehmenswert, der sich hauptsächlich aus der aktuellen Marktkapitalisierung berechnet, ergibt sich eine Zweiteilung, mit den drei großen Konzernen InBev, SABMiller und Heineken an der Spitze und den schwächeren Brauern Carlsberg und S\&N. Auffällig ist der im Verhältnis zum Umsatz relativ geringe Unternehmenswert von Heineken, was v.a. auf die schwache Aktienkursentwicklung der Niederländer über die vergangenen drei Jahre zurückzuführen ist. Wichtig ist der Wert eines Unternehmens hinsichtlich einer möglichen feindlichen Übernahme. So wurde im Schnitt der letzten Jahre das Zehnfache des EBITDAs bei Akquisitionen von den Käuferunternehmen gezahlt. Je höher also die Marktkapitalisierung, desto teurer wird das Unternehmen für potentielle Käufer. Dies spielt v.a. deshalb eine immer wichtigere Rolle, da gerade die letzten Jahre zeigten, dass die Transaktionsvolumina in der Weltbraubranche immer höher wurden. So beliefen sich die Transaktionswerte der SAB-Miller-Fusion auf gut 6 Mrd. $€$, der Interbrew-AmBev deal auf über 16 Mrd. €, Carlsbergs Erwerb der OrklaAnteile auf 7 Mrd. $€$ und die jüngste Fusion zwischen Coors und Molson auf etwa 3,5 Mrd. €. Auch in letzter Zeit dreht sich das Übernahme-Karrussel weiter. So wird derzeit spekuliert, wer wohl das Rennen um die Übernahme des kolumbianischen Grupo Empresarial Bavaria machen wird. Die üblichen Verdächtigen sind wieder einmal Anheuser-Busch, die sich jüngst vermehrt internationalisieren wollen, InBev, Heineken und SABMiller. Am aussichtsreichsten gelten die beiden letzteren, wobei der Verkaufspreis von Bavaria wohl jenseits von 7 Mrd. $€$ liegen wird.

Einen Eindruck von der außergewöhnlich starken Entwicklung sowohl InBevs als auch SABMillers über die letzten Jahre gibt Abbildung 3. Sie veranschaulicht vor allem eine deutliche Erhöhung des Vorsteuerergebnisses (engl. EBIT = earnings before interest and taxes) im Geschäftsjahr 2004. 
Abbildung 3: Vorsteuerergebnis (EBIT) - Top 5 Braukonzerne 2000-2004.

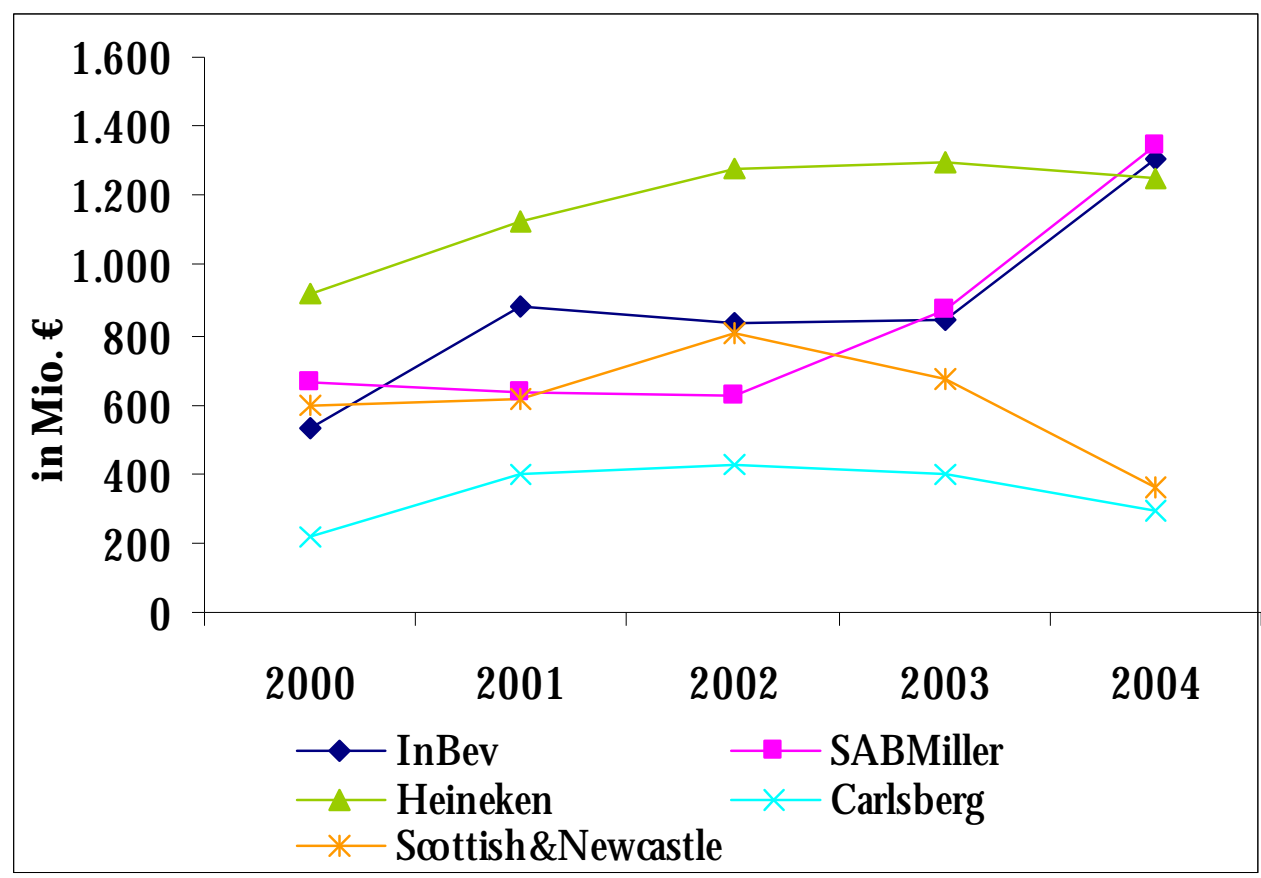

Quelle: Unternehmensdaten, eigene Berechnungen.

Da Absolutwerte jedoch immer irreführend sein können muss der Blick weiter gehen und bei Performancebewertungen von Unternehmen möglichst Kennzahlen in Form von Prozentzahlen bilden. In der Praxis ist es üblich, das Verhältnis des Ergebnisses zum Umsatz als Umsatzrentabilität oder Umsatzrendite $\mathrm{zu}$ bezeichnen. Aufgrund unterschiedlicher Rechnungslegungsnormen sei die im Folgenden dargestellte Umsatzrendite (engl. operating margin) als Relation von EBIT zu Nettoumsatz (= Umsatz abzüglich Verbrauchssteuern) definiert. EBITKennzahlen bieten den Vorteil, dass sie eine Vergleichbarkeit bei unterschiedlicher Kapitalstruktur und zwischen verschiedenen Rechtsformen und Perioden bilden. Abbildung 4 veranschaulicht die Entwicklung des EBITs in Relation zum erzielten Nettoumsatz. 
Abbildung 4: Umsatzrendite - Top 5 Braukonzerne 2000-2004.

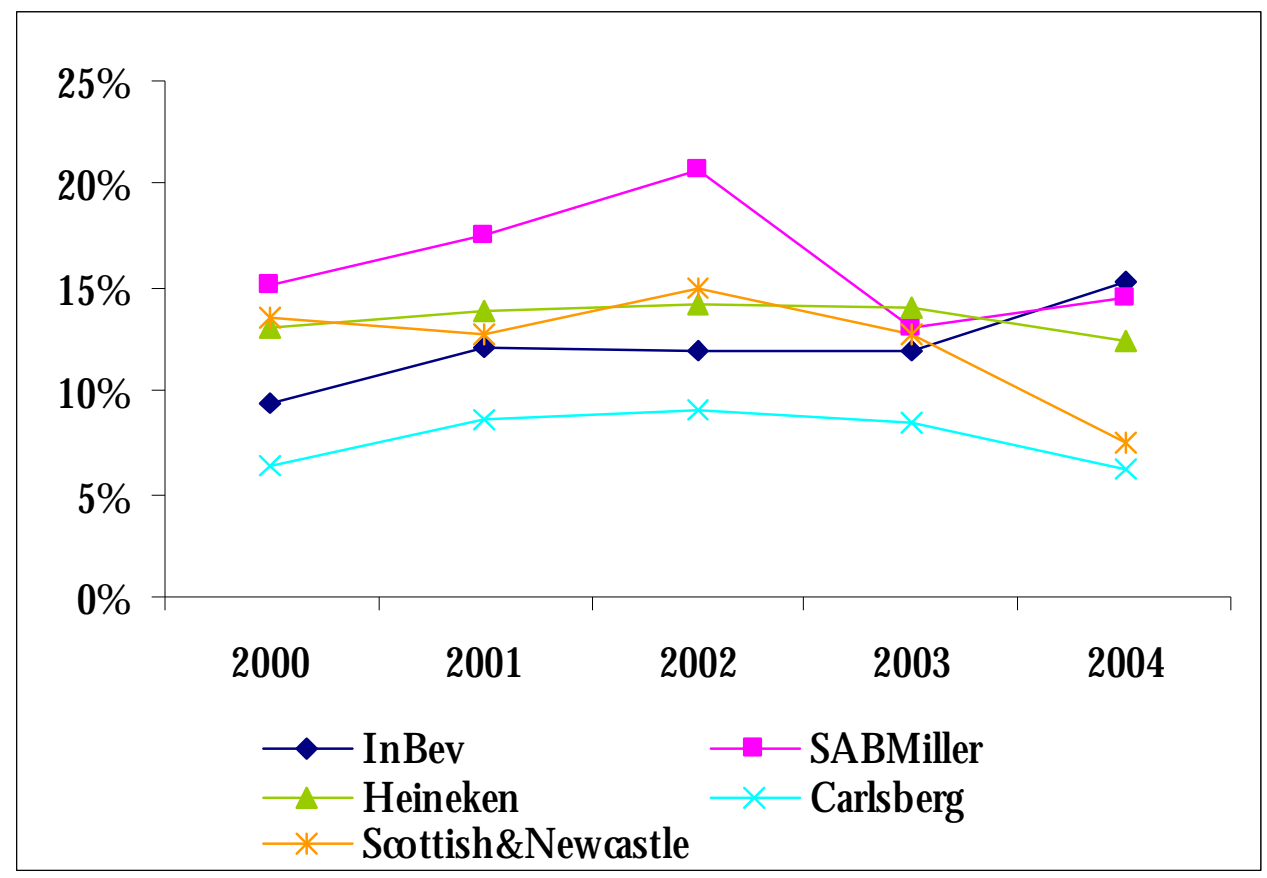

Quelle: Unternehmensdaten, eigene Berechnungen.

Wie auch in der vorherigen Abbildung wird deutlich, dass InBev und SABMiller die Gruppe der europäischen Fünf inzwischen anführen, doch sind Carlsberg und S\&N nicht so weit abgeschlagen wie bei den absoluten Werten des erzielten Vorsteuerergebnisses. In beiden Abbildungen wird deutlich, dass sich der frühere Branchenprimus Heineken in den vergangenen Jahren zwar nicht verschlechterte aber gegenüber den Hauptwettbewerbern InBev und SABMiller doch deutlich an Führung verloren hat.

Um die Strategien der einzelnen Braukonzerne zu bewerten, ist auch eine Betrachtung ihrer internationalen Ausrichtung nötig. Abbildung 5 gliedert die Aktivitäten der fünf Unternehmen in die verschiedenen Regionen des Weltbiermarktes auf. Die jeweilige Produktionsmenge bezieht sich dabei auf von den fünf Konzernen vor Ort über Tochtergesellschaften produziertes Bier. Weltweite Minderheitsbeteiligungen oder Exporte sind nur teilweise erfasst, weswegen die Zahlen von der Gesamtproduktionsmenge der Konzerne abweichen. 
Abbildung 5: Top 5 Europäische Braukonzerne - Produktion in Mio. hl nach Regionen.

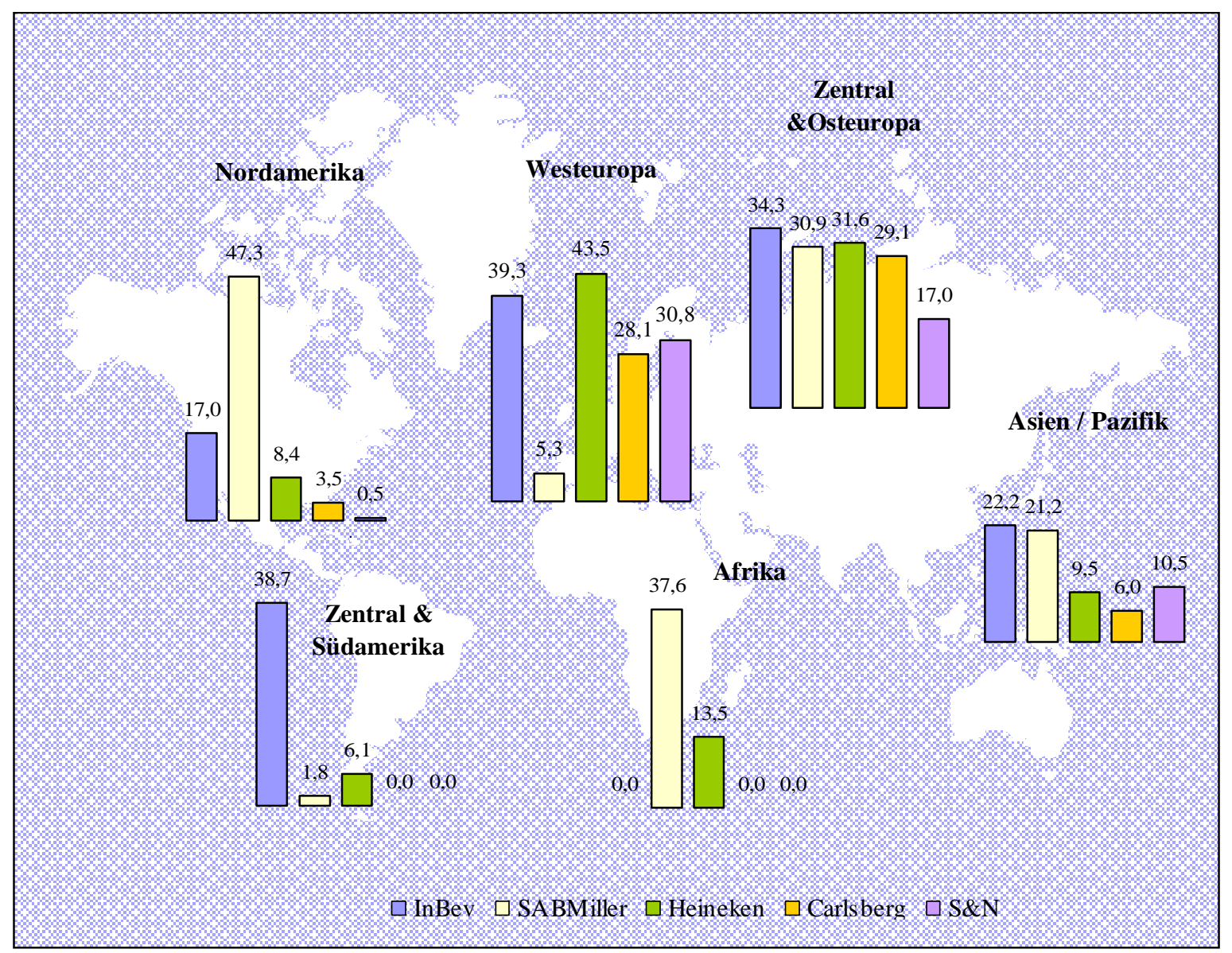

Heineken ist in nach wie vor europäischer Marktführer, mit einer besonders starken Position in Westeuropa. SABMiller ist der einzige Konzern, der dort relativ schwach lediglich über seine italienische Tochter Peroni vertreten ist.

In Osteuropa als einem der wichtigsten weltweiten Wachstumsmärkte haben sich inzwischen alle Unternehmen verhältnissmäßig stark aufgestellt. Besonders erfolgreich konnten sich Carlsberg und S\&N entwickeln, die dort seit einigen Jahren über ihr gemeinsames Joint Venture, die BBH agieren.

In Südamerika als weiteren wichtigen Wachstumsmarkt stellt die neue InBev nunmehr den entscheidenden Player dar. Durch die spektakuläre Übernahme von AmBev, die mit einer EBITDA-Marge von 37\% als profitabelster Braukonzern weltweit galt, hat sich die vormalige Interbrew auf einen Schlag Nr.1-Positionen in sechs mittel- und südamerikanischen Staaten verschafft. In Brasilien als dem weltweit viertgrößten Biermarkt verfügt InBev über eine marktbeherrschende Stellung von $68.1 \%$ der Bierproduktion. Nicht zuletzt durch diese Vor- 
machtstellung streben Heineken und SABMiller danach, durch die Übernahme der BavariaGruppe zumindest den Anschluss nicht zu verlieren.

Den nordamerikanischen Markt dominiert eindeutig SABMiller durch seine Nr.2-Position in den USA. Doch auch InBev verfügt v.a. über seine kanadische Tochter Labatt und ein Joint Venture mit der mexikanischen FEMSA über beträchtliche Marktanteile im weltweit profitabelsten Biermartkt. Heineken ist nach wie vor das erfolgreichste Importbier in den USA, doch leiden die Niederländer seit einigen Jahren unter dem schwachen Dollar, was sich deutlich in der Bilanz niederschlägt. So ging im Vergleich zum vergangenen Jahr der EBIT von 358 auf 269 Mio. $€$ zurück. Carlsberg und S\&N sind bisher lediglich über reine Exporte in Nordamerika vertreten.

An der Markt beherrschenden Stellung von SABMiller in Afrika wird sich wohl auch in den kommenden Jahren nichts ändern. Ihren Heimatmarkt haben die Südafrikaner zu 99\% konsolidiert und auch den wenigen restlichen relevanten Märkten wie Nigeria oder Kamerun halten sie hohe Marktanteile. Der zweite internationale Brauer, der sich traditionell auf dem schwarzen Kontinent engagiert ist Heineken. Ihren Schwerpunkt legen die Holländer dabei auf Nigeria, als dem zweitgrößten Biermarkt nach Südafrika, was sich jüngst an einer Anteilserhöhung an Consolidated Breweries niederschlug. Abgesehen von diesen wenigen größeren Teilmärkten wird sich am unprofitablen restlichen afrikanischen Biermarkt in den nächsten Jahren wohl wenig ändern, weshalb ein Engagement der übrigen Konzerne als ausgeschlossen gilt.

Seit Jahren allerdings richten die internationalen Bierleader ihre begehrlichen Blicke auf den asiatischen Markt, dessen Dynamik hauptsächlich von den alles andere in den Schatten stellenden chinesischen Wachstumsraten herrührt. So verdoppelten die Chinesen ihren Bierkonsum innerhalb eines Jahrzehnts auf mittlerweile etwa 270 Mio. Hektoliter. Dies erfolgte allerdings auf nach wie vor auf extrem niedrigen Preisniveau, was viele westliche Brauer nach wie vor gern übersehen. Deshalb dürfte bisher wohl abgesehen von der seit Jahrzehnten in Schwellenmärkten erfolgreich agierenden SABMiller noch keiner der großen Braukonzerne etwas in China verdient haben. Dies liegt auch in der immer noch atomisierten Marktstruktur. Abbildung 5 macht deutlich, dass noch keiner der internationalen Braukonzerne über eine marktbeherrschende Stellung verfügt, weshalb sich der Wettlauf um weitere Übernahmen zukünftig sicherlich noch beschleunigen wird. Letzte Übernahmen verzeichneten Heineken (Jiangsu DaFuHao Breweries) und InBev ( Hunan Debier Brewery). 
Abschließend lässt sich sagen, dass der Konsolidierungsprozess in der Weltbrauindustrie weit davon entfernt ist, an Dynamik zu verlieren. Vielmehr scheint es aufgrund zunehmender Transaktionsvolumina nicht unwahrscheinlich, dass sich das Konzentrationstempo in der Weltbrauwirtschaft in den nächsten Jahren noch einmal deutlich erhöhen wird. Es bleibt lediglich die Frage offen, wann es ähnlich wie bei SAB-Miller bzw. Coors-Molson zu weiteren Fusionen zwischen den Großen der Branche kommen wird. Die „Kriegskassen“ vieler Braukonzerne sind noch gut gefüllt. Anhand der beschriebenen Erfolgskennzahlen wird jedoch auch deutlich, dass externes Wachstum um jeden Preis langfristig nicht zum Erfolg führt. Wie für die deutsche Brauwirtschaft so heißt es auch für die weltweiten Braukonzerne nicht nur auf Hektoliter sondern vielmehr auf die Ertragskraft des Unternehmens zu schauen. So gibt John Brock, der CEO von InBev im aktuellen Jahresbericht folgende Devise für den weltweit größten Brauer aus:

„To move from Biggest to Best“, nicht nur die Größten, sondern auch die Besten werden!

\section{REFERENCES}

Joh.Barth\&Sohn (1999). The Barth Report. Hops 1998/99, Nuremberg. Joh.Barth\&Sohn (2005). The Barth Report. Hops 2004/05, Nuremberg. 
KAPITEL III

\author{
INTERNATIONALISIERUNG UND UNTERNEHMENSERFOLG \\ DISKUSSION UND OPERATIONALISIERUNG VERSCHIEDENER \\ MESSKONZEPTE
}


KAPITEL III.1

\section{Accelerating Growth Opportunities \\ Profit Pools in the World Brewing Industry}

\section{Oliver Ebneth}

Published in:

- Brewing and Beverage Industry International 1(2006): 10-13.

- Brewing and Beverage Industry Espagñol 1(2006): 10-14.

- Brewing and Beverage Industry China 1(2006): 6-9. 


\section{Accelerating Growth Opportunities Profit Pools in the World Brewing Industry}

This article strives to illustrate the major developments and trends in the world beer industry over the last decade which had transformed it from the local market it was into a global one. First, a general overview about trends in consumption, production and profitability is given. Then we describe the distinct features of certain beer markets around the globe. Not only do we focus on size and trends in the beer markets, but we also pay particular attention to the largest players in the world beer industry.

\section{Volumes by Market}

The world beer market totals 1.55 billion hectoliter in 2004. Generally, global beer consumption quickened over the last decade. The trend line picked up from just below $2 \%$ per annum to more than $2.4 \%(\mathrm{CAGR}=$ compound annual growth rate) since the beginning of the $1990 \mathrm{~s}$, reaching an extraordinary expansion of nearly $5 \%$ last year (see figure 1 ). Nowadays China is the largest beer market, outrunning the US two years ago. Both markets together are well ahead of the rest of the world. Six other markets have significant size: Germany, Brazil, Russia, Mexico, Japan, UK. After that there is a big drop to smaller markets Spain and Poland. Altogether these top ten markets account for $67.8 \%$ of the world beer production. In terms of volume growth, China is by far the most attractive, accounting for $45 \%$ of total world growth over the last decade, with Russia second at 20\%, Mexico and Brazil third at 7\% both. In terms of absolute sales value growth, we expect the US to account for 39\% of total world growth, with China second at $21 \%$ and Brazil third at $12 \%$. This leads us to an issue, often disregarded by the public (i.e. German) brewing scene: sales and value instead of pure volume figures.

Figure 1 illustrates the incremental growth of the world beer production over the last decade. 
Figure 1: World Volume Growth 1993 -2004.

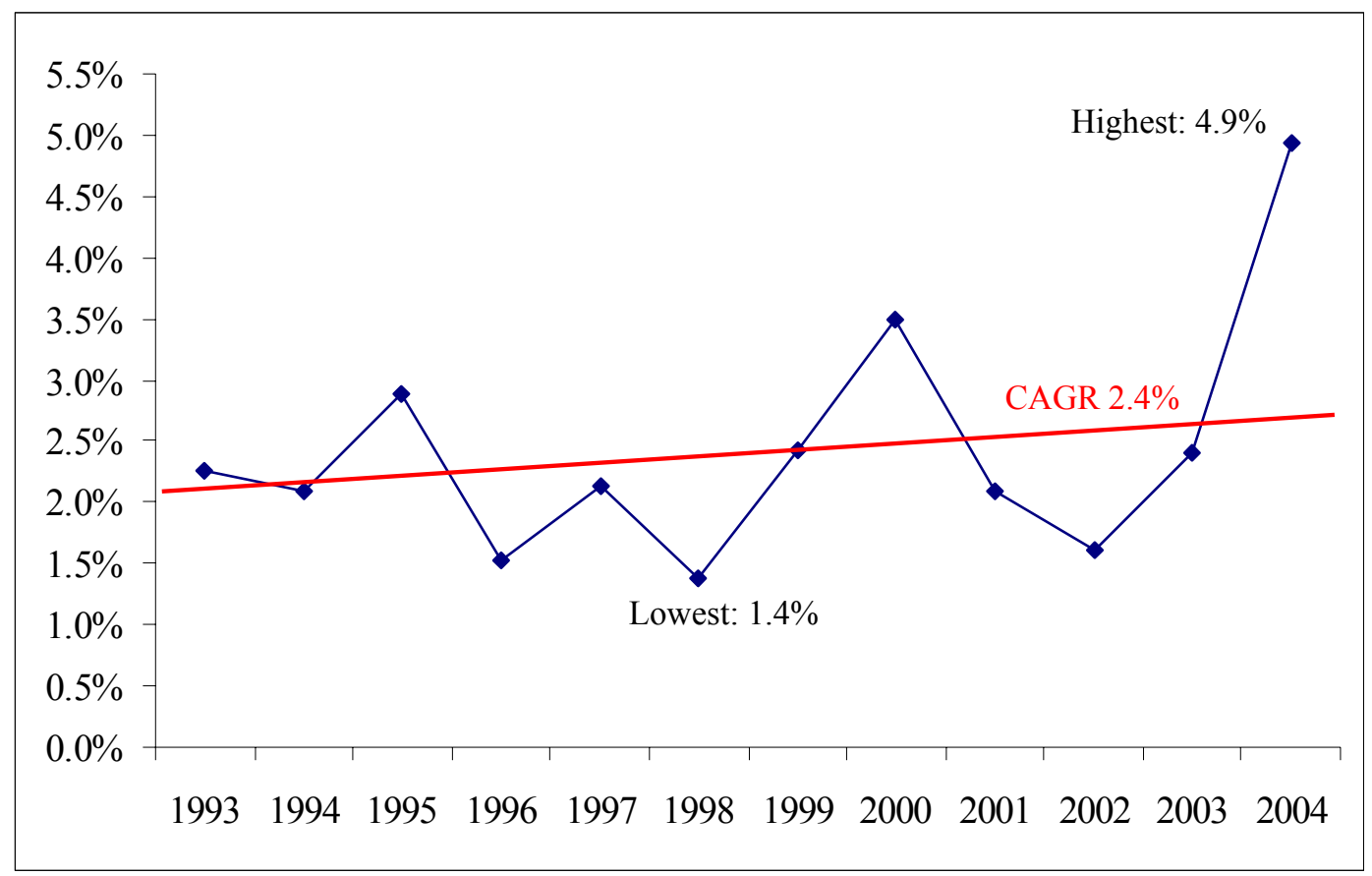

Source: (Joh.Barth\&Sohn, 1995; Joh.Barth\&Sohn, 2005).

\section{Values by Market}

The world beer market is worth US\$234 billion at retail. We do not have global figures for brewers' revenues (many are private companies and hence unpublished), so we use Euromonitor and some broker reports (DrKW, Morgan Stanley, Merrill Lynch). By far the largest market in the world is the US, which is worth US\$68 billion at retail. Eleven other markets are worth over US\$8 billion at retail: Japan, UK, Germany, China, Mexico, Brazil, Spain, Russia, Canada, France and South Korea. The sharp differences in the price of a liter of beer from one country to another are immediately apparent in that China is the largest beer market in size by volume, but only the seventh largest by value. By contrast, Japan is the seventh largest market by volume, but the second largest by value.

\section{What Drives Brewer Returns?}

A large share of profitable markets of course drives brewer returns. This seems to be stating the obvious, but we think it is a fact that is missed by many beer companies, as well as investors. Brewers and investors focus too much on volume growth, and not enough on value growth. Figure 2 illustrates the link between brewer's volume in million hectoliters and the turnover they generate. It attracts analyst's attention that companies like Anheuser-Busch, 
Heineken, S\&N as well as the Japanese brewers receive by far higher revenues per hl than some others, in particular Tsingtao, AmBev, Efes but more surprisingly, even the leading big, InBev and SABMiller perform comparatively weak in terms of sales.

Figure 2: Volume and Revenues - Top 21 Publicly Listed Brewers - 2004.

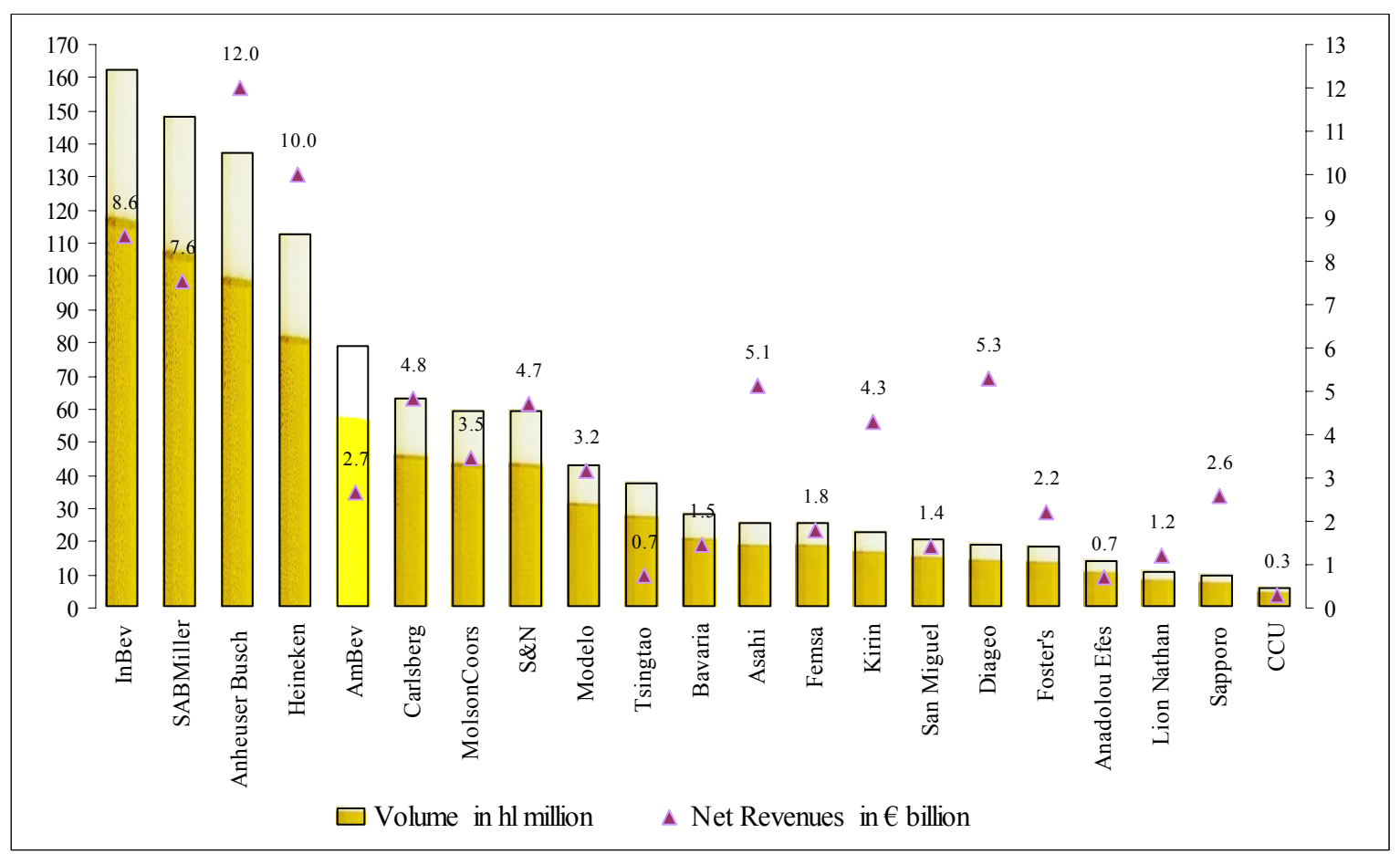

Source: Company data, author's calculations.

\section{But what indeed forces brewers’ financial success?}

Market concentration: There is a fairly well established link between beer market concentration and profitability. Despite this, many brewers seem to chase volume rather than profit growth markets. While it is true that a market's profitability changes dramatically with consolidation, this can be a slow process. Outside investors have been pilling money into China and Germany, two of the world's biggest beer markets for decades, and have yet to see any meaningful returns. By the way, although most of China's top 10 brewers are now profitable, their returns remains exceptionally low. Correspondingly, their profit per hl (as defined by EBITDA) is also very low. In addition there remain many hundreds of lossmaking breweries in China that need to be rationalized. Thus, there is a broad scale in terms of profit per hl in world-wide beer markets ranging from US\$1/hl (China), US\$4/hl (Germany), US\$6/hl (Rus- 
sia) to US\$26/hl (Japan), US\$31/hl (Canada) and US\$32/hl (Ireland, Australia).

Market leadership: Within each market, there can be dramatic differences in brewer profitability. Typically, these differences are highly correlated with scale - the brewers with the largest market shares are usually the most profitable. Brewers who are strongly positioned in most of their markets therefore have a sizeable advantage over brewers with global scale that do not have strong market positions. SABMiller is a top three brewer in 30 of its 40 markets. Carlsberg operates in far more markets, but would have top three positions in fewer than 10 . Figure 3 illustrates the link between highly dominated key markets of different brewers where they are driving there highest percentage of EBITDA compared to sales. Carlsberg's BBH operations for example account for $14 \%$ of Carlsberg's total sales but for $34 \%$ of its EBITDA.

Figure 3: Sales and EBITDA 2004 - Profit Pools in Selected Brewers' Main Markets.

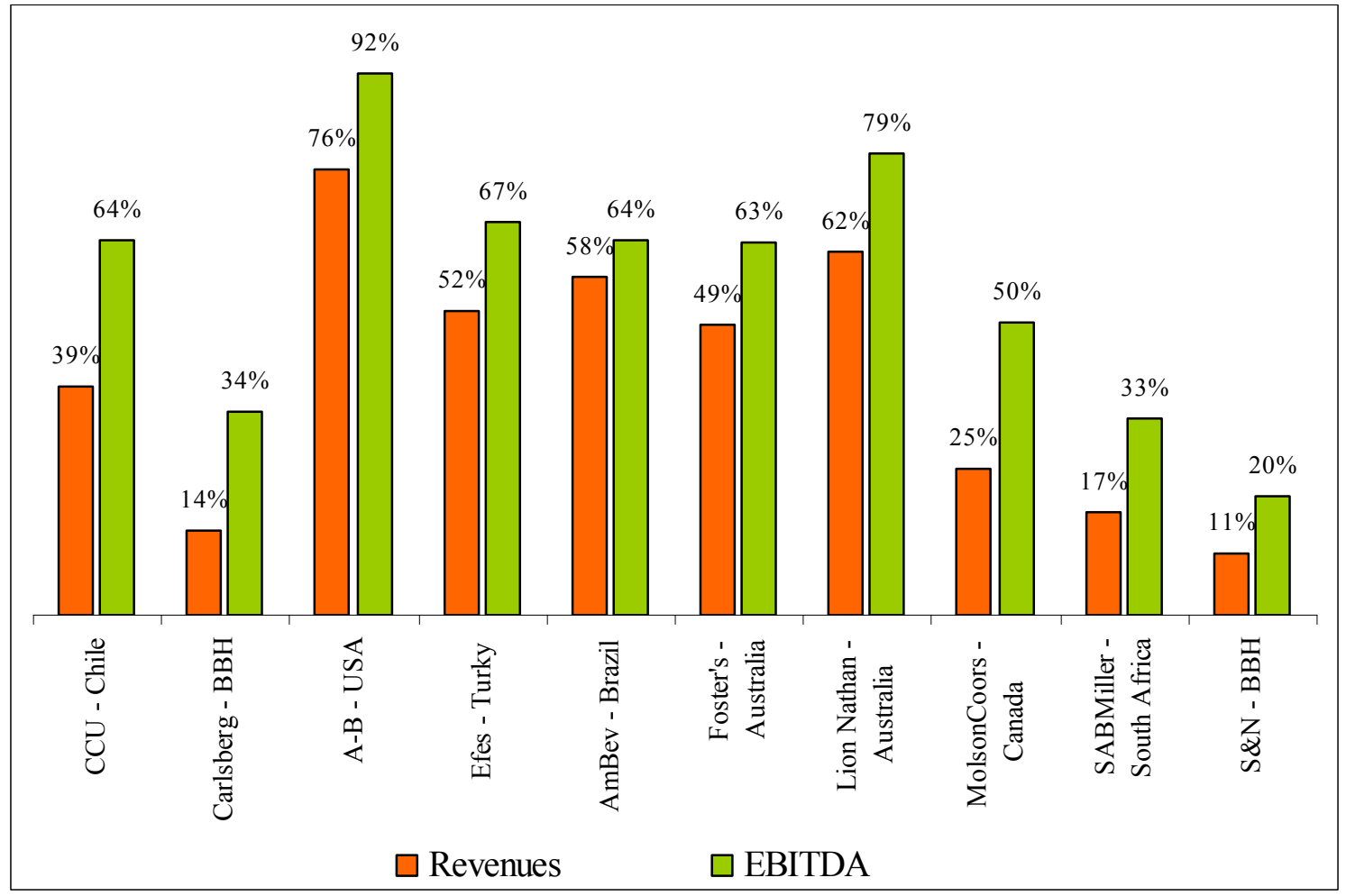

Source: Company data, author's calculations.

Market rationality: Concentrated markets are not always profitable. Often market profitability is partially dependent on strong price leadership from a particular brewer, concurrently with rational behavior from the other brewers. Countries that have one dominant brewer and a number of smaller, equally sized brewers are usually very profitable - essentially because 
everyone 'knows their place' ( $\rightarrow$ Brazil, Canada, Ireland). The last thing the market needs is a market share aspirant, often caused by too many brewers all seeking scale, or a consolidated market where share and price are the only levers of growth ( $\rightarrow$ Germany).

Market profitability: Big shares in fragmented markets are unlikely to drive high returns, even if the markets exhibit high levels of volume growth. The least attractive markets are unconsolidated markets with low growth - many of which are actually the historical beer drinking countries ( $\rightarrow$ Germany).

\section{What are the Big Profit Pools in the World Brewing Industry?}

To conclude, the perfect market is broadly one that is 1) large, 2) growing, 3) highly concentrated and 4) already very profitable. We would include the US, Brazil, South Africa, Mexico, UK, Japan, and even Australia and Canada in this group. These are eight of the largest profit pools in the world - accounting for $72 \%$ of the global profits (see figure 4 ).

Figure 4: Global Top 15 Profit Pools 2003 - 2013.
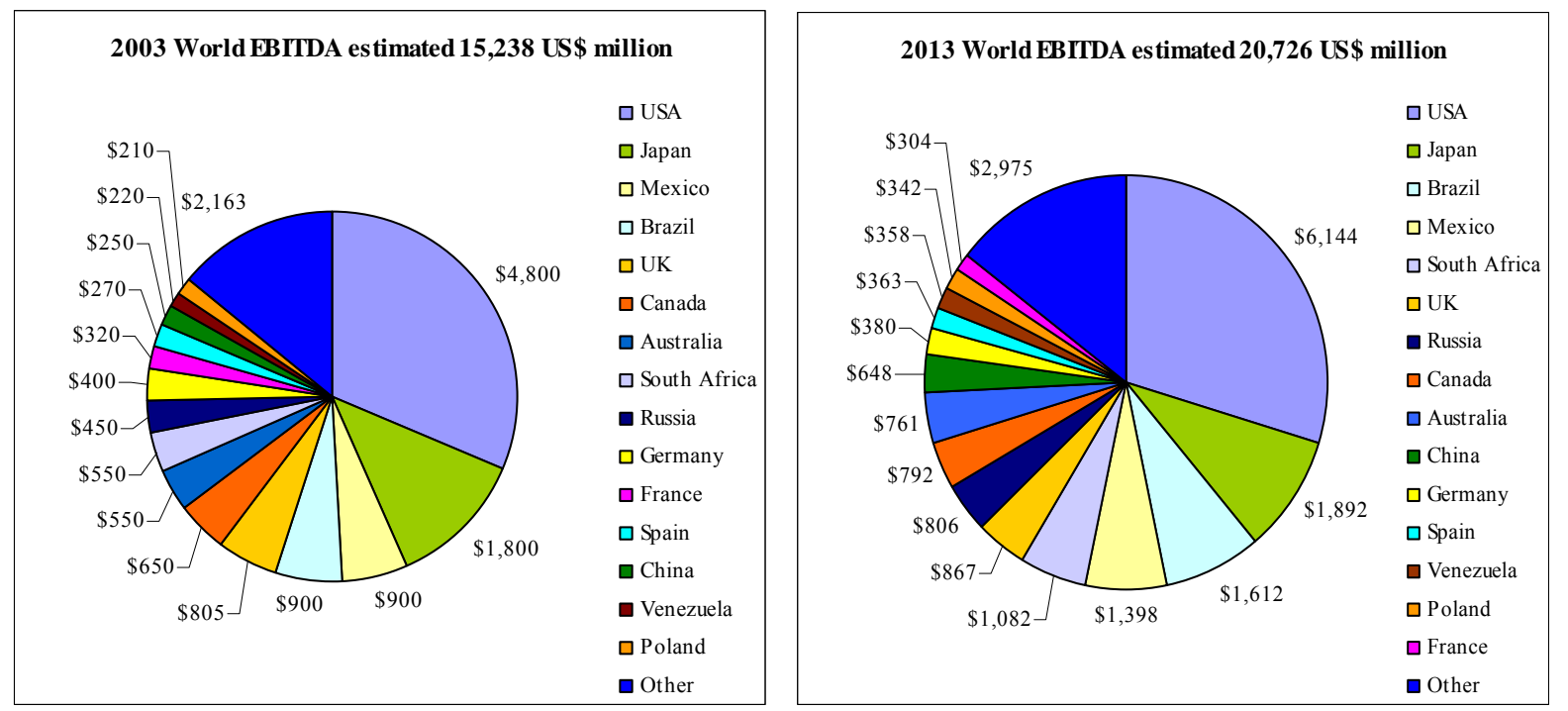

Source: (Jordan et al, 2004; Holland et al, 2005; Yule et al, 2005).

The US profit pool is about three times as large as the combined profit pools of the 'BRIC' economies - Brazil, Russia, India, China. In 10 years' time, we believe the US will probably still be almost twice as large.

China would have to show compound growth of over $37 \%$ of its profit pool to catch the US in the next 10 years, assuming compound growth of the US of only $2 \%$ per annum. 
Japan's profit pool is slightly larger than the entire market of Western Europe, and Latin America's is over 35\% bigger. In 10 years Latin America's profit pool should be about twice as large as Western Europe's.

In 10 year's time, we would expect South Africa's profit pool to be close to $30 \%$ larger than the UK's - despite being $30 \%$ smaller today.

\section{Brewer's Profitability: Scale is Key}

Our comparison of the 21 biggest world-wide leading and publicly listed companies' profit and loss account and cash flow statements highlights some very stark differences. The retail value per liter varies from $€ 7.87$ in Norway to $€ 0.46$ in Ukraine - a 17-fold difference. The 21 companies in our database have a difference in revenue per hl from $€ 269.6$ (Sapporo) to $€ 19.9$ (Tsingtao) - a 14-fold difference (see figure 5). More important, they also have a difference in profit per hl ranging from $€ 43.4$ (Kirin) to $€ 2.8$ (Tsingtao), a 16-fold difference (see figure 6).

\section{Revenue per hl}

The revenue per $\mathrm{hl}$ for each brewer reflects three factors: The retail price per $\mathrm{hl}$ in the markets in which the brewer operates. The structure of the market, i.e., how much mark-up is taken by the retailer and how much tax is taken by the government. Whether the brewer in question is a single-market operator or has a portfolio of markets. Figure 5 on the following page shows the differences from one company in terms of net revenue per hl (excise duties excluded).

We think it fair to subdivide the competitive set into three groupings: Developed Asia, developed world and developing world:

At the top of the range are several brewers with a strong presence in highly developed Asian markets. Sapporo, Asahi and Kirin are all Japanese brewers, where retail and wholesale prices are exceptionally high, reflecting the high costs of doing business in Japan.

We make two observations about the developed world brewers: At the top end of this range are brewers exposed to just one market, or primarily to developed markets. This applies to San Miguel, Foster's, Lion Nathan, Heineken and Anheuser-Busch. Lower in the range are brewers with a broader portfolio of markets that have strong developed-market businesses, but also have exposure to emerging markets that lower the average rate, such as Carlsberg, MolsonCoors and InBev.

The developing world brewers are those primarily exposed to emerging markets where 
beer is sold cheaply to improve its affordability. These include SABMiller, AmBev, Efes and Tsingtao.

Figure 5: Revenues per hl in $€$ - Top 21 Publicly Listed Brewers - 2004.

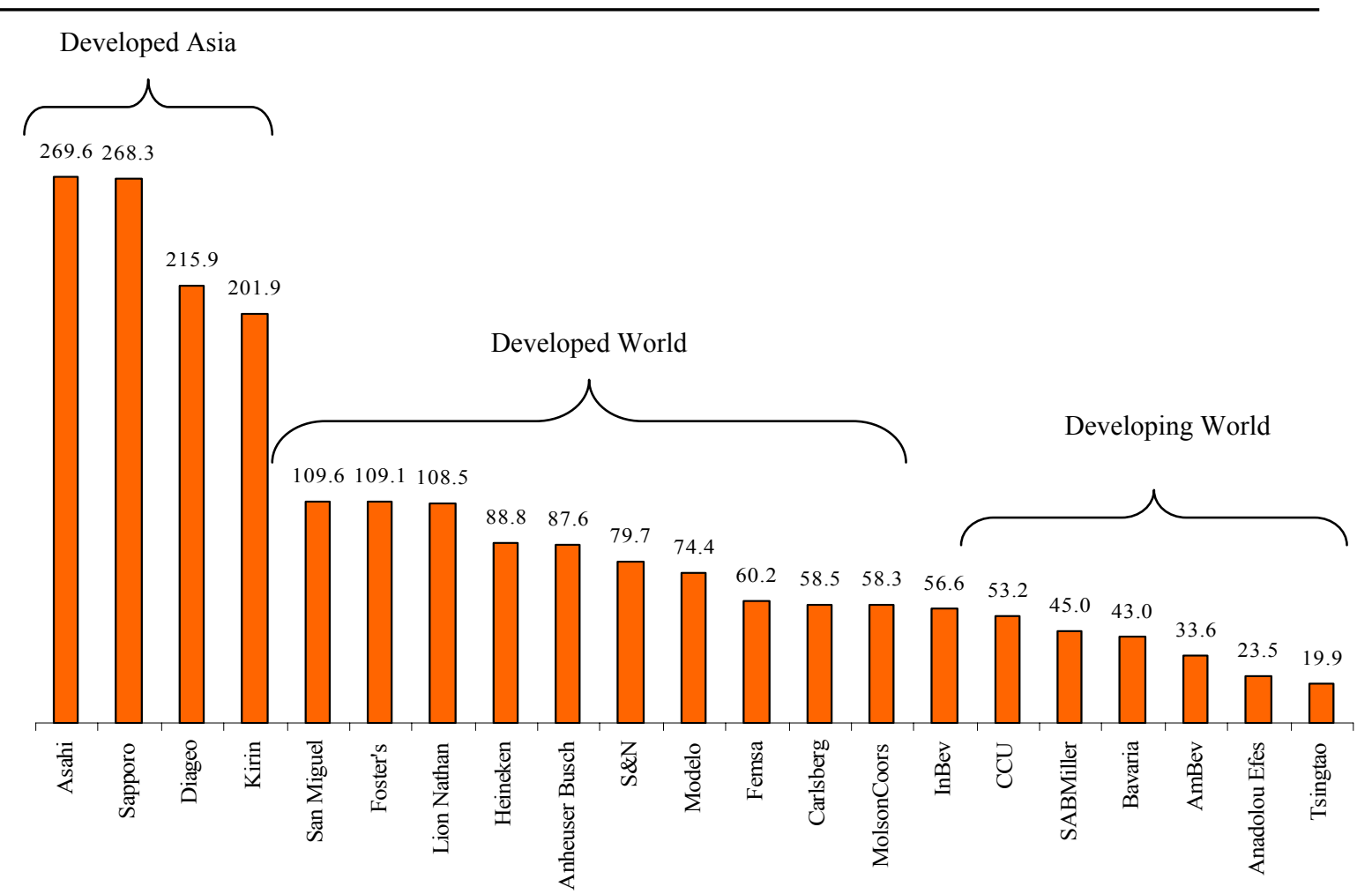

Source: Company data, author's calculations, (Jordan et al, 2004).

When comparing EBITDA with volumes, it is apparent that EBITDA per hl is a function of (1) a high level of revenue per hl and (2) scale. In figure 6 only the brewers that achieve the combination of both of these factors find themselves towards the higher end of the range. We have grouped the companies in to four clusters:

Strong scale combined with extreme rev/hl: Every brewer in this classification combines an extraordinary level of revenue per hl with strong scale in many of the markets in which it operates. Japanese brewers Kirin, Asahi and Sapporo as well as Diageo's beer segment (Guiness) fit this group.

Strong scale combined with strong rev/hl: These brewers all combine a quite high level of revenue per hl with strong scale in many of the markets in which it operates. Relative to the percentage EBITDA margins, Foster's, Modelo, A-B and Lion Nathan all look far better. 
They operate in markets with a high revenue per hl so their percentage margins are depressed, but their scale means their costs per hl are acceptable (or even highly) efficient, so the EBITDA per $\mathrm{hl}$ is attractive.

Benefiting from scale or high revenue per hl: Most notably AmBev, which offers exceptionally high EBITDA margins operates in low revenue per hl markets, so it cannot generate such a high level of EBITDA per hl, despite the high margin.

Lack of scale or low revenue per hl: Carlsberg and MolsonCoors suffer from a lack of scale, Efes suffers from a low revenue per hl and Tsingtao combines both problems.

Figure 6: EBITDA per hl in $€$ - Top 21 Publicly Listed Brewers - 2004.

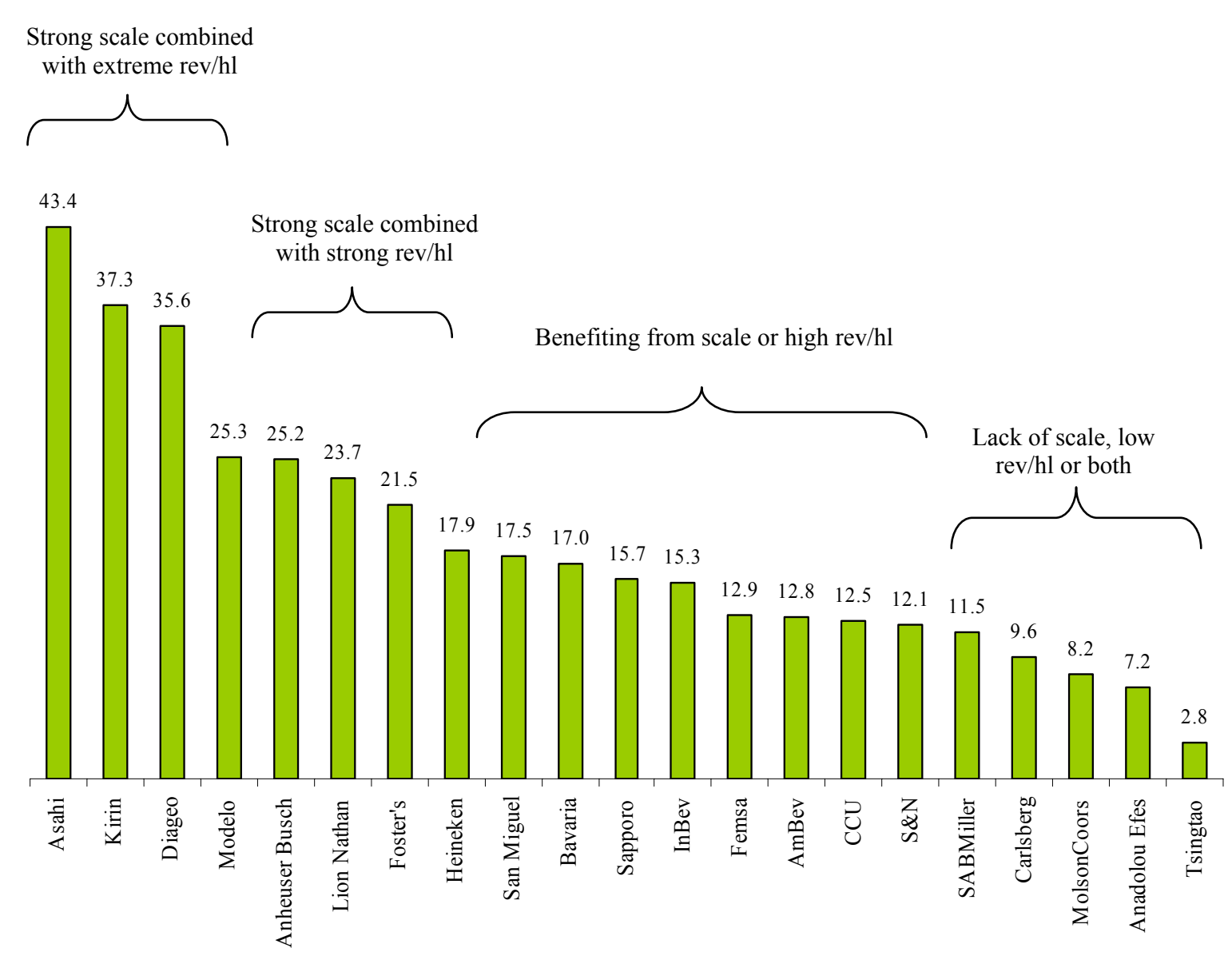

Source: Company data, own calculations, (Jordan et al, 2004; Oldroyd et al, 2005). 


\section{So Whereto Next?}

It is clear that in many of the currently unprofitable markets, consolidation will help profitability considerably. Russia seems to be consolidating fast, as does India. However, we would caution that the level of consolidation needed to drive a big step-change in profit can take much longer. Germany, Western-Europe's biggest volume market, still remains highly fragmented and unprofitable, despite decades of consolidation.

As we have argued, this analysis is still a good guide to those brewers with the best exposure to higher opportunities for profit growth. Market growth is of course not the only source of incremental profit to the brewers. Disproportionate profit shares in markets accrue to those that have the best businesses and brands.

\section{REFERENCES}

Holland, A. et al (2005): Beverages Quarterly: Q3 2005, Dresdner Kleinwort Wasserstein Equity Research, London.

Joh.Barth\&Sohn (1995): The Barth Report. Hops 1994/95, Nuremberg. Joh.Barth\&Sohn (2005): The Barth Report. Hops 2004/05, Nuremberg. Jordan, M. et al (2004): Global Beer Industry. Dresdner Kleinwort Wasserstein Research, London.

Oldroyd, A. et al (2005): Basking in the Brazilian Sunshine, Morgan Stanley Equity Research, London.

Yule, M. et al (2005): Beer Appreciation: Episode 1. Morgan Stanley Research, London. 
KAPITEL III.2

\section{Growth and Profit Opportunities \\ Analyzing the World Brewing Industry}

Oliver Ebneth and Ludwig Theuvsen

Poster will be presented at the $16^{\text {th }}$ Annual World Food and Agribusiness Symposium and Forum, $10^{\text {th }}-13^{\text {th }}$ of June 2006, Buenos Aires. 


\section{Growth and Profit Opportunities Analyzing the World Brewing Industry}

\section{Problem Statement}

This investigation strives to illustrate the major developments and trends in the world beer industry over the last decade which transformed it from a local market into a global one. A general overview about trends in consumption, production and profitability is provided as is a description of the distinct features of certain beer markets around the globe. Not only do we focus on size and trends in the beer markets, but we also pay particular attention to the largest players in the world beer industry. Generally, global beer consumption has increased over the last decade and the world beer market totaled 1.55 billion hectoliter (hl) in 2004 . The trend line has picked up from just below $2 \%$ growth per annum to more than $2.4 \%$ since the beginning of the 1990s (Joh.Barth\&Sohn, 2005). But far more important and interesting is an issue often disregarded by the public brewing scene, which tends to focus on pure volume figures, and that is sales and value.

\section{OBJECTIVES}

We ask two central questions: What drives brewer returns, and where are the big profit pools in the world brewing industry?

A brewer's share of profitable markets, of course, drives its returns (Yule et al, 2005). There is a fairly well established link between beer market concentration and profitability. Despite this, many brewers seem to chase volume rather than growth markets. Within each market there can be dramatic differences in brewer profitability. Typically, these differences are highly correlated with scale - the brewers with the largest market shares are usually the most profitable. Often market profitability is partially dependent on strong price leadership from a particular brewer coupled with rational behavior from the other brewers. Large shares in fragmented markets are unlikely to drive high returns, even if the markets exhibit high levels of volume growth (Hax, 2003; Wan and Hoskisson, 2003). 


\section{Procedures}

The above-mentioned issues seem to be stating the obvious, but we think it is a fact that is missed by many beer companies, as well as by investors. Brewers and investors focus too much on volume growth and not enough on value growth (Geringer et al, 1989). The poster will pinpoint both an overview of growth and profits in the world beer industry and a comparison of the leading brewing groups' performance in selected regional markets. The paper focuses on identifying differences in profitability between several beer markets and also illustrates brewers' financial performance through compiling significant key data and financial ratios. The assumption thereby is that a link can be revealed between the brewers' domination of certain markets and their corporate success (Fröhlich, 2004). This will be proved by collecting ratios covering financial data provided in the respective brewers' annual reports. The key data that give the deepest and most reliable insight into a beer market's profitability as well as a brewer's financial performance are as follows (Born, 2001):

- A comparison of Sales to EBITDA per market,

- Revenues per hectoliter,

- EBITDA per hectoliter.

Figure 1 presents the main operating statistics of our sample, which consists of the 21 largest publicly-listed brewing groups world-wide. Thus, our sample represents $67.8 \%$ of the total world beer production. 
Figure 1: Operating Statistics of the 21 World-Wide Leading Brewing Groups - 2004.

\begin{tabular}{|c|c|c|c|c|c|c|c|c|c|}
\hline & $\begin{array}{l}\text { Company } \\
\text { (Alphabetical order) }\end{array}$ & $\begin{array}{c}\text { Volume } \\
(\mathrm{mhl})\end{array}$ & $\begin{array}{c}\text { Market } \\
\text { Cap }(€ \mathbf{m}) \\
\end{array}$ & $\begin{array}{l}\text { Enterprise } \\
\text { Value }(€ \mathbf{m}) \\
\end{array}$ & $\begin{array}{c}\text { Net Revenues } \\
(€ \mathbf{m}) \\
\end{array}$ & $\begin{array}{c}\text { EBITDA } \\
(€ \mathrm{~m}) \\
\end{array}$ & $\begin{array}{r}\text { EBIT } \\
(€ m) \\
\end{array}$ & $\begin{array}{l}\text { EBITDA- } \\
\text { Margin } \\
\end{array}$ & $\begin{array}{l}\text { EBIT- } \\
\text { Margin }\end{array}$ \\
\hline 1 & AmBev & 79.2 & 17,721 & 19,274 & 3,302 & 1,255 & 781 & $38.0 \%$ & $23.6 \%$ \\
\hline 2 & Anadolou Efes & 14.4 & 2,309 & 2,360 & 756 & 231 & 153 & $30.6 \%$ & $20.2 \%$ \\
\hline 3 & Anheuser Busch & 137.1 & 27,874 & 29,689 & 12,006 & 3,468 & 2,724 & $28.8 \%$ & $22.5 \%$ \\
\hline 4 & Asahi & 25.8 & 5,083 & 7,069 & 6,624 & 1,135 & 753 & $17.1 \%$ & $11.4 \%$ \\
\hline 5 & Bavaria & 28.6 & 3,600 & 5,457 & 1,479 & 584 & 478 & $39.5 \%$ & $32.3 \%$ \\
\hline 6 & Carlsberg & 63.2 & 3,697 & 7,302 & 4,835 & 806 & 306 & $16.5 \%$ & $6.1 \%$ \\
\hline 7 & $\mathrm{CCU}$ & 5.9 & 1,377 & 1,569 & 607 & 142 & 81 & $23.4 \%$ & $13.4 \%$ \\
\hline 8 & Diageo & 19.2 & 37,206 & 41,013 & 9,823 & 2,861 & 2,534 & $29.1 \%$ & $25.8 \%$ \\
\hline 9 & Femsa & 25.7 & 7,075 & 8,602 & 6,680 & 1,436 & 977 & $21.5 \%$ & $14.6 \%$ \\
\hline 10 & Foster's & 18.8 & 6,789 & 9,367 & 2,348 & 595 & 463 & $25.3 \%$ & $19.7 \%$ \\
\hline 11 & Heineken & 112.6 & 13,190 & 18,798 & 10,005 & 2,021 & 1,248 & $20.2 \%$ & $12.5 \%$ \\
\hline 12 & InBev & 162.1 & 18,441 & 22,410 & 8,568 & 2,325 & 1,310 & $27.1 \%$ & $15.3 \%$ \\
\hline 13 & Kirin & 23.1 & 7,658 & 8,411 & 9,104 & 1,389 & 814 & $15.3 \%$ & $8.9 \%$ \\
\hline 14 & Lion Nathan & 10.8 & 2,646 & 3,421 & 1,190 & 267 & 211 & $22.4 \%$ & $17.1 \%$ \\
\hline 15 & MolsonCoors & 42.8 & 4,392 & 6,610 & 3,462 & 489 & 275 & $14.1 \%$ & $7.9 \%$ \\
\hline 16 & Modelo & 59.4 & 8,579 & 8,283 & 3,186 & 1,084 & 935 & $34.0 \%$ & $29.3 \%$ \\
\hline 17 & SABMiller & 59.2 & 20,484 & 21,855 & 8,422 & 2,156 & 1,424 & $25.6 \%$ & $16.9 \%$ \\
\hline 18 & San Miguel & 148.3 & 2,918 & 4,700 & 2,266 & 362 & 239 & $16.0 \%$ & $10.6 \%$ \\
\hline 19 & Sapporo & 20.7 & 1,336 & 3,138 & 3,519 & 202 & 176 & $9.9 \%$ & $4.8 \%$ \\
\hline 20 & Scottish \& Newcastle & 9.6 & 6,193 & 9,285 & 4,719 & 717 & 355 & $15.2 \%$ & $7.5 \%$ \\
\hline 21 & Tsingtao & 37.6 & 1,013 & 1,034 & 747 & 106 & 54 & $14.2 \%$ & $7.3 \%$ \\
\hline & $\Sigma$ & $1,104.1$ & 199,579 & 239,648 & 103,649 & 23,631 & 16,291 & & \\
\hline & $\operatorname{Max}$ & & 37,206 & 41,013 & 12,006 & 3,468 & 2,724 & $39.5 \%$ & $32.3 \%$ \\
\hline & Average & & 9,504 & 11,412 & 4,936 & 1,125 & 776 & $23.0 \%$ & $15.6 \%$ \\
\hline & Median & & 6,193 & 8,283 & 3,519 & 806 & 478 & $22.4 \%$ & $14.6 \%$ \\
\hline & Min & & 1,013 & 1,034 & 607 & 106 & 54 & $9.9 \%$ & $4.8 \%$ \\
\hline
\end{tabular}

Source: Company data, authors' calculations.

\section{Results}

To conclude, the perfect market is broadly one that is 1) large, 2) growing, 3) highly concentrated and 4) already very profitable. We would include the United States, Brazil, South Africa, Mexico, the United Kingdom, Japan, and even Australia and Canada in this group. These are eight of the largest profit pools in the world - accounting for $72 \%$ of the global profits in the brewing industry.

Our comparison of the profit and loss accounts and cash flow statements of the 21 largest leading publicly listed companies world-wide highlights some very stark differences. The 21 companies in our database have differences in revenue per hl from $€ 394.1$ (Sapporo) to $€ 19.9$ (Tsingtao) - a 20-fold difference. More important, they also have differences in profit per hl ranging from $€ 47.6$ (Kirin) to $€ 2.8$ (Tsingtao), a 17-fold difference. Besides, it attracts analyst's attention that companies like Anheuser-Busch, Heineken, S\&N and the Japanese brewers receive far higher revenues per hl than some others, in particular Tsingtao, AmBev, and Efes, but more surprisingly, even the leading large brewers InBev and SABMiller perform comparatively weakly in terms of sales. 


\section{CONCLUSIONS}

It is clear that, in many of the currently unprofitable markets, consolidation will help profitability considerably (Capar and Kotabe, 2003). Russia seems to be consolidating fast, as does India. However, we would caution that the level of consolidation needed to drive a big step change in profit can take much longer. Germany, Western-Europe's largest volume market, still remains highly fragmented and unprofitable despite decades of consolidation (Jordan et al, 2004).

As we have argued, this analysis is still a good guide to those brewers with the best exposure to higher opportunities for profit growth. Market growth is, of course, not the only source of growing profit to the brewers. Disproportionate profit shares in markets accrue to those that have the best businesses and brands.

\section{REFERENCES}

Born, K. (2001): Bilanzanalyse international. Deutsche und ausländische Jahresabschlüsse lesen und beurteilen (2nd ed.), Stuttgart.

Capar, N. and Kotabe, M. (2003): The Relationship between International Diversification and Performance in Service Firms, in: Journal of International Business Studies 34(2003): 345-355.

Fröhlich, C. (2004): Wertorientierte Kennzahlen und ihre Bedeutung bei der Ermittlung des Unternehmenswertes - ein Fallbeispiel, in: Unternehmensbewertung und Management 10(2004): 383-391.

Geringer, J. M. et al (1989): Diversification Strategy and Internationalization: Implications for MNE Performance, in: Strategic Management Journal 10(1989): 109-119.

Hax, H. (2003): Measuring the Firm's Performance: Accounting versus Market Value, in: Journal of Institutional and Theoretical Economics 159(2003): 675-682.

Joh.Barth\&Sohn (2005): The Barth Report. Hops 2004/05, Nuremberg.

Jordan, M. et al (2004): Global Beer Industry. Dresdner Kleinwort Wasserstein Research, London.

Wan, W. P. and Hoskisson, R. E. (2003): Home Country Environments, Corporate Diversification Strategies, and Firm Performance, in: Academy of Management Journal 46(2003): 27-45.

Yule, M. et al (2005): Beer Appreciation: Episode 1. Morgan Stanley Research, London. 


\section{Growth and Profit Opportunities - Analyzing the World Brewing Industry -}

Oliver Ebneth ${ }^{1}$ and Ludwig Theuvsen ${ }^{2}$

\section{Problem Statement and Objectives}

Generally, global beer consumption has increased over the last decade and the world beer market totaled 1.55 billion hectoliters (hl) in 2004. The trend line has picked up from just below $2 \%$ growth per annum to more than $2.4 \%$ since the beginning of the 1990s. But far more important and interesting is an issue often disregarded by the public brewing scene, which tends to focus on pure volume figures, and that is sales and value.

We ask two central questions: What drives brewer returns, and where are the big profit pools in the world

A brewer's share of profitable markets, of course, drives its returns. There is a fairly well established link between a are highly correlated with scale - the brewers with the largest market shares are usually the most profitable Often market profitability is partially dependent on strong price leadership from a particular brewer coupled with rational behavior from the other brewers. Large shares in fragmented markets are unlikely to drive high returns, even if the markets exhibit high levels of volume growth.

The above-mentioned issues seem to be stating the obvious, but we think it is a fact that is missed by many beer companies, as well as by investors. Brewers and investors focus too much on volume growth and not enough on value growth. This poster will pinpoint both an overview of growth and profits in the world beer industry and a comparison of the leading brewing groups' performance in selected regional markets. The paper focuses on performance through compiling significant key data and financial ratios. The assumption thereby is that a link can be revealed between the brewers' domination of certain markets and their corporate success. This will be proved by collecting ratios covering financial data provided in the respective brewers' annual reports. The key data that give the deepest and most reliable insight into a beer market's profitability as well as a brewer's financial performance are

A comparison of Sales and EBITDA per market, Revenues per hectoliter, EBITDA per hectoliter

Operating Statistics of the 21 World-Wide Leading Brewing Groups - 2004

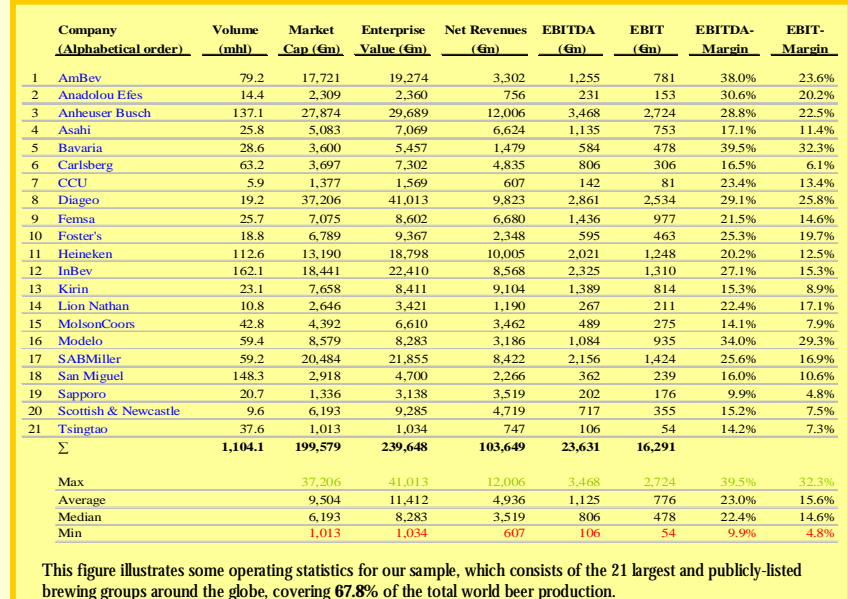

brewing groups around the globe, covering $67.8 \%$ of the total world beer production
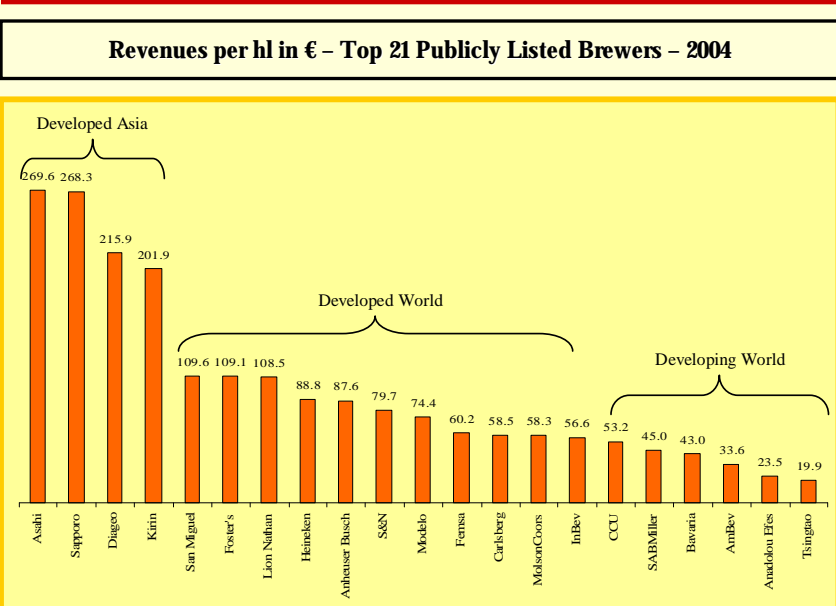

Sales and E BITDA 2004 - Profit Pools in Selected Brewers' Main Markets

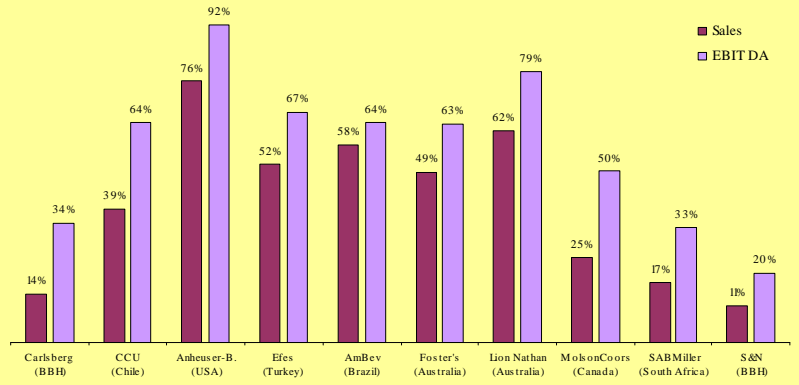

Market leadership

This figure illustrates the link between highly dominated key markets (in brackets) of different brewers where they are driving their highest percentage of EBITD A compared to sales. Carlsbery's BBH operations for example account for 14\% of Carlsberg's total sales but for 34\% of its EBITDA. Brewers who are strongly positioned in market positions. SABMiller, i.e. is a top three brewer in 30 of its 40 markets. Carlsbery operates in far more markets, but would have top three positions in fewer than 10 .
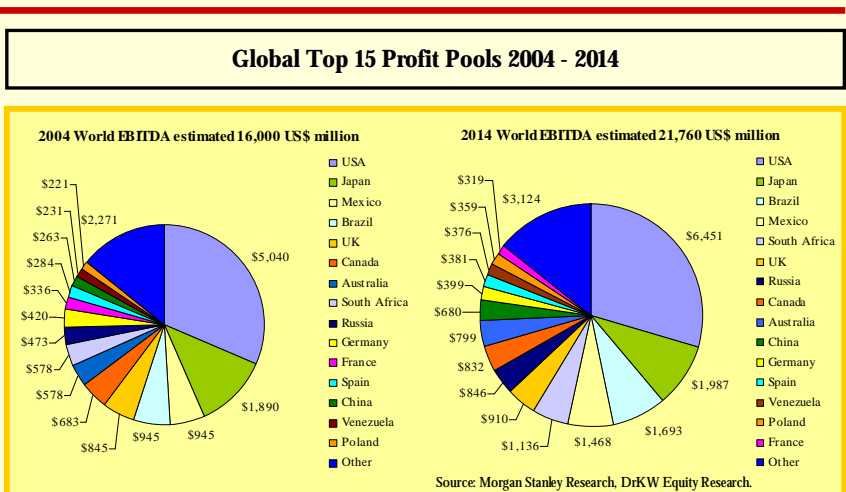

What are the Big Profit Pools in the World Brewing Industry?

These are the fifteen largest profit pools (expressed as EBITDA) in the world - accounting for $86 \%$ of the total global profits (Top 5: 60\%). The US profit pool is about three times as large as the combined profit pools of the twice as large. China would have to show compound growth of over $37 \%$ of its profit pool to catch the US in the next 10 years, assuming compound growth of the US of only $2 \%$ per annum. Japan's profit pool is slightly larger than the entire market of Westem Europe, and Latin America's is over 35\% bigger. In 10 years Latin America's profit pool should be about twice as large as Western Europe's and South Africa's profit pool is expected to be close to $25 \%$ larger than the UK's - despite being $45 \%$ smaller today.

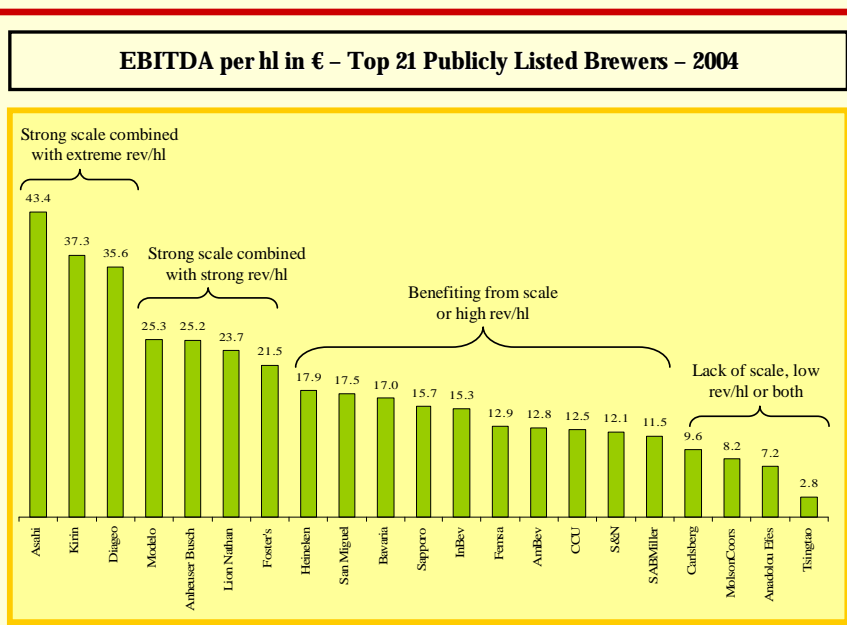

Results

Our comparison of the profit and loss accounts and cash flow statements of the 21 largest leading publicly listed companies world-wide highlights some very stark differences. The 21 companies in our database have differences in revenue per hl from €269.6 (Sapporo) to €19.9 (T singtao) - a 14-fold difference. More important, they also have differences in profit per hl ranging from €43.4 (Kirin) to €2.8 (Tsingtao), a 16-fold difference. Besides, it attracts analyst's attention that companies like Anheuser-Busch, Heineken, S\&N and the Japanese brewers receive far higher revenues per hl than some others, in particular Tsingtao, AmBev, and Efes, but more suprisingly, even the leading large brewers InBev and SABMiller perform comparatively weakly in terms of sales compared to their high production volume.

Conclusions

It is clear that, in many of the currently unprofitable markets, consolidation will help profitability considerably. Russia seems to be consolidating fast, as does India. However, we would caution that the level of consolidation needed to drive a big step change in profit can take much longer. G ermany, Westem-Europe's largest volume market, still remains highly fragmented and unprofitable despite decades of consolidation. As we have argued, this analysis is still a good guide to those brewers with the best exposure to higher opportunities for profit growth. Market growth is, of course, not the only source of growing profit to the brewers. Disproportionate profit

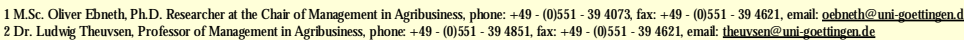

Poster presented at the $16^{\text {th }}$ World Forum, Symposium and Case Conference of the Intemational Food and Agribusiness Management Association (IAMA) - June, 10-13, 2006, Buenos Aires, Argentina. 


\section{KAPITEL III.3}

\section{Does Internationalization Pay Off? \\ Accounting-Based Evaluation of M\&As of European Brewers}

Oliver Ebneth and Ludwig Theuvsen

Poster presented at the $15^{\text {th }}$ Annual World Food and Agribusiness Symposium and Forum, $25^{\text {th }}-28^{\text {th }}$ of June 2005, Chicago. 


\section{Does Internationalization Pay Off?}

\section{Accounting-Based Evaluation of M\&As of European Brewers}

\section{Problem Statement}

Despite its long heritage as a local industry, the brewing sector is becoming a global market now, governed by cross-border takeovers and growth through acquisitions. This development arose from the fact that larger brewers faced low prospects for volume growth in developed markets leading them to seek growth either via acquisition of other brewers, by aggressive participation in developing markets or both [Lewis, 2001; Kaplan, 2003]. Thus, consolidation and acquisitions have been the growing trend in recent years as the major brewers like Heineken and Interbrew pursue growth outside of their own backyards [Benson-Armer et al., 1999; Todd, 2004]. The aggregate volume of the world's top brewers has grown at more than four times the pace of total industry volume since the mid-1990s. The following chart shows the above-average growth of the respective five largest brewing companies over the past five years. Also the $\mathrm{Cr} 5$ has arisen strongly compared to the preceding five years.

\begin{tabular}{|c|c|c|c|c|c|c|c|c|c|c|c|}
\hline \multicolumn{6}{|c|}{ Ranking of the world's biggest brewers } & \multicolumn{6}{|c|}{$\begin{array}{l}\text { Percentage of the world beer } \\
\text { production }\end{array}$} \\
\hline \multirow{2}{*}{$\begin{array}{l}\text { Rank } \\
2004\end{array}$} & \multirow{2}{*}{$\begin{array}{l}\text { in } \\
\text { mio. } \\
\text { hl }\end{array}$} & \multirow{2}{*}{$\begin{array}{l}\text { Rank } \\
1999\end{array}$} & \multirow{2}{*}{$\begin{array}{c}\text { in } \\
\text { mio. } \\
\text { hl }\end{array}$} & \multirow{2}{*}{$\begin{array}{l}\text { Rank } \\
1994\end{array}$} & \multirow{2}{*}{$\begin{array}{c}\text { in } \\
\text { mio. } \\
\text { hl }\end{array}$} & \multicolumn{2}{|c|}{$\begin{array}{c}\mathbf{2 0 0 4} \\
1,475 \text { mio. hl }\end{array}$} & \multicolumn{2}{|c|}{$\begin{array}{c}\mathbf{1 9 9 9} \\
1,365 \text { mio. hl }\end{array}$} & \multicolumn{2}{|c|}{$\begin{array}{c}1994 \\
1,222 \text { mio. hl }\end{array}$} \\
\hline & & & & & & $\%$ & $\mathrm{Cr}$ & $\%$ & $\mathrm{Cr}$ & $\%$ & $\mathrm{Cr}$ \\
\hline $\begin{array}{l}1 . \\
\text { InBev }\end{array}$ & 181 & $\begin{array}{l}\text { 1. } \\
\text { Anheuser- } \\
\text { Busch }\end{array}$ & 123 & $\begin{array}{l}\text { 1. } \\
\text { Anheuser- } \\
\text { Busch }\end{array}$ & 104 & 12.3 & $\begin{array}{l}\text { Cr1 } \\
12.3\end{array}$ & 9.0 & $\begin{array}{l}\text { Cr1 } \\
9.0\end{array}$ & 8.5 & $\begin{array}{r}\text { Cr1 } \\
8.5\end{array}$ \\
\hline $\begin{array}{l}2 . \\
\text { Anheuser- } \\
\text { Busch }\end{array}$ & 152 & $\begin{array}{l}\mathbf{2 .} \\
\text { Heineken }\end{array}$ & 91 & $\begin{array}{l}\mathbf{2 .} \\
\text { Heineken }\end{array}$ & 61 & 10.3 & $\begin{array}{l}\text { Cr2 } \\
22.6\end{array}$ & 6.7 & $\begin{array}{l}\text { Cr2 } \\
15.7\end{array}$ & 5.0 & $\begin{array}{r}\text { Cr2 } \\
13.5\end{array}$ \\
\hline $\begin{array}{l}3 . \\
\text { SAB } \\
\text { Miller }\end{array}$ & 138 & $\begin{array}{l}3 . \\
\text { Interbrew }\end{array}$ & 56 & $\begin{array}{l}3 . \\
\text { Miller }\end{array}$ & 54 & 9.4 & $\begin{array}{r}\text { Cr3 } \\
32.0\end{array}$ & 4.1 & $\begin{array}{l}\text { Cr3 } \\
19.8\end{array}$ & 4.4 & $\begin{array}{r}\text { Cr3 } \\
17.9\end{array}$ \\
\hline $\begin{array}{l}4 . \\
\text { Heineken }\end{array}$ & 120 & $\begin{array}{l}4 . \\
\text { Miller }\end{array}$ & 54 & $\begin{array}{l}4 . \\
\text { Kirin }\end{array}$ & 36 & 8.1 & $\begin{array}{l}\text { Cr4 } \\
41.1\end{array}$ & 4.0 & $\begin{array}{l}\mathrm{Cr} 4 \\
23.8\end{array}$ & 2.9 & $\begin{array}{l}\text { Cr4 } \\
20.8\end{array}$ \\
\hline $\begin{array}{l}\mathbf{5 .} \\
\text { Carlsberg }\end{array}$ & 89 & $\begin{array}{l}5 . \\
\text { AmBev }\end{array}$ & 43 & $\begin{array}{l}\mathbf{5 .} \\
\text { Foster's }\end{array}$ & 33 & 6.0 & $\begin{array}{l}\text { Cr5 } \\
47.1\end{array}$ & 3.2 & $\begin{array}{l}\text { Cr5 } \\
27\end{array}$ & 2.7 & $\begin{array}{r}\text { Cr5 } \\
23.5 \\
\end{array}$ \\
\hline
\end{tabular}

As aforementioned, amid the brewing industry acquisitions have been the growing trend in recent years, giving the opportunity to enhance remarkably a firm's degree of internationalization and its market share through diverse one-off deals. But according to various studies most 
of these cross-border deals did not meet the expectations [Agrawal et al., 1992; Jensen, 1992; Müller-Stewens, 2000; Müller-Stewens et al., 2002]. Recent spectacular acquisitions in the brewing industry have highlighted the apparent urgency of the major European brewers to build scale and improve their strategic positioning. Unfortunately for shareholders, this has mainly come at their expense. Assessing the corporate success and the financial performance of latest M\&As in the brewing sector, therefore, deserves more scientific attention.

\section{OBJECTIVES}

This poster only focuses on leading European brewers due to the fact that most global brewers are based there. Within the European brewery scene we consider five brewers as serious contestants in the global arena: Heineken, Interbrew, SABMiller, Carlsberg and Scottish\&Newcastle [Hoojimaijers, 2003]. Recently the number of mergers transacted by the Top 5 European breweries has increased. Several more changes can therefore be expected by the end of 2004 [Joh.Barth\&Sohn, 2004]. The following chart illustrates some of the high valuescaled cross-border deals, transacted by the European peer group members.

\section{Transaction Multiples}

\begin{tabular}{|c|c|c|c|c|c|c|c|}
\hline \multirow{2}{*}{$\begin{array}{c}\text { Date } \\
\text { Announced } \\
\end{array}$} & \multirow[b]{2}{*}{ Acquiror } & \multirow[b]{2}{*}{ Target } & \multirow[b]{2}{*}{$\%$ Acquired } & \multirow{2}{*}{$\begin{array}{r}\text { Transaction } \\
\text { Value (€m) } \\
\end{array}$} & \multicolumn{3}{|c|}{ Transaction Value as a Multiple of: } \\
\hline & & & & & Sales & EBITDA & HI (€/HI) \\
\hline 12-Aug-04 & Interbrew & SunInterbrew & $91 \%$ & 1,212 & 2.1 & 11.0 & 65 \\
\hline 03-Mar-04 & Interbrew & Ambev & $58 \%$ & 16,341 & 3.5 & 11.5 & 146 \\
\hline 19-Feb-04 & Carlsberg & Orkla & $40 \%$ & 7,054 & 1.5 & 8.6 & 87 \\
\hline 20-Jan-04 & Carlsberg & Holsten & $100 \%$ & 437 & 1.0 & 9.1 & 71 \\
\hline 07-Jan-04 & Interbrew & Oriental Brewery & $45 \%$ & 1,547 & - & 10.7 & 201 \\
\hline 18-Sep-03 & Interbrew & Spaten & $100 \%$ & 533 & 1.5 & 9.9 & 109 \\
\hline 11-Sep-03 & Interbrew & Apatinska Pivara & $100 \%$ & 229 & - & 6.3 & 95 \\
\hline 13-May-03 & $\mathrm{S} \& \mathrm{~N}$ & Centralcer & $51 \%$ & 828 & 3.3 & 11.4 & 251 \\
\hline 13-Мay-03 & SABMiller & Peroni & $60 \%$ & 563 & 1.1 & 12.6 & 128 \\
\hline 02-Мау-03 & Heineken & BBAG & $100 \%$ & 1,899 & 1.7 & 10.2 & 146 \\
\hline 28-Apr-03 & S\&N & Bulmer & $100 \%$ & 398 & 0.5 & 9.8 & 132 \\
\hline 14-Jan-03 & Heineken & $\mathrm{CCU}$ & $31 \%$ & 1,525 & 2.8 & 11.3 & 151 \\
\hline $15-$ Nov-02 & Interbrew & Gilde & $100 \%$ & 475 & 1.8 & 8.6 & 126 \\
\hline 12-Sep-02 & Heineken & Al Ahram & $100 \%$ & 273 & 2.7 & 8.0 & 260 \\
\hline 30-May-02 & $\mathrm{SAB}$ & Miller & $100 \%$ & 5,341 & 1.0 & 9.1 & 90 \\
\hline 18-Mar-02 & Heineken & Molson Brazil & $20 \%$ & 250 & 2.8 & 13.7 & 84 \\
\hline 14-Feb-02 & Scottish \& Newcastle & Hartwall & $100 \%$ & 2,273 & 2.8 & 10.1 & 142 \\
\hline 01-Feb-02 & Heineken & Bravo & $100 \%$ & 395 & 2.4 & 9.7 & 137 \\
\hline 29-Nov-01 & $\mathrm{SAB}$ & BevCo & $60 \%$ & 561 & 1.6 & 6.5 & 519 \\
\hline 06-Aug-01 & Interbrew & Becks & $100 \%$ & 1,790 & 2.1 & 13.0 & 206 \\
\hline 13-Jul-01 & Interbrew & Diebels & $80 \%$ & 100 & 1.4 & 8.3 & 83 \\
\hline 14-Jun-00 & Interbrew & Bass & $100 \%$ & 3,611 & 1.3 & 9.7 & 206 \\
\hline \multirow[t]{2}{*}{ 20-Mar-00 } & Scottish \& Newcastle & Kronenbourg & $100 \%$ & 2,744 & - & 11.3 & 183 \\
\hline & & & & $\begin{array}{r}\text { Summation: } \\
50,380\end{array}$ & & & \\
\hline
\end{tabular}

This study now attempts to evaluate cross-border acquisitions, transacted by the five leading European brewing companies using accounting-based measures. By calculating some meaningful indices further conclusions are to be drawn on the different strategies of external 
growth pursued by the leading brewing groups. The central question here is as follows: Which differences regarding the brewer's corporate success can be determined within the European peer group and what does this insinuate for gaining and sustaining long-term profit-growth regarding the level of sales and profitability?

\section{Procedures}

The poster shall pinpoint both, an overview about recently transacted cross-border acquisitions and a comparison of the European peer group member's acquisitioning performance. The main focus of the poster lies on the latter, to evaluate the brewer's financial performance through compiling significant key data and financial ratios. The assumption thereby is that a link between the brewer's increased international engagement through cross-border acquisitions and their corporate success can be revealed. This will be proved by compiling a time series over the last five years, covering financial data provided in the respective brewer's annual report. The key data which give the deepest and most reliable insight in a firm's financial performance are as follows:

- Return on Investment,

- Return on Net Assets,

- Ratio of Net Debt to EBITDA,

- Return on Capital Employed,

- Return on Equity [Fröhlich, 2004].

Additional attention will be turned on transaction multiples which are given by calculating the transaction value as a multiple of sales, EBITDA and hectoliters a target shows.

\section{RESULTS}

Taking a closer look at the transaction multiples (see first results given in the chart above) being paid by acquiring a target shows clear differences between several brewer's attitude towards external growth via cross-border acquisitions. Especially since the mid-1990s, some of the peer group members demonstrated their ability to increase margins and market shares by smart M\&A deals, other bought themselves into markets through extraordinary - and sometimes overpriced - acquisitions. Thus the leading European brewers pursue quite different internationalization strategies with diverse financial pay-offs. 


\section{CONCLUSION}

Recent acquisitions carried out by the European peer group members have confirmed that it is still a seller's market, with high multiples being paid, elongated periods of acceptable return and questionable strategic rationale in certain cases [Blythman et al., 2003]. Moreover our empirical study proves both, significant differences regarding the peer group members' level of acquisitioning and their impact on several brewers financial performance.

\section{REFERENCES}

Agrawal, A. et al. (1992): The Post-Merger Performance of Acquiring Firms: A Reexamination of an Anomaly, in: The Journal of Finane, 47(1992): 1605-1621.

Benson-Armer, R. et al. (1999): Global Beer: what's on tap? The McKinsey Quarterly 1999, No. 1.

Blythman, M. et al. (2003): Pan European Brewing Review - 2003, Merrill Lynch, London. Fröhlich, C. (2004): Wertorientierte Kennzahlen und ihre Bedeutung bei der Ermittlung des Unternehmenswertes - ein Fallbeispiel, in: Unternehmensbewertung und Management 10(2004): 383-391.

Hoojimaijers, M. (2003): European Breweries. Medium-Sized Brewery Targets in Europe. Julius Baer European Brokerage, Amsterdam.

Jensen, M. C. (1992): Market for Corporate Control, in: Newman, P. et al. (Edrs.), The New Palgrave Dictionary of Money and Finance, London: 657-665.

Joh.Barth\&Sohn (2004): The Barth Report. Hops 2003/04, Nuremberg.

Kaplan, A. (2003): Global beer: Tapping into Growth, in: Beverage World, 122(2003): 24-28.

Lewis, C. (2001): The Future of British Brewing: Strategies for Survival, in: Strategic Change, 10(2001): 151-161.

Müller-Stewens, G. (2000): Akquisitionen und der Markt für Unternehmenskontrolle: Entwicklungstendenzen und Erfolgsfaktoren, in: Picot, A. (Hrsg.), Management von Akquisitionen : Akquisitionsplanung und Integrationsmanagement, Stuttgart, S. 41-61.

Müller-Stewens, G. et al. (2002): Stand und Entwicklungstendenzen von Cross-BorderAkquisitionen, in: Krystek, U. und Zur, E. (Hrsg.) Handbuch Internationalisierung, S. 141-169.

Todd, H. (2004): Global Beer, in: Beverage World, 123(2004): 39-41. 


\section{D oes Intemationalizaltion Pay Off? - Accounting-Based Evaluation of M\&As of E uropean Brewers -}

Oliver Ebneth ${ }^{1}$ and Ludwig Theuvsen ${ }^{2}$

Problem Statement and Objectives

In the brewing industry acquisitions have been a wide-spread trend in recent years, giving the opportunity to enhance market share through several one-off deals. Amongst the world-wide leading brewers particularly the per coupt $f$ world-wide leading brewers particularly the peer group of the European brewery scene we consider five groups as serious contestants in the global arena: Heineken. InBev, Cartsbery Scottish\&Newcastle (S\&N) and SABMiller. These leading European brewing companies transacted a lot of cross-border acquisitions during recent years and thus could increase significantly their degree of intemationalization. This study now attempts to evaluate these accelerated intemationalization process by using aocounting-based measures and some the following question shall be answered. Which differneces

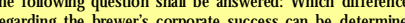
within the European peer group and what does this insinuate for gaining and sustaining long-term profit-growth regarding the level of sales and profitability?

Oline

\section{Evaluation of Corporate Success - Balance Sheet Analysis}

To evaluate the brewer's financial performance significant key data and financial ratios are built. The assumption thereby is that a link between the brewer's increased intemational engagement through cross-border acquisitions and their corporate success can be revealed. This will be proved by compiling a time series over the last five years, covering financial data provided in the respective brewer's annual report. The key data which give the deepest and most reliable insight in a firm's financia

Business Performance Measures

- Retum on Equity (ROE)

The ROE is defined as the accounting rate on shareholders' contributed capital. This is calculated by dividing the Net Income (= Net Profit) figure

and dividing it by Shareholders' Equity.

- Operating Margin

The Operating Margin serves as an indicator of management's pricing power and cost contro. economic returns but their lower visibility generally depresses valuation. It is calculated by
dividing the O perating Profit by Net Sales.

\author{
Ouality of Earnings Measures \\ - Cash Realization Ratio (CRR) \\ The CRR is defined as the Cash flow from \\ operating activities divided by Net Income. A \\ positive high value is consistent with conservative \\ depreciation policies, high reinvestment and \\ - Net Debt to Equity \\ Net Debt to Equity shows Net Debt as a \\ percentage of Equity Capital. It is the most \\ commonly used measure of financial leverage and \\ it provides an indicator of both financial risk and \\ capital efficiency.
}

\author{
Valuation Measures \\ - Enterprise Value/ EBITDA Multiple \\ The Enterprise Value (EV) / EBITDA multiple \\ gives the investor a broad indication of the cash- \\ Ture \\ Particularly in terms of acquisitions this is a useful \\ ratio of volume leverage, giving Enterprise Value \\ in relation to Sales (= Net Revenues). \\ -EV - Sales - EBITDA \\ These chart enables a comparison of EBITDA to \\ Sales ratio with the EV as a mainly capital-market \\ determined figure.
}

Recent Consolidation and Competition Issues in the World Brewing Industry

\begin{tabular}{|c|c|c|c|c|c|c|c|c|}
\hline \multirow{3}{*}{\multicolumn{2}{|c|}{$\begin{array}{l}\text { Top } 10 \text { Global Brewers } 1998 \\
\text { Share of Production Volume (1,301 mhl) } \\
\text { 37.6\% Market Share }\end{array}$}} & \multirow{3}{*}{$\begin{array}{l}\text { World Beer Production - Top } 10 \text { Brewers } \\
\text { mhl } \\
1,600\end{array}$} & \multirow{19}{*}{$\begin{array}{l}\text { Top } 10 \mathrm{Global} \text { Brewers } \mathbf{2 0 0 4} \\
\text { Share of Production Volume (1,475 mhl) } \\
\mathbf{6 1 . 8 \%} \text { Market Share }\end{array}$} & \multicolumn{5}{|c|}{ Major Mergers \& Acquisitions 2000-2005 } \\
\hline & & & & \multicolumn{2}{|c|}{$\begin{array}{ll}\text { Date } & \\
\text { Announced } & \text { Acquiror } \\
\end{array}$} & \multirow{2}{*}{$\begin{array}{l}\text { Target } \\
\begin{array}{l}\text { Sunnterbrew } \\
\text { Sunnterbrew }\end{array}\end{array}$} & \multirow{2}{*}{$\begin{array}{c}\% \\
\text { Acquired } \\
99 \% \\
91 \%\end{array}$} & \multirow{2}{*}{$\begin{array}{r}\begin{array}{r}\text { Transaction } \\
\text { Value }(\mathrm{fm})\end{array} \\
260 \\
1,212\end{array}$} \\
\hline & & & & 03-Jan-05 & InBev & & & \\
\hline & 口Anheuser-Bush & $\begin{array}{l}1,400- \\
1,200-\end{array}$ & & $\begin{array}{l}\text { 12-AAge-04 } \\
\text { 03-Mar-04 }\end{array}$ & $\begin{array}{l}\text { Intererowew } \\
\text { Interbrew }\end{array}$ & & $\begin{array}{l}91 \% \% \\
58 \%\end{array}$ & $\begin{array}{r}1,212 \\
16,341\end{array}$ \\
\hline & 口 H Hénelen & $\begin{array}{l}1,200- \\
1,000-\end{array}$ & & 19-Feb-04 & Carlsberg & Orkla & $40 \%$ & 7,054 \\
\hline & & $\begin{array}{l}1,000 \\
800-1\end{array}$ & & $\begin{array}{l}20-1 \operatorname{lan}-04 \\
07-\operatorname{lan}-04\end{array}$ & $\begin{array}{l}\text { Carlsherg } \\
\text { Interbrew }\end{array}$ & $\begin{array}{l}\text { Holsten } \\
\text { Oriental Brewery }\end{array}$ & $100 \%$ & $\begin{array}{r}437 \\
1.547\end{array}$ \\
\hline & 口Bradma & $600-\left(\begin{array}{c}C r 10 \\
1999\end{array}\right.$ & & 18-Sep-03 03 & Interbrew & Spaten & $100 \%$ & 533 \\
\hline & 口Intabresw & $400-32.2 \%$ & & $\begin{array}{l}\text { 11-5ep-03 } \\
\text { 13-May-03 }\end{array}$ & $\begin{array}{l}\text { Interbrew } \\
\text { S\&N }\end{array}$ & $\begin{array}{l}\text { AApaninksa i ivara } \\
\text { Centralcer }\end{array}$ & $\begin{array}{l}10 \% \% \\
51 \%\end{array}$ & 828 \\
\hline & G Grupo Moddo & 200 & & 13-May-03 & SABMiller & Peroni & $60 \%$ & 563 \\
\hline & $\mathrm{GKinn}$ & & & $\begin{array}{l}\text { 20-May-03 } \\
\text { 28-Apr-03 }\end{array}$ & $\begin{array}{l}\text { Heineken } \\
\text { S\&N }\end{array}$ & $\begin{array}{l}\text { BBAG } \\
\text { Bulmer }\end{array}$ & $\begin{array}{l}100 \% \\
100 \%\end{array}$ & ${ }_{398}^{1,899}$ \\
\hline & - Fosters & $\underbrace{+\infty}$ & & 14-Jan-03 & Heineken & $\mathrm{CCU}$ & $31 \%$ & 1,525 \\
\hline & & & & 15-Nov-02 & Interbrew & & $100 \%$ & 475 \\
\hline & & 口World Beer Production & & $\begin{array}{l}12-\text {-esp-02 } \\
\text { 30-May-02 }\end{array}$ & $\begin{array}{l}\text { Heineken } \\
\mathbf{S A B}\end{array}$ & $\begin{array}{l}\text { Al Alhram } \\
\text { Miller }\end{array}$ & $100 \%$ & $\begin{array}{r}273 \\
5341\end{array}$ \\
\hline Despite its long heritage a & al industry, the & ewing sector is becoming a global market now, govemed by cn & & 18-Mar-02 & Heineken & Molson Brazil & $20 \%$ & 250 \\
\hline acquisitions. This devel & las arisen from & e fact that larger brewers faced low prospects for volume grow & & $\begin{array}{l}14-\mathrm{F} b-02 \\
01-\mathrm{Feb}-02\end{array}$ & $\begin{array}{l}\text { S\&N } \\
\text { Heineken }\end{array}$ & $\begin{array}{l}\text { Hatrwall } \\
\text { Bravo }\end{array}$ & $\begin{array}{l}100 \% \\
100 \%\end{array}$ & $\begin{array}{l}2,273 \\
395\end{array}$ \\
\hline growth either via acquisitic & ther brewers, $\mathrm{b}$ & aggressive participation in developing markets or both. Thus, $\mathrm{c}$ & & 29-Nov-01 & & & $60 \%$ & 561 \\
\hline growing trend in recent ye & the major brew & s like Heineken and InBev pursue growth outside & & 06-Aug-01 & Interbrew & Becks & $100 \%$ & 1,790 \\
\hline The aggregate volume of $t$ & rld's top brewe & has grown at more than four times the pace of total industry v & & 14-Jun-00 & Interbrew & & $100 \%$ & 3,611 \\
\hline the above average growth & ten largest bre & ng companies over the past six years. Thereby the $\mathrm{Cr} 10$ has ari & & 20-Mar-00 & & Kronenbourg & $\frac{100 \%}{\text { Total }}$ & $\frac{2,744}{50,540}$ \\
\hline
\end{tabular}
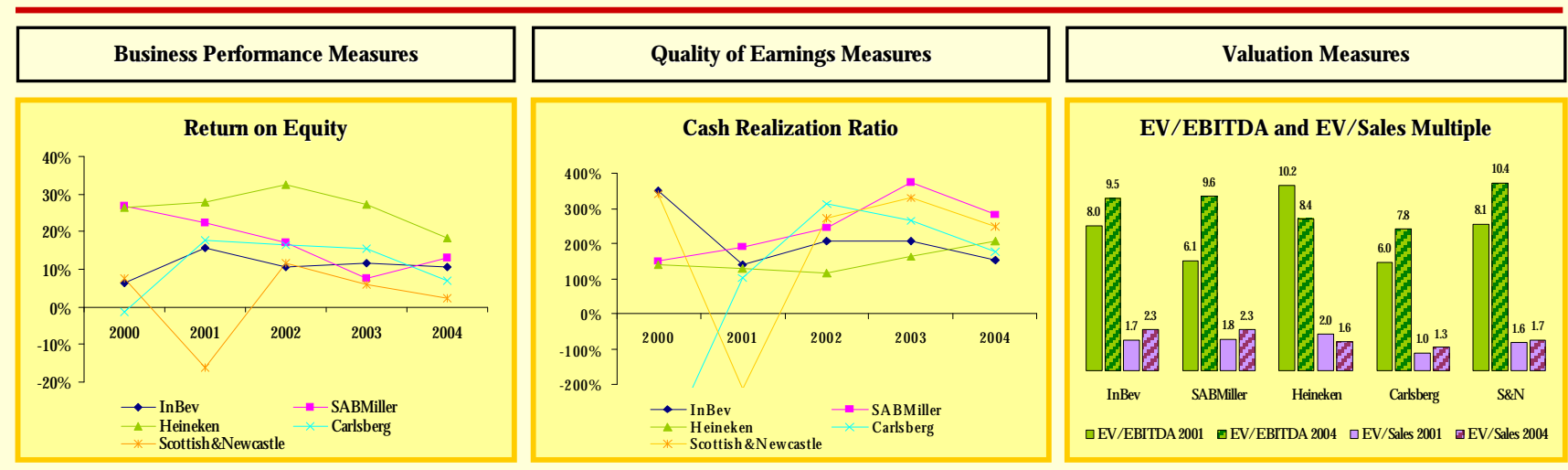

EV/ E BITDA and EV/ Sales Multiple
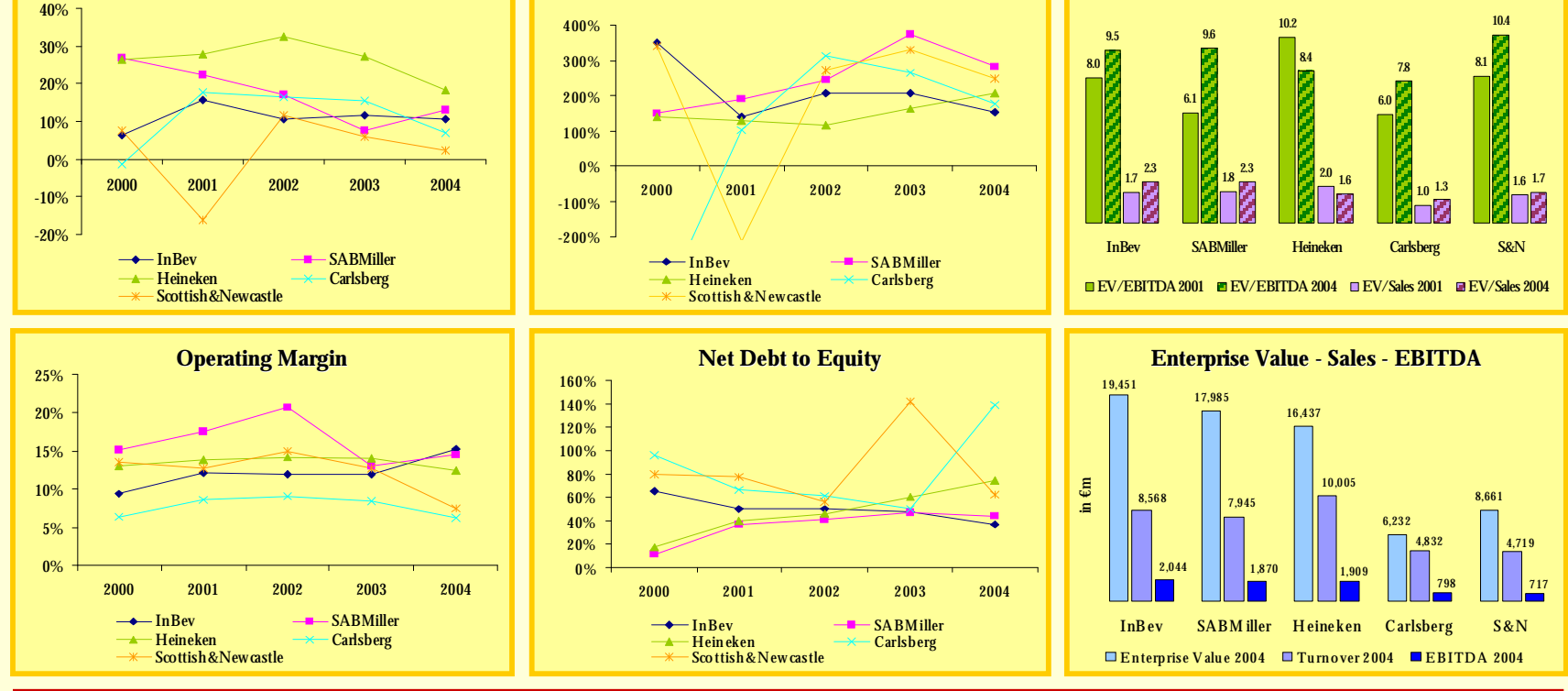

\section{Results}

Some of the E uropean brewers demonstrated their ability to increase margins and market shares by smart M\&A deals, other bought themselves into markets through extraordinary and overpriced acquisitions. Thus European brewers pursue quite different intemationalization strategies with diverse financial pay-offs. Our empirical study proves significant differences between the peer group members' financial performance regarding accounting and capital-market determined figures. $\ddot{y}$ Business Performance Measures: InBev's Return on Equity appears a little low, but this includes the impact of the AmBev transaction in 2004 in which it issued equity to Braco and took significant goodwill into the balance sheet. Similarly, the AmBev deal left InBev with the highest Operating Margin in the brewing sector, closely followed by SABMiller. The margin figure for Carlsherg is still low, due to the delay in delivering any cost savings through to profit improvement. ÿ Quality of Eamings Measures: Starting by looking at the Cash Realization Ratio all brewers perform quite strongly with a high value compared to the market. The two outliers of the group are S\&N and Carlsbery. Looking at the Net D ebt to Equity, Carlsberg and S\&N again stand out as having the highest net debt/ equity ratio. Carlsberg geared up in 2004 when acquiring Holsten and the Orkla minority. S\&N shows no sign of declining, due to its poor cash flow generation. $\ddot{y}$ Valuation Measures: These multiples and figures make a connection between accounting-based ratios and market value. In 2001 Heineken appeared the most expensive stock in the group. But unlike its European competitors Heineke downgraded its EV/EBITDA and EV/ Sales multiples over the last four years. The EV - Sales - EBITDA figure also illustrates Heineken's as well as Carlsberg's relatively weak market value compared to their level of sales and EBITDA. 
KAPITEL III.4

\section{Large Mergers and Acquisitions of European Brewing Groups Event Study Evidence on Value Creation}

\section{Oliver Ebneth and Ludwig Theuvsen}

Submitted for 'Agribusiness' in December 2005.

Paper presented in a similar form at the $15^{\text {th }}$ Annual World Food and Agribusiness Symposium and Forum, $25^{\text {th }}-28^{\text {th }}$ of June 2005, Chicago. 


\section{Large Mergers and Acquisitions of European Brewing Groups Event Study Evidence on Value Creation}

\section{EXECUTIVE SUMMARY}

The importance of mergers and acquisitions (M\&As) as strategies for achieving international market shares is evidenced by the increasing number of such activities in recent years. Even the brewing sector is becoming a global market now, governed by cross-border takeovers and growth through acquisitions. Whether a merger or acquisition in the brewing industry will in fact have a positive impact on shareholder wealth will be illustrated by applying advanced event study methodology.

The basic assumption underlying the use of stock market data to estimate the effects of M\&As is that share prices reflect expectations about future profit and dividend streams. The primary data employed in this paper are daily stock returns of five European brewing groups which announced 31 M\&As during the sample period from March 2000 through August 2005.

The results of this study provide empirical evidence on both significant differences regarding the peer group members' level of acquisitioning and their impact on several brewers' financial performance as expressed in increasing or decreasing stock prices. Some investor reactions were broadly negative, reflecting overpriced deals. But these high purchase prices may be justified by the immense strategic importance of entering markets like China or Russia. Unlike the results of event studies on other industries, no overall significant negative response to M\&As has been observed in the brewing industry despite some negative outliers. Obviously it is paramount in the brewing industry to make the right deals to enhance shareholders' value. Thus, there seems to be no doubt that the consolidation process will continue to gather pace: However there remains one unanswered question: When will the brewing scene witness large-scale deals due to mergers within the top ten?

Further research should be carried out comparing both indicators of a firm's true performance: capital-market oriented event study methodology and accounting-based measures. More work on MNCs in the brewing sector could also focus on evaluating the efficiency of various strategies according to their specialization or diversification along geographical lines. Lastly, it would be extremely helpful if analysis of M\&As in the brewing industry included the target company's abnormal returns. 


\section{Abstract}

Acquisitions have been the growing trend in recent years, giving brewers the opportunity to enhance their degree of internationalization and market share remarkably through diverse oneoff deals. Larger brewers are faced with low prospects for volume growth in developed markets leading them to seek growth either via acquisition of other brewers or by aggressive participation in developing markets or both. This study employs event study analysis to examine 31 mergers and acquisitions among leading European brewing groups. Differences regarding the brewer's corporate success can be determined within the European peer group. Managerial implications as well as future research propositions conclude this paper.

\section{JEL CLASSIFICATION SYSTEM}

G140 - Information and Market Efficiency; Event Studies

G340 - Mergers; Acquisitions; Restructuring; Voting;

\section{KEYWORDS}

European Brewing Groups, Internationalization, Corporate Success, Event Study Methodology, Mergers\&Acquisitions. 


\section{INTRODUCTION}

In recent decades, one of the most striking developments has been the ever-increasing globalization of markets and the internationalization of economic activities. Due to low growth rates on home markets, growing international competitive pressures, shortened product lifecycles and growing $\mathrm{R} \& \mathrm{D}$ and marketing investments, many companies have expanded their national activities and, thus, contributed to the further advancement of the intensity and dynamics of international competition (Scholl, 1989; Marx, 1998). This development has also seized brewers. Despite its long heritage as a local industry, the brewing sector is now becoming a global market governed by cross-border takeovers and growth through acquisitions. This development arose from the fact that larger brewers faced low prospects for volume growth in developed markets leading them to seek growth either via acquisition of other brewers, by aggressive participation in developing markets or both (Köhler and Hüttemann, 1989). Thus acquisitions have been the growing trend in recent years, giving firms the opportunity to enhance their degree of internationalization and market share remarkably through diverse oneoff deals (Lewis, 2001; Kaplan, 2003). But according to various studies about diverse industries, most cross-border deals have not met expectations (Agrawal et al, 1992; Jensen, 1992; Müller-Stewens, 2000; Müller-Stewens et al, 2002). Recent spectacular acquisitions in the brewing industry have highlighted the apparent urgency for the major European brewers to build scale and improve their strategic positioning. Unfortunately for shareholders, this has come mainly at their expense. Assessing the corporate success and financial performance of latest M\&As in the brewing sector, therefore, deserves more scientific attention (BensonArmer et al, 1999; Todd, 2004).

In this paper we apply an empirical tool called event study to measure the response of financial markets to changes in the global brewing industry resulting from M\&A activities over the last five years. Event study analysis uses data from daily stock price movements to determine whether an event - such as the announcement of a M\&A activity - generates a statistically significant change in firm valuations (King et al, 2002). Here event study methodology is applied to the announcement of $31 \mathrm{M} \& \mathrm{~A}$ events in the world brewing industry. This paper contributes to the existing literature in two ways. First of all, it broadens the regional scope of the literature on event study methodology by going beyond the United States as most event studies are focused on industries based in the U.S. Secondly, most of the existing literature focuses on banking or other financial service industries (Jensen, 1992; Agrawal and Jaffe, 2000; Putlitz, 2001; Beitel, 2002; Harrison et al, 2005) whereas this paper specifically ad- 
dresses the impact of mergers within the brewing industry on shareholders' return from acquiring firms.

\section{MERGERS AND ACQUISITIONS IN THE BREWING INDUSTRY}

Although globalization is a general trend in many industries, the brewing industry has long lagged behind and remained very fragmented. Globalization in the beer industry has proceeded at a much slower pace than in many related businesses. Just five years ago management consultants feared that "Even today's most global brewers are relatively small and vulnerable to takeovers, perhaps by other packaged goods companies aiming to extend their food brands or distribution" (Benson-Armer et al, 1999). Thus, during recent years pioneer enterprises like Heineken and InBev (former Interbrew) have started internationalizing their activities in order to continue growing and expanding in view of saturated home markets (Todd, 2004). According to various studies the aggregate volume of the world's top ten brewers (see also figure 1) has grown at more than four times the pace of total industry volume since the mid-1990s.

Figure 1: Top Ten Global Brewers (1998-2004).

Top 10 Global Brewers 1998 Share of Production Volume $\left(1,301 \mathrm{mhl}^{*}\right)$ $37.6 \%$ Market Share

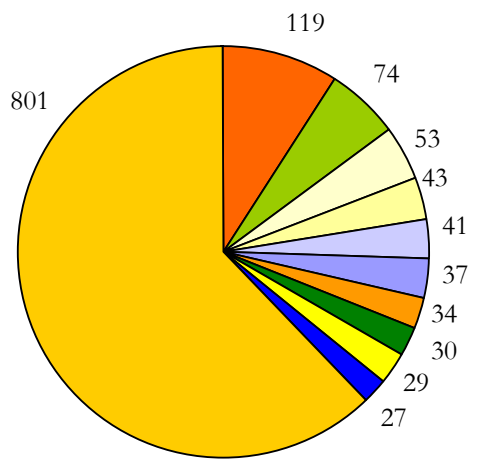

$\square$ Anheuser-Busch

$\square$ Heineken

$\square$ Miller

$\square \mathrm{SAB}$

$\square$ Brahma

$\square$ Interbrew

$\square$ Carlsberg

$\square$ Grupo Modelo

$\square$ Kirin

- Foster's

$\square$ Others
Top 10 Global Brewers 2004

Share of Production Volume (1,475 mhl) $61.8 \%$ Market Share

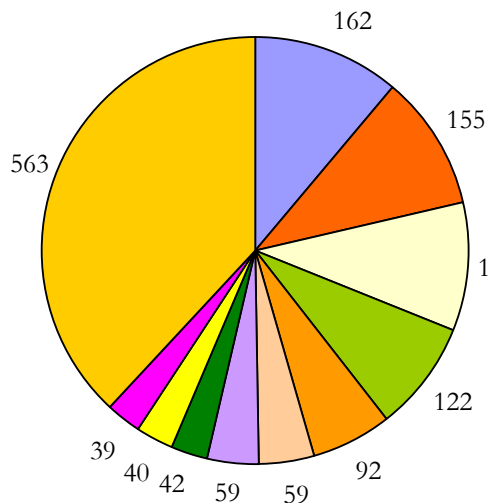

$\square \mathrm{InBev}$

$\square$ Anheuser-Busch

$\square$ SABMiller

$\square$ Heineken

$\square$ Carlsberg

$144 \square$ MolsonCoors

$\square$ Scottish\&Newcastle

$\square$ Grupo Modelo

$\square$ Kirin

$\square$ Tsingtao

$\square$ Others

\footnotetext{
$* \mathrm{mhl}=$ million hectoliters.
}

Source: Authors' calculations, company data, Joh.Barth\&Sohn (2005). 
In recent years the brewing industry has been undergoing an unprecedented drive for consolidation primarily effected through a series of high profile mergers and acquisitions. Thus, internationalization in the beer industry is now moving at a much higher speed. Within only a couple of years formerly medium-sized InBev has become the world's number one brewer by aggressively expanding and acquiring foreign competitors. Internationalization in the brewing sector, therefore, deserves more scientific attention. The following chart shows the aboveaverage growth of the five largest brewing companies over the past five years. It is noteworthy that the CR5 has risen strongly compared to the preceding five years.

Table 1: Ranking of the Leading Brewers and Concentration Ratio.

\begin{tabular}{|c|c|c|c|c|c|c|c|c|c|c|c|}
\hline \multicolumn{6}{|c|}{ Ranking of the world's biggest brewers } & \multicolumn{6}{|c|}{$\%$ of the world beer production } \\
\hline \multirow{2}{*}{$\begin{array}{l}\text { Rank } \\
\mathbf{2 0 0 4}\end{array}$} & \multirow[t]{2}{*}{ mhl } & \multirow{2}{*}{$\begin{array}{l}\text { Rank } \\
1999\end{array}$} & \multirow[t]{2}{*}{$\mathrm{mhl}$} & \multirow{2}{*}{$\begin{array}{l}\text { Rank } \\
\mathbf{1 9 9 4}\end{array}$} & \multirow{2}{*}{$\mathrm{mhl}$} & \multicolumn{2}{|c|}{$\begin{array}{c}\mathbf{2 0 0 4} \\
1,475 \mathrm{mhl}\end{array}$} & \multicolumn{2}{|c|}{$\begin{array}{c}1999 \\
1,365 \mathrm{mhl}\end{array}$} & \multicolumn{2}{|c|}{$\begin{array}{c}1994 \\
1,222 \mathrm{mhl}\end{array}$} \\
\hline & & & & & & $\%$ & $\mathrm{Cr}$ & $\%$ & $\mathrm{Cr}$ & $\%$ & $\mathrm{Cr}$ \\
\hline InBev & 162 & $\begin{array}{l}\text { Anheuser- } \\
\text { Busch }\end{array}$ & 123 & $\begin{array}{l}\text { Anheuser- } \\
\text { Busch }\end{array}$ & 104 & 11.0 & $\begin{array}{l}\text { Cr1 } \\
11.0\end{array}$ & 9.0 & $\begin{array}{c}\text { Cr1 } \\
9.0\end{array}$ & 8.5 & $\begin{array}{l}\text { Cr1 } \\
8.5\end{array}$ \\
\hline $\begin{array}{l}\text { Anheuser- } \\
\text { Busch }\end{array}$ & 155 & Heineken & 91 & Heineken & 61 & 10.5 & $\begin{array}{l}\text { Cr2 } \\
21.5\end{array}$ & 6.7 & $\begin{array}{l}\text { Cr2 } \\
15.7\end{array}$ & 5.0 & $\begin{array}{l}\text { Cr2 } \\
13.5\end{array}$ \\
\hline SABMiller & 144 & Interbrew & 56 & Miller & 54 & 9.8 & $\begin{array}{l}\text { Cr3 } \\
31.3\end{array}$ & 4.1 & $\begin{array}{l}\text { Cr3 } \\
19.8\end{array}$ & 4.4 & $\begin{array}{l}\text { Cr3 } \\
17.9\end{array}$ \\
\hline Heineken & 122 & Miller & 54 & Kirin & 36 & 8.3 & $\begin{array}{l}\text { Cr4 } \\
40.6\end{array}$ & 4.0 & $\begin{array}{l}\text { Cr4 } \\
23.8\end{array}$ & 2.9 & $\begin{array}{l}\text { Cr4 } \\
20.8\end{array}$ \\
\hline Carlsberg & 92 & AmBev & 43 & Foster's & 33 & 6.2 & $\begin{array}{l}\text { Cr5 } \\
46.8\end{array}$ & 3.2 & $\begin{array}{c}\text { Cr5 } \\
27\end{array}$ & 2.7 & $\begin{array}{l}\text { Cr5 } \\
23.5\end{array}$ \\
\hline
\end{tabular}

Source: Author's calculations.

Recently the number of mergers transacted by the Top 5 European breweries has increased. More changes can therefore be expected by the end of 2005 (Joh.Barth\&Sohn, 2005). According to various brokers the leading players in the brewing scene have spent more than $€ 75$ billion in around $400 \mathrm{M} \& \mathrm{~A}$ transactions in the course of only the last five years (Rössing, 2005). The 31 biggest deals alone have accounted for approximately $€ 64$ billion. The following table illustrates some of these high-value cross-border deals transacted by the European peer group members. In this figure the transaction values as multiples of sales, EBITDA and $€$ per hectoliter (hl) are highlighted. 
Table 2: Transaction Multiples of Major M\&As in the Brewing Industry - 2000-2005.

\begin{tabular}{|c|c|c|c|c|c|c|c|}
\hline \multirow{2}{*}{$\begin{array}{c}\text { Date } \\
\text { Announced }\end{array}$} & \multirow[b]{2}{*}{ Acquiror } & \multirow[b]{2}{*}{ Target } & \multirow[b]{2}{*}{$\%$ Acquired } & \multirow{2}{*}{$\begin{array}{c}\text { Transaction } \\
\text { Value (€m) }\end{array}$} & \multicolumn{3}{|c|}{ Transaction Value as a Multiple of: } \\
\hline & & & & & Sales & EBITDA & $\mathrm{hl}(€ / \mathrm{hl})$ \\
\hline 16-Aug-05 & Heineken & Taranov & $100 \%$ & 453 & - & 11,8 & - \\
\hline 19-Jul-05 & SABMiller & Bavaria & $100 \%$ & 6.473 & 4,38 & 10,0 & 243 \\
\hline 18-Jul-05 & InBev & Tinkoff & $100 \%$ & 167 & - & - & - \\
\hline 03-Jan-05 & InBev & SunInterbrew & $100 \%$ & 260 & 1,80 & 8,8 & 61 \\
\hline 16-Sep-04 & SABMiller & Lion Nathan China & $100 \%$ & 119 & 1,90 & 10,3 & 134 \\
\hline 12-Aug-04 & Interbrew & SunInterbrew & $91 \%$ & 1.212 & 2,05 & 11,0 & 65 \\
\hline 22-Jul-04 & Coors & Molson & $50 \%$ & 3.200 & 2,01 & 8,8 & 152 \\
\hline 15-Jun-04 & Anheuser-Busch & Harbin & $29 \%$ & 640 & - & - & - \\
\hline 03-Mar-04 & Interbrew & Ambev & $58 \%$ & 16.341 & 3,50 & 11,5 & 146 \\
\hline 19-Feb-04 & Carlsberg & Orkla & $40 \%$ & 7.054 & 1,52 & 8,6 & 87 \\
\hline 20-Jan-04 & Carlsberg & Holsten & $100 \%$ & 437 & 0,99 & 9,1 & 71 \\
\hline 07-Jan-04 & Interbrew & Oriental Brewery & $45 \%$ & 1.547 & - & 10,7 & 201 \\
\hline 18-Sep-03 & Interbrew & Spaten & $100 \%$ & 533 & 1,49 & 9,9 & 109 \\
\hline 11-Sep-03 & Interbrew & Apatinska Pivara & $100 \%$ & 262 & - & 6,3 & 95 \\
\hline 08-Sep-03 & Interbrew & Lion & $50 \%$ & 114 & 1,60 & 11,4 & 132 \\
\hline 13-May-03 & S\&N & Centralcer & $51 \%$ & 828 & 3,32 & 11,4 & 251 \\
\hline 13-May-03 & SABMiller & Peroni & $60 \%$ & 563 & 1,14 & 12,6 & 128 \\
\hline 02-May-03 & Heineken & BBAG & $100 \%$ & 1.899 & 1,73 & 10,2 & 146 \\
\hline 28-Apr-03 & S\&N & Bulmer & $100 \%$ & 398 & 0,50 & 9,8 & 132 \\
\hline 14-Jan-03 & Heineken & $\mathrm{CCU}$ & $31 \%$ & 1.525 & 2,83 & 11,3 & 151 \\
\hline 15-Nov-02 & Interbrew & Gilde & $100 \%$ & 575 & 1,80 & 8,6 & 126 \\
\hline 12-Sep-02 & Heineken & Al Ahram & $100 \%$ & 273 & 2,70 & 8,0 & 260 \\
\hline 05-Jun-02 & Heineken & Karlsberg & $45 \%$ & 135 & 1,80 & 10,8 & - \\
\hline 30-May-02 & SAB & Miller & $100 \%$ & 6.194 & 1,04 & 9,1 & 90 \\
\hline 18-Mar-02 & Heineken & Molson Brazil & $20 \%$ & 250 & 2,80 & 13,7 & 84 \\
\hline 14-Feb-02 & S\&N & Hartwall & $100 \%$ & 2.273 & 2,81 & 10,1 & 142 \\
\hline 01-Feb-02 & Heineken & Bravo & $100 \%$ & 395 & 2,43 & 9,7 & 137 \\
\hline 29-Nov-01 & SAB & BevCo & $60 \%$ & 561 & 1,60 & 6,5 & 519 \\
\hline 06-Aug-01 & Interbrew & Becks & $100 \%$ & 1.790 & 2,11 & 13,0 & 206 \\
\hline 13-Jul-01 & Interbrew & Diebels & $80 \%$ & 100 & 1,40 & 8,3 & 83 \\
\hline $11-F e b-01$ & Heineken & Schörghuber & $50 \%$ & 195 & 1,70 & 9,1 & - \\
\hline 14-Jun-00 & Interbrew & Bass & $100 \%$ & 3.611 & 1,25 & 9,7 & 206 \\
\hline 03-Nov-00 & Carlsberg & Feldschlösschen & $100 \%$ & 574 & 1,60 & 8,6 & 99 \\
\hline \multirow[t]{2}{*}{ 20-Mar-00 } & S\&N & Kronenbourg & $100 \%$ & 2.744 & 1,80 & 11,3 & 183 \\
\hline & & & & 63.696 & 1,99 & 10,0 & 153 \\
\hline
\end{tabular}

Source: Authors' calculations, company data.

Since most global brewers are based in Europe, this paper focuses only on the leading European brewers. Within the European brewery scene we consider five brewers as serious contestants in the global arena: Heineken, InBev, SABMiller, Carlsberg and Scottish\&Newcastle (S\&N). Especially since the mid-1990s, some members of this peer group have demonstrated their ability to increase margins and market shares by smart M\&A deals, and others have bought themselves into markets through extraordinary - and sometimes overpriced - acquisitions (Hoojimaijers, 2003). Figure 2 presents the latest annual financial data for the five leading European brewing groups in the sample. 
Table 3: Operating Statistics of European Brewing Groups Involved in M\&As - 2004.

\begin{tabular}{|c|c|c|c|c|c|c|c|c|c|c|}
\hline Company & $\begin{array}{l}\text { Volume } \\
(\mathrm{mhl})^{*}\end{array}$ & $\begin{array}{c}\text { Market } \\
\text { Cap } \\
(€ m)\end{array}$ & $\begin{array}{c}\text { Enterprise } \\
\text { Value } \\
(€ m)^{* *} \\
\end{array}$ & $\begin{array}{c}\text { Net } \\
\text { Sales } \\
(€ \mathbf{m})^{* * *}\end{array}$ & $\begin{array}{c}\text { EBITDA } \\
(€ \mathrm{~m}) \\
\end{array}$ & $\begin{array}{c}\text { EBIT } \\
(€ m)\end{array}$ & $\begin{array}{c}\text { Net } \\
\text { Profit } \\
(€ m) \\
\end{array}$ & $\begin{array}{c}\text { EBITDA- } \\
\text { Margin } \\
\end{array}$ & $\begin{array}{c}\text { EBIT- } \\
\text { Margin }\end{array}$ & $\begin{array}{c}\begin{array}{c}\text { Net } \\
\text { Profit- } \\
\text { Margin }\end{array} \\
\end{array}$ \\
\hline Heineken & 112,0 & 13.190 & 16.304 & 10.005 & 2.021 & 1.248 & 537 & $20,2 \%$ & $12,5 \%$ & $5,4 \%$ \\
\hline InBev & 183,7 & 19.430 & 24.878 & 8.568 & 2.325 & 1.310 & 719 & $27,1 \%$ & $15,3 \%$ & $8,4 \%$ \\
\hline SABMiller & 151,9 & 19.307 & 20.678 & 8.422 & 2.156 & 1.424 & 906 & $25,6 \%$ & $16,9 \%$ & $10,8 \%$ \\
\hline Carlsberg & 67,1 & 3.697 & 7.062 & 4.835 & 806 & 306 & 75 & $16,5 \%$ & $6,1 \%$ & $1,3 \%$ \\
\hline S\&N & 58,5 & 6.193 & 9.285 & 4.719 & 717 & 355 & 59 & $15,2 \%$ & $7,5 \%$ & $1,2 \%$ \\
\hline Max & 183,7 & 19.430 & 24.878 & 10.005 & 2.325 & 1.424 & 906 & $27,1 \%$ & $16,9 \%$ & $10,8 \%$ \\
\hline Average & 114,6 & 12.363 & 15.641 & 7.310 & 1.605 & 929 & 459 & $20,9 \%$ & $11,7 \%$ & $5,4 \%$ \\
\hline Median & 112,0 & 13.190 & 16.304 & 8.422 & 2.021 & 1.248 & 537 & $20,2 \%$ & $12,5 \%$ & $5,4 \%$ \\
\hline Min & 58,5 & 3.697 & 7.062 & 4.719 & 717 & 306 & 59 & $15,2 \%$ & $6,1 \%$ & $1,2 \%$ \\
\hline$\sum$ Total Sample & 573,2 & 61.817 & 78.207 & 36.548 & 8.025 & 4.643 & 2.296 & & & \\
\hline $\begin{array}{l}* \quad \text { Volume in } \\
* * \quad \text { Enterprise } \\
* * * \text { Excise dut }\end{array}$ & $\begin{array}{l}\text { ion hectol } \\
\text { le = Mark } \\
\text { educted }\end{array}$ & $\begin{array}{l}\text { ss (mhl) } \\
\text { Capitalizat }\end{array}$ & on + Net De & + Minoriti & S - Associ & & & & & \\
\hline
\end{tabular}

Source: Author's calculations.

In terms of volume, the sample represents $37 \%$ of the total world beer production. The combined market capitalization totals around $€ 62$ billion, the combined sales reached $€ 36.5$ billion and the sample's cumulated profit figures in 2004 have been as follows: $€ 8.0$ billion (EBITDA), €4.6 billion (EBIT), €2.3 billion (Net Profit).

The number of studies trying to answer the question of the motivation behind mergers has grown proportionally with the growth of M\&A activities in certain industries. Reasons for mergers include various efficiency improvements, such as replacing of inefficient management and gaining monopoly power, as well as agency motives, such as growth maximization, free cash flow and employment risk reduction. Moreover, strategy theory tells us that value is created in an M\&A through the identification and exploitation of synergies (Shusterman et al, 2001). Three broad classes of synergies are the usual focus of researchers. First, operating synergies arise when economies of scale and scope are captured across a variety of firm activities. Financial synergies are driven by reductions in the cost of capital. Collusive synergies ("market power") enable a firm either to extract a higher price for its products or services or pay suppliers a reduced price (Chatterjee, 1996). The recent burst of takeover activities has been viewed as a distinctly new wave driven by strategic, synergistic factors. For the brewing groups M\&As seem to be the fastest and most efficient approach to capturing the benefits with access to new markets and, at the same time, to being prepared for 
the competition in the world beer market (Bleakley et al, 2004). More specifically, the following M\&A-motives are thought to be good for shareholders:

- Economy of scale: The combined company can often reduce duplicative departments or operations, lowering the company's costs relative to theoretically the same revenue stream, thus increasing profit (Rall, 2002).

- Increased revenue (due to lack of competition): This motive assumes that the company will be getting rid of a major competitor and increasing its power to set prices.

- Synergies: Better use of complementary resources (Kutschker and Schmid, 2004).

- Taxes: A profitable company can buy a loss maker to exploit the target's tax shield.

- Geographical or other diversification: This is designed to smooth the earnings results of a company, which over the long term smooths the stock price of a company, giving conservative investors more confidence in investing in the company (Glaum et al, 2003).

It is widely agreed that the "success" of a M\&A may be defined as the creation of synergy: the value of the combined firms is greater than that of the two firms operating separately. This precondition reflects the simple observation that the price paid for a strategic asset must be lower than its expected value if it is to add economic value to the acquiring organization. If this assumption is met we can expect a higher valuation of the acquiring company. Thus, the central research question here is as follows: Does the stock market react positively to the announcement by listed brewing companies of a planned merger or acquisition?

\section{RESEARCH METHODOLOGY}

Using event study methodology let us now evaluate the cross-border acquisitions transacted by the five leading European brewing companies. The basic assumption underlying the use of stock market data to estimate the effects of M\&As is that share prices reflect expectations about future profit and dividend streams. In addition, any changes in future profit streams that an acquisition is expected to bring about are reflected in changes in prices and returns of the company's shares (Panayides and Gong, 2002). The relevant hypothesis in this paper is, that the increase in the peer groups' degree of internationalization has a positive impact on the companies' corporate success, expressed in increasing share prices.

Four principle methodologies have been employed to measure M\&A success: Event study, accounting-based measures, survey data and case studies. The event study methodology has a 
number of attractive features. First, data is often publicly available; second, it relies upon the well-respected efficient market hypothesis; and, third, because "abnormal" returns are calculated, the data is not subject to industry sensitivity, enabling a broad spectrum of industries to be studied (Cording et al, 2002). Event study is a research method developed thirty years ago that is appearing frequently in financial services studies to measure the impact of changes in corporate policy. Event studies attempt to measure abnormal changes in the stock prices of publicly traded companies that occur in conjunction with an "event"1 (Brown and Warner, 1980; Brown and Warner, 1985; Wells, 2004). The event study method relies on the assumption that over time individual stock returns can be predicted to some degree. The researcher then observes the actual stock returns over the period of interest and computes the difference between the returns that were predicted and the returns that actually occurred. If the difference between the actual results and the predicted results is determined to be statistically significant different from zero, it may be concluded that the event under study did impact stock returns and reflects an investor reaction to the event.

Acquisitions are complex, so that even researchers have difficulty assessing and understanding them. Although a few exceptions exist, most recent research on acquisitions were event studies centered on acquisition announcements. Harrison et al (2005) conclude that acquisitions are value-creating, with the lion's share of the gains going to target-firm shareholders. Acquiring-firm shareholders generally broke even or suffered small losses if stock consideration was offered. The above discussion raises the question of whether a merger or acquisition in the brewing industry will in fact have a positive impact on shareholder wealth.

\section{Assumptions and Theoretical Basis}

Readers can be confident that the conclusions from an event study are valid only if the inference of significance relies on the following assumptions:

\section{1) Market Efficiency:}

This assumption provides the basis for the use of event study methodology. Market efficiency implies that stock prices incorporate all relevant information that is available to market traders.

\footnotetext{
${ }^{1}$ This can be divestitures, corporate control changes, product recalls, earnings announcements, issues of new debt or equity, the appointment of top executives, announcements of dividend payments, profit expectations or half year results, strategic investment decisions, formation of joint ventures and strategic alliances, or in the case under consideration, the announcement of cross-border acquisitions.
} 
The assumption of market efficiency is difficult to reconcile with the use of a long event window. As many management studies use quite long event windows this implies that some researchers do not believe that the effects of events are quickly incorporated into stock prices (McWilliams and Siegel, 1997). Particularly in cases of acquisitions, information about the number of potential acquirers and their evaluation of the target may be revealed over a relatively long period. Where this is the case, it is the obligation of the researcher to explain why the effect would not be realized within a short period of time. Otherwise, the use of the event study method is inappropriate.

\section{2) Unanticipated Events:}

Usually, when M\&As are announced in the financial and business press the market has had no previous knowledge of the event. Abnormal returns then can be assumed to be the result of the stock market's reacting to new information. Difficulties occur when the event has been anticipated by traders or information leaked to the market in advance of a formal announcement (McWilliams and Siegel, 1999).

\section{3) Confounding Effects:}

The central and most critical claim is that researchers have isolated the effect of an event from the effects of other events. Confounding events can include any effect that may impact share price during an event window, such as announcements of dividend payments, profit expectations or the appointment of top executives. The longer the event window, the more difficult it is for researchers to claim they have controlled for confounding effects. With a short event window, one can be reasonably confident that abnormal returns are due to the event under consideration because it is quite easy to identify confounding events. Failing to control for confounding effects causes serious doubts about the validity of empirical results and calls into question any conclusions drawn.

In the following the design section describes the procedure of the event study conducted in this research.

\section{Modeling Normal Returns}

When events of interest have been defined and the length of the estimation window determined, the normal return must be estimated. There are two common choices for modeling normal returns $\left(\hat{R}_{i t}\right)$ : The so called "mean adjusted model" and the more sophisticated "market model". In the case under consideration, the former will be adopted as it provides the 
same reliability but is more user-friendly than the market model. The mean adjusted model uses the mean daily return on each individual firm's stock over a predetermined estimation period (estimation window). This period typically includes about 180 trading days immediately preceding the event date (Wells, 2004). These mean-adjusted returns are calculated by subtracting the average return for stock $i$ during the estimation period from the stock's return during the event period $s$ (Binder, 1998). The mean return is now used as a benchmark for the firm's daily stock returns during the event period (Brown and Warner, 1980; Brown and Warner, 1985; Kritzman, 1994).

The theoretical basis for the event study is fairly straightforward, albeit based on certain key assumptions. Researchers evaluate stock returns, which are the day-to-day changes in the value of a stock sold on the open market. Wells (2004) provides the following example: A stock that opens the day at $€ 50$ and closes at $€ 54$ will have an 8.00 percent return for the day $(€ 4 / € 50=0.080)$. If the stock price returns to $€ 50$ the next day, then the stock would have a 7.41 percent return for that day $(-€ 4 / € 54=-0.0741)$. Note that the average over the two-day period would actually be a positive value $-(0.0800+-0.0741) / 2=0.0059$, or $0.59 \%$; because of this arithmetic anomaly and to avoid this bias, natural logarithms are used in this study instead of simple percentage changes.

For each security $i$, the mean $\hat{R}_{i t}$ and standard deviation $\sigma\left(R_{i t}\right)$ of its return in days -180 through -11 are estimated as follows:

$$
\hat{R}_{i t}=\frac{1}{170} * \sum_{t=-180}^{t=-11} R_{i t}
$$

\section{Calculating Abnormal Returns}

Appraisal of the event's impact requires a measure of the Abnormal Return $(A R)$. The $A R$ is the ex post return of the security over the event window $\left(R_{i t}\right)$ minus the normal return of the firm over the estimation window $\left(\hat{R}_{i t}\right)$ (MacKinlay, 1997). For firm $i$ and event $t$ the abnormal return is

$$
A R=R_{i t}-\hat{R}_{i t} .
$$


Finally, the individual daily abnormal returns for the individual firms are aggregated across all firms in the sample for each day. These Average Abnormal Returns $\left(A A R_{t}\right)$ are examined to determine whether on average the event produces returns (good or bad) that are different from the returns that would be expected:

$$
A A R_{t}=\frac{1}{n} * \sum_{i=1}^{n} A R_{i t}
$$

Also, because it may be difficult to pinpoint a specific event day, a cumulative effect over a period may be present and observable. Cumulative Average Abnormal Returns $\left(C A A R_{t}\right)$ are calculated by summing daily $A A R_{t}$ over time:

$$
C A A R_{\left[t_{1} ; t_{2}\right]}=\sum_{\left[t_{1} ; t_{2}\right]} A A R_{t}=\sum_{\left[t_{1} ; t_{2}\right]} \frac{1}{n} * \sum_{i=1}^{n} A R_{i t}
$$

To determine whether $C A A R_{t}$ and $A A R_{t}$ are statistically significant different from zero, they need to be tested using a t-test. Prior to conducting the t-test, the aggregate of pre-event standard deviation of abnormal returns across all securities should be computed (Asquith et al, 1983; Ruback, 1983; Bühner, 1990). The following equation is the formula for estimating the standard deviation of daily ARs during the pre-event period (from -180 to -11):

$$
\sigma_{i, p r e}=\sqrt{\frac{1}{n-1} * \sum_{-180}^{-11}\left(A R_{i t}-A A R_{p r e}\right)^{2}} \text {, }
$$

where

$\sigma_{i, p r e}=\quad$ standard deviation of security-specific returns of security $i$ estimated from the preevent measurement period.

$A A R_{\text {pre }}=$ average of security-specific returns of security $i$ estimated from the pre-event measurement period.

$\mathrm{n}=\quad$ number of days in pre-event measurement period. 
The standard deviations are aggregated by squaring the standard deviation of each security's specific return estimated during the pre-event period, summing these values across all securities, taking the square root of this sum and then dividing by the number of securities:

$\sigma_{N, p r e}=\sqrt{\frac{\sum_{i=1}^{N} \sigma_{i, p r e}^{2}}{N}}$,

where

$\sigma_{N, p r e}=$ aggregate of the pre-event standard deviations of security-specific returns across all securities.

$\mathrm{N}=\quad$ number of securities in the sample.

Having completed all calculations for the aggregate standard deviation of pre-event abnormal return for all securities, the t-test for $A A R_{t}$ is (Kritzman, 1994; Kusnadi and Sohrabian, 1999)

$A A R_{t}$ t-statistic $=\frac{A A R_{t}}{\sigma_{N, p r e}}$,

and for cumulative abnormal returns, the t-test formula is:

CAARt t-statistic $=\frac{C A A R_{t}}{\sigma_{N, p r e} * \sqrt{t_{2}-t_{1}+1}}$,

where $t_{1}$ is the first day and $t_{2}$ is the last day of the period over which returns are cumulated.

The test statistic used in classical event study methodology is the sum of the event-period abnormal returns divided by the square root of the sum of all the securities' estimation-period residual variances. Under normality, independence, and stationarity assumptions, this is used to assess the significance of the event (Brockett et al, 1994). 


\section{SAMPLE DATA AND DESIGN}

This section is divided into two parts: data collection and design. The data sources section explains the criteria applied in this study and the data collection process.

\section{Sample Data}

The primary data employed in this paper are the daily stock returns for five European brewing groups which announced 31 M\&As during the sample period from March 2000 through August 2005. In fact, the brewing scene has seen many more M\&As during this period. But those 31 M\&As are the ones that meet the criteria for this study, which are as follows (Kusnadi and Sohrabian, 1999; Cybo-Ottone and Murgia, 2000; Putlitz, 2001):

(1) The transaction was announced between March 1, 2000 and August 31, 2005.

(2) The acquiring firm's stock is publicly traded ${ }^{2}$.

(3) The bidder is a member of the European brewers' peer group.

(4) The targets are brewers as well.

(5) The return on the acquiring firm's securities is available for at least from 180 days prior to the announcement date.

(6) The information related to the M\&A, such as transaction price and announcement date was publicly disclosed.

(7) The transaction volume exceeds a valuation of $€ 100 \mathrm{~m}$ in order to attract sufficient attention from capital markets.

The daily stock returns are obtained from Thomson Financial Datastream. To identify the M\&As' announcements exactly, an intensive search through diverse financial magazines and newspapers, such as The Wall Street Journal, The Financial Times Europe, Handelsblatt, Lebensmittel Zeitung, The Financial Times Deutschland was undertaken. As we used a relatively long event window (from $t=-10$ to +10$)^{3}$, which is justified because of uncertainty about when information was exactly revealed, we also checked this period for confounding

${ }^{2}$ Carlsberg's B-share is traded at the Copenhagen Stock Exchange (Reuters code: CARLb.CO), Heineken N.V. is listed at the Euronext Amsterdam (HEIN.AS), SABMiller (SAB.L) as well as S\&N (SCTN.L) are listed on the London Stock Exchange, and InBev's shares are traded at the Euronext Brussels (INTB.BR).

${ }^{3}$ This means 10 trading days prior to the event to 10 days after the event. 
events. We eliminated M\&As when we saw that any additional information (e.g., announcements of dividend payments, profit expectations or half year results) might have affected the share price on the event dates that we examined (Simpson and Hosken, 1998).

\section{Sample Design}

The initial task of conducting an event study is to define the event of interest (here: crossborder acquisitions during the last five years). The estimation window is the 170-day period $(-180$ to -11$)$ preceding the event date. Afterwards the period has to be identified over which the security prices of the firms involved in this event will be examined - the event window. In this study, we calculated the short-term as well as the intermediate-term effects of the brewing M\&A announcements. The short-term effects were calculated by using the usual three-day event window (from $t=-1$ to +1 ). Especially in cases where the event is an announcement of an acquisition, it is customary to define the event window as larger than the specific period of interest (McWilliams and Siegel, 1997). This permits examination of periods surrounding the event. Amendatory to the long event window (from $t=-10$ to +10 ), medium-term windows surrounding the event day are also taken in account, such as the elevenday (from $t=-5$ to +5 ) and the five-day window (from $t=-3$ to +1 ). The following figure illustrates the time line for conducting this event study.

Figure 2: Cycle of Implementation of the Event Study.

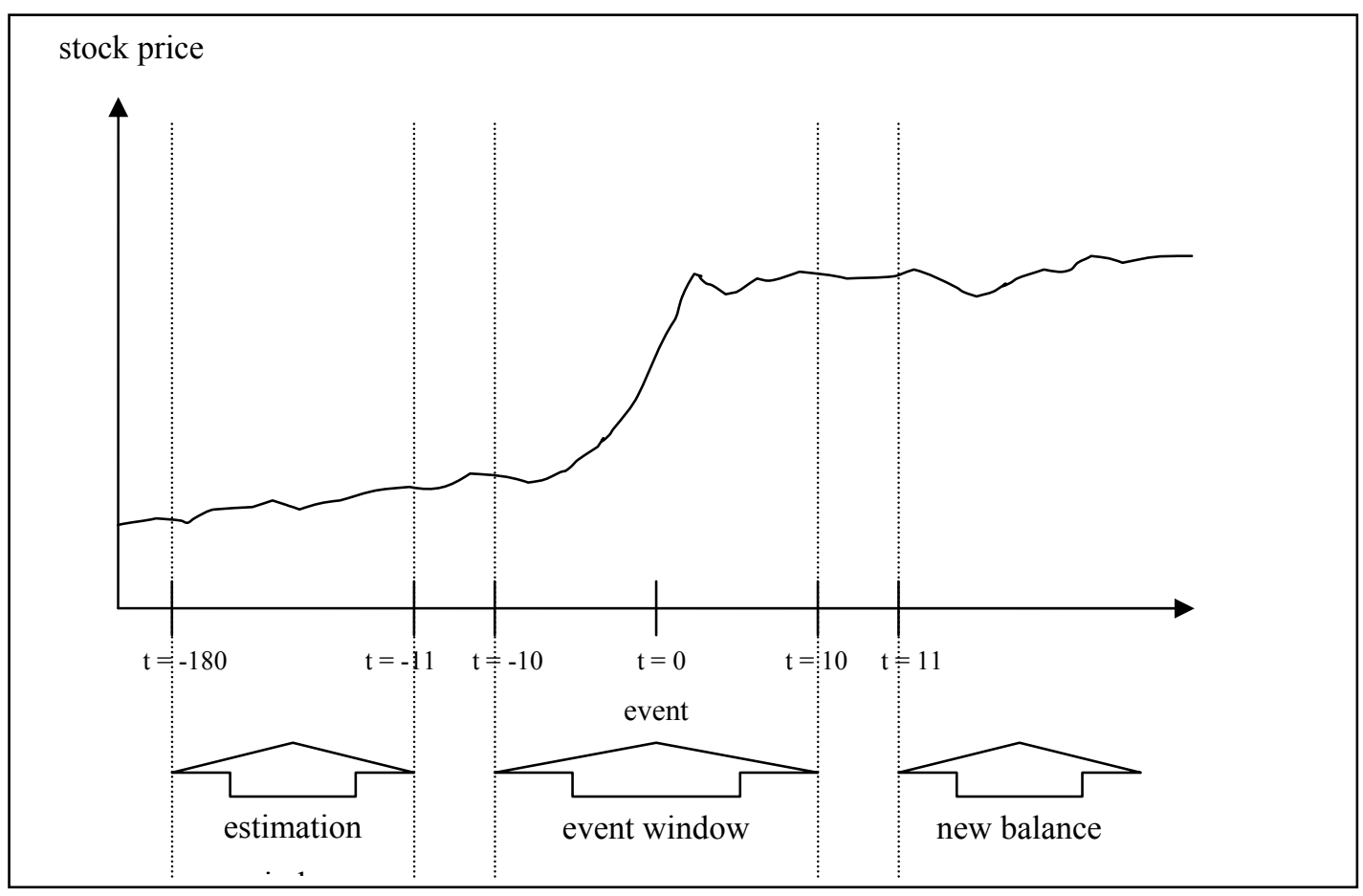




\section{EMPIRICAL RESULTS}

The final sample consists of five European brewing groups which have transacted $31 \mathrm{M} \& \mathrm{As}$ during the event period. CARs are computed for the whole sample as well as for each brewing group. The results of the 21-day window CARs are given below.

We start by discussing Carlsberg's three acquisitions over the last five years (see figure 4). The Orkla deal transacted in early 2004 was undoubtedly perceived positively by shareholders as it gave Carlsberg A/S sole ownership of Carlsberg Breweries (Bevan and Greenberg, 2004). The 100\% takeover of Feldschlösschen in November, 2000, first cumulated $8.5 \%$ positive abnormal returns near the announcement day, then recovered to zero, and finally resulted in $+3.3 \%$ gains. The Holsten deal in January, 2004, saw $-4.2 \%$ returns some days before the acquisition had taken place, recovering to zero on the announcement day and had finally had a decreasing trend, staying around $-7 \%$ till event day +10 . The latter two events were not statistically significant.

Figure 3: Carlsberg - Cumulative Abnormal Returns $(-10,+10)$.

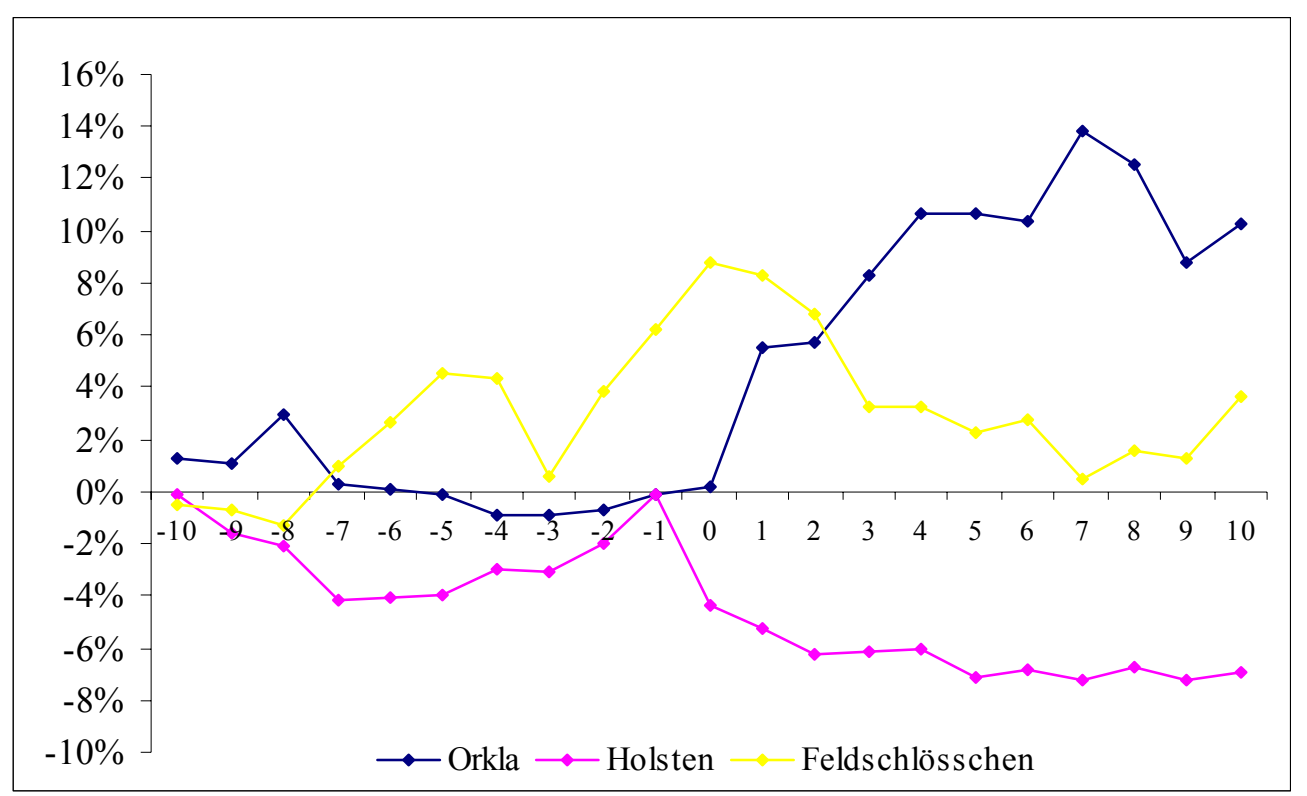

Apart from the BBAG deal Heineken has transacted a lot of mid-scaled acquisitions over the last five years, all resulting in moderately negative returns. Two outliers can be found in the sample. First, the acquisition of BBAG in May 2003 which had a transaction volume of around $€ 1.9$ billion, was negatively absorbed by the capital market, causing a $13 \%$ decrease in 
Heineken's stock price, which was statistically significant at the $1 \%$ level. In contrast the acquisition of Bravo in February, 2002, lead to a \% 10 increase in the stock price with a statistical significance at the $5 \%$ level.

Figure 4: Heineken - Cumulative Abnormal Returns (-10, +10).

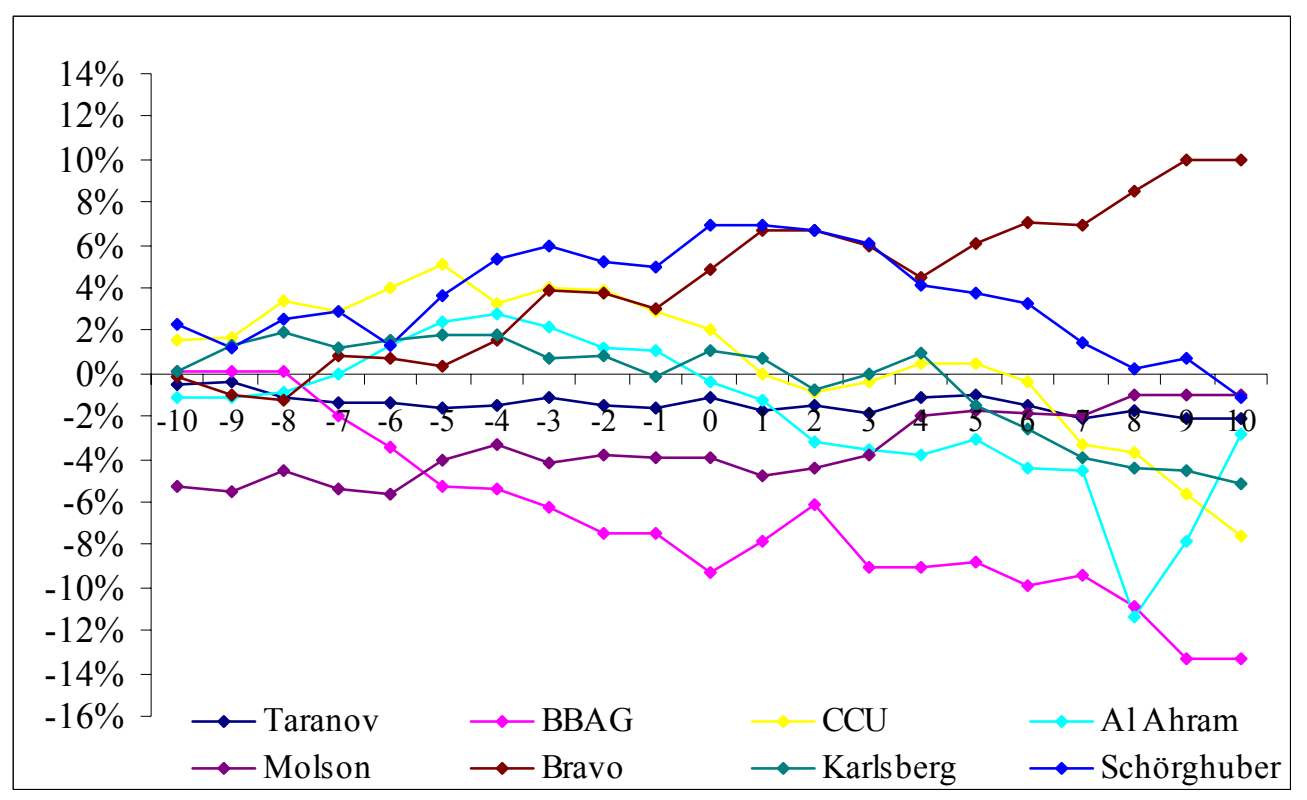

InBev was responsible for the bulk of M\&As in the sample, resulting in an aggregate transaction volume of more than $€ 23$ billion. Regarding all transactions, InBev experienced an average loss of $3.17 \%$, statistically significant at the $10 \%$ level. The acquisition of Beck's in August 2001 was the spectacular beginning of a roll up by leading brewing groups of the previously closed German beer market. It is noteworthy that most analysts evaluated the transaction value of $€ 1.8$ billion as overpriced, leading to an EBITDA multiple of 13.0. This resulted in a stock price loss of more than $12.5 \%$, statistically significant at the $5 \%$ level. 
Figure 5: InBev - Cumulative Abnormal Returns $(-10,+10)$.

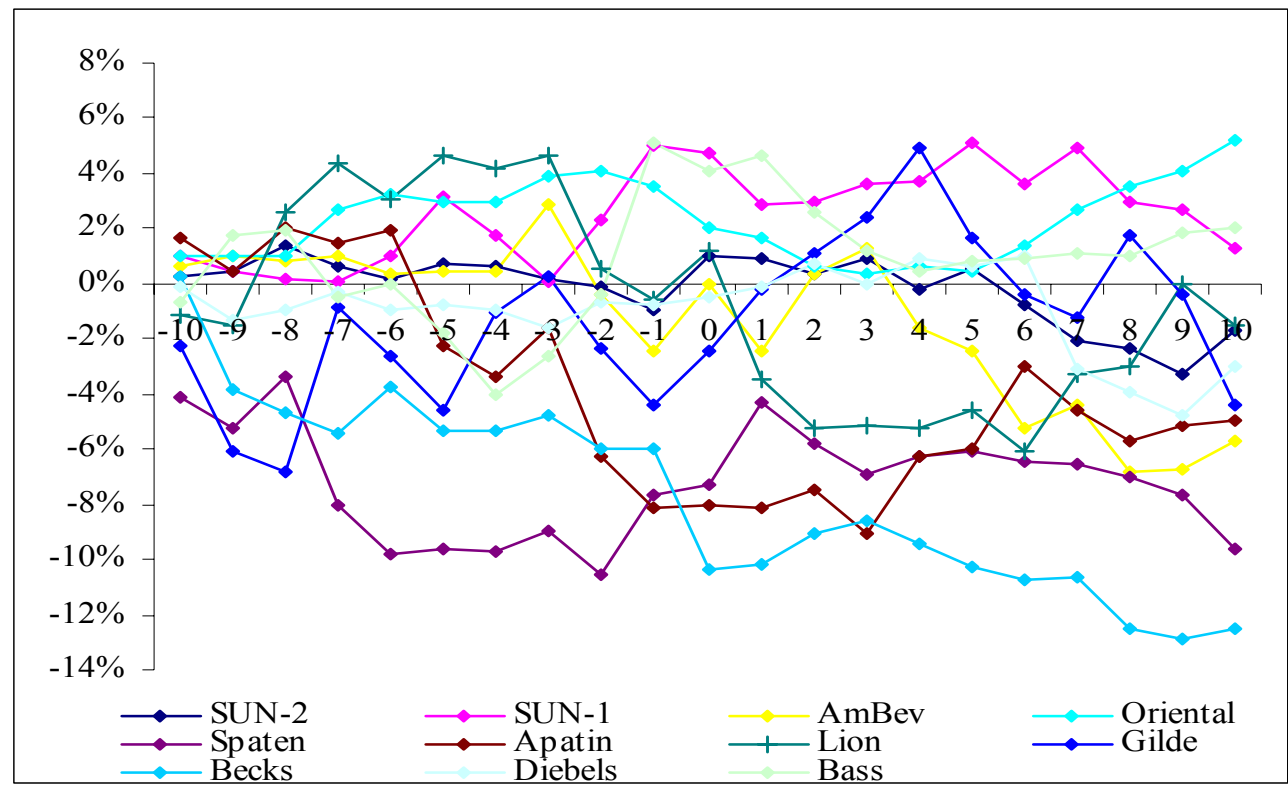

Unlike InBev, the smallest brewer within the European peer group Scottish\&Newcastle experienced an everage gain of $7.78 \%$ also statistically significant at the $10 \%$ level. In particularly the Kronenbourg $(+11.3 \%)$ and the Bulmers $(+12.9 \%)$ deals have been perceived quite positively by shareholders as these acquisitions were important steps for S\&N towards entering strategic markets in Western Europe.

Figure 6: S\&N - Cumulative Abnormal Returns $(-10,+10)$.

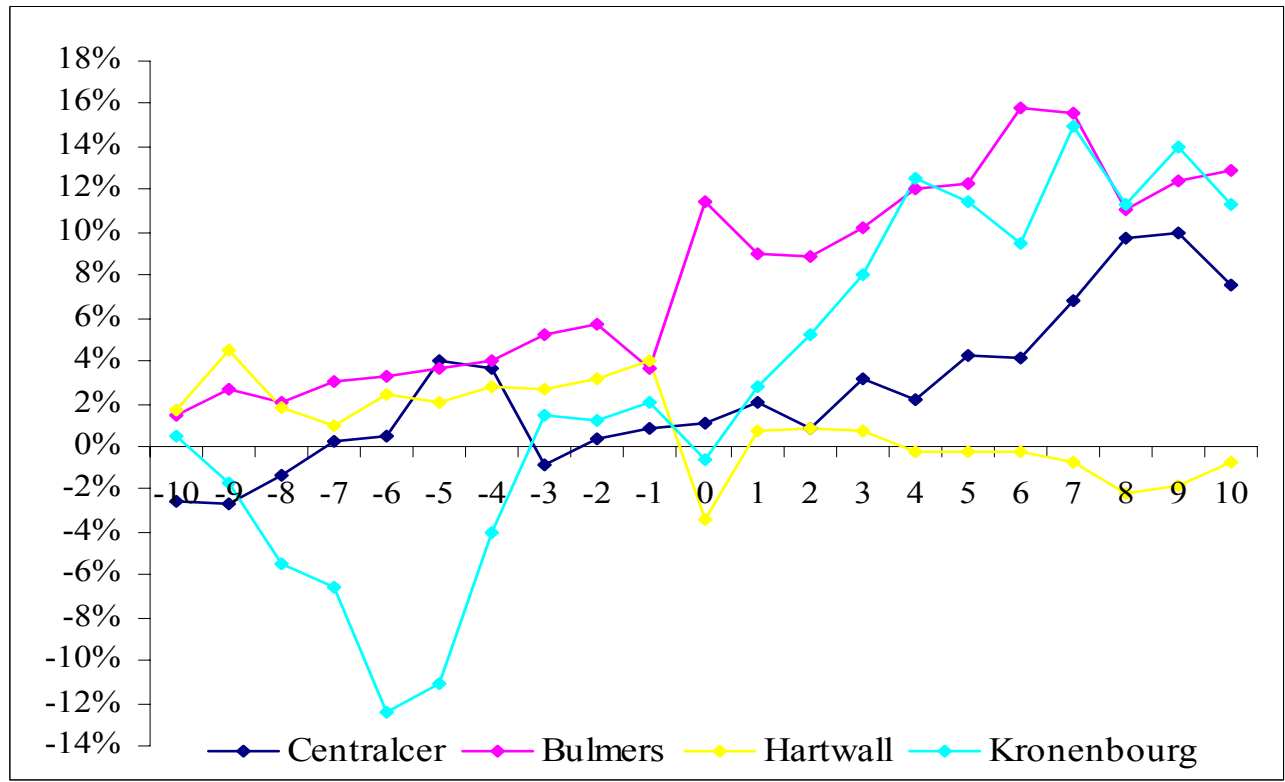


The last brewer in our sample London-based SABMiller executed three smaller-scale acquisitions apart from SABs merger with US-based brewer Miller in 2002. Referring again to the aforementioned EBITDA multiples, the relatively high-priced Peroni deal (12.6 x EBITDA) produced a negative CAR of $-5.7 \%$ while the "cheaper" Lion Nathan (10.3) and BevCo (6.5) transactions led to positive CARs of $1.6 \%$ and $8.5 \%$, respectively. But none of these acquisitions saw statistically significant CARs in the 21-day window. By far the most spectacular deal has been the takeover of Columbian-based brewer Bavaria for approximately $€ 6.5$ billion in July 2005. The transaction price has been 10 times the EBITDA. As this not really has been a bargain buy, the capital market has highly appreciated this deal resulting in a jump of SABMillers share price by $8.4 \%$, statistically significant at the $5 \%$ level. This leads from the fact that most investors expected SABMiller to pay much more for the Bavaria target as the market entry into the fast growing Southern American beer market has been of paramount strategic importance for SABMiller.

Figure 7: SABMiller - Cumulative Abnormal Returns $(-10,+10)$.

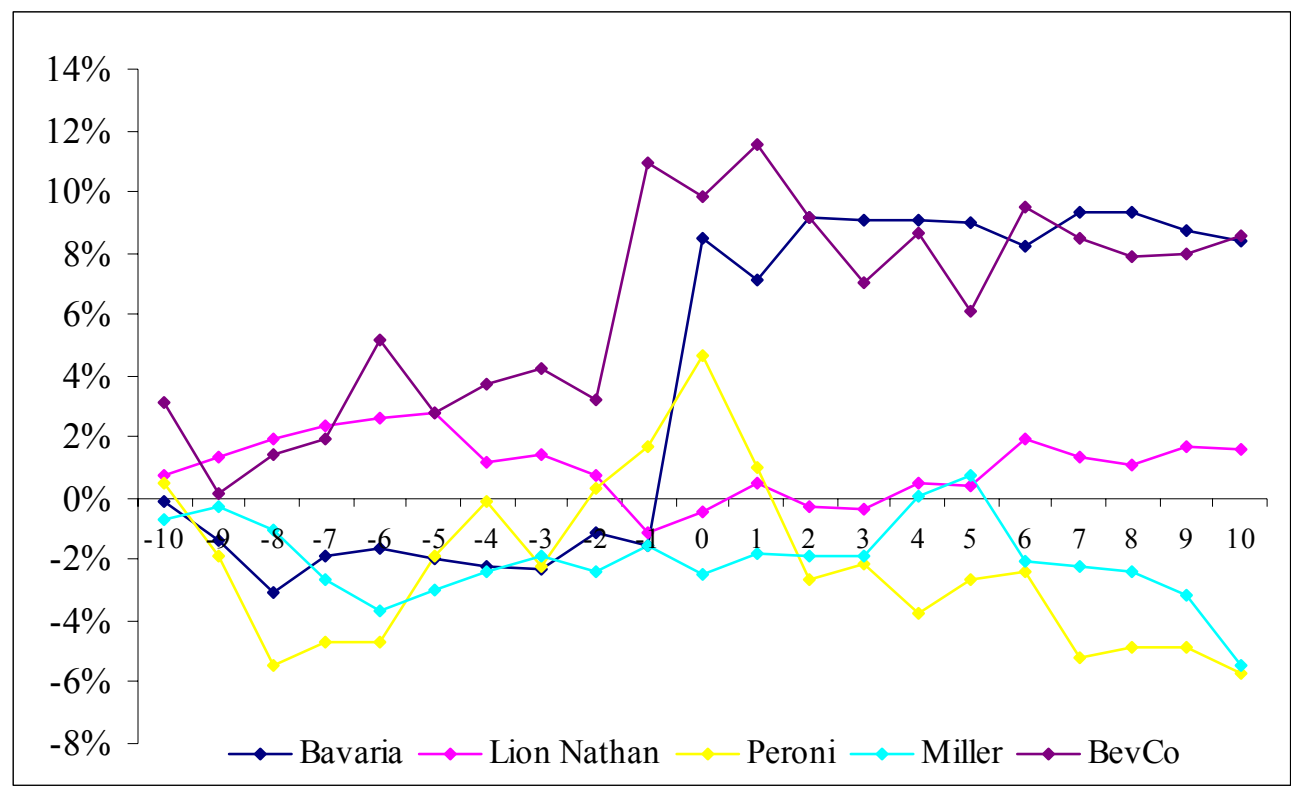

The average CARs calculated for the five bidders allow us to rank the companies in relation to the securities' perception of their strategies. 
Figure 8: Average CARs $(-10,+10)$ for the Five European Brewing Groups.

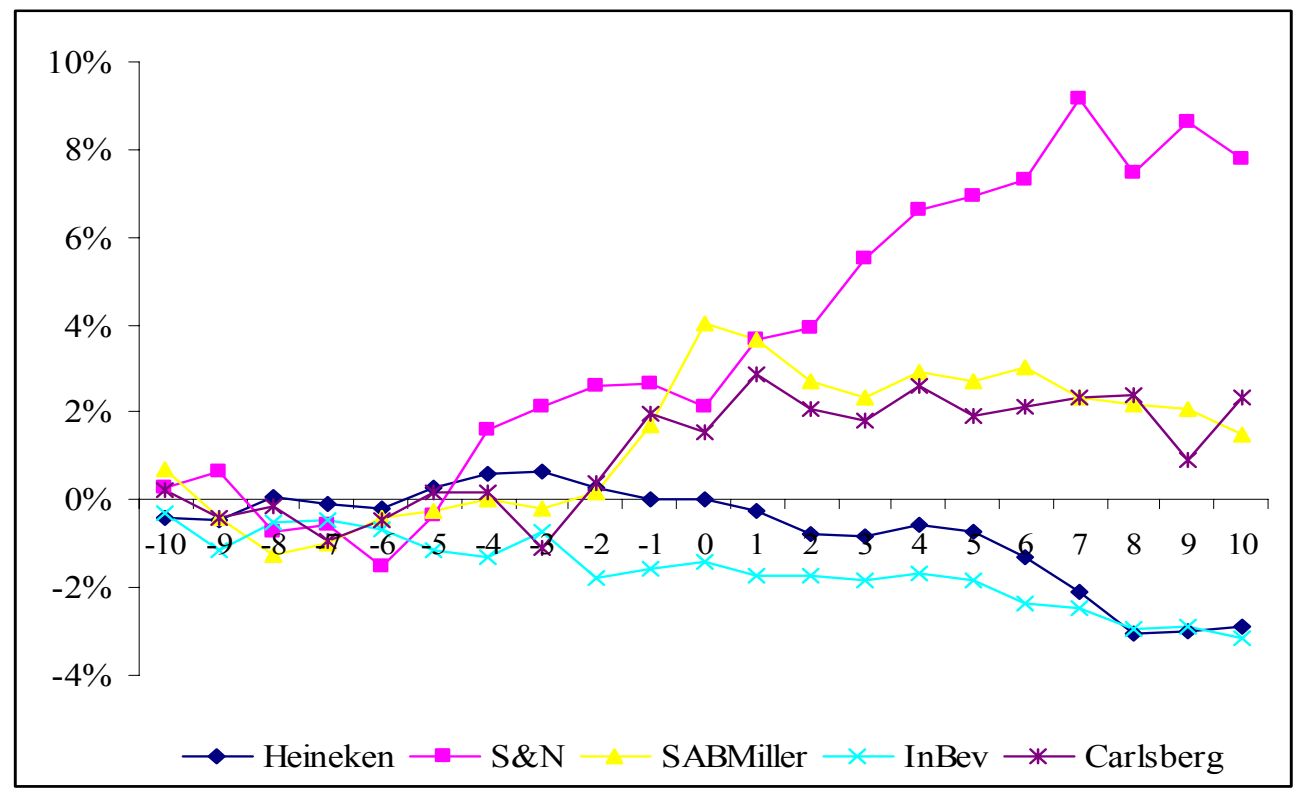

Scottish\&Newcastle is ranked at the top facing an average CAR21 of $+7.78 \%$. Carlsberg is next with $+2.33 \%$. Then comes SABMiller with slightly positive negative returns at $1.49 \%$. The other three brewing groups receive negative average CARs. Heineken's stock price oscillates around zero but has a decreasing trend five days after the event leading to $-2.87 \%$. The brewer with the worst performance in the sample is Belgium-based InBev, obtaining an average loss in the share price of $3.17 \%$.

An interesting link seems to exist between the transaction valuation as a multiple of the target's EBITDA and the stock market valuation of the M\&A. The average EBITDA multiple over the whole sample is 10.0 as can be seen in table 2. Thus, two subsamples are built: One with an EBITDA multiple above 10.0, the other with an EBITDA multiple below 10.0. The following figure illustrates the 31 transactions' CARs $(-5,+5)$ near the announcement day. The events are ranked from left to right by their EBITDA multiple in decreasing order. Heineken's acquisition of Molson Brazil i. e. has been transacted facing an EBITDA multiple of 13.7. The lowest EBITDA multiple resulted from Interbrew's Apatinska acquisition (6.3). 
Figure 9: CARs $(-5,+5)$ Ranked by the EBITDA Multiple.

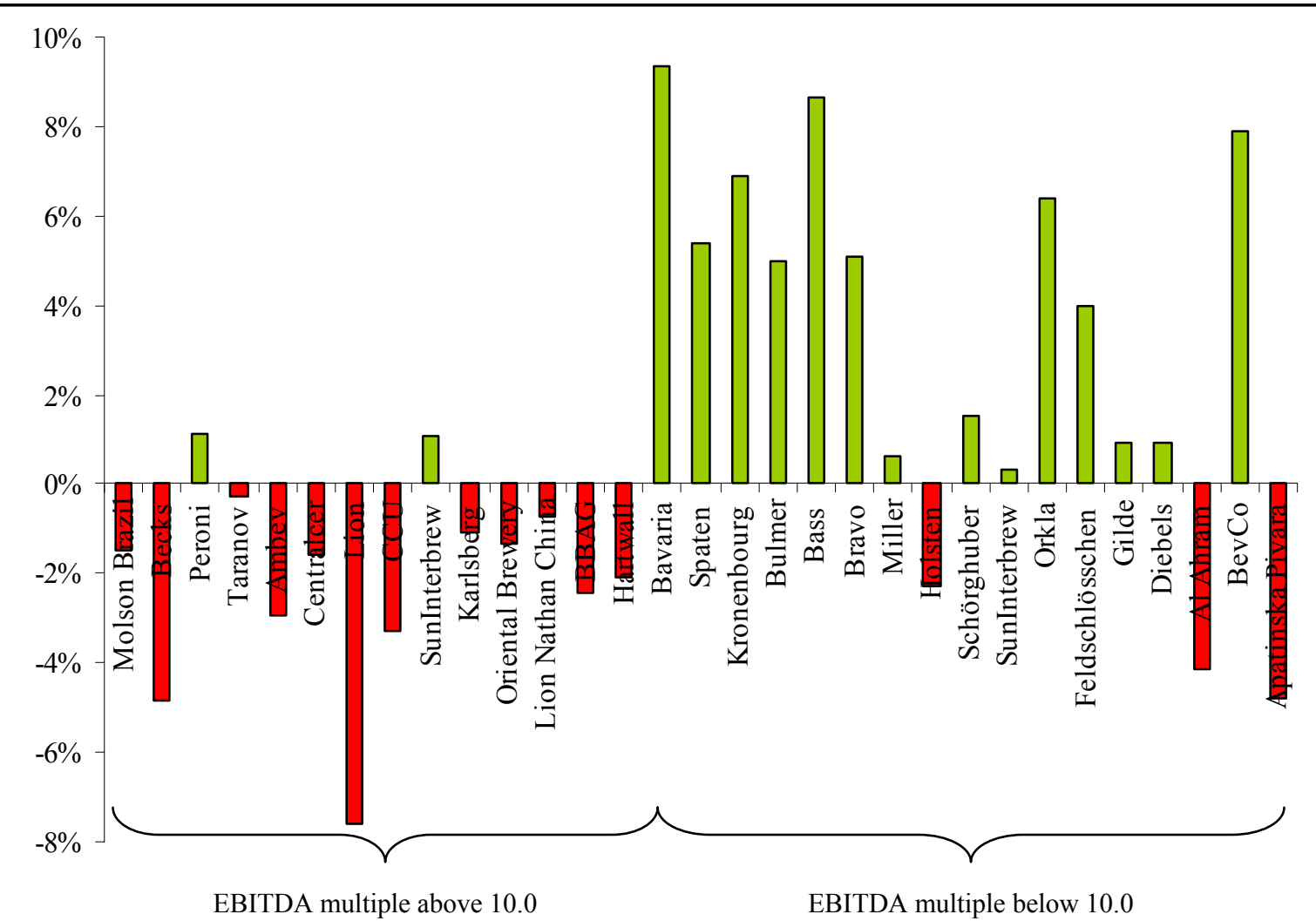

For the first subsample which includes the fourteen M\&As with above average prices, cumulative abnormal returns in the eleven-day windows are negative and statistically significant at the $1 \%$ level. For the second subsample, comprising seventeen transactions with an EBITDAmultiple less than 10.0, abnormal returns are positive with a statistical significance at the $5 \%$ level. Thus, the capital market reacts effectively to the several brewers' M\&A announcements. It seems that in the world brewing scene, the price of the publicly traded stocks reflects the reaction of the financial markets to the introduction of the new information efficiently. We can confirm the efficient markets hypothesis as overpriced transactions result in negative abnormal returns whereas the transactions with an EBITDA multiple below the average in our sample have been appreciated by rising stock prices. 


\section{SUMMARY AND DISCUSSION OF RESULTS}

In conclusion, our empirical study proves there are significant differences regarding the peer group members' level of acquisitioning and their impact on brewers' financial performance, expressed in increasing or decreasing stock prices. Some of the leading European brewers pursue a moderate and continuous expansion strategy while some seek growth via extraordinary and often overpriced acquisitions. The brewers' attitude towards external growth via cross-border acquisitions is reflected in the brewing group's financial performance on capital markets. The results of this study are summarized in the following figure.

Figure 10: Abnormal Returns and Cumulative Abnormal Returns - Total Sample.

\begin{tabular}{|c|c|c|c|c|c|c|c|c|c|c|}
\hline \multirow{2}{*}{$\begin{array}{l}\text { Event } \\
\text { Day }\end{array}$} & \multirow[b]{2}{*}{ Acquiror } & \multirow[b]{2}{*}{ Target } & \multirow{2}{*}{$\begin{array}{l}\text { EBITDA } \\
\text { Multiples }\end{array}$} & \multicolumn{4}{|c|}{ Cumulative Abnormal Returns } & \multicolumn{3}{|c|}{ Abnormal Returns } \\
\hline & & & & $(-10,+10)$ & $(-5,+5)$ & $(-3,+1)$ & $(-1,+1)$ & $\mathrm{t}=1$ & $\mathrm{t}=0$ & $\mathrm{t}=-1$ \\
\hline 16-Aug-05 & Heineken & Taranov & 11.8 & -0.0205 & 0.0033 & -0.0026 & -0.0026 & -0.0053 & 0.0042 & -0.0015 \\
\hline 19-Jul-05 & SABMiller & Bavaria & 10.0 & $0.0843^{* *}$ & 0.1064 ** & $0.0933^{* *}$ & 0.0819 *** & -0.0134 & 0.0998 & -0.0045 \\
\hline 03-Jan-05 & InBev & SunInterbrew & 8.8 & -0.0166 & 0.0036 & 0.0032 & 0.0102 & -0.0011 & 0.0197 & -0.0084 \\
\hline 16-Sep-04 & SABMiller & Lion Nathan China & 10.3 & 0.0158 & -0.0223 & -0.0071 & -0.0027 & 0.0087 & 0.0074 & -0.0188 \\
\hline 12-Aug-04 & Interbrew & SunInterbrew & 11.0 & 0.0132 & 0.0417 & 0.0109 & 0.0053 & -0.0192 & -0.0021 & 0.0266 \\
\hline 03-Mar-04 & Interbrew & Ambev & 11.5 & -0.0573 & -0.0274 & -0.0294 & -0.0207 & -0.0247 & 0.0246 & -0.0205 \\
\hline 19-Feb-04 & Carlsberg & Orkla & 8.6 & 0.1028 & 0.1057 & 0.0639 & 0.0622 & 0.0530 & 0.0036 & 0.0056 \\
\hline 20-Jan-04 & Carlsberg & Holsten & 9.1 & -0.0695 & -0.0301 & -0.0229 & -0.0322 & -0.0083 & -0.0428 & 0.0190 \\
\hline 07-Jan-04 & Interbrew & Oriental Brewery & 10.7 & 0.0521 & -0.0280 & -0.0133 & -0.0243 & -0.0037 & -0.0151 & -0.0055 \\
\hline 18-Sep-03 & Interbrew & Spaten & 9.9 & -0.0958 & 0.0368 & 0.0539 & 0.0620 & 0.0299 & 0.0031 & 0.0290 \\
\hline 11-Sep-03 & Interbrew & Apatinska Pivara & 6.3 & -0.0500 & -0.0787 & -0.0477 & -0.0188 & -0.0017 & 0.0012 & -0.0184 \\
\hline 08-Sep-03 & Interbrew & Lion & 11.4 & -0.0151 & -0.0767 & -0.0761 & -0.0398 & -0.0462 & 0.0178 & -0.0114 \\
\hline 13-May-03 & SABMiller & Peroni & 12.6 & -0.0567 & 0.0206 & 0.0112 & 0.0074 & -0.0364 & 0.0300 & 0.0137 \\
\hline 13-May-03 & S\&N & Centralcer & 11.4 & 0.0755 & 0.0370 & -0.0159 & $0.0169 *$ & 0.0097 & 0.0022 & 0.0049 \\
\hline 02-May-03 & Heineken & BBAG & 10.2 & -0.1332 *** & -0.0531 & -0.0245 & -0.0037 & 0.0145 & -0.0190 & 0.0008 \\
\hline 28-Apr-03 & S\&N & Bulmer & 9.8 & 0.1293 & 0.0895 & 0.0496 & 0.0332 & -0.0246 & 0.0781 & -0.0203 \\
\hline 14-Jan-03 & Heineken & $\mathrm{CCU}$ & 11.3 & -0.0754 & -0.0355 & -0.0329 & -0.0388 * & -0.0214 & -0.0079 & -0.0095 \\
\hline 15 -Nov-02 & Interbrew & Gilde & 8.6 & -0.0439 & 0.0423 & 0.0090 & 0.0215 & 0.0226 & 0.0198 & -0.0209 \\
\hline 12-Sep-02 & Heineken & Al Ahram & 8.0 & -0.0279 & -0.0441 & $-0.0413^{* *}$ & -0.0243 & -0.0090 & -0.0152 & -0.0001 \\
\hline 05-Jun-02 & Heineken & Karlsberg & 10.8 & -0.0510 & -0.0305 & -0.0110 & -0.0018 & -0.0045 & 0.0124 & -0.0097 \\
\hline 30-May-02 & SAB & Miller & 9.1 & -0.0542 & 0.0441 & 0.0060 & 0.0066 & 0.0071 & -0.0094 & 0.0089 \\
\hline 18-Mar-02 & Heineken & Molson Brazil & 13.7 & -0.0101 & 0.0387 & -0.0150 & -0.0101 & -0.0080 & -0.0008 & -0.0013 \\
\hline 14-Feb-02 & S\&N & Hartwall & 10.1 & -0.0069 & -0.0268 & -0.0206 & -0.0242 & 0.0414 & -0.0739 & 0.0083 \\
\hline 01-Feb-02 & Heineken & Bravo & 9.7 & $0.1000 * *$ & 0.0527 & $0.0511 *$ & 0.0294 & 0.0179 & 0.0188 & -0.0073 \\
\hline 29-Nov-01 & SAB & BevCo & 6.5 & 0.0852 & 0.0095 & 0.0789 & 0.0834 & 0.0177 & -0.0118 & 0.0775 \\
\hline 06-Aug-01 & Interbrew & Becks & 13.0 & $-0.1251 * \star$ & -0.0652 & -0.0483 & -0.0421 & 0.0020 & -0.0444 & 0.0002 \\
\hline 13-Jul-01 & Interbrew & Diebels & 8.3 & -0.0304 & 0.0158 & 0.0091 & 0.0063 & 0.0038 & 0.0032 & -0.0007 \\
\hline 25-May-01 & Interbrew & Bass & 9.7 & 0.0205 & 0.0080 & 0.0867 & 0.0509 & 0.0059 & -0.0105 & 0.0554 \\
\hline $11-F e b-01$ & Heineken & Schörghuber & 9.1 & -0.0116 & 0.0240 & 0.0153 & 0.0173 & 0.0003 & 0.0193 & -0.0022 \\
\hline 03-Nov-00 & Carlsberg & Feldschlösschen & 8.6 & 0.0365 & -0.0044 & 0.0396 & 0.0443 & -0.0048 & 0.0255 & 0.0236 \\
\hline \multirow[t]{2}{*}{ 20-Mar-00 } & S\&N & Kronenbourg & 9.9 & 0.1133 & 0.2388 ** & 0.0692 & 0.0159 & 0.0352 & -0.0279 & 0.0086 \\
\hline & & & 10.0 & & & & & & & \\
\hline \multirow{10}{*}{\multicolumn{3}{|c|}{$\begin{array}{l}*, * * \text { and } * * * \text { indicate significance at the } .10 \text { level, } \\
.05 \text { level, and } .01 \text { level, respectively. }\end{array}$}} & & CAAR21 & CAAR11 & CAAR5 & CAAR3 & AARt $=1$ & AARt $=0$ & AARt $=-1$ \\
\hline & & & & -0.0040 & 0.0128 & 0.0078 & 0.0087 & 0.0012 & 0.0036 & 0.0039 \\
\hline & & & Minimum & -0.1332 & -0.0787 & -0.0761 & -0.0421 & -0.0462 & -0.0739 & -0.0209 \\
\hline & & & Maximum & 0.1293 & 0.2388 & 0.0933 & 0.0834 & 0.0530 & 0.0998 & 0.0775 \\
\hline & & & Median & -0.0151 & 0.0080 & 0.0032 & 0.0063 & -0.0011 & 0.0031 & -0.0007 \\
\hline & & & Average & -0.0040 & 0.0128 & 0.0078 & 0.0087 & 0.0012 & 0.0036 & 0.0039 \\
\hline & & & Deviation & 0.0700 & 0.0641 & 0.0432 & 0.0345 & 0.0218 & 0.0322 & 0.0216 \\
\hline & & & & -0.3047 & 1.0720 & 0.9732 & 1.3517 & 0.2973 & 0.5937 & 0.9740 \\
\hline & & & Positive AR & 12 & 18 & 16 & 17 & 15 & 18 & 14 \\
\hline & & & Negative AR & 19 & 13 & 15 & 14 & 16 & 13 & 17 \\
\hline
\end{tabular}


In competitive acquisition markets such as the brewing industry, gains associated with combination synergies accrue almost exclusively to target firm shareholders. However, if a specific combination of acquiring and target firm is unique in its synergy potential, the acquiring firm may participate in the gains from the acquisition (Brooks et al, 2000).

Some securities show positive and some negative abnormal returns over all different event windows $(-10,+10 ;-5,+5 ;-3,+1 ;-1,+1)$. None of the abnormal returns over the whole sample are significantly different from zero since t-statistic results were less than the t-table at the required level of significance. Indeed, CAAR21 over the whole sample for all acquiring show slightly negative abnormal returns but at no statistical significance. These results are not surprising as they confirm many previous studies (Asquith et al, 1983; McWilliams and Siegel, 1997; Agrawal and Jaffe, 2000; Beitel, 2002). As we found no significant negative abnormal returns for acquiring firms in acquisitions, no evidence is provided on managerial self-interest or hubris theory. Interestingly, our results are in line with more detailed studies on different forms of conducting M\&As. Consistently with previous work, our results prove that horizontal acquisitions face slightly negative returns in fact (average CAAR21 $=-0.40 \%$ ), but were appraised comparatively optimistic compared to conglomerate takeovers (Gerke et al, 1995; Glaum and Lindemann, 2002).

Some caveats apply. First, conclusions based on financial data depend ultimately on the views of financial markets and rely on an assumption of market efficiency. However, stock markets can be notoriously indecisive. Event studies adjust for movements in the broad market, and care was taken to ensure that other confounding events did not interfere with the events in this study. Second, stock price is an aggregate measure of firm value and might capture influences on profitability other than M\&A effects. Third, the negative effects for bidder firms over the whole sample were not significantly different from zero (King et al, 2002). But this is consistent with most event studies, measuring the effects for the bidder unlike the significant positive effects for target firms. One explanation for the insignificance might be the difference in size between most bidders and targets. Given that the bidders among the brewing groups are on average twenty times larger than the target firms, it might be expected that it would be difficult to detect significant abnormal returns around the announcement of the acquisition for bidder firms (Putlitz, 2001). 


\section{MANAGERIAL IMPLICATIONS}

Mergers and acquisitions seem to be a fast and efficient approach for companies to capture the benefits associated with the access to new markets and to being prepared for competition that is, to gain economies of scale and scope. In the last five years, five leading European brewing groups claimed to have become the world-brewing industry leader in the new competitive environment. These European peer group members transacted more than $€ 50$ billion (about $\$ 65$ billion) in mergers and acquisitions. This study is the first empirical investigation of the stock market effects of brewing companies' strategic activities such as mergers and acquisitions. The objective of this study has been to find out how the stock market values of these companies' strategies by analyzing the bidders' stock price reactions to brewing M\&A announcements. The outcome of the study is generally consistent with findings in previous studies in the finance literature (Shusterman et al, 2001).

The results of event studies can be of particular importance for shareholders, investors and management. Shareholders and investors have a vested interest in the market's evaluation of important strategic decisions such as deciding on M\&As because these decisions affect the brewing companies' future competitive positions and worth. Management may obtain useful information from the market that will serve as feedback for past executive decisions as well as provide guidelines for future ones. Knowledge of whether the announcement and implementation of important strategic moves such as M\&As create or destroy wealth will underpin future courses of action - not only where the efficiency of such strategies is concerned, but also relating to the method and timing of announcement and implementation (Panayides and Gong, 2002). Initial managerial implications strive to some of the peer group brewers, who need to redesign their future transaction and implementation process for cross-border acquisitions.

As table 2 shows the brewing consolidation process is not drawing to a close or even losing momentum. The combined value of transactions undertaken in recent years (2003-2004) is the highest level of activity ever seen in the industry and equates to the total level of activity seen over the previous five years combined. Our research results showed both a relatively neutral shareholder reaction to the M\&As on average but clear differences regarding single acquisitions in terms of transaction costs. Some investor reactions were broadly negative, reflecting overpriced deals. However, these high purchase prices may have been justified by the immense strategic importance of entering markets like China or Russia. Unlike the results of event studies in other industries, despite some negative outliers, there has not been an overall significant negative response to M\&As in the brewing industry. Thus, future developments in 
the beer industry will undoubtedly display many of the characteristics of recent years - with intense consolidation activity driven by increasing M\&A engagement between the world's leading brewers. Undoubtedly, the consolidation process will continue to gather pace. One question remains: When will the brewing scene see large scale deals due to mergers within the top ten?

\section{FUTURE RESEARCH}

Further research should be done on comparing both indicators of the performance of a firm: event study methodology and accounting-based measures. The latter could complement the short-term and capital-market oriented impacts of M\&As as the definition of "success" begins to take on a longer-term perspective: It may take three to five years to fully reap the benefits of the combined firms. There is a hypothesis that the ability of top management teams to work together effectively will drive M\&A success, measured by return on assets (Krishnan et al, 1997).

M\&A activity in a competitive, contestable market should not be profitable for other firms. Firms that combine to realize competitive advantages (economies of scale and scope, etc.) do so in order to lower their costs or generate other efficiencies. These should create a positive effect on the aggregate profitability of the firms involved in the combination but lower profits for rivals (King et al, 2002). Therefore, future research on evaluating M\&As in the brewing sector should include the effects of one firm's merger or acquisition on the stock price of its main competitors. It follows that events affecting one company also affect the other even though the merger has not actually taken place. Another useful modification would be to assess different modes of M\&A transactions (i.e. friendly versus hostile acquisition, method of payment, domestic versus international, etc.).

Furthermore, as acquisitions are complicate events, the event windows could be amended through a further step using a longer window than the common 21-day one (e.g. - 10, +30 or $30,+30)$. Because of the complexity of M\&As, it takes longer than a few days surrounding the announcement for market participants to correctly determine the extent of the economic implications for the acquiring firm (Baltazar and Santos, 2003; Mueller and Sirower, 2003). At the time of announcement, investors will estimate those implications; however, as time passes by and information is released about an acquisition and its performance, investors will revise their initial estimates. To exemplify this assumption, Carlsberg's acquisition of Holsten should be mentioned. In the -10 to +10 -window, this deal has indeed seen negative returns but 
not at a statistically significant level. In the weeks following the announcement, the shares fell approximately $10 \%$ prompting a clearly negative response from shareholders. In an event study review over the last decade, Harrison et al (2005) found that less than $10 \%$ of the event studies published considered returns more than 31 days after the event. Hence, it is important to consider the relationship between short-term shareholder reactions and long-term outcomes of M\&As.

More work on MNCs in the brewing sector could also focus on evaluating the efficiency of various strategies and considering the structural consequences of different internationalization strategies. Another amendment would be to classify the M\&A transactions in the sample according to their specialization or diversification along the geographical lines (Lepetit et al, 2002). For instance, it may be the case that shareholders will react differently to mergers between competitors operating in similar geographical markets than to mergers between companies operating in different geographical markets. Hence, there are strategic factors that may be used to explain the variation in wealth gains.

This approach is useful to explain why the phenomenon of brewing M\&As occurs despite the fact that they do not increase firm value on average. Finally, it would be particularly helpful in analyzing M\&As in the brewing industry to include the target company's abnormal returns. There is strong empirical evidence in the bulk of event studies to indicate that target firms' shareholders receive significant increases in their stock prices in comparison to the shareholders of bidding firms.

\section{REFERENCES}

Agrawal, A. and Jaffe, J. F. (2000). The Post-Merger Performance Puzzle, in: Cooper, C. and Gregory, A. (Eds.): Advances in Mergers and Acquisitions, New York: 7-41.

Agrawal, A. et al (1992). The Post-Merger Performance of Acquiring Firms: A ReExamination of an Anomaly, in: The Journal of Finance 47(1992): 1605-1621.

Asquith, P. et al (1983). The Gains for Bidding Firms from Merger, in: Journal of Financial Economics 11(1983): 121-139.

Baltazar, R. and Santos, M. (2003). The Benefits of Banking Mega-Mergers: Event Study Evidence from the 1998 Failed Mega-Merger Attempts in Canada. in: Canadian Journal of Administrative Sciences 20(2003): 196-208.

Beitel, P. (2002). Akquisitionen und Zusammenschlüsse europäischer Banken. Wertsteigerung durch M\&A-Transaktionen, Wiesbaden.

Benson-Armer, R. et al (1999). Global Beer: What's on Tap? The McKinsey Quarterly 1999, No. 1.

Bevan, N. and Greenberg, M. (2004). Global Brewing - The Global Pitcher 2004 - Volume V, 
Deutsche Bank Report, London.

Binder, J. (1998). The Event Study Methodology since 1969, in: Review of Quantitative Finance and Accounting 11(1998): 111-137.

Bleakley, M. et al (2004). European Brewers - Going for Growth, Credit Suisse First Boston Equity Research.

Brockett, P. L. et al (1994). Event Study Methodology: A New and Stochastically Flexible Approach. University of Connecticut. Department of Economics. URL: http://ideas.repec.org/.

Brooks, L. D. et al (2000). The Impact of Shifts in Forecasted Earnings and Systemic Risk on Acquiring Firm Shareholder Wealth in Domestic and International Acquisitions, in: Journal of Financial and Strategic Decisions 13(2000): 1-7.

Brown, S. J. and Warner, J. B. (1980). Measuring Security Price Performance, in: Journal of Financial Economics 8(1980): 205-258.

Brown, S. J. and Warner, J. B. (1985). Using Daily Stock Returns: The Case of Event Studies, in: Journal of Financial Economics 14(1985): 3-31.

Bühner, R. (1990). Reaktionen des Aktienmarktes auf Unternehmenszusammenschlüsse, in: Zeitschrift für betriebswirtschaftliche Forschung 42(1990): 295-316.

Chatterjee, S. (1996). Types of Synergy and Economic Value: The Impact of Acquisitions on Merging and Rival Firms, in: Strategic Management Journal 7(1986): 119-140.

Cording, M. et al (2002). A Focus on Resources in M\&A Success: A Literature Review and Research Agenda to Resolve Two Pardoxes. URL: www.darden.virginia.edu/batten/pdf/WP0017.pdf.

Cybo-Ottone, A. and Murgia, M. (2000). Mergers and Shareholder Wealth in European Banking, in: Journal of Banking \& Finance 24 (2000): 831-860.

Gerke, W. et al (1995). Die Bewertung von Unternehmensübernahmen auf dem deutschen Aktienmarkt, in: zfbf 47 (9/1995), S. 805-820.

Glaum, M. et al (2003). Wachstumsstrategien internationaler Unternehmungen: 10 Thesen, in: Zeitschrift für betriebswirtschaftliche Forschung 55(2003): 823-846.

Glaum, M. and Lindemann, J. (2002). Externes Wachstum: Theoretische Überlegungen und empirische Befunde, in: Glaum et al. (Eds.), Wachstumsstrategien internationaler Unternehmungen. Internes vs. externes Unternehmenswachstum, Stuttgart: 269-299.

Harrison, J. et al (2005). Event Studies and the Importance of Longer-Term Measures in Assessing the Performance Outcomes of Complex Events. URL: http://www.indiana.edu.

Hoojimaijers, M. (2003). European Breweries. Medium-Sized Brewery Targets in Europe. Julius Baer European Brokerage, Amsterdam.

Jensen, M. C. (1992). Market for Corporate Control, in: Newman, P. et al. (Eds.), The New Palgrave Dictionary of Money and Finance, London: 657-665.

Joh.Barth\&Sohn (2005). The Barth Report. Hops 2004/05, Nuremberg.

Kaplan, A. (2003). Global Beer: Tapping into Growth, in: Beverage World 122(2003): 24-28.

King, J. L. et al (2002). A Tale of Two Mergers: What We Can Learn from Agricultural Biotechnology Event Studies, in: AgBioForum 5(2002): 14-19.

Köhler, R. and Hüttemann, H. (1989). Marktauswahl im internationalen Marketing, in: Macharzina, K. and Welge, M. (Eds.), Handwörterbuch Export und internationale Unternehmung, Stuttgart: 1428-1440.

Krishnan, H. A. et al (1997). Diversification and Top Management Team Complementarity: Is Performance Improved by Merging Similar or Dissimilar Teams?, in: Strategic Management Journal 18(1997): 361-374.

Kritzman, M. P. (1994). About Event Studies, in: Financial Analysts Journal 50(1994): 17-20. Kusnadi, J. and Sohrabian, A. (1999). The Impact of Insurance Mergers on Shareholder Re- 
turns. URL: http://www.csupomona.edu.

Kutschker, M. and Schmid, S. (2004). Internationales Management, München.

Lepetit, L. et al (2002). Diversification Versus Specialisation: An Event Study of M\&As in the European Banking Industry. URL: www.economics.bham.ac.uk/ research/dp2002/WP.Birmingham(2).pdf.

Lewis, C. (2001). The Future of British Brewing: Strategies for Survival, in: Strategic Change, 10(2001): 151-161.

MacKinlay, A. C. (1997). Event Studies in Economics and Finance, in: Journal of Economic Literature 35(1997): 13-39.

Marx, T. (1998). Internationale Marketingstrategien in der deutschen Brauwirtschaft, in: Europäische Hochschulschriften, Reihe V, Volks- und Betriebswirtschaft, Band 2233, Frankfurt am Main u.a.

McWilliams, A. and Siegel, D. (1997). Event Studies in Management Research: Theoretical and Empirical Issues, in: Academy of Management Journal 40(1997): 626-657.

McWilliams, A. and Siegel, D. (1999). Issues in the Use of the Event Study Methodology: A Critical Analysis of Corporate Social Responsibility Studies, in: Organizational Research Methods 2(1999): 340-365.

Mueller, D. C. and Sirower, M. L. (2003). The Causes of Mergers: Tests Based on the Gains to Acquiring Firm's Shareholders and the Size of Premia, in: Managerial and Decision Economics 24(2003): 373-391.

Müller-Stewens, G. (2000). Akquisitionen und der Markt für Unternehmenskontrolle: Entwicklungstendenzen und Erfolgsfaktoren, in: Picot, A. (Ed.), Management von Akquisitionen: Akquisitionsplanung und Integrationsmanagement, Stuttgart: 41-61.

Müller-Stewens, G. et al (2002). Stand und Entwicklungstendenzen von Cross-BorderAkquisitionen, in: Krystek, U. and Zur, E. (Eds.) Handbuch Internationalisierung: 141-169.

Panayides, P. M. and Gong, X. (2002). The Stock Market Reaction to Merger and Acquisition Announcements in the Liner Shipping, in: International Journal of Maritime Economics 4(2002): 55-80.

Putlitz, J. (2001). Internationalisierung europäischer Banken: Motive, Determinanten, Entwicklungsmuster und Erfolg, Gabler, Wiesbaden.

Rall, W. (2002). Internes versus externes Wachstum, in: Glaum, M. et al. (Eds.), Wachstumsstrategien internationaler Unternehmungen. Internes vs. externes Unternehmenswachstum, Stuttgart: 3-19.

Rössing, S. (2005). Verpasste Chancen, www.LZ-net.de.

Ruback, R. S. (1983). Assessing Competition in the Market for Corporate Acquisitions, in: Journal of Financial Economics 11(1983): 141-153.

Scholl, R. (1989). Internationalisierungsstrategien, in: Macharzina, K. und Welge, M. (Hrsg.), Handwörterbuch Export und internationale Unternehmung, Stuttgart: 983-1001.

Shusterman, T. G. et al (2001). Stock Market Reaction to Mergers and Acquisitions in the U.S. Telecommunications Industry. URL: http://www.norsworthy.net/uploads/PaperTatianaRandy3-14-01.pdf.

Simpson, J. D. and Hosken, D. (1998). Are Retailing Mergers Anticompetitive? An Event Study Analysis. URL: www.ftc.gov/be/workpapers/wp216.pdf.

Todd, H. (2004). Global Beer, in: Beverage World 123(2004): 39-41.

Wells, W. (2004). A Beginner's Guide to Event Studies, in: Journal of Insurance Regulation 22(2004): 61-70. 


\section{KAPITEL III.5}

\section{Multinationality and Financial Performance Findings from a Set of Listed Brewing Groups}

Oliver Ebneth and Ludwig Theuvsen

Paper submitted in different versions for:

- $46^{\text {th }} \mathrm{GeWiSoLa}$ Conference, $4^{\text {th }}-6^{\text {th }}$ of October 2006, Gießen,

- VIII ${ }^{\text {th }}$ IFSAM World Congress, $28^{\text {th }}-30^{\text {th }}$ of September 2006, Berlin. 


\title{
Multinationality and Financial Performance \\ Findings from a Set of Listed Brewing Groups
}

\begin{abstract}
Although there has been significant development in the theory of international business and there is a substantial empirical literature on the causes and outcomes of foreign direct investment (FDI), researchers still suffer from a limited understanding of how the degree of internationalization affects firm performance. The evidence is mixed in explaining the effects of overseas expansion on firm performance. In this paper, we research some of the controversies surrounding the internationalization-performance link. Using a company sample of worldwide leading stock-listed brewing groups (SBG) and with the help of multiple statistical techniques, we examine the relationship for the period 1999-2004. The outcome of the study is a slightly significant and negative internationalization-performance relationship that is generally consistent with findings in previous studies in the finance literature. This is related to the business expansion track of leading SBGs requiring large extraordinary investments. Our research represents a valuable step into the analysis of internationalization-performance patterns in the food industry and highlights some large variations between different brewers in their international involvement and their corporate success.
\end{abstract}

\section{KEYWORDS}

Degree of Internationalization, Financial Performance, Stock-Listed Brewing Groups. 


\section{Problem Statement AND OBJectives}

The relationship between the internationalization and the performance of corporations has triggered extensive interdisciplinary research throughout the last three decades. Researchers have examined the link between performance and the degree of internationalization (DOI), attempting to prove empirically the theoretical argument that international expansion represents a precondition for superior financial success (Annavarjula and Beldona, 2000; Ruigrok and Wagner, 2003). In the field of research about internationalization-performance links, researchers have studied the relationship between the degree of internationalization and performance from different perspectives, such as the portfolio investment theory (Markowitz, 1952), the resource-based view (Wernerfelt, 1984), or foreign direct investment (FDI) theories (Rugman, 1982). Indeed, findings based on these streams of research have been equivocal (Hsu and Boggs, 2003). From a corporate business policy perspective, the degree of a multinational company's (MNC) internationalization derives its importance as a corporate strategy from its potential to co-exist with higher or lower corporate financial performance. In contrast, the general tenet held by many industry executives and consultants is that more extensive international business operations coincide with above-average financial success. This view is hotly discussed in the academic community of international business researchers as clear conclusions concerning the internationalization-performance relationship could not be drawn from earlier work (Annavarjula and Beldona, 2000; Gerpott and Jakopin, 2005).

In recent years the brewing industry has been undergoing an unprecedented drive for consolidation primarily effected through a series of high profile mergers and acquisitions. Thus, leading SBGs account for increasingly larger shares of worldwide beer output as in the early 1990s progressed. The aggregate volume of the world's top ten brewers has grown at more than four times the pace of total industry volume since the mid-1990s. Subsequently, the concentration ratio (CR10) increased remarkably from 37\% in 1998 to 57\% in 2004 .

The international impetus of SBGs, parallel to that experienced by other MNCs, raises the question that has guided the present study: Has this process created value for the enterprises involved? It is important to ascertain whether firms that have focused mainly on domestic markets show different profitability levels than firms with a broader geographic scope. The answer to this question could enable us to highlight the consequences for the profitability of SBGs of the choice between a proximity and a global approach (Zucchella, 2001; Majocchi and Zucchella, 2003). In summary, this investigation addresses the following two main research questions related to the FDI-based multinational expansion processes of stock-listed 
brewing groups:

(1) How did the extent of internationalization of worldwide leading SBGs evolve between the late 1990 s and $2004 ?$

(2) What relationships exist between SBGs' degree of internationalization and their respective corporate financial performance?

\section{SAMPLE}

The aim of this research is to contribute to a better understanding of the problem through an analysis of the performance implications of the different internationalization modes in a sample of SBGs, which consists of the 18 largest publicly listed brewing groups around the globe. With more than $1 \mathrm{bn}$ hectoliters these 18 companies represent $65.6 \%$ of the total world beer market in terms of production volume. Figure 1 illustrates impressively the strong volume growth over recent years, mainly driven by the peer group of leading European brewers that spent more than $€ 60$ bn over the last six years in spectacular M\&As.

Figure 1: Top 18 Stock-Listed Brewing Groups (1998-2004).

Top 18 Global Brewers 1998 Share of Production Volume $(1,301 \mathrm{mhl})$ $40.1 \%$ Market Share

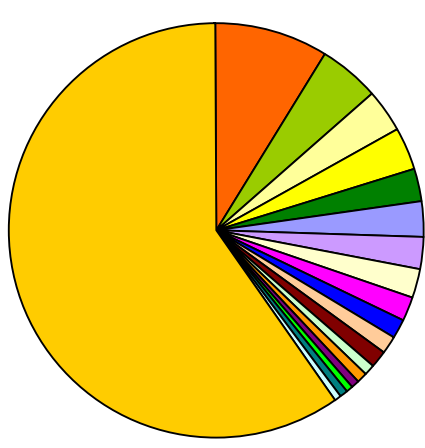

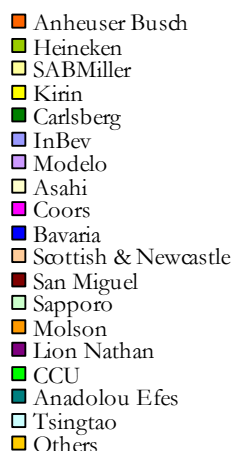

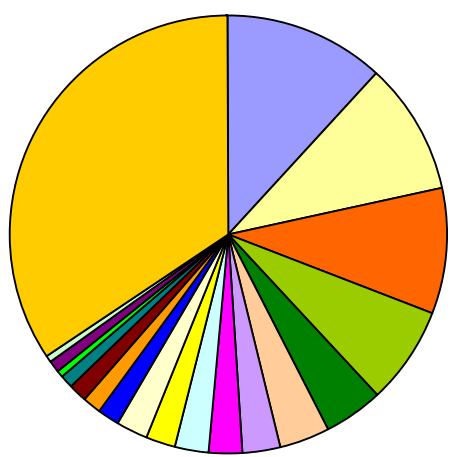

Top 18 Global Brewers 2004 Share of Production Volume $(1,552 \mathrm{mhl})$ $\mathbf{6 5 . 6} \%$ Market Share

Source: (Joh.Barth\&Sohn, 2005; Plato, 2005). 
The industry's CR18 (CR10) in 1998 has been 40.1\% (37.4\%) in 1998 and has risen to more than $65 \%(57.5 \%)$ in 2004 . As the figures in brackets prove, is this development for the most part boosted by the larger brewing groups.

The whole samples' market capitalization at year-end 2005 was around $€ 135 \mathrm{bn}$, of which the average market capitalization was $€ 7,451 \mathrm{mn}$ (s.d. $=€ 7,599 \mathrm{mn}$ ) and the total enterprise value ( $=$ market capitalization + net debt + minorities - associates) about $€ 165$ bn (average $=$ $€ 9,119 \mathrm{mn} ;$ s.d. $=€ 8,284 \mathrm{mn})$. Net sales were more than $€ 83 \mathrm{bn}$ (average $=€ 4,625 \mathrm{mn}$; s.d. $=$ $€ 3,634 \mathrm{mn}$ ), the EBITDA totaled ca. $€ 18 \mathrm{bn}$ (average $=€ 1,000 \mathrm{mn}$; s.d. $=€ 934 \mathrm{mn}$ ), the EBIT was $€ 12 \mathrm{bn}$ (average $=€ 659 \mathrm{mn}$; s.d. $=€ 678 \mathrm{mn}$ ) and the net profit generated by the sample's brewing groups was around $€ 6 \mathrm{bn}$ (average $=€ 329$; s.d. $=€ 454 \mathrm{mn}$ ). Indeed, these figures offer an impressive confirmation that today these companies are well-capitalized multinationals seeking business opportunities in a global environment. Table 1 illustrates operating statistics for our sample at financial year-end 2004.

Table 1: Operating Statistics of 18 World-Wide Leading Brewing Groups - 2004.

\begin{tabular}{|c|c|c|c|c|c|c|c|c|c|}
\hline Company & & $\begin{array}{l}\text { Country of } \\
\text { Incorporation }\end{array}$ & $\begin{array}{c}\begin{array}{c}\text { Volume } \\
(\mathrm{mhl})\end{array} \\
2004\end{array}$ & $\begin{array}{c}\begin{array}{c}\text { Market } \\
\text { Cap }(€ m)\end{array} \\
\text { Sep } 05\end{array}$ & $\begin{array}{c}\begin{array}{r}\text { Enterprise } \\
\text { Value }(€ \mathbf{m})\end{array} \\
\text { Sep } 05\end{array}$ & $\begin{array}{c}\begin{array}{c}\text { Net Sales } \\
(\boldsymbol{\epsilon m})\end{array} \\
2004\end{array}$ & $\begin{array}{c}\text { EBITDA } \\
(€ \mathbf{m}) \\
2004\end{array}$ & $\begin{array}{c}\begin{array}{c}\text { EBIT } \\
(€ \mathbf{m})\end{array} \\
2004\end{array}$ & $\begin{array}{c}\begin{array}{c}\text { Net Profit } \\
(€ \mathbf{m})\end{array} \\
2004\end{array}$ \\
\hline & & & (b) & & (c) & & & & \\
\hline Anheuser Busch & & USA & 144.2 & 27,874 & 29,689 & 12,006 & 3,468 & 2,724 & 1,830 \\
\hline Coors & (a) & USA & 38.4 & 4,392 & 6,610 & 3,462 & 489 & 275 & 166 \\
\hline Molson & (a) & Canada & 21.0 & 2,905 & 3,585 & 1,589 & 362 & 322 & 149 \\
\hline Heineken & & Netherlands & 112.0 & 13,190 & 16,304 & 10,005 & 2,021 & 1,248 & 537 \\
\hline InBev & & Belgium & 183.7 & 19,430 & 24,878 & 8,568 & 2,325 & 1,310 & 719 \\
\hline SABMiller & & UK & 151.9 & 19,307 & 20,678 & 8,422 & 2,156 & 1,424 & 906 \\
\hline Carlsberg & & Denmark & 67.1 & 3,697 & 7,062 & 4,835 & 806 & 306 & 75 \\
\hline Scottish \& Newcastle & & UK & 58.5 & 6,193 & 9,285 & 4,719 & 717 & 355 & 59 \\
\hline Kirin & & Japan & 36.0 & 7,658 & 8,411 & 9,104 & 1,389 & 814 & 365 \\
\hline Asahi & & Japan & 32.2 & 5,083 & 7,069 & 6,624 & 1,135 & 753 & 228 \\
\hline Sapporo & & Japan & 9.9 & 1,336 & 3,138 & 3,681 & 364 & 176 & 35 \\
\hline Lion Nathan & & Australia & 11.0 & 2,646 & 3,421 & 1,190 & 267 & 211 & 102 \\
\hline Modelo & & Mexico & 42.8 & 8,579 & 8,283 & 3,186 & 1,084 & 935 & 440 \\
\hline San Miguel & & Philippines & 20.7 & 3,406 & 5,188 & 2,266 & 362 & 239 & 116 \\
\hline Bavaria & & Colombia & 26.6 & 3,600 & 5,457 & 1,479 & 584 & 478 & -24 \\
\hline Anadolou Efes & & Turkey & 13.8 & 2,309 & 2,360 & 756 & 231 & 153 & 131 \\
\hline Tsingtao & & China & 37.1 & 1,134 & 1,155 & 747 & 106 & 54 & 28 \\
\hline $\mathrm{CCU}$ & & Chile & 11.4 & 1,377 & 1,569 & 607 & 142 & 81 & 65 \\
\hline Max & & & 183.7 & 27,874 & 29,689 & 12,006 & 3,468 & 2,724 & 1,830 \\
\hline Average & & & 56.6 & 7,451 & 9,119 & 4,625 & 1,000 & 659 & 329 \\
\hline Median & & & 36.6 & 4,044 & 6,836 & 3,572 & 651 & 339 & 140 \\
\hline S.d. & & & 54.0 & 7,599 & 8,284 & 3,634 & 934 & 678 & 454 \\
\hline Min & & & 9.9 & $1,134.2$ & $1,155.0$ & 606.7 & 106.1 & 54.4 & -24.3 \\
\hline$\sum$ Total Sample & & & $1,018.4$ & 134,114 & 164,143 & 83,246 & 18,008 & 11,859 & 5,925 \\
\hline
\end{tabular}

(a) Molson and Coors announced an agreement to combine in a merger of equals in July 2004

(b) Volume in million hectoliter (mhl)

(c) Enterprise Value $=$ Market Capitalization + Net Debt + Minorities - Associates

Source: Company data, author's calculations. 
Indeed, these figures underline impressively that these companies are nowadays really multinational and well capitalized companies seeking business opportunities in a global environment. It has to be mentioned that all financial figures are disclosed in euros as some of the largest companies report their annual results in the common European currency. All other currencies have been converted into euros using year-end exchange ratios for the balance sheet and average exchange ratios for the profit $\&$ loss account.

\section{Methodology}

The research period comprised the last six financial years (1999-2004). Various facets of SBG business activities outside their domestic markets are highlighted using two quantitative indicators: (a) the Foreign Sales to Total Sales Index, and (b) the Network Spread Index (Sullivan, 1994; Ietto-Gillies, 1998). The second research question, on associations between degrees of internationalization and SBG profitability, entails the measurement of two categories of additional variables: (a) performance variables and (b) control variables. To shed light on potential relationships between internationalization and financial performance, several regressions and correlations were run for the control variables and the various internationalizationperformance measure combinations.

\section{Measuring the Degree of Internationalization}

The most common ways of measuring the degree of internationalization are the ratio of foreign sales to total sales, the share of foreign assets to total assets (Reeb et al, 1998), the ratio of foreign pre-tax income to total pre-tax income (Chen et al, 1997), the number of countries with foreign operations and the shares of foreign employees, profits, value added or shareholders (Fisch and Oesterle, 2003). All the above measures tend to capture the depth of internationalization. There has also been some research that investigates the scope or breadth of internationalization by examining the geographic dispersion of operations across countries (Kogut, 1985; Hsu and Boggs, 2003). Therefore, the second internationalization index adopted in the current study covers the international dispersion of brewers' subsidiaries. The so-called Network Spread Index (NSI) is calculated by dividing the number of countries in which an enterprise maintains subsidiaries by the total number of countries that received direct investments in 2004 (Ietto-Gillies, 1998). For this reason, the following internationaliza- 
tion measures are applied in this paper:

(1) Foreign Sales Index (FSI): Ratio of foreign sales (exports and subsidiaries) to total sales.

(2) Network Spread Index ${ }^{1}$ (NSI) with $\mathrm{n}^{*}=167:$ NSI $=\frac{n}{n^{*}}=\frac{n}{167}$.

\section{Measuring Financial Performance - Balance Sheet Analysis}

In most previous empirical studies, authors have used traditional financial ratios as dependent variables. Properly interpreted, these ratios provide keen insight into the sources and adequacy of profits, the efficiency of assets committed to the firm, solvency risk and liquidity risk. Individually they tell very little about the whole, but taken together the entire picture of financial health comes into focus (Hsu and Boggs, 2003). They are only as good as the timeliness and accuracy of the financial data fed into them, and analyzing them also depends on a consideration of the company's industry and its position in the business cycle (McClure, 2004). To evaluate the sample firms' performance, we calculated the following financial indicators:

- EBITDA margin: EBITDA margin as a percentage of sales can be used to find companies that are the most efficient operators in an industry and measures the extent to which cash operating expenses use up revenue. The average EBITDA margin across the 18 SBGs and the six study years amounted to $20.68 \%$ (s.d. $=6.71 \% ; n=102$; see variable 3 in Table 5).

- Return on Sales (ROS): The total ROS (= EBIT margin) mean value across all firms and study years was $13.40 \%$ (s.d. $=6.62 \% ; \mathrm{n}=102$; see variable 4 in Table 5). Comparing EBIT to sales shows how successful a company's management has been in generating income from the operation of the business. High operating profits can mean the company has effective control of costs, or that sales are increasing faster than operating costs.

- Return on Equity (ROE): Of all the fundamental ratios that investors look at, one of the most important is return on equity. It's a basic test of how effectively a company's management uses investors' money - ROE shows whether management is growing the

\footnotetext{
${ }^{1}$ Indeed, the number of countries which received FDI in 2004 was 191. But in line with the purposes of the brewing industry, as not all 191 countries play a significant role in the brewing scene context, we decided to adjust the NSI in this examination. We took 167 countries as $n *$. These are 167 countries, which show a production volume of at least 4,000 hl (Joh.Barth\&Sohn, 2005).
} 
company's value at an acceptable rate. ROE, which is calculated by dividing annual net income through average shareholders' equity, offers a useful signal of financial success since it indicates whether a company is increasing profits without expending new equity capital. Overall ROE statistics mean value across the sample within the study period was $12.3 \%$ with a standard deviation of $14.2 \%(n=101$; see variable 5 in Table 5$)$.

- Return on Net Assets (RONA): The RONA reveals how much profit a company earns for every euro of its assets (cash \& cash equivalents, accounts receivable, property, plant \& equipment, etc.). It is calculated as follows: Net income / (fixed assets + net working capital). The total samples' average RONA from 1999 to 2004 was $8.79 \%$ with a standard deviation of $4.23 \%(n=101$; see variable 6 in Table 5).

\section{Control Variables}

According to Gerpott and Jakopin (2005) and based on a review of relevant literature on potential factors that should be neutralized before asking whether there are true internationalization-performance relationships for SBGs, we controlled for six major control factors, carrying out regression analysis. They were composed and calculated as follows:

(1) SBG size: Firm size, a common variable related to firm performance, was used to control for economies and diseconomies of scale at the corporate level (Hsu and Boggs, 2003). The average net sales of the 18 brewing groups included in the sample over the period from 1999-2004 was €4,506bn (s.d. $=€ 3,604 \mathrm{bn}, \mathrm{n}=102$ ).

(2) Year of observation: This variable was included as a black-box parameter to ensure that internationalization-performance correlations were distorted as little as possible by general time-related environmental changes (e.g., increasing competitive intensity, market saturation).

(3) Domestic market share: The domestic market share was measured by the production volume of an SBG in its home country. The average domestic market share of the 18 brewing groups included in the sample over the study period was $52.2 \%$ (s.d. $=27.7 \%$, $\mathrm{n}=108)$.

(4) Leverage: This ratio stands for the level of net debts to total assets and measures the capital structure of the SBG (Majocchi and Zucchella, 2003; Mayer and Whittington, 2003). In previous work leverage was found to be negatively associated with the performance measures of firms operating internationally (Gerpott and Jakopin, 2005). 
(5) Speed of international expansion: This rate was computed by dividing an SBG group's foreign sales to total sales (FSTS) share in a given year by its FSTS share in the preceding year for each of the six observation periods from 1999 to 2004. The overall sample average of the speed indicator amounted to $21.7 \%$ (s.d. $=76.6 \%, \mathrm{n}=108$ ). Earlier studies present evidence that a high speed of foreign business expansion coincides with lower corporate performance (Gerpott and Jakopin, 2005).

(6) Five-firm concentration ratio: In our investigation we use the five-firm concentration ratio (CR5) to measure the concentration in the total world brewing industry. The average CR5 in the brewing industry between 1999 and 2004 was $34.3 \%$ (s.d. $=7.1 \%$ ) and increased significantly from $25.1 \%$ in 1999 to $43.3 \%$ in 2004 . 


\section{RESULTS}

\subsection{Progress in SBGs’ Degrees of Internationalization}

The first research question concerns changes in the extent of internationalization of leading brewing groups since the end of the 1990s. To shed light on this issue, the SBGs' FSI and NSI were computed starting in the year 1999. FDI data have not been published in brewers' annual reports but were carefully calculated by analyzing company publications.

The FSI grew at a compound annual growth rate (CAGR) of $8.2 \%$ from $28.1 \%$ (s.d. = $31.6 \%$ ) in 1999 to $41.7 \%$ (s.d. $=33.8 \%$ ) in 2004 . The degrees of internationalization among leading brewing groups became slightly more inhomogeneous during the five-year period as a result of different paths as well as different speeds of international investment. In 2004 there were still strong differences in the DOI in the sample with FSI figures (NSI figures) ranging from $97.0 \%(45.6 \%)$ for InBev to $3.8 \%(0.6 \%)$ for Chinese Tsingtao. Table 2 highlights the development of the brewing group's FSI and NSI over the study period from 1999 to 2004.

Table 2: Development of the FSI and the NSI for the 18 Brewing Groups from 1999-2004.

\begin{tabular}{|c|c|c|c|c|c|c|c|c|c|c|c|c|c|c|c|c|}
\hline \multirow[b]{2}{*}{ Company } & & \multirow[b]{2}{*}{$\begin{array}{l}\text { Country of } \\
\text { Incorporation }\end{array}$} & \multicolumn{2}{|c|}{1999} & \multicolumn{2}{|c|}{2000} & \multicolumn{2}{|c|}{2001} & \multicolumn{2}{|c|}{2002} & \multicolumn{2}{|c|}{2003} & \multicolumn{2}{|c|}{2004} & \multicolumn{2}{|c|}{ countries (d) } \\
\hline & & & $\begin{array}{l}\text { FSI } \\
(\%) \\
\end{array}$ & $\begin{array}{l}\text { NSI } \\
(\%) \\
\end{array}$ & $\begin{array}{l}\text { FSI } \\
(\%) \\
\end{array}$ & $\begin{array}{l}\text { NSI } \\
(\%) \\
\end{array}$ & $\begin{array}{l}\text { FSI } \\
(\%) \\
\end{array}$ & $\begin{array}{l}\text { NSI } \\
(\%) \\
\end{array}$ & $\begin{array}{l}\text { FSI } \\
(\%) \\
\end{array}$ & $\begin{array}{l}\text { NSI } \\
(\%) \\
\end{array}$ & $\begin{array}{l}\text { FSI } \\
(\%) \\
\end{array}$ & $\begin{array}{l}\text { NSI } \\
(\%) \\
\end{array}$ & $\begin{array}{l}\text { FSI } \\
(\%) \\
\end{array}$ & $\begin{array}{l}\text { NSI } \\
(\%) \\
\end{array}$ & $\begin{array}{c}\text { MA } \\
2004 \\
\end{array}$ & MI \\
\hline Anheuser Busch & & USA & 5.2 & 1.2 & 5.0 & 1.2 & 5.1 & 1.2 & 5.1 & 1.8 & 12.9 & 2.4 & 13.9 & 3.0 & 2 & 3 \\
\hline Coors & & USA & 2.6 & 1.2 & 3.4 & 1.2 & 17.4 & 1.2 & 54.1 & 1.8 & 57.5 & 1.8 & 59.0 & 3.0 & 2 & 1 \\
\hline Molson & & Canada & 9.8 & 1.8 & 11.3 & 1.8 & 10.9 & 1.8 & 11.0 & 1.8 & 25.5 & 1.8 & 23.4 & 3.0 & 2 & 2 \\
\hline Heineken & & Netherlands & 90.0 & 27.5 & 91.0 & 30.5 & 92.2 & 36.5 & 94.0 & 38.3 & 94.5 & 39.5 & 94.9 & 35.9 & 33 & 27 \\
\hline InBev & & Belgium & 89.1 & 23.4 & 91.2 & 25.7 & 92.6 & 28.1 & 93.4 & 31.7 & 96.9 & 40.1 & 97.0 & 41.9 & 37 & 33 \\
\hline SABMiller & & UK & 57.4 & 8.4 & 61.5 & 9.0 & 81.6 & 9.6 & 82.7 & 12.0 & 83.2 & 12.6 & 85.7 & 13.8 & 8 & 12 \\
\hline Carlsberg & & Denmark & 89.2 & 15.0 & 91.7 & 15.6 & 92.3 & 15.6 & 92.9 & 16.2 & 94.1 & 17.4 & 94.7 & 18.0 & 19 & 13 \\
\hline Scottish\&Newcastle & & UK & 6.8 & 5.4 & 48.0 & 6.0 & 63.6 & 7.2 & 65.7 & 7.8 & 66.7 & 9.0 & 68.1 & 9.6 & 11 & 8 \\
\hline Kirin & (a) & Japan & 9.5 & 6.0 & 14.0 & 6.6 & 13.9 & 7.2 & 15.7 & 7.2 & 17.1 & 7.8 & 17.5 & 8.4 & 4 & 10 \\
\hline Asahi & & Japan & 0.2 & 2.4 & 0.4 & 2.4 & 0.5 & 2.4 & 0.6 & 2.4 & 0.9 & 3.0 & 2.2 & 3.0 & 3 & 5 \\
\hline Sapporo & (b) & Japan & 8.0 & 0.6 & 8.5 & 0.6 & 8.8 & 0.6 & 9.2 & 0.6 & 9.4 & 0.6 & 9.1 & 0.6 & 1 & 1 \\
\hline Lion Nathan & & Australia & 39.6 & 4.2 & 37.7 & 4.8 & 32.0 & 4.8 & 29.0 & 4.8 & 39.1 & 4.8 & 38.3 & 4.8 & 4 & 8 \\
\hline Modelo & & Mexico & 24.0 & 0.6 & 22.6 & 0.6 & 23.3 & 0.6 & 24.9 & 0.6 & 26.7 & 0.6 & 28.4 & 0.6 & 1 & 1 \\
\hline San Miguel & & Philippines & 16.9 & 1.2 & 18.2 & 1.2 & 14.3 & 1.2 & 10.5 & 1.8 & 10.2 & 1.8 & 13.2 & 1.8 & 3 & 3 \\
\hline Bavaria & (c) & Colombia & 22.4 & 1.8 & 22.8 & 2.4 & 23.5 & 2.4 & 24.7 & 2.4 & 47.0 & 2.4 & 42.7 & 2.4 & 4 & 1 \\
\hline Anadolou Efes & & Turkey & 19.3 & 4.8 & 26.1 & 5.4 & 34.7 & 6.0 & 39.2 & 6.0 & 43.3 & 6.6 & 48.1 & 6.6 & 7 & 9 \\
\hline Tsingtao & & China & 0.0 & 0.6 & 0.0 & 0.6 & 0.0 & 0.6 & 0.0 & 0.6 & 0.0 & 0.6 & 3.8 & 0.6 & 1 & 1 \\
\hline $\mathrm{CCU}$ & & Chile & 15.6 & 1.2 & 14.9 & 1.2 & 14.9 & 1.2 & 7.2 & 1.2 & 8.2 & 1.2 & 11.0 & 1.2 & 2 & 2 \\
\hline Max & & & 90.0 & 27.5 & 91.7 & 30.5 & 92.6 & 36.5 & 94.0 & 38.3 & 96.9 & 40.1 & 97.0 & 41.9 & 37 & 33 \\
\hline Mean & & & 28.1 & 6.0 & 31.6 & 6.5 & 34.5 & 7.1 & 36.7 & 7.7 & 40.7 & 8.5 & 41.7 & 8.8 & 8.0 & 7.8 \\
\hline S.d. & & & 31.6 & 8.0 & 31.9 & 8.8 & 33.7 & 10.1 & 34.6 & 10.9 & 34.0 & 12.3 & 33.8 & 12.0 & 3.5 & 4.0 \\
\hline Min & & & 0.0 & 0.6 & 0.0 & 0.6 & 0.0 & 0.6 & 0.0 & 0.6 & 0.0 & 0.6 & 2.2 & 0.6 & 1 & 1 \\
\hline
\end{tabular}

(a) No geographical analysis disclosed in annual report 1999, overseas business less than 10\%, 1999 estimated.

(b) No geographical analysis disclosed in annual reports, overseas business less than $10 \%$, figures estimated.

(c) 1999, 2000, 2001 figures estimated.

(d) Number of countries in which an LBG held majority (MA $>50 \%$ ) and minority (MI $<50 \%$ ) ownership stakes in currently active brewing companies at year-end 2004 .

Source: Company data, author's calculations. 
The NSI increased at a CAGR of $8.0 \%$ from $6.0 \%$ (s.d. $=8.0 \%$ ) in 1999 to $8.8 \%$ (s.d. $=$ $12.0 \%$ ) at year-end 2004. As we can derive from the rising standard deviation, dispersions regarding FDI engagement among leading SBGs enhanced over recent years. The spearhead behind this development have been the European brewing groups that have undergone an unprecedented boost in M\&A corporate activity in the course of the last years. Having a further glance at the NSI figures, the column to the extreme right in Table 2 reveals that in 2004 the total number of foreign majority- and minority-owned subsidiaries varied between 70 (InBev) and 2 (Sapporo, Modelo, Tsingtao) with an average of 15.8 FDI. At the financial year-end 2004, the only SBGs with truly global business activities were InBev (70 ownerships) and Heineken (60), followed by a second group, which consists of the other European brewers Carlsberg (32), SABMiller (20) and Scottish\&Newcastle (19). Anadolou Efes (16), Kirin (14) and Lion Nathan (12) can be grouped into a third category characterized by above-average geographical diversification of their business activities. The other brewers in the sample do not have relevant FDI that go beyond more than one other continent than that of their origin. In average these remaining groups held 4.1 subsidiaries abroad in 2004. Consequently, NSI figures vary between $41.9 \%$ for Belgium brewer InBev and $0.6 \%$ for Chinese brewer Tsingtao, the Mexican Grupo Modelo and Japanese brewer Sapporo.

\subsection{Development of SBgs’' Financial Performance}

The second research question on relationships between multinationality and financial performance before controlling for potential confounding impacts of other factors surrounding a brewing group's business expansion abroad entails the measurement of different categories of profitability variables. As most performance indicators include the operating profit (EBIT) in their denominators, we confine this chapter to analyzing the development of the SBGs' earnings before interest and taxes over the research period of the six financial years between 1999 and 2004. We examine the EBIT in two ways. First, we view the total amount of the EBIT growth; second, we look at the development of the EBIT-ratio.

The cumulated EBIT of our whole sample grew at a compound annual growth rate of $9.4 \%$ from $€ 7.6 \mathrm{bn}$ in 1999 to $€ 11.9 \mathrm{bn}$ in 2004 . The average EBIT in 1999 was $€ 541 \mathrm{mn}$ (s.d. = $€ 612 \mathrm{mn}$ ) and increased significantly to $€ 659 \mathrm{mn}$ in 2004 (s.d. =€978mn). Glancing at Table 3 in more detail reveals some large differences regarding brewing groups' growth speed. The world's largest brewer, InBev, for example, grew at a CAGR of $25.6 \%$ and more than tripled 
its EBIT within six years. This rapid growth was mainly driven by former Interbrew's aggressive external growth strategy, spending more than €25bn in M\&As in the course of recent years. Other companies have outperformed even this impressive increase by growing $292 \%$ (Anadolou Efes) or 371\% respectively (Tsangtao). But compared to InBev these brewers have enhanced their EBIT from a relatively low level. Our samples' cumulative EBIT pool grew by $45 \%$ within the study period of six financial years. This big step forward seems even more impressive compared to the growth of net sales, which grew by 'only' $27 \%$ from $€ 65.8 \mathrm{bn}$ to $€ 83.2 \mathrm{bn}$. This boost in profitability is caused mainly by the realization of synergies through intensive $M \& A$ activities, strict cost control and restructuring programs carried out by leading brewers. Moreover, the brewing industry's leader could assert higher selling prices through strong performances by major brands. Thus, organic growth in profits amounted to high single-digit percentages each year within the period under investigation. Table 3 on the following page highlights the development of the brewing groups' EBIT between 1999 and 2004 and the respective brewers' total growth rate. 
Table 3: Development of SBG's EBIT between 1999 and 2004.

\begin{tabular}{|c|c|c|c|c|c|c|c|}
\hline Company & $\begin{array}{r}1999 \\
(€ \mathrm{mn}) \\
\end{array}$ & $\begin{array}{r}2000 \\
(€ m n) \\
\end{array}$ & $\begin{array}{r}2001 \\
(€ \mathrm{mn}) \\
\end{array}$ & $\begin{array}{r}2002 \\
(€ \mathrm{mn}) \\
\end{array}$ & $\begin{array}{r}2003 \\
(€ \mathrm{mn}) \\
\end{array}$ & $\begin{array}{r}2004 \\
(€ \mathrm{mn}) \\
\end{array}$ & $\begin{array}{r}\text { GR } \\
(\%) \\
\end{array}$ \\
\hline Anheuser Busch & 2,451 & 2,697 & 3,017 & 3,151 & 2,827 & 2,724 & $11 \%$ \\
\hline Coors & 150 & 163 & 169 & 315 & 272 & 275 & $84 \%$ \\
\hline Molson & 122 & 151 & 193 & 268 & 333 & 322 & $165 \%$ \\
\hline Heineken & 799 & 921 & 1,125 & 1,282 & 1,296 & 1,248 & $56 \%$ \\
\hline InBev & 419 & 533 & 885 & 836 & 839 & 1,310 & $213 \%$ \\
\hline SABMiller & 687 & 702 & 708 & 878 & 1,147 & 1,424 & $107 \%$ \\
\hline Carlsberg & 231 & 209 & 396 & 430 & 396 & 306 & $33 \%$ \\
\hline Scottish \& Newcastle & 584 & 616 & 873 & 806 & 675 & 355 & $-39 \%$ \\
\hline Kirin & 767 & 937 & 689 & 760 & 775 & 814 & $6 \%$ \\
\hline Asahi & 659 & 760 & 714 & 587 & 603 & 753 & $14 \%$ \\
\hline Sapporo & $141 *$ & 162 & 182 & 93 & 102 & 176 & $9 \%$ \\
\hline Lion Nathan & 181 & 204 & 221 & 200 & 227 & 211 & $17 \%$ \\
\hline Modelo & 421 & 909 & 937 & 1,027 & 888 & 935 & $122 \%$ \\
\hline San Miguel & $159 *$ & 194 & 228 & 259 & 233 & 239 & $23 \%$ \\
\hline Bavaria & $271^{*}$ & $287 *$ & $321 *$ & 363 & 418 & 478 & $32 \%$ \\
\hline Anadolou Efes & 39 & 74 & 73 & 57 & 114 & 153 & $292 \%$ \\
\hline Tsingtao & 11 & 12 & 40 & 66 & 56 & 54 & $371 \%$ \\
\hline CCU & 66 & 63 & 70 & 52 & 65 & 81 & $23 \%$ \\
\hline Max & 2,451 & 2,697 & 3,017 & 3,151 & 2,827 & 2,724 & \\
\hline Average & 453 & 533 & 602 & 635 & 626 & 659 & \\
\hline Median & 251 & 248 & 358 & 397 & 407 & 339 & \\
\hline S.d. & 563 & 630 & 695 & 728 & 664 & 678 & \\
\hline Min & 11 & 12 & 40 & 52 & 56 & 54 & \\
\hline$\sum$ Total Sample & 8,157 & $\mathbf{9 , 5 9 4}$ & 10,842 & 11,431 & 11,264 & 11,859 & $45 \%$ \\
\hline
\end{tabular}

Source: Company data, author's calculations.

Investigating the EBIT-margin $(=\mathrm{ROS})$ of our sample exposes large differences regarding brewers' profitability. Interestingly, some of the most profitable brewing groups stem from emerging markets. Colombian-based Bavaria in particular is the most successful brewer in terms of EBIT generated from operations. Comparing Mexican Grupo Modelo's and Bavaria's financial performance data with the leading stock-listed brewing groups worldwide reveals that both these brewers are well ahead of the rest of their industry's peer group. The average EBIT-margin for the financial year 2004 was 14.7\%. Modelo (29.3\%) and Bavaria (32.3\%) outperform these figures by far. With an EBIT-margin of $32.3 \%$, Bavaria more than doubles the industry's average. These extraordinary financial ratios can be explained mainly by the enormous domestic market shares of Modelo and Bavaria. Modelo controls about 57\% of the fast growing Mexican market, the sixth largest beer market worldwide. Bavaria holds a 
99.6\% market share in both Colombia and Peru. Table 4 on the following page illustrates the change in our samples' EBIT from 1999 to 2004. The chart on the right side is ranked by the brewers' 2004 EBIT figures.

Table 4: EBIT-Margin for the 18 Brewing Groups from 1999-2004.

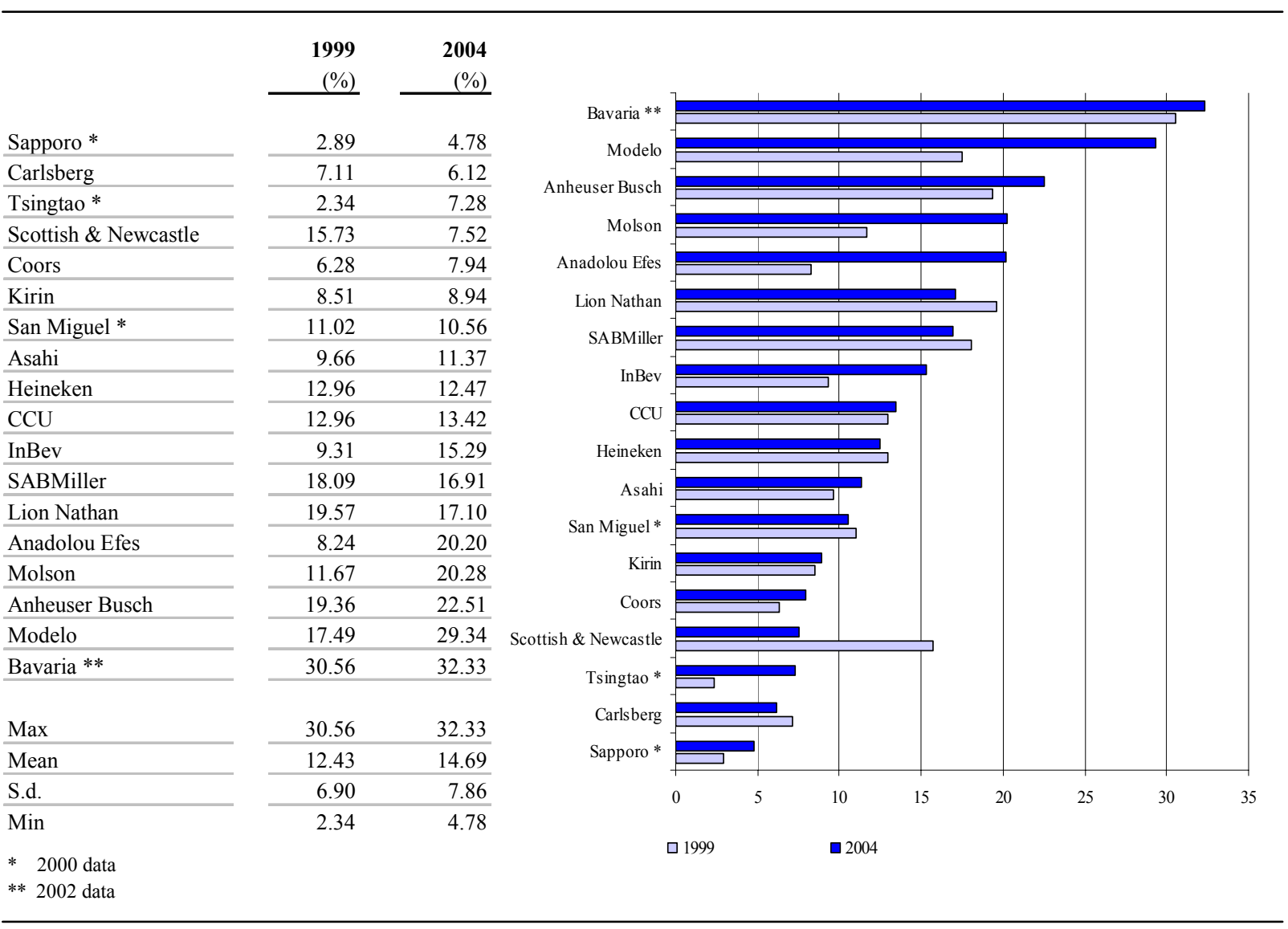

Source: Company data, author's calculations.

\subsection{INTERNATIONALIZATION-PERFORMANCE RELATIONSHIPS}

A methodological prerequisite of the possibility of finding significant internationalizationperformance linkages is that the brewing groups under investigation differ perceptibly regarding their internationalization and financial performance indicator values (Gerpott and Jakopin, 2005). A glance at the standard deviations (s.d.) in Table 5 discloses that the value dispersions of both types of variables were so large that it makes sense to conduct correlational analysis to look for significant internationalization-performance relationships.

We based our statistical approach on the ANOVA technique on averaged data, drawing (mean) performance comparisons among firms at different levels or ranges of internationali- 
zation $(\mathrm{n}=108 ; 18$ companies $\mathrm{x} 6$ financial years). Two analytical steps were taken to shed light on potential links between each of the four performance criteria selected for this investigation and the FSIs and NSIs as the two measures of an SBG group's degree of internationalization. In line with the approach of Gerpott and Jakopin (2005), we first calculated a multiple linear regression analysis (simple bivariate Pearson correlations) for each of the eight possible internationalization-performance measure combinations. Second, for each SBG performance criterion, two ordinary least-squares (OLS) multiple regressions were run, in which standardized beta-weights for the complete set of six control variables and either the FSI share or the NSI share internationalization indicators were obtained.

Table 5: Descriptive Statistics (Means, Standard Deviations and Correlations).

\begin{tabular}{|c|c|c|c|c|c|c|c|c|c|}
\hline $\begin{array}{l}\text { Measures of } \\
\text { internationalization }\end{array}$ & $\begin{array}{r}\text { Mean } \\
(\%)\end{array}$ & $\begin{array}{l}\text { S.d. } \\
(\%)\end{array}$ & $\mathbf{n}$ & 1 & 2 & & & & \\
\hline 1. FSI & 35.54 & 33.52 & 108 & - (a) & & & & & \\
\hline 2. NSI & 7.42 & 10.32 & 108 & $0.68 * *$ & - & & & & \\
\hline $\begin{array}{l}\text { Performance } \\
\text { indicators }\end{array}$ & $\begin{array}{r}\text { Mean } \\
(\%)\end{array}$ & $\begin{array}{l}\text { S.d. } \\
(\%)\end{array}$ & $\mathbf{n}$ & 3 & 4 & 5 & 6 & & \\
\hline 3. EBITDA-Margin & 20.68 & 6.71 & 102 & - & & & & & \\
\hline 4. $\operatorname{ROS}$ & 13.40 & 6.63 & 102 & $0.94 * *$ & - & & & & \\
\hline 5. $\mathrm{ROE}$ & 12.25 & 14.20 & 101 & $0.34 * *$ & $0.39 * *$ & - & & & \\
\hline 6. RONA & 8.79 & 4.23 & 101 & $0.62 * *$ & $0.62 * *$ & $0.70 * *$ & - & & \\
\hline Control variables & Mean & S.d. & $\mathbf{n}$ & 7 & 8 & 9 & 10 & 11 & 12 \\
\hline 7. Year & & & & - & & & & & \\
\hline 8. Net Sales $(€ \mathrm{mn})$ & 4,506 & 3,604 & 102 & 0.04 & - & & & & \\
\hline 9. $\quad$ CR5 $(\%)$ & 34.27 & 7.13 & 108 & $0.98 * *$ & 0.03 & - & & & \\
\hline 10. Market Share $(\%)$ & 52.21 & 27.74 & 108 & 0.05 & -0.13 & 0.05 & - & & \\
\hline 11. Leverage $(\%)$ & 76.32 & 81.09 & 101 & 0.04 & $0.24 *$ & 0.04 & $-0.34 * *$ & - & \\
\hline 12. Speed (\%) & 21.73 & 76.61 & 108 & -0.02 & 0.02 & -0.02 & -0.18 & 0.05 & - \\
\hline
\end{tabular}

In the multivariate regression analyses (see models $1 \mathrm{a}$ and $1 \mathrm{~b}$ in Table 6) several control variables displayed statistically significant beta-weights. Moreover, the FSI share as well as the NSI share internationalization measure saw statistically significant beta-weights $(p<0.10)$ in predicting two of the four SBG financial performance criteria. All the results of our models show an excellent explanatory power $\left(\mathrm{R}^{2}\right.$ in all models ranges between 0.21 and 0.33$)$. 
Table 6: Multiple Regression Analyses of SBG Performance-Internationalization Indicators.

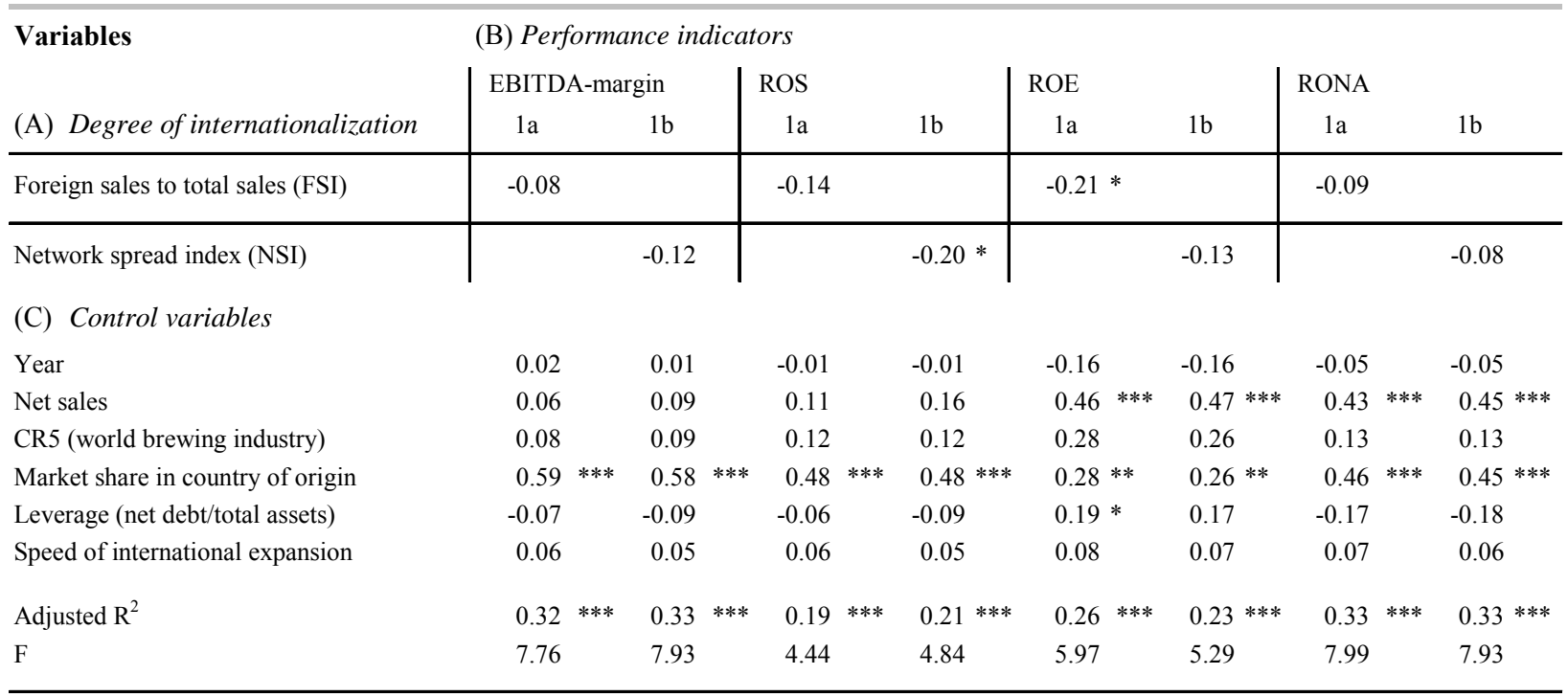

$\mathrm{p}<0.10, * * \mathrm{p}<0.05, * * * \mathrm{p}<0.01$ (two-tailed-tests).

Due to the correlations reported in table 2, additional tests for multicollinarity were run with variance inflation factors.

Figures are standardized beta-values.

In detail, the multivariate regression analyses (see Models 1a and 1b) in Table 6 indicate that:

(1) the negative bivariate EBITDA-margin-internationalization degree (FSI and NSI) relationships achieved no statistical significance after checking several control variables;

(2) the ROS criterion was slightly negatively significant $(p<0.10)$ in relation to the NSI measurement of the degree of internationalization but did not hold the market share control;

(3) the ROE in turn was negatively related to the FSI share at the $10 \%$ significance level but did not contain a set of control variables (net sales, market share, leverage);

(4) a negative relationship between the degree of internationalization and RONA was confirmed but not significantly correlated. Interestingly, the business performance measures ROE and RONA but not the EBITDA-margin and ROS were strongly $(\mathrm{p}<0.01)$ influenced by the net sales control variable (see 'Control variables' section in Table 5).

The relationships observed between increasing international engagement and company business performance were in large part consistent with existing literature. This study's results contributed to our understanding of multinational enterprise by extending research in this domain to a sample of SBGs in recent years. In summary, the present investigation of the whole sample of 18 leading SBGs worldwide did not detect any evidence that higher degrees of in- 
ternationalization coincided with higher profitability levels in the period from 1999 to 2004 (Gerpott and Jakopin, 2005). These findings are clearly at variance with popular opinion on the performance-enhancing effects of internationalization in the brewing industry. Nor do they support popular beliefs about the corporate success-enhancing effects of increasing internationalization of MNEs in general. But our findings are in line with the outcomes of many previous studies (Geringer et al, 1989; Gomes and Ramaswamy, 1999; Agrawal and Jaffe, 2000; Capar and Kotabe, 2003; Contractor et al, 2003).

\section{DisCUSSION OF RESULTS}

Results and empirical evidence on the internationalization degree-performance relationships are mixed. Arguments related to lower business risk/revenue volatility, exploitation of market imperfections, leveraging superior management skills reflected in above average home market performance, better capacity usage and higher market power through economies of scale, scope and learning are put forward as reasons to expect significantly positive internationalization-performance links (Capar and Kotabe, 2003; Hsu and Boggs, 2003; Ruigrok and Wagner, 2003). But, apart from these positive linkages, there are many reasons for a negative relationship. Causes of negative internationalization-performance associations include foreign entry investments/costs as well as higher transaction and complexity costs resulting from running businesses in economically and culturally heterogeneous countries.

Concepts that deal with the costs of raising internationalization are integrated into transaction cost and agency theory. Increasing degrees of internationalization and concomitant organizational and environmental complexity may eventually exhaust managerial capacity. Additionally, communication, coordination and motivation problems stemming from cultural diversity in a given firm cause further costs. As international expansion increases, governance and transaction costs increase exponentially due to the geographic and cultural dispersion of the various principals and agents in the multinational firm. Addressing external costs of internationalization, researchers further emphasize the financial and political risks accompanying foreign expansion (Hofstede and Spangenberg, 1989; Reeb et al, 1998; Ruigrok and Wagner, 2003).

Our results partly confirm these findings, as companies with the highest performance ratios, like Anheuser Busch (2004 ROS 22.5\%), Modelo (2004 ROS 29.3\%) or Bavaria (2004 ROS $32.3 \%$ ), are minimally internationalized but are characterized by high domestic market shares 
(see the influence of the control variable 'Market share' in Table 6), which form the basis for their extraordinary financial performance results. On the other hand, the SBGs that are most internationalized (such as the European peer group) do not per se produce the highest financial performances. Glancing at the ROE figures in our dataset, for instance, we can detect some outliers related to the business expansion track requiring large extraordinary investments (such as InBev's takeover of British brewer Bass in 2000 [ROE -30.5\%] or Scottish\&Newcastle's acquisition of French Kronenbourg in 2001 [ROE -12.7\%]). These extraordinary costs negatively influence brewers' $\mathrm{P} \& \mathrm{~L}$ accounts but are considered of paramount strategic importance for sustained future growth among internationalizing SBGs. Indeed, these multinational European brewing groups are not the most successful brewers in terms of performance ratios but have become the largest brewers in terms of production volume and sales through intensive M\&A activities in the course of recent years. In contrast, highly profitable brewers, like AmBev or Bavaria, did not maintain their independence due to a lack of market capitalization; they consequently became M\&A targets and were acquired by European groups. Thus, internationalization does not contribute to short-term profitability but guarantees future independence and growth perspectives in a globalizing business environment.

Obviously, SBGs encounter both the benefits and the costs of internationalization. Hence, internationalization per se is not a sufficient condition for superior performance. This argument is best exemplified by the theoretical framework and the assumption of incremental internationalization (Johanson and Vahlne, 1977). Brewing firms are said to internationalize on an evolutionary path, starting in geographically and culturally close markets, then successively progressing toward culturally and physically more distant environments. However, as soon as corporations enter unfamiliar territories that require major reconfiguration of internal processes, structures and mechanisms, the costs of internationalization increase dramatically and eventually exceed the benefits (Ruigrok and Wagner, 2003). For SBG groups, our results provide scholarly support for the common sense notion that higher degrees of multinationality are not per se accompanied by higher corporate financial success. Consequently, fast business internationalization is not a cure-all to counter profit and revenue growth erosion in an SBG's domestic market (Gerpott and Jakopin, 2005). 


\section{CONCLUSIONS, MANAGERIAL IMPLICATIONS AND FUTURE RESEARCH}

This paper contributes to the existing literature in two ways. First of all, it broadens the regional scope of the literature on the link between corporate success and internationalization by going beyond the United States as most studies focus on U.S.-based industries. Second, most of the existing literature focuses on the manufacturing, automotive or financial services industry (Jensen, 1992; Agrawal and Jaffe, 2000; Putlitz, 2001; Beitel, 2002; Harrison et al, 2005), whereas this paper specifically addresses the impact of the internationalization impetus within the food-processing industry on brewing firms' accounting performance. Moreover, this investigation represents the first study on the linkage between degree of internationalization and corporate success that covers a real global sample with SBGs from all continents, representing about $70 \%$ of the total world beer industry. The outcome of the study is generally consistent with findings in previous studies in the finance literature.

A major contribution of this study is that it computed not only the degree of internationalization of the sample firms but also each of the brewing groups' financial performance criterion. We did not rely on database sources but carefully accounted all financial ratios in the same manner, reconciling differences in the firms' accounting and valuation standards, which differ significantly around the globe. Moreover, unlike many previous studies, which have relied on one or two years of financial data, we pooled our data across six financial years. This not only increased the degree of freedom, but also allowed an examination of variations among cross-section units and within individual units over time (Majocchi and Zucchella, 2003; Gerpott and Jakopin, 2005).

For SBG executives facing substandard financial performance and/or low levels of internationalization, apparently it is not sufficient to ensure that their company's overall degree of internationalization at least matches that of their industry peers. Rather, a more differentiated approach to internationalization appears to be useful-one that entails the set-up of various strategies and processes supporting the identification of structurally attractive foreign markets (that is, markets characterized by growing population, growing beer consumption, the absence of cutthroat competition, and the like). Furthermore, attention should be paid to the appropriate mode of foreign market entry and the speed of the international expansion. Finally, brewing executives ought to be aware of the correct way to transform into actual improvements the value creation potentials stemming from foreign market opportunities and their fit with an SBG's idiosyncratic resource profile (Perlmutter, 1969; Glaum and Lindemann, 2002). 
Future work should broaden the scientific scope of internationalization-performance relationships and include capital market-based criteria to assess internationalization-performance linkages. Event study methodology is a research method that frequently appears in financial services studies to measure the impact of changes in corporate policy (Ebneth and Theuvsen, 2005). Thus, further research could look at whether specific modes of internationalization, like M\&As, coincide with negative or zero DOI-performance relationships, whereas other modes have positive associations. Additionally, we urge future investigators to consider alternative conceptualization and operationalization techniques for the 'DOI' variable. Researchers who can select from a wide range of measures could also choose indicators that more purely reflect cultural dissimilarity. Such replication would allow direct assessment of the validity of our assumption that different forms of the DOI-performance link can be explained by country-specific types of expansion (culturally related versus unrelated). Admittedly, gathering this information is difficult due to the very limited availability of data that go beyond FDI figures. In our investigation, for example, the foreign assets index (foreign employees index) was available only for 11 (19) out of 108 (= 18 companies x 6 financial years) potential data points.

In conclusion, this study has shed some empirical light on (1) the degree to which 18 major SBGs have internationalized their businesses since the late 1990s and (2) the relationship between degree of internationalization and financial performance for the sample. Furthermore, it shows that the leading brewing groups around the globe have undergone rapid international expansion in the 1999-2004 period but that there were still large variations between the brewers in the degree of internationalization achieved by 2004. Even though this research represents only a small step into the analysis of patterns of internationalization, it highlights some large variations between various brewers around the globe in their international involvement and corporate success. 


\section{REFERENCES}

Agrawal, A. and Jaffe, J. F. (2000): The Post-Merger Performance Puzzle, in: Cooper, C. and Gregory, A. (Eds.): Advances in Mergers and Acquisitions, New York: 7-41.

Annavarjula, M. and Beldona, S. (2000): Multinationality-Performance Relationship: A Review and Reconceptualization, in: International Journal of Organizational Analysis 8(2000): 48-67.

Beitel, P. (2002): Akquisitionen und Zusammenschlüsse europäischer Banken. Wertsteigerung durch M\&A-Transaktionen, Wiesbaden.

Capar, N. and Kotabe, M. (2003): The Relationship Between International Diversification and Performance in Service Firms, in: Journal of International Business Studies 34(2003): 345-355.

Chen, C. J. P. et al (1997): An Investigation of the Relationship Between International Activities and Capital Structure, in: Journal of International Business Studies, 28(1997): 563-577.

Contractor, F. J. et al (2003): A Three-Stage Theory of International Expansion: The Link Between Multinationality and Performance in the Service Sector, in: Journal of International Business Studies 34(2003): 5-18.

Ebneth, O. and Theuvsen, L. (2005): Internationalization and Corporate Success - Event Study Evidence on M\&As of European Brewing Groups. Paper presented at the 15 th Annual World Food and Agribusiness Symposium and Forum 2005, Chicago.

Fisch, J. H. and Oesterle, M.-J. (2003): Exploring the Globalization of German MNCs with the Complex Spread and Diversity Measure, in: Schmalenbach Business Review, 55(2003): 2-21.

Geringer, J. M. et al (1989): Diversification Strategy and Internationalization: Implications for MNE Performance, in: Strategic Management Journal 10(1989): 109-119.

Gerpott, T. J. and Jakopin, N. M. (2005): The Degree of Internationalization and the Financial Performance of Mobile Network Operators, in: Telecommunications Policy 29(2005): 635-661.

Glaum, M. and Lindemann, J. (2002): Externes Wachstum: Theoretische Überlegungen und empirische Befunde, in: Glaum et al. (Eds.), Wachstumsstrategien internationaler Unternehmungen. Internes vs. externes Unternehmenswachstum, Stuttgart: 269-299.

Gomes, E. and Ramaswamy, K. (1999): An Empirical Examination of the Form of the Relationship Between Multinationality and Performance, in: Journal of International Business Studies, 30(1999): 173-188.

Harrison, J. et al (2005): Event Studies and the Importance of Longer-Term Measures in Assessing the Performance Outcomes of Complex Events. URL: http://www.indiana.edu.

Hofstede, G. and Spangenberg, J. (1989): Technik der internationalen Vergleiche, in: Macharzina, K. und Welge, M. (Eds.), Handwörterbuch Export und internationale Unternehmung, Stuttgart: 948-963.

Hsu, C. and Boggs, D. J. (2003): Internationalization and Performance: Traditional Measures and their Decomposition, in: Multinational Business Review 11(2003): 23-49.

Ietto-Gillies, G. (1998): Different Conceptual Frameworks for the Assessment of the Degree of Internationalization: An Empirical Analysis of Various Indices for the Top 100 Transnational Corporations, in: Transnational Corporations, 7(1998): 17-39.

Jensen, M. C. (1992): Market for Corporate Control, in: Newman, P. et al. (Eds.), The New Palgrave Dictionary of Money and Finance, London: 657-665.

Joh.Barth\&Sohn (2005): The Barth Report. Hops 2004/05, Nuremberg.

Johanson, J. and Vahlne, J.-E. (1977): The Internationalization Process of the Firm: A Model 
of Knowledge Development and Increasing Foreign Market Commitments, in: Journal of International Business Studies 8(1977): 23-32.

Kogut, B. (1985): Designing Global Strategies: Profiting From Operational Flexibility, in: Sloan Management Review, 26(1985): 27-38.

Majocchi, A. and Zucchella, A. (2003): Internationalization and Performance. Findings From a Set of Italian SMEs, in: International Small Business Journal 21 (2003): 249-268.

Markowitz, H. (1952): Portfolio Selection, in Journal of Finance 7(1952): 77-91.

Mayer, M. and Whittington, R. (2003): Diversification in Context: A Cross-National and Cross-Temporal Extension, in: Strategic Management Journal, 24(2003): 773-781.

McClure, B. (2004): The Bottom Line on Margins, URL: www.investopedia.com.

Perlmutter, H. V. (1969): The Tortous Evolution of the Multinational Corporation, in: Columbia Journal of World Business, 1969(4): 9-18.

Plato (2005): World Datasheets 2005, Plato Logic Limited, London.

Putlitz, J. (2001): Internationalisierung europäischer Banken: Motive, Determinanten, Entwicklungsmuster und Erfolg, Gabler, Wiesbaden.

Reeb, D. M. et al (1998): Systemic Risk of the Multinational Corporation, in: Journal of International Business Studies, 29(1998): 263-279.

Rugman, A. M. (1982): New Theories of the Multinational Enterprise, New York, St. Martin's Press.

Ruigrok, W. and Wagner, H. (2003): Internationalization and Performance: An Organizational Learning Perspective, in: Management International Review 43(2003): 63-83.

Sullivan, D. (1994): Measuring the Degree of Internationalization of a Firm, in: Journal of International Business Studies, 25(1994): 325-342.

Wernerfelt, B. (1984): A Resource-Based View of the Firm; in: Strategic Management Journal 5(1984): 171-180.

Zucchella, A. (2001): The Internationalization of SMEs: Alternative Hypothesis and Empirical Survey, in: Berry, M. et al (Eds.), Multinational in a New Era, Palgrave: 24-48. 
KAPITEL III.6

\title{
The Relationship between Business Internationalization and Financial Performance of Listed Brewing Groups
}

\author{
Oliver Ebneth and Christian Wocken
}

This paper will be presented in a shorter version at the $16^{\text {th }}$ IAMA Annual World Forum, Symposium and Case Conference, $10^{\text {th }}-13^{\text {th }}$ of June 2006, Buenos Aires. 


\section{The Relationship between Business Internationalization and Financial Per- formance of Listed Brewing Groups}

\section{EXECUTIVE SUMMARY}

The purpose of this paper is to examine the relationship between the degree of internationalization (DOI) and the financial performance of a sample of worldwide leading listed brewing groups (LBGs). The study adds to the existing knowledge by utilizing a more updated firm sample than prior studies, and by covering a substantially different perspective. First, this study tests the relationship of LBGs' performance to their degree of internationalization - an issue still not yet clear in the literature. Second, it examines the effect of two different measures of internationalization - country scope and foreign sales as a percent of total sales (FSTS) - on various measures of performance (EBITDA-margin, operating profit margin, return on net assets, cash realization ratio and net debt ratio). In order to test the effect of internationalization on firm profitability, we define some independent variables that measure internationalization under a variety of definitions; then we introduce some control variables in order to gauge for other aspects that influence firms' performance. Furthermore, the interactive effects of depth of internationalization (FSTS) and breadth of internationalization (country scope) on LBGs' performance are also examined. We pay particular attention to selected brewing groups' differing paths of internationalization and their impact on the respective brewers' corporate success.

The contribution of the various internationalization strategies to value creation has been the object of a number of empirical studies. The international business literature has focused mainly on the performance implications of foreign direct investment (FDI), especially for larger firms. Although it is possible to argue that higher levels of international involvement would lead to better performance, the results of empirical studies do not reach definitive conclusions (Ramaswamy, 1992). We investigate the empirical relationship between accountingbased measures of performance and the degree of multinational diversification for a set of worldwide leading brewing firms. We find that for these firms the degree of multinational diversification is partly related to minor financial performance. The results hold for each of the eight sample years. The statistical findings do not suggest that more internationalized firms outperform firms focusing mainly on domestic and/or export markets. Despite these 
results an in-depth comparative analysis of two leading LBGs provides strong support for gains from multinational diversification. The relationship between profitability and internationalization has been widely explored in the literature with regard to multinational corporations (MNC) in the manufacturing, automotive and financial services industries, but seldom with reference to food processing companies in general and not at all for LBGs in particular.

Our findings should be of considerable interest to international business academicians as well as to practitioners in the brewing industry. On the practical level, our results suggest that managers should be eager to pursue new international opportunities but should be wary of over-expanding, or overextending, their firms - particularly geographically. Finally, we emphasize the principal impact of internationalization on the world brewing scene: gaining and securing sustainable corporate success through top-line internal and external growth.

\begin{abstract}
Previous empirical studies on the relationship between internationalization and firm performance have been mixed. This paper utilizes a sample of brewing firms and decomposes traditional and innovative financial performance measures, applying two different measures of the degree of internationalization, to measure the effects on financial performance of different degrees of internationalization. We analyzed data using the cross-section OLS regression method. Contrary to widespread belief in the performance-enhancing effects of rising internationalization, it was found that, at first glance, higher degrees of internationalization were accompanied by lower group profitability. Managerial implications and future research recommendations close this analysis. For the future it seems of paramount importance, in order to ensure greater opportunities for future profit growth, that LBGs have broad exposure to both fast-growing emerging markets and the largest profit pools.
\end{abstract}

\title{
JEL Classification System
}

F23 Multinational Firms; International Business.

L25 Firm Performance: Size, Age, Profit, and Sales.

M41 Accounting.

\section{KEYWORDS}

Internationalization, Financial Performance, Balance Sheet Analysis, Listed Brewing Groups. 


\section{Problem Statement}

The relationship between the internationalization and the performance of corporations has triggered extensive interdisciplinary research throughout the last three decades. Researchers have examined the link between performance and the degree of internationalization, attempting to prove empirically the theoretical argument that international expansion represents a precondition for superior financial success (Annavarjula and Beldona, 2000; Ruigrok and Wagner, 2003). In the field of research about internationalization-performance links, researchers have studied the relationship between the degree of internationalization and performance from different perspectives, such as portfolio investment theory (Markowitz, 1952), the resource-based perspective (Wernerfelt, 1984) and FDI theories (Rugman, 1982), but findings based on these streams of research have been equivocal (Hsu and Boggs, 2003).

The world's largest listed brewing groups (LBGs) accounted for increasingly larger shares of worldwide beer output as the early 1990s progressed. As part of this growth, managers of LBGs turned to international markets to seek new growth opportunities for their firms. The result of this foreign direct investment was a substantial strengthening of brewers' investment positions in several areas of the world: Asia, Eastern Europe and South America in particular (Delios, 2000). For many firms, the number of foreign subsidiaries increased dramatically in the course of the last decade. European brewers have been the driving force behind this development. Heineken, for instance, doubled its fully-consolidated foreign subsidiaries in the late 1990s. It had 31 main subsidiaries in 1998; but by 2005 this number had risen to 61. InBev had an even more impressive pace of international expansion during this period. Its FDI portfolio grew by $254 \%$ percent to 322 subsidiaries $^{1}$ in 2005 . While not all brewing groups around the globe undertook this rapid expansion, the general trend during this period was towards increasing growth in international markets. We explore the performance consequences of this growth and seek to answer the question of whether this expansion into international markets was accompanied by a positive impact on brewers' performance. At an empirical level, this question is of particular importance to firms that are beginning to move into international markets.

In this study, we attempted to further illuminate and to resolve some of the controversies

1 "The total number of companies consolidated (fully, proportional and equity method)" (InBev, 2006). 
surrounding the internationalization-performance link. Using a company sample of worldwide LBGs and with the help of multiple statistical techniques, we examined the relationship for the period from 1998 to 2005 . This investigation is structured as follows. First, we depict the settings and objectives of our analysis. In the literature review that follows, we discuss the conceptual frameworks and methodological procedures of previous inquiries on DOIperformance relationships. We then describe some operating statistics and financial figures for our company sample. We pay particular attention to the internationalization impetus in the world brewing scene. In particular, we consecutively discuss the ways leading brewers internationalize based on the theoretical foundations. The next chapter deals with applied variable conceptualization/operationalization and analysis techniques for measuring the multinationality and financial performance of brewing groups. This is followed by a presentation of the results. Sections on discussion/conclusions and limitations/recommendations close the article. 


\section{OBJECTIVES}

Accounting profit serves to evaluate a firm's performance. This measurement serves to create incentives for managers - directly through compensation depending on performance and indirectly through the influence of performance on reputation and professional career. Another important effect of publishing information on performance is market reaction, which may result in sanctions for negligent managers: a drop in market value may become a signal to replace management; moreover, it can make the firm attractive for a hostile takeover (Hax, 2003).

From a corporate business policy perspective, the degree of an LBG's internationalization derives its importance as a corporate strategy from its potential to co-exist with higher or lower corporate financial performance. The general tenet held by many industry executives and consultants is that more extensive international business operations coincide with aboveaverage financial success. This view is hotly discussed in the academic community of international business researchers (Glaum, 1996; Annavarjula and Beldona, 2000). Correspondingly, the second intention of this study is to explore the internationalization-performance relationship in a sample of LBGs as clear conclusions concerning this association could not be drawn from earlier work (Gerpott and Jakopin, 2005).

From a conceptual point of view, internationalization is a multidimensional construct (Sullivan, 1994; Ramaswamy et al, 1996; Fisch and Oesterle, 2003). The traditional distinction between export and foreign direct investment, as the two core expressions of an internationalization strategy, has been replaced by a wide range of entry-mode alternatives. Thus, internationalization may range from exports to inter-firm nonequity and equity arrangements to FDI and involves different levels of commitment and risk (Majocchi and Zucchella, 2003). Some studies have demonstrated that firm size and export intensity - as measured by the ratio of exports to sales - do not correlate. The international impetus of LBGs, parallel to that experienced by other MNCs, raises the question that has guided the present study: Has this process created value for the enterprises involved? This is not a new question for researchers, and is at present a crucial one for managers, though we are still far from drawing clear-cut conclusions in the matter. The aim of this research is to contribute to a better understanding of the problem through analysis of the performance implications of the different internationalization modes in a sample of LBGs. It is important to ascertain whether firms mainly focused on domestic markets show different profitability levels than firms with a broader geographic 
scope. The answer to this question could enable us to highlight the consequences for the profitability of LBGs of the choice between a proximity and a global approach (Zucchella, 2001; Majocchi and Zucchella, 2003).

As seen from the above discussion, there is no consensus for measuring the 'degree of internationalization,' nor is the exact nature of the relationship between internationalization and a firm's financial performance known. Given the overall absence of clear research findings in this area, this study examines the nature of the internationalization-performance relationship by subjecting different financial performance ratios and their decomposition to investigation. It is predicted that the relationship between internationalization and firms' traditional financial ratios (ROE, RONA,) will differ from the relationship between internationalization and the decomposition of these financial ratios (EBITDA margin, operating profit margin), and the results will thus reveal new information about the relationship between internationalization and financial performance. The findings should be of considerable interest to international business academicians and practitioners (Hsu and Boggs, 2003).

In summary, this investigation addresses the following two main research questions related to FDI-based multinational expansion processes of LBGs:

(1) How did the extent of internationalization of worldwide leading LBGs evolvebetween the late 1990 s and $2005 ?$

(2) What relationships exist between an LBG's degree of internationalization and its corporate financial performance? 


\section{Multinationality ANd Firm Performance: A ReView Of THE LITERATURE}

International business scholars have looked at the relationship between degree of internationalization and performance from a variety of theoretical perspectives. The benefits that accompany increasing internationalizing firm activities are suggested and described by two core theory streams: theories of foreign direct investment (FDI) and theories of the multinational corporation. FDI theories, which are primarily focused on factors located in the firm's external environment, seek to explain why multinational companies exist. Imperfections in international product, factor and financial markets are postulated to benefit firms that internationalize. Whereas scholars in international business and industrial organization economics normally emphasize economies of scale and scope, researchers in financial economics stress portfolio diversification and its effect on companies' risk-return performance (Buckley and Casson, 1976; Ruigrok and Wagner, 2003). Adopting a more managerial perspective, MNC theories focus on an organization's internal environment. The main source of benefits from internationalization lies in the proactive induction and exhaustion of intra-firm comparative advantages. The international resource transfer and the integration potential of worldwide corporate structures, systems and processes can provide MNCs with company-specific competencies not available to the firm operating domestically. Similarly, the resource-based view of the firm proposes that global resources and core competencies promote organizational learning and knowledge development. Finally, the theory of operational flexibility proposes arbitrage and leverage opportunities in MNCs, especially those that have successfully developed global network structures (Fayerweather, 1989; Ruigrok and Wagner, 2003).

For the most part, studies examining multinationality and firm performance argue that the superior performance of a multinational firm emerges from its ability to gain higher returns from exploiting proprietary assets, such as brand equity, patents or unique processes across a greater number of markets. In the process of developing a multinational network of subsidiaries, advantages accrue to the MNC from the ability to source low cost inputs across disparate geographical locations; from a diversification in revenue sources across host country markets which reduce the variability in these returns; and from an increase in market power. Advantages might also be gained from the larger volume of output and associated gains in economies of scale and scope that come with a greater international spread (Delios, 2000). Given the large set of advantages to be found in operating on a multinational basis, it is not surpris- 
ing that many studies have found a positive relationship between multinationality and performance.

There is also a growing literature that focuses on the shape of the relationship between DOI and performance. Contractor, Kundu, and Hsu (2003) list 15 studies that find the relationship between performance and DOI is linear: seven of the studies find a positive relationship, four a negative relationship and four no relationship. Two studies listed find a U-shaped relationship, and eight find an inverted U-shaped relationship. Contractor, Kundu, and Hsu (2003) and $\mathrm{Lu}$ and Beamish (2004) provide theoretical models for curvilinear relationships between DOI and performance (Hejazi and Santor, 2005).

There is a large volume of literature which provides conceptual reasons as to why the degree of internationalization and financial performance measures should be linked in a linearly positive, linearly negative or non-linear manner. Arguments related to lower business risk/revenue volatility, exploitation of market imperfections, leveraging superior management skills reflected in above-average home market performance, better capacity usage, and higher market power through economies of scale, scope, and learning are put forward as reasons for expecting significantly positive internationalization-performance links (Capar and Kotabe, 2003; Hsu and Boggs, 2003; Ruigrok and Wagner, 2003). Although it is possible to argue that higher levels of international involvement would lead to better performance, the results of empirical studies do not reach definitive conclusions (Ramaswamy, 1992).

Admittedly, expectations of finding significant positive internationalization-performance correlations can be criticized as overly naïve: International business expansion is not per se boosting corporate financial success levels. Therefore, results and empirical evidence on internationalization degree-performance relationships are mixed. According to Gerpott and Jakopin (2005), who recently conducted a literature review, 51 studies have deducted significantly positive linear, 12 significantly negative linear, 15 significant non-linear, and 18 insignificant associations. One explanation for the ambiguity of previous findings is that many earlier studies displayed weakness in using multivariate statistical methods that are sophisticated enough to capture whether there are true internationalization-performance linkages or not. To wit, the failure in finding correlations or the identification of spurious covariations could stem from not statistically controlling background variables associated with internationalization and performance measures (Gerpott and Jakopin, 2005). Most prominent researchers in the field of internationalization-performance linkages now argue that the omission of internationalization costs in the theoretical frameworks of early inquiries largely explain the am- 
biguous results (Sullivan, 1994b).

Whereas early researchers touched only lightly on the issue of internationalization costs, authors in the 1990s elaborated both, the benefits and the costs as well as their potential interplay. Apart from these positive linkages there is a bulk of reasons for a negative relationship. Causes for negative internationalization-performance associations include exaggerated foreign entry investments/costs as well as higher transaction and complexity costs resulting from running businesses in economically and culturally heterogeneous countries. These concepts, which deal with the costs of raising internationalization, are integrated in transaction cost and agency theories. Some studies conclude that performance appears to increase monotonically up to a critical zone, climaxes and then decreases monotonically. This argument is best exemplified by the theoretical framework and the assumption of incremental internationalization (Johanson and Vahlne, 1977). Increasing DOIs and concomitant organizational and environmental complexity may eventually exhaust managerial capacity. Additionally, communication, coordination, and motivation problems stemming from cultural diversity in the firm cause further costs. As international expansion increases, governance and transaction costs increase exponentially due to the geographical and cultural dispersion of the various principals and agents in the multinational firm. Addressing external costs of internationalization, researchers further emphasized the financial and political risks accompanying foreign expansion (Hofstede and Spangenberg, 1989; Reeb et al, 1998; Ruigrok and Wagner, 2003).

Analysis of a firm's performance drivers involves two fundamental complexities: the first deals with the concept of performance itself and the second refers to the blend of the many different drivers that influence performance, most of them evidencing a firm-specific nature and each of them difficult to isolate from the others. In its simplest and most commonly used meaning, performance can be expressed by profitability ratios. The concept of performance may refer to business dimensions that differ from traditional profitability ratios, but the international business literature has predominantly referred to ROE, RONA, and ROS because they can be easily extracted from common-size financial statements and compared in crosscountry surveys; such comparison is facilitated by the substantial improvements worldwide in approaches to the harmonization of financial analyses (Majocchi and Zucchella, 2003). The relationship between profitability and internationalization has been widely explored in the literature with regard to MNCs in the manufacturing, automotive or financial services industry, but rarely with reference to food processing companies in general and not at all concerning LBGs in particular. 


\section{SAMPLE}

Table 1 illustrates some operating statistics for our sample, which consists of the ten largest brewing groups around the globe, covering 62.5\% (994 million hectoliters (mhl) out of $1,589 \mathrm{mhl}$ ) of the total world beer production in terms of production volume. On average, the world's leading LBGs produced 97.9mhl in 2005 (s.d. $=61.5 \mathrm{mhl}$ ). The whole samples' market capitalization at year-end 2005 was around $€ 141$ billion (average $=€ 12,078$ million; s.d. $=$ $€ 8,717$ million) and the total enterprise value was about $€ 140$ billion (average $=€ 13,962$ million; s.d. $=€ 9,477$ million). Net sales totaled more than $€ 71$ billion (average $=€ 7,130$ million; s.d. $=€ 3,866 \mathrm{n}$ ), the EBITDA totaled ca. $€ 15.4$ billion (average $=€ 1,540$ million; s.d. $=€ 1,050$ billion), EBIT was $€ 10.9$ billion (average $=€ 1,086$ million; s.d. $=€ 814$ million) and the net profit generated by the sample's brewing groups rose above $€ 5.9$ billion (average $=€ 591$ million; s.d. $=€ 471$ million).

Table 1: Operating Statistics of the Ten Brewing Groups Investigated, 2005

\begin{tabular}{|c|c|c|c|c|c|c|c|c|}
\hline & Country of & $\begin{array}{c}\text { Volume } \\
\text { (mhl) }\end{array}$ & $\begin{array}{c}\text { Market } \\
\text { Cap (€mn) }\end{array}$ & $\begin{array}{c}\text { Enterprise } \\
\text { Value (€mn) } \\
\end{array}$ & $\begin{array}{c}\text { Net Sales } \\
(€ \mathbf{m n})\end{array}$ & $\begin{array}{c}\text { EBITDA } \\
(€ \mathbf{m n}) \\
\end{array}$ & $\begin{array}{l}\text { EBIT } \\
(€ \mathrm{mn}) \\
\end{array}$ & $\begin{array}{c}\text { Net Profit } \\
(€ \mathbf{m n}) \\
\end{array}$ \\
\hline \multirow[t]{2}{*}{ Company } & Incorporation & 2005 & Mar 06 & Mar 06 & 2005 & 2005 & 2005 & 2005 \\
\hline & & & (a) & (b) & (c) & & & \\
\hline InBev & Belgium & 223.5 & 23,448 & 28,689 & 11,656 & 3,132 & 2,198 & 904 \\
\hline SABMiller & UK & 183.9 & 17,068 & 18,502 & 9,196 & 2,602 & 2,159 & 1,149 \\
\hline Anheuser-Busch & USA & 151.6 & 27,842 & 29,354 & 12,073 & 2,891 & 2,105 & 1,477 \\
\hline Heineken & Netherlands & 118.6 & 15,591 & 18,705 & 10,796 & 1,959 & 1,249 & 761 \\
\hline Carlsberg & Denmark & 72.1 & 4,023 & 6,769 & 5,099 & 815 & 440 & 149 \\
\hline Scottish\&Newcastle & UK & 61.7 & 6,911 & 9,884 & 4,765 & 617 & 438 & 362 \\
\hline MolsonCoors & USA & 61.2 & 4,862 & 7,183 & 4,422 & 682 & 339 & 197 \\
\hline Modelo & Mexico & 44.9 & 9,097 & 8,785 & 3,564 & 1,218 & 1,050 & 503 \\
\hline Tsingtao & China & 41.0 & 1,407 & 1,429 & 875 & 122 & 62 & 32 \\
\hline Kirin & Japan & 35.3 & 10,531 & 10,321 & 8,855 & 1,360 & 816 & 374 \\
\hline \multicolumn{2}{|l|}{ Max } & 223.5 & 27,842 & 29,354 & 12,073 & 3,132 & 2,198 & 1,477 \\
\hline \multicolumn{2}{|l|}{ Average } & 99.4 & 12,078 & 13,962 & 7,130 & 1,540 & 1,086 & 591 \\
\hline \multicolumn{2}{|l|}{ Median } & 66.9 & 9,814 & 10,103 & 6,977 & 1,289 & 933 & 439 \\
\hline \multicolumn{2}{|l|}{ S.d. } & 66.5 & 8,717 & 9,477 & 3,866 & 1,050 & 814 & 471 \\
\hline \multicolumn{2}{|l|}{ Min } & 35.3 & 1,407 & 1,429 & 875 & 122 & 62 & 32 \\
\hline \multicolumn{2}{|l|}{$\sum$ Total Sample } & 993.8 & 120,780 & 139,622 & 71,300 & 15,398 & 10,856 & 5,908 \\
\hline \multicolumn{9}{|c|}{$\begin{array}{l}\text { (a) Market capitalization }=\text { Number of outstanding common shares of the firm * cur } \\
\text { (b) Enterprise Value }=\text { Market Capitalization }+ \text { Net Debt }+ \text { Minorities }- \text { Associates } \\
\text { (c) Excise duties deducted }\end{array}$} \\
\hline
\end{tabular}

Source: Company data, authors' calculations. 
It has to be pointed out that all financial figures are stated in $€$, as four of the largest companies (Heineken, InBev, Carlsberg, Scottish\&Newcastle [S\&N]) report their annual results in the European currency. The other currencies have been converted into $€$ using year-end exchange ratios for the balance sheet and average exchange ratios for the profit\&loss account.

As we can see from the high standard deviations (s.d.), there is a relatively wide dispersion of financial figures within our sample's companies. This is caused primarily by the fact that rankings in the world brewing industry still are made up by production volume in million hectoliters and not sales-based. Therefore the world's number 9 brewer is Chinese Tsingtao, which, while it produced more than $40 \mathrm{mhl}$ in 2005 , generated only $€ 747$ million. In contrast, the number 10 brewer, Kirin, from Japan, produced around $35 \mathrm{mhl}$ but had a turnover of more than $€ 9$ billion, twelve times the net sales of Tsingtao.

In the brewing industry most of the data available on the relative growth rates of countries, regions or continents focuses on volume. This is obviously the most easily measurable and available metric within the industry. But for investors it is not the most important - that has to be profitability ${ }^{2}$. As we can see from Figure 1 there are still stark differences within the largest brewers' financial figures. Indeed, Chinese Tsingtao is the ninth largest brewer in terms of production volume but still far behind its main competitors' financial key figures. InBev generates 35 times Tsingtao's EBIT. Figure 1 illustrates our sample's main financial figures in 2005: net sales, EBITDA and operating profit ( EBIT).

\footnotetext{
${ }^{2}$ We estimate that over $90 \%$ of the industry's profitability is now generated by public companies - the only significant exceptions being Polar in Venezuela, Mahou and Damm in Spain and a long list of small operators in the three main fragmented markets - China, Germany and Russia (Bevan and Greenberg, 2005).
} 
Figure 1: LBG's Net Sales, EBITDA and Operating Profit - 2005 (in € million)

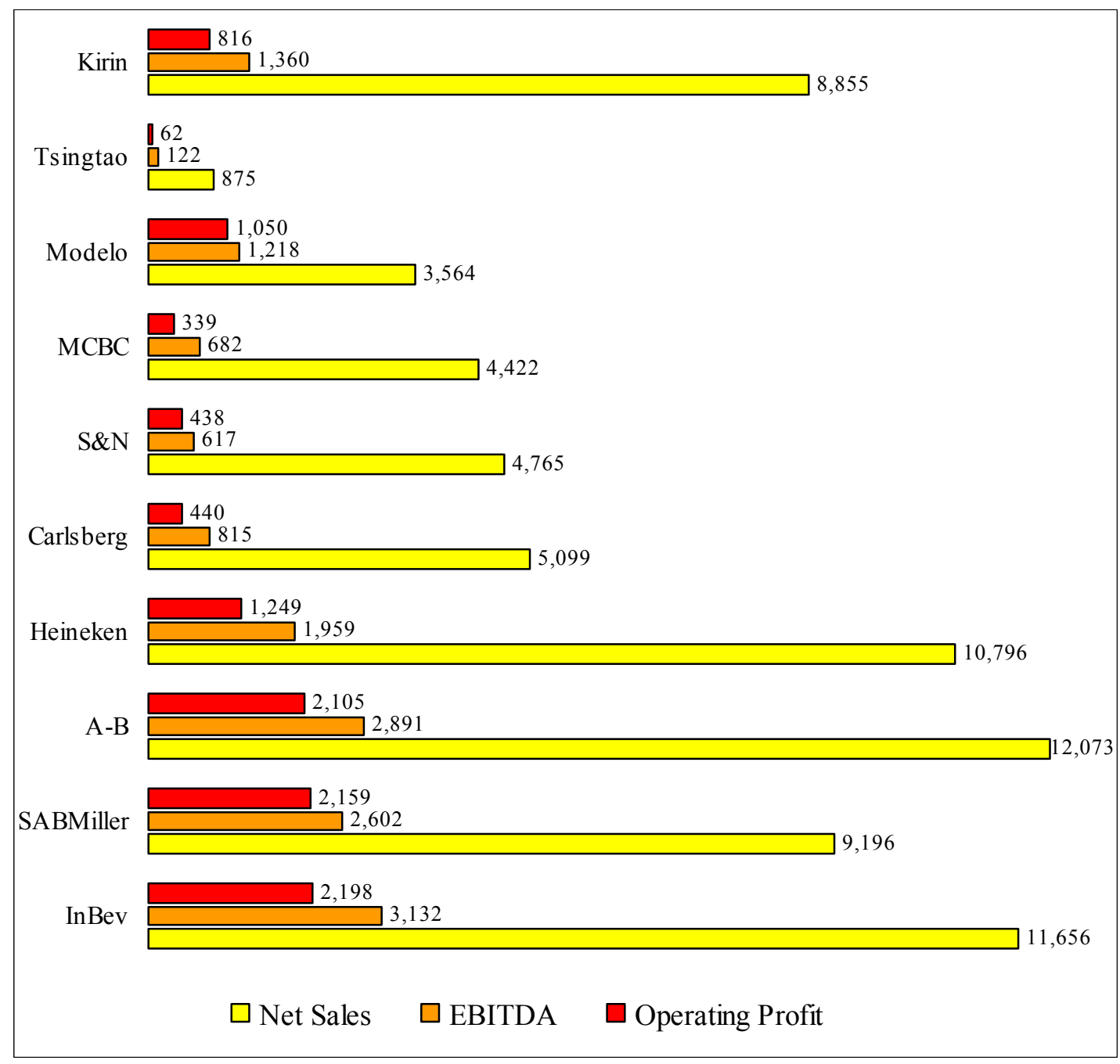

Source: Company data, author's calculations.

Figure 1 illustrates the aforementioned discrepancy. The brewers - ranked in increasing order by production volume - can be divided in two main groups. The leading four brewers are the largest companies both in terms of production volume and in terms of financial key figures. This peer group is followed by the medium-caps, like Carlsberg, S\&N, MCBC and Modelo. Our sample shows two outliers: the aforementioned Chinese Tsingtao and Japanese Kirin. The latter shows extraordinarily high financial figures compared to a production volume of "only" $35 \mathrm{mhl}^{3}$.

${ }^{3}$ The stark differences in Tsingtao's and Kirin's financial figures are based on the fact that Kirin generates an EBITDA of approximately $€ 43$ per hectolitre, whereas Tsingtao in China only generates $€ 3 / \mathrm{hl}$ - 1 14-fold difference. (See also Chapters III.1 and III.2.) 


\section{INTERNATIONALIZATION IMPETUS IN THE LBG CONTEXT}

In recent years the brewing industry has been undergoing an unprecedented drive for consolidation primarily effected through a series of high profile mergers and acquisitions. Thus, internationalization in the beer industry is now moving at a much higher speed.During recent years pioneer enterprises like Heineken, SABMiller and InBev (former Interbrew) have started internationalizing their activities in order to continue growing and expanding in the face of saturated home markets (Todd, 2004) ${ }^{4}$. The world beer market has changed significantly in the past ten years, with stagnation in mature markets in Western Europe and North America, and substantial growth in emerging markets. In 2005 the world beer industry produced a total of $1,589 \mathrm{mhl}$ and generated total profits of US\$19 billion. To put this in perspective, that is about 24 liters per capita, worldwide (Heineken, 2006b). The aggregate volume of the world's top ten brewers has grown at more than four times the pace of total industry volume since the mid-1990s, totaling $993 \mathrm{mhl}$ in 2005. Figure 2 on the following page illustrates the boost in concentration and consolidation within the world brewing scene driven by the industry's leading players over the last eight years.

\footnotetext{
${ }^{4}$ While Heineken's volumes have almost doubled over the last decade, the company's share of the global beer market has increased from 5.2\% in 1995 to $7.5 \%$ in 2005. During that period, both InBev (2.4\% in 1995 to $14.0 \%$ in 2005$)$ and SABMiller (2.9\% in 1995 to $11.6 \%$ in 2005 ) have grown far more quickly (Heineken, 2006b).
} 
Figure 2: Top Ten Global Brewers (1998-2005)

Top 10 Global Brewers 1998

Share of Production Volume (1,301 mhl)

$33.7 \%$ Market Share

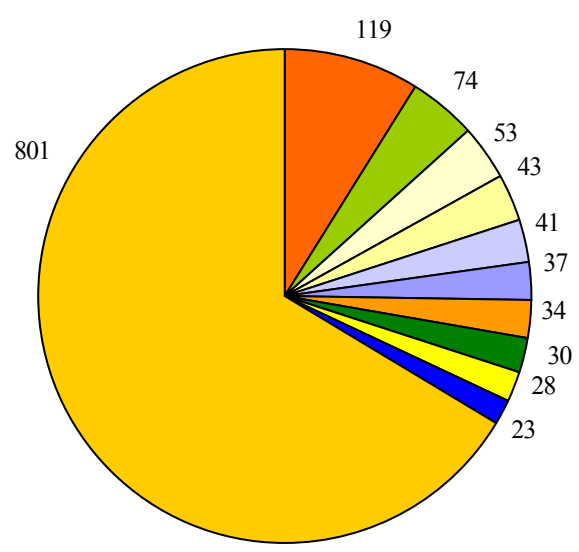

$\square 1$ Anheuser-Busch (USA)

$\square 3$ Miller (USA)

$\square 5$ Brahma (Brazil)

$\square 7$ Carlsberg (Denmark)

$\square 9$ Kirin (Japan)

$\square$ Others
Top 10 Global Brewers 2005

Share of Production Volume (1,589 mhl)

62.5\% Market Share

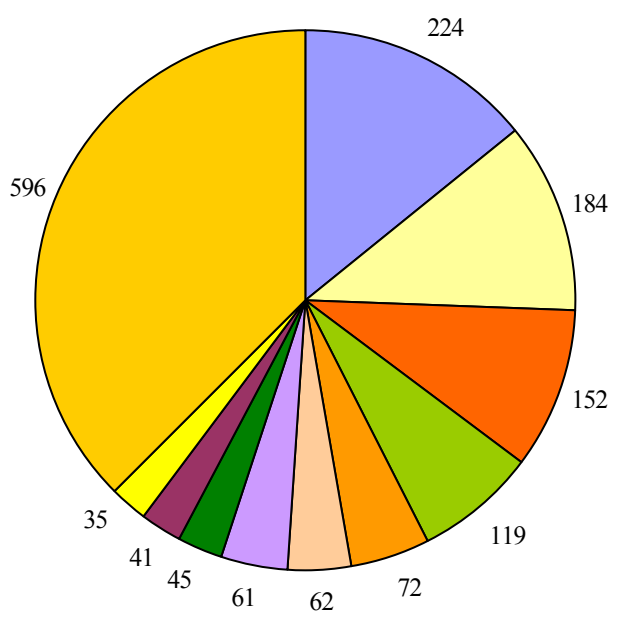

$\square 1 \operatorname{InBev}($ Belgium)

$\square 2$ SABMiller (UK)

$\square 3$ Anheuser-Busch (USA)

$\square 4$ Heineken (Netherlands)

$\square 5$ Carlsberg (Denmark)

$\square 6$ S\&N (UK)

$\square 7$ MolsonCoors (USA)

$\square 8$ Grupo Modelo (Mexico)

$\square 9$ Tsingtao (China)

$\square 10$ Kirin (Japan)

Source: Company data (Joh.Barth\&Sohn, 2005), author's research.

During the 1990 s, the CR $10^{5}$ hardly increased, going from $29 \%$ in 1992 to only $34 \%$ in 1998 . But in the course of recent years this development has picked up pace, driven by a boost in M\&A activity led by the world's largest brewing groups that enhanced the CR10 from $33.7 \%$ in 1998 to $62.5 \%$ in $2005^{6}$. Much more impressive is the growth of the CR3 and CR5. In 2005

${ }^{5}$ The concentration ratio is a way of measuring the concentration of market share held by particular suppliers in a market. It is the percentage of total market sales accounted for by a given number of leading firms. Thus, a ten-firm concentration ratio is the total market share of the five firms with the largest market shares (Bleymüller et al, 2000).

${ }^{6}$ The world's total beer production in 2005 has been estimated as these figures have not yet been published by the annual Barth Report. We have multiplied the 2004 volume figures with the CAGR over the last ten years, which was $2.4 \%$, resulting in a preliminary 2005 volume of $1,589 \mathrm{mhl}$. In the same way, we have estimated the 2005 production volume for Tsingtao, SABMiller and MCBC. 
the three largest brewers accounted for more than a third of the total beer production around the globe and the top five companies produced nearly $50 \%$ of the world beer market. Looking at the CR1, we can detect the shift between the world's number 1 position. For many years, Anheuser-Busch (AB) was the largest brewer, accounting for approximately $9 \%$ of the total market. But in 2003 AB was overtaken by InBev, which has grown extraordinarily over recent years, nowadays representing $14.1 \%$ of the world beer market. Figure 3 illustrates the development of the CR1, CR3, CR5 and CR10 over the last eight years.

Figure 3: Development of the Concentration in the World Brewing Industry, 1998-2005

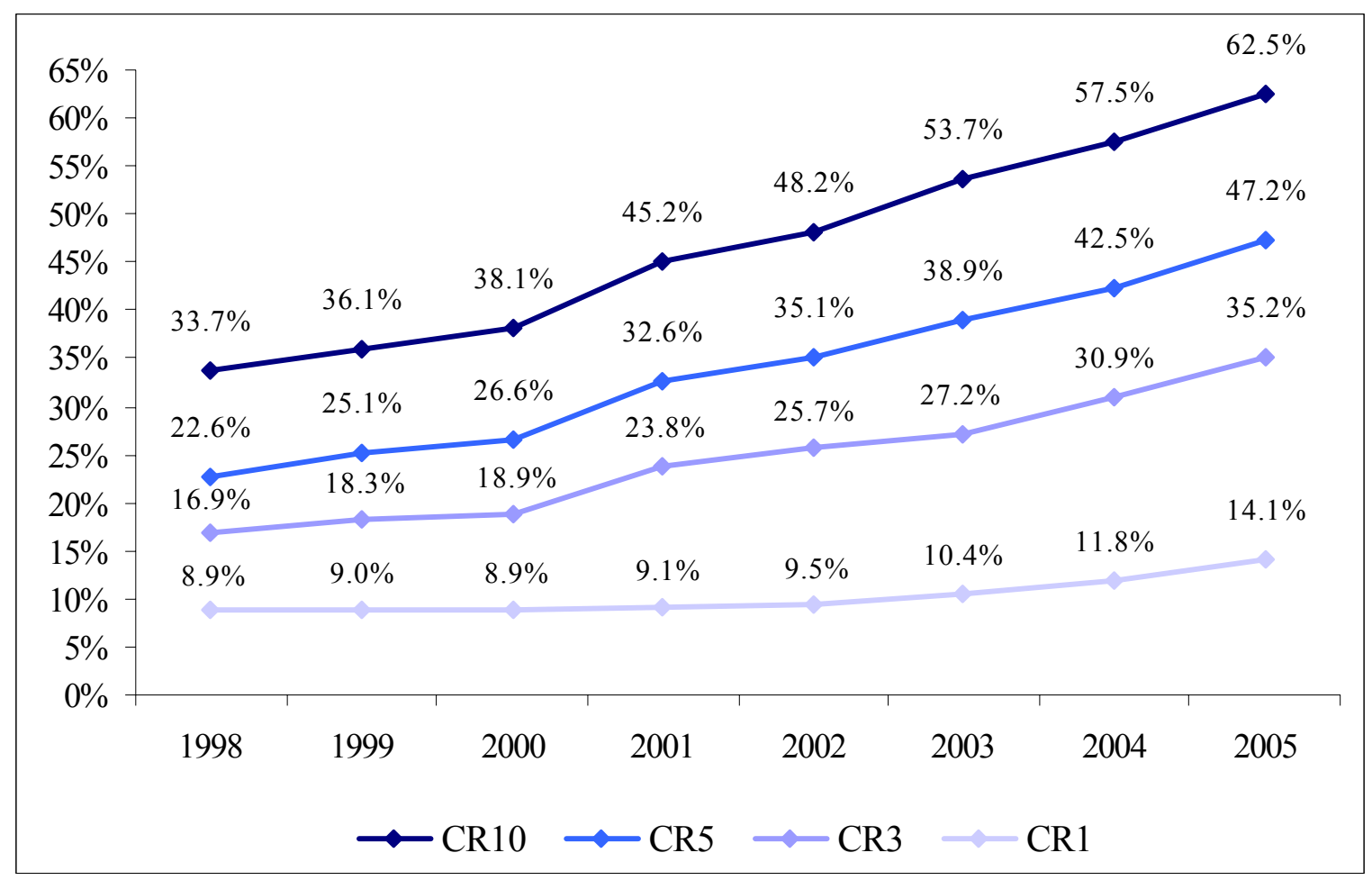

Source: Barth Reports (1999-2005), company data, author's calculations

In 2005 , for the fourth year in a row, the key trading and share price trend within the brewing industry was the out-performance of the emerging market brewers. This leads us to the thesis that the industry's profit growth is being driven by the emerging market regions. According to Bevan and Greenberg (2005) these emerging markets accounted for broadly one-third of the industry's profit pool (EBITDA) but contributed two thirds of the profit growth. Risks facing the global brewing scene vary, depending on the geographic location of the operations. In the developed markets, the main challenge is driving organic revenue growth, often against the background of a weak consumer environment and a rising demand for wine and spirits. In the 
emerging markets, the situation is generally more buoyant at present—although these regions will typically be more volatile over a period of time (Bevan and Greenberg, 2005). If we look back over the last eight years, the key theme in the global brewing sector has been the ever widening contrast in trading conditions between emerging and developed markets. We argue that these contrasting trading conditions have not only shaped the respective financial performances of the leading brewers but also influenced their strategic decision making. Specifically, the desire to gain an ever-increased exposure to these buoyant emerging markets has been reflected in the scramble to secure the remaining quality-independent assets in places as diverse as China, Colombia and Russia (Bevan and Greenberg, 2005). In our sample, SABMiller is the company with the largest exposure (ca. 75\%) to emerging markets among LBGs, followed by InBev (ca. 65\%), Heineken (ca. 39\%), Carlsberg (ca. 38\%) and Scottish\&Newcastle (ca. 21\%). Brewing groups like Anheuser-Busch (ca. 16\%), Kirin (ca. 12\%) and MCBC (ca. 5\%) are still mainly operating in their developed markets. Two brewers in our sample have their origins in developed markets, Modelo (Mexico) and Tsingtao (China) and therefore run their business primarily in their home countries. Modelo, admittedly, has a sound $30 \%$ export business segment (Corona).

International brewing groups became more and more interested in nondomestic markets when they believed that a foreign country offered locational advantages, i.e., it displayed characteristics (such as population size and growth, economic wealth and competitive structures) suggesting that the country was well suited for setting up and running profitable brewing firms. In a considerable number of cases, the internationalization pull resulting from the (absolute) locational attractiveness of a foreign market was amplified in relative terms by expected decreases in MNO domestic market attractiveness. Moreover, LBGs expand internationally since they see opportunities to exploit economies of scale and scope accruing from larger procurement volumes and to benefit from accelerated learning through bilateral transfers of technical and management best practices across the various subsidiaries. The extent to which different factors accounting for business internationalization are actually relevant for an individual LBG depend on the firm's idiosyncratic market environment and its fit with its resource base, which, in turn, results from its historical developmental path. This fit is likely to vary across LBGs. Therefore, in spite of potential bandwagon/fashion tendencies in LBG internationalization efforts, it is expected that, at any point in time, the degree of internationalization will vary substantially in a sample of LBGs (Gerpott and Jakopin, 2005). 


\section{LBG INTERNATIONALIZATION MODES}

Decades of intensive research within the field of international business have led to a huge body of theoretical approaches to explain and to describe the multinational firms in their evolution and being. In the brewing sector context, we focus not on the country-based perspective of international trade theory but rather on the firm-specific internationalization process (Andersen, 1993). The Uppsala Internationalization Model, developed by Johanson and Vahlne (Johanson and Vahlne, 1977), emphasizes explicitly not only the established national firm as the starting point of internationalization, but also the relevance of critical incidences within the process of internationalization and the process-inherent switch from export to higher forms of international activity (Oesterle, 1997).

A firm seeking to perform a business function outside its domestic market must choose the best mode of entry for the foreign market. The would-be entrant faces a large array of choices, including a wholly-owned subsidiary; a joint venture (j-v) in which the entrant could be majority, equal, or minority partner; or a non-equity arrangement, such as licensing or a contractual joint venture (Anderson and Gatignon, 1986; Frost and Han, 2002).

According to the process model of internationalization, developed by Meissner and Gerber (Meissner and Gerber, 1980), the internationalization process of a company can be described as a multi-step process in which capital and management is transferred step by step from a company's headquarter to foreign subsidiaries. Companies often start with pure export activities that do not require capital or management transfers to foreign countries. After some time they often decide to license their products, enter international joint ventures or move production overseas. In the end they may own full-scale foreign subsidiaries. Whereas in early phases (export, licensing) the relationship is very intensive with the home-country and very weak with export destinations, it is often the other way round when full-scale subsidiaries exist abroad (Swoboda, 2002). In a truly global company the recently dominant role of the headquarter has been replaced by an international network with changing strategic roles for foreign subsidiaries (Bartlett and Ghoshal, 1989).

Global beer market dynamics have driven the burst of financial ventures over recent years. In Europe, global brewers, such as InBev, Heineken and SABMiller, have sought direct entry to large beer markets to gain access to full income streams rather than royalty income streams from licensed brands (Lawler and Lee, 2003). 
To locate the focus of the present empirical study of LBG internationalization, it is helpful to systematize the generic opportunities of brewing groups to internationalize their business activities. In the following section we will point out the modes of internationalization and foreign market entry — but not company by company, as this would lead too far, faced with a sample of ten firms. Instead, we will examine in greater depth the peer group of the four largest European brewers in the sample as representatives of entire group; these four are responsible for the bulk of the internationalization process through intensive M\&A-activity in the run of the last decade ${ }^{7}$.

InBev was created in March 2004, when Interbrew announced its merger with the Latin American giant, AmBev, which transformed its scale, regional balance and medium-term growth prospects. As part of the transaction, Interbrew (now renamed InBev) issued $141.7 \mathrm{~m}$ shares to the founders of AmBev, which enabled them to establish joint-control of Stichting Interbrew, the private company which continues to hold a 53\% stake in InBev. Therefore, control of InBev is now jointly exercised between the founding Belgian families and the founders of AmBev. The merger saw Interbrew inject its Canadian operations into AmBev as part of the transaction. InBev now owns a $56 \%$ economic stake in AmBev ( $81 \%$ of votes). However, this is set to rise as InBev has indicated that it plans to acquire an additional $€ 500$ million of AmBev stock, as well as buying back $€ 300$ million of InBev stock (Bevan and Greenberg, 2005). In May 2004 Mexican brewer Femsa announced a buy-out of its 30\% stake from Interbrew. InBev nowadays runs several joint ventures (JVs), mainly in Central and Eastern Europe. Its spectacular market entry into the formerly closed German beer market through the acquisition of Beck's in August 2001 for approximately $€ 1.8$ billion was the starting shot for the consolidation of the long fragmented German market by foreign brewing groups. Just recently the Belgian brewing giant won the bidder's rivalry for the Chinese Fujian Sedrin Brewery. InBev acquired a majority of Fujian for approximately US\$750 million, according to The New York Times. InBev competed against the Dutch Heineken NV, the US brewery Anheuser-Busch and the Chinese Yanjing Brewery.

SABMiller was spectacularly created four years ago, when South African Breweries plc an-

\footnotetext{
${ }^{7}$ This peer group is represented by the four leading European brewers-InBev, SABMiller, Heineken and Carlsberg - together representing exactly a third of the total world beer market.
} 
nounced that it had entered into an agreement with Philip Morris Companies Inc. under which SAB acquired $100 \%$ of Miller Brewing Company ${ }^{8}$. Further important M\&A activity included the acquisitions of the largest Italian brewer, Peroni, in May 2003 for approximately $€ 560$ million and Lion Nathan China for approximately $€ 120$ million. Many analysts raised a glass to SABMiller's US\$7.8 billion purchase of Grupo Empresarial Bavaria ${ }^{9}$, South America's second-largest brewer, when the deal was announced on July 19'2005, because it gave SABMiller access to some of the world's fastest-growing beer markets at a time when drinkers in richer countries are ordering more wine and spirits (Anonymous, 2005). Apart from M\&A activity, SABMiller maintains license agreements with Guinness for the East African markets. In China SABMiller formed a JV with China Resources Enterprises (CRE) to hold 55\% in the Shenyang Brewery in 1994. In $2001 \mathrm{CRE}$ and SAB announced a JV to create China Resources Breweries, incorporating all the breweries in Sichuan province (Plato, 2005). Nowadays, CRB is the second-largest brewer in China. In addition, it is noteworthy that SABMiller holds one of the few strategic alliances (SAs) on the world brewing scene. In February 2001 it announced an SA with Castel Group in Africa. SAB acquired a 20\% stake in the beer division of the Castel Group and Castel acquired a 38\% stake in SAB-Africa through a share change. Through this SA, diverse new 50/50 JVs have been established for many African markets.

Heineken has numerous investments with partners around the world and remains the world's most international brewing group. Production takes place in more than 110 breweries in over 65 countries $^{10}$. In February 2001 Heineken announced its partnership with Bayerische Brau Holding, acquiring $49.9 \%$ of the newly created joint venture company Brau Holding International. This JV owns $50 \%$ of Paulaner, $63 \%$ of Kulmbacher and $50 \%$ of IRSA, the company

${ }^{8}$ In consideration, SAB had issued to Philip Morris 430 million shares. The implied enterprise value of Miller was US\$4,993 million, including net debt of US\$2,000 million. The combined entity has been called SABMiller plc.

${ }^{9}$ The transaction was effected by way of a merger and resulted in Bavaria owning an economic interest of approximately $15.1 \%$ in SABMiller. The implied equity value of Bavaria is US\$4.8 billion and, including net debt and minority interests, the total implied enterprise value for $100 \%$ of the Bavaria group is approximately US\$7.8 billion.

${ }^{10}$ In 2005 Heineken held majority ownership stakes in companies spread over 36 countries and minority ownership stakes in 29 countries around the globe. 
that holds the majority stake in Chilean brewer CCU (Plato, 2005). Heineken's biggest deal so far has been the majority takeover of Austrian-based brewing group BBAG in May 2003 for approximately $€ 1.9$ billion. Following the completion, Heineken and BBAG combined all their operations in Central Europe. Moreover, Heineken runs major export activities in the United States. In March 2005 Heineken launched its Premium Light Lager. The Americas accounted for just $8 \%$ of Heineken's total production volume but for $20 \%$ of its profits in 2004. Furthermore, Heineken took over the distribution of Femsa brands in the US from 2005. At the same time, Heineken brought off a new license agreement with Lion Nathan to distribute the Heineken brand in Australia.

Carlsberg has been developing its business, particularly in Eastern Europe and Asia. In May 2000 Carlsberg announced a merger of its brewing business with the Norwegian consumer goods processor Orkla. All brewing operations were pooled into a new company, Carlsberg Breweries, to be owned $60 \%$ by Carlsberg A/S and $40 \%$ by Orkla. In February 2004, Carlsberg announced the acquisition of Orkla's 40\% stake for €7,054 million. In January 2004 Carlsberg acquired German-based brewer Holsten for approximately €440 million. Since 1997 the Carlsberg brand has been brewed mainly under license by Brahma Brazil. The Baltic Beverage Holding (BBH), a 50/50 joint venture with Scottish\&Newcastle, represents Carlsberg's biggest investment and is the most successful and largest joint venture in the world brewing scene. BBH in turn became Baltika's main shareholder in the early 1990s. It currently holds $75 \%$ of the ordinary and $14 \%$ of the preferred shares. Baltika is the leading player on the Russian beer market with a domestic market share of 25\% (Bevan and Greenberg, 2005). 


\section{Procedures ANd Methodology}

Commonly, there exist two avenues of research that examined the relationship between firm's degree of internationalization and its performance. The first, classified as comparative studies, compares the profitability and risk levels of multinational corporations to that of domestic corporations. The second group of studies uses control variables (research and development intensity, firm size, industry effect and so on) to examine the relationship of internationalization to performance. The significant findings in the above-mentioned studies were the existence of some kind of 'internationalization threshold' for multinational enterprises. Geographic diversification would lead to enhanced corporate performance up to a point beyond which the organizational costs and complexity associated with managing widely scattered operations would outweigh the advantage (Gomes and Ramaswamy, 1999; Hsu and Boggs, 2003). Over a research period of the last eight financial years (1998-2005) various facets of LBG business activities outside their domestic markets are highlighted by using two quantitative indicators: (a) the Foreign Sales to Total Sales Index, and (b) the Network Spread Index (Sullivan, 1994a; Ietto-Gillies, 1998).

The second research question, on associations between internationalization degrees and LBG profitability, entails the measurement of two categories of additional variables: (a) performance variables and (b) control variables. Financial performance is represented by four accounting-based and widely recommended measures: (1) the ratio of earnings before interest, taxes, depreciation and amortization (EBITDA-Margin), (2) the Operating Profit Margin, (3) the Return on Net Assets, (4) the Cash Realization Ratio, and, (5) the Net Debt Ratio. Control variables include the year of observation within the study period, LBG size, domestic market share, the assessment of the industry effect and several other variables.

To shed light on potential relationships between internationalization and financial performance several regressions and correlations were run for the control variables and the various internationalization-performance measure combinations (Gerpott and Jakopin, 2005).

\subsection{MEASURING THE DEGREE OF INTERNATIONALIZATION}

Internationalization describes the process by which an economy, an industry or a company becomes increasingly integrated into international economic activities. The degree of internationalization is the measure of the degree to which this economic integration has already taken place. When determining a company's degree of internationalization, activities on foreign 
markets are compared to home-market or world-wide activities (Germann et al, 1996). The degree of internationalization determines to what extent a company is confronted with problems of managing international activities and to what extent international environments are relevant for a specific company (Schmidt, 1981). Measuring the degree of internationalization, therefore, is of high managerial relevance. Most approaches to measuring the degree of internationalization rely on a single indicator, multidimensional approaches on two or more indicators representing different dimensions of the internationalization phenomenon. The result of combining two or more internationalization indicators by, for instance, calculating a mean value is often called an internationalization index (Glaum, 1996). The most common ways of measuring the degree of internationalization are the ratio of foreign to total sales, the share of foreign assets to total assets (Reeb et al, 1998), the ratio of foreign pre-tax income to total pretax income (Chen et al, 1997), the number of countries with foreign operations and the shares of foreign employees, profits, value added or shareholders (Fisch and Oesterle, 2003). All the above measures tend to capture the 'depth of internationalization'. There also has been some research that investigates the 'scope/breadth of internationalization' by examining the geographical dispersion of operations across countries. Multinational enterprises tend to leverage location-based advantages to enhance their performance (Kogut, 1985; Hsu and Boggs, 2003). Other scholars measured configuration as the number of overseas plants and found a significant positive relationship to performance. Therefore, the second internationalization index, adopted in the current study covers the international dispersion of brewers' subsidiaries. The so called Network Spread Index (NSI) is calculated by the number of countries in which an enterprise maintains subsidiaries, divided by the total number of countries that received direct investments in $2004^{11}$ (Ietto-Gillies, 1998). For this reason, the following internationalization measures are applied in this paper:

\footnotetext{
${ }^{11}$ Indeed, the number of countries which received FDI in 2004 was 191 (UNCTAD, 2004). But according to the brewing industry purposes we decided to adjust the Network Spread Index in this examination, as not all these 191 countries play a significant role in the brewing scene context. We took 167 countries as $n^{*}$. These 167 countries, that show a production volume of at least 4,000 hl (Cayman Islands), are listed in the 2005 Barth Report (Joh.Barth\&Sohn, 2005).
} 
(1) Foreign Sales Index (FSI): Ratio of foreign sales (exports and subsidiaries) to total sales.

(2) Network Spread Index (NSI) with $\mathrm{n}^{*}=167:$ NSI $=\frac{n}{n^{*}}=\frac{n}{167}$.

In the brewing setting, selecting the operationalization technique for the internationalization variable is largely restricted by the non-availability of data. As noted above, reliable and complete internationalization data for the five-year period under study was obtainable only in FSI and NSI form; measures like the share of foreign assets to total assets or the shares of foreign employees could not been gathered as hardly any brewer discloses these information in its company publications. We therefore had to depend on the financial dimension of the degree of internationalization. This has its merits. As mentioned above, next to structural internationalization it captures a core dimension of the degree of firms' foreign activity. Equally important, comparing foreign sales to total sales (FSTS) is the DOI measure most used in previous inquiries, facilitating valid cross-study comparison of findings and therefore sustained research progress (Ruigrok and Wagner, 2003).

\subsection{Measuring Financial Performance - Balance Sheet analysis}

In most previous empirical studies, authors used traditional financial ratios, such as return on net assets (RONA), return on equity (ROE), or return on sales (ROS), as dependent variables. Properly interpreted, these ratios provide keen insight into the sources and adequacy of profits, the efficiency of assets committed to the firm, solvency risk, and liquidity risk. Individually they tell only a little about the whole but, taken together, the entire picture of financial health comes into focus (Hsu and Boggs, 2003).

To evaluate the firms' performance, we choose accounting profit, as it is a useful basis of incentives for agents who decide about capital investments in the firm; among such agents are the managers of investment centers, but also top management, which decides about the investment policy of the whole firm. In the context of corporate governance a measure of performance has several related functions. It serves to create incentives for managers, directly by compensation depending on performance, and indirectly by the influence of performance upon reputation and professional career. Another important effect of publishing information on performance is market reaction, which may result in sanctions for negligent managers: a 
drop in market value may become a signal to replace management; moreover, it can make the firm attractive for a hostile takeover (Hax, 2003).

Relying on net earnings alone to gauge profitability does not always provide a clear picture of a company, and using such income figures as the sole measure of profitability can have big repercussions. Profit-margin ratios, on the other hand, can give investors deeper insight into management efficiency. Margins, quite simply, are earnings expressed as a ratio - for instance a percentage of sales. A percentage allows investors to compare the profitability of different companies, while net earnings - an absolute number - cannot.

Margin analysis is a useful way to understand the profitability of companies. It tells how effectively management can wring profits from sales, and how much room a company has to withstand a downturn, fend off competition and make mistakes. But, like all ratios, margin ratios never offer perfect information. They are only as good as the timeliness and accuracy of the financial data that gets fed into them, and analyzing them also depends on a consideration of the company's industry and its position in the business cycle (McClure, 2004). For our sample under investigation we chose the following ratios:

\section{EBITDA-Margin}

The EBITDA is calculated by taking operating income and adding back to it interest, depreciation and amortization expenses. EBITDA is used to analyze a company's operating profitability before non-operating expenses (such as interest and 'other' non-core expenses) and non-cash charges (depreciation and amortization). The EBITDA can be used to analyze the profitability between companies and industries (Born, 2001). Because it eliminates the effects of financing and accounting decisions, EBITDA can provide a relatively good 'apples-toapples' comparison.

$$
\text { EBITDA-Margin }=\frac{\text { EBITDA }}{\text { Net Sales }}
$$

For example, EBITDA as a percent of sales (the higher the ratio, the higher the profitability) can be used to find companies that are the most efficient operators in an industry. The ratio can also be used to evaluate different industry trends over time (Wayman, 2002). EBITDA-Margin measures the extent to which cash operating expenses use up revenue. 


\section{Operating Profit Margin}

The most important goal of a company is to make money and keep it, which depends on liquidity and efficiency. Because these characteristics determine a company's ability to pay investors a dividend, profitability is reflected in share price. As such, investors should know how to analyze various facets of profitability including how efficiently a company uses its resources and how much income it generates from operations. Calculating a company's profit $\operatorname{margin}^{12}$ is a great way to gain insight into these and other aspects of how well a company generates and retains money.

By comparing earnings before interest and taxes (EBIT) to sales, operating profit margin shows how successful a company's management has been in generating income from the operation of the business. Operating Profit Margin is the ratio of earnings (before interest and taxes) to sales:

$$
\text { Operating Profit Margin }=\frac{\text { EBIT }}{\text { Net Sales }} \text {. }
$$

This ratio is a rough measure of the operating leverage a company can achieve in the conduct of the operational part of its business. It indicates how much EBIT is generated per Euro of sales. High operating profits can mean the company has effective control of costs, or that sales are increasing faster than operating costs (Born, 2001). Operating profit also gives investors an opportunity to do profit-margin comparisons between companies that do not issue a separate disclosure of their cost of goods sold figures which are needed to do gross margin analysis (McClure, 2004). Operating Profit or EBIT-Margin is a measure of profitability on sales, most useful when compared against other firms in the same industry. It is also meaningful when evaluated over time to show where corporate profitability is headed.

\section{Return on Net Assets}

Return on Net Assets (RONA) is a bottom-line measurement showing performance relative to strategic goals and objectives. More and more successful companies are utilizing RONA measurement. It provides an apples-to-apples comparison of performance that is understood

\footnotetext{
12 'Profit margin', 'Operating Profit Margin', 'Return on Sales' (ROS) and 'EBIT-Margin' are used synonymously.
} 
by non-financial professionals. It is calculated as follows:

$$
\text { RONA }=(\text { EBITA }+ \text { Income from Associates }) * \frac{1-\text { Corporate Tax Rate }}{\text { Average Total Net Liabilities }} .
$$

RONA expresses the higher the return, the better the profit performance for the company (Ritchie, 2003; McClure, 2005). The Return on Net Assets, which, offering a different take on management's effectiveness, reveals how much profit a company earns for every Euro of its assets. Assets include things like cash in the bank, accounts receivable, property, plant \& equipment, land, inventory and furniture. In previous studies, scholars have relied upon Return on Equity (ROE) rather than on RONA. Indeed, both measure a kind of return, and, at first glance, gauge a company's ability to generate earnings from its investments. But they do not exactly represent the same thing. A closer look at these two ratios reveals some key differences (McClure, 2005). The big factor that separates ROE and RONA is financial leverage, or debt. The balance sheet's fundamental equation shows how this is true: assets $=$ liabilities + shareholders' equity. This equation tells that if a company carried no debt, its shareholders' equity and its total assets would be the same.

\section{Cash Realization Ratio}

The Cash Realization Ratio (CRR) is defined as the cash flow from operating activities divided by net income. A positive high value is consistent with conservative depreciation policies, high reinvestment and effective working capital management (Blythman et al, 2003). The cash generated from the operations of a company is generally defined as revenues less all operating expenses, but calculated through a series of adjustments to net income. The operating cash flow (OCF) can be found on the statement of cash flows. It is also known as 'cash flow provided by operations' or 'cash flow from operating activities'.

$$
C R R=\frac{\text { Operating Cash Flow }}{\text { Net Sales }}
$$

While EBITDA is sometimes called 'cash flow', it is really earnings before the effects of financing and capital investment decisions. It does not capture the changes in working capital (inventories, receivables, etc.). Operating cash flow is the lifeblood of a company and the most important barometer that investors have. OCF is a better metric of a company's financial health than net income. First, cash flow is harder to manipulate under GAAP and IFRS than 
net income. Second, 'cash is king' and a company that does not generate cash over the long term is on its deathbed due to a lack of liquidity (Wayman, 2003).

\section{Net Debt Ratio}

The net debt ratio (NDR) is a measure of a company's financial leverage calculated by dividing long-term debt by shareholder's equity. It indicates what proportion of equity and debt the company is using to finance its assets. A high debt/equity ratio generally means a company has been aggressive in financing its growth with debt. This can result in volatile earnings as a result of the additional interest expense. If a lot of debt is used to finance increased operations (high debt to equity), the company could potentially generate more earnings than it would have without this outside financing. However, the cost of this debt financing may outweigh the return that the company generates on the debt through investment and business activities and become too much for the company to handle, which may result in bankruptcy and leave shareholders with nothing, so it is a delicate balance. This is what the leverage effect is about and what the debt/equity ratio measures. The debt/equity ratio will also be dependent on the industry the company operates $\mathrm{in}^{13}$.

$$
N D R=\frac{\text { Net Debts }}{\text { Total Shareholder's Equity }}
$$

Net debt to equity shows net debt as a percentage of equity capital. It is the most commonly used measure of financial leverage and it provides an indicator of both financial risk and capital efficiency (Winston et al, 2005). In this ratio, debt is defined as all interest-bearing liabilities, finance leases, and all non-equity capital instruments or equity-linked instruments (including those issued by subsidiaries). Net debt is simply debt less all cash and cash equivalents. The equity component is made up of total shareholder's equity.

\subsection{CONTROL VARIABLES}

According to Gerpott and Jakopin (2005) and based on a review of relevant literature on potential factors that should be neutralized before asking whether there are true internationalization-performance associations for LBG corporate groups, we controlled for six major control

\footnotetext{
${ }^{13}$ For example, capital-intensive industries such as auto manufacturing tend to have a debt-to-equity ratio above 2 , while personal computer companies have a debt to equity of under 0.5 .
} 
factors, carrying out regression analysis. They were composed and calculated as follows:

(1) LBG size: Firm size, a common variable related to firm performance, was used to control for economies and diseconomies of scale at the corporate level. According to the rationale behind the 'firm size effect' as firm size increases, it becomes more difficult to sustain impressive performance. Thus, when a firm is large, financial performance is expected to be lower. In this study, firm size was measured by the natural logarithm of net sales for all models. Natural logarithm transformation is usually applied to the variables that are not taken as ratios. Log transformation will not only make the results easy to interpret because the changes in the logarithm domain represent relative (percentage) changes in the original metric, but it also makes the distribution of the data closer to a normal distribution. (Banz, 1981; Hsu and Boggs, 2003). The average net sales of the ten brewing groups included in the sample over the period from 1998 to 2005 was $€ 7,130 \mathrm{mn}$ (s.d. $=€ 3,866 \mathrm{mn}$, $\mathrm{n}=80)$.

(2) Year of observation: This variable was included as a 'black-box parameter' to ensure that internationalization-performance associations were distorted as little as possible by general time-related environmental changes (e.g. increasing competitive intensity, market saturation) (Gerpott and Jakopin, 2005).

(3) Domestic market share: The market share in the country of origin was measured by the ratio of a LBG's sales in its home country. The average domestic market share of the ten brewing groups in the sample over the study period was $45.8 \%$ (s.d. $=24.4 \%, \mathrm{n}=80$ ).

(4) Leverage: This ratio stands for the level of net debts to total assets and measures the capital structure of the LBG (Majocchi and Zucchella, 2003). In previous work leverage was found to be negatively associated with performance measures of firms operating internationally (Mayer and Whittington, 2003; Gerpott and Jakopin, 2005).

(5) Industry effect: Prior research has showed that industry effects have important impacts on cross-sectional variation of firm performance. Some studies have measured the industry effect by a set of industry dummies. However, many studies on diversification and in the strategy field have associated performance effects with relative industry growth rates; hence, an industry variable, measured as the compound annual industry growth rate over the period of 1998 to 2005 is included (Hsu and Boggs, 2003). Hereby, we equate the increase in the world beer markets' production volume with the industry growth (CAGR 1998 to $2005=2.59 \%$ ). 
(6) Speed of international expansion: This rate was computed by dividing a LBG group's FSTS share in a given year by its FSTS share in the preceding year for each of the six observation periods from 1998 to 2005. The overall sample average of the speed indicator amounted to $31.1 \%$ (s.d. $=92.8 \%, \mathrm{n}=70$ ). Early studies present evidence that a high speed of foreign business expansion coincides with lower corporate performance (Gerpott and Jakopin, 2005).

(7) Concentration ratio: Generally, the more highly concentrated a market, the more profitable it is. Merrill Lynch equity research deducted a strongly significant correlation between the Hirschmann-Herfindahl-Index (HHI) and the EBITA margins in single brewing country markets (Winston et al, 2004). The HHI is a specific measurement of market concentration, that is, of the extent to which a small number of firms account for a large proportion of output (Buntinas and Funk, 2005). In our investigation we use the five-firm concentration ratio (CR5) as well as the ten-firm concentration ratio (CR10) to measure the concentration in the total world brewing industry. The average CR5 in the brewing industry between 1998 and 2005 was 33.8\% (s.d. = 8.2\%) and enhanced remarkably from $22.6 \%$ in 1998 to $47.2 \%$ in 2005 . The average CR10 in the brewing industry between 1998 and 2005 has been $46.9 \%$ (s.d. $=9.9 \%$ ) and enhanced remarkably from $33.7 \%$ in 1998 to $62.5 \%$ in 2005 .

\section{Results}

\subsection{Progress In LBGs’’ Degrees OF INTERNATIONALIZATION}

The first research question concerns changes in the extent of internationalization of worldwide leading brewing groups since the end-1990s. To shed light on this issue, the LBGs' Foreign Sales Index and Network Spread Index were traced starting in the year 1998 (see table 2 after the following page). FSTS and FDI data have not been published in brewers' annual reports but were carefully calculated analyzing company publications.

The FSI grew at a compound annual growth rate of $7.1 \%$ from $39.7 \%$ in 1998 to $64.3 \%$ in 2005. Further, we can detect a grand spreading among the different brewing groups. Whereas the European brewers already have been quite internationalized in 1998, companies from the 
rest of the world hardly have been internationally involved. Within the full sample dispersions of FSI shares became imperceptibly higher in the study period. Indeed, firms from other parts of the world could catch up in the period from 1998 to 2005, but the European brewers could enhance their foreign investments over again disproportionate. Having a closer look at the different brewers' FSI reveals an extraordinary jump for Scottish\&Newcastle in 2000 (acquisition of Kronenbourg), SABMiller in 2001 (merger of SAB with US-brewer Miller) and former Coors Brewing Company in 2002 (acquisition of Carling breweries business in UK). Caused to these first large-scaled M\&A activities undertaken by several brewers, the FSI shares standard deviation amounted to $40.4 \%$ in 1998 and had a value of $33.6 \%$ in 2005 . This means that the degrees of internationalization among leading brewing groups became slightly less inhomogeneous during the 8-year-period, proving different paths as well as an increased speed of international investment. Nevertheless, in 2005, there were still strong differences in the DOI in the sample with FSI and NSI figures ranging from $97.2 \%$ for InBev to $4.9 \%$ for Chinese Tsingtao.

The more impressive DOI figure represents the NSI. The NSI grew at a CAGR of $7.2 \%$ from $9.3 \%$ (s.d. $=9.4 \%$ ) in 1998 to $15.1 \%$ in 2005 (s.d. $=15.2 \%$ ). The high standard deviations prove the wide dispersion of FDI activities among leading brewing groups. In opposite to the FSI figures, the NSI's standard deviations became wider within the 8-year study period. That proves that some of the most internationalized LBGs have created a dense network of global subsidiaries whereas other brewers rely on less sophisticated forms of market entry and business internationalization such as licence agreements or pure exporting. The extent to which brewing groups have been developing a broad net of affiliated companies and subsidiaries ranges between a sluggish NSI of $0.6 \%$ (Modelo and Tsingtao) and a remarkable NSI share of $44.9 \%$ for InBev. These $44.9 \%$ NSI for InBev, the largest brewer world-wide in terms of production volume, means that the Belgium/Brazilian brewing giant has subsidiaries and participations in 75 countries, spread over all five continents. Conceptually, large deviations in the degrees of internationalization among the ten LBGs may be taken to indicate that the corporations do not experience identical pressures to expand their business abroad but also try to link the amount of FDI to their resources and to market opportunities (Gerpott and Jakopin, 2005). The column to the extreme right in table 2 on the following page reveals that in 2005 the total number of foreign majority- and minority-owned subsidiaries varied between 75 (InBev) and 2 (Modelo, Tsingtao) with an average of $15.8 \%$ FDI. At the financial year-end 2005, the only 
LBGs with truly global business activities were InBev (75 ownerships) and Heineken (65), followed by a second group, which consists of the other European brewers Carlsberg (31), SABMiller (23) and Scottish\&Newcastle (22). Apart from Kirin that still has mentionable FDI activities in 14 different countries the other brewers in the sample do not have relevant FDI that go beyond more than one other continent than that of their origin. In average these remaining brewing groups (A-B, MCBC, Modelo and Tsingtao) hold 3.8 subsidiaries abroad in 2005. Dispersion of majority (average $=13.9$ ) and minority (average $=12.4$ ) ownership positions for the ten brewers are all about the same.

Table 2: Development of the FSI and the NSI for the 10 Brewing Groups from 1998-2005.

\begin{tabular}{|c|c|c|c|c|c|c|c|c|c|c|c|c|c|c|c|c|c|c|}
\hline \multirow[b]{3}{*}{ Company } & \multicolumn{2}{|c|}{1998} & \multicolumn{2}{|c|}{1999} & \multicolumn{2}{|c|}{2000} & \multicolumn{2}{|c|}{2001} & \multicolumn{2}{|c|}{2002} & \multicolumn{2}{|c|}{2003} & \multicolumn{2}{|c|}{2004} & \multicolumn{2}{|c|}{$2005 * * *$} & \multirow{2}{*}{\multicolumn{2}{|c|}{$\begin{array}{c}2005 \\
\text { countries }\end{array}$}} \\
\hline & FSI & NSI * & FSI & NSI & FSI & NSI & FSI & NSI & FSI & NSI & FSI & NSI & FSI & NSI & FSI & NSI & & \\
\hline & $(\%)$ & $(\%)$ & $(\%)$ & $(\%)$ & $(\%)$ & $(\%)$ & $(\%)$ & $(\%)$ & $(\%)$ & $(\%)$ & $(\%)$ & $(\%)$ & $(\%)$ & $(\%)$ & $(\%)$ & $(\%)$ & MA & MI \\
\hline InBev & 86.3 & 21.2 & 89.1 & 23.4 & 91.2 & 25.7 & 92.6 & 28.1 & 93.4 & 31.7 & 96.9 & 40.1 & 97.0 & 41.9 & 97.2 & 44.9 & 39 & 36 \\
\hline SABMiller & 56.8 & 8.2 & 57.4 & 8.4 & 61.5 & 9.0 & 81.6 & 9.6 & 82.7 & 12.0 & 83.2 & 12.6 & 85.7 & 13.8 & 86.1 & 13.8 & 10 & 13 \\
\hline Anheuser-Busch & 4.9 & 1.2 & 5.2 & 1.2 & 5.0 & 1.2 & 5.1 & 1.2 & 5.1 & 1.8 & 12.9 & 2.4 & 13.9 & 3.0 & 17.1 & 3.6 & 2 & 4 \\
\hline Heineken & 89.4 & 25.9 & 90.0 & 27.5 & 91.0 & 30.5 & 92.2 & 36.5 & 94.0 & 38.3 & 94.5 & 39.5 & 94.9 & 35.9 & 95.1 & 38.9 & 36 & 29 \\
\hline Carlsberg & 89.0 & 14.3 & 89.2 & 15.0 & 91.7 & 15.6 & 92.3 & 15.6 & 92.9 & 16.2 & 94.1 & 17.4 & 94.7 & 18.0 & 95.3 & 18.6 & 17 & 14 \\
\hline Scottish\&Newcastle & 6.7 & 5.0 & 6.8 & 5.4 & 48.0 & 6.0 & 63.6 & 7.2 & 65.7 & 7.8 & 66.7 & 9.0 & 68.1 & 9.6 & 73.1 & 9.6 & 13 & 9 \\
\hline $\mathrm{MCBC}$ & 2.5 & 1.2 & 2.6 & 1.2 & 3.4 & 1.2 & 17.4 & 1.2 & 54.1 & 1.8 & 57.5 & 1.8 & 59.0 & 3.0 & 59.8 & 3.0 & 3 & 2 \\
\hline Modelo & 22.8 & 0.6 & 24.0 & 0.6 & 22.6 & 0.6 & 23.3 & 0.6 & 24.9 & 0.6 & 26.7 & 0.6 & 28.4 & 0.6 & 30.3 & 0.6 & 1 & 1 \\
\hline Tsingtao $* *$ & 0.0 & 0.6 & 0.0 & 0.6 & 0.0 & 0.6 & 0.0 & 0.6 & 0.0 & 0.6 & 0.0 & 0.6 & 3.8 & 0.6 & 4.9 & 0.6 & 1 & 1 \\
\hline Kirin & 9.1 & 5.7 & 9.5 & 6.0 & 14.0 & 6.6 & 13.9 & 7.2 & 15.7 & 7.2 & 17.1 & 7.8 & 17.5 & 8.4 & 17.9 & 8.4 & 4 & 10 \\
\hline Max & 89.4 & 25.9 & 90.0 & 27.5 & 91.7 & 30.5 & 92.6 & 36.5 & 94.0 & 38.3 & 96.9 & 40.1 & 97.0 & 41.9 & 97.2 & 44.9 & 39 & 36 \\
\hline Average & 39.7 & 9.3 & 40.2 & 9.9 & 47.3 & 10.7 & 53.4 & 12.0 & 59.6 & 13.0 & 61.6 & 14.2 & 62.8 & 14.4 & 64.3 & 15.1 & 13.9 & 12.4 \\
\hline Median & 16.0 & 5.4 & 16.8 & 5.7 & 35.3 & 6.3 & 43.5 & 7.2 & 59.9 & 7.5 & 62.1 & 8.4 & 63.5 & 9.0 & 66.5 & 9.0 & 7.0 & 9.5 \\
\hline S.d. & 40.4 & 9.4 & 40.7 & 10.1 & 38.7 & 11.1 & 38.0 & 12.9 & 35.1 & 13.7 & 34.4 & 14.9 & 34.0 & 14.0 & 33.6 & 15.2 & 11.5 & 9.5 \\
\hline Min & 0.0 & 0.6 & 0.0 & 0.6 & 0.0 & 0.6 & 0.0 & 0.6 & 0.0 & 0.6 & 0.0 & 0.6 & 3.8 & 0.6 & 4.9 & 0.6 & 1 & 1 \\
\hline * $\quad \mathrm{FSI}=$ Foreign $\mathrm{Sa}$ & dex & ICI - & res & read & & & & & & & & & & & & & & \\
\hline ** 1998,1999 and 2 & 5 figure & s estimat & $\mathrm{d}$ as no & annual & orts ar & publis & & & & & & & & & & & & \\
\hline *** 2005 figures estir & ed for & ssingtao, & $\mathrm{ABMi}$ & ler and & pdelo a & these & npanies & have $\mathrm{n}$ & ret pub & ished $t$ & annua & repor & til end & -Marcl & & & & \\
\hline$* * * *$ Number of count & in whi & ch an LB & held $\mathrm{r}$ & hajority & $\mathrm{IA}>5 \mathrm{C}$ & $\%)$ and & inority & $(\mathrm{MI}<$ & owne & rship & in $\mathrm{cl}$ & rently & ve brev & $\operatorname{ving} \mathrm{c}$ & anies at & 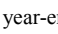 & 2005. & \\
\hline
\end{tabular}

Source: Company data, annual reports, authors' calculations.

In fact facing the early boost in European brewers' international activities one can argue that these companies all are located in small countries with a comparatively small home market for beer. Since the possible degree of specialization is mainly determined by the size of the market (Stigler, 1951), companies from small countries are forced to internationalize their activities by acquiring or founding local subsidiaries in order to become cost-efficient 
(Kutschker and Schmid, 2004). In this respect, brewers from larger countries (i.e. A-B or the Japanese groups) which offer more opportunity for economies of scale and specialization experience lower pressures to internationalize their activities and have systematic disadvantages with respect to the measurement of degrees of internationalization. Thus, the small-country argument is a convincing explanation for the high FSI of European LBGs. Nonetheless, also brewers from other regions sought to increase their international commitment in the course of the last years, facing low volume prospects in their saturated home markets. Because of the low level of FDI for newly internationalizing firms (e.g. MCBC, S\&N), the pace of foreign investment growth, when measured on a percentage level, can be quite rapid (Delios, 2000).

\subsection{Progress in LBGs Growth and Financial Performance}

This chapter analyses the development of the world's largest brewing groups' accounting figures over the eight financial years from 1998 to $2005^{14}$. The second research question on relationships between multinationality and financial performance before controlling for potential confounding impacts of other factors surrounding a brewing groups' business expansion abroad entails the measurement of different categories of profitability variables. As stated above, we measure both, so called business performance measures such as EBITDA-Margin and ROS as well as quality earnings measures like CRR and RONA. As most performance indicators include the operating profit $(=$ EBIT) in their denominators, we confine this chapter to analyze the development of LBG's net sales, EBITDA and operating profit over the research period of eight financial years between 1998 and 2005. This descriptive empirical analysis also entails the comparison of our sample's development in the respective ratios: the EBITDA-Margin as well as the Operating Profit-margin. It has to be mentioned, that all our financial analyses are based on a conversion into $€$ as the European brewers operate internationally and report in $€$. Currency fluctuations, especially relating to the US dollar, materially affect overall company results. Heineken e.g. has a clear policy on hedging transactional ex-

\footnotetext{
${ }^{14}$ It has to be mentioned that three LBGs have not yet published (SABMiller's financial year end is March 31) their annual results for the financial year 2005 until this investigation has been undertaken. Therefore, we based our 2005 figures for the descriptive part of our investigation on broker forecasts of DrKW, ING and Merrill Lynch (Chen and Luo, 2005; Holland et al, 2005; Urrutia and Aramoni, 2005). For the internationalization-performance correlation we did not take these three results into account.
} 
change risks, which postpones the impact on financial results. Hedging transactions to limit exchange risks are entered into only in respect of actual amounts receivable and payable and highly probable future cash flows in foreign currencies. The instruments used are forward contracts and options (Heineken, 2005). Indeed, companies that run their business more domestically - like Anheuser Busch - have been affected much more by the fluctuations in the $€ /$ US\$ exchange ratio. But to make our results comparable it is inevitable to report all results in one common currency.

Cumulated net sales of our whole sample grew at a compound annual growth rate of $6.9 \%$ and a total growth rate of $59.8 \%$ from $€ 44$.6bn in 1998 to $€ 71.3 \mathrm{bn}$ in 2005 . The average net sales in 1998 has been $€ 4,462 \mathrm{mn}$ (s.d. $=€ 3,555 \mathrm{mn}$ ) and increased remarkably to $€ 7,130 \mathrm{mn}$ in 2005 (s.d. $=€ 3,866 \mathrm{mn}$ ). The fact that the standard deviation grew less as the average net sales indicates that the dispersion among leading brewers decreased over recent years. The predominant position of Anheuser-Busch has changed into a peer group of equals (A-B, InBev, Heineken, SABMiller). Glancing at table 3 in more detail reveals some large differences regarding brewing group's growth speed. The world's largest brewer in terms of production volume, InBev, grew at a CAGR of $18.3 \%$ and could more than triple its net sales within eight years from $€ 3.6 \mathrm{bn}$ in 1998 to $€ 11.7 \mathrm{bn}$ in 2005 . This rapid growth was mainly driven by former Interbrew's aggressive external growth strategy, spending more than $€ 25 \mathrm{bn}$ in M\&As in the course of the last years. Apart from InBev, there is a second group that shows nearly equal doubledigit growth rates of around $10 \%$ each year and that is SABMiller, Heineken, MCBC and Modelo. 
Table 3: Development in Leading Brewing Groups’ Net Sales 1998-2005.

\begin{tabular}{|c|c|c|c|c|c|c|c|c|c|c|}
\hline Company & $\begin{array}{r}1998 \\
(€ \mathrm{mn}) \\
\end{array}$ & $\begin{array}{r}1999 \\
(€ \mathrm{mn}) \\
\end{array}$ & $\begin{array}{r}2000 \\
(€ \mathrm{mn}) \\
\end{array}$ & $\begin{array}{r}2001 \\
(€ \mathrm{mn}) \\
\end{array}$ & $\begin{array}{r}2002 \\
(€ \mathrm{mn}) \\
\end{array}$ & $\begin{array}{r}2003 \\
(€ \mathrm{mn}) \\
\end{array}$ & $\begin{array}{r}2004 \\
(€ \mathrm{mn}) \\
\end{array}$ & $\begin{array}{r}2005 \\
(€ \mathrm{mn}) \\
\end{array}$ & $\begin{array}{r}\text { Total } \\
\text { Growth } \\
\end{array}$ & CAGR \\
\hline InBev & 3,600 & 4,500 & 5,657 & 7,303 & 6,992 & 7,044 & 8,568 & 11,656 & $223.8 \%$ & $18.3 \%$ \\
\hline SABMiller & 4,688 & 3,798 & 3,234 & 3,425 & 6,735 & 7,945 & 8,422 & 9,196 & $96.2 \%$ & $10.1 \%$ \\
\hline Anheuser-Busch & 13,170 & 12,665 & 13,514 & 14,400 & 14,345 & 12,499 & 12,006 & 12,073 & $-8.3 \%$ & $-1.2 \%$ \\
\hline Heineken & 5,453 & 6,164 & 7,014 & 8,107 & 9,011 & 9,255 & 10,005 & 10,796 & $98.0 \%$ & $10.2 \%$ \\
\hline Carlsberg & 2,968 & 3,246 & 3,439 & 4,616 & 4,781 & 4,650 & 4,835 & 5,099 & $71.8 \%$ & $8.0 \%$ \\
\hline Scottish\&Newcastle & 3,756 & 3,711 & 4,553 & 5,696 & 5,356 & 5,320 & 4,719 & 4,765 & $26.8 \%$ & $3.5 \%$ \\
\hline MolsonCoors & 2,225 & 2,190 & 2,611 & 2,710 & 3,993 & 3,534 & 3,462 & 4,422 & $98.8 \%$ & $10.3 \%$ \\
\hline Modelo & 1,815 & 2,410 & 3,498 & 3,836 & 3,948 & 3,306 & 3,186 & 3,564 & $96.4 \%$ & $10.1 \%$ \\
\hline Tsingtao & 356 & 416 & 494 & 632 & 790 & 716 & 747 & 875 & $145.7 \%$ & $13.7 \%$ \\
\hline Kirin & 6,594 & 9,009 & 10,657 & 10,105 & 9,625 & 8,892 & 9,104 & 8,855 & $34.3 \%$ & $4.3 \%$ \\
\hline Max & 13,170 & 12,665 & 13,514 & 14,400 & 14,345 & 12,499 & 12,006 & 12,073 & & \\
\hline Average & 4,462 & 4,811 & 5,467 & 6,083 & 6,558 & 6,316 & 6,505 & 7,130 & & \\
\hline Median & 3,678 & 3,755 & 4,025 & 5,156 & 6,046 & 6,182 & 6,628 & 6,977 & & \\
\hline S.d. & 3,555 & 3,619 & 3,956 & 4,034 & 3,764 & 3,474 & 3,599 & 3,866 & & \\
\hline Min & 356 & 416 & 494 & 632 & 790 & 716 & 747 & 875 & & \\
\hline$\sum$ Total Sample & 44,623 & 48,110 & 54,670 & 60,829 & 65,577 & 63,161 & 65,054 & 71,300 & $59.8 \%$ & $6.9 \%$ \\
\hline
\end{tabular}

Source: Company data, author's calculations.

One of the most important profitability figures that attracts analysts' attention is EBITDA. Since the distortionary accounting and financing effects on company earnings do not factor into EBITDA, it is a good way of comparing companies within and across industries. This measure is also of interest to a company's creditors, since EBITDA is essentially the income that a company has free for interest payments. Within the last eight years the total sample's EBITDA pool grew at a CAGR of $8.0 \%$ and a total growth of $71.5 \%$ from $€ 8,980 \mathrm{mn}$ in 1998 to $€ 15,398 \mathrm{mn}$ in 2005 . The average EBITDA in 1998 amounted $€ 898 \mathrm{mn}$ (s.d. $=€ 923 \mathrm{mn}$ ) and augmented to $€ 1,540 \mathrm{mn}$ in 2005 (s.d. $=€ 1,050 \mathrm{mn}$ ). Again, InBev is well ahead of the rest of the world's largest brewers, as the Belgium brewing group grew at a CAGR of $25.2 \%$ consecutively reaching $€ 3,132 \mathrm{mn}$ in 2005 , that is nearly the fivefold $(+381 \%)$ of its 1998 EBITDA of $€ 651 \mathrm{mn}$. This rapid growth was mainly driven by former Interbrew's aggressive external growth strategy, spending more than $€ 25 \mathrm{bn}$ in M\&As in the course of recent years. But beyond its external growth these impressive figures reflect InBev's motto, to move 'from biggest to best' (InBev, 2005). Apparently, InBev's strategy yielded success. Table 4 illustrates the sample brewers' EBITDA development between 1998 and 2005. It strikes, that not 
only InBev but also SABMiller and MCBC have performed quite well over these eight financial years (CAGR 15.4\% and 15.0\% respectively). The weakest performance has been offered by Kirin, A-B and S\&N, the latter two even decreased their EBITDA remarkably. 2005 has been the first year, when Anheuser-Busch was not only taken over by InBev in terms of production volume but also by profitability. For decades, A-B has reigned supreme in terms of sales and profits. Nowadays, InBev is not only the biggest brewer in terms of beer production but also in terms of EBITDA.

Table 4: Development in Leading Brewing Group's EBITDA 1998-2005.

\begin{tabular}{|c|c|c|c|c|c|c|c|c|c|c|}
\hline Company & $\begin{array}{r}1998 \\
(€ \mathrm{mn}) \\
\end{array}$ & $\begin{array}{r}1999 \\
(€ \mathrm{mn}) \\
\end{array}$ & $\begin{array}{r}2000 \\
(€ \mathrm{mn}) \\
\end{array}$ & $\begin{array}{r}2001 \\
(€ \mathrm{mn}) \\
\end{array}$ & $\begin{array}{r}2002 \\
(€ \mathrm{mn}) \\
\end{array}$ & $\begin{array}{r}2003 \\
(€ \mathrm{mn}) \\
\end{array}$ & $\begin{array}{r}2004 \\
(€ \mathrm{mn}) \\
\end{array}$ & $\begin{array}{r}2005 \\
(€ \mathrm{mn}) \\
\end{array}$ & $\begin{array}{r}\text { Total } \\
\text { Growth } \\
\end{array}$ & CAGR \\
\hline InBev & 651 & 756 & 1,084 & 1,534 & 1,504 & 1,498 & 2,325 & 3,132 & $381.0 \%$ & $25.2 \%$ \\
\hline SABMiller & 952 & 926 & 945 & 1,023 & 1,512 & 1,870 & 2,156 & 2,602 & $173.5 \%$ & $15.4 \%$ \\
\hline Anheuser-Busch & 3,354 & 3,279 & 3,566 & 3,988 & 4,047 & 3,602 & 3,452 & 2,891 & $-13.8 \%$ & $-2.1 \%$ \\
\hline Heineken & 1,032 & 1,227 & 1,389 & 1,601 & 1,811 & 1,940 & 2,021 & 1,959 & $89.8 \%$ & $9.6 \%$ \\
\hline Carlsberg & 394 & 438 & 405 & 775 & 834 & 806 & 807 & 815 & $106.9 \%$ & $10.9 \%$ \\
\hline Scottish\&Newcastle & 863 & 864 & 967 & 1,250 & 1,091 & 1,060 & 717 & 617 & $-28.5 \%$ & $-4.7 \%$ \\
\hline MolsonCoors & 257 & 283 & 303 & 304 & 555 & 481 & 495 & 682 & $165.3 \%$ & $15.0 \%$ \\
\hline Modelo & 493 & 555 & 1,080 & 1,152 & 1,223 & 1,047 & 1,084 & 1,218 & $146.8 \%$ & $13.8 \%$ \\
\hline Tsingtao & 49 & 53 & 64 & 119 & 127 & 113 & 106 & 122 & $149.0 \%$ & $13.9 \%$ \\
\hline Kirin & 935 & 1,325 & 1,686 & 1,391 & 1,422 & 1,394 & 1,389 & 1,360 & $45.5 \%$ & $5.5 \%$ \\
\hline Max & 3,354 & 3,279 & 3,566 & 3,988 & 4,047 & 3,602 & 3,452 & 3,132 & & \\
\hline Average & 898 & 970 & 1,149 & 1,314 & 1,413 & 1,381 & 1,455 & 1,540 & & \\
\hline Median & 757 & 810 & 1,023 & 1,201 & 1,323 & 1,227 & 1,237 & 1,289 & & \\
\hline S.d. & 923 & 904 & 984 & 1,061 & 1,053 & 970 & 1,022 & 1,050 & & \\
\hline Min & 49 & 53 & 64 & 119 & 127 & 113 & 106 & 122 & & \\
\hline$\sum$ Total Sample & 8,980 & 9,705 & 11,487 & 13,137 & 14,127 & 13,811 & 14,552 & 15,398 & $71.5 \%$ & $8.0 \%$ \\
\hline
\end{tabular}

Source: Company data, author's calculations.

Earnings before interest and taxes are a measure of a company's earning power from ongoing operations, equal to earnings before deduction of interest payments and income taxes. EBIT (also called operating profit) is watched closely by creditors, since it represents the amount of cash that such a company will be able to use to pay off creditors. Over the study period of eight financial years from 1998 to 2005, the LBGs' combined EBIT grew imposingly at a CAGR of $8.4 \%$ and a total growth rate of $76.4 \%$ from $€ 6,153 \mathrm{mn}$ in 1998 to $€ 10,856 \mathrm{mn}$ in 2005. The average EBIT was $€ 615 \mathrm{mn}$ (s.d. $=€ 699 \mathrm{mn}$ ) in 1998 and increased to $€ 1,086 \mathrm{mn}$ in 2005 (s.d. $=€ 814 \mathrm{mn}$ ). Not surprisingly, InBev is again the absolute out-performer in the peer 
group of LBGs in the world brewing industry. The Belgium brewing group jumped astonishingly forward at a CAGR of $28.4 \%$, amounting $€ 2,198 \mathrm{mn}$ in 2005 , a plus of $474 \%$ compared to its 1998 EBIT. Thus, InBev is the most profitable brewing group in terms of operating profit, followed by SABMiller $(€ 2,159 \mathrm{mn})$ and Anheuser-Busch $(€ 2,105 \mathrm{mn})$. Far behind this peer group, we find Heineken with $€ 1,249 \mathrm{mn}$ and Modelo with $€ 1,050 \mathrm{mn}$. Noteworthy, Chinese brewer Tsingtao could more than six-fold $(+516 \%)$ its EBIT, admittedly from a really low 1998 basis of $€ 10 \mathrm{mn}$.

Table 5: Development in Leading Brewing Group's Operating Profit 1998-2005.

\begin{tabular}{|c|c|c|c|c|c|c|c|c|c|c|}
\hline Company & $\begin{array}{r}1998 \\
(€ \mathrm{mn}) \\
\end{array}$ & $\begin{array}{r}1999 \\
(€ \mathrm{mn}) \\
\end{array}$ & $\begin{array}{r}2000 \\
(€ \mathrm{mn}) \\
\end{array}$ & $\begin{array}{r}2001 \\
(€ \mathrm{mn}) \\
\end{array}$ & $\begin{array}{r}2002 \\
(€ \mathrm{mn}) \\
\end{array}$ & $\begin{array}{r}2003 \\
(€ \mathrm{mn}) \\
\end{array}$ & $\begin{array}{r}2004 \\
(€ \mathrm{mn}) \\
\end{array}$ & $\begin{array}{r}2005 \\
(€ \mathrm{mn}) \\
\end{array}$ & $\begin{array}{r}\text { Total } \\
\text { Growth } \\
\end{array}$ & CAGR \\
\hline InBev & 383 & 419 & 533 & 885 & 836 & 839 & 1,310 & 2,198 & $473.6 \%$ & $28.4 \%$ \\
\hline SABMiller & 673 & 687 & 702 & 708 & 878 & 1,147 & 1,424 & 2,159 & $220.9 \%$ & $18.1 \%$ \\
\hline Anheuser-Busch & 2,489 & 2,451 & 2,697 & 3,017 & 3,151 & 2,827 & 2,702 & 2,105 & $-15.4 \%$ & $-2.4 \%$ \\
\hline Heineken & 659 & 799 & 921 & 1,125 & 1,282 & 1,296 & 1,248 & 1,249 & $89.5 \%$ & $9.6 \%$ \\
\hline Carlsberg & 219 & 231 & 209 & 396 & 430 & 396 & 307 & 440 & $101.4 \%$ & $10.5 \%$ \\
\hline Scottish\&Newcastle & 683 & 584 & 616 & 873 & 806 & 675 & 355 & 438 & $-35.8 \%$ & $-6.1 \%$ \\
\hline MolsonCoors & 122 & 151 & 163 & 169 & 315 & 272 & 281 & 339 & $178.7 \%$ & $15.7 \%$ \\
\hline Modelo & 401 & 421 & 909 & 937 & 1,027 & 888 & 935 & 1,050 & $161.6 \%$ & $14.7 \%$ \\
\hline Tsingtao & 10 & 11 & 12 & 40 & 66 & 56 & 54 & 62 & $515.5 \%$ & $57.8 \%$ \\
\hline Kirin & 514 & 767 & 937 & 689 & 760 & 775 & 814 & 816 & $58.7 \%$ & $6.8 \%$ \\
\hline Max & 2,489 & 2,451 & 2,697 & 3,017 & 3,151 & 2,827 & 2,702 & 2,198 & & \\
\hline Average & 615 & 652 & 770 & 884 & 955 & 917 & 943 & 1,086 & & \\
\hline Median & 458 & 503 & 659 & 791 & 821 & 807 & 874 & 933 & & \\
\hline S.d. & 699 & 685 & 755 & 826 & 849 & 772 & 785 & 814 & & \\
\hline Min & 10 & 11 & 12 & 40 & 66 & 56 & 54 & 62 & & \\
\hline$\sum$ Total Sample & 6,153 & 6,521 & 7,698 & 8,840 & 9,551 & 9,170 & 9,430 & 10,856 & $76.4 \%$ & $8.4 \%$ \\
\hline
\end{tabular}

Source: Company data, author's calculations.

Compared to table 3 ('net sales'), we can see that the sample's brewers could seemingly increase their profitability as the EBITDA and still more impressively the EBIT both grew stronger than net sales (59.8\% versus $71.5 \%$ and $76.4 \%$ respectively).

Throwing a glance at the following table reveals changes within the brewing groups profitability margins expressed as EBITDA per net sales. We can see several shifts within our sample over the 8-year study period. The clearest leader in terms of EBITDA-Margin is Mexicanbased Grupo Modelo that already was the most profitable brewer in 1998 but still could en- 
hance its profitability from $27.2 \%$ in 1998 to impressive $34.2 \%$ in 2005 . Modelo is followed by two other strong performer InBev and SABMiller, not only the two largest brewers in the world brewing industry but also some of the most successful in terms of accounting profitability. The number 4 brewer is US Anheuser-Busch that has deteriorated indeed over recent years. In the same way, the long-year number 2 brewer Heineken could not enhance its profitability but faced comparatively slight $18.1 \%$ in 2005. Carlsberg, MCBC and Kirin could improve their EBITDA-Margins more or less strongly. The clearest looser in our sample is S\&N that remarkably had to loose efficiency. The sample's average EBITDA-Margin in 1998 was $19.1 \%(\mathrm{n}=9$; s.d. $=5.5 \%)$ and increased to $20.5 \%$ in $2005(\mathrm{n}=10$; s.d. $=7.3 \%)$. Noteworthy, the 1998 average EBITDA-Margin would have been slightly weaker, if we had Tsingtao data to include. May it be as it is we can see that both did increase the average margin as well as the standard deviation which indicates that some brewers could improve their profitability above-proportional.

Table 6: Development in Leading Brewing Groups’ EBITDA-Margin 1998-2005.

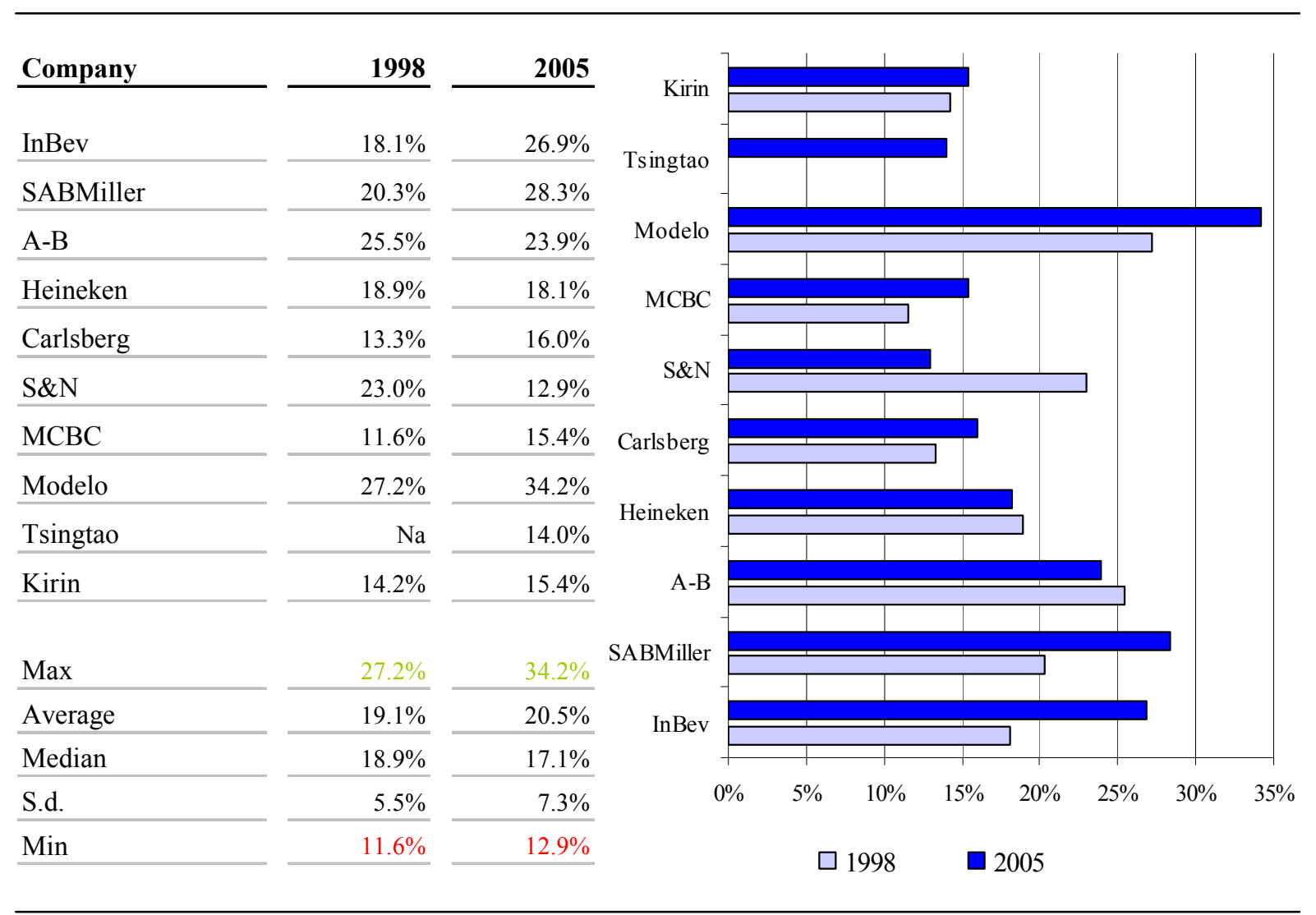

Source: Company data, author's calculations. 
Consequently we analyze the operating profit margin of our sample's brewers. Again, Modelo shows extraordinary strong figures with an EBIT-Margin of $29.5 \%$, followed by SABMiller (23.5\%), InBev (18.9\%) and A-B (17.4\%). With some distance, Heineken follows with 11.6\% not far ahead of the other brewing groups whose ROS ranges between $7.0 \%$ and $9.2 \%$. The sample's average EBITDA-Margin in 1998 was $13.0 \%(\mathrm{n}=9$; s.d. $=5.8 \%)$ and increased to $14.3 \%$ in $2005(\mathrm{n}=10$; s.d. $=7.7 \%)$. As aforementioned we could not calculate the 1998 ROS figures for Tsingtao due to data non-availability.

Table 7: Development in Leading Brewing Groups' Operating Profit-margin 1998-2005.

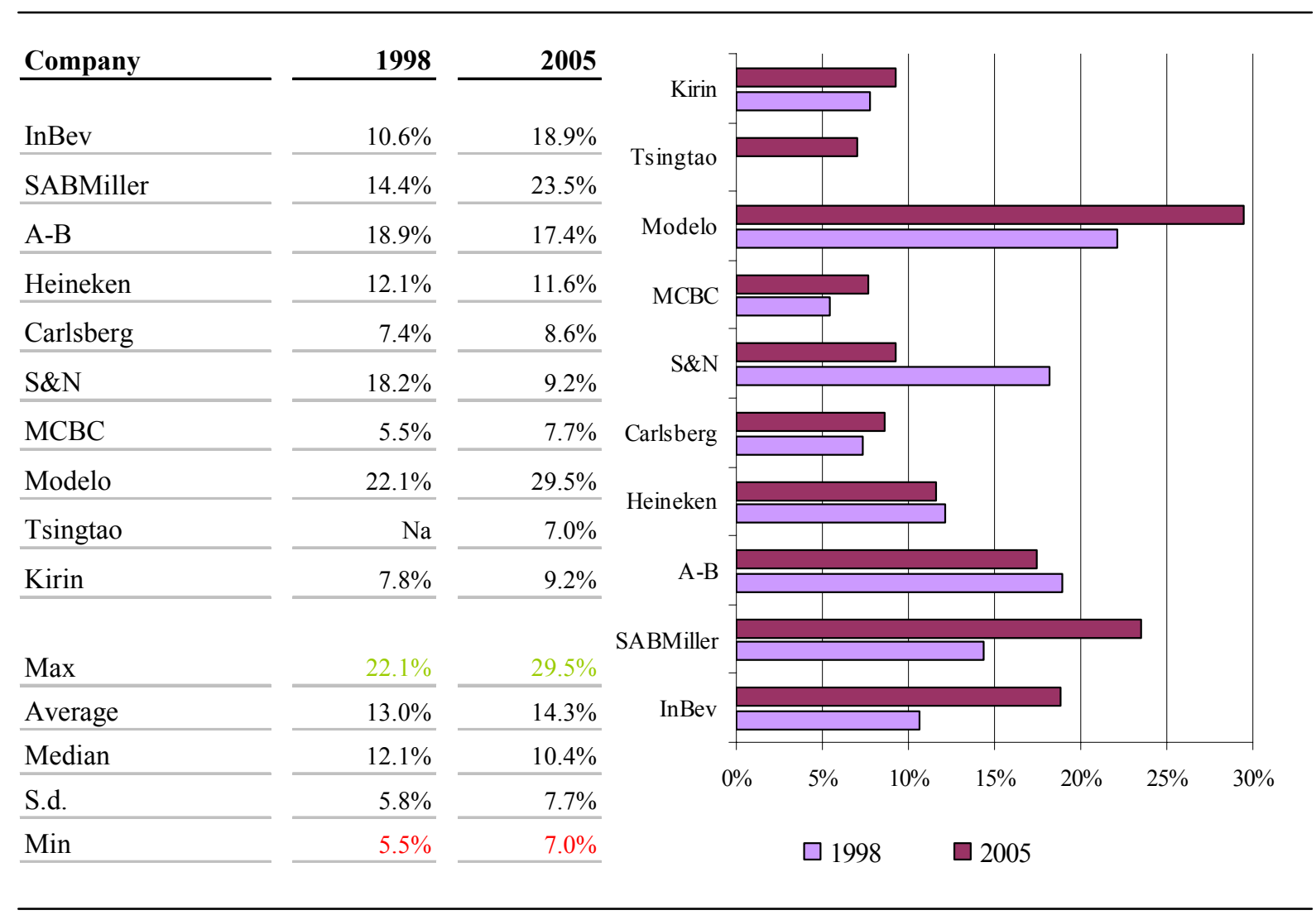

Source: Company data, author's calculations.

Table 8 illustrates the way we have calculated the financial ratios of our sample's companies. We choose InBev, the world-largest brewer as a proxy for the whole sample. The first three sections show InBev's profit\&loss account, balance sheet and cash flow statement in Euro million, the lower section discloses the five financial ratios we have calculated for our internationalization-performance relationship analysis. It has to be stressed out, that these figures could not been taken directly out of the respective annual reports but have been carefully cal- 
culated for each financial year and for each brewing group in the same way. Thus, the possibility of an industry comparison between the leading brewing groups is guaranteed.

Table 8: Analysis of InBev's Profit\&Loss Account, Balance Sheet and Cash Flow Statement.

\begin{tabular}{|c|c|c|c|c|c|c|c|c|}
\hline \multirow{2}{*}{$\begin{array}{l}\text { Profit \& Loss Statement } \\
\text { Year end Dec }\end{array}$} & \multicolumn{8}{|c|}{ Euro million } \\
\hline & 1998 & 1999 & 2000 & 2001 & 2002 & 2003 & 2004 & 2005 \\
\hline Net revenues & 3.600 & 4.500 & 5.657 & 7.303 & 6.992 & 7.044 & 8.568 & 11.656 \\
\hline Cost of goods sold & 1.563 & 2.117 & 2.703 & 3.593 & 3.418 & 3.385 & 3.996 & 5.082 \\
\hline Gross profit & 2.037 & 2.383 & 2.954 & 3.710 & 3.574 & 3.659 & 4.572 & 6.574 \\
\hline Operating costs & 1.386 & 1.627 & 1.870 & 2.176 & 2.070 & 2.161 & 2.247 & 3.442 \\
\hline EBITDA & 651 & 756 & 1.084 & 1.534 & 1.504 & 1.498 & 2.325 & 3.132 \\
\hline Depreciation & 237 & 303 & 451 & 569 & 535 & 540 & 799 & 934 \\
\hline Amortization & 31 & 34 & 100 & 80 & 133 & 119 & 216 & 0 \\
\hline EBIT & 383 & 419 & 533 & 885 & 836 & 839 & 1.310 & 2.198 \\
\hline Income/(expense) from associates & 30 & 61 & 50 & 67 & 71 & 35 & 23 & 46 \\
\hline Net interest income/(expense) & -103 & -112 & -258 & -176 & -134 & -131 & -172 & -451 \\
\hline Non operating income/(expense) & -21 & -43 & -1.235 & 161 & -108 & 0 & 0 & 0 \\
\hline Pre-tax income & 289 & 324 & -910 & 937 & 665 & 743 & 1.161 & 1.793 \\
\hline $\operatorname{Tax}$ & 94 & 93 & 75 & 179 & 162 & 185 & 263 & 391 \\
\hline Reported net profit pre minorities & 195 & 230 & -985 & 758 & 503 & 558 & 898 & 1.402 \\
\hline Minority interest & 5 & 0 & 21 & -59 & -36 & -53 & -179 & -498 \\
\hline Reported net profit & 201 & 230 & -964 & 699 & 467 & 505 & 719 & 904 \\
\hline Plus: Goodwill amortisation & 31 & 34 & 100 & 80 & 133 & 119 & 190 & 0 \\
\hline Less: Exceptionals (post-tax) & 0 & 0 & 0 & 161 & -78 & 0 & -98 & 120 \\
\hline Normalised cash net profit & 231 & 264 & -864 & 618 & 678 & 624 & 811 & 1.024 \\
\hline Balance Sheet & \multicolumn{8}{|c|}{ Euro million } \\
\hline Year end Dec & 1998 & 1999 & 2000 & 2001 & 2002 & 2003 & 2004 & 2005 \\
\hline Shareholders equity & 1.464 & 1.536 & 4.066 & 4.818 & 4.694 & 4.720 & 8.530 & 11.471 \\
\hline Minority interest & 153 & 454 & 414 & 497 & 463 & 410 & 412 & 379 \\
\hline Accumulated goodwill amortization & 1.243 & 1.386 & 1.458 & 1.201 & 375 & 480 & 670 & 670 \\
\hline Provisions & 429 & 697 & 823 & 839 & 868 & 791 & 1.588 & 2.056 \\
\hline Net debt & 1.866 & 2.100 & 2.952 & 2.662 & 2.583 & 2.434 & 3.271 & 4.867 \\
\hline Total Net Liabilities & 5.156 & 6.174 & 9.713 & 10.017 & 8.983 & 8.835 & 14.471 & 19.443 \\
\hline Cash Flow Statement & \multicolumn{8}{|c|}{ Euro million } \\
\hline Year end Dec & 1998 & 1999 & 2000 & 2001 & 2002 & 2003 & 2004 & 2005 \\
\hline Operating cash flow & 706 & 860 & 1.195 & 1.349 & 1.225 & 1.357 & 1.790 & 3.136 \\
\hline Interest & -498 & 5 & -231 & -178 & -114 & -106 & -177 & -422 \\
\hline $\operatorname{Tax}$ & -87 & -89 & 117 & -145 & -91 & -158 & -237 & -371 \\
\hline Net capital expenditures & -245 & -314 & -465 & -535 & -515 & -544 & -812 & -1.037 \\
\hline Free cash flow & -124 & 462 & 616 & 491 & 505 & 549 & 564 & 1.306 \\
\hline Net M\&A & -678 & -301 & -4.445 & -135 & -454 & -383 & -892 & -1.643 \\
\hline Dividends & -45 & -56 & -80 & -106 & -142 & -168 & -229 & -568 \\
\hline Net Financing & 283 & -6 & 4.440 & -626 & -188 & 241 & -132 & -65 \\
\hline Other & 195 & 138 & -198 & 57 & 28 & 46 & 1.209 & 607 \\
\hline Net change in cash & -368 & 120 & 333 & -319 & -251 & 285 & 520 & -363 \\
\hline \multicolumn{9}{|l|}{ Financial Ratio's } \\
\hline Year end Dec & 1998 & 1999 & 2000 & 2001 & 2002 & 2003 & 2004 & 2005 \\
\hline EBITDA-margin & $18,1 \%$ & $16,8 \%$ & $19,2 \%$ & $21,0 \%$ & $21,5 \%$ & $21,3 \%$ & $27,1 \%$ & $26,9 \%$ \\
\hline ROS & $10,6 \%$ & $9,3 \%$ & $9,4 \%$ & $12,1 \%$ & $12,0 \%$ & $11,9 \%$ & $15,3 \%$ & $18,9 \%$ \\
\hline Cash Realization Ratio & $19,6 \%$ & $19,1 \%$ & $21,1 \%$ & $18,5 \%$ & $17,5 \%$ & $19,3 \%$ & $20,9 \%$ & $26,9 \%$ \\
\hline RONA (after tax) & $2,4 \%$ & $5,5 \%$ & $5,7 \%$ & $6,9 \%$ & $7,2 \%$ & $7,4 \%$ & $8,8 \%$ & $8,7 \%$ \\
\hline Leverage & $127,4 \%$ & $136,7 \%$ & $72,6 \%$ & $55,3 \%$ & $55,0 \%$ & $51,6 \%$ & $38,3 \%$ & $42,4 \%$ \\
\hline
\end{tabular}

Source: InBev's annual reports 1998-2005, author's calculations. 


\subsection{INTERNATIONALIZATION-PERFORMANCE RELATIONSHIPS}

In order to test the effect of internationalization on firm profitability, we define some independent variables that measure internationalization under different definitions; then we introduce some control variables in order to gauge for other aspects that influence firms' performance. The first independent variable we introduce is the ratio of foreign sales to total sales, a typical measure of the degree of internationalization (Ramaswamy et al, 1996). In order to have another measure of internationalization, we had a glance at foreign direct investments, which defines whether or not firms maintain foreign affiliates and subsidiaries. We define FDI as any investment involving a lasting control on a foreign enterprise. Therefore, we calculated the network spread index (NSI) which covers the country scope of a company's foreign affiliates (Tallman and Li, 1996; Ietto-Gillies, 1998; Majocchi and Zucchella, 2003).

Strategic benefits stem from being able to compete across many markets, and to reduce the risk in a firm's revenue and profit streams. Operational benefits include the opportunity to source geographically dispersed sources of knowledge, as well as the ability to source lower cost inputs. A methodological prerequisite of the possibility to find significant internationalization-performance linkages is that the brewing groups under investigation differ perceptibly regarding their internationalization and financial performance indicator values (Gerpott and Jakopin, 2005).

Table 9 reports the means, standard deviations (s.d.) and Pearson Product Moment Correlations $^{15}$ as well as Kendall Rank Correlation among the variables used. The correlation provides a first check of how far the sample's companies differ regarding their internationalization and accounting profitability values. Throwing a glance at the standard deviations in table 9 discloses that the value dispersions of both types of variables were so large that it makes

\footnotetext{
${ }^{15}$ The correlation between two variables reflects the degree to which the variables are related. The most common measure of correlation is the Pearson Product Moment Correlation (called Pearson' correlation for short). When computed in a sample, it is designated by the letter ' $r$ ' and is sometimes called 'Pearson's r'. Pearson's Correlation reflects the degree of linear relationship between two variables. It ranges from +1 to -1 . A correlation of +1 means, that there is a perfect positive linear relationship between the variables. Kendall's Rank Correlation provides a distribution-free test of independence and a measure of the strength of dependence between two variables.
} 
sense to conduct correlational analysis seeking for significant internationalizationperformance relationships (Hsu and Boggs, 2003; Gerpott and Jakopin, 2005). Noteworthy, the NDR shows no correlation with other performance measures.

As table 9 shows the sample's mean EBITDA-Margin in the study period was $20.3 \%$ (s.d. $=6.1 \% ; \mathrm{n}=78)$. The average Operating profit margin has been $13.4 \%$ with a standard deviation of $6.4 \%(\mathrm{n}=78)$. The average RONA was $9.4 \%($ s.d. $=4.5 \% ; \mathrm{n}=78)$. The NDR totalled $62.2 \%$ (s.d. $64.1 \% ; \mathrm{n}=78$ ). The fifth performance indicator under investigation, CRR was in average $19.6 \%$ (s.d. $=7.0 \% ; \mathrm{n}=78$ ). Likewise, both internationalization measures have shown similar standard deviations but already have been explained in chapter 8.1.

Table 9: Descriptive Statistics (Means, Standard Deviations and Correlation).

\begin{tabular}{|c|c|c|c|c|c|c|c|c|c|c|c|}
\hline $\begin{array}{l}\text { Measures of } \\
\text { internationalization }\end{array}$ & $\begin{array}{r}\text { Mean } \\
(\%)\end{array}$ & $\begin{array}{l}\text { S.d. } \\
(\%)\end{array}$ & $\mathbf{n}$ & 1 & 2 & & & & & & \\
\hline 1. FSI & 48.4 & 37.5 & 80 & - (a) & $0.80 * *$ & & & & & & \\
\hline 2. NSI & 11.3 & 12.4 & 80 & $0.73 * *$ & - & & & & & & \\
\hline $\begin{array}{l}\text { Performance } \\
\text { indicators }\end{array}$ & $\begin{array}{r}\text { Mean } \\
(\%)\end{array}$ & $\begin{array}{l}\text { S.d. } \\
(\%)\end{array}$ & $\mathbf{n}$ & 3 & 4 & 5 & 6 & 7 & & & \\
\hline 3. EBITDA-Margin & 20.3 & 6.1 & 78 & - & $0.97 * *$ & $0.77^{* *}$ & 0.02 & $0.91 * *$ & & & \\
\hline 4. Operating Profit Margin & 13.4 & 6.4 & 78 & $0.83 * *$ & - & $0.79 * *$ & -0.02 & $0.91 * *$ & & & \\
\hline 5. RONA & 9.4 & 4.5 & 78 & $0.55 * *$ & $0.58 * *$ & - & 0.17 & $0.74 * *$ & & & \\
\hline 6. NDR & 62.2 & 64.1 & 78 & -0.02 & -0.04 & 0.00 & - & 0.41 & & & \\
\hline 7. $\mathrm{CRR}$ & 19.6 & 7.0 & 78 & $0.73 * *$ & $0.73 * *$ & $0.53 * *$ & -0.05 & - & & & \\
\hline Control variables & Mean & S.d. & $\mathbf{n}$ & 8 & 9 & 10 & 11 & 12 & 13 & 14 & 15 \\
\hline 8. Firm Size $(€)$ & 5,917 & 3,673 & 80 & - & $0.22 *$ & $0.22 *$ & $0.29 * *$ & -0.06 & -0.06 & 0.06 & $0.23 *$ \\
\hline 9. CR10 $(\%)$ & 46.9 & 9.9 & 8 & $0.20 *$ & - & $0.99 * *$ & 0.03 & 0.06 & -0.12 & $0.36^{* *}$ & $0.99 * *$ \\
\hline 10. CR5 (\%) & 33.8 & 8.2 & 8 & $0.20 *$ & $0.99 * *$ & - & 0.02 & 0.06 & -0.12 & $0.36 * *$ & $0.99 * *$ \\
\hline 11. Market Share (\%) & 45.8 & 24.4 & 80 & $0.18 *$ & 0.06 & 0.06 & - & 0.08 & 0.22 & 0.00 & 0.02 \\
\hline 12. Growth Speed (\%) & 11.1 & 17.9 & 73 & -0.08 & 0.07 & 0.07 & 0.01 & - & 0.19 & -0.09 & 0.07 \\
\hline 13. Speed of expansion (\%) & 31.1 & 92.8 & 70 & $-0.17 *$ & -0.09 & -0.09 & -0.02 & -0.03 & - & 0.01 & -0.10 \\
\hline 14. Industry Growth (\%) & 2.6 & 1.1 & 8 & 0.02 & $0.26 * *$ & $0.26 * *$ & -0.01 & 0.09 & 0.01 & - & $0.41 * *$ \\
\hline 15. Year & & & & $0.20 *$ & $0.99 * *$ & $0.99 * *$ & 0.06 & 0.07 & -0.09 & $0.26 * *$ & - \\
\hline
\end{tabular}

$* \mathrm{p}<0.10, * * \mathrm{p}<0.05, * * * \mathrm{p}<0.01$ (two-tailed-tests).

(a) Coefficients above (below) the diagonal are Pearson correlations (Kendall-Tau-b correlations). 
Three analytical steps were taken to shed light on potential links between each of the five performance criteria selected for this investigation and the FSI and NSI shares as the two measures of a LBG group's degrees of internationalization. According to the approach of Gerpott and Jakopin (2005), we first calculated a simple bivariate Pearson correlation as well as a Kendall rank correlation for each of the ten possible internationalization-performance measure combinations. Secondly, for these combinations a first-order partial correlation (= betavalue) was accounted controlling for the 'year within the study period' to neutralize general time-related environmental changes (e.g. changes in consumers' attitudes, new products and/or markets, intensified M\&A activity) not reflected completely in the remaining seven control variables. Lastly, for each LBG performance criterion, two ordinary least-squares (OLS) multiple regressions were run in which standardized beta-weights for the complete set of eight control variables and either the FSI share or the NSI share internationalization indicators were obtained. Table 10 shows the results of the multiple regression analysis of LBG internationalization-performance indicators. 
Table 10: Regression Analyses of LBG Performance-Internationalization Indicators.

\begin{tabular}{|c|c|c|c|c|c|c|c|c|c|c|c|c|c|}
\hline \multirow{3}{*}{$\begin{array}{l}\text { Variables } \\
\text { DOI (b) }\end{array}$} & \multicolumn{13}{|c|}{ Performance indicators (a) } \\
\hline & \multicolumn{3}{|c|}{$\begin{array}{c}\text { EBITDA } \\
\text { Margin }\end{array}$} & \multicolumn{2}{|c|}{$\begin{array}{c}\text { Operating Profit } \\
\text { Margin }\end{array}$} & \multicolumn{4}{|c|}{ RONA } & \multicolumn{2}{|c|}{ NDR } & \multicolumn{2}{|c|}{ CRR } \\
\hline & 1a & $1 \mathrm{~b}$ & & $1 \mathrm{a}$ & $1 \mathrm{~b}$ & 1a & & $1 b$ & & $1 \mathrm{a}$ & $1 b$ & 1a & $1 b$ \\
\hline FSI & $-0.51 * * *$ & & & $-0.54 * * *$ & & -0.35 & $* * *$ & & & 0.07 & & $-0.51 * * *$ & \\
\hline NSI & & $-0.31^{* *}$ & $* * *$ & & $-0.38 * * *$ & & & -0.25 & $* *$ & & -0.16 & & $-0.28 * *$ \\
\hline \multicolumn{14}{|c|}{ Control variables (b) } \\
\hline Firm Size & 0.03 & 0.18 & * & 0.02 & 0.20 & 0.23 & $* *$ & $0.34 *$ & $* * *$ & $0.47^{* * *}$ & $0.48^{* * *}$ & 0.03 & 0.18 \\
\hline CR10 & -0.34 & -0.03 & & -1.63 & -1.29 & 0.28 & & 0.50 & & 1.27 & 1.30 & -0.63 & -0.34 \\
\hline CR5 & 0.21 & 0.13 & & 1.19 & 1.08 & -0.95 & & -1.02 & & -1.45 & -1.50 & -0.01 & -0.09 \\
\hline Market share & $0.75^{* * *}$ & $0.58^{* *}$ & $* * *$ & $0.67^{* * *}$ & $0.51^{* * *}$ & 0.60 & $* * *$ & $0.50 *$ & $* * *$ & -0.10 & -0.02 & 0.69 *** & $0.51 * * *$ \\
\hline Growth speed & -0.03 & -0.06 & & -0.10 & -0.13 & -0.07 & & -0.09 & & 0.01 & 0.03 & -0.02 & -0.06 \\
\hline Speed of expansion & 0.08 & 0.13 & & 0.10 & 0.14 & -0.03 & & -0.01 & & -0.10 & -0.14 & 0.10 & 0.15 \\
\hline Industry growth & -0.04 & -0.02 & & -0.07 & -0.05 & -0.08 & & -0.07 & & -0.04 & -0.04 & 0.00 & 0.01 \\
\hline Year & 0.32 & 0.03 & & 0.63 & 0.33 & 0.74 & & 0.55 & & 0.23 & 0.28 & 0.80 & 0.51 \\
\hline Adjusted $R^{2}$ & $0.47 * * *$ & $0.35^{* * *}$ & & $0.42 * * *$ & $0.32 * * *$ & 0.34 & $* * *$ & 0.30 ** & $* * *$ & 0.11 & $0.13 *$ & $0.42 * * *$ & $0.28 * * *$ \\
\hline $\mathbf{F}$ & 7.42 & 4.88 & & 6.16 & 4.29 & 4.70 & & 4.09 & & 1.88 & 2.06 & 6.16 & 3.77 \\
\hline
\end{tabular}

$* \mathrm{p}<0.10, * * \mathrm{p}<0.05, * * * \mathrm{p}<0.01$ (two-tailed-tests).

(a) Due to the correlations reported in table 9, additional tests for multicollinarity were run with variance inflation factors.

(b) Figures are standardized beta-values.

The FSI as well as the NSI internationalization indicators show statistically significant betaweights at the $5 \%$-level in forecasting all five financial performance criteria. Noteworthy, four out of five results of our models show sound explanatory power $\left(R^{2}\right.$ ranges between 0.28 and 0.47). The NDR-internationalization indicator does not show remarkable $\mathrm{R}^{2}$-values (no statistical significance for the FSI/NDR relationship $\mathrm{R}^{2}=0.11$; significance at the $10 \%$-level for the NSI/NDR relationship $\mathrm{R}^{2}=0.13$ ).

Our findings of the multivariate regression analysis indicate that four performance measures (EBITDA-Margin, Operating Profit Margin, RONA, CRR) were strongly and negatively related to rising degrees of internationalization and achieved statistical significance at the $1 \%$ level, respectively the 5\%-level (NSI/RONA, NSI/CRR). Only the NDR performance indicator did not show any statistically significance regarding an internationalization-performance 
linkage. The simple and first-order correlations in table 10 (see models 1a and 1b) indicate that the four significant and negative bivariate performance-internationalization degree relationships achieved statistical significance at $\mathrm{p}<0.01$ and $\mathrm{p}<0.05$ before and after controlling the 'year within observation' covariate as a proxy capturing unspecified general time-related changes. With the exception of the variables 'market share' and, in parts, 'firm size', table 10 shows that the level of correlation between variables is quite low, thereby suggesting that multicollinearity is not a problem. Indeed, the variable 'market share' has had significant influence on the multinationality-performance linkages. Another correlation could be observed between the 'firm size' variable on RONA as well as NDR/internationalization relationships.

\section{Discussion OF Results}

With some qualifications, we can conclude that the observed outcome of our investigation into the relationships between ever-increasing international engagement and corporate business performance was in large part consistent with the existing finance and international management literature. Our results seem to be in line with finance theory (Majocchi and Zucchella, 2003), which predicts a negative relationship between FDI and firms' returns. Furthermore, this study's results contributed to our understanding of multinational enterprise by extending research in this domain to a sample of LBGs in recent years.

It is worth noting that these negative associations did not hold the impact of some control variables on the internationalization-LBG performance relationship. The strongest influence came from the 'firm size' variable and, to an even greater extent, the 'market share' variable. These findings are not surprising as a large domestic market share guarantees high LBG profitability. Anheuser-Busch, for example, is the least internationalized company among the largest brewers (FSI in 2005: 17.1\%) but still dominates 50\% of the US beer market, which is the most profitable in the world brewing industry. Similarly, there is a trade-off between firm size and performance. It seems obvious that larger firms achieve better financial results due to their ability to realize economies of scale, scope and speed, their first-mover-advantages in entering profitable markets and their greater market power versus their smaller competitors (Calof, 1994).

Another observation regards one of our performance criteria, the net debt ratio (NDR). First, the NDR is positively related to FSI-performance relationships but negatively related to 
the NSI-performance link. Moreover, it shows far weaker explanatory power than the other performance indicators. This may be caused by the fact that the NDR is not correlated with any of the other performance measures (see Table 9). Another reason might be that the NDR is a balance sheet performance figure, composed of borrowings and liabilities. In contrast, the other performance measures are calculated from the profit and loss account and the cash flow statement, which more directly concern a company's operating success. Thus, it seems advisable to replace the NDR figure with another EBIT-based performance measure in future research. In contrast, we strongly recommend the RONA, which is of paramount importance for a company's sustained corporate success in general and for brewing groups in particular. The RONA represents a bracket between the P\&L account and the balance sheet as it expresses the ability of a company to cover the weighted average cost of capital (WACC). As the brewing industry is a high asset industry, the strongest virtue of using RONA compared to traditional methods for measuring company success is that it also considers the assets a company uses to achieve its output.

For LBG groups, our results provide scholarly support for the common-sense notion that higher degrees of multinationality are not per se accompanied by higher (or lower) corporate financial success. Consequently, fast business internationalization is not a cure-all to counter profit and revenue growth erosions in a LBG domestic market (Gerpott and Jakopin, 2005). Obviously, MNCs encounter both the benefits and the costs of internationalization. Hence, internationalization per se is not a sufficient condition for superior performance. Brewers are said to internationalize on an evolutionary path, starting in geographically and culturally close markets, then successively progressing toward cognitively and physically more distant environments. However, as soon as corporations enter unfamiliar territories that require major reconfiguration of internal processes, structures and mechanisms, the costs of internationalization dramatically increase and eventually exceed the benefits (Ruigrok and Wagner, 2003). This phenomenon was clearly identified on the brewing scene.

Beyond the statistical linkages we detected in our descriptive statistical part of the brewing group's balance sheet analysis, there are clear movements and changes among the world brewing scene's leading companies. Of particular interest in this context is the question concerning the most promising ways and paths of internationalization. To shed light on this question, which is of paramount relevance for LBG executives and, similarly, of highest impor- 
tance for capital market participants, we will take a closer look at the two most widely internationalized brewing groups, which both face severe competition in many markets: Belgian InBev and Dutch Heineken. Figure 4 illustrates both brewing groups' latest financial figures according to the geographic spread of each brewer's corporate activities.

Figure 4: InBev and Heineken: Geographic Segment Analysis, 2005

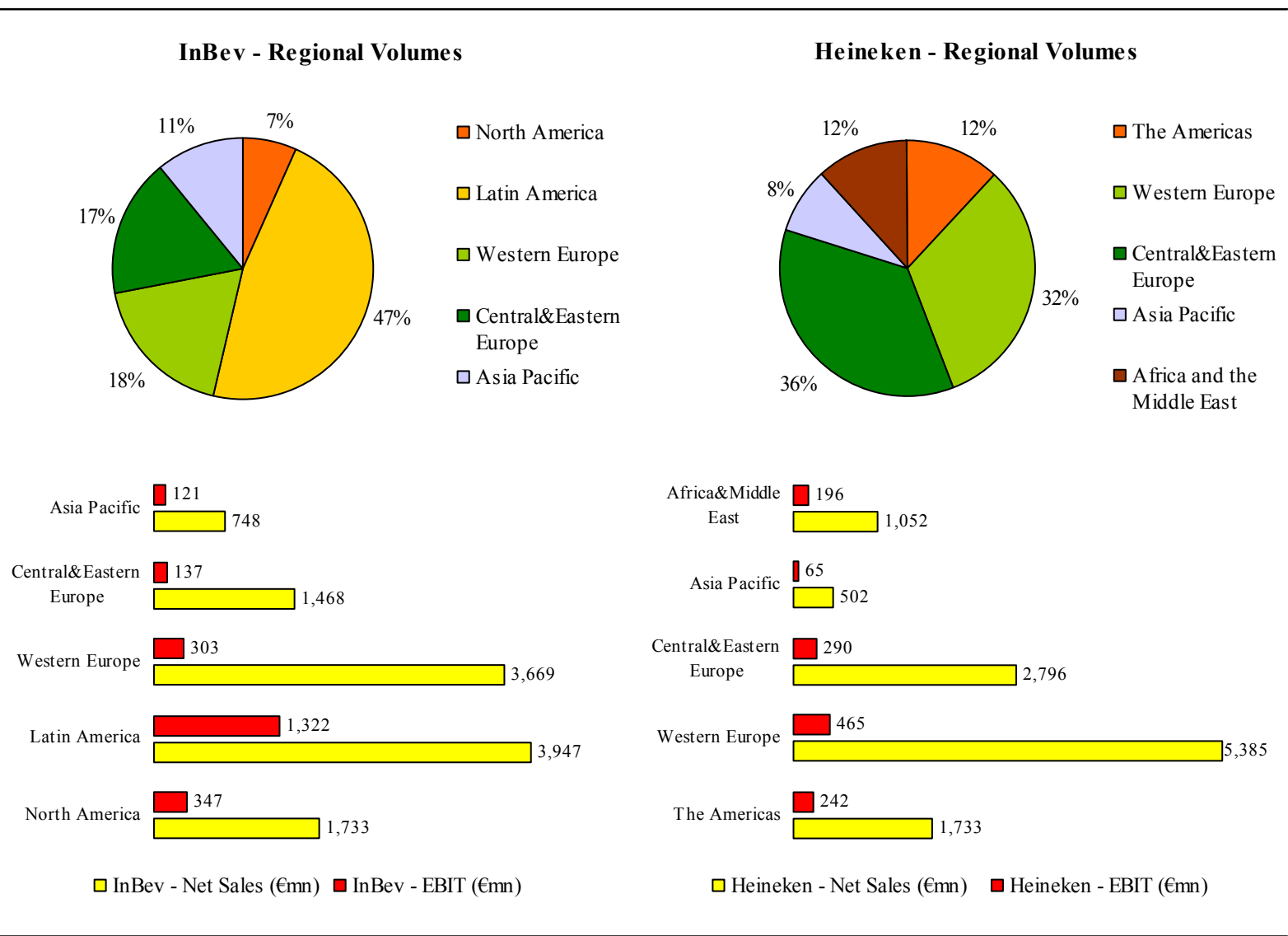

Source: (Heineken, 2006; InBev, 2006), author's calculations

In terms of growth, InBev is clearly outpacing the industry but, much more impressive, its profitability is setting new benchmarks. As Figure 4 highlights, InBev is characterized by a good balance between developing and developed markets, which seems to be the guarantee for InBev's sustained business success. InBev's net sales are well spread out between developed markets, like Western Europe and North America (47\%), and emerging markets, like Latin America (34\%), Eastern Europe (13\%) and Asia-Pacific (6\%). In contrast, Heineken still dominates the European beer market in general and Western Europe in particular. The Dutch brewing group distributes $68 \%$ of its total production volume in European markets and generates $71 \%$ of its net sales there. The Western European markets alone account for $47 \%$ of 
Heineken's net sales. Thus, every change in Western Europe's consumer environment and every damp European summer season directly affects Heineken's results. In contrast, through its global spread of operating activities, InBev is able to attenuate adverse business and economic climates in certain markets.

As there is a fairly well established link between beer market concentration and profitability, a market's profitability changes dramatically with consolidation. Within each market, there can be dramatic differences in brewer profitability. Typically, these differences are highly correlated with scale - the brewers with the largest market shares are usually the most profitable. Admittedly, this is not always the case, but it is usually a good thing to be one of the largest players (and preferably the largest). Brewers who are strongly positioned in most of their markets therefore have a sizeable advantage over brewers with global scale that do not have strong market positions ${ }^{16}$ (Yule et al, 2005). The following figure illustrates InBev's and Heineken's return on sales in the world's main geographic beer markets.

Figure 5: InBev and Heineken: Return on Sales by Geographic Segment, 2005

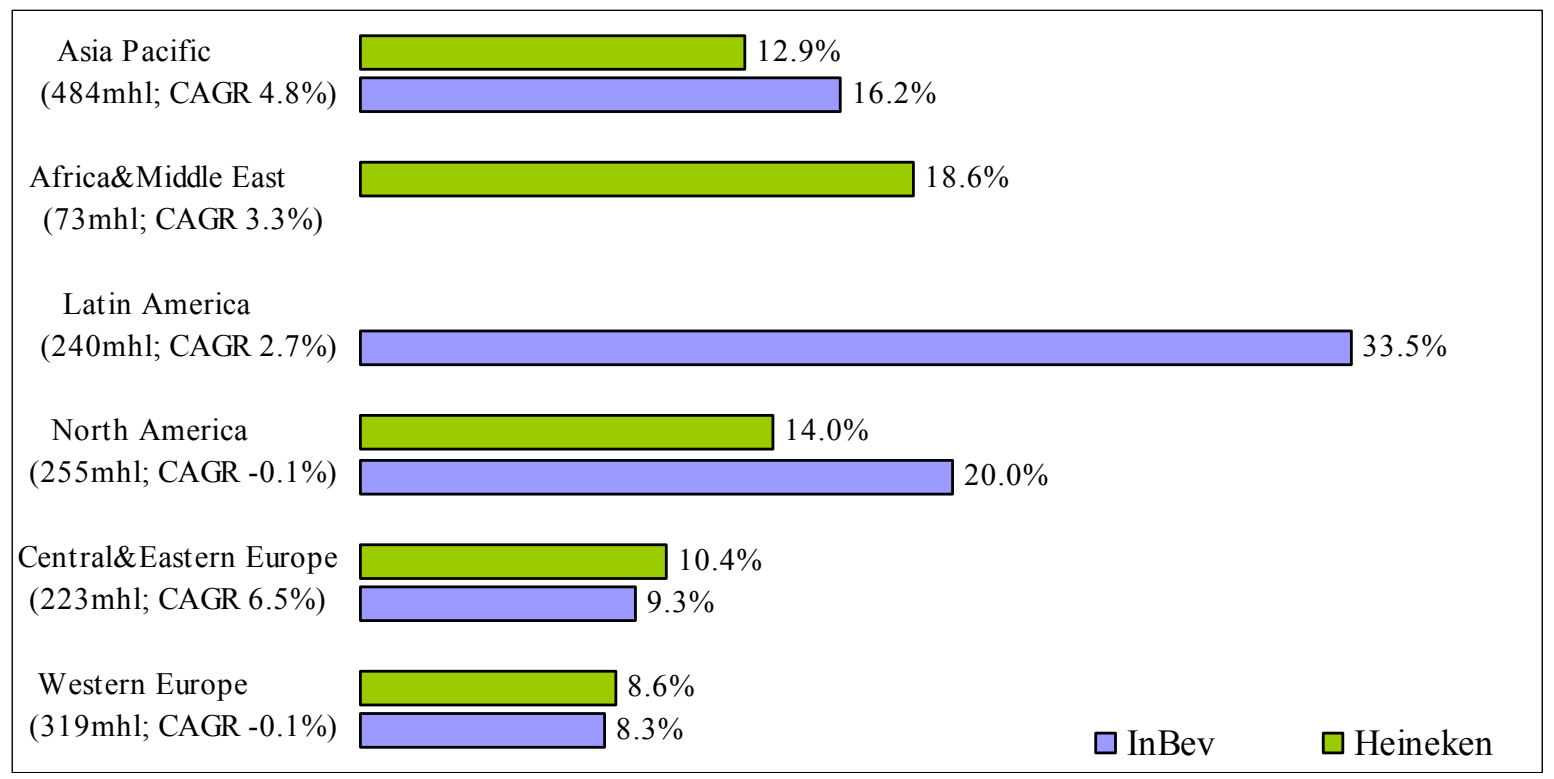

Source: (Heineken, 2006; InBev, 2006), author's calculations

As we can see from Figure 5, InBev is well ahead of its main rival Heineken. In fact, Heineken still dominates the European markets (see Figure 4) and also shows slightly better mar-

\footnotetext{
${ }^{16}$ See also chapter III.1 'Accelerating Growth Opportunities'.
} 
gins. But InBev shows better performance in North America and Asia-Pacific and benefits from a overwhelmingly strong business in South (Latin) America, where Heineken hardly does any business ${ }^{17}$. The left column in Figure 4 provides further important information (in brackets): the market size of the different regional markets as well as the CAGR over the last ten years. The fastest growing region in the world beer industry is Central\&Eastern Europe. This represents Heineken's greatest advantage against InBev as Heineken does more business in Eastern Europe than InBev (net sales of $€ 2,796$ million versus $€ 1,468$ million) and is still more profitable (ROS $10.4 \%$ versus $9.3 \%$ ). Moreover, Heineken traditionally does comparatively strong business in Africa and in the Middle East, where InBev does not have notable activities. Indeed, Africa\&Middle East is still growing (CAGR 3.3\%) but at a relatively low level (73mhl in 2005). In contrast, InBev is extraordinarily strongly positioned in South America through its merger with Brazilian AmBev in 2004 and dominates this fast growing region. Nearly $60 \%$ of InBev's 2005 operating profit is delivered by Latin America (EBIT $€ 1,322$ million), caused by an enviable ROS of $33.5 \%$. In Asia-Pacific Heineken in fact generates more net sales than InBev ( $€ 1,052$ million versus $€ 748$ million) but has a weaker ROS (12.9\% versus $16.2 \%)$. In conclusion, Heineken has missed some opportunities to gain market shares in the fast growing emerging markets that represent more and more of the brewing world's profit pool, whereas InBev has secured its position in many important markets around the globe. This is of principal importance as it seems that geographic diversification is one of the keys to InBev's impressive and continuing success. Not every market always performs at peak levels, and the best way to hedge against a downturn in any given market is to maintain a strong presence in many markets (InBev, 2006). Heineken, in turn, continues to suffer from the sluggish economic growth in some key European markets, which clearly affects the spending attitude of consumers in markets such as the Netherlands, France and Germany. Moreover, Heineken's previous 'cash cow', the US beer exporting business, has become a stumbling block to further profitability. For many years, this export business represented around $6 \%$ of Heineken's volume but about $20 \%$ of its EBIT. Today, Heineken's US business suffers from the unfavorable US\$/€ exchange rate, on the one hand, while, on the other, the mounting competition from wine and spirits and the competition for share of throat in the US beer market drives fierce price competition among the national players (Heineken, 2006).

\footnotetext{
${ }^{17}$ Heineken's South and Central American brewing and exporting business is included in North America. In Heineken's annual reports it is called 'The Americas'.
} 
Furthermore, it seems to be of paramount importance - as in other industries - for brewers to have leading market positions in central markets around the globe. InBev, for example, owns No. 1 positions in 13 markets (including Brazil, Argentina, Belgium and the Ukraine.) and No.2 positions in 16 markets (among them, the U.K., Germany and the Netherlands). Admittedly, InBev's 'cash cows' are its overwhelmingly strong South American base in the large, fast growing Brazilian market, with a $68.3 \%$ market share, and a $77.8 \%$ market share in Argentina, as can be seen in Figure 4.

Thus, it seems obvious that Heineken relies too much on its main strongholds - its dominance in Europe and its (formerly highly) profitable export of the Heineken brand. InBev, in turn, has implemented a global network of strong and sound regional market shares in many of the fastest growing beer markets.

\section{CONCLUSIONS, Limitations OF RESEARCH AND FUTURE RESEARCH}

Internationalization of firm activities through exporting and FDI makes it possible for successful firms to enhance their performance and to grow further. At the same time, however, internationalization also implies that unsuccessful firms are likely to suffer as a result of fierce global competition. Therefore, the aggregate productivity effects of firms' internationalization crucially depend on how efficiently resources are reallocated from declining firms to growing firms, whether within an industry or across industries. Our findings in this paper comparing LBG financial data suggest that both challenges and opportunities from internationalization increase as internationalization advances from the earlier stage of exporting to the later stage of horizontal and vertical FDI (Ahn et al, 2005).

In summary, the present investigation of the whole sample of ten worldwide leading LBGs did not detect any evidence that higher degrees of internationalization coincided with higher profitability levels in the period from 1998 to 2005 (Gerpott and Jakopin, 2005). Indeed, these findings did not reflect the impact of the set of control variables we took into account, but nevertheless they are not in line with popular opinion on the performance-enhancing effects of internationalization in the brewing industry. These findings are obviously out of tune with popular opinion concerning the benefits of increasing internationalization among MNEs for corporate success, but our findings are in line with the outcomes of may previous studies (Geringer et al, 1989; Gomes and Ramaswamy, 1999; Agrawal and Jaffe, 2000; Capar and 
Kotabe, 2003; Contractor et al, 2003).

Regarding the decomposition of our profitability ratios, our work is new and adds detail to our knowledge of the internationalization-performance relationship. Operating profit margin is a pure profitability ratio, while RONA is an efficiency ratio. Important conclusions can be inferred from our findings regarding these two constructs. Even though we did not observe a 0.05 level of significance in the relationship between profitability and internationalization (measured by FSI and NSI), we did find a positively linear slope in both cases. Based on the theoretical foundation of the advantages of internationalization, many international business scholars have concluded that a firm's performance is expected to improve with an increase in its level of foreign involvement. Theories that support diversification into international markets propose reduced risk of bankruptcy and increased sales and, in turn, a higher accrued value to the firm. Our Profit Margin findings do not contradict this existing research. We would caution managers to be alert for international expansion opportunities, but to be wary of geographic overexpansion.

A further contribution is the broadening of regional scope on the link between corporate success and internationalization by going beyond the United States. If one looks at the country of origin of the data analyzed in previous studies, it becomes evident that researchers have predominantly relied on US organizations. Few author teams have included non-US firms in their analyses, merging firms from several European countries into one aggregate sample and thus largely blurring nation-specific impacts (Geringer et al, 1989; Majocchi and Zucchella, 2003; Campa and Hernando, 2005). Moreover, most of the existing literature focuses on financial service and manufacturing industries, whereas our investigation focuses on the food processing industry and, more specifically, on the brewing industry.

In summary, the evidence suggests that the advantages of internationalization are greater than the disadvantages as long as the multinational corporation can efficiently manage its resources. At higher degrees of internationalization, however, it may be that sometimes the advantages are offset by the disadvantages of managerial constraints and resource limits or by the problems of efficiently managing the resources (Hsu and Boggs, 2003). Altogether, the impression given by previous research that the costs of internationalization inevitably outweigh the potential benefits beyond a certain threshold is not confirmed by our findings. Instead, our findings suggest that MNCs pass through an organizational learning process characterized by internal reconfiguration that allows for superior performance development at high DOIs. On a practical level, our results suggest that managers should be eager to pursue 
new international opportunities, but should be wary of over-expanding or overextending their firms_-particularly geographically (Hsu and Boggs, 2003).

While this investigation provides a valuable new perspective on the question of the internationalization-performance relationship, some caveats remain, and one word of caution is merited in relation to our empirical findings. Given that companies learn on their way to high degrees of internationalization, a central question arises: Which are the organizational capabilities most critical for successful operation in increasingly complex foreign market environments? Our findings obviously fall short of advising companies as to the exact nature of decisive areas for reconfiguration. Here, researchers are called upon to quantitatively identify core organizational moderators of the DOI-performance relationship (such as organizational structure, top management team composition and control system).

Admittedly, we are obliged to mention one limitation to our analysis as we had to rely on FSTS as the DOI measure due to the non-availability of data. As pointed out by Sullivan (1994), single-item measures of firms' DOI may be less suitable than multi-criterion composites (Ruigrok and Wagner, 2003). Organizational culture and transaction cost theory both lead to the hypothesis that multinational companies' expansion into countries that are culturally and spatially close to their domestic markets will be accompanied by higher corporate success than international diversification patterns with the opposite characteristics (King et al, 2002; Gerpott and Jakopin, 2005). Therefore, we urge future investigators to consider alternative conceptualization and operationalization techniques for the "degree of internationalization" variable. Researchers who can select from a wide range of measures could also choose indicators that more purely reflect cultural dissimilarity (such as entropy measures or Herfindahl variants based on Hofstede's (1980) culture scores). Such replication would be able to directly assess the validity of our assumption that different forms of the DOI-performance link can be explained by country-specific types of expansion (culturally related versus unrelated). Admittedly, this information is hard to come by. There are two common ways of acquiring corporate financial data: Either investigators trust in purchasable databases provided by professional financial services or - and this requires greater diligence and detail — conduct their own data collection and calculation. In the latter case, as we have done in this study, the researcher has to rely on information published in the company's annual reports. For instance, all the brewers in our sample are listed and are therefore obliged by disclosure requirements to publish reports of their corporate dealings. Most previous studies suggest and urge future re- 
searchers not to rely solely on FSTS and country scope measures (like the network spread index) but to include more sophisticated measurements of DOI. The problem is the aforementioned non-availability or at least limited availability of further data that go beyond FSTS and FDI figures ${ }^{18}$.

Beyond these limitations, resulting from difficulties of data collection, there are further amendments to the research question of investigating DOI-performance relations. As our study relies exclusively on accounting-based measures, we face several balance sheet related restrictions. However, it must be pointed out that, especially by adopting a transnational comparative analysis, key figures are strongly influenced by different valuation and accounting regulations. Therefore, future work ought to broaden the scientific scope by including capitalmarket-based criteria to assess internationalization-performance associations ${ }^{19}$. Event study methodology is a research method that appears frequently in financial and international management studies to measure the impact of changes in corporate policy, such as cross-border M\&As (Ebneth and Theuvsen, 2005).

In conclusion, this study has shed some empirical light on (1) the degree to which the ten major LBGs have internationalized their businesses since the mid-1990s and (2) the relationship between degree of internationalization and financial performance for the sample. Furthermore, it shows that the leading brewing groups around the globe underwent rapid international expansion in the 1998-2005 period but that there were still large variations between the brewers in the DOI achieved until 2005. The European brewers in particular have spearheaded the world brewing industry's consolidation in the late 1990s. Even though this research represents only a small step into the analysis of patterns of internationalization, it highlights some large variations between different brewers around the globe in their internationalization strategies and their corporate success.

What many broker forecasts imply is that the overall global beer profit pool continues to grow marginally ahead of global economic growth. Conceptually, this makes sense, as the

\footnotetext{
${ }^{18}$ The share of foreign assets to total assets (FA) or the share of foreign employees to total employees (FE) are seldom disclosed in brewing groups' annual reports. For example, the FA index and FE index were available for only six (eleven) out of eighty (ten companies $\mathrm{x}$ eight years) potential data points.

${ }^{19}$ Chapter III.7 combines both methodologies of measuring a set of listed brewing group's corporate success.
} 
industry's consolidation process is still ongoing and beer consumption is growing globally. Thus, for the future it seems to be of paramount importance to have a broad exposure to both fast growing emerging markets and the largest profit pools to ensure higher opportunities for future profit growth. Market growth is, of course, not the only source of incremental profit to the brewers. Disproportionate profit shares in markets accrue to those that have the best businesses and brands. Finally, we emphasize the principal impact of internationalization in the world brewing scene: gaining and securing sustainable corporate success through top-line internal and external growth.

\section{REFERENCES}

Agrawal, A. and Jaffe, J. F. (2000): The Post-Merger Performance Puzzle, in: Cooper, C. and Gregory, A. (Eds.): Advances in Mergers and Acquisitions, New York: 7-41.

Ahn, S. et al (2005): The Internationalization and Performance of Korean and Japanese Firms: An Empirical Analysis Based on Micro-data. RIETI Discussion Paper Series 05-E-008.

Andersen, O. (1993): On the Internationalization Process of Firms: A Critical Analysis, in: Journal of International Business Studies 24(1993): 209-231.

Anderson, E. and Gatignon, H. (1986): Modes of foreign entry: a transaction cost analysis and propositions, in: Journal of International Business Strudies, 1986 (17): 1-26.

Annavarjula, M. and Beldona, S. (2000): Multinationality-Performance Relationship: A Review and Reconceptualization, in: International Journal of Organizational Analysis 8(2000): 48-67.

Anonymus (2005): Andean Thirst. SABMiller Buys South America's Second-Biggest Brewer, in: Economist Vol. 376 (8436): 61.

Banz, R. R. (1981): A Possible Explanation of the Small Firm Effect, in: The Journal of Finance, 36(1981): 879-889.

Bartlett, C. A. and Ghoshal, S. (1989): Managing Across Borders. The Transnational Solution, Boston.

Bevan, N. and Greenberg, M. (2005): Global Brewing - The Global Pitcher 2005 - Volume VI, Deutsche Bank Report, London.

Bleymüller, J. et al (2000): Statistik für Wirtschaftswissenschaftler. 12th Edition. Vahlen, München.

Blythman, M. et al (2003): Pan European Brewing Review - 2003, Merrill Lynch, London.

Born, K. (2001): Bilanzanalyse international. Deutsche und ausländische Jahresabschlüsse lesen und beurteilen, Second Edition, Stuttgart.

Buckley, P. J. and Casson, M. C. (1976): The Future of the Multinational Enterprise, London, MacMillan.

Buntinas, M. and Funk, G. M. (2005): Statistics for the Sciences, Belmont.

Campa, J. M. and Hernando, I. (2005): M\&As Performance in the European Financial Industry, Documentos de Trabajo No. 0516, Banco de España, Madrid.

Capar, N. and Kotabe, M. (2003): The Relationship Between International Diversification and Performance in Service Firms, in: Journal of International Business Studies 34(2003): 


\section{5-355.}

Chen, C. J. P. et al (1997): An Investigation of the Relationship Between International Activities and Capital Structure, in: Journal of International Business Studies, 28(1997): 563-577.

Chen, J. and Luo, C. (2005): Tsingtao Brewery, 22.08.2005, Merrill Lynch, Shanghai.

Contractor, F. J. et al (2003): A Three-Stage Theory of International Expansion: The Link Between Multinationality and Performance in the Service Sector, in: Journal of International Business Studies 34(2003): 5-18.

Delios, A. (2000): Multinationality and Performance in Japanese Firms, in: Asian Management Matters: Regional Relevance and Global Impact, Imperial College Press, London: 61-78.

Ebneth, O. and Theuvsen, L. (2005): Internationalization and Corporate Success - Event Study Evidence on M\&As of European Brewing Groups. Paper presented at the 15 th Annual World Food and Agribusiness Symposium and Forum 2005, Chicago.

Fayerweather, J. (1989): Begriff der internationalen Unternehmung, in: Macharzina, K. and Welge, M. (Eds.), Handwörterbuch Export und internationale Unternehmung, Stuttgart: 926-948.

Fisch, J. H. and Oesterle, M.-J. (2003): Exploring the Globalization of German MNCs with the Complex Spread and Diversity Measure, in: Schmalenbach Business Review, 55(2003): 2-21.

Frost, F. and Han, P. C. (2002): Foreign Market Entry Modes. An Empirical Study of Singaporean SMEs, in: Journal of Global Business, 13(2002): 5-15.

Geringer, J. M. et al (1989): Diversification Strategy and Internationalization: Implications for MNE Performance, in: Strategic Management Journal 10(1989): 109-119.

Germann, H. et al (1996): Globalisierung der Wirtschaft: Begriff, Bereiche, Indikatoren, in: Steger, U. (Hrsg.), Globalisierung der Wirtschaft: Konsequenzen, für Arbeit, Technik und Umwelt, Berlin: 18-55.

Gerpott, T. J. and Jakopin, N. M. (2005): The Degree of Internationalization and the Financial Performance of Mobile Network Operators, in: Telecommunications Policy 29(2005): 635-661.

Glaum, M. (1996): Internationalisierung und Unternehmenserfolg, Wiesbaden.

Gomes, E. and Ramaswamy, K. (1999): An Empirical Examination of the Form of the Relationship Between Multinationality and Performance, in: Journal of International Business Studies, 30(1999): 173-188.

Hax, H. (2003): Measuring the Firm's Performance: Accounting versus Market Value, in: Journal of Institutional and Theoretical Economics 159(2003): 675-682.

Heineken (2005): Heineken N.V. Annual Report 2004, Amsterdam.

Heineken (2006a): Heineken N.V. Annual Report 2005, Amsterdam.

Heineken (2006b): Heineken N.V. Horizon, 1(2006), Amsterdam.

Hejazi, W. and Santor, E. (2005): Degree of Internationalization and Performance: An Analysis of Canadian Banks. Bank of Canada Working Paper 2005-32, Toronto, Ontario.

Hofstede, G. and Spangenberg, J. (1989): Technik der internationalen Vergleiche, in: Macharzina, K. und Welge, M. (Eds.), Handwörterbuch Export und internationale Unternehmung, Stuttgart: 948-963.

Holland, A. et al (2005): SABMiller plc, 09.08.2005, Dresdner Kleinwort Wasserstein Equity Research, London.

Hsu, C. and Boggs, D. J. (2003): Internationalization and Performance: Traditional Measures and their Decomposition, in: Multinational Business Review 11(2003): 23-49.

Ietto-Gillies, G. (1998): Different Conceptual Frameworks for the Assessment of the Degree 
of Internationalization: An Empirical Analysis of Various Indices for the Top 100

Transnational Corporations, in: Transnational Corporations, 7(1998): 17-39.

InBev (2005): InBev Annual Report 2004, Brussels.

InBev (2006): InBev Annual Report 2005, Brussels.

Joh.Barth\&Sohn (2005): The Barth Report. Hops 2004/05, Nuremberg.

Johanson, J. and Vahlne, J.-E. (1977): The Internationalization Process of the Firm: A Model

of Knowledge Development and Increasing Foreign Market Commitments, in: Journal of International Business Studies 8(1977): 23-32.

King, J. L. et al (2002): A Tale of Two Mergers: What We Can Learn from Agricultural Biotechnology Event Studies, in: AgBioForum 5(2002): 14-19.

Kogut, B. (1985): Designing Global Strategies: Profiting From Operational Flexibility, in: Sloan Management Review, 26(1985): 27-38.

Kutschker, M. and Schmid, S. (2004): Internationales Management, München.

Lawler, K. and Lee, K., -P. (2003): Brewing, in: Industries in Europe: Competitions, Trends and Policy Issues, Cheltenham.

Majocchi, A. and Zucchella, A. (2003): Internationalization and Performance. Findings From a Set of Italian SMEs, in: International Small Business Journal 21 (2003): 249-268.

Markowitz, H. (1952): Portfolio Selection, in Journal of Finance 7(1952): 77-91.

Mayer, M. and Whittington, R. (2003): Diversification in Context: A Cross-National and Cross-Temporal Extension, in: Strategic Management Journal, 24(2003): 773-781.

McClure, B. (2004): The Bottom Line on Margins, URL: www.investopedia.com.

McClure, B. (2005): Understanding the Subtitles of ROA vs ROE, URL: www.investopedia.com.

Meissner, H. G. and Gerber, S. (1980): Die Auslandsinvestition als Entscheidungsproblem, in: BFuP, 3(1980): 217-228.

Oesterle, M.-J. (1997): Time-Span until Internationalization: Foreign Market Entry as a BuiltIn-Mechanism of Innovations, in: Managament International Review 37(1997): 125149.

Plato (2005): World Brewer Factfile: International Groups - Datasheets \& Consolidations, Plato Logic Limited, London.

Ramaswamy, K. (1992): Multinationality and Performance: A Synthesis and Redirection, in: Advances in International Comparative Management 7(1992): 241-267.

Ramaswamy, K. et al (1996): Measuring the Degree of Internationalization of a Firm: A Comment, in: Journal of International Business Studies 27(1996): 167-177.

Reeb, D. M. et al (1998): Systemic Risk of the Multinational Corporation, in: Journal of International Business Studies, 29(1998): 263-279.

Ritchie, L. (2003): RONA, URL: www.isixsigma.com.

Rugman, A. M. (1982): New Theories of the Multinational Enterprise, New York, St. Martin's Press.

Ruigrok, W. and Wagner, H. (2003): Internationalization and Performance: An Organizational Learning Perspective, in: Management International Review 43(2003): 63-83.

Schmidt, R. (1981): Zur Messung des Internationalisierungsgrades von Unternehmen, Kiel.

Stigler, G. (1951): The Division of Labor is Limited by the Extent of the Market. Journal of Political Economy, 59(1951): 185-193.

Sullivan, D. (1994a): Measuring the Degree of Internationalization of a Firm, in: Journal of International Business STUDIES, 25(1994): 325-342.

Sullivan, D. (1994b): The 'Threshold of Internationalization': Replication, Extension, and Reinterpretation, in: Management INTERNATIONAL Review, 34(1994): 165-186.

Swoboda, B. (2002): Dynamische Prozesse der Internationalisierung. Managementtheore- 
tische und empirische Perspektiven des unternehmerischen Wandels, Wiesbaden.

Tallman, S. and Li, J. (1996): Effects of International Diversity and Product Diversity on the Performance of Multinational Firms, in: Academic of Management Journal 39(1996): 179-196.

Todd, H. (2004): Global Beer, in: Beverage World 123(2004): 39-41.

UNCTAD (2004): World Investment Report, 2003. FDI Policies for Development: National and International Perspectives. United Nations: New York and Geneva.

Urrutia, R. and Aramoni, M. (2005): Grupo Modelo. Lime Time? 30.08.2005, ING Equity Markets, Mexico City.

Wayman, R. (2002): EBITDA: The Good, the Bad, and the Ugly, URL: www.investopedia.com.

Wayman, R. (2003): Operating Cash Flow: Better Than Net Income?, in www.investopedia.com.

Wernerfelt, B. (1984): A Resource-Based View of the Firm; in: Strategic Management Journal 5(1984): 171-180.

Winston, C. et al (2005): European Beverages Valuations. The Sweet Sixteen - Standardising Valuations Across the Beverages Sector, Merrill Lynch, London.

Winston, C. et al (2004): Brand New World. Marketing and Brands in the Global Beer Industry, Merrill Lynch, London.

Yule, M. et al (2005): Beer Appreciation: Episode 1. Morgan Stanley Research, London.

Zucchella, A. (2001): The Internationalization of SMEs: Alternative Hypothesis and Empirical Survey, in: Berry, M. et al (Eds.), Multinational in a New Era, Palgrave: 24-48. 


\section{KAPITEL III.7}

\section{Performance and Internationalization}

\section{A Comparison of Capital Market-Based and Accounting-Based Analysis}

\section{Oliver Ebneth}

Paper submitted in a shorter version to the USDA and AIEA2 International Meeting, $15^{\text {th }}$ $16^{\text {th }}$ of June 2006, Bologna. 


\section{Performance and Internationalization}

\section{A Comparison of Capital Market-Based and Accounting-Based Analysis}

\section{EXECUTIVE SUMMARY}

This study sheds some empirical light on the relationship between the degree of internationalization (DOI) and financial performance for the sample of the largest North American and European stock-listed brewing groups (LBG). This study is innovative in two ways. First, as most studies in economics and international management focus on financial service and manufacturing industries, hardly any literature exists at all about internationalizing agribusiness, food processing and consumer goods companies. Second, we do not confine our investigation to the common accounting-based procedures as has been done by the bulk of previous scholars but will enlarge this investigation by including capital market-oriented measurements. We discuss the use of event study methodology as a potentially important and useful way to assess the dynamic effects of mergers and acquisitions (M\&A) in the brewing scene. The event study data employed in the empirical analysis are daily stock returns for the brewing groups involved in 21 M\&As between March 2000 and July 2005. The accounting-based examination is based on financial data provided in the brewing groups' annual reports for the six financial years from 1999 to 2004 .

The outcomes of both methodologies adopted in this investigation are in line with the existing literature on internationalization-performance studies in the finance and economic literature. Shareholder returns to the bidding companies oscillate around zero on the announcement day although cumulated abnormal returns for the whole sample have not shown any statistical significance. Indeed, there are some brewers whose M\&A transactions did not meet shareholder's expectations. But, at the same time, many acquisitions have created significant value for some of the fastest growing brewers in our sample. Furthermore, there seems to be a clear link between speed of external growth and a significant boost in brewer's profitability. Brewing groups show substantial improvements in both their net sales and EBITDA figures following this period of intensive external growth. Regarding the decomposition of our performance indicators, our work is new and adds detail to our knowledge about the internationalization-performance relationship. Furthermore, we discuss the development of the brewers' market capitalization, which is of paramount importance and represents the core element of an 
LBG's sustained corporate success by calculating and assessing the enterprise value of a brewing group. In particular, European competitors showing aggressive growth made astonishing progress over recent years. The comparison of capital market-based analysis with accounting-based measures provides evidence for the fact that both methods should not be seen as alternative ways to evaluate performance, but as complementary ones.

The implications for central management look apparent as brewing executives face a new paradigm: They have to grow faster than the market in terms of market capitalization and enterprise valuation, whether to become a global company or to remain one. Otherwise, they run the risk of losing their independance and of being acquired by more successful brewing groups. Heineken, SABMiller and InBev in particular have proven how effective M\&A transactions can contribute to satisfying this new paradigm.

\begin{abstract}
This analysis will assess the internationalization-performance relationship with regard to both aspects of measuring corporate success: Firstly, a capital market-based event study on the performance implications of 21 recently transacted cross-border mergers and acquisitions by leading stock-listed brewing groups, and secondly, an evaluation of the investigated brewers' financial performance through compiling significant key data and financial ratios. The assumption thereby is that a link between the brewers' increased international engagement through intensive cross-border mergers and acquisitions and their corporate success can be revealed. Managerial implications as well as future research propositions conclude this paper.
\end{abstract}

\title{
JEL Classification System
}

G140 Information and Market Efficiency; Event Studies

G340 Mergers; Acquisitions; Restructuring; Voting

L25 Firm Performance: Size, Age, Profit, and Sales

M41 Accounting

\section{KEYWORDS}

Internationalization, Corporate Success, Mergers\&Acquisitions, Brewing Industry, Balance Sheet Analysis, Event Study Methodology. 


\section{Problem Statement}

Acquisitions have been the growing trend in recent years, giving brewers the opportunity to enhance their degree of internationalization and market share remarkably through diverse oneoff deals. Larger brewers are faced with low prospects for volume growth in developed markets, leading them to seek growth through either acquisition of other brewers or aggressive participation in developing markets, or both. In the world brewing scene over recent years, we can detect a clear shift towards increased speed of global consolidation. The spearhead of international business expansion has been the peer group of leading European brewing groups. But there are clear signs that this concentration process has also seized North American groups, resulting in an increased drive to close the gap between themselves and their European rivals.

Although there has been significant development in the theory of international business and there exists a substantial empirical literature on the causes and outcomes of foreign direct investment, researchers still suffer from a limited understanding of how the degree of internationalization affects firm performance. The evidence is mixed in explaining the effects of overseas expansion on firm performance. This paper contributes to the existing literature in two ways. First of all, it broadens the industrial scope of the literature on the link between corporate success and internationalization as most of the existing literature focuses on banking or other financial service industries (Jensen, 1992; Agrawal and Jaffe, 2000; Putlitz, 2001; Beitel, 2002), whereas this paper specifically addresses the impact of the internationalization impetus within the food industry and, more specifically, the brewing industry on firms' accounting performance. The outcome of the study is generally consistent with findings in many previous studies. Second, we do not confine our investigation to the common accountingbased procedures as has been done by previous studies but enlarge our investigation by including capital market-oriented measurements.

Our paper is structured as follows. Chapter 2 discusses the general scientific and practical objectives. Chapter 3 gives an overview of the relevant literature on previous findings of internationalization-performance investigations. Chapter 4 illustrates the competition background within the North American and European brewing industries. Chapter 5 portrays our study's sample. Chapter 6 summarizes recent developments in M\&A activities within the global brewing sector. Chapter 7 describes the methodologies adopted in this analysis. Chapter 8 presents the results, which are discussed in Chapter 9. A concluding section closes our 
examination and gives managerial implications as well as recommendations for future research.

\section{OBJECTIVES}

The general tenet held in this investigation is that we assume that a link-whether positive or negative - can be detected between rising internationalization and companies' financial performance. As external expansion through M\&A activities is the fastest way to boost corporate growth, we hypothesize that M\&As in the brewing scene are the core instrument adopted by leading players in globalizing the world brewing industry. It follows that a boost in M\&A activity should correlate with a brewer's financial performace-whether measured in rising stock prices or in accounting profit.

What are the primary motivations for mergers and acquisitions in the brewing scene? The amount of research necessary to answer this question has grown proportionately with the growth of M\&A activities (Shusterman et al, 2001). The proponents of the value-creation of M\&As claim that mergers and acquisitions enable an enterprise to enhance economies of scale and scope that give it the capability to remain competitive in an increasingly global economy. Berger et al identify three categories of benefits that may motivate cross-border M\&As: scale and scope economies, access to safety net subsidies and market power. These categories assume wealth-maximation as the primary motive for the M\&A deal. The efficiency argument is that M\&As can change input or output quantities in ways that reduce costs, increase revenues, and/or reduce risks to increase value for a given set of prices (Berger et al, 1999; Carow and Kane, 2001). Specific efficiencies are economies of scale, which permit combined institutions to obtain more business or produce services at lower average costs; economies of scope, which are created when the combination of two or more separate products or services enhances revenues or costs; and x-efficiency gains, which result when the efficient LBG merge and reform the practices of less efficient brewers (Baltazar and Santos, 2003). Further reasons for M\&As are gain of monopoly power and tax synergies, as well as a number of agency motives such as growth maximization, free cash flow and employment risk reduction (Mueller and Sirower, 2003). In the brewing industry context, these synergies include discontinued operations and closures of breweries, plant optimization strategies for remaining brewing facilities and programs to improve operating productivity and procurement 
synergy around the globe for all direct and indirect purchases (InBev, 2005). Last but not least, there is a 'defensive' reason for M\&A activity: to avoid becoming the target of a hostile takeover.

The methodology often used implicitly assumes that internationalization is the cause of observed firm value or firm performance - that is, it is implicitly assumed that increasing DOI has a direct impact on firm performance. Although it is true that, in part, the causality may move from DOI to performance, the aforementioned assumption ignores a very important aspect of the theory in international business that firms go abroad to exploit firm-specific advantages. That is, firms develop techniques and products that give them some competitive advantage, which then allows the innovating firm to perform well in the domestic market. These firms then move abroad through FDI and other modes to exploit these firm-specific advantages (Contractor et al, 2003; Hsu and Boggs, 2003; Hejazi and Santor, 2005).

Indeed, the specific internationalization steps of single-stock-listed brewing groups, such as SABMillers' recent spectacular acquisition of Columbian brewer Bavaria for $€ 6.5$ billion, have received substantial public interest. Nevertheless, there has been a lack of rigorous research using quantitative indicators to capture facets of the degree of internationalization and relationships between the extent of internationalization and financial performance measures (Gerpott and Jakopin, 2005). Moreover, as most studies focus on financial service and manufacturing industries, there is hardly any literature at all about internationalizing agribusiness, food and consumer goods companies. Therefore, the first purpose of this investigation is to analyze the internationalization patterns of brewing groups over a number of years.

The international business literature offers a simple framework in which to measure the link between the degree of a brewing group's internationalization and its performance. The idea is that, as firms increase the share of their operations abroad, thus increasing their degree of internationalization, they experience higher levels of performance. DOI can be measured in terms of the share of foreign sales to total sales (FSTS) or the number of foreign affiliates of an LBG. Performance can be measured as return on assets, return on investment, return on equity or profitability (Hejazi and Santor, 2005). Correspondingly, the second intention of this study is to explore the internationalization-performance correlation in the sample of LBGs and to detect differences regarding this relationship between North American and European brewers.

Another goal of this paper is to provide an assessment of the success in merger and acquisition activities involving North American and European brewing enterprises. For this pur- 
pose, we further analyze the stock market response of the brewers involved in M\&A deals in the international brewing scene. More precisely, we conduct event studies of the stock market performance of these firms at different time horizons around the announcement of the respective deals. We then go beyond the event studies and perform a detailed analysis by identifying the European brewers' basic financial characteristics in terms of their operating and accounting performance (Campa and Hernando, 2005).

Implicitly assumed in studies that use DOI as a predictor of firm performance is the idea that a positive relationship is somehow sufficient to justify the movement abroad. In other words, the positive relationship is taken to imply that the move abroad has "paid off". If the movement abroad increases the risk profile of a particular firm's operations, then an increase in performance is the minimum that would be expected by shareholders. The question is whether the increase in performance is sufficient to compensate shareholders for the increased risk (Hejazi and Santor, 2005). Our analysis suggests that there is a significant but weak negative relationship between DOI and performance. However, the composition of foreign claims, in terms of risk, vis-à-vis more loans (as opposed to greater claims in the form of relatively risk-free securities), has higher returns. In other words, much of the increase in accounting performance could be compensation for the higher risk associated with negative capitalmarket-response to intensive M\&As. The implications for brewing managers and their boards are clear. If one believes that internationalization somehow improves firm performance, then corporate strategists may be led to believe that expanding abroad will lead to improvements in firm value. On the other hand, to the extent that firm values are high to begin with because of firm-specific advantages, corporate strategists will realize that internationalization is a reflection of underlying firm-specific advantages and hence high market values (Hejazi and Santor, 2005).

To summarize, we have three objectives in this paper. First, we trace the respective brewers' path of internationalization and the amount to which some brewers have driven their international engagement. Second, we strive to investigate the relationship between rising DOI and the accounting performance of our sample's companies. Finally, we argue that companies that have undergone intensive external growth over recent years due to exhaustive M\&A activities should at least benefit from accounting profit even if the stock markets' response to their M\&As has been negative. 


\section{REVIEW OF THE RELEVANT LITERATURE}

The first studies dealing with internationalization-performance relationships go back at least to Vernon and hypothesized a positive link at that time. Many studies have followed. It is generally hypothesized that internationalization is good for firms and leads to better performance for several reasons (Vernon, 1971; Dunning, 1977). First, going international implies that firms can spread fixed costs, such as operating overhead and research and development expenditures through increased scale and scope. Second, internationalization allows firms to learn about domestic markets from their international market experience, thus improving performance (Kobrin, 1991). Third, operating in foreign jurisdictions allows firms to access factors at lower cost (Porter, 1990); this is particularly true for instances of FDI and other modes of direct involvement in foreign markets. Fourth, internationalization allows firms to crosssubsidize their domestic operations and provides greater opportunities for price discrimination and tax and price arbitrage (Hejazi and Santor, 2005).

The literature so far contains many fascinating insights from experienced M\&A executives. M\&As possess several important advantages: speed of entry and speed to market, the ability to acquire critical assets, platforms for future growth and entry into geographical markets (Cooper and Gregory, 2001). Speed of entry is felt to be particularly critical in the brewing scene. At the same time, its disadvantages are significant: Asset value goes down as well as up, and cultural barriers in international acquisitions often mean that M\&As do not replace internal growth and alliances. However, the failure of M\&As is frequently very visible, whereas startups and new venture failures often go unnoticed (Ghauri and Buckley, 2003).

Although theory hypothesizes a positive relationship, the empirical evidence of the effects of DOI on performance is mixed (Hsu and Boggs, 2003). For example, Sullivan (1994a) lists 17 studies that test the relationship between DOI and financial performance, six of which find a positive relationship and five a negative one. The remaining six find no relationship between the two. This reflects the consensus in the literature that the empirical results are highly dependent on the sample, the measures of DOI, the measures of performance used and the industry in which the investigation is conducted.

Event studies are a research tool developed thirty years ago to measure the impact of regulatory events; this method is used frequently in financial services studies. An event study attempts to measure abnormal changes in the stock prices of publicly traded companies that 
occur in conjunction with an "event", such as an M\&A announcement. Since the price of those stocks is set in an auction market that is as close as we can come to the economic concept of a "perfect market", the price of those publicly traded stocks should reflect the reaction of the financial markets to the introduction of new corporate information (Wells, 2004). As event study methodology is a widely used research methodology in the field of financial studies and international management sciences, there is a plethora of investigations into the financial services industries in general. At the same time, the food-processing industry is widely under-investigated. With this in mind, we confine our literature review to the relevant studies conducted in the food industries.

There are a number of event studies that deal with food and agribusiness concerns. The relevant event studies can be grouped as follows (Golub et al, 2004). The first group of studies includes research devoted to quantifying the effects of food safety issues on the prices of contaminated products (McKenzie and Thomsen, 2001; Lusk and Schroeder, 2002). Lusk and Schroeder (2002) examined the effect of meat recalls on live cattle and lean hog futures prices. They found that the announcement of recalls did not have a strong effect on either price series. McKenzie and Thomsen (2001) examined the impact of beef recalls due to contamination on wholesale and farm-level beef prices and found that the prices of boneless beef reacted negatively to recalls. However, there was no reaction in the live cattle prices and very little reaction in the boxed beef prices. The second group includes literature investigating the effects of food safety events on the stock prices of the involved firms (Salin and Hooker, 2001; Henson and Mazzocchi, 2002). Salin and Hooker (2001) quantified the firm-specific effects of incidents of microbiological contamination of food, which lead to immediate health concerns for consumers. They found that returns fell after the recall only for the smallest firm in the study and concluded that financial markets reacted in a limited way to certain food recalls. Henson and Mazzocchi (2002) examined the impact on agribusinesses of the United Kingdom government's announcement of a possible link between Bovine Spongiform Encephalopathy and human health. In order to assess the diffusion impact in the industry, another recent study investigated the effects of Starlink food safety events on the risks and the returns of food and agribusiness firms that were not only directly involved but also indirectly affected by the incidents (Golub et al, 2004).

As one can see from the aforementioned studies, some investigations into the agribusiness industry have been conducted, but these focused primarily on food recalls and contaminations. Nevertheless, as our literature review has revealed, there are no regular event studies on the 
impacts of changes in corporate policy, such as M\&As, among food processing companies. The only study about M\&A activity in the overall agribusiness industry was conducted by King et al in 2002. They measured the response of financial markets to changes in the agricultural biotechnology industry resulting from mergers and acquisitions. Their results detected positive cumulative deviations but were not significantly different from zero (King et al 2002).

However, the lion's share of event studies in the finance and economic literature concern financial services and, less frequently, manufacturing MNCs in general (Bromiley et al 1988; Simpson and Hosken, 1998; Lepetit et al 2002; Panayides and Gong, 2002; Baltazar and Santos, 2003; Harrison et al 2005).

In a deep review of the relevant literature, we found only one study in the finance and international management literature that dealt with both measures of corporate success. It was carried out by Campa and Hernando (2005) and investigated M\&A performance in the European financial industry. Therefore, our study breaks new scientific ground in two ways: Firstly, it broadens the industrial scope of event studies to the consumer goods industry, and, secondly, it combines both measures of corporate success - capital market-oriented and accountingoriented methodologies.

\section{COMPETITION BACKGROUND: US VERSUS WeSTERn EuROPEAN BEER MAR- KETS}

The world beer market totaled 1.55 billion hectoliters in 2004. Generally, global beer consumption has quickened over the last decade. The trend line picked up from just below $2 \%$ per annum to more than $2.4 \%$ (CAGR = compound annual growth rate) since the beginning of the 1990 s, reaching an extraordinary expansion of nearly $5 \%$ in 2004 . But this positive trend is being driven mainly by the increasing importance of fast growing emerging markets, such as China and Russia. Matured markets, like the US and particularly Western Europe, have been stagnating or even shrinking for more than a decade. But still, taken together, the size of these markets' is well ahead of the rest of the world. Together, the North American (USA and Canada) and Western European markets account for $36.9 \%$ of world beer production (16.5\% and $20.4 \%$ respectively). Thus, more than a third of the total world beer volume is still consumed in these Western markets. Figures 1 and 2 illustrate the developments in beer consumption and per capita consumption in the North American and Western European beer markets. 
Figure 1: North American Beer Consumption and Per Capita Consumption.

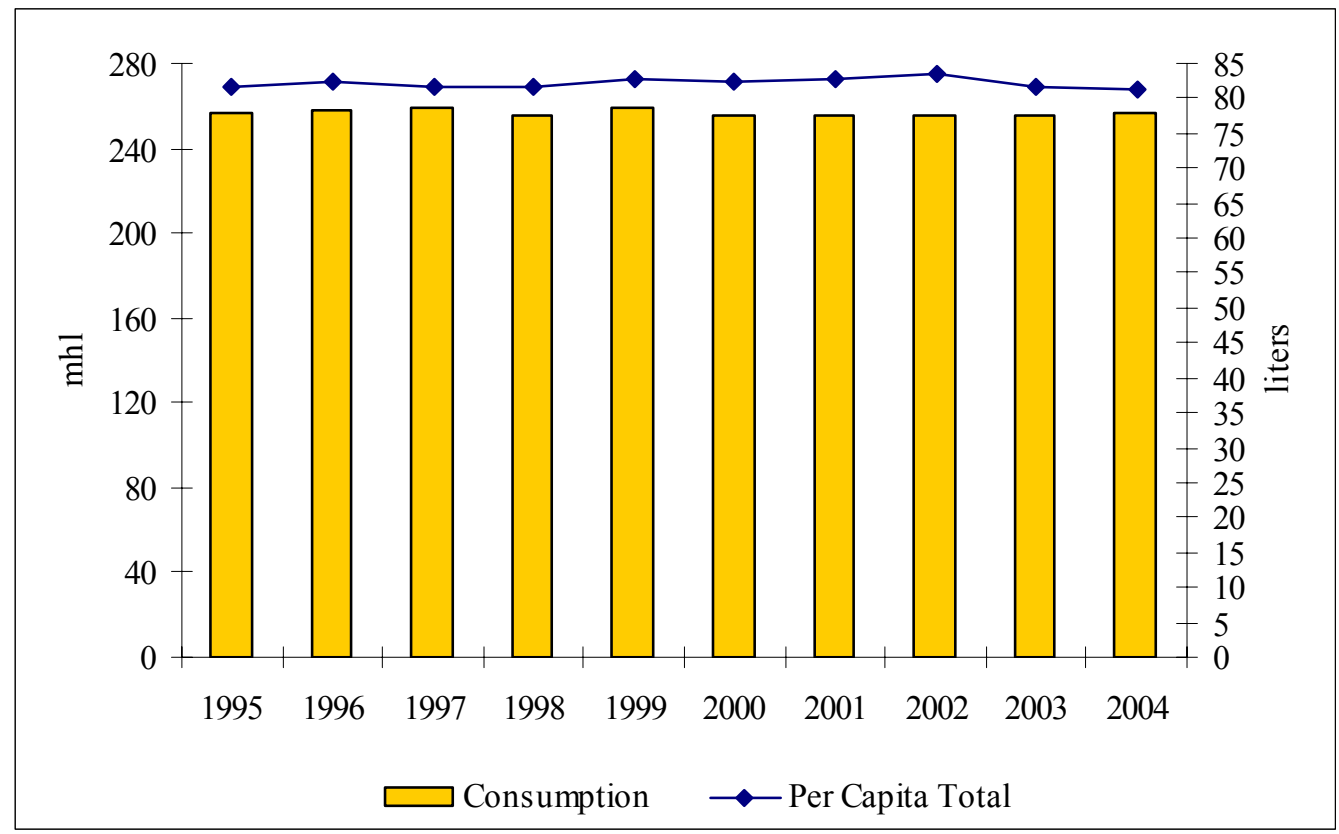

Source: (Joh.Barth\&Sohn, 2005; Plato, 2005a).

Figure 2: West European Beer Consumption and Per Capita Consumption.

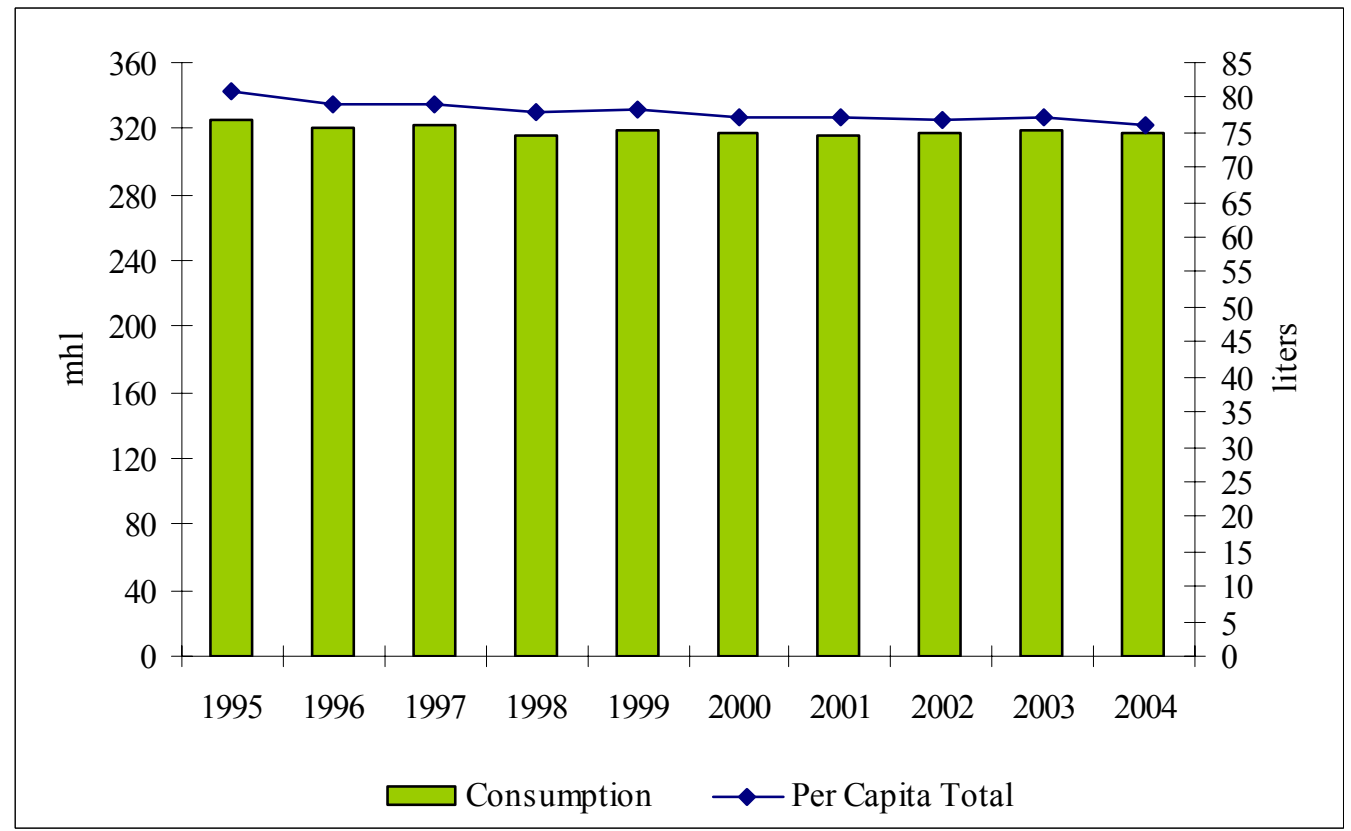

Source: (Joh.Barth\&Sohn, 2005; Plato, 2005b).

In both markets we can see relatively stagnant or slightly decreasing trends in terms of total production volume. But, in the Western European beer markets, a clearly declining trend line in per capita consumption can be detected. This negative trend is being driven mainly by the 
still dominant but declining German market, whose volume is decreasing by $1 \%$ each year. This development has early led large European brewing groups to seek business opportunities beyond their small domestic markets. In contrast, the USA has long guaranteed enough scope for modest but satisfying growth and high-scaled profitability. The USA is now the second largest beer market worldwide, having been overtaken by China in 2003 but still offering a consumption of 233 million hectoliters (mhl). This amount is based on a relatively high, stable per capita consumption as well as slight population growth. Much more impressive than total volume figures is total consumer expenditure on beer, which exceeded US\$60 billion in 2004, and the total US profit-pool (expressed in EBITDA) is estimated at more than US\$5 billion (Plato, 2005a). Thus, the USA is still by far the most profitable beer market worldwide. Consenquently, US brewers were forced to go abroad later than their European counterparts. That seems to be a central reason why North American brewers are much less internationalized than their European competitors, as will be shown in the following chapters.

\section{SAMPLE}

\subsection{NORTH AMERICAN BREWING GROUPS}

\section{Molson Coors Brewing Company}

Molson Coors Brewing Company (MCBC) is the third largest brewer in the US and ranks fifth internationally, producing a combined $59 \mathrm{mhl}$ in 2004 . Coors began as a regional brand in the American West, expanding to all fifty states by 1991. Coors' brands are now available in 31 international markets. In February 2002, Coors closed its acquisition of Carling Brewers in the UK for $€ 1.9$ billion, and in February 2005, completed a $€ 3.2$ billion merger with Molson, creating Molson Coors. Having started as a company with $75 \%$ of its volume in one brand (Coors Light) and 95\% in one market (the US), MCBC has now become the second largest brewer in the UK behind S\&N and the number one market share leader in Canada, with 58\% of its sales outside the U.S in 2004 (Bevan and Greenberg, 2005).

\section{Anheuser-Busch}

Anheuser-Busch (A-B) is the largest brewer in the world as measured by sales revenue, profitability or market value, despite being overtaken by InBev in volume terms. AB's annual beer sales of $144 \mathrm{mhl}$ in 2004 are equal to around $9 \%$ of total global beer production. Its operations are, however, heavily concentrated on the United States, which accounts for $77 \%$ of its beer volume. $\mathrm{AB}$ has indirect exposure, however, to the Argentine, Chilean, Chinese and Mexican 
markets through its 20\% stake in Chilean brewer CCU (operations in Chile and Argentina), its $27 \%$ ownership of Chinese Tsingtao and 100\% ownership of Harbin Brewery in China and its $50.2 \%$ stake in the Mexican brewer Modelo (Bevan and Greenberg, 2005).

\subsection{EUROPEAN BREWING GROUPS}

\section{InBev}

Belgium/Brazilian InBev is the largest brewer in the world by volume, having been created in September 2004 by the merger of Interbrew with AmBev, the leading player in Brazil. It has pursued an acquisition-led strategy, with a concentration on acquiring local breweries and brands in each market in order to attain a strong market share with mainstream-priced local beers. It leverages its local distribution strength by adding its worldwide flagship premium brands, Stella Artois and Becks, the German lager brand acquired in August 2001 for approximately $€ 1.8$ billion. The company has operations spanning Western and Eastern Europe, North and South America and the Asia Pacific region. It is the largest brewer in Belgium, Bulgaria, Croatia, Cuba, Luxembourg, Montenegro and the Ukraine, and the number two brewer in Canada, the Czech Republic, Hungary, Holland, Russia and South Korea. It is also number one in Brazil and Argentina following the acquisition of AmBev. It sold its stake in Mexican brewer Femsa in 2004 (Holland et al, 2005; Oldroyd et al, 2005).

\section{SABMiller}

SABMiller is the world's second-largest brewer by volume with $152 \mathrm{mhl}$ in 2004. SABMiller has a 98\% market share in South Africa and operates in more than forty other countries. The company's brands include the Miller brand family in the U.S., Peroni in Italy, Pilsner Urquell in the Czech Republic and Castle in South Africa. Incorporated in London in 1999, the company was renamed SABMiller in 2002 after the merger of South African Breweries and the US-based Miller Brewing Company for approximately $€ 6.2$ billion. Miller contributed 23\% of group EBITDA in the financial year 2004 in its second full year under the ownership of SABMiller. In July 2004, SABMiller announced its acquisition of the listed Colombian brewer Bavaria at an enterprise value of $€ 6.5$ billion. Bavaria has dominant market positions in Colombia, Peru, Ecuador and Panama and offers SABMiller a platform to expand in the attractive South American continent (Bevan and Greenberg, 2005).

\section{Heineken}

Heineken is the world's fourth-largest brewer by volume after InBev, SABMiller and An- 
heuser-Busch. Heineken sold $112 \mathrm{mhl}$ of beer in 2004 through wholly owned companies, affiliates or license agreements. This ranks the group as the fourth-largest international brewer but the number one brewer in Europe. This European leadership position was strengthened by the acquisition of the Central European brewer, BBAG, in 2003. Its main brands are the most international beer brand, Heineken (22\% of group volume), and Amstel (11\%), and it also holds a full portfolio of local brands in many markets. Heineken's largest region is Western Europe, where it is the number one brewer in the Netherlands, Spain, Italy, Poland and Slovakia and number two in France, Ireland, Switzerland and Bulgaria. It is one of the largest brewers in Africa, with a concentration in the Central and Western states. It has a large-scale import business in the US. In Asia its 42.5\%-owned associate Asia Pacific Breweries is either number one or number two across a broad spread of markets. In 2004 and 2005 the Dutch brewing group acquired several breweries in Russia, making it number three there, with a market share of $10 \%$ in that fast growing market (Holland et al, 2005).

\section{Carlsberg}

Carlsberg is the world's fifth-largest brewing group; 90\% of its beer sales are made outside Denmark. It produces beer at 67 sites in 42 countries. In 2000 it merged its brewing operations with those of Orkla by acquiring 100\% control of the merged entity Carlsberg Breweries in 2004. Its main markets are located primarily in Western and Northern Europe, Russia and Asia. The company also owns an attractive 50\% stake in Baltic Beverage Holding (BBH), the foremost brewer in Russia with additional interests in the Ukraine, the Baltic states and Kazakhstan, which represents an important growth engine (Holland et al, 2005). In addition, Asia is a region where Carlsberg has already been present for some years. The Asian markets present future potential for growth, but they still generate a small percentage of group profits (Lastra and Wiliamson, 2005).

\section{Scottish\&Newcastle}

Scottish\&Newcastle (S\&N) is the seventh-largest brewer in the world, with activities concentrated in Western and Northern Europe. It is the largest brewer in the UK, with a $29 \%$ market share and a focus on the Fosters brand (to which it has just recently bought $80 \%$ of the European rights in perpetuity, John Smith's and Kronenbourg. Due to its ownership of Brasseries Kronenbourg, it is the largest brewer in France, with a $40 \%$ market share. It owns $50 \%$ of $\mathrm{BBH}$, the leading brewer in Russia. (Carlsberg owns the other 50\%.) It also owns brewing operations in Finland, Belgium and Portugal and has minority investments in Greece, India 
and China (Holland et al, 2005).

Figure 3 shows the ranking of the world's twenty largest publicly listed brewing groups and the proportionate share of our sample's brewers. The CR20 (CR10) in 2004 was 69.7\% (57.5\%). It has to be pointed out that we have provided Coors' and Molsons' volume figures separately even though these companies merged into MCBC in 2004. This is because each continued to publish its own annual report until 2004.

Figure 3: Ranking of the World's Largest Brewers in Terms of 2004 Production Volume

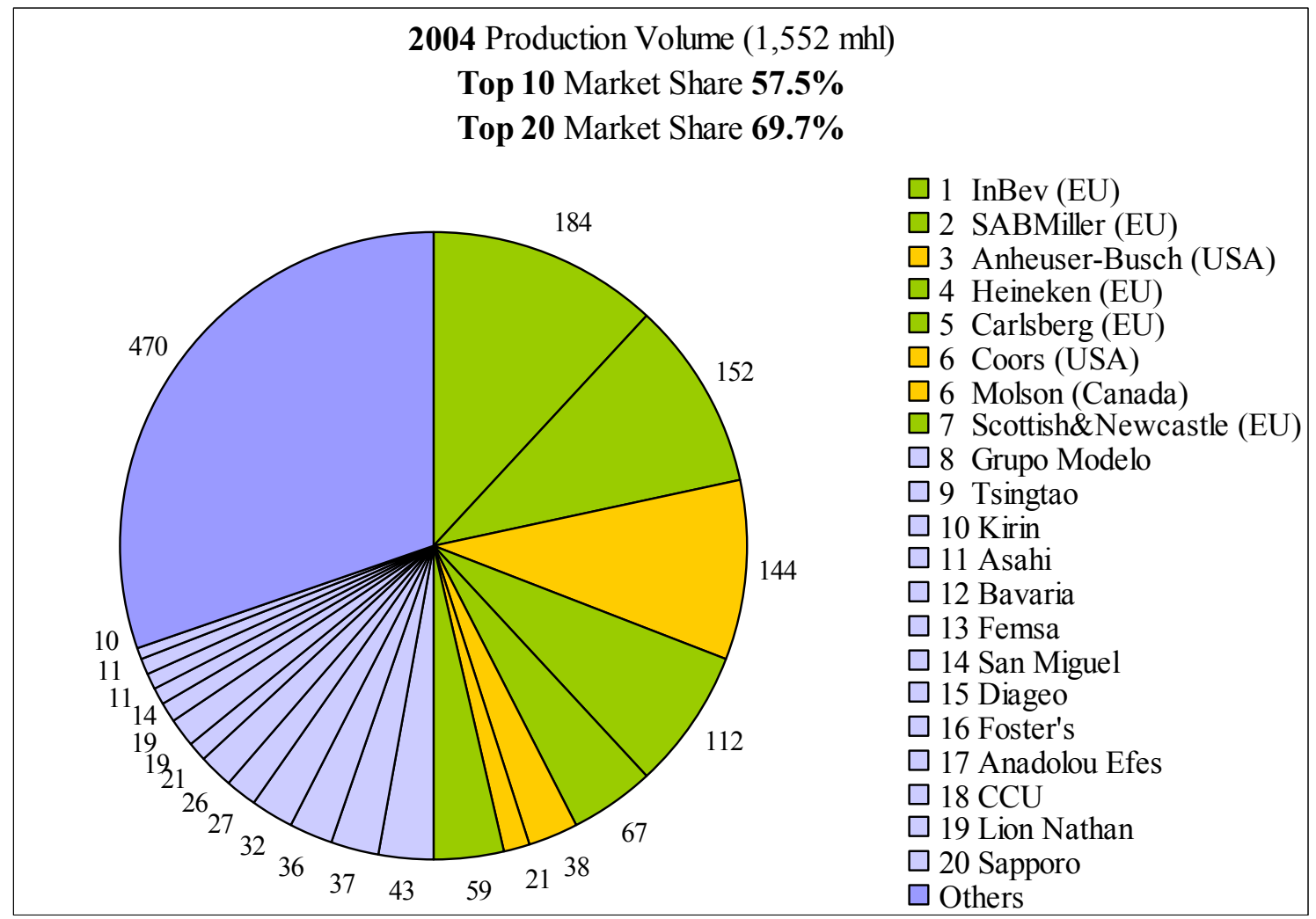

Source: (Joh.Barth\&Sohn, 2005; Plato, 2005c).

Table 1 illustrates some operating statistics for our sample, which consists of the three largest North American brewing groups as well as the five leading European brewers. As mentioned above, we have analyzed Molson and Coors separately as MCBC will produce its first joint annual report for the financial year 2005. Together, the sample's brewing groups cover exactly $50 \%$ of the total world beer production in terms of production volume $(777 \mathrm{mhl}$ out of $1,552 \mathrm{mhl})$. The whole sample's market capitalization at year-end 2004 was roughly $€ 97$ billion (average $=€ 12,123$ million; s.d. $=€ 9,298$ million) and the total enterprise value (= mar- 
ket capitalization + net debt + minorities - associates) was about $€ 118$ billion (average $=$ $€ 14,761$ million; s.d. $=€ 9,586$ million). Net sales totaled more than $€ 53$ billion (average $=$ $€ 6,701$ million; s.d. $=€ 3,577 \mathrm{n}$ ), the EBITDA totaled roughly $€ 12$ billion (average $=€ 1,541$ million; s.d. $=€ 1,108$ billion), EBIT was $€ 8$ billion (average $=€ 993$ million; s.d. $=€ 855$ million) and the net profit generated by the sample's brewing groups rose above $€ 4$ billion (average $=€ 551$ million; s.d. $=€ 597$ million)

Table 1: Operating Statistics of the Investigated Brewing Groups, 2004

\begin{tabular}{|c|c|c|c|c|c|c|c|c|c|}
\hline & Country of & & $\begin{array}{c}\text { Volume } \\
(\mathrm{mhl}) \\
\end{array}$ & $\begin{array}{c}\text { Market } \\
\text { Cap }(€ m) \\
\end{array}$ & $\begin{array}{r}\text { Enterprise } \\
\text { Value }(€ \mathrm{~m}) \\
\end{array}$ & $\begin{array}{c}\text { Net Sales } \\
(€ \mathbf{E m}) \\
\end{array}$ & $\begin{array}{c}\text { EBITDA } \\
(€ \mathbf{\epsilon m}) \\
\end{array}$ & $\begin{array}{l}\text { EBIT } \\
(€ \mathrm{~m}) \\
\end{array}$ & $\begin{array}{c}\text { Net Profit } \\
(€ \mathrm{~m}) \\
\end{array}$ \\
\hline Company & Incorporation & & 2004 & Sep 05 & Sep 05 & 2004 & 2004 & 2004 & 2004 \\
\hline & & & (b) & (c) & (d) & & & & \\
\hline Anheuser Busch & USA & & 144.2 & 27,874 & 29,689 & 12,006 & 3,452 & 2,702 & 1,801 \\
\hline Coors & USA & & 38.4 & 4,392 & 6,610 & 3,462 & 489 & 275 & 166 \\
\hline \multirow[t]{2}{*}{ Molson } & Canada & & 21.0 & 2,905 & 3,585 & 1,589 & 362 & 322 & 149 \\
\hline & & $\sum$ & 203.6 & 35,170 & 39,884 & 17,058 & 4,303 & 3,299 & 2,116 \\
\hline Heineken & Netherlands & & 112.0 & 13,190 & 16,304 & 10,005 & 2,021 & 1,248 & 537 \\
\hline InBev & Belgium & & 183.7 & 19,430 & 24,878 & 8,568 & 2,325 & 1,310 & 719 \\
\hline SABMiller & UK & & 151.9 & 19,307 & 20,678 & 8,422 & 2,156 & 1,424 & 906 \\
\hline Carlsberg & Denmark & & 67.1 & 3,697 & 7,062 & 4,835 & 806 & 306 & 75 \\
\hline \multirow[t]{2}{*}{ Scottish \& Newcastle } & UK & & 58.5 & 6,193 & 9,285 & 4,719 & 717 & 355 & 59 \\
\hline & & $\Sigma$ & 573.2 & 61,817 & 78,207 & 36,548 & 8,025 & 4,643 & 2,296 \\
\hline Max & & & 183.7 & 27,874 & 29,689 & 12,006 & 3,452 & 2,702 & 1,801 \\
\hline Average & & & 97.1 & 12,123 & 14,761 & 6,701 & 1,541 & 993 & 551 \\
\hline Median & & & 89.6 & 9,692 & 12,794 & 6,628 & 1,414 & 802 & 351 \\
\hline S.d. & & & 59.2 & 9,298 & 9,586 & 3,577 & 1,108 & 855 & 597 \\
\hline Min & & & 21.0 & 2,905 & 3,585 & 1,589 & 362 & 275 & 59 \\
\hline$\sum$ Total Sample & & & 776.9 & 96,987 & 118,091 & 53,606 & 12,328 & 7,942 & 4,412 \\
\hline
\end{tabular}

\footnotetext{
(a) Molson and Coors announced an agreement to combine in a merger of equals in July 2004

(b) Volume in million hectoliters (mhl)

(c) Market capitalization $=$ Number of outstanding common shares of the firm * current price of those shares

(d) Enterprise Value $=$ Market Capitalization + Net Debt + Minorities - Associates
}

Source: Company data, authors' calculations 


\section{ReCENT TREnds IN M\&A ACTIVITIES WITHIN THE BREWING SECTOR}

Risks facing the global brewing scene vary depending on the geographic location of the operations. In the developed markets the main challenge is driving organic revenue growth, often against the background of a weak consumer environment and a rising demand for wine and spirits. In the emerging markets the situation is more buoyant at present-although these regions will typically be more volatile over a period of time. If we look back over recent years, the key theme in the global brewing sector has been the ever widening contrast in trading conditions between emerging and developed markets. We would argue that these contrasting trading conditions have not only shaped the respective financial (and share price) performances of the leading brewers but have also influenced the companies' strategic decision making. Specifically, the desire to gain an increased exposure to these buoyant emerging markets has been reflected in the scramble to secure the remaining independent quality assets in places as diverse as China, Colombia and Russia (Bevan and Greenberg, 2005). In our sample, SABMiller is the company with the largest exposure (ca. $75 \%$ ) to emerging markets among leading brewing groups, followed by InBev (ca. 65\%), Heineken (ca. 39\%), Carlsberg (ca. $38 \%$ ) and Scottish \& Newcastle (ca. 21\%). The North American groups Anheuser-Busch (ca. $16 \%$ ) and $\mathrm{MCBC}$ (ca. 5\%) are still mainly operating in their developed markets.

In the course of the last decade, the brewing industry has evolved from an industry that concentrated on domestic markets to one that views itself as part of a global market (Karrenbrock, 1990; Yule et al, 2005). Overall M\&A activity among the peer group of worldwide leading brewing groups, measured by transaction value, experienced a significant boost after 1998. This increase was also perceptible in the steep rise in the number of transactions over the last decade, which has been a major force behind the brewing industry's consolidation. In the early 2000 s, invested volumes increased as these transactions were more aggressive in pursuing market access and enhancing the competitive position of the firms involved in the by now more integrated national markets (Campa and Hernando, 2005; Yule et al, 2005). The consolidation process has picked up pace in recent years, and there are some clear signs that this process will continue to gather speed due to the aforementioned hunt for businesses going on in the fast-growing emerging markets, which still offer opportunities for M\&A activities. One reason for this phenomenon is that the biggest international brewing groups are well capitalized and are all seeking further external growth. Second, there has never been a more opportune time for them to try to establish stronger positions in the emerg- 
ing markets or to attempt to find synergistic deals in their home territories (Bevan and Greenberg, 2004).

According to various brokers the leading players in the brewing scene have spent more than $€ 75$ billion in around $400 \mathrm{M} \& \mathrm{~A}$ transactions in the course of only the last five years (Rössing, 2005). Table 2 underscores these impressive figure and discloses transaction multiples of the $21 \mathrm{M} \& \mathrm{~A}$ deals investigated in the study period between 2000 and 2005. The total transaction value of these 21 deals added up to $€ 62$ billion, whereas the average transaction price was $€ 2,955$ million (s.d. $=€ 3,667$ million). The M\&A's average sales-multiple ${ }^{1}$ was 2.11, the EBITDA-multiple (or hl-multiple) totaled 10.2 (171).

Table 2: Transaction Multiples of the 21 M\&As Analyzed in the Brewing Scene, 2000-2005

\begin{tabular}{|c|c|c|c|c|c|c|c|c|}
\hline \multirow{2}{*}{$\begin{array}{l}\text { Date } \\
\text { Announced }\end{array}$} & \multirow[b]{2}{*}{ Acquiror } & \multirow{2}{*}{$\begin{array}{l}\text { Country of } \\
\text { Incorporation }\end{array}$} & \multirow[b]{2}{*}{ Target } & \multirow[b]{2}{*}{$\%$ Acquired } & \multirow{2}{*}{$\begin{array}{r}\text { Transaction } \\
\text { Value (€m) }\end{array}$} & \multicolumn{3}{|c|}{ Transaction Value as a Multiple of: } \\
\hline & & & & & & Sales & EBITDA & $\mathrm{hl}(€ / \mathrm{hl})$ \\
\hline 19-Jul-05 & SABMiller & UK & Bavaria & $100 \%$ & 6,473 & 4.38 & 10.0 & 243 \\
\hline 12-Aug-04 & InBev & Belgium & SunInterbrew & $91 \%$ & 1,212 & 2.05 & 11.0 & 65 \\
\hline 22-Jul-04 & Coors & USA & Molson & $50 \%$ & 3,200 & 2.01 & 8.8 & 152 \\
\hline 15-Jun-04 & Anheuser-Busch & USA & Harbin & $29 \%$ & 640 & - & - & - \\
\hline 03-Mar-04 & Interbrew & Belgium & Ambev & $58 \%$ & 16,341 & 3.50 & 11.5 & 146 \\
\hline 19-Feb-04 & Carlsberg & Denmark & Orkla & $40 \%$ & 7,054 & 1.52 & 8.6 & 87 \\
\hline 07-Jan-04 & Interbrew & Belgium & Oriental Brewery & $45 \%$ & 1,547 & - & 10.7 & 201 \\
\hline 18-Sep-03 & Interbrew & Belgium & Spaten & $100 \%$ & 533 & 1.49 & 9.9 & 109 \\
\hline 13-May-03 & S\&N & UK & Centralcer & $51 \%$ & 828 & 3.32 & 11.4 & 251 \\
\hline 13-May-03 & SABMiller & UK & Peroni & $60 \%$ & 563 & 1.14 & 12.6 & 128 \\
\hline 02-May-03 & Heineken & Netherlands & BBAG & $100 \%$ & 1,899 & 1.73 & 10.2 & 146 \\
\hline 14-Jan-03 & Heineken & Netherlands & $\mathrm{CCU}$ & $31 \%$ & 1,525 & 2.83 & 11.3 & 151 \\
\hline 15-Nov-02 & Interbrew & Belgium & Gilde & $100 \%$ & 575 & 1.80 & 8.6 & 126 \\
\hline 30-May-02 & SAB & South Africa & Miller & $100 \%$ & 6,194 & 1.04 & 9.1 & 90 \\
\hline 14-Feb-02 & $\mathbf{S \& N}$ & UK & Hartwall & $100 \%$ & 2,273 & 2.81 & 10.1 & 142 \\
\hline 24-Dec-01 & Coors & USA & Carling & $100 \%$ & 1,919 & - & - & - \\
\hline 29-Nov-01 & SAB & South Africa & BevCo & $60 \%$ & 561 & 1.60 & 6.5 & 519 \\
\hline 06-Aug-01 & Interbrew & Belgium & Becks & $100 \%$ & 1,790 & 2.11 & 13.0 & 206 \\
\hline 14-Jun-00 & Interbrew & Belgium & Bass & $100 \%$ & 3,611 & 1.25 & 9.7 & 206 \\
\hline 03-Nov-00 & Carlsberg & Denmark & Feldschlösschen & $100 \%$ & 574 & 1.60 & 8.6 & 99 \\
\hline 20-Mar-00 & $\mathbf{S} \& \mathbf{N}$ & UK & Kronenbourg & $100 \%$ & 2,744 & 1.80 & 11.3 & 183 \\
\hline Max & & & & $100 \%$ & 16,341 & 4.38 & 13.0 & 519 \\
\hline Average & & & & $77 \%$ & 2,955 & 2.11 & 10.2 & 171 \\
\hline Median & & & & $100 \%$ & 1,790 & 1.80 & 10.1 & 146 \\
\hline S.d. & & & & $28 \%$ & 3,667 & 0.91 & 1.6 & 99 \\
\hline Min & & & & $29 \%$ & 533 & 1.04 & 6.5 & 65 \\
\hline
\end{tabular}

Source: Company press releases, author's calculations

\footnotetext{
${ }^{1}$ This means that the average sample's transaction value was 2.11 times the target's net sales value.
} 
Thus, the world brewing sector has been going through a period of unprecedented change in recent years with a burst of corporate activity-mainly M\&As - in the last few years. The acquisitions of BBAG by Heineken and Centralcer by Scottish\&Newcastle, Carlsberg's Orkla deal, the merger between AmBev and Interbrew (which created the world's largest brewer, InBev) and Coors' and Molson's merger have highlighted the apparent urgency among major brewers to build scale and improve their strategic positioning (Blythman et al, 2003).

Thus, takeover activities have played an important role in the consolidation of the world brewing industry over the past decade and appear likely to continue to do so in the future. A brewer's objective in such a takeover is to extend its business into geographic areas where the firm has so far had no presence. This tactic is typically employed by firms that are rolling up a fragmented industry (Schweiger and Very, 2003). The general tenet held by many industry executives and consultants is that more extensive international business operations coincide with above-average financial success (Glaum, 1996; Annavarjula and Beldona, 2000).

Concerning the geographical scope of the merger deals in the world brewing industry, most of the transactions were cross-border deals, caused by the expansion of brewers into different emerging economic areas, like Latin America, Eastern Europe and South-East Asia. It seems probable that focus will remain on the potential for deals in Russia and China. InBev, SABMiller, Heineken, Anheuser-Busch and S\&N have all been involved in consolidating these high-growth markets in recent years, and many industry observers expect the pace of consolidation to continue in these future core markets (Bleakley et al, 2006). 


\section{Procedures ANd Methodology}

Over a research period of six financial years (1999-2004), various aspects of LBG business activities are highlighted by applying two different but complementary financial science approaches: (1) Balance sheet analysis and (2) event study methodology. Our methodological approach is as follows. First, we explain our procedure in measuring a company's degree of internationalization. We then provide scientific insight through our accounting analysis, and, lastly, discuss the event study approach.

\subsection{MEASURING THE DEGREE OF INTERNATIONALIZATION}

By using two quantitative indicators, (a) the Foreign Sales to Total Sales Index and (b) the Network Spread Index, we will give a methodological framework for measuring the DOI (Sullivan, 1994a; Ietto-Gillies, 1998). Regarding tests of the internationalization-performance link, the literature has moved in two distinct directions. First, to address a measurement issue, Sullivan (1994) attempts to more reliably measure the DOI of a firm by developing a novel index measure of internationalization that captures three of its attributes: structure, performance, and attitude. As Ramaswamy et al (1996) show, there are several limitations to the empirical and theoretical underpinnings of Sullivan's work. As such, many studies continue to use a one-dimensional measure of DOI: the share of either assets, revenues, profits, or employment that locates abroad (Sullivan, 1994b; Ramaswamy et al, 1996; Hejazi and Santor, 2005). To stay in line with the bulk of studies about internationalization-performance relationships, we apply the foreign sales index (FSI), which is calculated as follows:

\section{(1) FSI: Ratio of foreign sales (exports and subsidiaries) to total sales}


A second internationalization index adopted in the current study covers the international dispersion of brewers' subsidiaries. The so-called Network Spread Index (NSI) is calculated by dividing the number of countries in which an enterprise maintains subsidiaries by the total number of countries that received direct investments in $2004^{2}$ (Ietto-Gillies, 1998):

(2) NSI with $\mathbf{n}^{*}=167: \quad N S I=\frac{n}{n^{*}}=\frac{n}{167}$.

\subsection{Measuring Corporate SuCCESS-Balance Sheet analysis}

To answer the research question on relationship between degree of internationalization and LBG accounting profitability, we have chosen the measurement of two categories of additional variables: (a) performance variables (EBITDA-margin, RONA, Net Debt Ratio, Cash Realization Ratio) and (b) a set of various control variables. To shed light on potential relationships between internationalization and financial performance, several regressions and correlations were run for the control variables and the various internationalization-performance measure combinations (Gerpott and Jakopin, 2005). We applied ANOVA techniques on averaged data ( $\mathrm{n}=48 ; 6$ financial years $* 8$ companies), drawing mean performance comparisons over the two subsamples at different levels of internationalization.

In most previous empirical studies, scholars used financial ratios, such as return on net assets (RONA), EBITDA-margin, return on sales (ROS), or net debt to equity as dependent variables. Properly interpreted, these ratios provide keen insight into the sources and adequacy of profits, the efficiency of assets committed to the firm, solvency risk, and liquidity risk. Individually they tell only a little about the whole but, taken together, the entire picture of financial health comes into focus (Hsu and Boggs, 2003).

Balance sheet analysis is a systematic procedure using the information potential of the balance sheet as well as the profit and loss account. The aim is to attain insights into the economic situation and future prospects of an enterprise. However, it must be pointed out that, especially in a transnational analysis, key figures are strongly influenced by different valua-

\footnotetext{
${ }^{2}$ Indeed, the number of countries which received FDI in 2004 was 191 (UNCTAD, 2004). But for brewing industry purposes, we have adjusted the NSI in this examination, as not all 191 countries play a significant role in the brewing scene context. We took 167 countries as $n^{*}$. These 167 countries, each of which shows a production volume of at least 4,000 hl (Cayman Islands), are listed in the 2004/05 Barth Report (Joh.Barth\&Sohn, 2005).
} 
tion and accounting regulations. Thus, when adopting a cross-border comparative analysis, one must always consider the basic conditions and the limitations of the informational value of balance sheet analysis (Küting and Weber, 2004). The intention of balance sheet analysis is not to make final judgments but to point out tendencies and prognoses for a company's future prospects (Gräfer, 2001). If one keeps track of these borders and possible misinterpretations and formulates and rates the extracted insights and statements with the required caution, balance sheet analysis can be an important aid in the evaluation and estimation of companies. Through adept dressing of the data using key figures, enterprises can be assessed. For our analysis we chose the following financial ratios:

\section{(3) EBITDA-Margin}

EBITDA is calculated by taking operating income and adding back to it interest, depreciation and amortization expenses. EBITDA is used to analyze a company's operating profitability before non-operating expenses and non-cash charges. Since the distortionary accounting and financing effects on company earnings do not factor into EBITDA, it is a good way of comparing companies within and across industries. This measure is also of interest to a company's creditors, since EBITDA is essentially the income that a company has free for interest payments. In EBITDA margin measures the extent to which cash operating expenses use up revenue (Anonymous, 2006a). EBITDA as a percentage of sales (the higher the ratio, the higher the profitability) can be used to find those companies in an industry that operate most efficiently. It is calculated as follows:

$$
\text { (1) EBITDA-margin }=\frac{\text { EBITDA }}{\text { Net Sales }}
$$

\section{(4) Return on Net Assets}

Return on net assets gauges a company's ability to generate earnings from its investments. RONA is one bottom-line measurement showing performance relative to strategic goals and objectives. More and more successful companies are utilizing RONA measurement. It provides an apples-to-apples comparison of performance that is understood by non-financial professionals (McClure, 2005). The return on net assets offers a different take on management's effectiveness, revealing how much profit a company earns for every euro of its assets. The RONA represents a bracket between the P\&L account and the balance sheet as it expresses the ability of a company to cover the weighted average cost of capital (WACC). In our analy- 
sis we calculate the RONA as follows:

(2) $R O N A=(E B I T A+$ Income from Associates $) * \frac{1-\text { Corporate Tax Rate }}{\text { Average Total Net Liabilities }}$.

\section{(5) Net Debt Ratio}

The Net Debt Ratio (NDR) is a measure of a company's financial leverage calculated by dividing long-term debt by stockholder equity. The ratio is further used for measuring solvency and researching the capital structure of a company. It is closely watched by investors and creditors, because it reveals the extent to which a brewing group is willing to fund its operations with debt rather than equity (Anonymous, 2006b). It is calculated as follows:

(3) NDR $=\frac{\text { Net Debts }}{\text { Total Shareholder's Equity }}$.

\section{(6) Cash Realization Ratio}

Operating cash flow is used to determine the extent to which cash flow differs from the reported level of operating income. It checks on the quality of a company's earnings. Operating cash flow is arguably a better measure of a brewing group's profits than earnings because a company can disclose positive net earnings and still not be able to pay its debts (Anonymous, 2006c). The Cash Realization Ratio (CRR) is defined as the cash flow from operating activities divided by net sales. A positive high value is consistent with conservative depreciation policies, high reinvestment and effective working capital management (Blythman et al, 2003).

(4) $C R R=\frac{\text { Operating Cash Flow }}{\text { Net Sales }}$

\section{Control Variables}

Based on a review of relevant literature on potential factors that should be neutralized before asking whether there are 'true' internationalization-performance relationships for LBG corporate groups, data were collected on six control variables (Hsu and Boggs, 2003; Gerpott and Jakopin, 2005). They were measured as follows:

(1) Year of observation: This variable was included as a 'black-box parameter' to ensure that internationalization-performance associations were distorted as little as possible by general time-related environmental changes (Gerpott and Jakopin, 2005). 
(2) Firm size: According to the rationale behind the "firm size effect", as firm size increases, it becomes more difficult to sustain impressive performance. Thus, when a firm is large, financial performance is expected to be lower (Banz, 1981). The average net sales for the eight brewing groups included in the sample over the period 1999 to 2004 was $€ 5,972$ billion (s.d. $=€ 3,612$ billion). In this study, firm size was then converted into the natural logarithm (LN). This transformation is usually applied to variables that are not taken as ratios. Not only will LN transformation make the results easy to interpret because the changes in the logarithm domain represent relative (percentage) changes in the original metric, but it also makes the distribution of the data closer to a normal distribution (Hsu and Boggs, 2003). The average LN (net sales) over the study period was 8.488 (s.d. $=0.674 ; \mathrm{n}=48)$.

(3) CR10: The ten-firm concentration ratio is a common indicator to measure the concentration in the world brewing scene. The average CR10 over the six years was $46.5 \%$ with a standard deviation of $7.8 \%$.

(4) Domestic market share: The market share in the country of origin was quantified by a LBG's production volume in its home country and has on average reached a remarkable $49.7 \%$ (s.d. $23.8 \% ; \mathrm{n}=48$ ).

(5) Growth speed: As some studies conclude that rapid international expansion through intensive external growth might overlap with weaker financial performance, this control variable was taken in account. It was measured by the growth in an LBG's net sales growth from 1999 to 2004 . On average the eight brewing groups have grown by $11.9 \%$ each year (s.d. $=20.9 \% ; \mathrm{n}=47)$. The high standard deviation indicates large differences in the brewer's annual business expansion.

(6) Industry growth: This variable has been calculated as a control variable for the total brewing industry's annual growth in terms of production volume. The average annual growth has been $2.8 \%($ s.d. $=1.1 \% ; \mathrm{n}=6)$.

\subsection{Measuring Corporate Success-Event Study Methodology}

In measuring a firm's performance an alternative profit is market value. The great advantage of market value is that it can be observed currently and reflects information from market agents as well as their judgment about the firm's further prospects. But the information content of market value is, at the same time, a weak point. Market value is a perfect measure of 
performance if we assume that the market is informationally efficient in a strict sense. Unfortunately, the assumption that the market price reflects all available information at any point in time is in contradiction to the assumption of asymmetric information. If management has more information than shareholders and other investors, then this information will not necessarily be reflected in the market price.

Moreover, the high volatility of stock-market prices gives reason to doubt the informational efficiency of the market, in the short run at least; in fact, there is evidence of strong overreaction. However, in the long run, we may expect the market value will reflect the benefits reaped by shareholders in the future (Hax, 2003).

Despite these caveats, event studies have become an increasingly popular tool for financial research by giving researchers a tool for measuring the notoriously elusive value of foreign direct investment (FDI). Event study methodology tries to measure the stock-market reaction to changes in corporate policy. Event studies in economics/finance/accounting research are an analysis of whether there was a statistically significant reaction in financial markets to past occurrences of a given type of event that is hypothesized to affect public firms' market values. Event study methodology assumes that stock markets swiftly incorporate new information in valuing a firm's stock. Thus, the announcement of a merger or acquisition between two firms would convey information that would prompt investors to bid up the price (Simpson and Hosken, 1998). Despite the empirical evidence that, on average, M\&As fail to create value for the acquiring firm's shareholders, corporations continue to employ this strategy at everincreasing rates (the 'success-paradox'). Some scholars hypothesize that failures to understand the characteristics of resource bundles lead to a tendency to overvalue the targets and the possibility of future value creation. Empirical evidence has shown that creating value for the acquiring firm's shareholders is a 50/50 at best. Other studies raise more serious concerns about the stock price performance of acquirers: Only about $35 \%$ of acquiring companies report positive market gains on the announcement date. These event study results, however, may be due to the methodology's reliance on the assumption that investors can accurately predict the combined firm's future cash flows. This assumption embodies the attractive feature of ensuring that non-M\&A-related factors have no influence on the incremental stock behavior. Abandoning this assumption represents a direct challenge to the efficient market hypothesis (Sirower, 1997; Cording et al, 2002).

A variety of models have been proposed, analyzed and used in practice to measure the normal rate of return, depending on certain variables, and then to generate abnormal return 
estimates. These abnormal returns have been measured as 1) mean-adjusted returns, 2) market-adjusted returns, 3) deviations from the Capital Asset Pricing Model and 4) deviations from the Arbitrage Pricing Model. We chose the mean-adjusted model, which uses the mean daily return on each individual firm's stock over a predetermined estimation period. These returns are calculated by subtracting the average return for stock i during the estimation period (180 trading days immediately preceding the event date) from the stock's return during the event period s. Although the ("constant") mean-adjusted return model is perhaps the simplest, many prominent researchers find it often yields results similar to those of more sophisticated models (Brown and Warner, 1980; MacKinlay, 1997; Binder, 1998). The significance of an event study's results relies on the following assumptions: (1) Markets are efficient (that means any new information will be quickly reflected in the market value of the affected firms), (2) the event was unanticipated by the capital market and (3) there were no confounding effects (such as other changes in corporate policy, product recalls, earnings announcements, half-year results or strategic investment decisions) surrounding the event window.

However, the first step in conducting an event study is simply to define the 'event' under investigation. After the event date is determined, the event window-the period over which the security prices of the firms involved in the event will be examined - and mean-adjusted model estimation period are identified. The event that affects a firm's market value may be within the firm's control, such as the announcement of a stock split. Alternatively, the event may be outside the firm's control, such as a legislative act being passed or a regulatory ruling being announced that will affect the firm's future operations in some way.

The M\&A data employed in this paper are the daily stock returns ${ }^{3}$ for five European and three North American brewing groups, which collectively announced 21 M\&As during the sample period from March 2000 through August 2005. These M\&As have been selected as they meet the criteria for this study (Kusnadi and Sohrabian, 1999; Cybo-Ottone and Murgia, 2000), which are as follows: (1) The return on the acquiring firm's securities is available for at least 180 days prior to the announcement date, (2) the information related to the M\&A, such as transaction price and announcement date, was publicly disclosed in the financial press, (3) there have not been any other confounding effects and (4) the transaction volume exceeds a valuation of $€ 500$ million in order to attract sufficient attention from the capital markets.

\footnotetext{
${ }^{3}$ The daily stock returns are obtained from Thomson Financial Datastream.
} 
The event study methodology outlined in this paper can be found applied to many economic and financial issues. In most instances, there is not one correct technique to apply, but many appropriate ones (Peterson, 1989). Consecutively, we shortly conclude our investigation steps by conducting the event study:

(1) Determination of the 'event': We investigate cross-border mergers and acquisitions in the global brewing scene.

(2) Identification of companies that might be impacted by the event: North American and European stock-listed brewing groups that have been involved in M\&As exceeding a transaction value of $€ 500$ million.

(3) Once the event days and the affected firms have been identified, the next step is to estimate the "normal" change in stock prices for those firms. The mean-adjusted model uses daily returns on each individual firm's stock price over a predetermined period (Wells, 2004). We choose the common 180 trading day estimation window. For each security $\mathrm{i}$, the mean $\hat{R}_{\text {it }}$ and standard deviation $\sigma\left(\mathrm{R}_{\mathrm{it}}\right)$ of its return in days -180 through -11 are estimated as follows:

$$
\hat{R}_{i t}=\frac{1}{170} * \sum_{t=-180}^{t=-11} R_{i t}
$$

(4) The abnormal return (AR) for an individual security for a given period is the difference between the observed return for that period and the expected or predicted return for that period. The difference between the two returns is computed for each event day (Peterson, 1989). The AR is the ex post return of the security over the event window $\left(\mathrm{R}_{\mathrm{it}}\right)$ minus the normal return of the firm over the estimation window ( $\left.\hat{R}_{\mathrm{it}}\right)$ (MacKinlay, 1997). For firm $i$ and event $t$ the abnormal return is

$$
A R=R_{i t}-\hat{R}_{i t}
$$

(5) Because it may be difficult to pinpoint a specific event day, a cumulative effect over a period may be present and observable. Cumulative Abnormal Returns $\left(\mathrm{CAR}_{t}\right)$ are calculated by summing daily $\mathrm{AR}_{\mathrm{t}}$ over time:

$$
C A R_{\left[t_{1} ; t_{2}\right]}=\sum_{\left[t_{1} ; t_{2}\right]} A R_{t}
$$


(6) To assess the total sample's average response on M\&A-related changes in corporate policy one can build the Cumulative Average Abnormal Returns (CAARt) that are calculated by adding up the daily average abnormal returns $\left(\mathrm{AAR}_{\mathrm{t}}\right)$ over time:

$$
C A A R_{\left[t_{1} ; t_{2}\right]}=\sum_{\left[t_{1} ; t_{2}\right]} \frac{1}{n} * \sum_{i=1}^{n} A A R_{i t}
$$

Acquisitions are complicated events, and, because of their complexity, it takes longer than the few days surrounding the announcement for market participants to correctly determine the extent of the economic implications for the acquiring firm. Researchers have the luxury of looking at longer-term share price movements and should do so before they assume that they have uncovered support for the particular theories they are advancing (Harrison et al, 2005). Thus, to fully capture the effects of the event of interest, a 3 -day $(-1,+1)$ event window is used, centering on the first announcement day. Additional longer-term event windows include a 5-day $(-2,+2)$, an 11-day $(-5,+5)$ and an extended 21-day $(-10,+10)$ event window.

Most event study research on M\&As concluded that acquisitions are value-creative, with the lion's share of gains goinr to target-firm shareholders. Acquiring-firm shareholders generally break even, or, if stock consideration is offered, suffer small losses. Consequently, we also hypothesize the same results for our sample of leading stock-listed brewing groups. 


\section{RESULTS}

\subsection{RISING INTERNATIONAL ENGAGEMENT}

The first scientific question in this investigation deals with the development in the extent of internationalization among leading brewing groups in the study period between 1999 and 2004. The mean foreign sales index for the whole sample over the six financial years 1999 to 2004 was $57.8 \%$ (s.d. $=36.2 \% ; \mathrm{n}=48$; see variable 1 in Table 7$)$ ). For the whole sample, the foreign sales index grew at a CAGR of $8.9 \%$ from $43.8 \%($ s.d. $=41.7 \%)$ in 1999 to $67.1 \%$ in 2004 (s.d. $=32.9 \%$ ). By looking at the two subsamples one can detect a broad spread among the various brewing groups. The large standard deviations reflect the division of our sample into the widely internationalized European brewers and their North American competitors, whose international involvement is relatively low. Indeed, the FSI for the North American groups grew at a notable CAGR of $40.3 \%$ but from a comparatively low level of $5.9 \%$ in 1999 to $32.1 \%$ in 2004 (see table 3). The extraordinary jump in the North American sample is caused mainly by the acquisition of UK-based Carling breweries by Coors Brewing Company in December 2001 (Coors' FSI 2001: 17.4\%, 2002: 54.1\%). Whereas the European peer group brewers were already quite internationalized in the late $1990 \mathrm{~s}$, companies from North America had hardly any international involvement at that time. In fact, within the full sample, dispersions of FSI shares did not become perceptibly higher in the study period. Nevertheless, European brewers enhanced their foreign investments again, disproportionately advancing their cumulated FSI from $66.5 \%$ in 1999 to $88.1 \%$ in 2004 . The total sample's most internationalized company in 2004 was InBev with a FSI of 97\%; the least internationalized brewer with regard to FSI was Anheuser-Busch (13.9\%).

The mean network spread index for the whole sample over the six financial years 1999 to 2004 totaled $13.3 \%$ (s.d. $=13.0 \% ; \mathrm{n}=48$; see variable 2 in Table 7). The NSI for the North American groups increased slightly from $1.4 \%$ in 1999 to $3.0 \%$ in 2004 reflecting the fact that North American brewers have long been domestically oriented. Only in recent years did AB seek to follow its European competitors by entering future core markets like China through intensive FDI activity. European brewing groups are much more internationalized in terms of foreign affiliates and wholly-owned subsidiaries. Consequently, their average NSI rose from $15.9 \%$ in 1999 to $23.8 \%$ in 2004 . In 2004 , there were still strong differences in the sample with FSI figures (NSI figures) ranging from 97.0\% (41.9\%) for InBev to $13.9 \%(3.0 \%)$ for 
Anheuser-Busch. The column to the extreme right in Table 3 reveals that, at the financial year-end 2004, the total number of foreign majority- and minority-owned subsidiaries varied between 70 (InBev) and 3 (Coors) with an average of 26 foreign direct investments. The disparity between North American brewers and European brewers are observable: The European LBGs run truly global business activities and are characterized by above-average geographical diversification, whereas the North American groups do not have relevant FDI that go beyond more than one other continent than that of their origin. Table 3 highlights the development of the brewing group's FSI and NSI over the study period between 1999 and 2004.

Table 3: Development of the FSI and NSI for the 10 Brewing Groups from 1999-2004

\begin{tabular}{|c|c|c|c|c|c|c|c|c|c|c|c|c|c|c|c|}
\hline \multirow[b]{2}{*}{ Company } & \multirow[b]{2}{*}{$\begin{array}{l}\text { Country of } \\
\text { Incorporation }\end{array}$} & \multicolumn{2}{|c|}{1999} & \multicolumn{2}{|c|}{2000} & \multicolumn{2}{|c|}{2001} & \multicolumn{2}{|c|}{2002} & \multicolumn{2}{|c|}{2003} & \multicolumn{2}{|c|}{2004} & \multicolumn{2}{|c|}{ countries * } \\
\hline & & $\begin{array}{l}\text { FSI } \\
(\%)\end{array}$ & $\begin{array}{l}\text { NSI } \\
(\%) \\
\end{array}$ & $\begin{array}{l}\text { FSI } \\
(\%)\end{array}$ & $\begin{array}{l}\text { NSI } \\
(\%) \\
\end{array}$ & $\begin{array}{l}\text { FSI } \\
(\%)\end{array}$ & $\begin{array}{l}\text { NSI } \\
(\%) \\
\end{array}$ & $\begin{array}{l}\text { FSI } \\
(\%)\end{array}$ & $\begin{array}{l}\text { NSI } \\
(\%) \\
\end{array}$ & $\begin{array}{l}\text { FSI } \\
(\%)\end{array}$ & $\begin{array}{l}\text { NSI } \\
(\%) \\
\end{array}$ & $\begin{array}{l}\text { FSI } \\
(\%)\end{array}$ & $\begin{array}{l}\text { NSI } \\
(\%) \\
\end{array}$ & $\begin{array}{l}\text { MA } \\
2004\end{array}$ & MI \\
\hline Anheuser Busch & USA & 5.2 & 1.2 & 5.0 & 1.2 & 5.1 & 1.2 & 5.1 & 1.8 & 12.9 & 2.4 & 13.9 & 3.0 & 2 & 3 \\
\hline Coors & USA & 2.6 & 1.2 & 3.4 & 1.2 & 17.4 & 1.2 & 54.1 & 1.8 & 57.5 & 1.8 & 59.0 & 3.0 & 2 & 1 \\
\hline Molson & Canada & 9.8 & 1.8 & 11.3 & 1.8 & 10.9 & 1.8 & 11.0 & 1.8 & 25.5 & 1.8 & 23.4 & 3.0 & 2 & 2 \\
\hline Mean & & 5.9 & 1.4 & 6.6 & 1.4 & 11.1 & 1.4 & 23.4 & 1.8 & 32.0 & 2.0 & 32.1 & 3.0 & 2 & 2 \\
\hline Heineken & Netherlands & 90.0 & 27.5 & 91.0 & 30.5 & 92.2 & 36.5 & 94.0 & 38.3 & 94.5 & 39.5 & 94.9 & 35.9 & 33 & 27 \\
\hline InBev & Belgium & 89.1 & 23.4 & 91.2 & 25.7 & 92.6 & 28.1 & 93.4 & 31.7 & 96.9 & 40.1 & 97.0 & 41.9 & 37 & 33 \\
\hline SABMiller & UK & 57.4 & 8.4 & 61.5 & 9.0 & 81.6 & 9.6 & 82.7 & 12.0 & 83.2 & 12.6 & 85.7 & 13.8 & 8 & 12 \\
\hline Carlsberg & Denmark & 89.2 & 15.0 & 91.7 & 15.6 & 92.3 & 15.6 & 92.9 & 16.2 & 94.1 & 17.4 & 94.7 & 18.0 & 19 & 13 \\
\hline Scottish \& Newcastle & UK & 6.8 & 5.4 & 48.0 & 6.0 & 63.6 & 7.2 & 65.7 & 7.8 & 66.7 & 9.0 & 68.1 & 9.6 & 11 & 8 \\
\hline Mean & & 66.5 & 15.9 & 76.7 & 17.4 & 84.5 & 19.4 & 85.7 & 21.2 & 87.1 & 23.7 & 88.1 & 23.8 & 22 & 19 \\
\hline Max & & 90.0 & 27.5 & 91.7 & 30.5 & 92.6 & 36.5 & 94.0 & 38.3 & 96.9 & 40.1 & 97.0 & 41.9 & 37 & 33 \\
\hline Mean & & 43.8 & 10.5 & 50.4 & 11.4 & 57.0 & 12.6 & 62.3 & 13.9 & 66.4 & 15.6 & 67.1 & 16.0 & 14 & 12 \\
\hline S.d. & & 41.7 & 10.4 & 39.5 & 11.5 & 39.3 & 13.3 & 36.5 & 14.1 & 32.5 & 16.0 & 32.9 & 15.2 & 14 & 12 \\
\hline Min & & 2.6 & 1.2 & 3.4 & 1.2 & 5.1 & 1.2 & 5.1 & 1.8 & 12.9 & 1.8 & 13.9 & 3.0 & 2 & 1 \\
\hline
\end{tabular}

Source: Company data, annual reports, authors' calculations 


\subsection{Development in Brewers' Growth and Financial Performance}

This section analyzes the accounting and economic evolution of the LBGs involved in the recent M\&A transactions and compares their performance development over the study period between the financial years 1999 and 2004. We first look at the absolute measures of profitability (net sales and EBITDA). As stated above, we measure the EBITDA-margin, RONA, NDR and CRR. Not to go beyond the scope of this investigation, we limit the descriptive part of the accounting analysis to the development of net sales and EBITDA as representative of the other financial ratios. The following two sections detail the value of net sales and EBITDA respectively for the research period of thesix financial years from 1999 to 2004.

The cumulated net sales of the total sample grew at a CAGR of $7.4 \%$ from $€ 37.5$ billion in 1999 to $€ 53.6$ billion in 2004 . The average net sales in 1999 was $€ 4,688$ million (s.d. $=€ 3,552$ million) and increased by $42.9 \%$ to $€ 6,701$ million in 2004 (s.d. $=€ 3,577$ million). The fact that the standard deviation in net sales did not increase despite the overall boost in total net sales indicates that smaller brewers were able to keep up. European competitors in particular were nearly able to close the gap between the formerly dominant Anheuser-Busch and the rest of the industry. A glance at Table 4 reveals some large differences regarding brewing groupss rate of growth. SABMiller, the world's second-largest brewer in terms of production volume, grew at a CAGR of $17.3 \%$ and more than doubled its net sales within six years $(+121.7 \%)$. Admittedly, this extraordinary growth was driven mainly by former South African Breweries' merger with US-based Miller for $€ 6.2$ billion in May 2002. It is noteworthy that the European brewers increased their combined sales volume by $70.7 \%$, while their North American competitors grew by only $6.0 \%$. 
Table 4: Development in Brewing Groups' Net Sales, 1999-2004

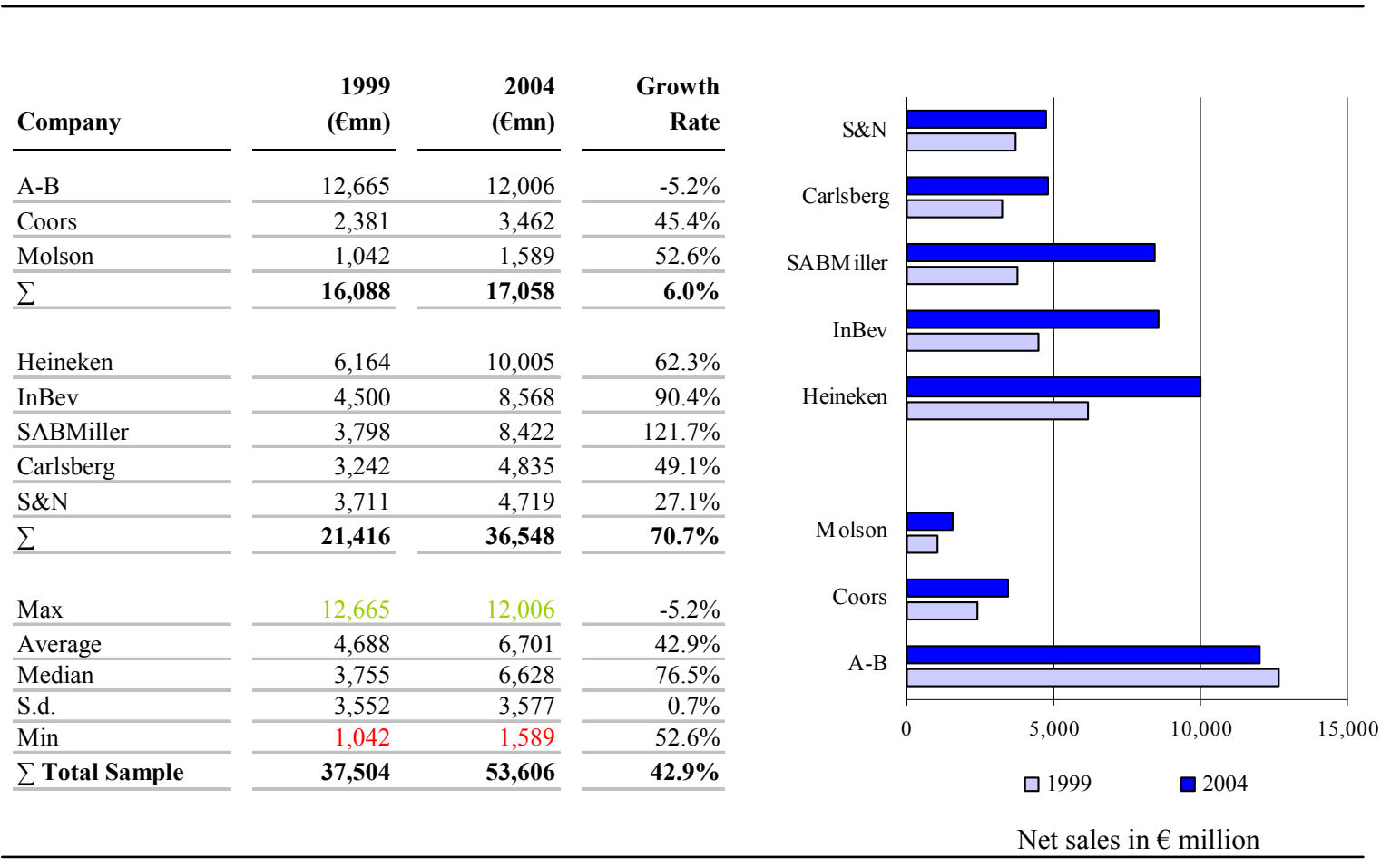

Source: Company data, author's calculations.

The combined EBITDA pool of the total sample grew at a CAGR of 7.3\% from $€ 7.9$ billion in 1999 to $€ 12.3$ billion in 2004. The average EBITDA in 1999 was $€ 993$ million (s.d. $=€ 989$ million) and rose by 55.2\% to $€ 1,541$ million in 2004 (s.d. $=€ 1,108$ million). Looking at Table 4 ("net sales"), we can see that the sample's brewers increased their profitability as the EBITDA grew stronger than net sales $(55.2 \%$ versus $42.9 \%)$. InBev, the world's largest brewer in terms of production volume, grew at a CAGR of $25.2 \%$ and more than tripled its EBITDA within six years $(+207.7 \%)$. This rapid growth was driven mainly by former Interbrew's aggressive external growth strategy; the company has spent more than $€ 25$ billion in M\&As in the course of recent years. But, beyond its external growth, these impressive figures also reflect InBev's motto - to move 'from biggest to best' (InBev, 2005). Apparently, InBev's strategy yielded success. Table 5 illustrates the sample brewers' EBITDA development between 1999 and 2004. It is striking that not only InBev but also the other members of the European peer group (Heineken, SABMiller, Carlsberg) performed quite well over these six financial years. Only S\&N deteriorated, which was due mainly to extraordinarily weak 2004 results. Combined, the European brewers enhanced their EBITDA pool by more than $90.8 \%$, whereas the North American LBGs added only 15.2\% to their 1999 EBITDA. 
Table 5: Development in Brewing Groups’ EBITDA, 1999-2004

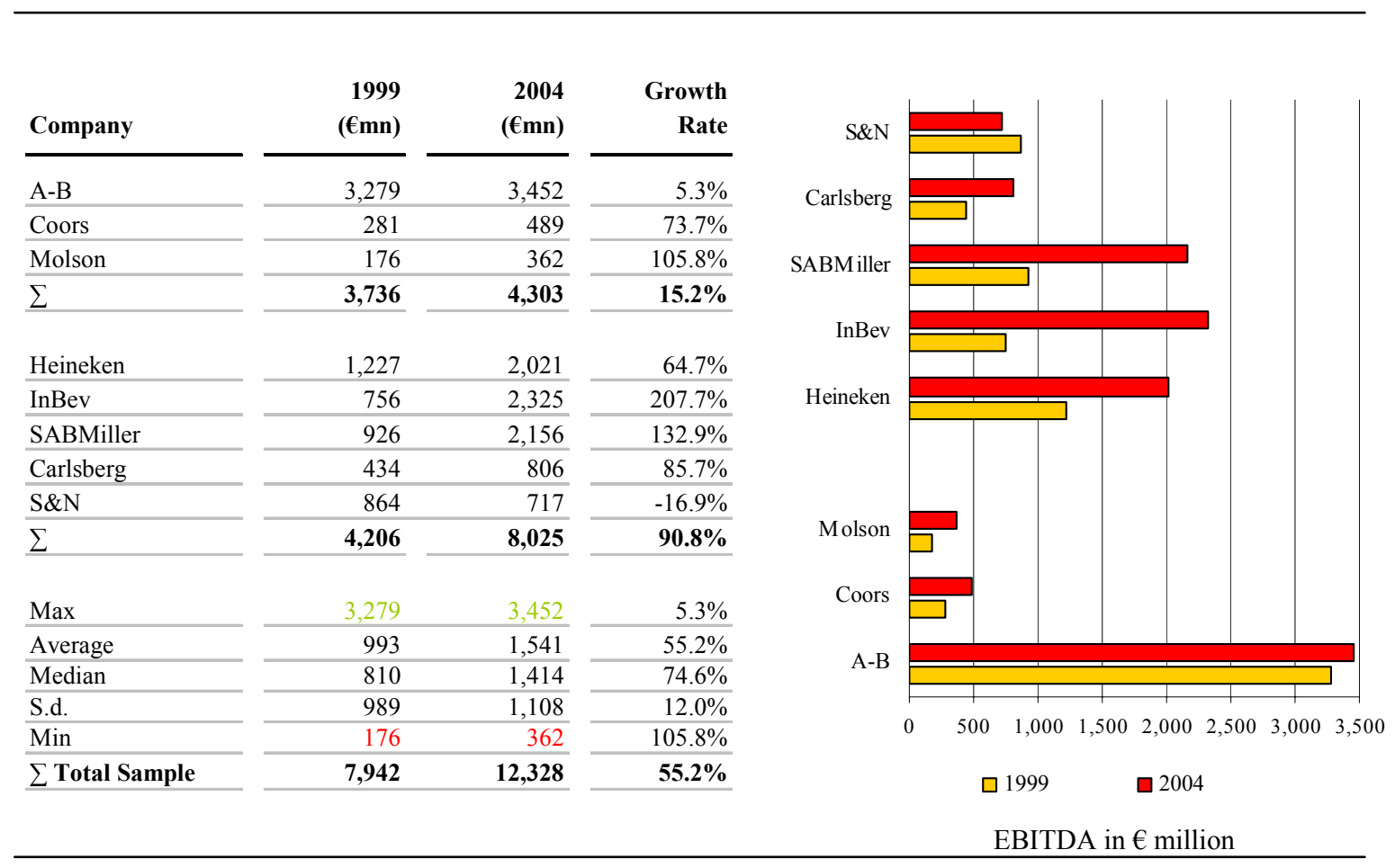

Source: Company data, author's calculations

Table 6 illustrates the mannerin which we have calculated the financial ratios of our sample's companies. We chose Anheuser-Busch, the world's largest brewer in terms of net sales, as a proxy for the whole sample. The left column shows AB's profit\&loss account, balance sheet and cash flow statement in US\$ million; the right column discloses the figures converted into $€$ million. It must be stressed that these figures were not taken directly from the respective annual reports but have been carefully calculated for each financial year and for each brewing group in the same way. 
Table 6: Analysis of AB's Profit\&Loss Account, Balance Sheet and Cash Flow Statement

\begin{tabular}{|c|c|c|c|c|c|c|c|c|c|c|c|c|}
\hline \multirow{2}{*}{$\begin{array}{l}\text { Profit \& Loss Statement } \\
\text { Year end Dec }\end{array}$} & \multicolumn{6}{|c|}{ US\$ million } & \multicolumn{6}{|c|}{ Euro million } \\
\hline & 1999 & 2000 & 2001 & 2002 & 2003 & 2004 & 1999 & 2000 & 2001 & 2002 & 2003 & 2004 \\
\hline Net revenues & 11,895 & 12,499 & 12,912 & 13,566 & 14,147 & 14,934 & 12,665 & 13,514 & 14,400 & 14,345 & 12,499 & 12,006 \\
\hline Cost of goods sold & 6,669 & 7,026 & 7,116 & 7,284 & 7,572 & 8,050 & 7,101 & 7,597 & 7,936 & 7,702 & 6,690 & 6,472 \\
\hline Gross profit & 5,226 & 5,473 & 5,796 & 6,282 & 6,575 & 6,884 & 5,565 & 5,917 & 6,464 & 6,643 & 5,809 & 5,535 \\
\hline Operating costs & 2,147 & 2,175 & 2,220 & 2,455 & 2,498 & 2,591 & 2,286 & 2,351 & 2,476 & 2,596 & 2,207 & 2,083 \\
\hline EBITDA & 3,079 & 3,298 & 3,576 & 3,827 & 4,077 & 4,294 & 3,279 & 3,566 & 3,988 & 4,047 & 3,602 & 3,452 \\
\hline Depreciation & 777 & 804 & 835 & 847 & 877 & 933 & 827 & 869 & 931 & 896 & 775 & 750 \\
\hline Amortization & 0 & 0 & 36 & 0 & 0 & 0 & 0 & 0 & 40 & 0 & 0 & 0 \\
\hline EBIT & 2,302 & 2,495 & 2,705 & 2,980 & 3,199 & 3,361 & 2,451 & 2,697 & 3,017 & 3,151 & 2,827 & 2,702 \\
\hline Income/(expense) from associates & 158 & 200 & 254 & 352 & 345 & 404 & 168 & 216 & 284 & 372 & 305 & 325 \\
\hline Net interest income/(expense) & -285 & -314 & -333 & -350 & -375 & -400 & -304 & -339 & -372 & -370 & -332 & -322 \\
\hline Non operating income/(expense) & -9 & -1 & 6 & -6 & 0 & 39 & -10 & -1 & 6 & -7 & 0 & 31 \\
\hline Pre-tax income & 2,165 & 2,380 & 2,632 & 2,975 & 3,169 & 3,404 & 2,305 & 2,573 & 2,935 & 3,146 & 2,800 & 2,736 \\
\hline $\operatorname{Tax}$ & 763 & 828 & 928 & 1,042 & 1,093 & 1,163 & 812 & 896 & 1,034 & 1,101 & 966 & 935 \\
\hline Reported net profit pre minorities & 1,402 & 1,552 & 1,705 & 1,934 & 2,076 & 2,240 & 1,493 & 1,678 & 1,901 & 2,045 & 1,834 & $\overline{1,801}$ \\
\hline Minority interest & 0 & 0 & 0 & 0 & 0 & 0 & 0 & 0 & 0 & 0 & 0 & 0 \\
\hline Reported net profit & 1,402 & 1,552 & 1,705 & 1,934 & 2,076 & 2,240 & 1,493 & 1,678 & 1,901 & 2,045 & 1,834 & 1,801 \\
\hline Plus: goodwill amortisation & 0 & 0 & 36 & 0 & 0 & 0 & 0 & 0 & 40 & 0 & 0 & 0 \\
\hline Less: Exceptionals (post-tax) & 0 & 0 & 4 & -6 & 0 & 39 & 0 & 0 & 5 & -7 & 0 & 31 \\
\hline Normalised cash net profit & 1,402 & 1,552 & 1,736 & 1,940 & 2,076 & 2,202 & 1,493 & 1,678 & 1,936 & 2,052 & 1,834 & 1,770 \\
\hline Balance Sheet & \multicolumn{6}{|c|}{ US\$ million } & \multicolumn{6}{|c|}{ Euro million } \\
\hline Year end Dec & 1999 & 2000 & 2001 & 2002 & 2003 & 2004 & 1999 & 2000 & 2001 & 2002 & 2003 & 2004 \\
\hline Shareholders equity & 3,922 & 4,129 & 4,062 & 3,052 & 2,711 & 2,668 & 3,938 & 4,383 & 4,584 & 2,912 & 2,159 & 1,956 \\
\hline Minority interest & 0 & 0 & 0 & 0 & 0 & 0 & 0 & 0 & 0 & 0 & 0 & 0 \\
\hline Accumulated goodwill amortization & 0 & 0 & 0 & 0 & 0 & 0 & 0 & 0 & 0 & 0 & 0 & 0 \\
\hline Provisions & 1,800 & 1,905 & 2,163 & 2,676 & 2,835 & 3,258 & 1,807 & 2,023 & 2,441 & 2,553 & 2,258 & 2,388 \\
\hline Net debt & 5,123 & 5,203 & 5,821 & 6,414 & 7,094 & 8,051 & 5,144 & 5,523 & 6,570 & 6,119 & 5,650 & 5,900 \\
\hline Total Net Liabilities & 10,844 & 11,237 & 12,046 & 12,143 & 12,641 & 13,976 & 10,889 & 11,928 & 13,596 & 11,583 & 10,067 & 10,244 \\
\hline Cash Flow Statement & \multicolumn{6}{|c|}{ US\$ million } & \multicolumn{6}{|c|}{ Euro million } \\
\hline Year end Dec & 1999 & 2000 & 2001 & 2002 & 2003 & 2004 & 1999 & 2000 & 2001 & 2002 & 2003 & 2004 \\
\hline Operating cash flow & 3,184 & 3,400 & 3,637 & 3,996 & 4,310 & $\overline{4,317}$ & 3,390 & 3,676 & 4,056 & 4,225 & 3,808 & $\overline{3,470}$ \\
\hline Interest & -285 & -314 & -333 & -350 & -375 & -400 & -304 & -339 & -372 & -370 & -332 & -322 \\
\hline Tax & -763 & -828 & -943 & -881 & -964 & -976 & -812 & -896 & $-1,052$ & -932 & -852 & -785 \\
\hline Net capital expenditures & -865 & $-1,075$ & $-1,022$ & -835 & -993 & $-1,090$ & -921 & $-1,162$ & $-1,140$ & -883 & -877 & -876 \\
\hline Free cash flow & 1,271 & 1,183 & 1,339 & 1,931 & 1,978 & $\overline{1,851}$ & 1,353 & 1,279 & 1,493 & 2,041 & 1,747 & 1,356 \\
\hline Net M\&A & 53 & -43 & -260 & -19 & -157 & -425 & 56 & -46 & -290 & -20 & -139 & -342 \\
\hline Dividends & -545 & -571 & -614 & -650 & -685 & -743 & -580 & -617 & -685 & -687 & -606 & -597 \\
\hline Net Financing & -851 & -561 & -461 & $-1,236$ & $-1,133$ & -646 & -906 & -607 & -515 & $-1,307$ & $-1,001$ & -519 \\
\hline Other & 0 & 0 & 0 & 0 & 0 & 0 & 0 & 0 & 0 & 0 & 0 & 0 \\
\hline Net change in cash & -73 & 8 & 3 & 26 & 2 & 37 & -77 & 8 & 3 & 28 & 2 & 27 \\
\hline & 1999 & 2000 & 2001 & 2002 & 2003 & 2004 & & & & & & \\
\hline Year end exchange rate US\$/EUR & 0.996 & 0.942 & 0.886 & 1.048 & 1.256 & 1.364 & & & & & & \\
\hline Average exchange rate US\$/EUR & 0.939 & 0.925 & 0.897 & 0.946 & 1.132 & 1.244 & & & & & & \\
\hline
\end{tabular}

Source: Anheuser-Busch's annual reports (1999-2004), author's calculations.

It should be noted that all financial figures are stated in $€$, as most of our sample's companies report in $€$. The other currencies have been converted into $€$ using year-end exchange ratios for the balance sheet and average exchange ratios for the profit \& loss account as well as the cash flow statement (see lower section of Table 6). It is noteworthy that Anheuser-Busch's results are especially materially affected by currency movements in the US\$/€ exchange ratio. Indeed, this has much influence on AB's financial figures. Therefore, we have both disclosed AB's accounting analysis in US\$ and converted it into $€$. The more internationalized European groups widely use hedging policy on transactional exchange risks, using such instruments as forward contracts and options. 


\subsection{Internationalization And ACCOUnting-Based Performance Relationships}

The subsequent research question regards internationalization-performance relationships and demands the measurement of different profitability ratios. Table 7 reports the means, standard deviations (s.d.) and Pearson product-moment correlations among the variables used. The correlation provides a first check on how far the sample's companies differ regarding their internationalization and accounting profitability values. The rather high correlations between some of the performance dependent variables are to be expected, but the high dispersions of all types of indicators point out that it makes sense to conduct correlation analysis for significant internationalization-performance associations (Hsu and Boggs, 2003; Gerpott and Jakopin, 2005). No correlation with other performance measures shows the NDR.

Table 7: Descriptive Statistics: Means, Standard Deviations and Intercorrelations

\begin{tabular}{|c|c|c|c|c|c|c|c|c|c|c|c|}
\hline $\begin{array}{l}\text { Measures of } \\
\text { internationalization }\end{array}$ & $\begin{array}{r}\text { Mean } \\
(\%)\end{array}$ & $\begin{array}{l}\text { S.d. } \\
(\%)\end{array}$ & $\mathbf{n}$ & 1 & 2 & & & & & & \\
\hline 1. FSI & 57.82 & 36.21 & 48 & - (a) & 0.80 & $* *$ & & & & & \\
\hline 2. NSI & 13.33 & 12.99 & 48 & $0.83 * *$ & - & & & & & & \\
\hline $\begin{array}{l}\text { Performance } \\
\text { indicators }\end{array}$ & $\begin{array}{r}\text { Mean } \\
(\%)\end{array}$ & $\begin{array}{l}\text { S.d. } \\
(\%)\end{array}$ & $\mathbf{n}$ & 3 & 4 & & 5 & 6 & & & \\
\hline 3. EBITDA-Margin & 20.41 & 5.08 & 48 & - & 0.74 & $* *$ & $0.32 *$ & $0.79 * *$ & & & \\
\hline 4. RONA & 10.26 & 4.14 & 48 & $0.53 * *$ & - & & $0.29 *$ & $0.74 * *$ & & & \\
\hline 5. NDR & 89.37 & 58.20 & 48 & 0.06 & -0.02 & & - & 0.24 & & & \\
\hline 6. CRR & 18.36 & 6.53 & 48 & $0.61 * *$ & 0.49 & $* *$ & -0.04 & - & & & \\
\hline Control variables & Mean & S.d. & $\mathbf{n}$ & 7 & 8 & & 9 & 10 & 11 & & 12 \\
\hline 7. Firm Size (LN) & 8.49 & 0.67 & 48 & - & 0.23 & & 0.24 & 0.08 & 0.02 & & 0.23 \\
\hline 8. $\mathrm{CR} 10(\%)$ & 46.47 & 7.79 & 6 & $0.21 *$ & - & & 0.03 & 0.02 & 0.34 & $*$ & $0.99 * *$ \\
\hline 9. Market Share $(\%)$ & 49.70 & 23.81 & 48 & 0.16 & 0.03 & & - & 0.15 & 0.00 & & 0.03 \\
\hline 10. Growth Speed (\%) & 11.95 & 20.95 & 47 & 0.02 & -0.06 & & 0.02 & - & -0.21 & & 0.02 \\
\hline 11. Industry Growth (\%) & 2.83 & 1.11 & 6 & -0.03 & 0.14 & & -0.02 & -0.06 & - & & $0.39 * *$ \\
\hline 12. Year & & & & $0.21 *$ & 1.00 & $* *$ & 0.03 & -0.06 & 0.14 & & - \\
\hline
\end{tabular}

$* \mathrm{p}<0.10, * * \mathrm{p}<0.05, * * * \mathrm{p}<0.01$ (two-tailed-tests).

(a) Coefficients above (below) the diagonal are Pearson correlations (Kendall-Tau-b correlations).

As Table 7 shows, the sample's mean EBITDA-margin in the study period was $20.4 \%$ (s.d. = $5.1 \% ; \mathrm{n}=48)$. The average RONA was $10.3 \%$ (s.d. $=4.1 \% ; \mathrm{n}=48)$. The NDR totaled $89.4 \%$ (s.d. $58.2 \% ; n=48$ ). The fourth performance indicator under investigation, CRR, was on average $18.4 \%$ (s.d. $=6.5 \% ; \mathrm{n}=48$ ). Likewise, both DOI measures showed similar standard 
deviations but were already explained above, in Chapter 8.1. The means, standard deviations and correlations ${ }^{4}$ of the six control variables can be seen in the lower section of Table 7.

Table 8 highlights the results of the multiple regression analysis of LBG internationalizationperformance indicators. The FSI as well as the NSI internationalization indicators show statistically significant beta-weights in forecasting all four financial performance criteria. It is worth mentioning that three out of four results of our models show excellent explanatory power $\left(\mathrm{R}^{2}\right.$ ranges between 0.51 and 0.71$)$. The NDR-internationalization indicator shows remarkable $\mathrm{R}^{2}$-values of 0.25 (FSI) and $0.24(\mathrm{NSI})$, at least.

Table 8: Multiple Regression Analysis of LBG Internationalization-Performance Indicators

\begin{tabular}{|c|c|c|c|c|c|c|c|c|c|c|c|c|}
\hline \multirow{3}{*}{$\begin{array}{l}\text { Variables } \\
\text { Degree of internationalization }\end{array}$} & \multicolumn{12}{|c|}{ Performance indicators (a) } \\
\hline & \multicolumn{3}{|c|}{ EBITDA-margin } & \multicolumn{3}{|c|}{ RONA } & \multicolumn{3}{|c|}{ NDR } & \multicolumn{3}{|c|}{ CRR } \\
\hline & $1 \mathrm{a}$ & $1 \mathrm{~b}$ & & $1 \mathrm{a}$ & & $1 \mathrm{~b}$ & 1a & & $1 \mathrm{~b}$ & 1a & & $1 b$ \\
\hline Foreign sales to total sales (FSI) & $-0.54 * * *$ & & & -0.55 & $* * *$ & & -0.55 & $* * *$ & & -0.38 & $* * *$ & \\
\hline Network spread index (NSI) & & -0.34 & $* * *$ & & & $-0.36 * * *$ & & & $-0.53 * * *$ & & & $-0.30^{* * *}$ \\
\hline \multicolumn{13}{|l|}{ Control variables } \\
\hline Firm Size & $0.42 * * *$ & 0.47 & $* * *$ & 0.56 & $* * *$ & $0.61 * * *$ & 0.29 & $* *$ & $0.40 * * *$ & 0.66 & $* * *$ & $0.72 * * *$ \\
\hline CR10 & 0.01 & -0.04 & & -0.15 & & -0.20 & -0.50 & & -0.53 & -0.58 & & -0.61 \\
\hline Market Share & $0.70^{* * *}$ & 0.58 & $* * *$ & 0.53 & $* * *$ & $0.40^{* * *}$ & 0.05 & & -0.04 & 0.53 & $* * *$ & $0.45 * * *$ \\
\hline Growth speed & -0.15 & -0.21 & $*$ & -0.14 & & $-0.20 *$ & -0.01 & & -0.08 & -0.13 & & $-0.17 *$ \\
\hline Industry growth & -0.05 & -0.04 & & -0.11 & & -0.11 & -0.16 & & -0.15 & -0.06 & & -0.05 \\
\hline Year & 0.16 & 0.14 & & 0.20 & & 0.19 & 0.82 & & 0.80 & 0.50 & & 0.49 \\
\hline Adjusted $\mathbf{R}^{2}$ & $0.67^{* * *}$ & 0.51 & $* * *$ & 0.63 & $* * *$ & $0.47^{* * *}$ & 0.25 & $* * *$ & $0.24 * * *$ & 0.71 & $* * *$ & $0.66^{* * *}$ \\
\hline $\mathbf{F}$ & 14.10 & 7.85 & & 12.02 & & 6.72 & 3.19 & & 3.10 & 16.94 & & 13.95 \\
\hline
\end{tabular}

$* \mathrm{p}<0.10, * * \mathrm{p}<0.05, * * * \mathrm{p}<0.01$ (two-tailed-tests).

(a) Due to the correlations reported in table 7, additional tests for multicollinarity were run with variance inflation factors.

(b) Figures are standardized beta-values.

\footnotetext{
${ }^{4}$ The correlation between two variables reflects the degree to which the variables are related. The most common measure of correlation is the Pearson product-moment correlation (called the Pearson's correlation for short). When computed in a sample, it is designated by the letter " $r$ " and is sometimes called "Pearson's r". Pearson's correlation reflects the degree of linear relationship between two variables. It ranges from +1 to -1 . A correlation of +1 means that there is a perfect positive linear relationship between the variables. Kendall's Rank Correlation provides a distribution-free test of independence and a measure of the strength of dependence between two variables.
} 
Our findings of the multivariate regression analysis indicate that all four performance measures (EBITDA-margin, RONA, NDR, CRR) were strongly and negatively related to rising degrees of internationalization and achieved statistical significance at the $1 \%$ level.

It is worth noting that these negative associations did not hold the impact of some control variables on LBG internationalization-performance relationships. The strongest influence had both the firm size variable and the market share variable. These findings are not surprising as a large domestic market share guarantees high LBG profitability. Anheuser-Busch, for example, is the least internationalized company in our sample (FSI in 2004: 13.9\%) but still dominates $50 \%$ of the US beer market, which is the most profitable around the globe. Similarly, there is a trade-off between firm size and performance. It seems obvious that larger firms achieve better financial results due to the possibility of their realizing economies of scale, scope and speed, first-mover advantages in entering profitable markets and more market power versus their smaller competitors (Calof, 1994). The growth speed control variable did not have that significant an impact on the internationalization-performance investigation but did affect the EBITDA-margin/NSI relationship, the RONA/NSI relationship and the CRR/NSI relationship all at the $10 \%$ level.

Another observation relates to one of our performance criteria, the net debt ratio (NDR). Indeed, the NDR is negatively related to rising internationalization but shows far weaker explanatory power. This may be caused by the fact that the NDR is not correlated with any of the other performance measures (see Table 7). Another reason might be that the NDR is a financial performance figure, composed of borrowings and liabilities. In contrast, the other performance measures are calculated out of the P\&L account and the cash flow statement, both of which more directly concern a company's operating success. Thus, it seems advisable to replace the NDR figure with another EBIT-based performance measure in future accounting-based investigations.

Hence, contrary to the practical beliefs in the performance-enhancing effects of rising internationalization, it was found that, at a first glance, higher degrees of internationalization go along with lower group profitability (Gerpott and Jakopin, 2005).

Before we conclude this analysis, we should recognize some possible shortcomings. We included a set of many of the most common control variables that have been found to affect performance in many studies in the past. We could have included more, but we were trying to select widely-used variables that were also widely available in an effort to maintain a large enough sample, which we believe was critical to the issue at hand. 


\subsection{EVEnT STUdy ANALYSIS}

To divulge the impact of the announcement of mergers or acquisitions on the analyzed brewers' stock price performance, we decided to illustrate this impact with one example that is representative of all $21 \mathrm{M} \& A s$ under investigation. The following figure displays SABMiller's stock price reaction to the acquisition announcement of Colombian-based brewer Bavaria in July 2005 for about US\$7.8 billion (see also Table 2). The chart pictures the real stock price of SABMiller in British pounds. On the announcement day, July 19, the share price jumped by more than $10 \%$, and the abnormal return totaled $9.98 \%$. This means a boost in SABMillers' market capitalization from $£ 11,25$ billion to $£ 12,37$ billion, a gain of more than $£ 1,12$ billion at that time.

Figure 4: SABMiller's Stock Price Reaction to the Acquisition Announcement of Bavaria

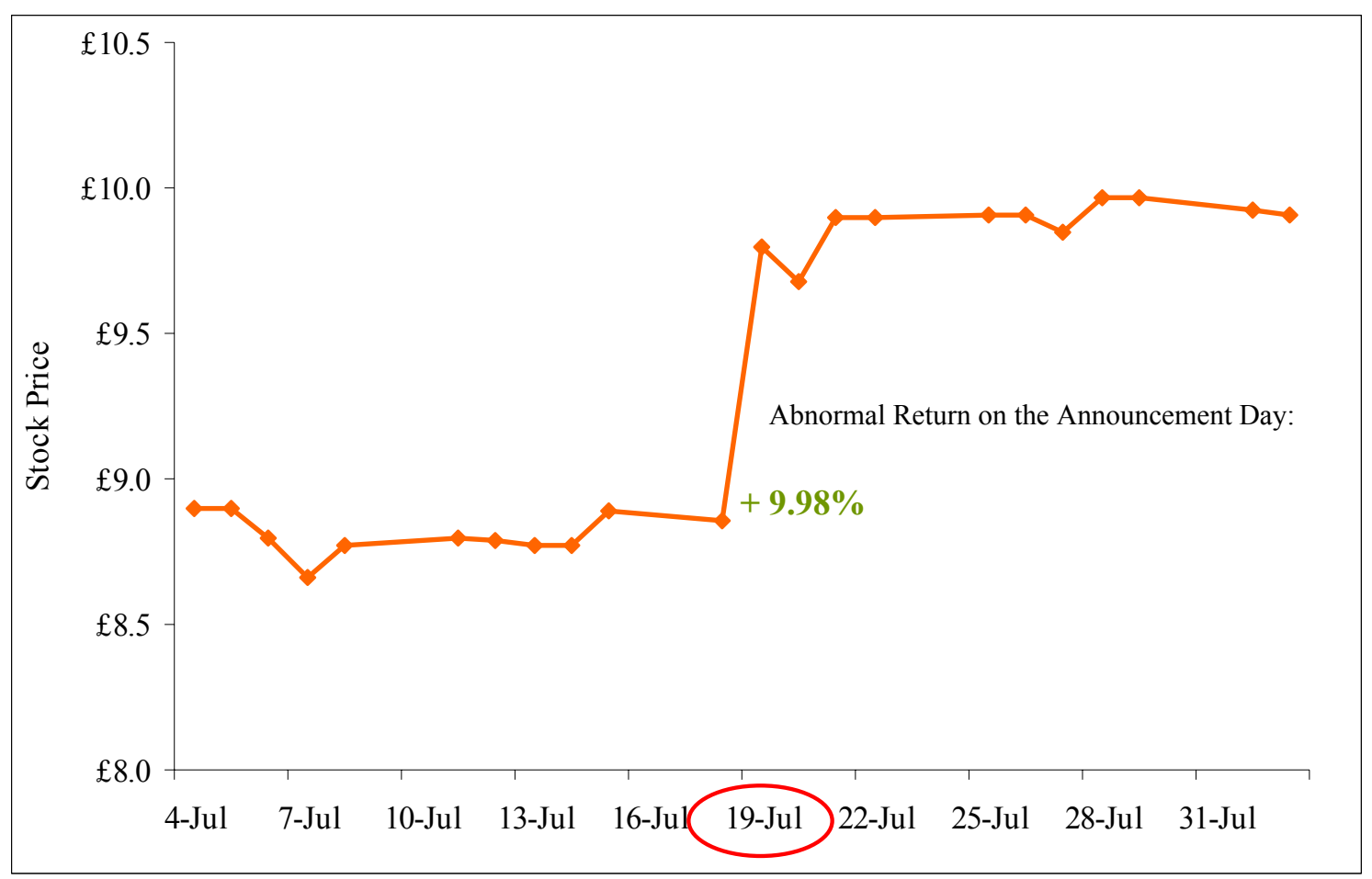

Source: Thomson Financial Datastream

The following figure displays the CAR21 for the five European brewers. The chart lines consist of InBev's seven M\&As and SABMiller's three M\&As as well as S\&N (3), Heineken (2) and Carlsberg (2). Table 7 discloses further information and results on each of these M\&A deals. In the following section the main M\&A deals will be outlined in detail for each respective brewing group. Figures 5 and 6 disclose aggregate results (CAAR) for the various brewers' M\&As within the study period. 
Figure 5: CAARs $(-10,+10)$ for the European Brewing Groups

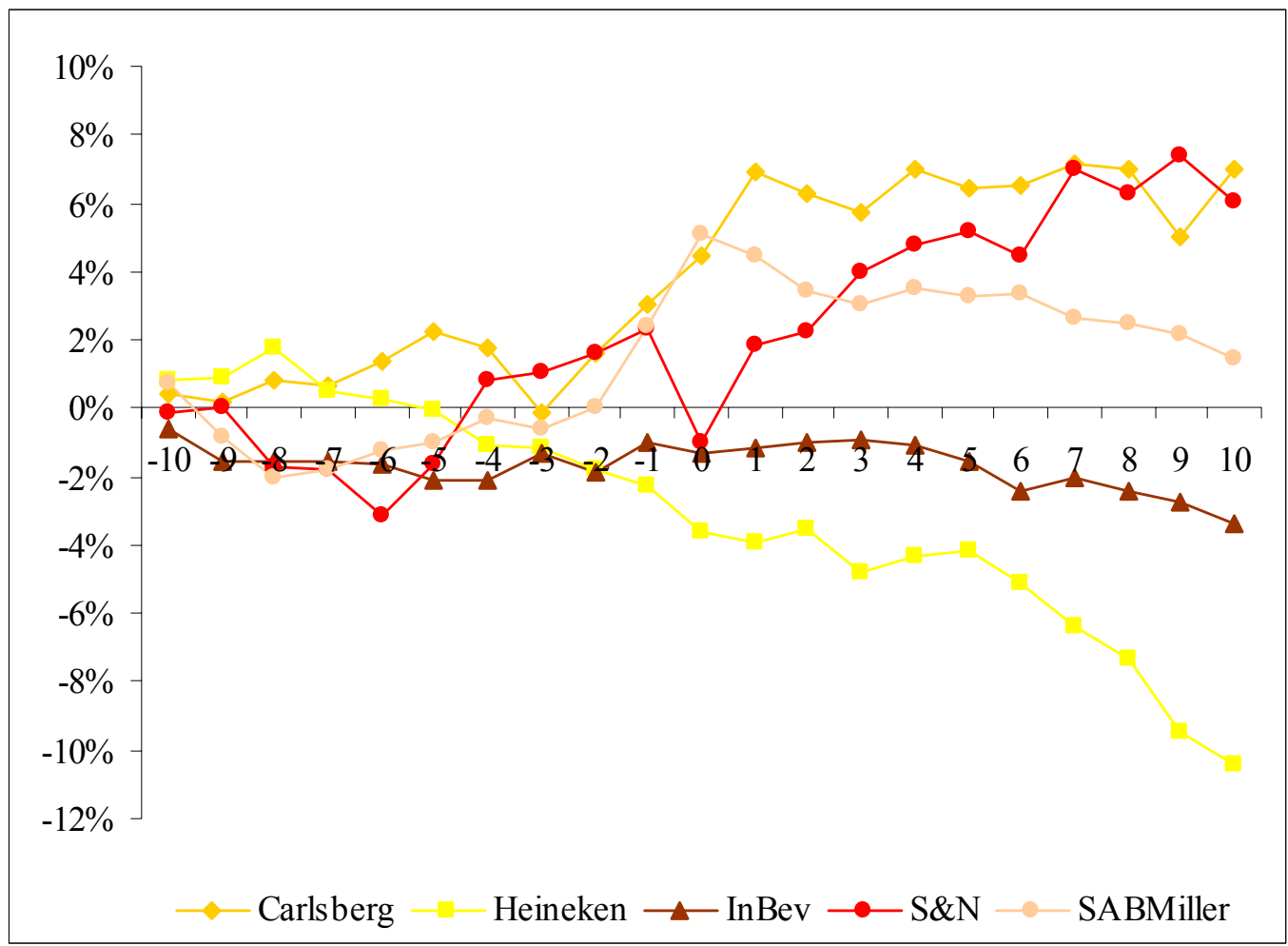

Of Carlsberg's two acquisitions, the Orkla share buyback transacted in February 2004 was undoubtedly perceived positively by shareholders, resulting in positive returns of $10.3 \%$. Carlsberg A/S has signed an agreement to buy Orkla ASA's 40\% shareholding in Carlsberg Breweries A/S for a total of approximately DKK14.8 billion. On completion of the transaction, the partnership between Carlsberg and Orkla ASA ("Orkla") will end and Carlsberg will be the sole shareholder of Carlsberg Breweries.

Heineken's negative abnormal excess returns are mainly affected by the pessimistic response of the capital-market to the announcement of the $€ 1.9$ billion acquisition of Austrian brewing group BBAG in May 2003. Even though BBAG offered Heineken the possibility not only to grow further but to command the lead in the strategically important Central \& Eastern European market, it seems that shareholders found the EBITDA-multiple of 10.2 too high (Hall and Jones, 2003). On May 2, 2003, Heineken realized a negative abnormal return of $2.45 \%$ during the period around the announcement day. This abnormal return cumulated to $5.3 \%(-13.3 \%)$ within the 11-day (21-day) event-window. The CAR21 return has been negative at the $1 \%$ level.

One of InBev's first spectacular deals was the acquisition of the 'pearl' among the German brewers, Beck's, in August 2001 for around $€ 1.8$ billion. Nowadays, Beck's represents In- 
Bev's major international flagship brand, but at that time investors did not appreciate Interbrew's bid. On the day the announcement about the Beck's deal appeared in the financial press (Financial Times Deutschland on July 19, 2001), Interbrew realized a negative abnormal return of $-4.4 \%$, cumulating to $-12.5 \%$ in the 21 -day event window, which was statistically significant at the $5 \%$ level using a t-test. This reaction can be explained by the high acquisition price, which was 13 times Beck's EBITDA.

Scottish\&Newcastle faced a positive abnormal return of $23.9 \%(11.3 \%)$ in the 11-day (21day) event window for its acquisition of French brewer Kronenbourg, whereof the CAR11 was statistically significant at the 1\% level. In March 2000, this represented the largest and strategically most important cross-border step for S\&N so far and resulted in an overall positive recognition by S\&N's shareholders.

As mentioned above, SABMiller's acquisition of Bavaria was one of the most spectacular recent deals in the brewing scene. The event study indicates that SABMiller realized abnormal returns of nearly $9 \%$ when news of its tender offer for Bavaria reached the market. These abnormal returns around the announcement day remained relatively stable, oscillating between $8 \%$ and $10 \%$. All cumulative abnormal returns (CAR3, 5, 11 and 21 ) were positive and statistically significant at the 1\% level (CAR3) and 5\& level respectively (CAR5, 11 and 21) as can be seen in Table 7. 
Figure 6: CAARs $(-10,+10)$ for the North American Brewing Groups

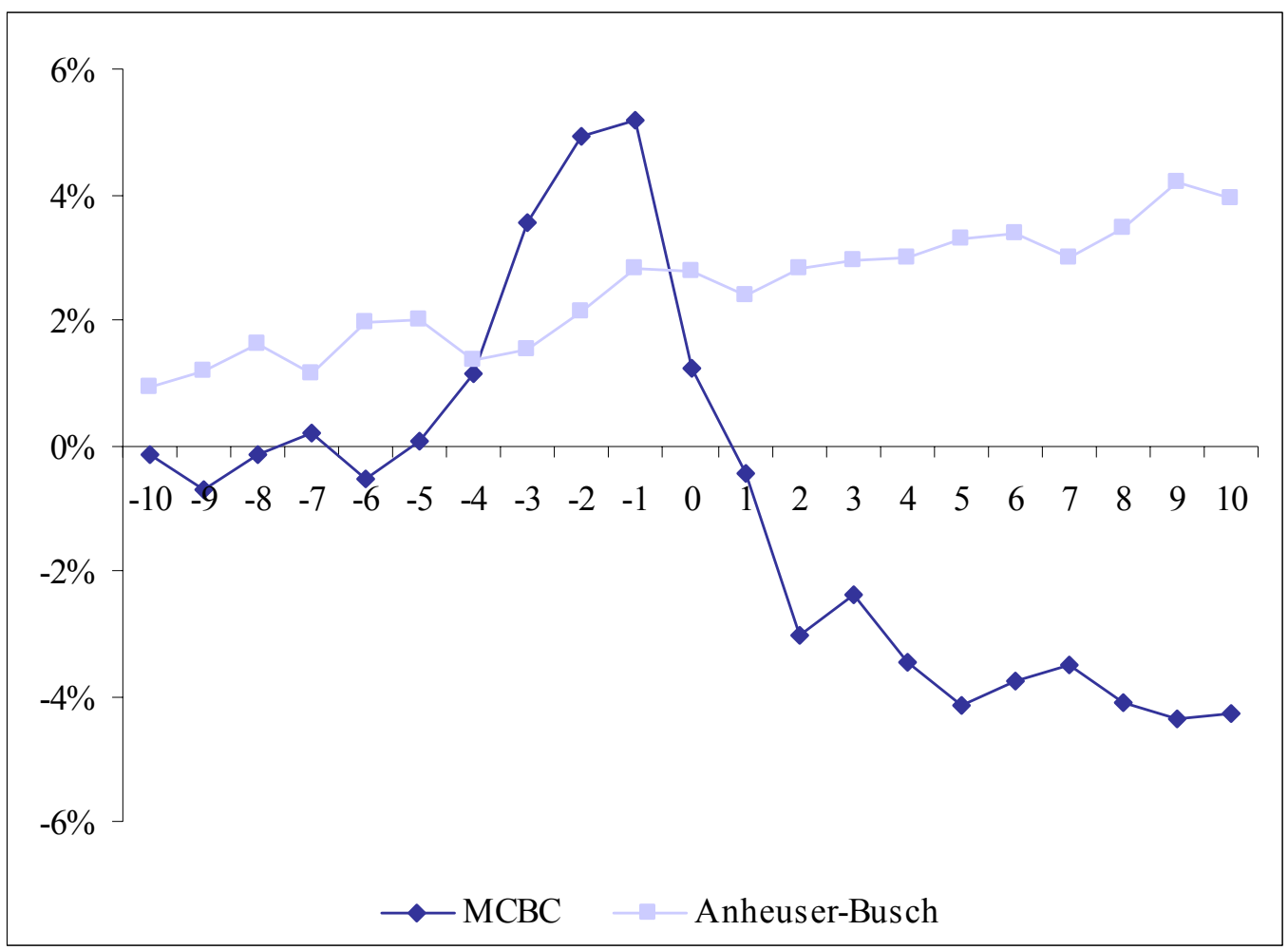

MCBC, the successor of the former Adolph Coors Company, announced in December 2001 that it would buy the Carling operations of Bass Brewers from Interbrew for US\$1.7 billion. The deal was seen as an effort by Coors, a distant third in the U.S. market, to expand to the beer-drinking nations of Europe. In acquiring the Carling line, Coors became an overnight player in the British beer market, going from almost no share to an $18 \%$ stake and becoming the second-largest brewer in Great Britain, behind only Scottish \& Newcastle. Coor's shareholders faced negative abnormal returns of $-4.6 \%$ around the announcement day, statistically significant at the 10\% level. The merger between Canadian brewer Molson and US brewer Coors created Molson Coors Brewing Company in July 2004, forming the world's fifthlargest brewing company by volume, with combined beer sales of 59mhl, pro forma combined net sales of US\$6.0 billion, EBITDA of US\$1.0 billion and free cash flow of US\$707 million. The transaction brought together Molson, Canada's leading brewer with a $43 \%$ market share and the third-largest brewer in Brazil, where it has an 11\% market share, with North America's oldest beer company, Coors, the third-largest brewer in the United States with an $11 \%$ market share and the second-largest in the United Kingdom with a 21\% market share. Industry observers viewed this transaction as an overdue and necessary step toward creating a stronger company in a consolidating global industry able to compete with its ever-growing 
direct competitors in its main markets: $\mathrm{AB}$ and SABMiller in the USA, InBev in Canada and Brazil and S\&N in the UK. Nevertheless, the Molson-Coors merger faced overall negative returns of which the CAR3 was statistically significant at the $1 \%$ level, causing negative abnormal returns of $-6.2 \%$.

Anheuser-Busch has long been virtually active solely in its domestic market. As the USA has been the world's largest market for decades and nowadays is still the largest profit pool around the globe, this may be understandable. But eventually $\mathrm{AB}$ recognized that it cannot rely only on its large and profitable domestic market not only because InBev took the global leadership in 2004 but also since AB was recently overtaken by its direct US rival SABMiller. In front of this competitive background and an ever faster consolidating world brewing scene, $\mathrm{AB}$ has been eager to catch up in order not to lose too much ground to its peers. Consequently, AB announced its purchase of $29 \%$ of Harbin Brewery Group Ltd., a leading brewer in China, for approximately $€ 640$ million in June 2004. Stock markets appreciated this essential step, showing positive abnormal returns of around $4 \%$.

Table 9 discloses the cumulative abnormal returns (CAR3, 5, 11 and 21) over all securities. 
Table 9: Cumulative Abnormal Returns for 21 M\&As between 2000 and 2005

\begin{tabular}{|c|c|c|c|c|c|c|c|}
\hline \multirow{2}{*}{$\begin{array}{l}\text { Event } \\
\text { Day }\end{array}$} & \multirow[b]{2}{*}{ Acquiror } & \multirow[b]{2}{*}{ Target } & \multirow{2}{*}{$\begin{array}{c}\text { Transaction } \\
\text { Value }\end{array}$} & \multicolumn{4}{|c|}{ Cumulative Abnormal Returns } \\
\hline & & & & $(-10,+10)$ & $(-5,+5)$ & $(-3,+1)$ & $(-1,+1)$ \\
\hline 19-Jul-05 & SABMiller & Bavaria & 6,473 & $0.0843 * *$ & $0.1064 * *$ & $0.0933 * *$ & $0.0819 * * *$ \\
\hline 12-Aug-04 & Interbrew & SunInterbrew & 1,212 & 0.0132 & 0.0417 & 0.0109 & 0.0053 \\
\hline 22-Jul-04 & Coors & Molson & 3,200 & -0.0427 & -0.0446 & -0.0109 & $-0.0618 * * *$ \\
\hline 15-Jun-04 & Anheuser Busch & Harbin & 640 & 0.0392 & 0.0136 & 0.0100 & 0.0024 \\
\hline 03-Mar-04 & Interbrew & Ambev & 16,341 & -0.0573 & -0.0274 & -0.0294 & -0.0207 \\
\hline 19-Feb-04 & Carlsberg & Orkla & 7,054 & 0.1028 & 0.1057 & 0.0639 & 0.0622 \\
\hline 07-Jan-04 & Interbrew & Oriental Brewery & 1,547 & 0.0521 & -0.0280 & -0.0133 & -0.0243 \\
\hline 18-Sep-03 & Interbrew & Spaten & 533 & -0.0958 & 0.0368 & 0.0539 & 0.0620 \\
\hline 13-May-03 & $\mathbf{S \& N}$ & Centralcer & 828 & 0.0755 & 0.0370 & -0.0159 & $0.0169 *$ \\
\hline 13-May-03 & SABMiller & Peroni & 563 & -0.0567 & 0.0206 & 0.0112 & 0.0074 \\
\hline 02-May-03 & Heineken & BBAG & 1,899 & $-0.1332 * * *$ & -0.0531 & -0.0245 & -0.0037 \\
\hline 14-Jan-03 & Heineken & $\mathrm{CCU}$ & 1,525 & -0.0754 & -0.0355 & -0.0329 & $-0.0388 *$ \\
\hline 15-Nov-02 & Interbrew & Gilde & 575 & -0.0439 & 0.0423 & 0.0090 & 0.0215 \\
\hline 30-May-02 & SAB & Miller & 6,194 & -0.0542 & 0.0441 & 0.0060 & 0.0066 \\
\hline 14-Feb-02 & S\&N & Hartwall & 2,273 & -0.0069 & -0.0268 & -0.0206 & -0.0242 \\
\hline 24-Dec-01 & Coors & Carling & 1,919 & -0.0429 & -0.0278 & -0.0216 & $-0.0460 *$ \\
\hline 29-Nov-01 & SAB & BevCo & 561 & 0.0852 & 0.0095 & 0.0789 & 0.0834 \\
\hline 06-Aug-01 & Interbrew & Becks & 1,790 & $-0.1251 * *$ & -0.0652 & -0.0483 & -0.0421 \\
\hline 25-May-01 & Interbrew & Bass & 3,611 & 0.0205 & 0.0080 & 0.0867 & 0.0509 \\
\hline 03-Nov-00 & Carlsberg & Feldschlösschen & 574 & 0.0365 & -0.0044 & 0.0396 & 0.0443 \\
\hline 20-Mar-00 & S\&N & Kronenbourg & 2,744 & 0.1133 & $0.2388 * *$ & 0.0692 & 0.0159 \\
\hline \multirow{8}{*}{\multicolumn{3}{|c|}{$\begin{array}{l}* \quad \text { indicates significance at the } .10 \text { level, } \\
* * \text { indicates significance at the } .05 \text { level, } \\
* * \text { indicates significance at the } .01 \text { level, }\end{array}$}} & & CAAR21 * & CAAR11 & CAAR5 & CAAR3 \\
\hline & & & Min & -0.1332 & -0.0652 & -0.0483 & -0.0618 \\
\hline & & & Max & 0.1133 & 0.2388 & 0.0933 & 0.0834 \\
\hline & & & Average & -0.0053 & 0.0187 & 0.0150 & 0.0095 \\
\hline & & & Median & -0.0069 & 0.0095 & 0.0090 & 0.0066 \\
\hline & & & S.d. & 0.0750 & 0.0687 & 0.0435 & 0.0424 \\
\hline & & & Positive CAR & $48 \%$ & $57 \%$ & $57 \%$ & $62 \%$ \\
\hline & & & Negative CAR & $52 \%$ & $43 \%$ & $43 \%$ & $38 \%$ \\
\hline
\end{tabular}

As we can see from Table 9, the distribution for the acquiring brewing groups around the M\&A announcement day is substantially different. Average excess returns (CAR21) are slightly negative and of the order of less than $-1 \%$. There does not appear to be a significant excess return during the run-up period to an announcement. Excess returns also experience a wide dispersion around this average number $(\operatorname{Min}=-13.3 \%$; $\operatorname{Max}=11.3 \%$; s.d. $=7.5 \%)$. The median excess return is very close to zero, indicating the presence of a few events of significant large positive returns (Campa and Hernando, 2005). Astonishingly, the percentage of acquirers that experienced negative abnormal returns around the announcement day was only $38 \%$ (3-day) but increases the longer the event window becomes (21-day: 52\%). 
Interestingly, our results are also in line with more detailed studies on different forms of conducting M\&As. Consistent with previous work, our results prove that horizontal acquisitions face slightly negative returns (average CAAR21 $=-0.53 \%$ ), which is a comparatively optimistic appraisal compared to conglomerate takeovers (Gerke et al, 1995; Glaum and Lindemann, 2002).

\section{Discussion OF ReSUlts}

According to the fact that the peer group of European brewers is much more internationalized than their North American counterparts, there is reason to assume that MNCs from many European nations have been obliged to address organizational needs at high degrees of internationalization and in culturally diverse business environments at an earlier stage than their US-based competitors. Furthermore, LBGs located in many European countries have been forced to direct most of their business operations toward foreign countries, mainly because of their small home markets. Particularly, we may be able to simplify and speed up the identification of intra-firm organizational needs in the course of foreign expansion by examining European pioneers that have already managed to achieve a "transnational" capability at high degrees of internationalization and in culturally diverse markets (Ruigrok and Wagner, 2003).

This study is the first empirical investigation of the stock market effects of brewing groups' strategic activities, such as mergers and acquisitions. Only about one-third of event studies on M\&A effects on bidders' shareholder value were found to have positive effects on the shareholder value of the buying firm in different studies. Performance results reported by earlier research are quite mixed (Sirower, 1997). In general M\&As are value creative, but the lion's share of gains go to target-firm shareholders. The brewers we investigated were not targets but bidder companies, which broke even or suffered small losses. These results are generally in line with findings in previous studies in the finance literature and are consistent with the predictions of economic and financial theory. In general, brewing mergers and acquisitions show behavior consistent with theoretical predictions. Most of the bidders faced slightly negative short-term and intermediate-term cumulative abnormal returns. But, in contrast to previous studies, our results have been strongly mixed as many M\&A deals had positive responses from the capital markets. We demonstrate what we believe to be a result of a heuristic that 
certain horizontal acquisitions of paramount strategic importance lead to positive performance for the acquiring brewing firm. This heuristic is most likely a result of widely-held beliefs about the increased market power and efficiency improvements available to merging firms that were previously competing in the same business (Harrison et al, 2005).

But our results also suggest that, if firms decide to move abroad to improve performance and their decision is based only on an assumed positive relationship between DOI and performance, then such a strategy may not result in improved performance. Furthermore, it seems to be obvious that both challenges and opportunities from internationalization increase as internationalization advances from earlier stages of exporting to the higher stage of horizontal FDI.

In terms of accounting profitability, brewing groups show improvements in both their net sales and EBITDA figures following this period of intensive external growth. These improvements are substantial. Indeed, they can be drawn not only from increased M\&A activity but also seem to be caused in large part by external growth effects. Thus, our results contradict other studies in some ways, as a survey of 22 accounting data studies from nine countries showed that the average acquiring firm does not earn a significantly higher profit than the industry average (Ghauri and Buckley, 2003). As we have seen from the accounting-based analysis of brewing groups' internationalization-performance links, this relationship seems to be negative in practice. One possible explanation might be that we can detect a major outlier in the sample: Anheuser-Busch. The leading US brewer is still one of the most profitable companies in the global brewing scene but only recently undertook its first internationalization steps. In contrast, the widely internationalized European brewers have long lagged behind AB's profitability benchmark but caught up over recent years, as our descriptive accounting results have proven (see Tables 4 and 5). That the internationalization-performance association is still negative may be caused by the fact that European LBGs have fostered their international expansion at a higher speed than the improvement of their profitability ratios. Nevertheless, they were able to close ranks over recent years, and it is probable that future research that includes the 2005 and 2006 financial results will discern a positive internationalizationperformance relationship.

Despite some caveats, we can conclude that the outcomes of both methodologies applied to internationalization-LBG performance links in this investigation are consistent with the exist- 
ing literature on internationalization-performance studies. But, apart from statistical links, we detected in our descriptive statistic part of the brewing group's balance sheet analysis that there are clear movements and changes among the world brewing scene's leading companies. As mentioned, Anheuser-Busch still outperforms its aggressively growing European competitors, but those competitors have caught up to an astonishing degree over recent years. In this context, one further aspect catches the analyst's eye and will be discussed in the following paragraphs: the development of the brewer's market capitalization (market cap).

The market cap is the core element of calculating the enterprise value, which is of paramount importance to assessing the value of a brewing group. Admittedly, even leading brewers are far from being as valuable as large soft drink bottlers, like Coca-Cola, but the brewing industry's principal companies were able to catch up at high speed over recent years. For many years, Anheuser-Busch has been the absolutely dominant LBG in terms of market cap and enterprise value, which always oscillated around $€ 30$ billion - three times the worth of its European competitors. But the European brewers have been closing in even more over the last five years, reflecting their boost in M\&A activities. Figure 7 exemplifies the development of the sample's market cap between December 2004 and March 2006.

Figure 7: Development of the Sample's Market Cap between Dec 2004 and Mar 2006

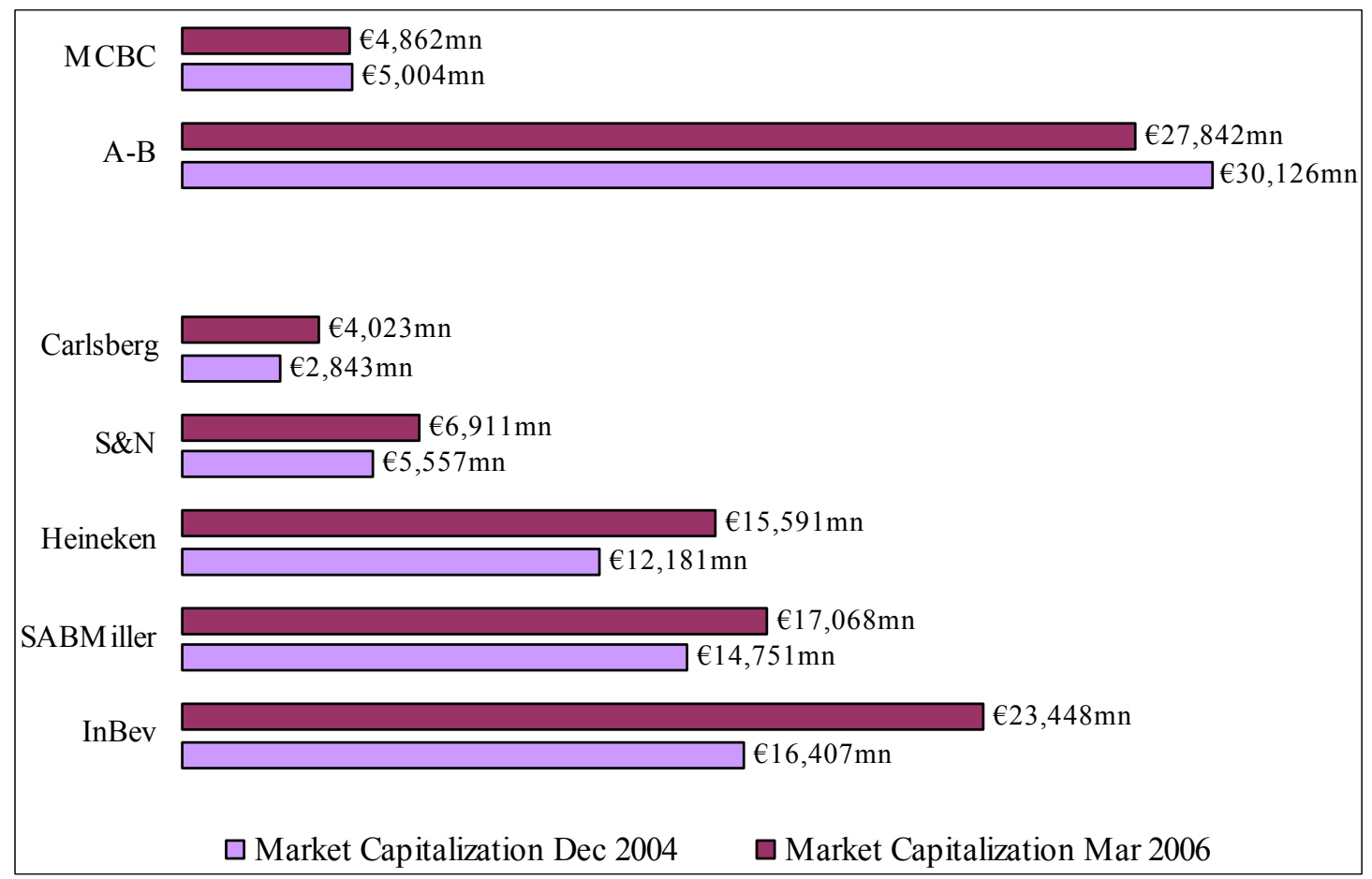

Source: Reuters.com, company publications, author's calculations 
On average, European brewers improved their aggregate corporate values by $29.6 \%$ from $€ 51.7$ billion to $€ 67.0$ billion in contrast to the North American groups, which noticeably lost cumulated market cap by $-6.9 \%$ from $€ 35.1$ billion to $€ 32.7$ billion. The latter is caused mainly by a $-7.6 \%$ decrease in AB's market cap. The major winner over recent months has unsurprisingly been InBev, which has gained a $42.9 \%$ boost in its corporate value, followed by Carlsberg (41.5\%), Heineken (28.0\%) and S\&N (24.4\%). SABMiller has been the weakest performer among the European brewers with a gain of $15.7 \%$. Thus, the peer group of European brewers improved their values extraordinarily, whereas AB lost market capitalization. These values are driven by the generally friendly environment in European capital markets over recent months, but they also reflect the financial market's appreciation and recognition of the European peers' corporate strategies.

The size and growth of a firm's market capitalization is often one of the critical measurements of a public company's success or failure. In particular the instrument of the exchange of shares and the issue of new shares is a financial instrument applied more and more often in large cross-border $\mathrm{M} \& \mathrm{As}^{5}$. Consequently, a rapidly rising market cap is of paramount importance for large brewing groups. More than $80 \%$ of M\&A transactions that exceed a volume of more than $€ 1$ billion are conducted through the exchange of shares or by issuing new shares. At the same time, a high market capitalization is still the best way to protect a brewing group against a hostile takeover (Dibelius, 2002). In contrast, a low market cap implies an increased risk of being taken over. Moreover, more highly capitalized brewers have to issue only comparatively few new shares to finance further acquisitions.

\footnotetext{
5 The creation of InBev in March 2004, for example, was transacted as a share exchange between Interbrew's and AmBev's controlling shareholder holding companies. Former Interbrew issued 141.7 million new shares to Braco S.A., which owned a 52.8\% voting interest in AmBev. As a result, Interbrew received a 21.8\% economic interest in AmBev. AmBev in turn issued new ordinary and preferred shares to Interbrew in exchange for Interbrew's wholly owned Canadian subsidiary, Labatt. The total transaction was valued $€ 16.3$ billion (InBev, 2004). The same instrument was used by SABMiller's acquisition of Colombian brewer Bavaria in July 2005. Pursuant to a merger between a subsidiary of Bevco (BC), the holding company of the Santo Domingo Groups's (SDG) interest in Bavaria and a wholly owned subsidiary of SABMiller, SABMiller will obtain BC's indirect 71.8\% interest in Bavaria and will issue 225 million SABMiller ordinary shares ( "Consideration Shares") with a value of approximately US\$3.5 billion to the SDG based on a weighted average US\$ SABMiller share price agreed between the parties. As a result, BC will own an economic interest of approximately $15.1 \%$ in SABMiller and will be subject to a five-year lock-up, subject to certain exceptions including limited disposals from the third year (SABMiller, 2005).
} 
Admittedly, it is not clear that market value is superior in all cases to accounting profit as a yardstick by which to measure firm performance. Comprehensive and reliable information in the hands of market agents can deter manipulation and reduce volatility, thereby improving the quality of market value as an indicator of LBG performance. This includes the information contained in accounting and financial statements (Mohnen, 2002; Hax, 2003). Therefore, accounting information and enterprise valuation should not be seen as alternative ways to evaluate performance but as complementary.

\section{CONCLUSIONS AND FUTURE RESEARCH}

This paper examines M\&A activities in the brewing sector that drove leading brewers' further internationalization paths during the period from 1999 through 2004. This period was characterized by increases in the average value of transactions as well as the total number of M\&As that took place in the global brewing scene. Even brewers that were formerly domestically oriented have moved tenuously from domestic consolidation towards a larger process of international integration. We have looked at both the value created to shareholders around the announcement of the transactions and the medium-term development of business performance during this period. The recent burst of takeover activities can be seen as a distinctly new wave that is driven by both strategic and synergistic factors. For the brewing companies, mergers and acquisitions seem to be the fastest and most efficient approach to capturing the benefits associated with access to new markets and, at the same time, to being prepared for the competition emerging in each regional market in the world brewing industry (Shusterman et al, 2001). To conclude, the motives that drive the industry's leading firms to further consolidate today originate from medium-term strategic and competitive forces rather than from pursuit of monopoly power and short-term profit seeking.

That M\&As on average have not lived up to the financial expectations of those transacting them has become a common theme in both the academic and the practitioner literatures. Many reasons have been advanced for this, ranging from lack of strategic fit of acquisition or merger partners to poor due diligence (Schweiger and Very, 2003). The results of our study confirm this common view only in part. Obviously, LBGs encounter both the benefits and the costs of internationalization. Hence, internationalization per se is not a sufficient condition for superior performance. Indeed, there are some brewers whose M\&A transactions did not meet 
shareholders' expectations. But, at the same time, many acquisitions have created significant value for some of the fastest growing brewers in our sample. Indeed, our event study results indicate that shareholder returns to the bidding companies are slightly negative on average upon the announcement of the transaction although cumulated abnormal returns for the whole sample have not shown any statistical significance from zero caused by the wide differences that brewers have experienced from their M\&A activities. Hence, it is important to consider the relationship between short-term shareholder reactions and long-term outcomes of mergers and acquisitions ${ }^{6}$.

Future researchers using event studies on consumer goods companies should consider whether the market has accurately impounded all information into market price at the conclusion of their event window. The underlying assumption hereby is that market participants were, at the date of the original acquisition announcement, able to anticipate whether resources would be transferred from the target to the acquirer and make adjustments to their valuations based on this information. It is difficult for us to see how investors could predict these future resource transfers at the time the acquisition was first announced (Harrison et al, 2005). The addition of longer-term measures (e.g. CAR31; CAR61) to future studies would be very helpful. Furthermore, including the target company's abnormal returns would shed more light on the implications of M\&As in the brewing industry. There is strong empirical evidence in many event studies that target firms' shareholders receive significant increases in their stock prices in comparison to the shareholders of bidding firms (Mathesius, 2003). However, as proved in related studies, the operating improvements observed after the M\&As are not correlated with the excess returns shareholders receive upon the announcement days of a deal. This lack of correlation suggests that the ability to obtain such operating gains is difficult for financial markets to predict (Campa and Hernando, 2005). Thus, longer-term, marketbased measures should be included alongside an event study. Contradictory results from short-term and long-term studies indicate that investors are incorrectly adjusting for longerterm performance implications (Harrison et al, 2005). As we detected in our event study analysis, CAARs around the announcement day still are slightly positive (CAAR3: 0.95\%; CAAR5: $1.50 \%$; CAAR11: $1.87 \%$ ) but, the longer the event window becomes, the more negative the CAARs (CAAR21: $-0.53 \%$ ). This kind of result is very interesting as it indicates that the market needs education with regard to the event being studied.

\footnotetext{
${ }^{6}$ See also Chapter III.4 for future research recommendations on event study methodology.
} 
Concerning our accounting-based analysis, future research could include other profitability ratios and take into account further control variables. A helpful amendment would be to prolong the study period by additional financial years (for example, 1998-2005). The decomposition of our DOI measures, which could be constructed from published written sources, represented progress compared to the approaches of earlier investigations, but they were nevertheless still rather crude. Therefore, we urge future investigators to consider alternative conceptualization and operationalization techniques for the "degree of internationalization" variable. Researchers who can select from a wide range of measures could also choose indicators that more purely reflect cultural dissimilarity (such as entropy measures or Herfindahl variants based on Hofstede's [1980] culture scores). Such replication would be able to directly assess the validity of our presumption that different forms of the DOI-performance link can be explained by country-specific types of expansion (culturally related versus unrelated) (Hofstede, 1980; Gerpott and Jakopin, 2005). Admittedly, limited availability of further data that go beyond FSTS and FDI figures make it almmost impossible to gather this information.

Despite the fact that extensive research was directed toward the internationalization paths and processes over recent decades, the performance effects of the internationalization paths and processes remain largely under-investigated. Therefore, future research could develop a finer-grained approach to the positions, paths and processes of internationalization in order to explain differential performance effects. Furthermore, an interesting extension would be to investigate a potential link between the speed of external growth and brewers' accounting profitability.

Our results also have implications for practice and in particular implications for the top echelons of management in the brewing scene. Knowledge of whether the announcement and implementation of important strategic movements creates or destroys value will underpin future courses of action, not just with the issue of the efficacy of such strategies, but with the method of timing of announcement and implementation as well (Panayides and Gong, 2002). As we have seen from the discussion about brewing groups' market capitalization, a central managerial implication seems to be clear as brewing executives face new paradigms. First, they have to ensure high valuations of their enterprises and, second, they have to grow faster than the market in terms of market capitalization to become a global company or to remain such an MNC. Otherwise, they run the risk of losing their independence and becoming acquired by more successful brewing groups. Heineken, SABMiller and InBev in particular have proven 
how effective M\&A transactions can contribute to satisfing these new paradigms.

Thus, it seems obvious that future developments within the beer industry will display many of the characteristics of recent years. There will continue to be intense consolidation activity as the leading brewing groups' benefit can only be maximized if the brewers are both well positioned to tap into the largest profit pools and have critical mass in the best growth markets. With the top five spirits players now estimated to account for around $50 \%$ of the global premium spirits market and the top five beer players for more than $40 \%$ of the world beer market, some brokers believe the scope for further industry consolidation lies not only in the brewing sector but also with a long-term view on a cross-sector level with deals likely to move to the next level of beer/spirits (possibly Heineken/Diageo) or beer/soft drink bottlers (for instance, Heineken or SABMiller/Femsa) in order to extract cost or marketing synergies (Bleakley et al, 2006). It seems obvious that InBev, SABMiller, S\&N and Anheuser-Busch remain active in consolidating the future core beer markets in Russia and China. These days Anheuser-Busch is still number three in the global brewing arena, but when Heineken will catch up seems to be only a question of time as the Dutch brewer is eager not to lose too much ground against InBev and SABMiller. Mexican brewer Femsa appears to be the next big M\&A target in the global brewing arena.

In conclusion, this study has shed some empirical light on (1) the degree to which eight major European and North American brewing groups have internationalized their businesses since the late 1990s, (2) on the relationship between the degree of internationalization and accounting performance and (3) on event study assessment of brewing groups acquisitioning performance for the study period between 1999 and 2004. On the one hand, this research represents only a small step further into the analysis of patterns of internationalization; on the other, our study represents one of the first investigations into both methods of measuring corporate success: accounting-based and capital-market-based analysis of leading brewers around the globe. From a methodological viewpoint, the central conclusion of our study reads as follows: Accounting profit and market value are complementary measures of a firm's performance. 


\section{REFERENCES}

Agrawal, A. and Jaffe, J. F. (2000): The Post-Merger Performance Puzzle, in: Cooper, C. and Gregory, A. (Eds.): Advances in Mergers and Acquisitions, New York: 7-41.

Annavarjula, M. and Beldona, S. (2000): Multinationality-Performance Relationship: A Review and Reconceptualization, in: International Journal of Organizational Analysis 8(2000): 48-67.

Anonymus (2006a): EBITDA Margin, in: www.investorwords.com, March 11, 2006.

Anonymus (2006b): Measuring Solvency and Capital Structure. Debt to Equity Ratio, in: www.valuebasedmanagement.net, March 13, 2006.

Anonymus (2006c): Operating Cash Flow, in: www.valuebasedmanagement.net, March 13, 2006.

Baltazar, R. and Santos, M. (2003): The Benefits of Banking Mega-Mergers: Event Study Evidence from the 1998 Failed Mega-Merger Attempts in Canada. in: Canadian Journal of Administrative Sciences 20(2003): 196-208.

Banz, R. R. (1981): A Possible Explanation of the Small Firm Effect, in: The Journal of Finance, 36(1981): 879-889.

Beitel, P. (2002): Akquisitionen und Zusammenschlüsse europäischer Banken. Wertsteigerung durch M\&A-Transaktionen, Wiesbaden.

Berger, A. N. et al (1999): The Consolidation of the Financial Services Industry: Causes, Consequences, and Implications for the Future, in: Journal of Banking \& Finance, 23(1999): 135-194.

Bevan, N. and Greenberg, M. (2004): Global Brewing - The Global Pitcher 2004 - Volume V, Deutsche Bank Report, London.

Bevan, N. and Greenberg, M. (2005): Global Brewing - The Global Pitcher 2005 - Volume VI, Deutsche Bank Report, London.

Binder, J. (1998): The Event Study Methodology since 1969, in: Review of Quantitative Finance and Accounting 11(1998): 111-137.

Bleakley, M. et al (2006): European Beverages. Themes for 2006, Credit Suisse First Boston Equity Research.

Blythman, M. et al (2003): Pan European Brewing Review - 2003, Merrill Lynch, London.

Bromiley, P. et al (1988): On Using Event-Study Methodology in Strategic Management Research, in: Technovation 8(1988): 25-42.

Brown, S. J. and Warner, J. B. (1980): Measuring Security Price Performance, in: Journal of Financial Economics 8(1980): 205-258.

Calof, J. L. (1994): The Relationship Between Firm Size and Export Behavior Revisited, in: Journal of International Business Studies, 25(1994): 367-388.

Campa, J. M. and Hernando, I. (2005): M\&As Performance in the European Financial Industry, Documentos de Trabajo No. 0516, Banco de Espana, Madrid.

Carow, K. A. and Kane, E. J. (2001): Event-Study Evidence on the Value of Relaxing Longstanding Regulatory Restraints on Banks, 1970-2000. Boston College Working Paper.

Contractor, F. J. et al (2003): A Three-Stage Theory of International Expansion: The Link Between Multinationality and Performance in the Service Sector, in: Journal of International Business Studies 34(2003): 5-18.

Cooper, C. and Gregory, A. (2001): Advances in Mergers and Acquisitions. Connecticut: JAI Press.

Cording, M. et al (2002): A Focus on Resources in M\&A Success: A Literature Review and Research Agenda to Resolve Two Pardoxes. URL: www.darden.virginia.edu/batten/pdf/WP0017.pdf. 
Cybo-Ottone, A. and Murgia, M. (2000): Mergers and Shareholder Wealth in European Banking, in: Journal of Banking \& Finance 24 (2000): 831-860.

Dibelius, A. (2002): Mergers \& Acquisitions: Schnittstelle zwischen Unternehmen und Kapitalmärkten, in: Picot, G. (Ed.), Handbuch Mergers \& Acquisitions. Planung, Durchführung, Integration, second edition, Schäffer-Poeschel, Stuttgart: 39-68.

Dunning, J. H. (1977): Trade, Location of Economic Activity and MNE: A Search for an Eclectic Approach, in: Ohlin, B. et al. (Eds.), The International Allocation of Economic Activity, London: Macmillan: 345-418.

Gerke, W. et al (1995): Die Bewertung von Unternehmensübernahmen auf dem deutschen Aktienmarkt, in: zfbf 47 (9/1995), S. 805-820.

Gerpott, T. J. and Jakopin, N. M. (2005): The Degree of Internationalization and the Financial Performance of Mobile Network Operators, in: Telecommunications Policy 29(2005): 635-661.

Ghauri, P. N. and Buckley, P. J. (2003): International Mergers and Acquisitions: Past, Present and Future, in: Cooper, C. and Gregory, A. (Eds.), Advances in Mergers and Acquisitions. Connecticut: JAI Press: 207-229.

Glaum, M. (1996): Internationalisierung und Unternehmenserfolg, Wiesbaden.

Glaum, M. and Lindemann, J. (2002): Externes Wachstum: Theoretische Überlegungen und empirische Befunde, in: Glaum et al. (Eds.), Wachstumsstrategien internationaler Unternehmungen. Internes vs. externes Unternehmenswachstum, Stuttgart: 269-299.

Golub, A. et al (2004): Who Cares About GMO Foods? Not Walls Street. Paper presented at the 14th Annual World Food and Agribusiness Forum, 12th - 15th of June, Montreux.

Gräfer, H. (2001): Bilanzanalyse, Eighth Edition, Berlin.

Hall, W. and Jones, A. (2003): Heineken Shows Thirst for Central Europe, in: Financial Times, May 3/May 4 2003: 8.

Harrison, J. et al (2005): Event Studies and the Importance of Longer-Term Measures in Assessing the Performance Outcomes of Complex Events. URL: http://www.indiana.edu.

Hax, H. (2003): Measuring the Firm's Performance: Accounting versus Market Value, in: Journal of Institutional and Theoretical Economics 159(2003): 675-682.

Hejazi, W. and Santor, E. (2005): Degree of Internationalization and Performance: An Analysis of Canadian Banks. Bank of Canada Working Paper 2005-32, Toronto, Ontario.

Henson, S. and Mazzocchi, M. (2002): Impact of Bovine Spobgiform Encephalopathy on Agribusiness in the United Kingdom: Results of an Event Study of Equity Prices, in: American Journal of Agricultural Economics, 84(2002): 370-386.

Hofstede, G. (1980): Culture's Consequences: International Differences in Work-Related Values, Beverly Hills, Sage Publications.

Holland, A. et al (2005): Beverages Quarterly: Q3 2005, Dresdner Kleinwort Wasserstein Equity Research, London.

Hsu, C. and Boggs, D. J. (2003): Internationalization and Performance: Traditional Measures and their Decomposition, in: Multinational Business Review 11(2003): 23-49.

Ietto-Gillies, G. (1998): Different Conceptual Frameworks for the Assessment of the Degree of Internationalization: An Empirical Analysis of Various Indices for the Top 100 Transnational Corporations, in: Transnational Corporations, 7(1998): 17-39.

InBev (2004): Interbrew and AmBev Establish InterbrewAmBev, The World's Premier

Brewer, Press Rreleases Archives. www.inbev.com, March 14, 2006.

InBev (2005): InBev Annual Report 2004, Brussels.

Jensen, M. C. (1992): Market for Corporate Control, in: Newman, P. et al. (Eds.), The New Palgrave Dictionary of Money and Finance, London: 657-665.

Joh.Barth\&Sohn (2005): The Barth Report. Hops 2004/05, Nuremberg. 
Karrenbrock, J. D. (1990): The Internationalization of the Beer Brewing Industry, in: Review - Federal Reserve Bank of St. Louis, 72(1990): 3-19.

King, J. L. et al (2002): A Tale of Two Mergers: What We Can Learn from Agricultural Biotechnology Event Studies, in: AgBioForum 5(2002): 14-19.

Kobrin, S. J. (1991): An Empirical Analysis of the Determinants of Global Integration, in: Strategic Management Journal 12(1991): 17-37.

Kusnadi, J. and Sohrabian, A. (1999): The Impact of Insurance Mergers on Shareholder Returns. URL: http://www.csupomona.edu.

Küting, K.-H. and Weber, C.-P. (2004): Die Bilanzanalyse. Lehrbuch zur Beurteilung von Einzel- und Konzernabschlüssen, Seventh Edition, Stuttgart.

Lastra, J. G. and Wiliamson, J. (2005): Carlsberg. Probably Half Full?, ABN AMRO, Amsterdam.

Lepetit, L. et al (2002): Diversification Versus Specialisation: An Event Study of M\&As in the European Banking Industry. URL: www.economics.bham.ac.uk/ research/dp2002/WP.Birmingham(2).pdf.

Lusk, J. L. and Schroeder, T. C. (2002): Effects of Meat Recalls on Future Markets Prices, in: Agricultural and Resource Economics Review, 31(2002): 47-58.

MacKinlay, A. C. (1997): Event Studies in Economics and Finance, in: Journal of Economic Literature 35(1997): 13-39.

Mathesius, J. (2003): Wertmanagement durch Equity Carve-out, Flensburg.

McClure, B. (2005): Understanding the Subtitles of ROA vs ROE, URL: www.investopedia.com.

McKenzie, A. M. and Thomsen, M. R. (2001): The Effect of E. Coli 0157:H7 on Beef Prices, in: Journal of Agricultural Resource Economics, 26(2001): 431-444.

Mohnen, A. (2002): Performancemessung und die Steuerung von Investitionsentscheidungen, Deutscher Universitäts Verlag, Wiesbaden.

Mueller, D. C. and Sirower, M. L. (2003): The Causes of Mergers: Tests Based on the Gains to Acquiring Firm's Shareholders and the Size of Premia, in: Managerial and Decision Economics 24(2003): 373-391.

Oldroyd, A. et al (2005): Basking in the Brazilian Sunshine, Morgan Stanley Equity Research, London.

Panayides, P. M. and Gong, X. (2002): The Stock Market Reaction to Merger and Acquisition Announcements in the Liner Shipping, in: International Journal of Maritime Economics 4(2002): 55-80.

Peterson, P. P. (1989): Events Studies: A Review of Issues and Methodology, in: Quarterly Journal of Business and Economics, 28(1989): 36-66.

Plato (2005a): Country Draughts: USA Beer Market, Plato Logic Limited, London.

Plato (2005b): Country Draughts: West Europe, Plato Logic Limited, London.

Plato (2005c): World Datasheets 2005, Plato Logic Limited, London.

Porter, M. E. (1990): The Competitive Advantage of Nations. New York: The Free Press.

Putlitz, J. (2001): Internationalisierung europäischer Banken: Motive, Determinanten, Entwicklungsmuster und Erfolg, Gabler, Wiesbaden.

Ramaswamy, K. et al (1996): Measuring the Degree of Internationalization of a Firm: A Comment, in: Journal of International Business Studies 27(1996): 167-177.

Rössing, S. (2005): Verpasste Chancen, www.LZ-net.de.

Ruigrok, W. and Wagner, H. (2003): Internationalization and Performance: An Organizational Learning Perspective, in: Management International Review 43(2003): 63-83.

SABMiller (2005): SABMiller and Bavaria Announce a Major Transaction in Latin America. SABMiller Group News. www.sabmiller.com, March 13, 2006. 
Salin, V. and Hooker, N. H. (2001): Stock Market Reaction to Food Recalls, in;: Review of Agricultural Economics, 23(2001): 33-46.

Schweiger, D. and Very, P. (2003): Creating Value Through Merger and Acquisition Integration, in: Cooper, C. and Gregory, A. (Eds.), Advances in Mergers and Acquisitions. Connecticut: JAI Press: 1-26.

Shusterman, T. G. et al (2001): Stock Market Reaction to Mergers and Acquisitions in the U.S. Telecommunications Industry. URL: http://www.norsworthy.net/uploads/PaperTatianaRandy3-14-01.pdf.

Simpson, J. D. and Hosken, D. (1998): Are Retailing Mergers Anticompetitive? An Event Study Analysis. URL: www.ftc.gov/be/workpapers/wp216.pdf.

Sirower, M. L. (1997): The Synergy Trap: How Companies Loose the Acquisition Game. New York, The Free Press.

Sullivan, D. (1994a): Measuring the Degree of Internationalization of a Firm, in: Journal of International Business Studies, 25(1994): 325-342.

Sullivan, D. (1994b): The 'Threshold of Internationalization': Replication, Extension, and Reinterpretation, in: Management International Review, 34(1994): 165-186.

UNCTAD (2004): World Investment Report, 2003. FDI Policies for Development: National and International Perspectives. United Nations: New York and Geneva.

Vernon, R. (1971): Sovereignty at Bay: The Multinational Spread of US Enterprises. New York, Basic Books.

Wells, W. (2004): A Beginner's Guide to Event Studies, in: Journal of Insurance Regulation 22(2004): 61-70.

Yule, M. et al (2005): Beer Appreciation: Episode 1. Morgan Stanley Research, London. 


\section{KAPITEL IV}

EXKURS: INTERNATIONALISIERUNG ANDERER SUBSEKTOREN DES Agribusiness 


\section{KAPITEL IV.1}

\section{Internationalization of Cooperatives in the Agribusiness \\ Concepts of Measurement and their Application}

Ludwig Theuvsen and Oliver Ebneth

Published in:

Strategies for Cooperatives, Theurl, Th. and Meyer, E. C. (Eds.), Aachen: 395-419. 


\title{
Internationalization of Cooperatives in the Agribusiness Concepts of Measurement and their Application
}

\begin{abstract}
Increasingly, cooperatives in the agribusiness are being confronted with the globalization of agri-food markets. Cooperatives adapt to this development by internationalizing their activities. This paper presents several methods of measuring the degree of internationalization and their application to European cooperatives in the meat and dairy sectors and ends with a discussion of why German cooperatives are noticeably less internationalized than their European competitors.
\end{abstract}

\section{INTERNATIONALIZATION: CHALLENGES FOR GERMAN COOPERATIVES}

In recent decades, one of the most striking developments has been the ever-increasing globalization of markets and the internationalization of economic activities. Due to low growth rates on home markets, growing international competitive pressures, shortened product lifecycles and growing $\mathrm{R} \& \mathrm{D}$ and marketing investments, many companies have expanded their national activities and, thus, contributed to the further advancement of the intensity and dynamics of international competition (Scholl, 1989; Marx, 1998). This development has also seized cooperatives. Due to strong pressures for reducing costs and developing new markets, mergers and acquisitions have become a common practice in the German cooperative sector. Cooperatives in the agribusiness sector, in particular, often located in rural areas, are forced to become more competitive by joining forces through mergers and acquisitions (Stappel and Hennigsen, 2003). Against this background, the (often neglected) internationalization of business activities is considered one of the most promising ways of gaining access to new markets and sustained economic success in the cooperative sector.

In this paper we empirically investigate the internationalization strategies of European cooperatives and explain differences between German cooperatives and their European competitors. In doing so, we focus on the dairy and meat sectors which are among the most important subsectors of the German food industry. We start by briefly introducing both sectors and then proceed by discussing different ways of measuring a cooperative's degree of internationalization. Some of these measure are then applied to a small sample of German and European co- 
operatives in both sectors. A discussion of results and managerial implications as well as some ideas for future research close this paper.

\section{ThE GERMAN DAIRY AND MEAT SECTOR}

The food sector is one of Germany's most important industries. In 2003, 5,880 companies employed about half a million people and had a turnover of 128 billion Euros. Although there are remarkable differences between subsectors, the food industry is for the most part still dominated by small and medium-sized companies. In 2001, the top ten German food companies represented only about $15 \%$ of the total turnover in their industry (Eichner Lisboa, 2002; BVE, 2004). Nevertheless, recent developments - at least in some subsectors - indicate the growing speed of the consolidation process and rising degrees of concentration. Leaving out other subsectors, this paper focuses on the dairy and meat sectors. This is for two reasons. First, cooperatives play a predominant role in both sectors and have leading positions not only in Germany but also in other European (and some non-European) countries. Second, both are characterized by high competitive pressures. Strong international competitors and a high degree of concentration in the retail sector force companies in both industries to become more competitive and to rethink their strategies (Weyhofen and Strecker, 2004). This also includes the critical analysis of internationalization strategies.

\subsection{The Dairy Sector}

In 2002 , the German dairy sector represented a turnover of 19.2 billion Euros, $16 \%$ of the total turnover in the German food industry. The dairy industry is characterized, on the one hand, by three large dairy cooperatives and a large privately-owned dairy company and, on the other, a considerable number of medium-sized dairy companies. The two largest dairy companies, Nordmilch and Humana, are both organized as cooperatives. In the European context, Nordmilch is the fifth and Humana the ninth largest milk processor (see figure 1). Recently, the German dairy industry has been characterized by dynamic structural change. From 2000 to 2002, the number of companies shrank from 250 to 118 . Nevertheless, the degree of concentration is still low compared, for instance, to the retail sector. The five largest companies have a combined market share of $42 \%$; the top ten companies represent $58 \%$ of the market. This small difference between CR5 and CR10 highlights the aforementioned division of the industry into four large competitors and a remarkable number of small and medium-sized enterprises. 
Figure 1: Top 10 in the European Dairy Industry

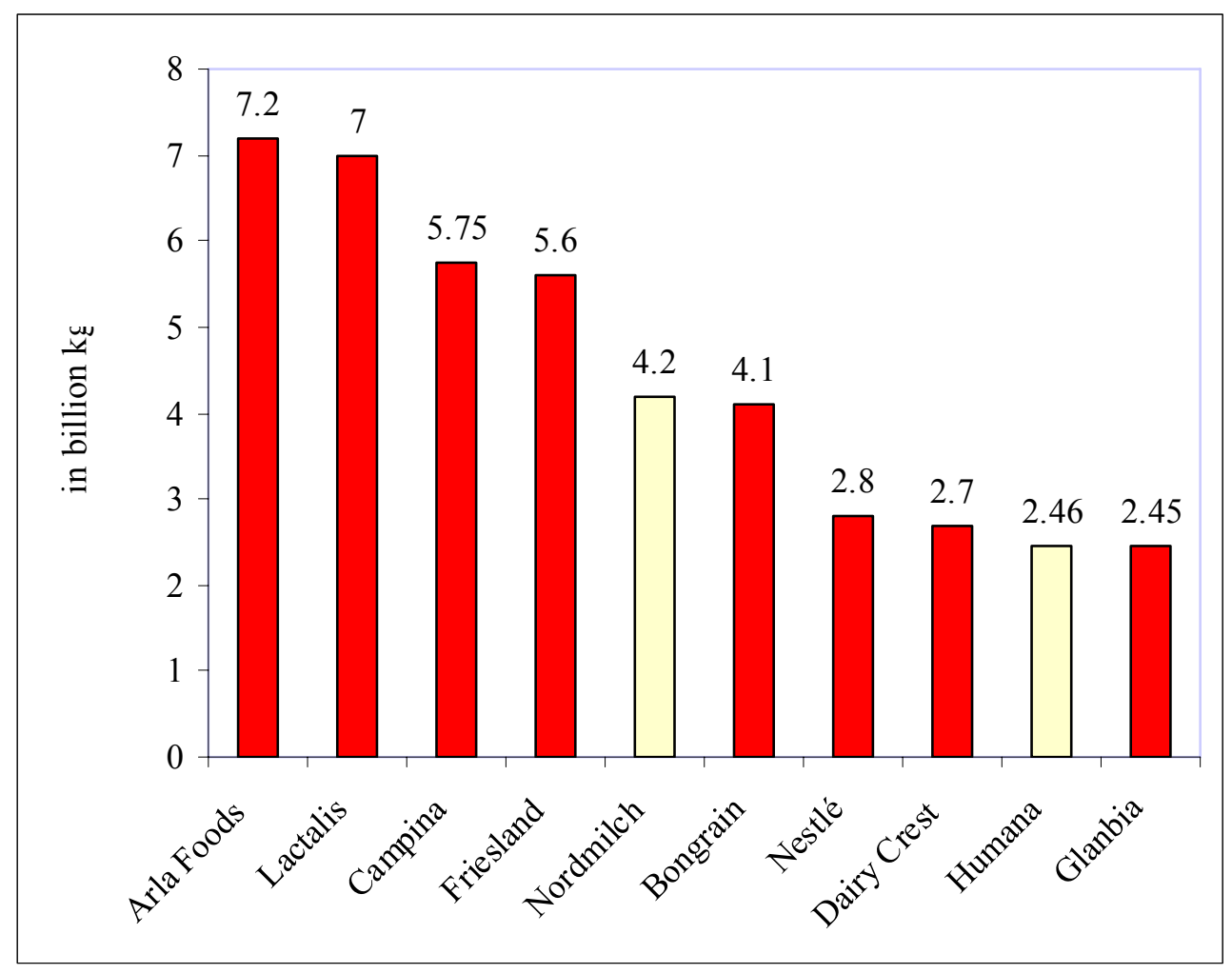

Source: Data compiled from annual reports.

The future competitiveness of the German dairy industry is influenced by several forces. Among the most important is the EU CAP (Common Agricultural Policy) reform, which, on the one hand, will reduce costs for dairy companies due to declining milk prices while, on the other hand, also inducing lower export prices. Taking market entries by foreign competitors and the internationalization strategies of large German retailers into account, as well, ongoing structural changes are expected in the dairy sector. Thus, in the near future, more mergers and acquisitions are expected to be announced so that the industry will slowly tranform into an oligopoly (Bridts and Köttl, 2003). Many authors expect that only about thirty companies will survive the next decade.

\subsection{The Meat Sector}

The meat industry is another subsector of the German food industry in which cooperatives have a high market share. In 2003 the total turnover of slaughtering, deboning and cutting companies was about 23 billion Euros. Like the dairy industry, the meat sector is characterized by a huge structural variety. The top four companies have a market share of about $30 \%$ (cattle) or $40 \%$ (pork), but compared to leading European competitors (Danish Crown, Best- 
meat) these companies (such as Westfleisch and Tönnies: see figure 2) are comparatively small. The overall industry struture is still polypolistic. Remarkable over-capacities and cost disadvantages due to the underutilization of fixed assets result in competitive disadvantages for German meat companies. This makes market entry quite easy for foreign competitors. In 2003, for instance, the Dutch Bestmeat Group acquired two major German competitors, Moksel and Nordfleisch (Schramm et al, 2004).

Figure 2: Top 10 in the European Meat Sector

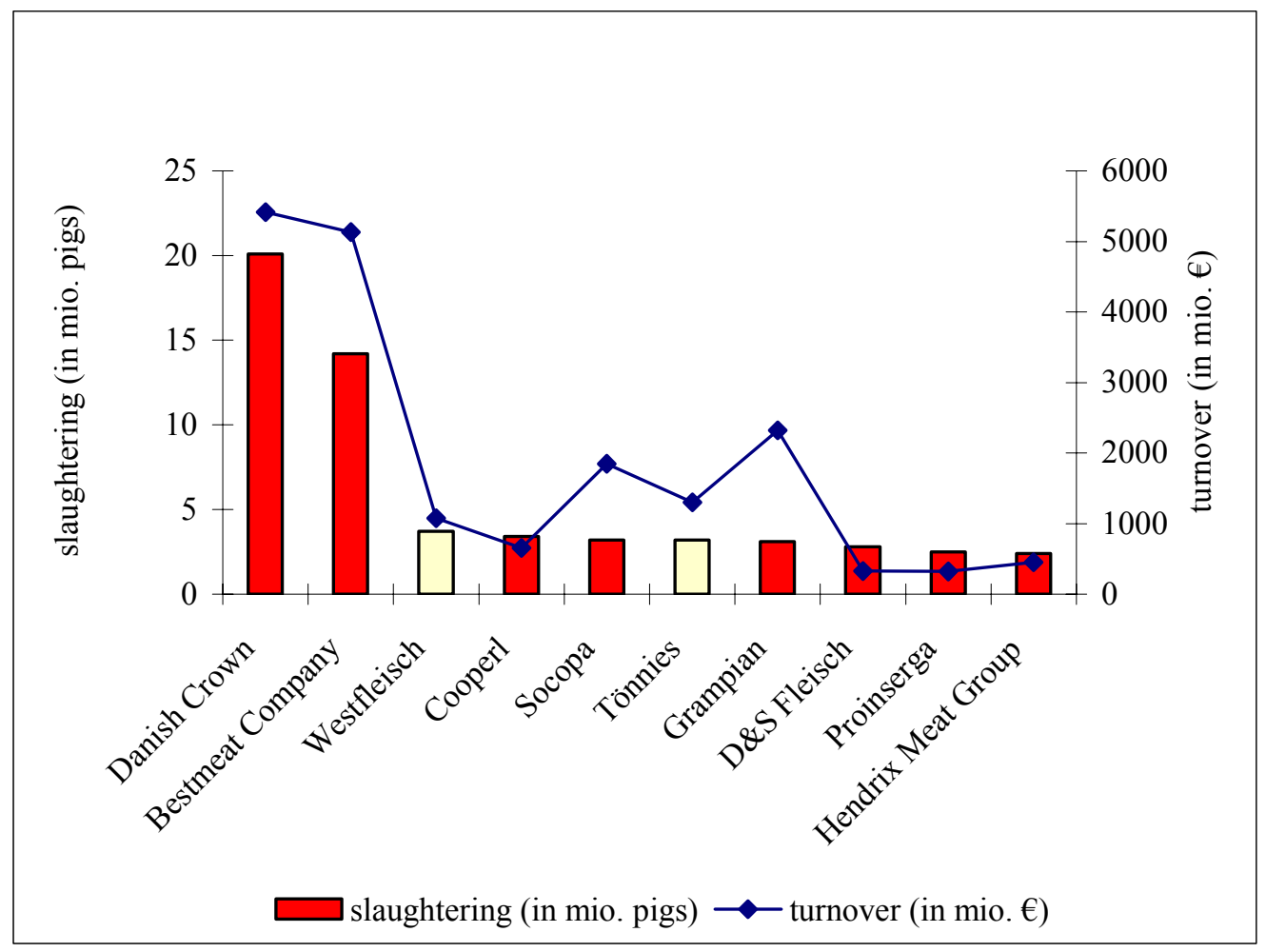

Source: Data compiled from annual reports.

The future development of the industry will be characterized by strong pressure on prices as well as the high quality expectations of customers. Companies in a vertically integrated meat chain are expected to have competitive advantages. In Denmark, the Netherlands and Belgium, highly competitive industry structures on the farm level as well as on the industry level have already emerged (Windhorst, 2004). From this point of view, the German meat industry is lagging behind in a catch-up position. 


\section{Methodology}

Internationalization describes the process by which an economy, an industry or a company becomes increasingly integrated into international economic activities. The degree of internationalization is the measure of the degree to which this economic integration has already taken place. When determining a company's degree of internationalization, activities on foreign markets are compared to home-market or world-wide activities (Germann et al, 1996). The degree of internationalization determines to what extent a company is confronted with problems of managing international activities and to what extent international environments are relevant for a specific company (Schmidt, 1981). Measuring the degree of internationalization, therefore, is of high managerial relevance.

There are unidimensional and multidimensional approaches to measuring the degree of internationalization. Unidimensional approaches rely on a single indicator, multidimensional approaches on two or more indicators representing different dimensions of the internationalization phenomenon. The result of combining two or more internationalization indicators by, for instance, calculating a mean value (Schmidt, 1981; Schüle, 1992) is often called an internationalization index (Glaum, 1996). The most common ways of measuring the degree of internationalization are quite simple, for example, calculating:

- the ratio of foreign sales to total sales or the share of foreign assets to total assets (Reeb et al, 1998),

- the ratio of foreign pre-tax income to total pre-tax income (Chen et al, 1997),

- the number of countries with foreign operations and the shares of foreign employees, profits, value added or shareholders (Fisch and Oesterle, 2003).

Advantages of these unidimensional approaches are the availability of information compiled from databases with open access. Furthermore, foreign ratios are clear indicators of international activities and they are easy to handle in mathematical operations. But this simplicity implies an obvious disadvantage: such measures capture only a single dimension of internationalization (Fisch and Oesterle, 2003). In contrast multidimensional measures try to cover a larger and more representative range of the concept internationalization. Some of these are discussed below.

\section{Sullivan's Degree of Internationalization}

Sullivan chooses five variables to measure the degree of internationalization (Sullivan, 1994): foreign sales as a percentage of total sales, overseas subsidiaries as a percentage of total sub- 
sidiaries, foreign assets as a percentage of total assets, psychic (cultural) dispersion of international operations and top managers' international experience. All indicators are calibrated from zero to one, receive equal weights, are summed up and result in a Degree of Internationalization (DOI) in the interval of $[0,5]$ (Fisch and Oesterle, 2003). Although innovative, this approach has faced severe criticism. Ramaswamy et al. [1996], for instance, point out that indicators which are located on different levels and measure very diverse aspects of internationalization are treated equally and combined to an internationalization index. They also argue that internationalization is more complex than envisioned by this index and suggest that further refinement of the concept is necessary before calculating indices. The authors question whether Sullivan's "index could be supported on the basis of content validity with the inclusion of an attitudinal component for which there is no logical justification and a seemingly narrow focus on subsidiary operating modes" [Ramaswamy et al., 1996, p. 167].

\section{UNCTAD Transnationality Index}

The Transnationality Index (TNi) was first published in 1995. In the UNCTAD approach the degree of internationalization is defined as the mean value of three unidimensional indicators: ratio of foreign assets to total assets (Ai), ratio of foreign sales to total sales ( $\mathrm{Si}$ ) and ratio of foreign employees to total employees (Ei):

$\mathrm{TNi}=[\mathrm{Ai}+\mathrm{Si}+\mathrm{Ei}]: 3$

with: $\mathrm{Ai}=$ Foreign Assets Index

$\mathrm{Si}=$ Foreign Sales Index

$\mathrm{Ei}=$ Foreign Employment Index

One of the most common criticisms of the Transnationality Index as well as Sullivan's approach is that a share of foreign activities contains no information about the distribution of these activities. If, for instance, a company with foreign sales shifts its activities from a few neighboring countries to many countries on different continents without increasing the share of foreign sales, the Transnationality Index does not depict this shift at all.

\section{Ietto-Gillies' Network Spread Index}

Grazia Ietto-Gillies' Network Spread Index is a remarkable attempt to overcome the foreign/home country dichotomy that is typical of other foreign ratio approaches (Ietto-Gillies, 1998). As has been mentioned before, the pure share of foreign activities does not reflect their international dispersion. Therefore, Ietto-Gillies calculates the Network Spread Index (NSi) by dividing the number of countries in which a company owns subsidiaries (n) by the maxi- 
mum number of countries in which a company could have subsidiaries $\left(\mathrm{n}^{*}\right)$. Ietto-Gillies defines $\mathrm{n}^{*}$ as the number of countries which have received foreign direct investments in a particular year. ${ }^{1}$ In 1998 when she developed her index, $n^{*}$ was 178:

$$
N S i=\frac{n}{n^{*}}=\frac{n}{178}
$$

Since the Network Spread Index does not contain any information about, for instance, foreign sales, it is more appropriate to consider NSi a weighting factor for, for instance, the UNCTAD Transnationality Index. The resulting Transnational Activities Spread Index (TASi) can be considered a combination of two dimensions of internationalization: ratios of foreign inputs and outputs to home-country inputs and outputs (TNi), on the one hand, and the country ratio (NSi), on the other (Ramaswamy et al, 1996; Fisch and Oesterle, 2000; Hassel et al, 2003). It is defined as follows:

$$
\mathrm{TASi}=[\mathrm{ASi}+\mathrm{SSi}+\mathrm{ESi}]: 3=\mathrm{TNi} \times \mathrm{Nsi}=\mathrm{TNi} \times\left(\frac{n}{n^{*}}\right)
$$

with: $\mathrm{ASi}=$ Assets Spread Index $=$ Ai x NSi

$$
\begin{aligned}
& \mathrm{SSi}=\text { Sales Spread Index }=\mathrm{Si} \times \mathrm{NSi} \\
& \mathrm{ESi}=\text { Employment Spread Index }=\mathrm{Ei} \times \mathrm{NSi}
\end{aligned}
$$

\section{Fisch and Oesterle's Degree of Globalization}

In 2000 Fisch and Oesterle proposed the most advanced measurement concept to date. The Degree of Globalization concept is based on the idea that the geographical spread of international activities as well as the cultural diversity of the countries a company is engaged in is relevant for discriminating different internationalization strategies. The geographical spread ( $g s^{\prime}$ ) of foreign activities is the ratio between the geographical spread of all economic activities in the world $\left(g_{G N P}\right)$ and a multinational company's (MNC) spread of international activities $\left(g_{M N C}\right)$. Both spreads are calculated as the Gini coefficients $g$ which describe the areas below the respective Lorenz curves (see figure 3). The ratio of these areas delivers the geographical spread measure:

$$
g s^{\prime}=\frac{1-g_{M N C}}{1-g_{G N P}}
$$

which is a number in the interval of $[0,1]$ and combines two aspects of internationalization:

1 Instead, $n *$ could also be defined as the total number of independent countries in the world. The difference between the two definitions is usually very small. 
the share of foreign activities and the number of countries these activities are spread over.

Figure 3: Geographical Spread of International Activities

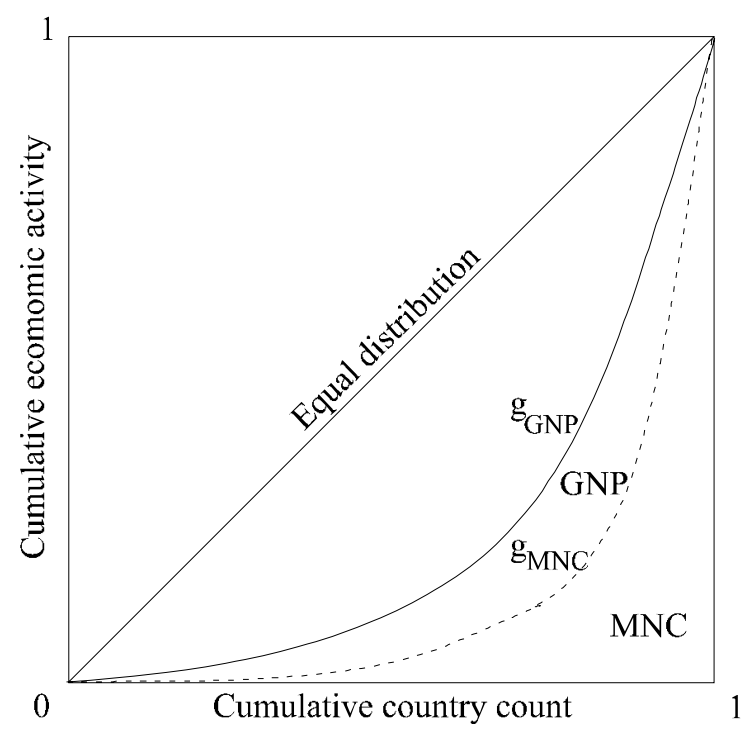

Source: (Fisch and Oesterle, 2003).

The economic activities of a company are considered perfectly globalized if their geographical spread equals the geographical spread of all economic activities in the world. In this case gs' is 1 whereas gs' of a truely national company without any foreign activities is 0 .

Cultural diversity ( $\left.c d^{\prime}\right)$ is measured by referring to Geert Hofstede's concept. Hofstede investigated cultural differences between fifty countries and three regions in four dimensions: power distance, individualism versus collectivism, masculinity versus femininity and uncertainty avoidance (Hofstede, 1980; Hofstede, 1993). Let $\sigma_{\text {Hofstede }}^{2}$ be the variance of all cultural indexes through all countries and regions considered by Hofstede and $\sigma_{M N C}^{2}$ be the corresponding variance through all countries in which a MNC has subsidiaries. Then cultural diversity is calculated as:

$$
c d^{\prime}=\frac{\sigma_{M N C}^{2}}{\sigma_{\text {Hofstede }}^{2}}
$$

Just like $g s^{\prime}, c d^{\prime}$ is a number in the interval of $[0,1]$.

To simultaneously compare different MNCs in more than one dimension of internationalization, the authors combine the geographical spread measure gs' and the culture diversity measure $c d^{\prime}$ in a complex number dog' (Degree of Globalization). A complex number consists of a real part and an imaginary part and is the only way to integrate independent dimensions of internationalization. The Degree of Globalization is then defined as: 


$$
\left|\operatorname{dog}^{\prime}\right|=\sqrt{g s^{\prime 2}+c d^{\prime 2}}
$$

The maximum absolute value of the Degree of Globalization indicating full globalization is:

$$
\left|\operatorname{dog}_{\max }\right|=\sqrt{1+1}=\sqrt{2} \approx 1,41 \text {. }
$$

To avoid the counterintuitive result of $141 \%$ being the maximum Degree of Globalization, $g s$ and $c d$ are finally defined as

$$
g s=\frac{g s^{\prime}}{\sqrt{2}} \text { and } c d=\frac{c d^{\prime}}{\sqrt{2}} \text {. }
$$

This operation limits the Degree of Globalization to the intuitively more appealing maximum value of 1 or $100 \%$ (see figure 4). The angle $\varphi$ defines which influence (geographical dispersion or cultural diversity) on a company's Degree of Globalization is stronger. If $\varphi$ is greater than $45^{\circ}$, the influence of cultural diversity is stronger than the influence of geographical dispersion and vice versa.

Figure 4: Degree of Globalization

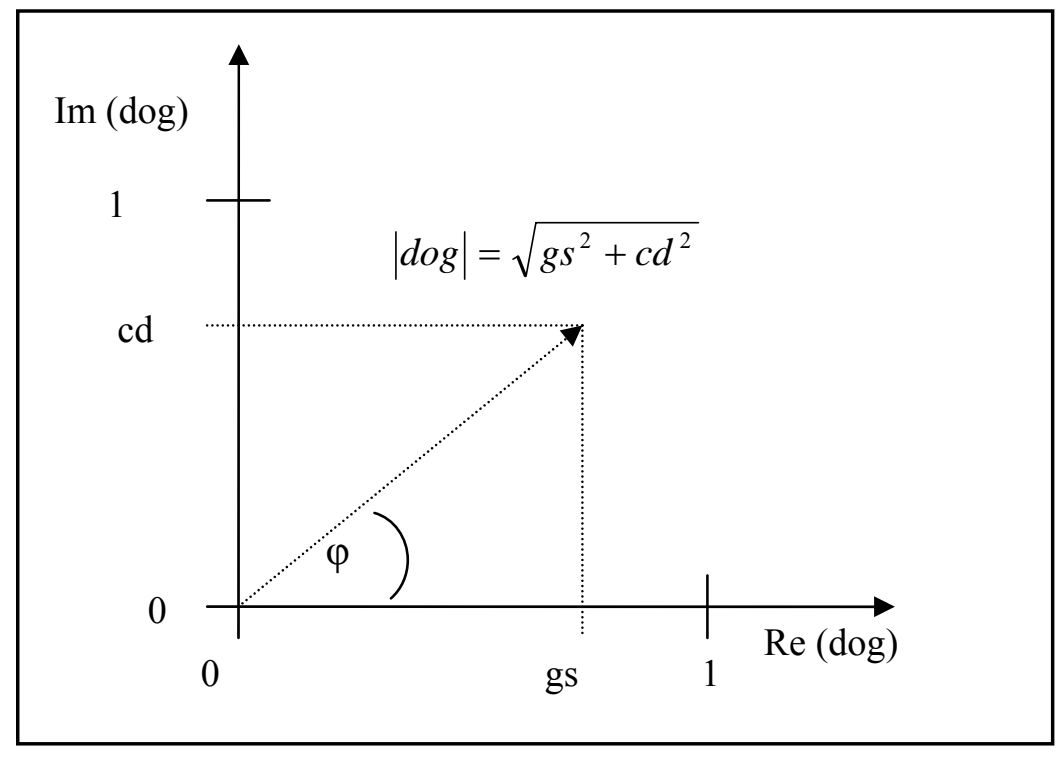

source: (Fisch and Oesterle, 2003).

Fisch and Oesterle's approach is slightly more complex than traditional measures of internationalization. The advantages of this new concept are that the Degree of Globalization effectively combines three widely used measures (share of foreign activities, number of countries and cultural diversity) in one single measure and that it can be used for any numerical operation. The most severe problem is the availability of data. Hofstede's concept, for instance, has not been applied to all countries, thus leaving empty spaces in determining cultural diversity. 


\section{Process Model of Internationalization}

An additional and somewhat differerent way of describing a company's degree of internationalization is the reference to a process model of internationalization. Meissner and Gerber argue that the internationalization process of a company can be described as a multi-step process in which capital and management is transfered step by step from a company's headquarter to foreign subsidiaries (Meissner and Gerber, 1980). Companies often start with pure export activities that do not require capital or management transfers to foreign countries. After some time they often decide to license their products, enter international joint ventures or move production overseas. In the end they may own full-scale foreign subsidiaries (see figure 5).

Figure 5: Process Model of Internationalization

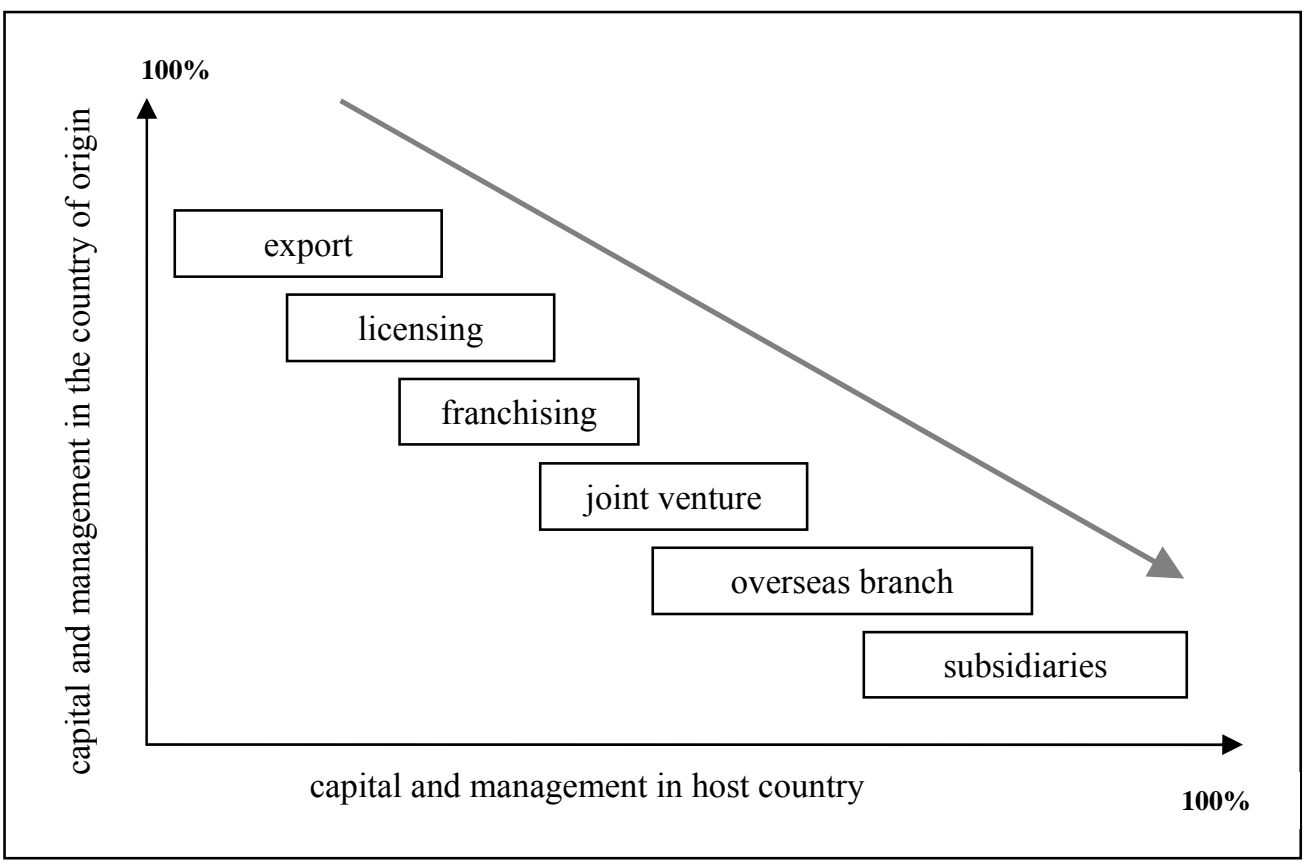

source: (Meissner and Gerber, 1980).

Whereas in early phases (export, licensing) the relationship is very intensive with the homecountry and very weak with export destinations, it is often the other way round when fullscale subsidiaries exist abroad (Swoboda, 2002). In a truly global company the recently dominant role of the headquarter has been replaced by an international network with changing strategic roles for foreign subsidiaries (Bartlett and Ghoshal, 1989).

When applying the measurement concepts discussed above to cooperatives, availability of data turns out to be a severe problem, which clearly limits the analysis. The concepts for measuring the degree of internationalization, especially the multidimensional approaches, 
have been developed for application to leading global companies like Daimler-Chrysler, Shell or Sony which have to publish financial and non-financial data extensively due to legislation or heavy reliance on external capital markets and which are very intensively covered by the media. Compared to these companies, even leading cooperatives in the meat and dairy industry are small and information about them is much scarcer for two reasons. First, cooperatives are neither listed on stock exchanges nor heavily dependent on bond markets. Due to this, legal and market pressures on them to disclose information are comparatively low. Second, due to their limited size and economic importance, cooperatives are widely overlooked by the media. Unlike the brewing industry, in which several global players applying the International Financial Reporting Standards (IFRS) have emerged (Theuvsen and Ebneth, 2004), the dairy and meat sectors are still comparatively "hidden" industries in which public access to information is limited. For this reason, only the following internationalization measures are applied in this paper:

- Foreign Sales Index: Ratio of foreign sales to total sales

- Network Spread Index with $\mathrm{n}^{*}=191(\mathrm{UNCTAD}, 2004): N S i=\frac{n}{n^{*}}=\frac{n}{191}$

- Degree of Internationalization: $\mathrm{DoI}=\left(\frac{\text { Foreign sales }}{\text { Total sales }}+\mathrm{NSi}\right): 2$

Additional references are made to Meissner and Gerber's process model of internationalization in order to describe the cooperatives' internationalization activities in more detail.

\section{SAMPLE}

In the European dairy and meat industries quite a large number of cooperatives can be found. In this early phase of research we focus on four cooperatives: Campina (the Netherlands) and Nordmilch (Germany) in the dairy industry and Danish Crown (Denmark) and Westfleisch (Germany) in the meat sector.

\section{Campina}

Campina BV and Friesland Coberco Dairy Foods are the leading Dutch dairy companies. Campina was founded in 1989 through a merger of two Dutch dairy cooperatives. In 2003, Campina processed 5.75 billion $\mathrm{kg}$ milk, had a turnover of 3.66 billion Euros and employed around 7,000 people. Today 7,000 dairy farmers in Western, Central and Southern Netherlands, 2,500 dairy farmers in the Western parts of Germany and some seventy dairy farmers in the Belgian province of Antwerpen are members of the dairy cooperative Campina. 


\section{Nordmilch}

Nordmilch eG is Germany's largest dairy company. Its 12,300 members and all of its production facilities are located in Northern Germany, mainly in the Northwest. The Nordmilch cooperative was founded through a merger of the "old" Nordmilch eG and Milch-Erfassung Nordmilch eG, Molkereizentrale Oldenburg-Ostfriesland eG (MZO), Molkerei BremerlandNordheide eG and Hansano Milchhof Niedersachsen eG. Today the Nordmilch group processes more than four billion $\mathrm{kg}$ milk per year and has an annual total turnover of around 2.1 billion Euros.

\section{Danish Crown}

With more than 19,800 members Danish Crown is Europe's largest abattoir and meat processor. It was founded on October 1, 1990, when Wenbo, Tulip and østJyske merged. Between 1994 and 2001, four more companies merged with Danish Crown: Syd and Skærbæk in 1994, Vestjyske in 1998 and Steff-Houlberg in 2001. In 2002 Danish Crown slaughtered 418,000 cattle and 21.4 million pigs. The cooperative has 14,300 employees and generates a turnover of 5.4 billion Euros.

\section{Westfleisch}

Westfleisch eG was founded in 1928 and still belongs to Germany's leading abattoirs. The cooperative and its about 3,250 members operate four slaughterhouses for pork and cattle. In recent years Westfleisch has diversified its activities and is now also operating in the convenience and poultry sectors. The cooperative has a turnover of just over one billion Euros and has more than 1,100 employees.

\section{DEGREE OF INTERNATIONALIZATION OF EUROPEAN MEAT AND DAIRY}

\section{COOPERATIVES}

\section{Campina}

Campina started internationalizing its activities in 1993, when it acquired the German Südmilch AG. Today Campina operates subsidiaries in sixteen countries of which five are nonEuropean. Just recently Campina strengthened its activities in Asia by acquiring Parmalat's Thai subsidiary (Anonymus, 2004b). Nevertheless, the Netherlands and Germany are still Campina's dominant markets (see figure 6). In Germany Campina operates through several local subsidiaries, i.e. former Südmilch, Milchwerke Köln/Wuppertal, Emzett and Molkerei Strothmann. 
Figure 6: Campina - Turnover Analyzed by Market

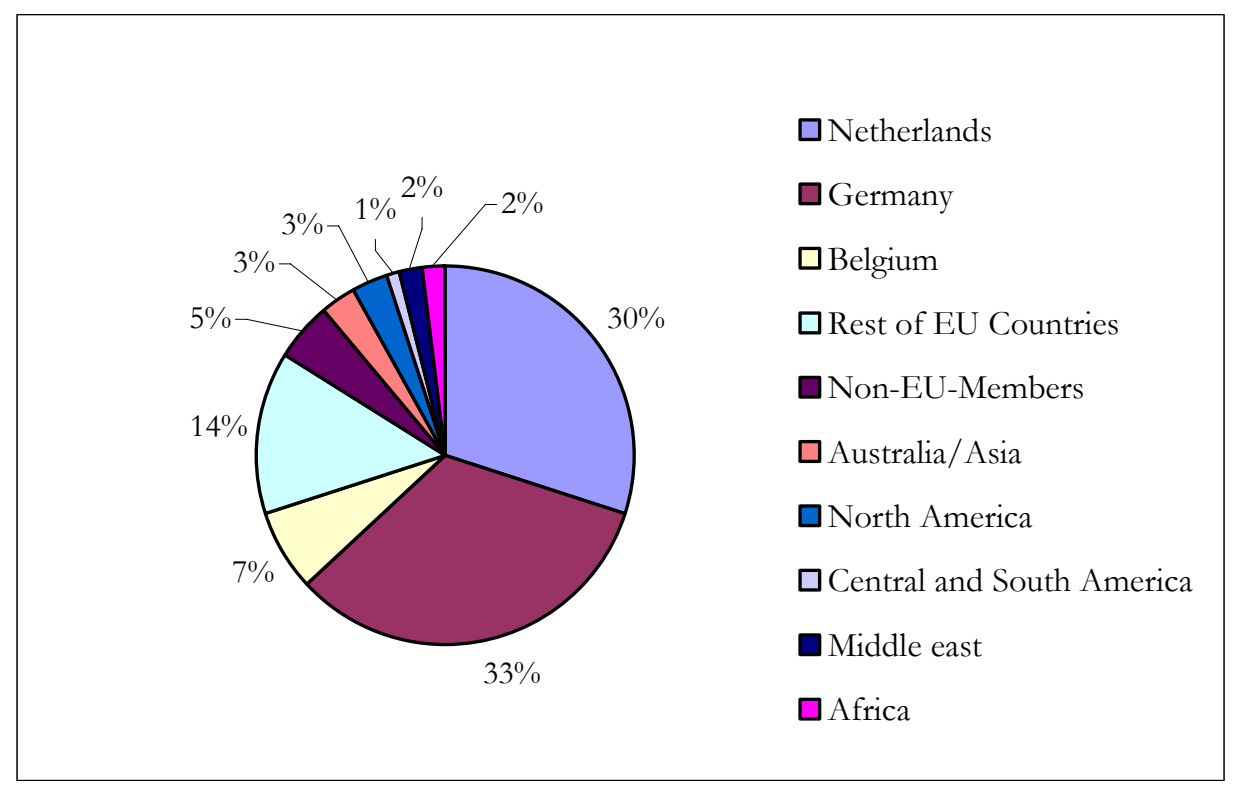

Figure 7 reveals that in 2003 Campina generated $70 \%$ of its turnover outside the Netherlands. The Degree of Internationalization was nearly 0.4. In recent years Campina has only slightly increased its Degree of Internationalization. Although the export quota has risen only moderately, Campina has succeeded in expanding via subsidiaries, which is the most intensive form of internationalization.

Figure 7: Campina - Degree of Internationalization

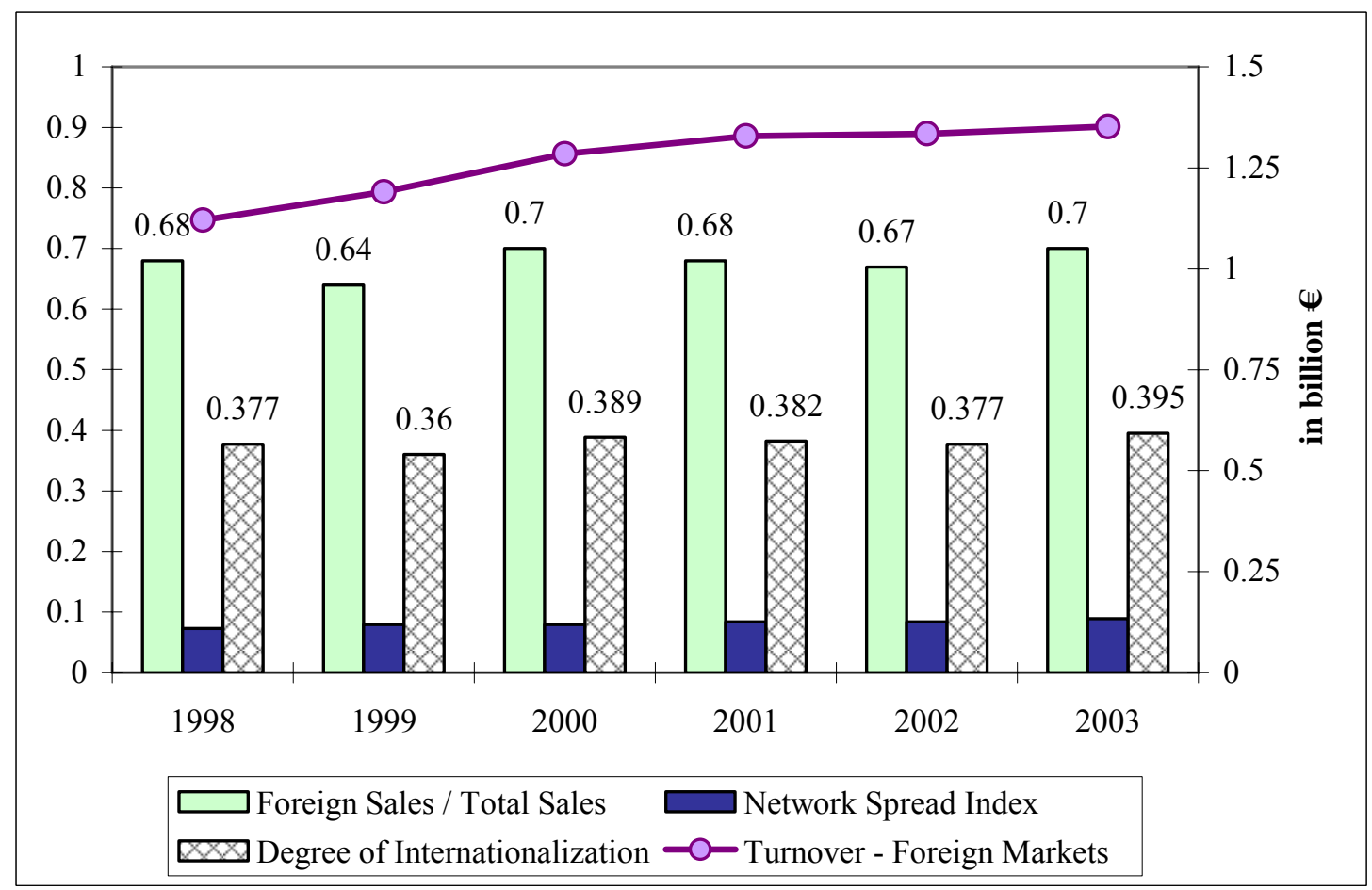




\section{Nordmilch}

Although nearly the same size as Campina, Nordmilch's internationalization strategy is very different from that of its important Dutch competitor. Only about 20\% of its turnover is generated through exports. Nearly $4 / 5$ of its exports are generated in the EU and only $1 / 5$ comes from non-EU countries. The internationalization strategy focuses only on export activities. Nordmilch does not have any foreign subsidiaries at all so that the Network Spread Index is zero. According to Meissner and Gerber, this is the least intensive form of international activities since neither capital nor management know-how are transferred to foreign countries (Meissner and Gerber, 1980). Consequently, the degree of internationalization displayed in figure 8 is much lower than that of Campina and has remained low in recent years.

Figure 8: Nordmilch - Degree of Internationalization

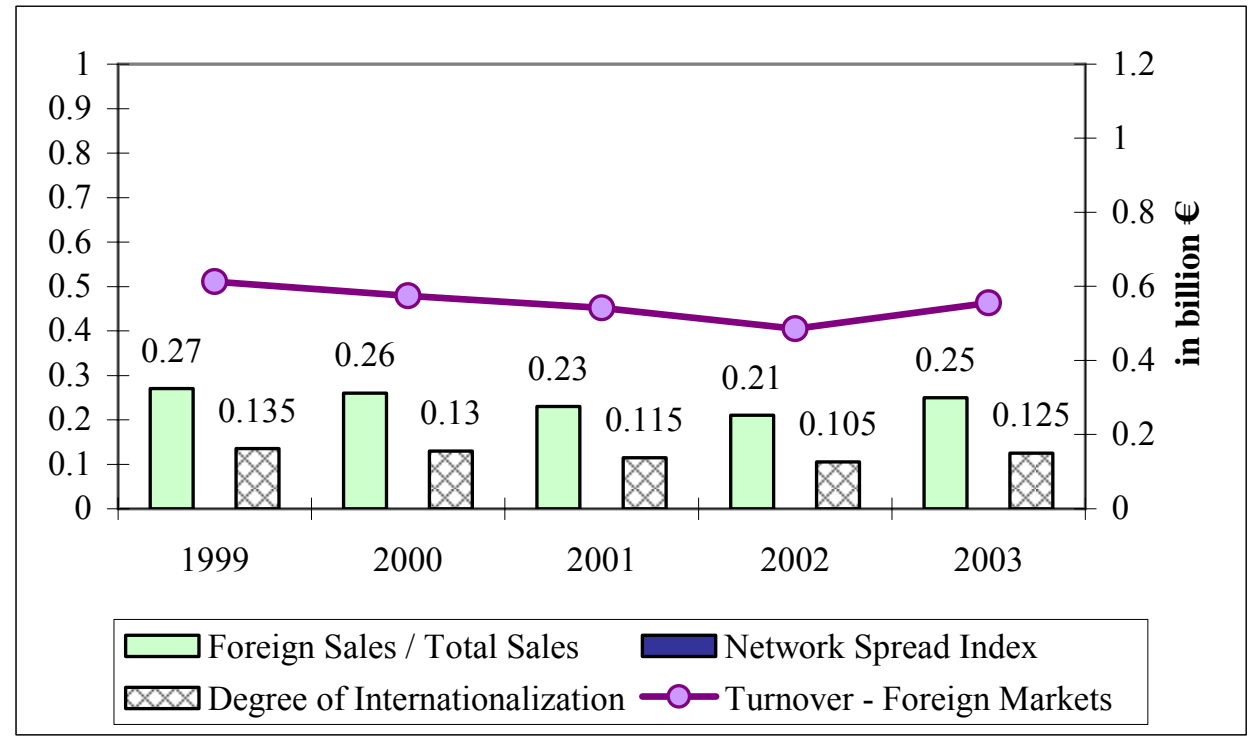

\section{Danish Crown}

Danish Crown dominates its home market and slaughters $60 \%$ of all cattle and $77 \%$ of all pork in Denmark. Some 90\% of pork and pork products produced by Danish Crown in Denmark are distributed internationally. Main export destinations for Danish pork are Great Britain, Germany, Japan, Eastern Europe and the United States (see figure 9). 
Figure 9: Danish Crown - Turnover Analyzed by Market

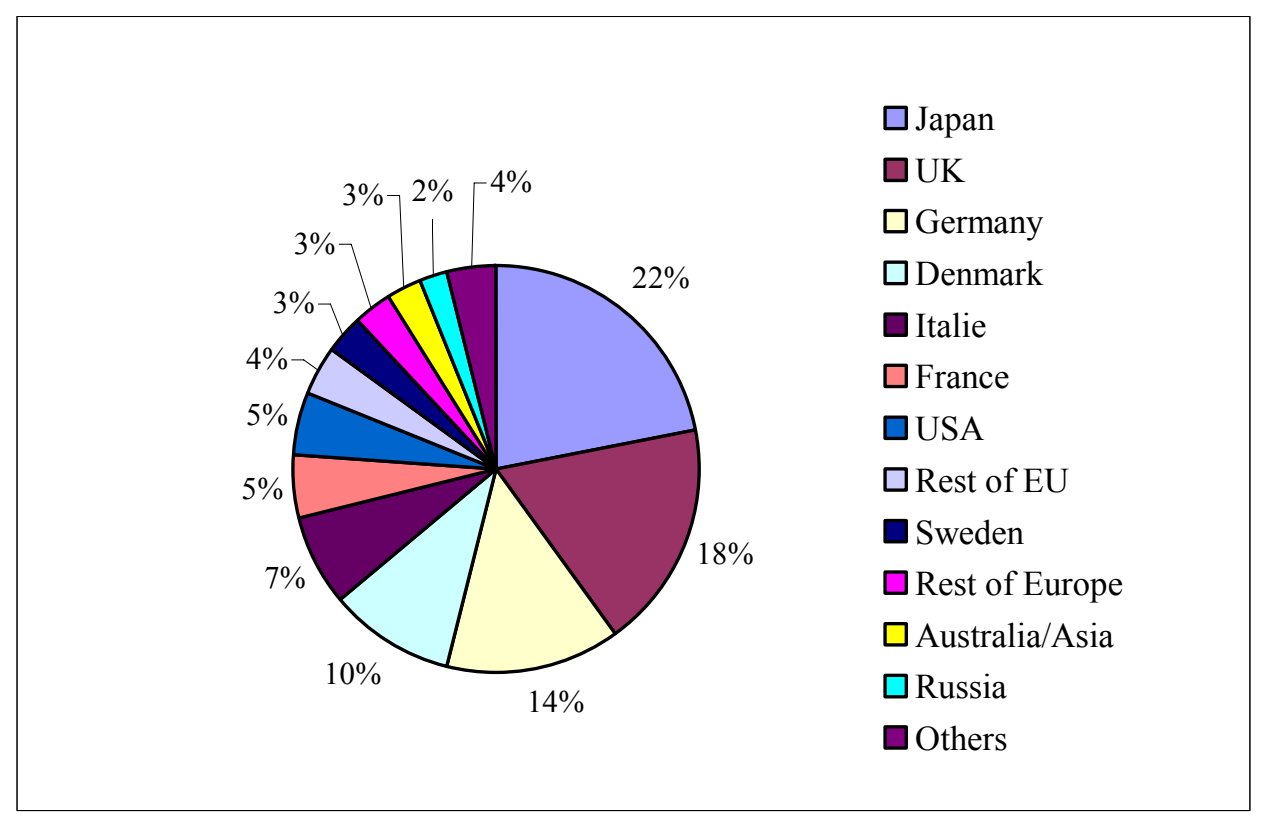

Danish Crown generates $89 \%$ of its turnover outside Denmark. It is Denmark's most important exporter in the agribusiness sector. The cooperative generates $54.5 \%$ of all Danish food exports and $6 \%$ of total exports. Figure 10 shows that in recent years Danish Crown's export quota has always been between $85 \%$ and $90 \%$. But Danish Crown also implements more advanced internationalization concepts, like foreign subsidiaries. These contribute to a continuous increase in Danish Crown's Degree of Internationalization. In addition to its twenty-one European subsidiaries the cooperative now operates in six non-European countries. Nevertheless, Danish Crown is still accelerating its internationalizing activities. Just recently it took over Flagship Foods Ltd. in Great Britain and announced that it would be moving parts of its meat processing activities from Denmark to Schüttorf and Oldenburg in Germany. 
Figure 10: Danish Crown - Degree of Internationalization

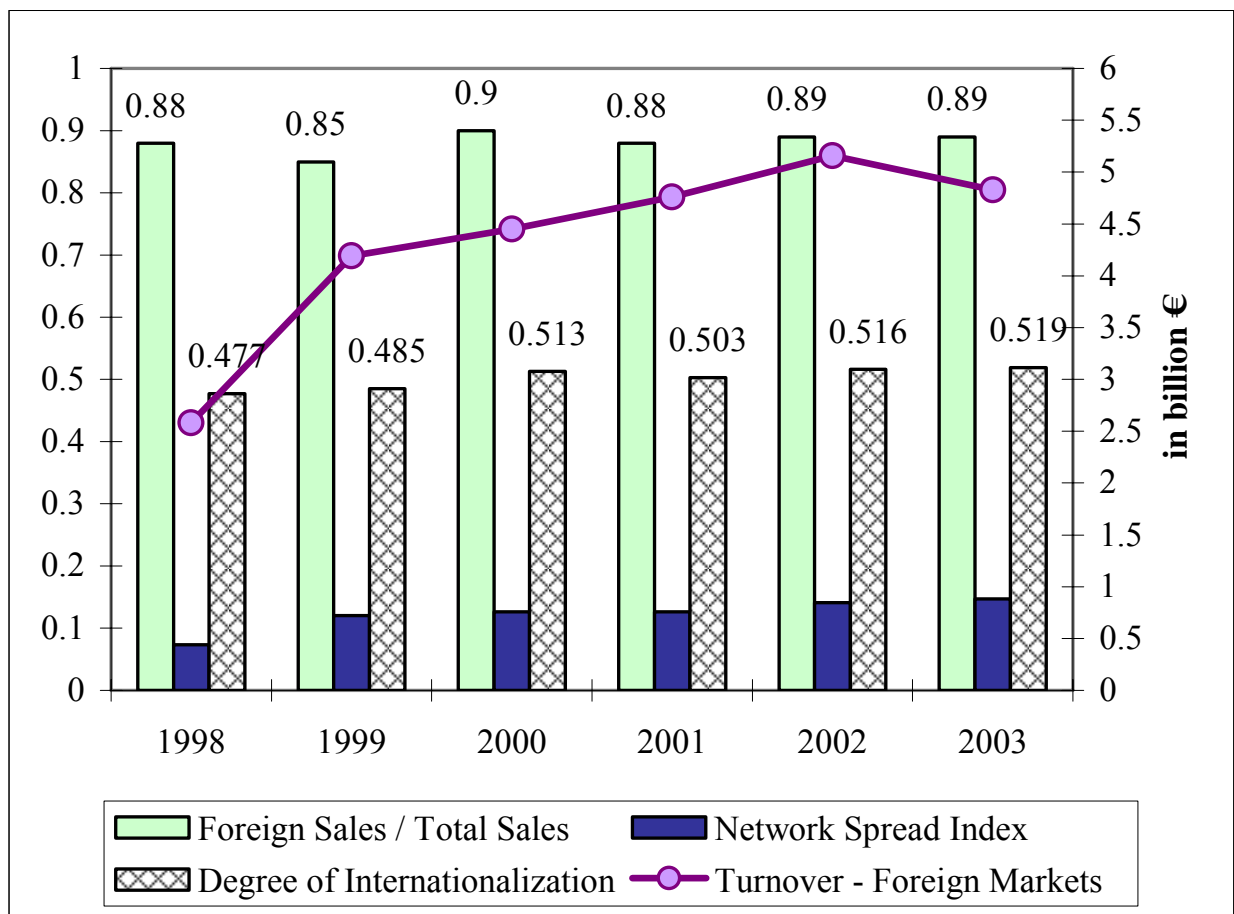

\section{Westfleisch}

Westfleisch generates about $20 \%$ of its turnover outside Germany. In recent years, Westfleisch's export quota has fluctuated noticeably due to great dependance on a few core export markets such as Russia. Westfleisch holds minority stakes in two foreign companies in Poland and the Netherlands but does not control any wholly-owned subsidiary. Thus, it is characterized by a much lower Degree of Internationalization than Danish Crown (see figure 11).

Figure 11: Westfleisch - Degree of Internationalization

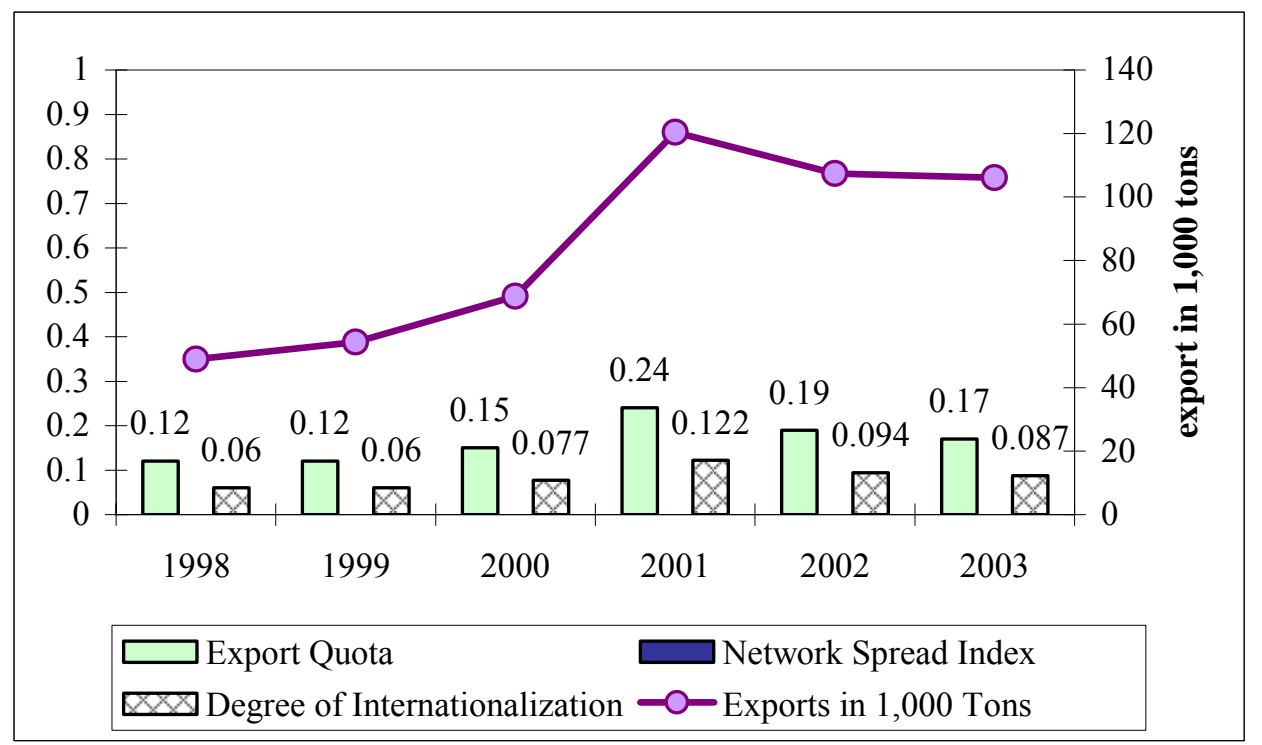




\section{DISCUSSION AND MANAGERIAL IMPLICATIONS}

Although this paper presents only preliminary results on a small-sized database from an ongoing research project, one trend has already become obvious: German cooperatives are far less internationalized than their counterparts from neighboring countries. How can we explain this distinctive difference between German and other European cooperatives?

One explanation is that Danish Crown as well as Campina are located in small countries with a comparatively small home market for meat and dairy products. Since the possible degree of specialization is mainly determined by the size of the market (Stigler, 1951), companies from small countries are forced to internationalize their activities in order to become cost-efficient (Kutschker and Schmid, 2004). In this respect, cooperatives from larger countries which offer more opportunity for economies of scale and specialization experience lower pressures to internationalize their acitivities and have systematic disadvantages with respect to the measurement of degrees of internationalization. Thus, the small-country argument is a convincing explanation for the high export quota of Danish as well as Dutch cooperatives. But it cannot explain why both Campina and Danish Crown seek business opportunities in European and non-European countries by acquiring or founding local subsidiaries, while their German competitors stick to their national and sometimes even regional markets.

Dairy industry insiders often argue that certain characteristics of fresh products like milk for instance, limited shelf-life - complicate the internationalization of business activities and require a mainly regional focus of production and distribution in the dairy industry (Krijger, 2004). Since only 32 million tons out of a worldwide milk production of 590 million tons are traded internationally, there is obviously something in this argument. But it cannot explain why some cooperatives have built up a far-reaching network of international subsidiaries whereas others refrain from investing abroad. Campina and other internationalized dairy companies, such as Skandinavian Arla Foods, no longer put their trust in exports but have subsidiaries in several other countries. It is clear, therefore, that product characteristics do not explain low degrees of internationalization but only certain internationalization modes. Referring to Meissner and Gerber's process model, we can say that product characteristics like limited shelf-life prevent only such half-hearted internationalization strategies as exporting products. In fact they require more intensive internationalization strategies based on investments abroad. Some authors expect that at the end of the consolidation process in the dairy sector only ten to twenty companies will have survived. Due to certain product characteristics, these multinational companies will be interwoven with a dense network of local partners which 
support local milk production and collection as well as distribution of end products (Krijger, 2004). Again, obvious differences between German cooperatives and their European competitors cannot be explained by this argument.

A third argument refers to the economic situation of German cooperatives. The German meat sector, for instance, is well-known for overcapacities and missing opportunities to adequately utilize investments in fixed assets (Traupe, 2002). Furthermore, strong European (Danish Crown, Bestmeat) and non-European competitors (for instance from Brazil) are increasingly challenging the market positions of German meat companies. A slow restructuring of the industry favors inefficient firm size and hampers the emergence of companies which have sustained competitive advantages in national and international markets. All these problems result in low returns on investments in the German meat sector. Therefore, most German meat companies lack sufficient financial and managerial resources to quickly and successfully internationalize their acitivities. Instead, they are increasingly threatened by their larger and more internationally oriented competitors from neighboring countries. It is doubtful whether German cooperatives will belong to those five or six companies which are expected to dominate the European meat sector in about ten years (Buchenau, 2003).

In the German dairy industry the market situation is similar. This favors strong price competition vis à vis a highly concentrated retail sector. Whereas privately-owned dairy companies often have strong brands and comparatively strong market positions, German dairy cooperatives usually pursue cost leadership strategies and focus on standardized low-cost and lowprice mass market articles like milk, milk powder and butter. This results in a very weak market position (Schramm et al, 2004) and limited financial resources for establishing international business activities. Dutch and Danish cooperatives operate on more consolidated home markets in which they have high market shares. This situation gives them a better starting position for internationalization.

A fourth strand of thought stems from the economic literature on the peculiarities of cooperatives. Most of this literature argues from the point of view of new institutional economics. Property rights theory, transaction cost theory and agency theory all come up with similar and somewhat interrelated arguments concerning the efficiency of cooperatives. With reference to internationalization strategies, the most important arguments can be summarized as follows (Staatz, 1989; Cook, 1995; Horsthemke, 2000):

- Free-riding problem: An external free-riding problem occurs when a cooperative treats non-members exactly the same as members by offering, for instance, the same terms of 
trade. In this case there is no need to become a member of the cooperative and to financially support its internationalization strategy. An internal free-riding problem occurs when new members of a cooperative are treated in the same way as "old" members. Again, the resulting free-riding problem undermines the motivation to invest since (mainly long-term) investments are not safeguarded against attenuation due to an imperfect definition of property rights. The reluctance to invest undermines a cooperative's ability to internationalize.

- Investment problem: The property rights of members in their cooperative are very difficult to trade. As a consequence, members who want to leave the cooperative cannot quickly get rid of their investments. Therefore, many cooperatives have a large number of inactive members who still hold their property rights even though they no longer interact economically with the cooperative. These investors are more interested in short-term returns on investments than in long-term investments in, for instance, internationalization strategies.

- Transaction cost problem: In large cooperatives members pursue very diverse goals. In such cases reaching decisions on, for instance, internationalization strategies becomes costly and cooperatives have severe problems reacting effectively to new market conditions by shifting the regional center of gravity of their business.

- Control problem: The "one man - one vote" principle and the non-tradeability of property rights prevent the accumulation of votes and capital shares which would lower transaction costs and help to overcome the free-riding problem in controlling the organization. So it is very difficult for individual members of a cooperative to initiate internationalization strategies which might be considered necessary but which are not supported by reluctant members or managers.

- Human resource problem: In many cooperatives part-time board members with low managerial know-how and severe time constraints have considerable influence on strategic decisions. Due to the transaction cost and the control problem, it is difficult for members who do not agree with a cooperative's internationalization strategy to get rid of these board members and to implement better top management.

The diverse capabilities of cooperatives in solving these problems may explain different degrees of internationalization. Some cooperatives stick to their traditional structure and the aforementioned problems remain largely unsolved. Others have revolutionized their corporate governance mechanisms by implementing new organizational forms. Some cooperatives, for instance, have transformed into holding companies by outsourcing their day-to-day business activities into a professionally managed joint-stock company. The members of the cooperative 
then focus only on managing the holding company which holds the shares in the newly founded daughter company.

Successful examples of the latter can be found in the German sugar industry, in which cooperatives have much more successfully internationalized than in the dairy and meat sectors. A similar solution can be found in the Dutch food industry. Dumeco in the meat sector as well as Campina in the dairy industry have both established a separation of cooperative elements and management issues (Horsthemke, 2000). On the other hand, Nordmilch and Westfleisch have relied on more traditional corporate governance mechanisms, which seem to have disadvantages with respect to long-term investments in internationalization strategies.

Assuming that our diagnosis is correct, the managerial implications are obvious. German cooperatives in the meat and dairy industries have to solve their financial problems and redesign their corporate governance mechanisms in order to overcome the above-mentioned problems hampering their further internationalization. Recent developments indicate that such a change of mind is, in fact, going on in leading German cooperatives and that a redesign and professionalization of management structures is taking place. Nordmilch, for instance, has just recently announced that since June 22, 2004, board membership is restricted to professional, full-time members.

Since many cooperatives lack the financial performance to internationalize their activities, cross-border mergers in the cooperative sector might be unavoidable. Such mergers result in transnational cooperatives with several geographic centers of gravity (Borgström, 2003). Arla Foods' chief executive, for instance, wants to double the turnover of his cooperative within the next few years. A further consolidation of European dairy cooperatives is considered paramount for competing successfully with multinational privately-owned food companies like Nestlé, Danone or Unilever (Anonymus, 2004a). Thus, Arla might become the core of the transnationalization of Europe's cooperative sector in the agribusiness.

\section{FUTURE RESEARCH}

In this paper we have presented some preliminary results of our analysis of internationalization strategies of European dairy and meat cooperatives. This provides a good starting point for future research acitivites. First, the sample should become much larger in order to obtain more representative results. Second, research should be extended beyond the meat and the dairy sector. Furthermore, future research should strive to apply more advanced measurement concepts like Fisch and Oesterle's Degree of Globalization. Last but not least, the financial 
efficiency of different internationalization strategies reflected by different degrees of internationalization should be analyzed.

\section{REFERENCES}

Anonymus (2004a). Fusion Arla und MD Foods. Milchriese entsteht in Dänemark, in: Handelsblatt (193): 13.

Anonymus (2004b). LZ-Net, Firmenprofile: Industrie, Campina b.v., http://www.lznet.de/companies/firmindustrie/pages/show.prl?params=stype $\% 3 \mathrm{D} 2 \% 26$ currChar $\% 3 \mathrm{D}$ C\&id=148\&currPage=, Abrufdatum: 26.05.2004.

Bartlett, C. A. and Ghoshal, S. (1989). Managing Across Borders. The Transnational Solution, Boston.

Borgström, M. H. (2003). Die Lage und Entwicklung landwirtschaftlicher Genossenschaften in Europa, http://www.iru.de/de/publikationen/courier/3-03/Deutsch-4.html, Abrufdatum: 27.05.2004.

Bridts, C. and Köttl, P. (2003). Steht die Milchwirtschaft vor der heißen Phase ihres Konzentrationsprozesses?, in: M\&A Intern, Zeitschrift der HypoVereinsbank (HBV Consult), 9(2003): 8-15.

Buchenau, M.-W. (2003). Deutscher formt neuen Fleisch-Konzern. Bestmeat-Chef gelingt Übernahme von Nordfleisch, in: Handelsblatt (217): 22.

BVE (2004). Zahlen, Daten, Fakten, http://www.bve-online.de/, Abrufdatum: 25.05.2004.

Chen, C. J. P. et al (1997). An Investigation of the Relationship Between International Activities and Capital Structure, in: Journal of International Business Studies, 28(1997): 563-577.

Cook, M. L. (1995). The Future of U.S. Agricultural Cooperatives: A Neo-Institutional Approach, in: American Journal of Agricultural Economics, 77(1995): 1153-1159.

Eichner Lisboa, S. (2002). Chancen nutzen - Kooperationen für den Mittelstand, http://www.bve-online.de/, Abrufdatum: 25.05.2004.

Fisch, J. H. and Oesterle, M.-J. (2000). Globalisation can be Measured: Unveiling Tales of Mystery and Globalisation With a New Integrative and Metric Measurement Concept, Paper Submitted to the 26th Annual Conference of the European International Business Academy, Maastricht.

Fisch, J. H. and Oesterle, M.-J. (2003). Exploring the Globalization of German MNCs with the Complex Spread and Diversity Measure, in: Schmalenbach Business Review, 55(2003): 2-21.

Germann, H. et al (1996). Globalisierung der Wirtschaft: Begriff, Bereiche, Indikatoren, in: Steger, U. (Hrsg.), Globalisierung der Wirtschaft: Konsequenzen, für Arbeit, Technik und Umwelt, Berlin: 18-55.

Glaum, M. (1996). Internationalisierung und Unternehmenserfolg, Wiesbaden.

Hassel, A. et al (2003). Two Dimensions of the Internationalization of Firms, in: Journal of Management Studies 40(2003): 705-723.

Hofstede, G. (1980). Culture's Consequences: International Differences in Work-Related Values, Beverly Hills, Sage Publications.

Hofstede, G. (1993). Cultural Constraints in Management Theories, in: Academy of Management Executive 7(1993): 81-94.

Horsthemke, A. (2000). Mitgliederbindung und Kapitalaufbringung im Strukturwandel länd- 
licher Genossenschaften, Stuttgart-Hohenheim.

Ietto-Gillies, G. (1998). Different Conceptual Frameworks for the Assessment of the Degree of Internationalization: An Empirical Analysis of Various Indices for the Top 100 Transnational Corporations, in: Transnational Corporations, 7(1998): 17-39.

Krijger, A. (2004). Auf die Strategie kommt es an, in: DLG-Mitteilungen, 5(2004): 26-29. Kutschker, M. and Schmid, S. (2004). Internationales Management, München.

Marx, T. (1998). Internationale Marketingstrategien in der deutschen Brauwirtschaft, in: Europäische Hochschulschriften, Reihe V, Volks- und Betriebswirtschaft, Band 2233, Frankfurt am Main u.a.

Meissner, H. G. and Gerber, S. (1980). Die Auslandsinvestition als Entscheidungsproblem, in: BFuP, 3(1980): 217-228.

Ramaswamy, K. et al (1996). Measuring the Degree of Internationalization of a Firm: A Comment, in: Journal of International Business Studies 27(1996): 167-177.

Reeb, D. M. et al (1998). Systemic Risk of the Multinational Corporation, in: Journal of International Business Studies, 29(1998): 263-279.

Schmidt, R. (1981). Zur Messung des Internationalisierungsgrades von Unternehmen, Kiel.

Scholl, R. (1989). Internationalisierungsstrategien, in: Macharzina, K. und Welge, M. (Hrsg.), Handwörterbuch Export und internationale Unternehmung, Stuttgart: 983-1001.

Schramm, M. et al (2004). Brand Orientation in der Ermährungsindustrie. Erfolgsdeterminanten der Markenführung am Beispiel genossenschaftlicher Hersteller, Wiesbaden.

Schüle, F. M. (1992). Diversifikation und Unternehmenserfolg: eine Analyse empirischer Forschungsergebnisse, Wiesbaden.

Staatz, J. M. (1989). Farmer Cooperative Theory. Recent Developments, in: USDA (Ed.): ACS Research Report Nr. 84.

Stappel, M. and Hennigsen, E. (2003). Die deutschen Genossenschaften 2003. Entwicklungen - Meinungen - Zahlen, Neuwied.

Stigler, G. (1951). The Division of Labor is Limited by the Extent of the Market. Journal of Political Economy, 59(1951): 185-193.

Sullivan, D. (1994). Measuring the Degree of Internationalization of a Firm, in: Journal of International Business Studies, 25(1994): 325-342.

Swoboda, B. (2002). Dynamische Prozesse der Internationalisierung. Managementtheoretische und empirische Perspektiven des unternehmerischen Wandels, Wiesbaden.

Theuvsen, L. and Ebneth, O. (2004). Exploring Globalization in the Brewing Industry. Internationalization Strategies of World-wide Leading Brewers. Poster presented at the 14th Annual World Food and Agribusiness Forum, 12th - 15th of June, Montreux.

Traupe, C. (2002). Schlachtschweinevermarktung in Niedersachsen: Stand, Defizite, Entwicklungsmöglichkeiten, Göttingen.

UNCTAD (2004). World Investment Report, 2003. FDI Policies for Development: National and International Perspectives. United Nations: New York and Geneva.

Weyhofen, C. and Strecker, O. (2004). Das planen die Unternehmen, in: DLG-Mitteilungen 5(2004): 17-19.

Windhorst, H.-W. (2004). Ohne Anpassung keine Zukunft, in: DLG-Mitteilungen 5(2004): 20-23. 
KAPITEL IV.2

\section{Internationalisierung europäischer Molkereigenossenschaften}

\section{Oliver Ebneth}

Erschienen in ähnlicher Form in folgenden Praxiszeitschriften:

- Wertschöpfungskette Milch muss an einem Strang ziehen, in: Land\&Forst 35(2005): 5-7,

- Am Ausland führt kein Weg vorbei, in: DLZ Agrarmagazin 12(2005): 132-135,

- Genossenschaften im Wettbewerb. Internationalisierung genossenschaftlicher Molkerei- und Schlachtunternehmen, in: Neue Landwirtschaft 1(2005): 22-26,

- Genossenschaften. Können sie mithalten?, in: DLG-Mitteilungen 9(2004): 70-71. 


\section{Internationalisierung europäischer Molkereigenossenschaften}

\section{MOLKEREIGENOSSENSCHAFTEN IM INTERNATIONALEN WETTBEWERB}

Viele Unternehmen der Agrar- und Ernährungswirtschaft sind in den letzten Jahren aus verschiedenen Gründen - geringes Marktwachstum, Wandel der Verbrauchergewohnheiten, Konzentration im Handel usw. - unter verstärkten wirtschaftlichen Druck geraten. Die angespannte wirtschaftliche Lage hat auch im deutschen Genossenschaftssektor den Konzentrations- und Konsolidierungsprozess vorangetrieben, wobei in den letzten Jahren zahlreiche Zusammenschlüsse zu verzeichnen waren. Zu den Zielen der Fusionen zählten die Reduzierung von Kosten sowie die Erschließung zusätzlicher Absatzpotentiale. Vor allem Molkereigenossenschaften stehen zunehmend unter dem Einfluss eines veränderten Verbraucherverhaltens sowie eines verstärkten Wettbewerbs auf den Agrarmärkten seitens Wettbewerbern aus anderen EU-Staaten (Stappel and Hennigsen, 2003).

Angesichts des schwierigen wirtschaftlichen Umfelds gilt die Internationalisierung im Agribusiness als eine Erfolg versprechende Maßnahme, langfristig das Unternehmenswachstum und den Unternehmenserfolg durch Erschließung neuer Märkte zu sichern. Ausländische Wettbewerber haben diesen Weg vielfach schon erfolgreich beschritten und sich auf diese Weise zu schlagkräftigen Einheiten entwickelt. Die deutsche Agrar- und Ernährungsindustrie ist dagegen in weiten Teilen noch stark regional bzw. national ausgerichtet. Dies gilt aufgrund ihrer langen Historie und ihres Zwecks - Verarbeitung und Absatz in der Region erzeugter landwirtschaftlicher Produkte - gerade auch für die deutschen Genossenschaften im Agribusiness. Bemerkenswert ist dabei, dass Genossenschaften aus anderen EU-Staaten ihre Aktivitäten bereits sehr viel stärker internationalisiert haben und sich insofern einen großen Vorsprung vor ihren deutschen Konkurrenten erarbeiten konnten. Dieser Beitrag zeigt an ausgewählten Beispielen, wie groß die Unterschiede im Bereich der Internationalisierung aber auch hinsichtlich einiger unternehmerischer Kennzahlen zwischen deutschen und ausländischen Molkereigenossenschaften aktuell sind, und diskutiert mögliche Ursachen dieser Entwicklung. 
Stehen auf der Seite der Primärproduktion in der Wertschöpfungskette Milch die Kosten im Fokus, so sind es auf Seiten der Verarbeiter eher die Erlöse, die optimiert werden können, sei es durch Marktausweitung ins Ausland oder durch höher verarbeitete Produkte im Premiumbereich. Die deutsche Milchindustrie erreichte 2004 einen Umsatz von 20,3 Mrd. € (Milchwirtschaft, 2005). Dies entspricht einem Anteil von etwa $16 \%$ am Gesamtumsatz der deutschen Ernährungsindustrie. Die Branchenstruktur ist zweigeteilt: Neben drei großen genossenschaftlichen Unternehmen und einer großen Privatmolkerei existieren eine Reihe mittelständisch geprägter sowie eine Vielzahl kleinerer Molkereien, von denen sich einige eine regionale Nische erschlossen haben. Trotz eines dynamischen Strukturwandels im Laufe der letzten Jahre konkurrieren immer noch 108 Molkereien in einem ruinösen Verdrängungswettbewerb miteinander. Somit ist auch der Konzentrationsgrad der Milchbranche immer noch vergleichsweise niedrig. Im Jahr 2004 sind die Konzentrationsraten $\mathrm{CR}_{5}\left(\mathrm{CR}_{10}\right)$ (Umsatzanteil der fünf (zehn) größten Molkereien am Gesamtumsatz) im Vergleich zum Vorjahr von $41 \%$ auf $38 \%$ (56\% auf $53 \%$ ) zurückgegangen, was mit Umsatzrückgängen der fünf größten Molkereien zusammenhängt. Knapp 50 \% der gesamten Milch wurde 2003 in den 16 Unternehmen verarbeitet, die eine Milchverarbeitungsmenge von mehr als 500 Mio. kg aufweisen; 2000 waren dies lediglich $38 \%$ in 13 Molkereiunternehmen mit mehr als 500 Mio. kg Milchmenge (Gerlach et al, 2006). Ein wichtiges Standbein für den Absatz von Milch und Milchprodukten deutscher Milchverarbeiter ist der Export mit einem Umsatzanteil von $18,9 \%$ in 2004, der bisher allerdings überwiegend auf Basisprodukten beruht.

Da sowohl der EU-Milchmarkt wie auch der deutsche Markt gesättigt sind und allenfalls einige wenige Produktsegmente noch langsam wachsen, können die einzelnen Unternehmen ihre Geschäftsaktivitäten nur durch eine Internationalisierung ihres Geschäfts ausdehnen. Abbildung 1 veranschaulicht anhand zweier Internationalisierungsmaße das relativ schwache Ausmaß grenzüberschreitender Geschäftstätigkeit der deutschen Molkereigenossenschaften. Vor allem dänische und niederländische Genossenschaften, wie die Molkereikonzerne Arla Foods, Friesland oder Campina, bauten in der Vergangenheit ein dichtes weltweites Netzwerk von Tochterunternehmen auf, das die lokale Milcherfassung, -verarbeitung und -distribution gewährleistet. Die deutschen Genossenschaften beschränken ihr internationales Engagement bisher dagegen ausschließlich auf reine Exporttätigkeit, ohne weitergehende Internationalisierungsschritte zu unternehmen. So verfügt die dänisch/schwedische Arla über mittlerweile 26 Tochtergesellschaften weltweit, wohingegen die Nordmilch gerade einmal erste Auslandsrep- 
räsentanzen in Prag und jüngst allerdings auch in China eröffnet hat (Anonymus, 2006a). Abbildung 1 gibt diese Unterschiede nochmals im Überblick wieder. Dabei stehen zwei Kennzahlen im Vordergrund: der Export- bzw. Auslandsanteil am Umsatz sowie die Anzahl ausländischer Tochtergesellschaften und Minderheitsbeteiligungen.

Abbildung 1: Internationalisierungskennzahlen europäischer Molkereigenossenschaften.

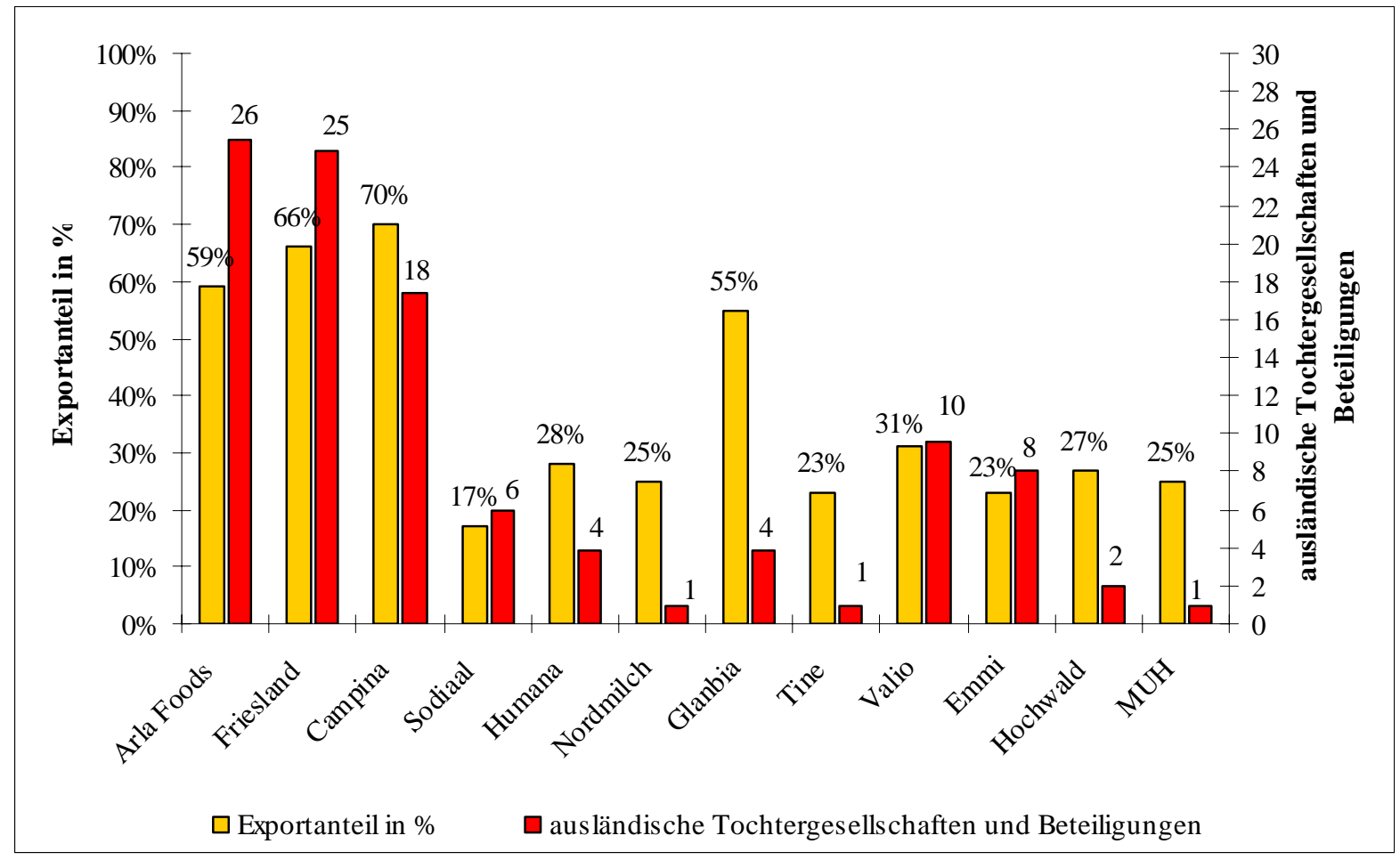

Quelle: Unternehmensangaben aus Geschäftsberichten, eigene Berechnungen.

Abbildung 2 zeigt die elf größten europäischen Molkereigenossenschaften anhand von Milchverarbeitung und Umsatz im Jahr 2004. Selbst die größten deutschen Milchkonzerne Nordmilch, Humana und Hochwald stehen im europäischen Vergleich aufgrund ihres begrenzten Auslandsengagements gemessen am Umsatz nur auf den Plätzen fünf, sechs und elf. Die Milchunion Hocheifel bildet die europäische Nummer 23, wurde als viertgrößte deutsche Molkereigenossenschaft allerdings mit in die Untersuchung aufgenommen. 
Abbildung 2: Umsatz und Milchverarbeitung europäischer Molkereigenossenschaften - 2004.

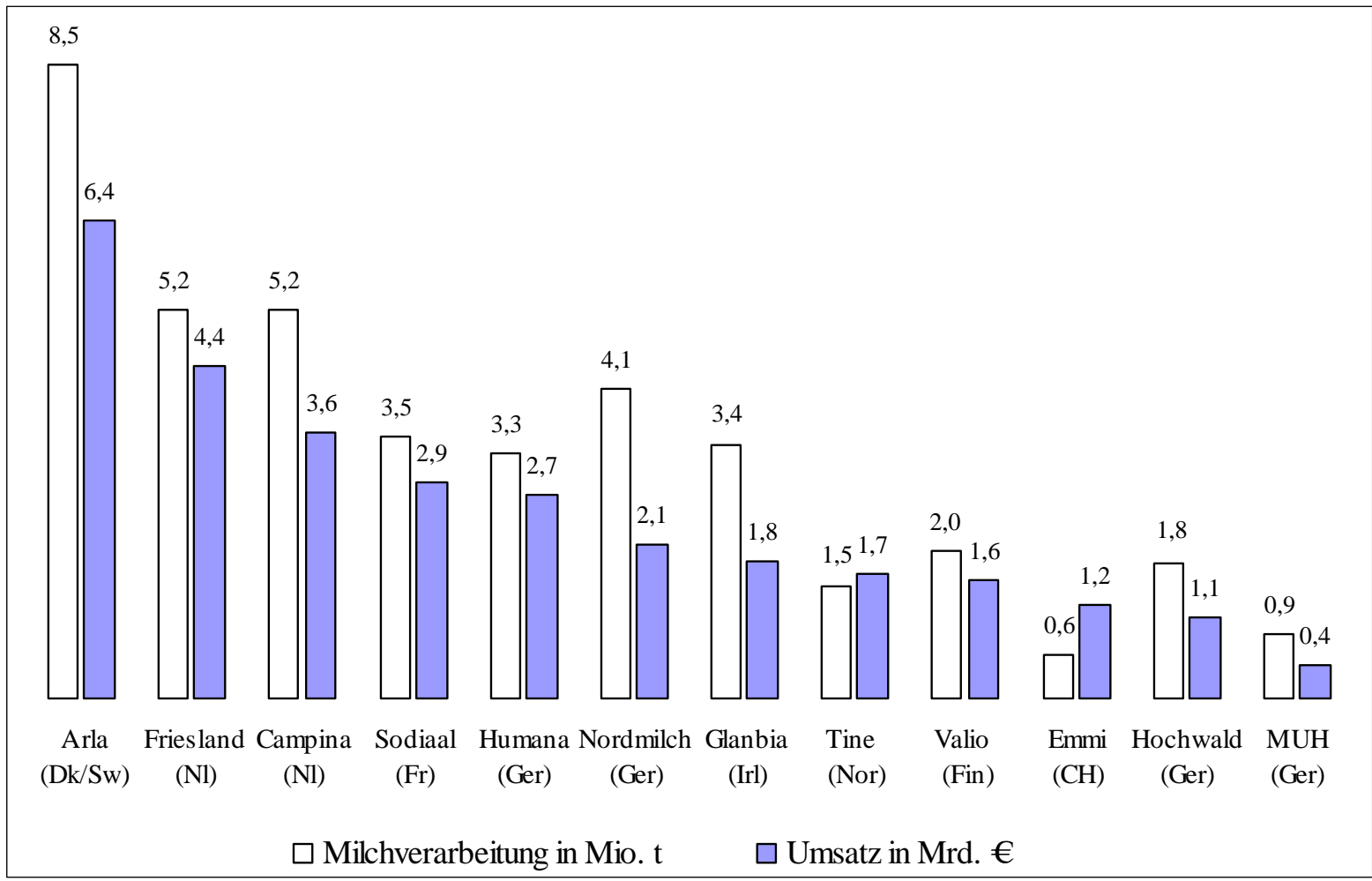

Quelle: Unternehmensdaten, eigene Berechnung.

Beim Vergleich mit den übrigen europäischen Genossenschaften fällt auf, dass v.A. beim deutschen Marktführer Nordmilch aus der großen Milchverarbeitungsmenge nur ein relativ geringer Umsatz resultiert. Dies rührt daher, dass das Unternehmen vor allem standardisierte Low-cost- und Low-price-Ware wie Butter, Magermilchpulver und Konsummilch produziert, bei denen es aufgrund der Austauschbarkeit der Produkte gegenüber den Abnehmern in einer besonders schlechten Verhandlungsposition ist. Eindrucksvoll veranschaulichen diese Schwäche Gerlach et al. (2006), die die deutschen Käse-Importe und -Exporte analysiert haben. Über die wichtigsten Ländermärkte hinweg ergibt sich für die Importe ein durchschnittlicher Preis von 4,02 €/kg, für Exportkäse lediglich 2,84€/kg. Mithin sind die deutschen Exporte im Wesentlichen niedrigpreisige Standardkäsesorten, die Importe dagegen überwiegend Spezialitäten. Der Blick auf einzelne Länder verdeutlicht dies. Insbesondere die als Käsespezialisten bekannten Länder Schweiz, Italien und Frankreich führen teure Käsesorten nach Deutschland ein wohingegen Italien und Frankreich im Gegenzug aus Deutschland Standardware wie Gouda, Feta und Mozzarella geliefert bekommen. Dies spiegelt die Handelsmarken- und Kostenführerschaftsstrategie deutscher Molkereien wider. 
Gerade im Zuge von Planung bzw. Durchführung erster oder weiterer Internationalisierungsschritte werden verstärkt finanzielle Mittel benötigt. Tabelle 1 verdeutlicht anhand ausgewählter Unternehmenkennzahlen, dass gerade den deutschen Marktführern Nordmilch und Humana oftmals diese Finanzmittel aufgrund relativ schwacher Bilanzdaten fehlt. Zwar weist die Nordmilch im Jahr 2003 noch eine äußerst schwache Eigenkapitalquote von etwa 22\% auf, doch greifen anscheinend nach und nach die umfangreichen Restrukturierungsmaßnahmen, was sich in einem Anstieg der Eigenkapitalquote auf 28\% nieder schlägt. Damit stehen die größten deutschen Molkereien Nordmilch und Humana inzwischen gleich auf mit den führenden europäischen Wettbewerbern. Doch anders als beim Eigenkapital fallen die deutschen Molkereigenossenschaften durch eine sehr geringe Umsatzrendite auf, die oftmals gerade einmal einer „schwarzen Null“ entspricht, im Gegensatz zu etwa 2-5\% der europäischen Mitbewerber. Problematisch ist auch das nach wie sehr geringe Anlagevermögen pro Tonne Milchverarbeitung der deutschen Großmolkereien. Dies kann darauf zurückzuführen sein, dass die deutschen Genossenschaften mit bereits abgeschriebenen Anlagen arbeiten und möglicherweise den Anschluss an den technischen Fortschritt verpasst haben. Umfangreiche Ersatzinvestitionen in der Zukunft erscheinen deshalb unvermeidlich.

Tabelle 1: Kennzahlen europäischer Molkereigenossenschaften - 2004.

\begin{tabular}{|c|c|c|c|c|c|c|}
\hline & $\begin{array}{l}\text { Arla } \\
\text { (Dk/Swe) }\end{array}$ & $\begin{array}{l}\text { Friesland } \\
(\mathrm{Nl})\end{array}$ & $\begin{array}{l}\text { Campina } \\
(\mathrm{Nl})\end{array}$ & $\begin{array}{l}\text { Glanbia } \\
\text { (Irl) }\end{array}$ & $\begin{array}{l}\text { Nordmilch } \\
\text { (D) }\end{array}$ & $\begin{array}{l}\text { Humana } \\
\text { (D) }\end{array}$ \\
\hline Eigenkapitalquote & $28,30 \%$ & $30,90 \%$ & $32,10 \%$ & $38,40 \%$ & $28,00 \%$ & $30,90 \%$ \\
\hline Anlagevermögen / $\mathrm{t}$ Milchverarbeitung & $233 €$ & $270 €$ & $171 €$ & $126 €$ & $57 €$ & $113 €$ \\
\hline Umsatz / t Milchverarbeitung & $752 €$ & $856 €$ & $684 €$ & $543 €$ & $500 €$ & $744 €$ \\
\hline Umsatzrendite & $2,40 \%$ & $3,60 \%$ & $0,20 \%$ & $4,60 \%$ & $1,20 \%$ & $-1,30 \%$ \\
\hline$\varnothing$ Auszahlungspreis 1999-2004 & 31,9 cent & 31,2 cent & 31,0 cent & 29,2 cent & 29,2 cent & 30,2 cent \\
\hline Auslandsanteil am Umsatz & $59 \%$ & $66 \%$ & $70 \%$ & $55 \%$ & $25 \%$ & $28 \%$ \\
\hline Ausländische Tochtergesellschaften & 26 & 25 & 19 & 5 & 1 & 4 \\
\hline
\end{tabular}

Quelle: Unternehmensangaben, eigene Berechnungen.

Interessant ist auch, dass sich der unternehmerische Erfolg der stark internationalisierten Molkereien nicht nur in bilanziellen Zahlen, sondern auch in höheren Auszahlungspreisen an die Genossen niederschlägt. 
Schon bei flüchtiger Betrachtung werden erhebliche Unterschiede zwischen den Internationalisierungsstrategien der beispielhaft vorgestellten deutschen und ausländischen Molkereigenossenschaften deutlich. Es wird deutlich, dass die betrachteten ausländischen Genossenschaften sehr viel stärker internationalisiert sind als ihre deutschen Wettbewerber. Campina, Arla Foods oder auch Royal Friesland haben durchweg höhere Exportquoten und vor allem wesentlich mehr Auslandsbeteiligungen. Doch scheint es auch einen Zusammenhang zwischen dieser stärkeren internationalen Verflechtung des operativen Geschäfts und dem daraus resultierenden unternehmerischen Erfolg zu bestehen. Warum ist das so? Zur Beantwortung dieser Frage werden im Folgenden vier Erklärungsansätze diskutiert: die unterschiedliche Größe des Heimatmarkts, Besonderheiten der betrachteten Produkte, die schwache Wettbewerbsposition deutscher Genossenschaften sowie Spezifika der Corporate Governance in Genossenschaften.

\section{Größe des Heimatmarktes}

Unternehmen wie Danish Crown, Bestmeat und Campina verfügen nur über einen relativ kleinen Heimatmarkt und sind somit gezwungen, ihre Geschäftsaktivitäten zu internationalisieren, um effiziente Betriebsgrößen zu erreichen. Dagegen bedienen die deutschen Genossenschaften einen großen inländischen Markt, der lange Zeit sowohl ausreichend Möglichkeiten zur Realisierung von Größenvorteilen für größere Unternehmen als auch genügend Nischen für kleine Anbieter bot. Der Druck auf die Unternehmen, sich zu internationalisieren, war dementsprechend deutlich geringer als in den kleineren Nachbarstaaten. Dieses so genannte „small-country“-Argument erklärt die höheren Exportquoten etwa niederländischer und dänischer Genossenschaften. Es liefert allerdings keine Begründung dafür, warum z.B. Danish Crown und Campina Unternehmenswachstum vermehrt durch den Aufbau ausländischer Produktionsstätten, die Beteiligung an ausländischen Gesellschaften oder die Gründung eigener ausländischer Tochtergesellschaften suchen. Als alleinige Erklärung für die stärkere Internationalisierung ausländischer Genossenschaften ist es daher nicht ausreichend.

\section{Produktmerkmale}

Gelegentlich wird eingewandt, dass bestimmte Produkteigenschaften der Milch wie begrenzte Haltbarkeit und großes Volumen der Globalisierung Grenzen setzen würden. Tatsächlich werden bei einer jährlichen weltweiten Milcherzeugung von etwa 590 Mio. Tonnen nur rund 32 Mio. Tonnen grenzüberschreitend gehandelt. Dem Argument muss allerdings entgegengehalten werden, dass die begrenzte Transportfähigkeit von Milch zwar den Export, jedoch nicht 
andere Formen der Internationalisierung behindert. Große europäische Wettbewerber wie Campina, Arla und Lactalis haben dies erkannt. Ihre Internationalisierung vollzieht sich nicht mehr allein über vermehrten Export, sondern vor allem über den Erwerb und die Gründung ausländischer Tochtergesellschaften. Produktcharakteristika können somit ebenfalls nicht abschließend die Defizite deutscher Genossenschaften im Bereich anspruchsvoller Internationalisierungsstrategien erklären.

\section{Wettbewerbsposition deutscher Genossenschaften}

Durch ihren saturierten Heimatmarkt befindet sich die deutsche Milchwirtschaft in einem extrem schwierigen Wettbewerbsumfeld, das zusätzlich noch durch die zunehmenden Markteintritte ausländischer Wettbewerber verschärft wird. Zwar ist der Konsolidierungsprozess weiter vorangeschritten als im genossenschaftlichen Fleischbereich, doch sehen sich die Unternehmen auch hier einem harten Preiskampf und einem hoch konzentrierten Lebensmitteleinzelhandel gegenüber. Ein Indiz für die schwache Wettbewerbsposition deutscher Molkereigenossenschaften liefert die Gegenüberstellung von verarbeiteter Milchmenge und Umsatz (siehe Abbildung 2). Beim Vergleich mit europäischen Genossenschaften, aber auch mit deutschen Privatmolkereien fällt auf, dass beim deutschen Marktführer Nordmilch aus der großen Milchverarbeitungsmenge nur ein relativ geringer Umsatz resultiert. Dies rührt daher, dass die Nordmilch vor allem standardisierte Low-cost- und Low-price-Ware wie Butter, Magermilchpulver und Konsummilch produziert, bei denen sie aufgrund der Austauschbarkeit der Produkte gegenüber den Abnehmern in einer besonders schlechten Verhandlungsposition ist. Für eine kapitalintensive Internationalisierungsstrategie ist dies keine gute Voraussetzung.

\section{Besonderheiten der Corporate Governance}

In der wirtschaftswissenschaftlichen Literatur ist wiederholt die Vermutung geäußert worden, dass die Rechtsform der Genossenschaft unter Managementgesichtspunkten Nachteile gegenüber anderen Rechtsformen, namentlich der Aktiengesellschaft, aufweist. Mit Blick auf die Internationalisierung der Geschäftsaktivitäten lassen sich die wichtigsten Argumente wie folgt zusammenfassen: In Genossenschaften gibt es externe und interne Trittbrettfahrer-Problem. Neben dem Trittbrettfahrer-Problem haben Genossenschaften auch ein Investitionsproblem, da Geschäftsanteile an Genossenschaften nicht handelbar sind. Ein Transaktionskostenproblem ist vor allem für große Genossenschaften charakteristisch, in denen die Mitglieder sehr unterschiedliche Interessen verfolgen. Das Kontrollproblem ergibt sich aus dem sog. Demokratieprinzip, nach dem alle Mitglieder unabhängig von der Höhe ihrer Geschäftsanteile 
gleich behandelt werden und so z.B. in den Generalversammlungen auch jeweils nur über eine Stimme verfügen. Ein Managementproblem resultiert aus der Beteiligung ehrenamtlicher Mitglieder mit wenig Managementerfahrung und begrenzter Zeit an der Geschäftsführung. Für eine verstärkte Internationalisierung der Geschäftsaktivitäten sind dies schlechte Voraussetzungen.

Die traditionelle Genossenschaftsstruktur wird aufgrund der genannten Probleme im Vergleich zu den Leitungsstrukturen privatwirtschaftlichen Unternehmen oft als starr und unflexibel empfunden. Genossenschaften, die an traditionellen Strukturen festhalten, haben daher Probleme, internationale Aktivitäten zu entwickeln. Dies haben viele ausländische Genossenschaften erkannt und deshalb das operative Geschäft über Holding-Strukturen ausgegliedert, um dem Management größere Handlungsspielräume zu verschaffen und schnellere, flexiblere und unabhängigere Entscheidungen zu ermöglichen. Beispiele dafür sind Campina. So beschränken sich bei Campina die bäuerlichen Eigner viel konsequenter als bei deutschen Molkereigenossenschaften auf eine allgemeine Erfolgskontrolle in Vorstand, Aufsichts- und Beirat, während das operative Geschäft von einer Hauptgeschäftsführung geführt wird. Damit wurden zugleich die Voraussetzungen dafür verbessert, mehr finanzielle Mittel den Rücklagen zuzuführen und auf diese Weise die finanziellen Grundlagen für die Internationalisierung des Geschäfts zu schaffen. Beispiele ausländischer Genossenschaften wie Campina, Emmi oder Glanbia zeigen, dass die Genossenschaft als Organisationsprinzip erfolgreich sein kann, wenn durch entsprechende Leitungsstrukturen Nachteile der genossenschaftlichen Rechtsform bei der Implementierung von Internationalisierungsstrategien überwunden werden. Auch in Deutschland lassen sich einige zukunftsweisende Beispiele finden. So wurde bei der Südzucker ebenfalls schon vor Jahren das operative Geschäft ausgelagert. Südzucker firmiert inzwischen als Aktiengesellschaft, deren Haupanteilseigner aber mit $56 \%$ die Süddeutsche Zuckerrübenverwertungs-Genossenschaft eG ist. Auch die deutsche Molkereigenossenschaft Hochwald hat rechtzeitig eine Abtrennung des operativen Geschäfts herbeigeführt.

\section{ZUKUNFTSPERSPEKTIVEN}

In sich globalisierenden Branchen müssen sich die Unternehmen mit geeigneten Internationalisierungsstrategien dem internationalen Wettbewerb stellen. Dies gilt auch für Genossenschaften in der Agrar- und Ernährungswirtschaft. Solange nach wie vor mehr als 100 Molkereien auf dem Markt agieren, steht es schlecht um die Verhandlungsposition der Molkereiwirtschaft. Um im Wettbewerb bestehen zu können, bleibt insbesondere den deutschen Mol- 
kereigenossenschaften daher nichts anderes übrig, sich schneller als bisher erfolgt zu konsolidieren und möglichst rasch ihre strukturellen Probleme zu lösen, um finanziellen Handlungsspielraum für eventuelle weitere Internationalisierungsschritte zu schaffen.. $\mathrm{Zu}$ diesem Ergebnis kommt auch ein Gutachten des deutschen Raiffeisenverbandes, das den Molkereigenossenschaften Einsparpotentiale von 600 Millionen Euro pro Jahr verspricht. Dazu solle es in einigen Jahren allerdings nur noch zehn statt der bisherigen 63 Genossenschaften geben, die Zahl der Betriebsstätten solle halbiert und ein Drittel der Arbeitsplätze gestrichen werden (Murmann, 2005). Die gewählten Internationalisierungsstrategien, die Unternehmen wie etwa Campina wählten, hätten bei entsprechender Konsolidierung des Heimatmarktes auch die deutschen Genossenschaften verfolgen können. Mit der Öffnung der osteuropäischen Märkte hätten sie bspw. mittels einer so genannten Wasserfallstrategie ausgehend von einer starken Durchdringung des Heimatmarktes sukzessive die mittel- und osteuropäischen Nachbarländer erschließen können (Welge and Holtbrügge, 2001; Kutschker and Schmid, 2004). Während die Strukturprobleme der Branche nur begrenzt dem Einfluss der einzelnen Genossenschaft unterliegen, müssen Defizite der genossenschaftlichen Rechtsform möglichst umgehend beseitigt werden. Dazu sind neue Organisationslösungen einzuführen, die den wachsenden Herausforderungen an die Unternehmensführung im internationalen Wettbewerb gerecht werden. Erste Anzeichen für ein Umdenken in der Praxis gibt es bereits, wie die vom neuen Nordmilch-Vorstand eingeleiteten umfangreichen Restrukturierungsmaßnahmen beweisen. Dabei wurde wenig Rücksicht auf frühere Strukturen genommen, die Geschäftsführung ausschließlich in den Händen hauptamtlicher Manager übertragen das Mitspracherecht der Genossenschaftsmitglieder eingeschränkt. Im Januar 2006 kam es schließlich zu einem, für den deutschen Molkereigenossenschaftssektor revolutionären Schritt. Die Nordmilch eG will das operative Geschäft in eine Aktiengesellschaft auslagern, ähnlich dem Vorbild europäischer Wettbewerber. Dieser Schritt solle laut Vorstandsvorsitzendem Tomat die Expansion in Europa und Übersee vorantreiben. Weitere Vorteile seinen eine größere Flexibilität und leichtere Kapitalbeschaffung (Anonymus, 2006c).

Zusammenfassend ist festzuhalten, dass die deutschen Genossenschaften in der Vergangenheit nur bedingt in der Lage waren, dem internationalen Wettbewerb standzuhalten und im Zuge der Globalisierung der Märkte eine aktive Rolle zu spielen. Vor großen strategischen Herausforderungen stehen dabei v.a. die Kosten fokussierten großen deutschen Genossenschaften, die bisher weder eine reine Marken- noch eine reine Kostenführerschaftsstrategie realisieren konnten. So wurde in der Vergangenheit weit gehend versäumt, in Marketing, 
Neuproduktentwicklungen, aber v.a. in die Internationalisierung durch Direktinvestitionen in Wachstumsmärkten zu investieren. Die Wahl letzterer Strategie fordert, Synergieeffekte zukünftig noch konsequenter zu nutzen und sich auf die Rolle eines schlanken Handelsmarkenund Industrieprodukte Spezialisten zu konzentrieren. Dies würde allerdings ebenfalls eine deutlich stärkere Internationalisierung voraussetzen (Gerlach et al, 2005). Da jedoch viele deutsche Genossenschaften alleine nicht die notwendige finanzielle Kraft zur Internationalisierung ihrer Aktivitäten besitzen, sind in der Zukunft weitere, auch grenzüberschreitende Fusionen zu erwarten. Zwar kam es bisher noch zu keiner derartigen Fusion unter den großen europäischen Molkereigenossenschaften, doch schlossen erst kürzlich die finnische Valio und die deutsche Nordmilch ein Abkommen, künftig ihre Produktionskapazitäten für bestimmte Erzeugnisse zu bündeln, spezielle Geschäftsbereiche und Produktkonzepte gemeinsam zu entwickeln und ihre Vermarktungsaktivitäten sowohl auf den jeweiligen Heimatmärkten als auch international zu stärken (Anonymus, 2006b). Spekulationen über eine gegenseitige Beteiligung oder sogar Fusion werden zwar bisher verneint, langfristig erscheint dies allerdings unerlässlich, um mit den führenden europäischen Genossenschaftsmolkereien, vor allem aber mit den internationalen Konzernen wie Danone, Nestlé und Unilever konkurrieren zu können. Die Schaffung eines großen deutschen Molkereikonzerns nach Vorbild der dänisch/schwedischen Arla Foods oder der niederländischen Campina würde es den Milchverarbeitern weiterhin ermöglichen, auf Augenhöhe mit dem LEH zu verhandeln.

\section{LITERATUR}

Anonymus (2006a). Nordmilch mit Repräsentanz in China, in: LZ-net, 04.01.2006.

Anonymus (2006b). Nordmilch und Valio kooperieren, in: LZ-net, 23.02.2006.

Anonymus (2006c). Nordmilch wird Aktiengesellschaft, in: LZ-net, 26.01.2006.

Gerlach, S. et al (2005). Der Markt für Milch und Milcherzeugnisse, in: Agrarwirtschaft 54(2005): 35-48.

Gerlach, S. et al (2006). Der Markt für Milch und Milcherzeugnisse, in: Agrarwirtschaft 55(2006): 29-50.

Kutschker, M. and Schmid, S. (2004). Internationales Management, München.

Milchwirtschaft, D. (2005). Die umsatzstärksten Mopro-Anbieter 2005. Deutsche Milchwirtschaft-Spezial. Gelsenkirchen.

Murmann, C. (2005). Einsparpotenzial scheint gigantisch, in: LZ-net, 15.12.2005.

Stappel, M. and Hennigsen, E. (2003). Die deutschen Genossenschaften 2003. Entwicklungen - Meinungen - Zahlen, Neuwied.

Welge, M. and Holtbrügge, D. (2001). Internationales Management, Second Edition, Landsberg am Lech. 


\section{KAPITEL IV.3}

\section{Internationalization and Corporate Success Empirical Evidence from the European Dairy Sector}

\section{Oliver Ebneth and Ludwig Theuvsen}

Paper presented in different variations at the following conferences:

- $45^{\text {th }}$ GeWiSoLa Conference, $5^{\text {th }}-7^{\text {th }}$ of October 2005, Göttingen,

- $X I^{\text {th }}$ International Congress of the European Association of Agricultural Economists (EAAE), $24^{\text {th }}-27^{\text {th }}$ of August 2005, Copenhagen,

- $V^{\text {th }}$ International PENSA Conference, $27^{\text {th }}-30^{\text {th }}$ of July 2005, Ribeirão Preto (Brazil),

- $15^{\text {th }}$ Annual World Food and Agribusiness Symposium and Forum, $25^{\text {th }}-28^{\text {th }}$ of June 2005, Chicago. 


\section{Internationalization and Corporate Success Empirical Evidence from the European Dairy Sector}

\section{EXECUTIVE SUMMARY}

During recent years, the agribusiness sector has more and more been confronted with the challenges of internationalizing food markets. This development has also seized dairy cooperatives which are forced to become more competitive by joining forces through international mergers and acquisitions. In this paper the authors empirically investigate the internationalization and corporate success of European cooperatives. We start by discussing different ways of measuring a cooperative's degree of internationalization. Having determined the mode and amount of the cooperatives' international activities, we assess their financial performance by applying balance sheet analysis.

A cooperative's internationalization strategy is reflected in its degree of internationalization. In this paper the authors take into account the Foreign Sales Index and the Network Spread Index which are combined into a measure for the degree of internationalization. This methodology is applied to a sample of leading European dairy cooperatives. Empirical results show remarkable differences regarding the degree of internationalization between German cooperatives and their European competitors.

Balance sheet analysis is a systematic procedure using the information potential of the balance sheet as well as the profit and loss account. The aim is to attain insights into the economic situation and future prospects of an enterprise. This investigation emphasizes the description of the enterprises' capital resources since these represent a crucial factor for the economic prosperity of a dairy company. In the course of planning and implementing internationalization strategies, financial resources in particular are increasingly important.

The results are discussed by referring to the small-country argument, the serious economic situation of German cooperatives, and arguments stemming from the economic literature on the peculiarities of cooperatives and the strategic management literature. Economists argue that cooperatives are characterized by free-riding, investment, transaction cost, control, and human resource problems. The authors argue that diverse capabilities of cooperatives in solving these problems explain different degrees of internationalization and differences in their financial performance. 
The managerial implications are obvious: German cooperatives in the dairy industry have to solve their financial problems and redesign their outdated corporate governance mechanisms by professionalizing their management structures.

\begin{abstract}
Increasingly, cooperatives in the agribusiness are being confronted with the globalization of agri-food markets. Cooperatives adapt to this development by internationalizing their activities. This paper presents a method of measuring the degree of internationalization (DoI) and its application to European cooperatives in the dairy sector. Then, the financial performance of these cooperatives is measured by applying balance sheet analysis. The paper ends with a discussion of why German cooperatives are noticeably less internationalized and show weaker financial performance than their European competitors.
\end{abstract}

\title{
KEYWORDS
}

Globalization, Dairy Industry, Cooperatives, Degree of Internationalization, Corporate Success. 


\section{INTERNATIONALIZATION: CHALlENGES FOR COOPERATIVES}

In recent decades, one of the most striking developments has been the ever-increasing globalization of markets and the internationalization of economic activities. Due to low growth rates on home markets, growing international competitive pressures, shortened product lifecycles and growing R\&D and marketing investments, many companies have expanded their national activities and, thus, contributed to the further advancement of the intensity and dynamics of international competition (Ger, 1999; Barney, 2002; Dhanaraj and Beamish, 2003). This development has also seized cooperatives. Due to strong pressures to reduce costs and develop new markets, mergers and acquisitions have become a common practice in the cooperative sector. Cooperatives in the agribusiness sector, in particular, often located in rural areas, are forced to become more competitive by joining forces through mergers and acquisitions. During recent years Danish and Dutch Cooperatives in particular have forced competition and formed strong groups (Stappel and Hennigsen, 2003). Against this background, the (often neglected) internationalization of business activities is considered one of the most promising ways of gaining access to new markets and sustained economic success in the cooperative sector.

In this paper we investigate the internationalization strategies and corporate success of European dairy cooperatives. We start by discussing different ways of measuring a cooperative's degree of internationalization. Having determined the mode and amount of the cooperatives' international activities, we assess their corporate success by applying balance sheet analysis. A discussion of the results explains differences between German cooperatives and their European competitors. Managerial implications as well as some ideas for future research close this paper.

\section{Methodology}

\section{Determining the Degree of Internationalization}

Internationalization describes the process by which an economy, industry or company becomes increasingly integrated into international economic activities. The degree of internationalization measures to what extent this economic integration has already taken place. When determining a company's degree of internationalization, activities on foreign markets are compared to home-market or worldwide activities (Germann et al, 1996). The degree of internationalization determines to what extent a company is confronted with problems of man- 
aging international activities and to what extent international environments are relevant for a specific company (Schmidt, 1981).

Measuring the degree of internationalization (DoI), therefore, is of high managerial relevance. During recent years a growing number of more or less complex methodologies have been developed but all for multinational publicly listed groups (Sullivan, 1994; Ietto-Gillies, 1998; Germann et al, 1999; Fisch and Oesterle, 2003; Hassel et al, 2003). Compared to these companies, even leading dairy cooperatives are quite small, and hardly any cooperative is listed on stock exchanges or heavily dependent on bond markets. For this reason, legal and market pressures on them to disclose information are comparatively low. Therefore a less sophisticated measure of the DoI will be applied in which two figures - the ratio of foreign sales to total sales and the international dispersion of subsidiaries - flow into a key figure with equivalent weighting. The dispersion of subsidiaries is calculated by adopting the Network Spread Index of Garcia Ietto-Gillies [1998]: the number of countries in which an enterprise maintains subsidiaries is divided by the total number of countries that received direct investments in 2003. For this reason, the following internationalization measure is applied in this paper:

1) Degree of Internationalization: $D o I=(F S i+N S i): 2$, with

2) Foreign Sales Index (FSi): Ratio of foreign sales (exports and subsidiaries) to total sales.

3) Network Spread Index with $\mathrm{n}^{*}=191$ (UNCTAD, 2004): $N S i=\frac{n}{n^{*}}=\frac{n}{191}$.

In the context of determining the DoI it should also be noted that implementing more advanced internationalization concepts like foreign subsidiaries represents a more advanced level of international engagement than pure exporting. Companies often start with pure export activities that do not require capital or management transfers to foreign countries. After some time they then enter international joint ventures or move production overseas. In the end they may own full-scale foreign subsidiaries. Whereas in early phases (export) the relationship of the company with its home country is very intensive while its relationship with export destinations is very weak, it is often the other way round when full-scale subsidiaries exist abroad (Bartlett and Ghoshal, 1989; Swoboda, 2002). 


\section{Determining Corporate Success}

In management literature there are in principle two approaches for the quantification of entrepreneurial success: balance sheet-oriented and capital market-oriented concepts (Germann et al, 1996; Schwab, 1997; Glaum and Lindemann, 2002). Since most of the enterprises discussed in this paper are not listed on stock markets, a capital market-oriented analysis is impossible; therefore, a balance sheet-analytic process is used.

\section{Balance Sheet Analysis}

Balance sheet analysis is a systematic procedure using the information potential of the balance sheet as well as the profit and loss account. The aim is to attain insights into the economic situation and future prospects of an enterprise. However it must be pointed out that especially in a transnational analysis key figures are strongly influenced by different valuation and accounting regulations. Thus by adopting a cross-border comparative analysis one must always consider the basic conditions and the limitations of the informational value of balance sheet analysis (Küting and Weber, 2004). The intention of balance sheet analysis is not to make final judgements but to point out tendencies and prognoses for a company's future prospects (Gräfer, 2001). If one keeps track of these limitations and possible misinterpretations and formulates and rates the extracted insights and statements with the required caution, balance sheet analysis can be an important aid in the evaluation and estimation of companies. Through adept dressing of the data using key figures enterprises can be assessed. Whenever possible, companies from the same industry should be analyzed in order to receive an additional evaluation criterion and thus to enhance the information value of the balance sheet analysis.

In this analysis we start by evaluating the earning potential of cooperatives. Profitability key figures provide information about the success or failure of business activities and thus form a basis for the decisions of management, shareholders and creditors:

The Return on Equity reflects a company's potential to realize profits and income (Born, 2001). The formula for the determination of the Return on Equity reads as follows:

1) $\frac{\text { Profit before Tax }}{\text { Equity }}=$ Return on Equity.

The Return on Assets eliminates the influence of different capital structures on success and 
thus improves the meaningfulness of comparisons. It is more suitable than the Return on Equity for judging the efficiency of an enterprise, since the external analyst is more interested in the return on equity capital than in the profitability of the assigned total capital (Gräfer, 2001):

2) $\frac{\text { Profit before Taxes }+ \text { Interests }}{\text { Total Equity and Liablilities }}=$ Return on Assets.

Another important key figure is the Net Profit Ratio or Return on Sales. This ratio reflects both market determined parameters such as sales volumes and prices (turnover $=$ sales volume $\mathrm{x}$ price) as well as internally influenceable factors, such as expenditures, seized in the operating result:

3) $\frac{\text { Operating Profit }}{\text { Turnover }}=$ Net Profit Ratio.

Additionally, the Added Value Index is calculated. This reflects the ratio of volume and turnover generated by the milk processed (Schramm et al, 2004; Gerlach et al, 2005).

Financial key figures provide information about the composition of the total capital employed. These ratios allow both estimation of financial risks and evaluation of the financial strength and credit-worthiness of enterprises.

Here, analysis of financial strength on equity by accounting the Equity Ratio is the focus. As a share of equity of the total capital employed, the equity ratio represents a measure for the "loss absorption capacity" of the enterprise (Küting and Weber, 2004).

The Net Debt to Equity Ratio shows Net Debt as a percentage of Equity Capital. It is the most commonly used measure of financial leverage and it provides an indicator of both financial risk and capital efficiency.

Finally, the following proprietary key figure is also formed:

The Fixed Assets in $€ /$ ton Milk Processed depends on the production program, degree of mechanization, capacity utilization and amortization of the plants. Very low assets/ton milk processed can be attributed to the fact that an enterprise operates with amortized plants and has possibly refrained from investing in the latest technologies (Gabler, 2003).

This investigation emphasizes the description of the enterprises' capital resources since these 
represent a crucial factor for the economic prosperity of a dairy company. In the course of planning and implementing internationalization strategies, financial resources in particular are increasingly important.

\section{OBJECT OF INVESTIGATION: EUROPEAN DAIRY COOPERATIVES}

The sample consists of twelve leading dairy cooperatives from nine European countries. These are the eleven biggest companies ranked by turnover and, additionally, European's number 23 Milchunion Hochwald (MUH) (in order to include the four largest German cooperatives). Table 1 gives an overview over the twenty largest dairy cooperatives and their sales development.

Table 1: Top 20 European Dairy Cooperatives.

\begin{tabular}{|c|c|c|c|c|c|c|c|c|}
\hline & company & country & $\begin{array}{r}\text { turnover } \\
2001 \\
\text { in } € \mathrm{mn} \\
\end{array}$ & $\begin{array}{r}\text { turnover } \\
2002 \\
\text { in } € \mathrm{mn} \\
\end{array}$ & $\begin{array}{r}\text { turnover } \\
2003 \\
\text { in } € \mathrm{mn} \\
\end{array}$ & $\begin{array}{r}\text { turnover } \\
2004 \\
\text { in } € \mathrm{mn} \\
\end{array}$ & $\begin{array}{c}\Delta \\
2004 / \\
2001 \\
\end{array}$ & $\begin{array}{r}\text { milk } \\
\text { processed } \\
\text { in } \mathrm{kg} \mathrm{mn} \\
\end{array}$ \\
\hline 1 & Arla Foods* & $\mathrm{Dk} / \mathrm{Sw}$ & $5,129.9$ & $5,308.7$ & $5,476.2$ & $6,400.8$ & $24.8 \%$ & 8,512 \\
\hline 2 & Friesland Foods & $\mathrm{Nl}$ & $4,370.2$ & $4,723.0$ & $4,575.0$ & $4,449.0$ & $1.8 \%$ & 5,200 \\
\hline 3 & Campina & $\mathrm{N} 1$ & $3,933.0$ & $3,733.0$ & $3,655.0$ & $3,559.0$ & $-9.5 \%$ & 5,205 \\
\hline 4 & Sodiaal $* * *$ & $\mathrm{Fr}$ & $2,730.0$ & $2,374.0$ & $2,823.0$ & $2,890.0$ & $5.9 \%$ & 3,500 \\
\hline 5 & Humana & Ger & $2,392.7$ & $2,530.0$ & $2,681.0$ & $2,723.0$ & $13.8 \%$ & 3,300 \\
\hline 6 & Nordmilch & Ger & $2,390.0$ & $2,307.1$ & $2,225.5$ & $2,150.0$ & $-10.0 \%$ & 4,143 \\
\hline 7 & Glanbia & Irl & $2,625.4$ & $2,316.7$ & $2,041.1$ & $1,846.0$ & $-29.7 \%$ & 3,400 \\
\hline 8 & Tine & Nwg & $1,480.0$ & $1,735.2$ & $1,596.9$ & $1,680.3$ & $13.6 \%$ & 1,491 \\
\hline 9 & Valio & Fin & $1,519.3$ & $1,600.0$ & $1,566.0$ & $1,581.0$ & $4.1 \%$ & 1,989 \\
\hline 10 & Emmi & $\mathrm{Ch}$ & 852.1 & 955.7 & $1,205.4$ & $1,247.7$ & $46.4 \%$ & 590 \\
\hline 11 & Hochwald $* * * *$ & Ger & 625.0 & 617.6 & 704.5 & $1,100.0$ & $76.0 \%$ & 1,800 \\
\hline 12 & Milk Link** & UK & 480.0 & 491.7 & 581.5 & 930.0 & $93.8 \%$ & 1,600 \\
\hline 13 & Dairygold & Irl & $1,069.6$ & 989.0 & 914.3 & 876.0 & $-18.1 \%$ & 850 \\
\hline 14 & Granarolo & Ita & 665.7 & 685.5 & 731.0 & 852.0 & $28.0 \%$ & N.a. \\
\hline 15 & First Milk** & UK & 800.0 & 652.8 & 821.9 & N.a. & N.a. & 2,300 \\
\hline 16 & Bayernland & Ger & 716.0 & 789.0 & 758.0 & 723.0 & $1.0 \%$ & N.a. \\
\hline 17 & Milcobel***** & Bel & 533.0 & 552.0 & 571.7 & 750.0 & $40.7 \%$ & N.a. \\
\hline 18 & Capsa & Spa & 632.0 & 651.9 & 674.7 & N.a. & N.a. & N.a. \\
\hline 19 & Lactogal & Ptg & 601.5 & 652.4 & 661.8 & N.a. & N.a. & N.a. \\
\hline 20 & Dairy Far. B** & UK & 720.0 & 573.4 & 654.5 & N.a. & N.a. & N.a. \\
\hline$\cdots$ & $\cdots$ & & & & & & & \\
\hline \multirow[t]{3}{*}{23} & MUH & Ger & 392.9 & 416.9 & 431.2 & 446.2 & $13.6 \%$ & 874 \\
\hline & & $\sum$ Top10 & $27,422.6$ & $27,583.4$ & $27,845.1$ & & & 36,774 \\
\hline & & $\sum$ Top20 & $34,756.4$ & $34,713.7$ & $35,327.3$ & & & \\
\hline \multicolumn{9}{|c|}{ * Book year ending September. >September 2003 = "2003" } \\
\hline \multicolumn{9}{|c|}{ ** Book year ending March. >March 2004 = "2003" } \\
\hline \multicolumn{9}{|c|}{ *** 50\%-daughter Yoplait. Book year ending June. } \\
\hline & oforma calca & Leons & 10 & & & & & \\
\hline
\end{tabular}

Source: Nyenrode (2005), company data. 


\section{RESULTS: DEGREE OF INTERNATIONALIZATION OF EUROPEAN DAIRY COOPERATIVES}

When computing the degree of internationalization, it becomes obvious that the three largest cooperatives are also the most internationalized ones. It is worth mentioning that the Network Spread index exhibits the most notable differences between the enterprises. The dairy companies Arla (26), Friesland (25) and Campina (18) possess many more foreign subsidiaries than Nordmilch (1), Humana (4) or MUH (1). Above all, so far German cooperatives have limited their international commitment exclusively to export activities without undertaking further internationalization steps. Figure 1 presents the results.

Figure 1: Degree of Internationalization of European Dairy Cooperatives.

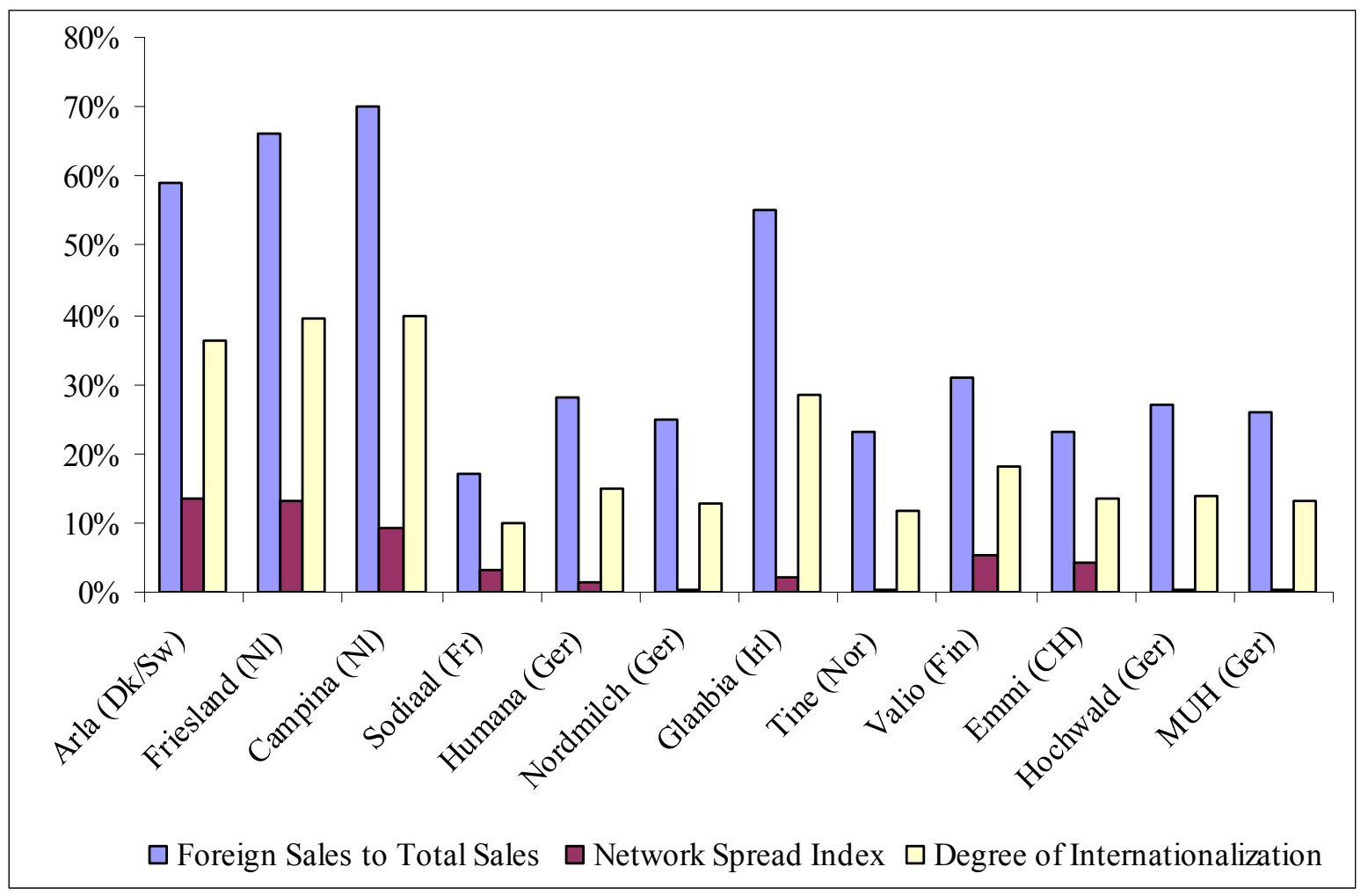




\section{Results: CORPORATE SUCCESS OF EUROPEAN DAIRY COOPERATIVES}

The larger cooperatives, above all the Swiss Emmi and the Norwegian Tine are characterized by relatively high Fixed Assets/ton Milk Processed. The large range of the fixed assets in $€ /$ ton milk processed suggests a quite different equipment with fixed assets. Particularly the German cooperatives run their business with largely depreciated facilities. This low intensity of investments arises from a depletion of assets and leads to a backlog demand of future investment. Figure 2 illustrates the cooperatives' intensity of production facilities.

Figure 2: Fixed Assets in €/ton Milk Processed of European Dairy Cooperatives.

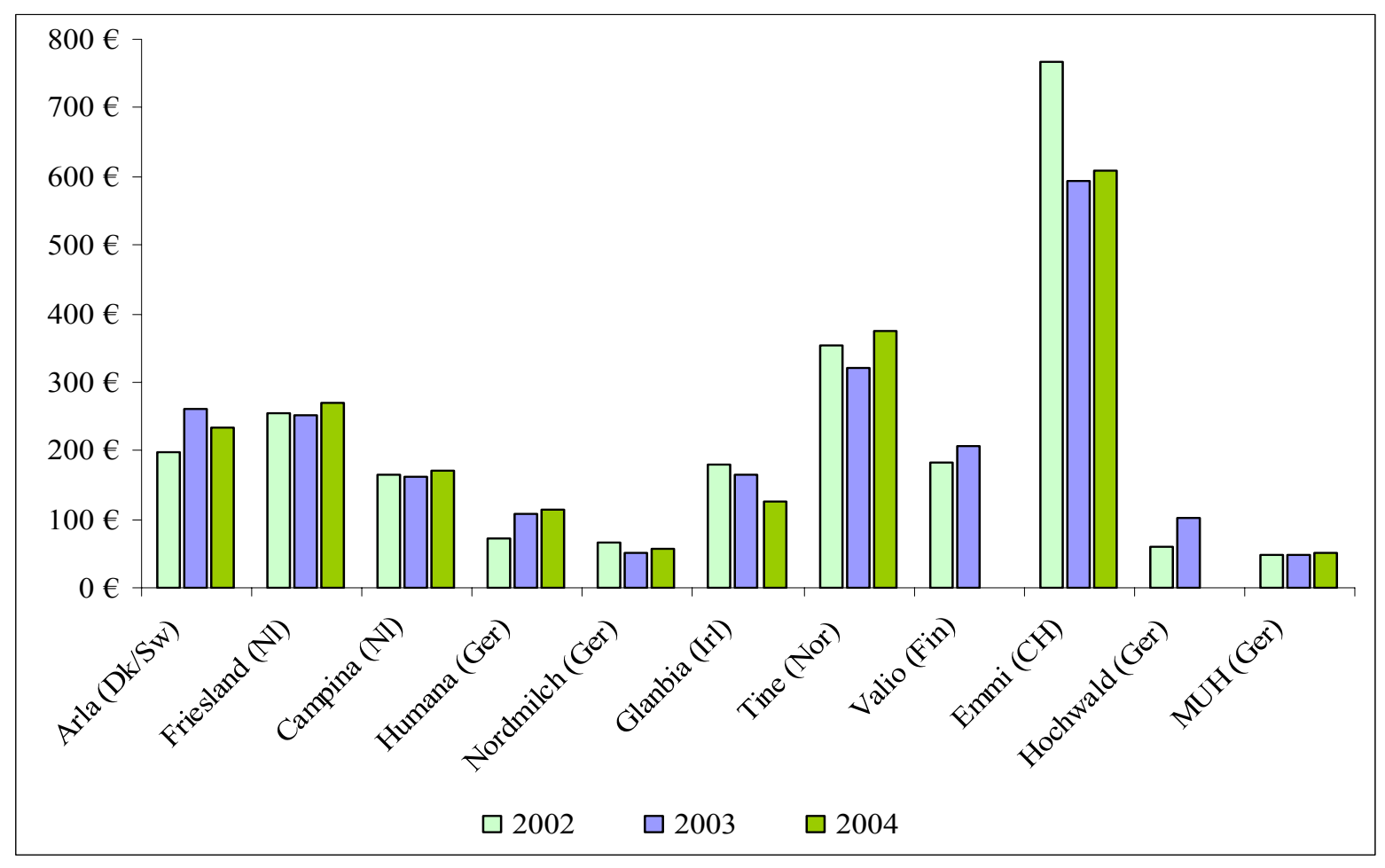

The comparison of German and other European cooperatives shows that the German market leader Nordmilch as well as Hochwald and MUH are generating a relatively small turnover per $\mathrm{kg}$ milk processed (see figure 3). This is caused by the fact that German dairy cooperatives usually pursue cost leadership strategies and focus on standardized low-cost and lowprice mass market articles like milk, milk powder and butter, whereas privately-owned dairy companies often own strong brands and have comparatively strong market positions. 
Figure 3: Added Value Index of European Dairy Cooperatives - 2004.

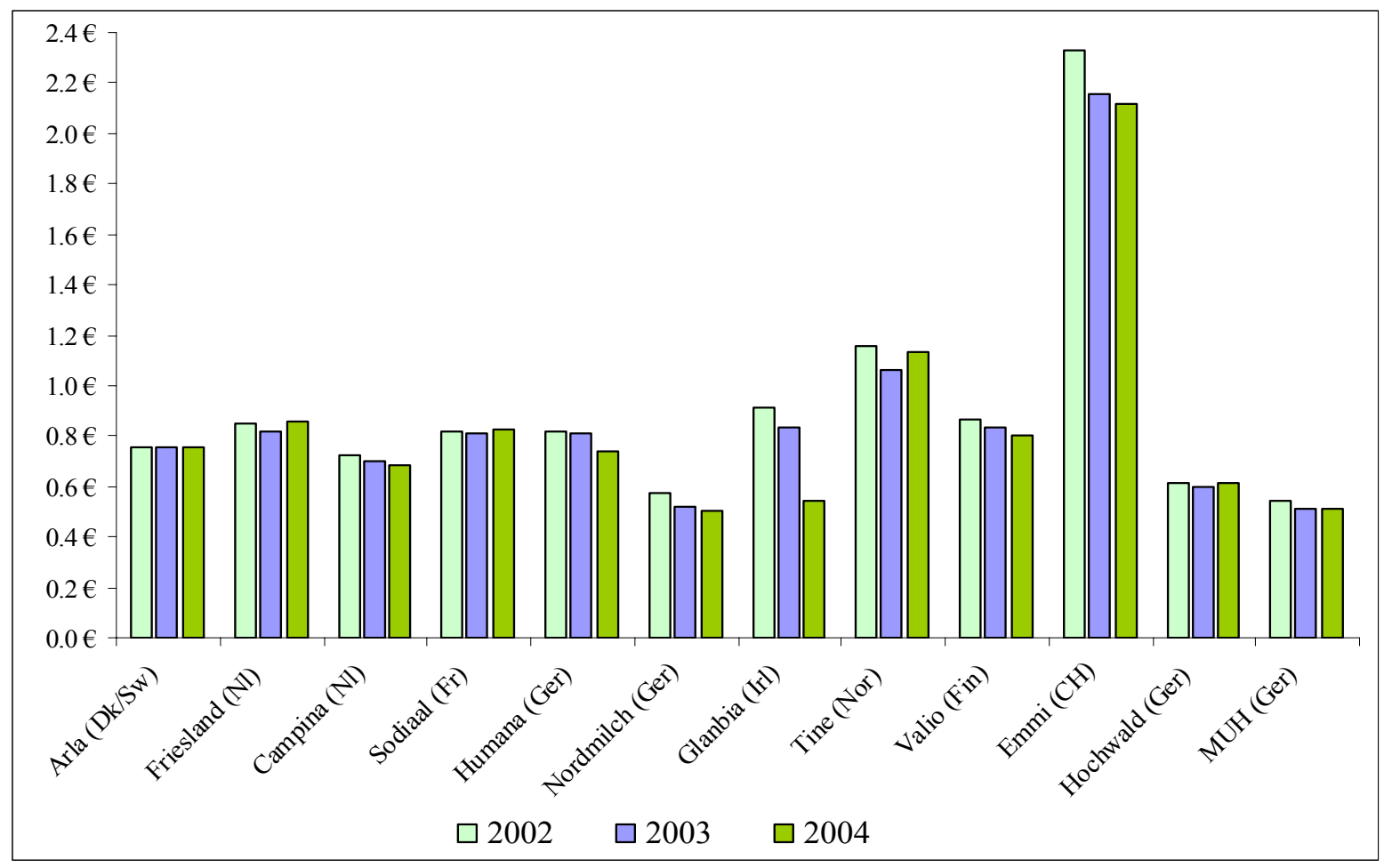

With the calculation of the equity ratio the smaller cooperatives in the sample come off somewhat better. Only the German Nordmilch exhibits a relatively low value for the year 2003, which is due to extensive restructuring measures. It has to be mentioned that it is not possible to calculate the equity ratio or any profitability key figures for the largest French cooperative Sodiaal which does not publish any financial statements. Good to see that all companies show an increasing trend regarding their equity ratio. This is urgently needed as most dairy industry experts require a level of $30 \%$ equity and more for future growth and prosperity. 
Figure 4: Equity Ratio of European Dairy Cooperatives.

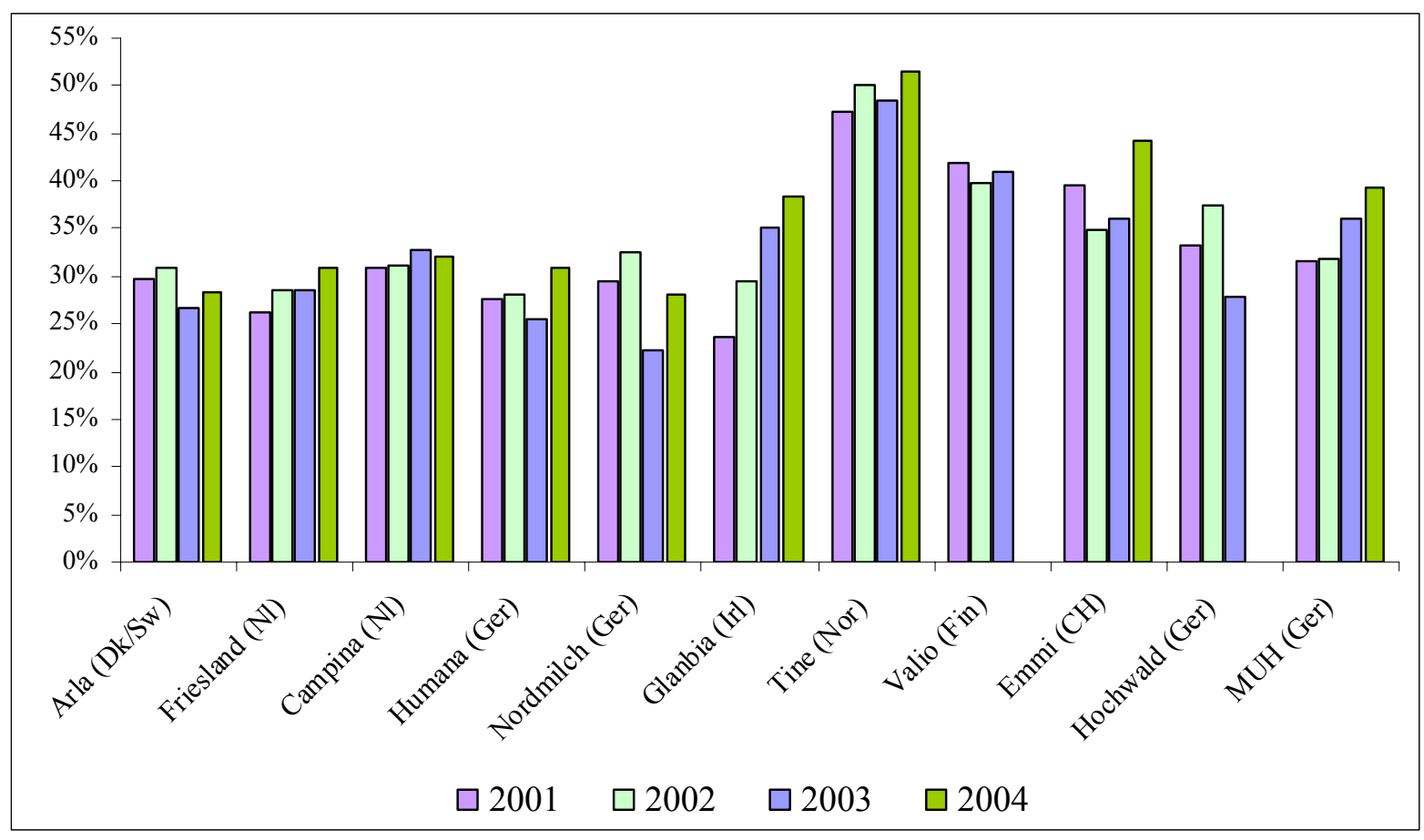

Regarding the ratio between Net Debt and Equity of the cooperatives under consideration, two aspects have to be highlighted. First, it strikes out, that the leading cooperative Arla as well as Tine, Valio and Emmi show a sound ratio between Net Debt and Equity. Nordmilch and Glanbia, but more surprisingly Friesland still perform quite week but with a positive trend over the last three financial years. The only outlier is the German Hochwald. In its 2002 balance sheet they display more cash than debts to credit institutions resulting in a negative Net debt to Equity ratio. The smallest company in the sample, German based MUH shows a quite low level of equity, indeed, but owes hardly debts to creditors on the other hand. 
Figure 5: Net Debt to Equity of European Dairy Cooperatives.

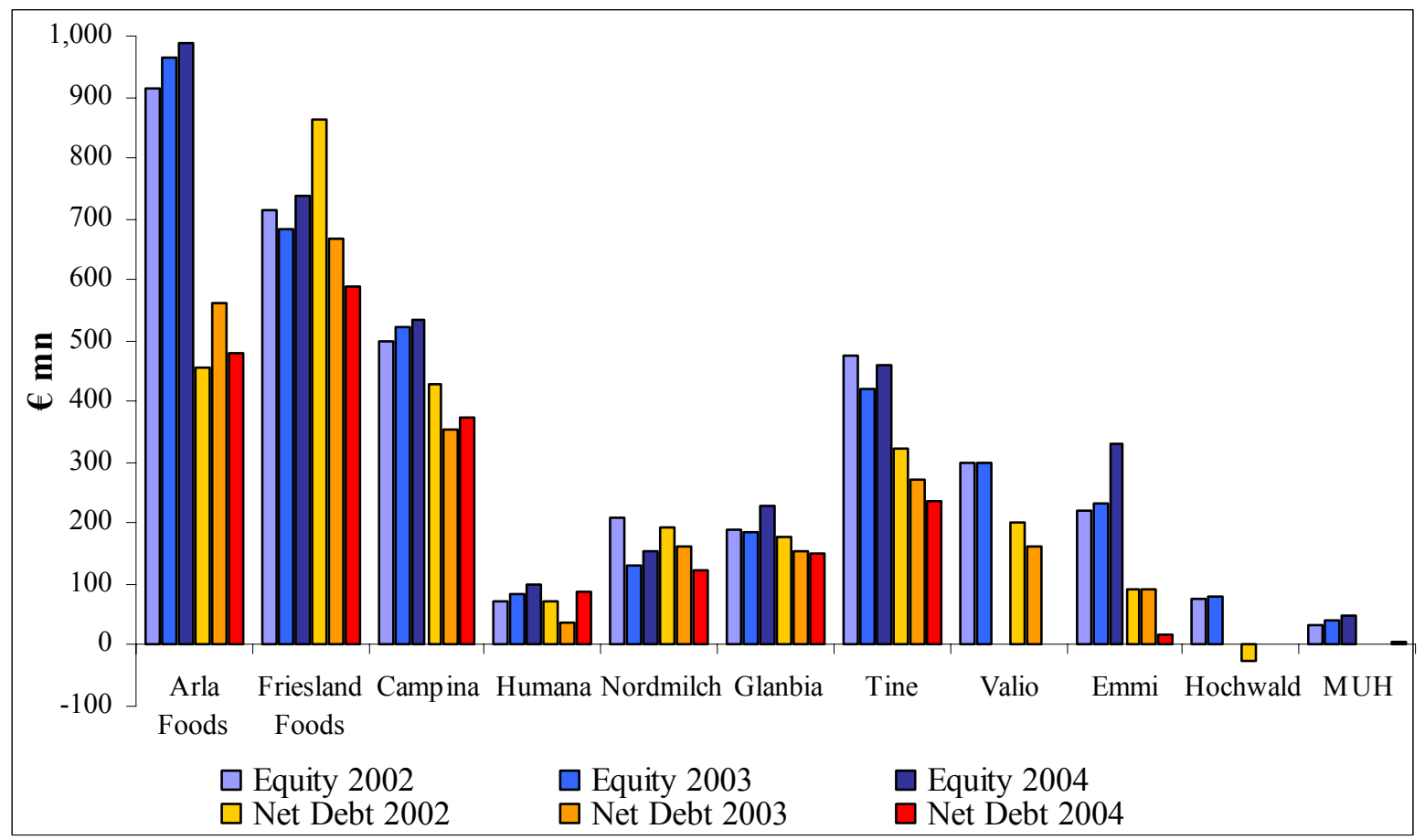

On the one hand, the two largest cooperatives as well as Glanbia and Emmi display above average profitability ratios (see table 2). On the other hand, all the four German enterprises demonstrate comparatively low performance expressed by the Net Profit Ratio. This ratio is the best indicator for a company's future ability to invest, either into internationalization strategies as well as into product innovations which enable the dairy company to improve its results.

Table 2: Profitability Ratios of European Dairy Cooperatives.

\begin{tabular}{lrrrrrrrrrrrr}
\hline & \multicolumn{3}{c}{ Return on Equity } & \multicolumn{4}{c}{ Return on Assets } & \multicolumn{3}{c}{ Net Profit Ratio } \\
\hline & $\mathbf{2 0 0 2}$ & $\mathbf{2 0 0 3}$ & $\mathbf{2 0 0 4}$ & $\boldsymbol{\varnothing}$ & $\mathbf{2 0 0 2}$ & $\mathbf{2 0 0 3}$ & $\mathbf{2 0 0 4}$ & $\boldsymbol{\emptyset}$ & $\mathbf{2 0 0 2}$ & $\mathbf{2 0 0 3}$ & $\mathbf{2 0 0 4}$ & $\boldsymbol{\emptyset}$ \\
\hline Arla Foods & $17.0 \%$ & $16.7 \%$ & $15.6 \%$ & $16.4 \%$ & $5.2 \%$ & $4.5 \%$ & $4.4 \%$ & $4.7 \%$ & $3.6 \%$ & $3.1 \%$ & $2.4 \%$ & $3.0 \%$ \\
Friesland & $22.0 \%$ & $17.3 \%$ & $17.3 \%$ & $18.8 \%$ & $6.3 \%$ & $4.9 \%$ & $5.3 \%$ & $5.5 \%$ & $4.2 \%$ & $3.3 \%$ & $3.6 \%$ & $3.7 \%$ \\
Campina & $2.3 \%$ & $7.2 \%$ & $0.6 \%$ & $3.3 \%$ & $0.7 \%$ & $2.4 \%$ & $0.2 \%$ & $1.1 \%$ & $1.6 \%$ & $0.5 \%$ & $0.2 \%$ & $0.8 \%$ \\
Humana & $7.5 \%$ & $-1.1 \%$ & $25.5 \%$ & $10.7 \%$ & $2.1 \%$ & $-0.3 \%$ & $7.9 \%$ & $3.2 \%$ & $0.2 \%$ & $-0.7 \%$ & $-1.3 \%$ & $-0.6 \%$ \\
Nordmilch & $7.3 \%$ & $4.3 \%$ & $13.6 \%$ & $8.4 \%$ & $2.4 \%$ & $1.0 \%$ & $3.8 \%$ & $2.4 \%$ & $1.0 \%$ & $0.5 \%$ & $1.2 \%$ & $0.9 \%$ \\
Glanbia & $-4.3 \%$ & $-8.0 \%$ & $34.7 \%$ & $7.4 \%$ & $-1.3 \%$ & $-2.8 \%$ & $13.3 \%$ & $3.1 \%$ & $3.8 \%$ & $3.7 \%$ & $4.6 \%$ & $4.0 \%$ \\
Tine & $1.0 \%$ & $5.2 \%$ & $6.0 \%$ & $4.1 \%$ & $0.5 \%$ & $2.5 \%$ & $3.1 \%$ & $2.0 \%$ & $0.9 \%$ & $1.9 \%$ & $2.0 \%$ & $1.6 \%$ \\
Valio & $0.0 \%$ & $4.1 \%$ & $\mathrm{~N} . \mathrm{a}$. & $2.1 \%$ & $0.0 \%$ & $1.7 \%$ & $\mathrm{~N} . \mathrm{a}$. & $0.8 \%$ & $0.5 \%$ & $1.1 \%$ & $\mathrm{~N} . \mathrm{a}$. & $0.8 \%$ \\
Emmi & $8.1 \%$ & $11.0 \%$ & $7.7 \%$ & $8.9 \%$ & $2.8 \%$ & $3.9 \%$ & $3.4 \%$ & $3.4 \%$ & $2.6 \%$ & $2.6 \%$ & $2.5 \%$ & $2.6 \%$ \\
Hochwald & $0.7 \%$ & $1.5 \%$ & $\mathrm{~N} . \mathrm{a}$. & $1.1 \%$ & $0.3 \%$ & $0.4 \%$ & $\mathrm{~N} . \mathrm{a}$ & $0.3 \%$ & $0.1 \%$ & $0.3 \%$ & $\mathrm{~N} . \mathrm{a}$. & $0.2 \%$ \\
MUH & $5.8 \%$ & $4.6 \%$ & $2.9 \%$ & $4.4 \%$ & $1.8 \%$ & $1.7 \%$ & $1.2 \%$ & $1.6 \%$ & $0.5 \%$ & $0.5 \%$ & $0.4 \%$ & $0.5 \%$ \\
\hline
\end{tabular}


Indeed, one could argue that the paramount objective of dairy cooperatives is to enhance farmer's income by maximizing the milk prices. Thus, high milk prices come along with low net profits. But glancing at the average milk prices over the last six years, even the most profitable cooperatives also pay higher prices than their German competitors. Deleting the three Scandinavian and Swiss outliers Tine, Valio and Emmi, the average milk price for the other nine dairy cooperatives between 1999 and 2004 has been 30.50 Euro Cent. The lowest prices are paid by German Nordmilch and Humana.

Figure 6: Average Milk Prices of European Dairy Cooperatives 1999-2004.

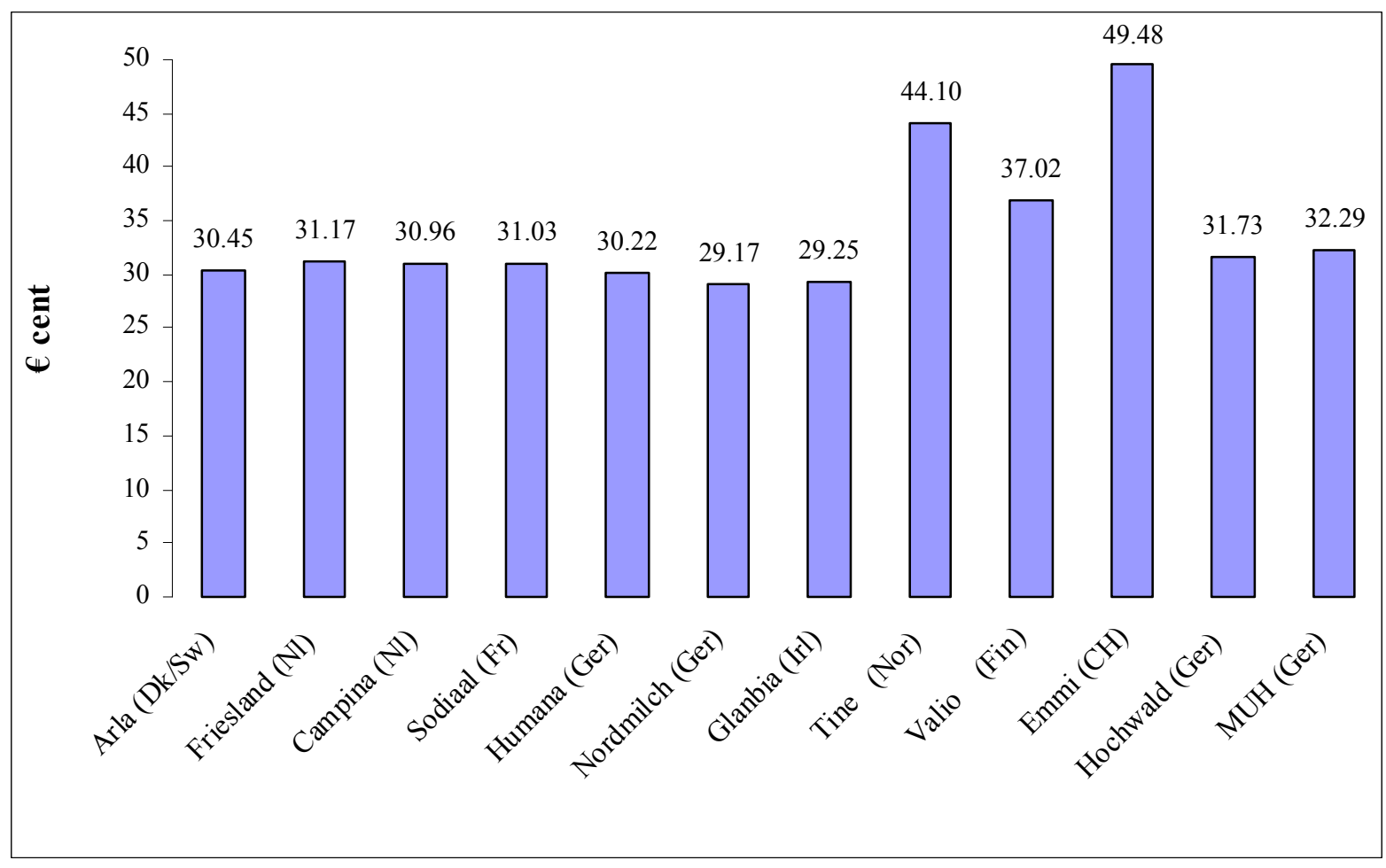

Source: Company data, author's calculations.

\section{DISCUSSION}

This study focuses on 12 leading European dairy cooperatives. Obviously there are many more dairy cooperatives in Europe. But since the smaller cooperatives have only a national or even a regional focus, they are irrelevant from the point of their internationalization. The remaining sample of actual or potential European players is too small to allow the use of any advanced statistics. Nevertheless, it is striking that German cooperatives are less internationalized than their European competitors and are, furthermore, less successful financially. What are the reasons for these discrepancies and what should German cooperatives do differently in 
order to improve their future prospects? These questions are discussed in the following paragraphs, and three explanations are given: the weak competitive position of German cooperatives, the differing sizes of different home markets and the peculiarities of corporate governance in cooperatives.

\section{Explanation of the Weak Financial Performance of German Cooperatives}

The competitive position of German cooperatives is relatively weak compared to many European competitors. Despite a dynamic structural change in the German dairy market, 108 dairies still are engaged in a ruinous predatory competition against each other. The concentration ratio within the dairy industry has remained comparatively low. The five largest dairies combine $42 \%$ of the whole industry's turnover, while the CR10 is only about $58 \%$ (Bridts and Köttl, 2003). This favors strong price competition vis à vis a highly concentrated retail sector. The comparison of German and other European cooperatives shows that, for example, the German market leader Nordmilch as well as Hochwald are drawing out a relatively small turnover per $\mathrm{kg}$ milk processed (see again figure 3). This is caused by the fact that, whereas privately-owned dairy companies often own strong brands and have comparatively strong market positions, German dairy cooperatives usually pursue cost leadership strategies and focus on standardized low-cost and low-price mass market articles like milk, milk powder and butter. This results in a very weak market position (Schramm et al, 2004) and limited financial resources for establishing international business activities. Dutch and Danish cooperatives operate on more consolidated home markets in which they have high market shares. This situation gives them a better starting position for internationalization.

\section{Explanation of the Low Degree of Internationalization of German Cooperatives}

Companies like Friesland, Campina, Valio and Arla are located in small countries with a comparatively small home market for dairy products. Since the possible degree of specialization is mainly determined by the size of the market (Stigler, 1951), companies from small countries are forced to internationalize their activities in order to become cost-efficient (Kutschker and Schmid, 2004). In this respect, cooperatives from larger countries which offer more opportunity for economies of scale and specialization experience lower pressures to internationalize their activities and have systematic disadvantages with respect to the measurement of degrees of internationalization. Thus, the small-country argument is a convincing 
explanation for the high export quota of Danish as well as Dutch cooperatives. But it cannot explain why companies like Arla, Friesland and Campina seek business opportunities in European and non-European countries by acquiring or founding local subsidiaries, while their German competitors stick to their national and sometimes even regional markets.

Dairy industry insiders often argue that certain characteristics of fresh products like milk for instance, limited shelf-life - complicate the internationalization of business activities and require a mainly regional focus of production and distribution in the dairy industry (Krijger, 2004). Since only 32 million tons out of a worldwide milk production of 590 million tons are traded internationally, there is obviously something in this argument. But it cannot explain why some cooperatives have built up a far-reaching network of international subsidiaries whereas others refrain from investing abroad. Campina and other internationalized dairy companies, such as Skandinavian Arla Foods, no longer put their trust in exports but have subsidiaries in several other countries. It is clear, therefore, that product characteristics do not explain low degrees of internationalization but only certain internationalization modes. Referring to Meissner and Gerber's process model (Meissner and Gerber, 1980), we can say that product characteristics like limited shelf-life prevent only such half-hearted internationalization strategies as exporting products. In fact they require more intensive internationalization strategies based on investments abroad. Some authors expect that at the end of the consolidation process in the dairy sector only ten to twenty companies will have survived. Due to certain product characteristics, these multinational companies will be interwoven with a dense network of local partners which support local milk production and collection as well as distribution of end products (Krijger, 2004). Again, obvious differences between German cooperatives and their European competitors cannot be explained by this argument.

\section{Deficits of Corporate Governance in German Cooperatives}

A central strand of thought stems from the economic literature on the peculiarities of cooperatives. Most of this literature argues from the point of view of new institutional economics. Property rights theory, transaction cost theory and agency theory all come up with similar and somewhat interrelated arguments concerning the efficiency of cooperatives. With reference to internationalization strategies, the most important arguments can be summarized as follows (Staatz, 1989; Cook, 1995; Horsthemke, 2000): 
Free-riding problem. An external free-riding problem occurs when a cooperative treats nonmembers exactly the same as members by offering, for instance, the same terms of trade. In this case there is no need to become a member of the cooperative and to financially support its internationalization strategy. An internal free-riding problem occurs when new members of a cooperative are treated in the same way as "old" members. Again, the resulting free-riding problem undermines the motivation to invest since (mainly long-term) investments are not safeguarded against attenuation due to an imperfect definition of property rights. The reluctance to invest undermines a cooperative's ability to internationalize or to invest in branding.

Investment problem. The property rights of members in their cooperative are very difficult to trade. As a consequence, members who want to leave the cooperative cannot quickly get rid of their investments. Therefore, many cooperatives have a large number of inactive members who still hold their property rights even though they no longer interact economically with the cooperative. These investors are more interested in short-term returns on investments than in long-term investments in, for instance, internationalization strategies and strong brands.

Transaction cost problem. In large cooperatives members pursue very diverse goals. In such cases reaching decisions on, for instance, internationalization strategies becomes costly, and cooperatives have severe problems reacting effectively to new market conditions by shifting the regional center of gravity of their business.

Control problem. The "one man - one vote" principle and the non-tradeability of property rights prevent the accumulation of votes and capital shares, which would lower transaction costs and help to overcome the free-riding problem in controlling the organization. Thus, it is very difficult for individual members of a cooperative to initiate internationalization strategies, which might be considered necessary but which are not supported by reluctant members or managers.

Human resource problem. In many cooperatives part-time board members with low managerial know-how and severe time constraints have considerable influence on strategic decisions. Due to the transaction cost and the control problem, it is difficult for members who do not agree with a cooperative's internationalization strategy to get rid of these board members and to implement better top management.

The diverse capabilities of cooperatives in solving these problems may explain different degrees of internationalization and different financial performance. Some cooperatives stick to 
their traditional structures, and the aforementioned problems remain largely unsolved. Others have revolutionized their corporate governance mechanisms by implementing new organizational forms. Some cooperatives, such as Campina, Arla, Valio and Friesland, have transformed into holding companies by outsourcing their day-to-day business activities to a professionally managed joint-stock company ${ }^{1}$. The members of the cooperative then focus only on managing the holding company that holds the shares in the newly founded daughter company. It has often been argued that structure follows strategy (Chandler, 1962). From this theoretical perspective the argument that structural deficits may prevent German cooperatives from internationalizing their activities is somewhat surprising. But there is also ample empirical evidence that in many cases strategy follows structure. Rumelt (Rumelt, 1974) argues that reorganizations can be a precondition for allowing top management to address certain strategy decisions. Furthermore, specific organization structures can induce a strategic behaviour which is in line with the organizational contingencies in order to avoid a strategy-structuremisfit (Mintzberg, 1981). This argument parallels early findings of contingency theory that an organization's differentiation influences the cognitive orientation of its managers (Lawrence and Lorsch, 1967). European and especially German dairy cooperatives and their internationalization strategies obviously support this theoretical strand.

\section{MANAGERIAL IMPLICATIONS}

In the future the German dairy industry must consolidate faster than before and solve its structural problems. A glance at leading European cooperatives reveals that the more successful ones have high market shares in their home markets, which provide the necessary financial resources for further internationalization ${ }^{2}$. The internationalization strategies pursued by companies like Campina could also have been implemented by German cooperatives if they had

\footnotetext{
${ }^{1}$ The most recent example is the Swiss cooperative Emmi, which enhanced its equity by 100 million francs through an initial public offering on the Zurich stock exchange. Almost the only shareholder is the cooperative of central Swiss milk producers; thus, the enterprise remains in farmers' hand. At the same time, they are much better positioned for further growth and internationalization steps through the new holding structure.

${ }^{2}$ For instance, Campina has a market share of 32\% in the Netherlands, Arla has more than 60\% in Denmark and more than $90 \%$ in Sweden, Tine in Norway and Valio in Finland each have around $80 \%$ in their respective countries, and Glanbia holds approximately $46 \%$ market share in Ireland. For comparison, here are the market shares for the German cooperatives: Nordmilch (16\%), Humana (12\%), Hochwald (5\%).
} 
also consolidated their home market. With the fall of the iron curtain and the opening of the Eastern European markets they could have penetrated their Central and Eastern European neighboring countries step by step, following a so called "cascade strategy" (Welge and Holtbrügge, 2001). Unfortunately, German cooperatives have forgone these opportunities.

Assuming that our diagnosis is correct, the managerial implications are obvious. German cooperatives in the dairy industry have to solve their financial problems and redesign their corporate governance mechanisms in order to overcome the above-mentioned problems hampering their financial success and further internationalization. Recent developments indicate that such a change of mind is, in fact, going on in leading German cooperatives and that a redesign and professionalization of management structures is taking place. Nordmilch, for instance, has announced that since June 22, 2004, board membership is restricted to professional, full-time members.

In summarizing it has to be noted that in the past German cooperatives have only very limitedly been able to withstand international competition and play an active role in the globalization of markets. Particularly the cost focused large German cooperatives facing large strategic challenges. So far these cooperatives have produced neither a pure brand leader nor a pure cost leadership strategy. The choice of the latter strategy demands using synergies more consistently in the future and concentrating on the role as a specialist focused on private brands and manufactured products. However this would clearly require stronger internationalization (Gerlach et al, 2005). Since many German cooperatives lack the financial performance to internationalize their activities, cross-border mergers in the cooperative sector might be unavoidable. Such mergers result in transnational cooperatives with several geographic centers of gravity (Borgström, 2003). A further consolidation of European dairy cooperatives is considered of paramount importance for successful competition with multinational privately-owned food companies like Nestlé, Danone or Unilever (Anonymus, 2004). The merger between Campina and Arla, announced last December, could have become the core of the transnationalization of Europe's cooperative sector in the agribusiness. But the more sobering it is to see, that the two cooperatives stooped their final merger negotiations. The Supervisory Boards and Executive Boards of the two companies concluded that joint definitive merger proposals cannot be put to the Members' Councils of the two co-operatives at present. It seems that not even these leading cooperatives are free of corporate governance problems. 


\section{REFERENCES}

Anonymus (2004): Fusion Arla und MD Foods. Milchriese entsteht in Dänemark, in: Handelsblatt (193): 13.

Barney, J. B. (2002): Gaining and Sustaining Competitive Advantage, New Jersey: Pearson Education, Inc.

Bartlett, C. A. and Ghoshal, S. (1989): Managing Across Borders. The Transnational Solution, Boston.

Borgström, M. H. (2003): Die Lage und Entwicklung landwirtschaftlicher Genossenschaften in Europa, http://www.iru.de/de/publikationen/courier/3-03/Deutsch-4.html, Abrufdatum: 27.05.2004.

Born, K. (2001): Bilanzanalyse international. Deutsche und ausländische Jahresabschlüsse lesen und beurteilen, Second Edition, Stuttgart.

Bridts, C. and Köttl, P. (2003): Steht die Milchwirtschaft vor der heißen Phase ihres Konzentrationsprozesses?, in: M\&A Intern, Zeitschrift der HypoVereinsbank (HBV Consult), 9(2003): 8-15.

Chandler, A. (1962): Strategy and Structure: Chapters in the History of Industrial Enterprise. Cambridge, Mass.-London.

Cook, M. L. (1995): The Future of U.S. Agricultural Cooperatives: A Neo-Institutional Approach, in: American Journal of Agricultural Economics, 77(1995): 1153-1159.

Dhanaraj, C. and Beamish, P. W. (2003): A Resource-Based Approach to the Study of Export Performance, in: Journal of Small Business Manegement, 41(2003): 242-256.

Fisch, J. H. and Oesterle, M.-J. (2003): Exploring the Globalization of German MNCs with the Complex Spread and Diversity Measure, in: Schmalenbach Business Review, 55(2003): 2-21.

Gabler, S. (2003): Möglichkeiten und Probleme der Eigenkapitalbildung und der langfristigen Fremdfinanzierung in Molkereiunternehmen der rechtsform Genossenschaft, FreisingWeihenstephan.

Ger, G. (1999): Localizing in the Local Village: Local Firms Competing in Global Markets, in: California Management Review, 41(1999): 64-83.

Gerlach, S. et al (2005): Der Markt für Milch und Milcherzeugnisse, in: Agrarwirtschaft 54(2005): 35-48.

Germann, H. et al (1999): Messung der Globalisierung: ein Paradoxon, in: Steger, U. (Ed.), Facetten der Globalisierung: ökonomische, soziale und politische Aspekte, Berlin etc.: $1-25$.

Germann, H. et al (1996): Globalisierung der Wirtschaft: Begriff, Bereiche, Indikatoren, in: Steger, U. (Hrsg.), Globalisierung der Wirtschaft: Konsequenzen, für Arbeit, Technik und Umwelt, Berlin: 18-55.

Glaum, M. and Lindemann, J. (2002): Externes Wachstum: Theoretische Überlegungen und empirische Befunde, in: Glaum et al. (Eds.), Wachstumsstrategien internationaler Unternehmungen. Internes vs. externes Unternehmenswachstum, Stuttgart: 269-299.

Gräfer, H. (2001): Bilanzanalyse, Eighth Edition, Berlin.

Hassel, A. et al (2003): Two Dimensions of the Internationalization of Firms, in: Journal of Management Studies 40(2003): 705-723.

Horsthemke, A. (2000): Mitgliederbindung und Kapitalaufbringung im Strukturwandel ländlicher Genossenschaften, Stuttgart-Hohenheim. 
Ietto-Gillies, G. (1998): Different Conceptual Frameworks for the Assessment of the Degree of Internationalization: An Empirical Analysis of Various Indices for the Top 100 Transnational Corporations, in: Transnational Corporations, 7(1998): 17-39.

Krijger, A. (2004): Auf die Strategie kommt es an, in: DLG-Mitteilungen, 5(2004): 26-29. Küting, K.-H. and Weber, C.-P. (2004): Die Bilanzanalyse. Lehrbuch zur Beurteilung von Einzel- und Konzernabschlüssen, Seventh Edition, Stuttgart.

Kutschker, M. and Schmid, S. (2004): Internationales Management, München.

Lawrence, P. R. and Lorsch, J. W. (1967): Organization and Environment. Homewood, Ill.

Meissner, H. G. and Gerber, S. (1980): Die Auslandsinvestition als Entscheidungsproblem, in: BFuP, 3(1980): 217-228.

Mintzberg, H. (1981): Organization Design: Fashion or Fit? Harvard Business Review, Vol. 59, Issue 1: 103-115.

Rumelt, R. P. (1974): Strategy, Structure and Economic Performance, Boston.

Schmidt, R. (1981): Zur Messung des Internationalisierungsgrades von Unternehmen, Kiel.

Schramm, M. et al (2004): Brand Orientation in der Ermährungsindustrie. Erfolgsdeterminanten der Markenführung am Beispiel genossenschaftlicher Hersteller, Wiesbaden.

Schwab, J. A. (1997): Begriff und Messung der Globalisierung. Ein Vorschlag zur Integration von Intensitäts- und Streuungskonzepten in der empirischen Globalisierungsanalyse, Mainz.

Staatz, J. M. (1989): Farmer Cooperative Theory. Recent Developments, in: USDA (Ed.): ACS Research Report Nr. 84.

Stappel, M. and Hennigsen, E. (2003): Die deutschen Genossenschaften 2003. Entwicklungen - Meinungen - Zahlen, Neuwied.

Stigler, G. (1951): The Division of Labor is Limited by the Extent of the Market. Journal of Political Economy, 59(1951): 185-193.

Sullivan, D. (1994): Measuring the Degree of Internationalization of a Firm, in: Journal of International Business Studies, 25(1994): 325-342.

Swoboda, B. (2002): Dynamische Prozesse der Internationalisierung. Managementtheoretische und empirische Perspektiven des unternehmerischen Wandels, Wiesbaden.

UNCTAD (2004): World Investment Report, 2003. FDI Policies for Development: National and International Perspectives. United Nations: New York and Geneva.

Welge, M. and Holtbrügge, D. (2001): Internationales Management, Second Edition, Landsberg am Lech. 


\section{FAZIT}

ENTWICKLUNG UND AUSBLICK EINER SICH KONSOLIDIERENDEN BRANCHE 


\title{
Entwicklung und Ausblick einer sich konsolidierenden Branche
}

\author{
,Was die Weltwirtschaft angeht, so ist sie verflochten'
}

Kurt Tucholsky

Die Weltbrauindustrie unterzog sich in der vergangenen Dekade einem grundlegenden Wandel und wandelte sich von einer vormals relativ regional bzw. national geprägten Branche in eine mittlerweile von global tätigen Braukonzernen geprägte Industrie. Die Treiber dieser Entwicklung waren die großen börsennotierten - vor allem europäischen - Brauunternehmen, die einen rasanten, vor allem externen Wachstumsprozess durchliefen und durch eine Vielzahl von teils spektakulären M\&A-Transaktionen seit Ende der 90er Jahre die Weltbrauindustrie konsolidierten und weiter konzentrierten. Mit Marktkapitalisierungen jenseits der 20 Milliarden $€$ und jährlichen Umsätzen von teilweise über 10 Milliarden $€$ handelt es sich dabei heute um multinationale Unternehmen, die in einem internationalen Umfeld hochprofitabel agieren.

Nach der Darstellung der wesentlichen Ergebnisse der vorliegenden Dissertation in den Kapiteln I bis IV folgt nun an dieser Stelle eine Schlussbetrachtung, mittels derer die einzelnen Kapitel thematisch zusammengefasst und vergleichend betrachtet werden. Damit wird der ,rote Faden', der diese Arbeit methodisch und thematisch durchzieht, nochmalig abschließend aufgegriffen. Hierzu erfolgt zunächst eine kurze Einzelbetrachtung der Kernergebnisse der Arbeit, die den Themenblöcken der Arbeit folgend gegliedert werden. Dabei wird insgesamt allerdings nicht zu sehr in die Tiefe gegangen, um unnötige Wiederholungen zu den ,Results'-, ,Managerial Implications'- und ,Future Research'-Abhandlungen der einzelnen Unterkapitel zu vermeiden. Es soll vielmehr auf die Kernaussagen der Dissertation fokussiert werden, wobei gesondert auf die Ergebnisse der angewandten Methodiken der empirischen Untersuchung eingegangen wird. Daran schließen sich Implikationen und Anregungen für die weitere Forschung im Bereich des Internationalen Managements im Speziellen und der empirischen Bilanz- und Kapitalmarktforschung im Agri-Food-Business im Besonderen an. 


\section{GLOBALISIERUNG UND KONSOLIDIERUNG DER WELTBRAUBRANCHE}

Der Weltbiermarkt umfasste im Jahre 2005 ein Produktionsvolumen von etwa 1,59 Milliarden Hektoliter (hl). Dabei erhöhte sich die jährliche Wachstumsrate der Bierproduktion im Laufe der letzten Dekade von etwa 2\% auf nunmehr 2,5\%. In den Wirtschaftsjahren 2004 und 2005 stieg dieses Wachstum nochmals deutlich an, was Grund zu der Annahme gibt, dass sich der Wachstumstrend der Brauindustrie in den kommenden Jahren eher noch verstärken wird. Vor drei Jahren übertraf die chinesische Bierproduktion (291 Mio. hl in 2004) zum ersten Mal die US-amerikanische (233 Mio. hl in 2004). Beide Länder zusammen liegen deutlich vor den übrigen großen Biermärkten. Die folgenden sechs Märkte befinden sich alle in etwa in der gleichen Größenklasse zwischen 60 und 100 Millionen Hektolitern; zu diesen Märkten zählt u. a. Deutschland. Danach folgen mit einigem Abstand Spanien und Polen. Zusammen kommen die Top 10 Länder auf einen Anteil von knapp 70\% der weltweiten Bierproduktion. Im Hinblick auf das Volumenwachstum ist China bei weitem der attraktivste Markt, der für $45 \%$ des Gesamtwachstums der letzten Dekade verantwortlich ist; danach folgen Russland mit 20\% sowie Mexiko und Brasilien mit jeweils 7\%.

Neben Produktion bezogenen Daten wurden in Kapitel I der Dissertation vor allem auch auf wesentliche Profitabilität bezogene Unterschiede weltweiter Biermärkte herausgearbeitet. So gibt es hinsichtlich der EBITDA-Margen, die in verschiedenen Biermärkten erzielt werden können, erhebliche Differenzen. Die Margen reichen dabei von etwa $1 € /$ hl (China), 4€/hl (Deutschland), 6€/hl (Russland) bis zu 26€/hl (Japan), 31€/hl (Kanada) und 32€/hl (Irland, Australien). Dabei konnte ein eindeutiger Zusammenhang zwischen hoher Profitabilität und starkem Konzentrationsgrad der Teilmärkte festgestellt werden.

Innerhalb der Weltbrauindustrie begann dieser Konzentrations- und Konsolidierungsprozess später als in anderen Consumer Goods-Industrien. Noch bis Ende der 90er Jahre verlief das Wachstum der zehn größten Braukonzerne fast parallel zu dem des gesamten Weltbiermarktes mit jährlichen Wachstumsraten von gut zwei Prozent. So erhöhte sich der Konzentrationsgrad (CR10) von 1989 bis 1998 lediglich von 29,6\% auf 33,7\%. Doch in den acht Jahren bis zum Jahr 2005 hat sich diese Konzentrationsrate auf mittlerweile 62,5\% nahezu verdoppelt. Durch diese Konsolidierungswelle haben Fusionen und Übernahmen als wichtigste Instrumente des externen Unternehmenswachstums in den letzten Jahren deutlich an Bedeutung gewonnen. Daraus resultierten eine deutliche Zunahme der internationalen Verflechtung und eine signifikante Erhöhung des Ausmaßes globaler Unternehmenstätigkeit börsennotierter Braukonzerne. 


\section{INTERNATIONALISIERUNG FÜHRENDER BRAUKONZERNE}

Selbst die größten deutschen Privatbrauereien und Braugruppen nehmen sich relativ bescheiden im Vergleich zu den weltweiten Braukonzernen aus. Doch jenseits der in der Branche hinlänglich bekannten Volumenzahlen und Hektoliter-Rankings ist wenig bekannt über finanzielle Performance und internationale Ausrichtung der Global Player in der Weltbrauindustrie. In Kapitel II der vorliegenden Arbeit wurde die zunehmende Internationalisierung weltweit führender Braukonzerne behandelt. Neben verschiedenen Möglichkeiten, Internationalisierungsgrade methodisch zu bestimmen, wurde auch rein deskriptiv-statistisch beschrieben, in wie weit führende Braukonzerne inzwischen internationalisiert sind und ihre Geschäftstätigkeit in einer zunehmend globalen Branche ausgedehnt haben. Es wurde deutlich, dass die führenden Unternehmen in der Weltbraubranche sich in den vergangenen Jahren zu multinationalen Konzernen entwickelt haben, die ihr operatives Geschäft auf ein dichtes Netz weltweit agierender Tochtergesellschaften stützen. So verfügt der weltgrößte Brauer InBev inzwischen über 322 Beteiligungen und Braustätten in 75 Ländern. Auch der niederländische Brauer Heineken operiert auf Basis eines globalen Netzwerkes und betreibt 110 Brauereien in 65 Ländern. Eine weitere Ausdehnung der Aktivitäten verläuft bei den führenden Braukonzernen immer stärker auf dem Wege des externen Wachstums über M\&As. Abbildung 1 veranschaulicht graphisch das teils enorme Unternehmenswachstum der vier weltgrößten Braukonzerne. Trotz einer Zunahme des Weltbiermarktes von 1,30 Milliarden hl in 1998 auf 1,59 Milliarden hl in 2005 konnten die Brauunternehmen ihre Marktanteile überproportional steigern.

Abbildung 1: Marktanteile führender Braukonzerne auf dem Weltbiermarkt.

1998

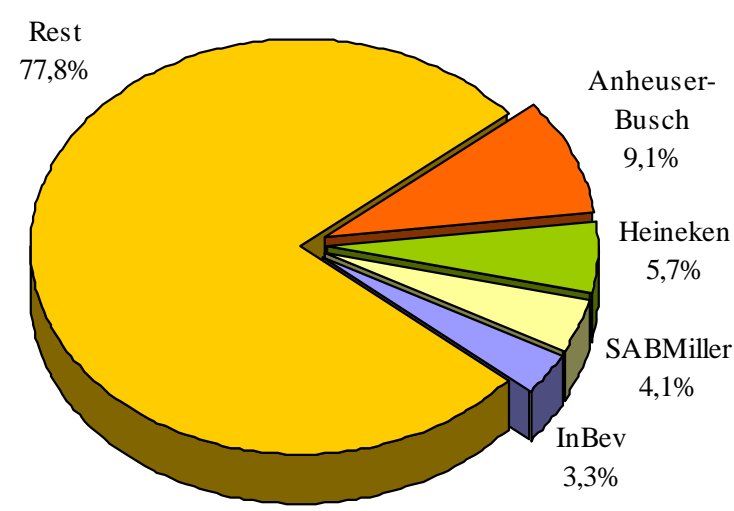

2005

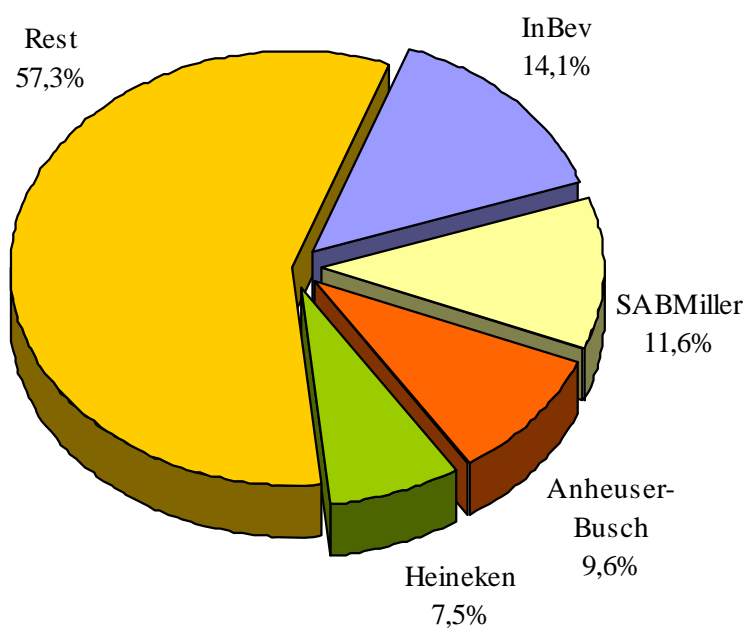


Unternehmensgröße bzw. Marktanteil werden dabei in der branchenüblichen ProduktionsgröBe Hektoliter gemessen. Stellte der US-amerikanische Brauer Anheuser-Busch für Jahrzehnte die unumschränkte Nummer 1 des Weltbiermarktes dar, stets gefolgt vom niederländischen Brauer Heineken - dem europäische Marktführer -, so verschoben sich seit Ende der 1990er Jahre die Gewichte nachhaltig. Die aggressiv extern gewachsenen Konzerne InBev und SABMiller dominieren heute mit 223 Millionen Hektolitern bzw. 184 Millionen Hektolitern die globale Brauszene. Deutlich abgeschlagen folgen Anheuser-Busch und Heineken, die mit dem Konsolidierungstempo der zwei größten Wettbewerber nicht mehr Schritt halten konnten, dies jedoch aus unterschiedlichen Gründen. Anheuser-Busch verließ sich über Jahre auf seine dominante Stellung im nordamerikanischen Biermarkt und bemüht sich angesichts eines stagnierenden Heimatmarktes erst seit kurzem um eine Zunahme seiner internationalen Aktivitäten. Heineken war es in den vergangenen Jahren aufgrund seiner Aktionärsstruktur nicht möglich, bei den wirklich großen Akquisitionen zum Zuge zu kommen.

Im folgenden soll nochmals auf M\&As als dem zentralen Wachstumsinstrument innerhalb der Weltbrauindustrie eingegangen werden, da die verschiedenen Transaktionen das Tempo und die Dynamik bestimmt haben, mit der die großen börsennotierten Konzerne die globale Braubranche in den vergangenen Jahren konsolidiert haben.

\section{MERGERS \& ACQUISITIONS ALS STRATEGISCHES INSTRUMENT EXTERNEN UNTERNEHMENSWACHSTUMS}

Akquisitionen sind ein wichtiges Instrument der strategischen Unternehmensentwicklung. Motive für diese Form des intensivierten externen Wachstums sind Marktmachtsteigerungen und Kosteneinsparungen aufgrund von restrukturierten und konsolidierten Einzelmärkten. Im Falle der vorliegenden Untersuchung zur Brauindustrie können Hybristhesen aus der Agencytheorie wohl verworfen werden. Zwar kam es kurzfristig nicht unbedingt zu einer signifikanten Steigerung des Shareholder Values, doch kann aus mittelfristiger Retrospektive geurteilt werden, dass es gerade den Braumanagern, die sich durch aggressive M\&A-Politik ausgezeichnet haben auch gelungen ist, ihre jeweiligen Unternehmenswerte - sowohl hinsichtlich der Marktkapitalisierung als auch bezüglich bilanzieller Profitabilitätskennzahlen - in besonders erfolgreichem Maße zu steigern. 
Für die Zukunft bleibt abzuwarten, wann es zur nächsten großen Fusion unter den führenden Braukonzernen kommt. Auch wird seit einiger Zeit von Branchen übergreifenden Fusionen bspw. mit Soft Drinks-Herstellern oder Spirituosenkonzernen ausgegangen. Als Möglichkeit gelten beispielsweise eine Fusion zwischen Heineken und Anheuser-Busch oder ein Zusammengehen von SABMiller mit dem mexikanischen Bier- und Getränkekonzern Femsa, dem größten Coca-Cola-Lizenznehmer Südamerikas. Auch erscheint eine Fusion zwischen dem weltgrößten Spirituosen- und Bierkonzern Diageo (Guinness) und Heineken strategisch sinnvoll. In Branchen, in denen weltweit nur noch wenige große Unternehmen von wesentlicher Bedeutung sind, werden Akquisitionen oder Fusionen als strategisch notwendig angesehen, um nicht selbst Übernahmeobjekt zu werden. In einer sich zunehmend konsolidierenden Industrie und vor dem Hintergrund möglichst schnell $\mathrm{zu}$ realisierender Wachstumsschritte kommt vor allem der Marktkapitalisierung eine wesentliche Bedeutung zu. Viele M\&As werden erst vor dem Hintergrund einer ausreichend hohen Marktkapitalisierung möglich. Insbesondere das Instrument des Aktientauschs, das mittlerweile in zunehmendem Maße auch bei grenzüberschreitenden Transaktionen Verwendung findet, lässt einer hohen Marktkapitalisierung eine überragende Bedeutung im M\&A-Wettrennen zukommen. Beim Bieterwettstreit um die kolumbianische Bavaria zwischen Heineken, Anheuser-Busch und SABMiller musste letztlich Heineken zurücktreten, da es den Niederländern aufgrund ihrer Aktionärsstruktur (50,005\% der Aktien an Heineken N.V. hält die im Besitz der Familie Heineken befindliche Holding) nicht möglich war, den von SABMiller gebotenen Kaufpreis von 6,2 Milliarden $€$ zu überbieten. Bei einer Kapitalerhöhung durch Ausgabe neuer Aktien verlöre die Heineken Holding die Stimmenmehrheit am Konzern, sofern die Familie die Aktien nicht zeichnen würde.

Im Gegensatz dazu bedeutet eine zu geringe Marktkapitalisierung zumeist ein erhöhtes Risiko, selbst Opfer einer feindlichen Übernahme zu werden, zumal hoch kapitalisierte Unternehmen nur relativ wenige neue Aktien bei einer Akquisition gegen Aktien ausgeben müssen. Das Management börsennotierter Braukonzerne sieht sich damit einem neuen Paradigma gegenüber: Sie müssen sich hohe Bewertungen sichern und in Bezug auf ihre Marktkapitalisierungen und Unternehmenswerte möglichst schneller wachsen als der Markt, um entweder den Schritt zu global tätigen Unternehmen zu gehen oder ein solches zu bleiben. Andernfalls laufen sie Gefahr, die Kontrolle über ihre Eigenständigkeit an erfolgreicher agierende Unternehmen zu verlieren. Als Beispiel aus der Braubranche seien die beiden südamerikanischen Bierkonzerne AmBev und Bavaria genannt. Zwar generierten sie die höchsten Profitabilitätskenn- 
zahlen der gesamten Weltbraubranche (EBITDA-Margen von 38,0\% bzw. 39,5\% in 2004), doch konnten sie aufgrund ihrer Fokussierung auf einzelne südamerikanische Kernmärkte zu wenig wachsen und wurden deshalb in den Jahren 2004 und 2005 jeweils von den europäischen Konzernen Interbrew (AmBev) und SABMiller (Bavaria) aufgekauft. Beide Transaktionen wurden mittels Kapitalerhöhung und Aktientausch durchgeführt.

Betrachtet man die Marktkapitalisierung der weltgrößten Brau- und Getränkekonzerne, fällt auf, dass es sich dabei inzwischen tatsächlich um so genannte ,big caps' handelt, also Unternehmen, die durch eine Marktkapitalisierung jenseits der 10 Milliarden US\$ gekennzeichnet sind. Vor allem Unternehmen wie InBev oder SABMiller konnten in den vergangenen Jahren nachhaltig Ihre Unternehmenswerte steigern. Dadurch ist die Weltbraubranche heutzutage durch multinational operierende Konzerne geprägt, deren Konsolidierungs-, Wachstums- und Konzentrationsbestrebungen keine Anzeichen für ein Nachlassen der bestehenden Dynamik erkennen lassen. Abbildung 2 veranschaulicht die Marktkapitalisierungen der größten börsennotierten Brau- und Getränkekonzerne zum März 2006.

Abbildung 2: Marktkapitalisierung weltweit führender Brau- und Getränkekonzerne.

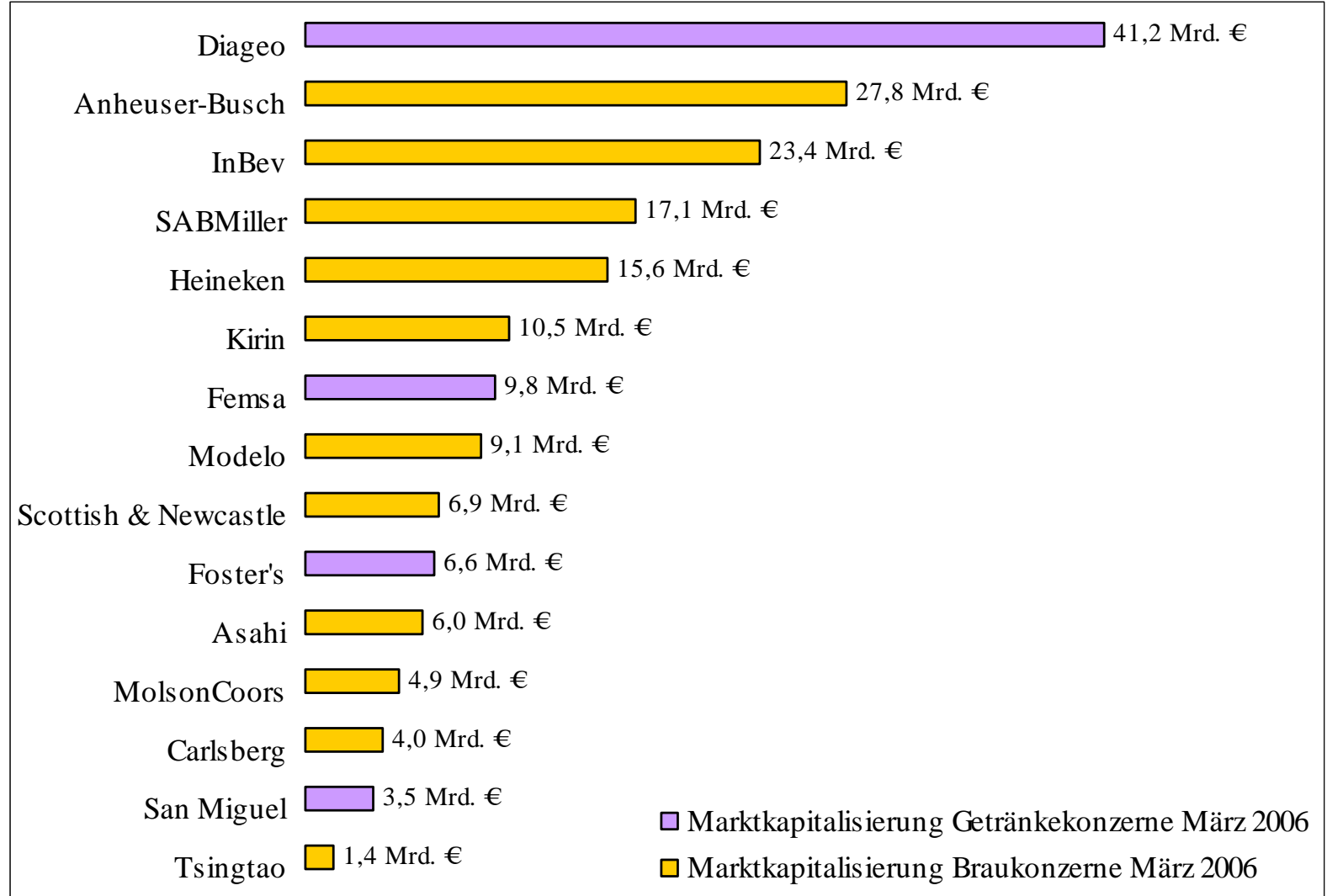




\section{INTERNATIONALISIERUNG UND UNTERNEHMENSERFOLG}

\section{Ergebnisse der bilanziellen Untersuchungen zu börsennotierten Braukonzernen}

Die Ergebnisse der bilanziellen Untersuchungen zu einer Verbindung zwischen steigender Internationalisierung und unternehmerischem Erfolg zeigen zwar über die verschiedenen Datensamples einen leicht negativen, statistisch teilweise durchaus signifikanten Zusammenhang, doch konnten aufgrund verschiedener Kontrollvariablen aufgezeigt werden, wodurch dieser Zusammenhang beeinflusst wird. Vor allem der heimische Marktanteil (,domestic market share') hatte einen hoch signifikanten Einfluss auf die Untersuchungsergebnisse. Dies ist nicht verwunderlich, wenn man einzelne Unternehmen näher betrachtet. Der USamerikanische Brauer Anheuser-Busch beispielsweise - über Jahrzehnte der größte und mit Abstand profitabelste Braukonzern weltweit - stützt seine Geschäftstätigkeit auf einen 50\%igen Marktanteil im US-amerikanischen Biermarkt, nach China volumenmäßig der zweitgrößte Biermarkt der Welt, nach Umsatz und EBITDA allerdings nach wie vor mit großem Abstand der größte profit pool im Weltbiermarkt. Ähnlich verhält es sich mit Brauunternehmen wie Grupo Modelo (57\% in Mexiko), Bavaria (99\% in Kolumbien) oder Anadolou Efes (79\% in der Türkei). Hierbei muss beachtet werden, dass die Höhe des Marktanteils nur bei einem ausreichenden Verhältnis der Größe des Heimatmarktes zur Gesamtproduktion des Unternehmens eine Rolle spielt. Zwar verfügt auch InBev über einen 56\%-igen Marktanteil in Belgien, doch repräsentieren diese dort abgesetzten 8,7 Millionen Hektoliter nur etwa 4\% von InBevs Gesamtbierausstoß von 223 Millionen Hektolitern in 2005. Bereinigt man jedoch die Ergebnisse der Untersuchung um die starken ,outlier' der genannten Unternehmen, bekommt man ein relativ klares Bild. So konnten vor allem die großen europäischen Braukonzerne InBev, SABMiller und Heineken einerseits ihre Profitabilitätskennzahlen verbessern und dies zusätzlich in Verbund mit einem überdurchschnittlichen Wachstum des Volumens der gesamten Geschäftstätigkeit. Dies veranschaulicht nochmals abschließend die folgende Abbildung, in der deutlich zum Ausdruck kommt, welche Dynamik hinter der unternehmerischen Entwicklung der führenden europäischen Braukonzerne steckte. Während Anheuser-Busch durch fehlendes bzw. verspätetes internationales Engagement Ergebnisrückgänge über die vergangenen Jahre verzeichnen musste, konnten Heineken, aber vor allem SABMiller und InBev außerordentliches Wachstum verbuchen, das auf die aggressiv verfolgte M\&A-Politik der europäischen Brauunternehmen zurück zu führen ist. 
Abbildung 3: Umsatz und EBITDA der vier weltgrößten Braukonzerne (in Mrd. €).

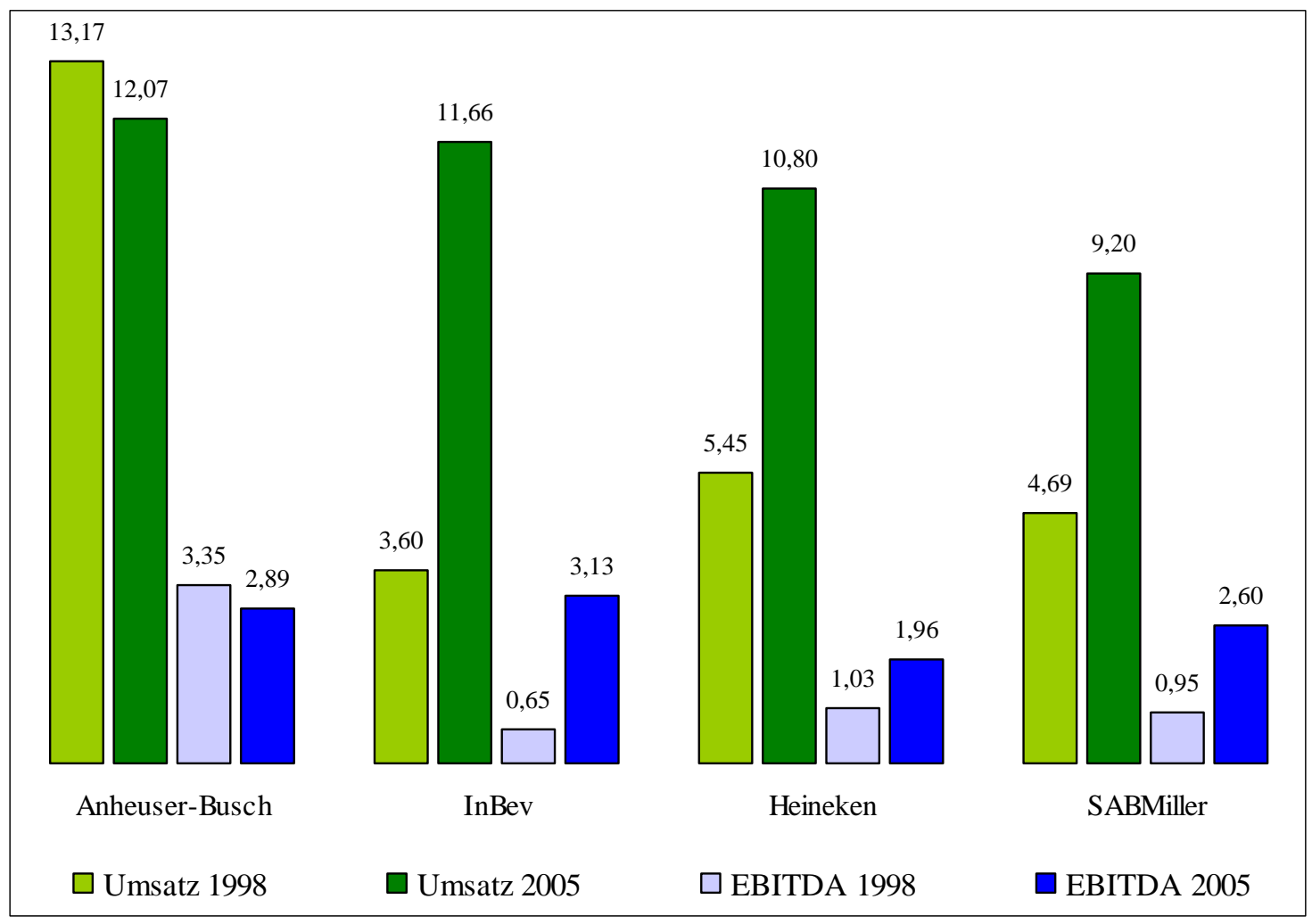

\section{Ergebnisse der Ereignisstudien zu börsennotierten Braukonzernen}

Übereinstimmend mit früheren Arbeiten zur Bewertung von M\&As mittels Ereignisstudien zeigen die Ergebnisse der vorliegenden Arbeit, dass horizontale Übernehmen zwar leicht negative Überrenditen für die Käuferunternehmen erzielen, solche Akquisitionen vom Kapitalmarkt aber noch vergleichsweise günstig bewertet werden im Gegensatz zu konglomeraten Transaktionen. Die Untersuchungsergebnisse für insgesamt 34 M\&As im Zeitraum von März 2000 bis Juli 2005 in der Weltbraubranche ergaben im Ereigniszeitraum von 21 Tagen negative kumulierte Überrenditen von $-0,50 \%$ und positive abnormale Renditen von $0,48 \%$ am Ankündigungstag. In die empirische Analyse wurden nur Fusionen und Akquisitionen einbezogen, die ein Transaktionsvolumen von 100 Millionen $€$ überstiegen, um eine ausreichende Reaktion der Kapitalmarktteilnehmer zu gewährleisten. Die Schlussfolgerungen der event study-Ergebnisse stimmen dabei tendenziell mit den Aussagen der meisten Ereignisstudien der empirischen Kapitalmarktforschung überein. Allerdings zeigt die vorliegende Untersuchung kein pauschal negatives Bild externer Wachstumsschritte innerhalb der Weltbraubranche. In der Tat sind die Ergebnisse recht heterogen. Interessant erscheint in diesem Zusam- 
menhang die Tatsache, dass es eine sehr breite Streuung positiver wie negativer Kapitalmarktreaktionen auf die M\&A-Ankündigungen verschiedener Braukonzerne gibt. Ein hochsignifikanter Zusammenhang bestand im Untersuchungssample hinsichtlich der Höhe der gezahlten Transaktionspreise und der abnormalen Rendite: Je höher die Übernahmepreise, desto negativer die Kapitalmarktreaktion. Ein weiterer Beleg für die Existenz effizienter Kapitalmärkte ist die Tatsache, dass die Shareholder durchaus auch strategisch besonders bedeutsame Übernahmen positiv aufnahmen, wie z.B. die Akquisition der kolumbianischen Braugruppe Bavaria durch SABMiller im Juli 2005 für 6,2 Milliarden €. Zwar lag die Kaufsumme laut Analystenmeinung relativ hoch, doch stellte dieser Deal für SABMiller den Einstieg in den hoch profitablen und wachsenden südamerikanischen Biermarkt dar. Somit zeigt sich, dass das Vorhandensein effizienter Kapitalmärkte als zentrale Voraussetzung für Validität und Reliabilität von Ereignisstudien im vorliegenden Fall durchaus gegeben war.

\section{Exkurs: Internationalisierung anderer Subsektoren des Agribusiness}

Die Untersuchung des genossenschaftlichen Schlachtsektors, vor allem aber die Analyse von Molkereigenossenschaften ergab, dass die deutschen Genossenschaften in der Vergangenheit nur bedingt in der Lage waren, dem internationalen Wettbewerb standzuhalten und im Zuge der Globalisierung der Märkte eine aktive Rolle zu spielen. Auch weisen die großen deutschen Molkereigenossenschaften nach wie vor schwächere bilanzielle Kennzahlen auf als die führenden europäischen Wettbewerber. Vor großen strategischen Herausforderungen stehen deshalb v.a. die Kosten fokussierten großen deutschen Genossenschaften, die es in der Vergangenheit weitgehend versäumten, in die Internationalisierung durch Direktinvestitionen in Wachstumsmärkte zu investieren. Die Wahl dieser Strategie würde allerdings fordern, Synergieeffekte zukünftig noch konsequenter zu nutzen. Ausländische Wettbewerber haben diesen Weg vielfach schon erfolgreich beschritten und sich auf diese Weise zu schlagkräftigen Einheiten entwickelt. Große europäische Genossenschaften in der Schlachtbranche (Danish Crown, Vion Food) als auch in der Molkereibranche (Arla Foods, Campina, Royal Friesland Foods) stützen ihre Geschäftstätigkeit inzwischen auf ein Netz weltweit operierender Tochtergesellschaften und sichern dadurch nachhaltig ihren Unternehmenserfolg. Erste Anzeichen für ein Umdenken zumindest innerhalb der deutschen Molkereigenossenschaften lassen mittlerweile die Branchengrößen Nordmilch und Hochwald erkennen, die deutliche Bestrebungen unternehmen, um das bisherige Auslandsengagement zu verstärken. 


\section{HANDLUNGSEMPFEHLUNGEN UND IMPLIKATIONEN FÜR DIE WEITERE FORSCHUNG}

Als ,Managerial Implications' sollen an dieser Stelle keine Lösungsvorschläge für CEOs börsennotierter Braukonzerne unterbreitet werden, doch vielleicht finden die Ergebnisse dieser Untersuchung in der deutschen - in weiten Teilen mittelständisch geprägten - Bier-, Schlachtund Molkereibranche Beachtung. In der Tat ist dort nach wie vor eine unsystematische und teils relativ konzeptionslose Erschließung von Auslandsmärkten ohne strategisch abgesicherte Entscheidungsbasen weit verbreitet. Im Zuge einer beschleunigten Weltmarktexpansion haben gerade auch viele deutsche Unternehmen konzeptionelle Management-Lücken im Rahmen ihrer Internationalisierungsbemühungen erkennen lassen. Dies trifft sowohl auf die Brauwirtschaft als auch auf den genossenschaftlichen Molkerei- und Schlachtsektor zu. Während allerdings die deutsche Schlacht- und Zerlegebranche, aber auch große Teile der Brauwirtschaft inzwischen von finanzstarken multinationalen Konzernen aus europäischen Nachbarländern kontrolliert werden, gibt es in der deutschen Molkereibranche noch berechtigte Hoffnung auf das Entstehen schlagkräftiger Strukturen in deutscher Hand, die auch im europäischen und transnationalen Wettbewerb eine entscheidende Rolle spielen könnten. Eine der am Erfolg versprechendsten Maßnahmen ist sicherlich nach wie vor die (bisher) vernachlässigte Internationalisierung der Geschäftstätigkeit, die vielen Unternehmen immer noch die Möglichkeit böte, Marktwachstum zu erzielen, um somit eine nachhaltige Sicherung des Unternehmenserfolges zu gewährleisten. Dabei kommt für viele Brauereien wohl lediglich eine Forcierung der Exportaktivitäten - eventuell durch ein joint venture mit einem ausländischen Braukonzern - in Frage. Den größeren deutschen Molkereigenossenschaften stünde der Weg zu einem weiter gehenden internationalen Engagement theoretisch noch offen, sollten sie ihre strukturellen Probleme in absehbarer Zeit lösen. Da jedoch viele deutsche Genossenschaften alleine nicht die notwendige finanzielle Kraft zur Internationalisierung über Direktinvestitionen besitzen, sind in der Zukunft auch grenzüberschreitende Fusionen innerhalb der Branche zu erwarten.

Implikationen für die weitere Forschung auf dem Gebiet des strategischen Internationalen Managements werden in zweierlei Weise gegeben: zum einen Limitationen, zum anderen Erweiterungsvorschläge. Limitationen betreffen die Methodik zur Bestimmung von Internationalisierungsgraden. Zwar sind in dieser Hinsicht mittlerweile durchaus anspruchsvolle Konzepte wie das von Fisch und Oesterle (siehe dazu Kapitel IV.1) gediehen, doch lässt sich eine wesentliche Hauptkritik an all diesen mehrdimensionalen Konzepten anbringen, und dies ist 
die mangelnde bzw. sogar völlig fehlende Datengrundlage. Deshalb bleibt es trotz allem Anspruch an eine umfassendere Abbildung, Messung und Operationalisierung des Phänomens der wirtschaftlichen Internationalisierung bei einer weitgehenden Beschränkung auf das in den jeweiligen annual reports veröffentlichten Zahlenmaterial. Dieses bezieht sich in der überwiegenden Mehrheit der Unternehmen auf Daten zu Umsatz, Gewinn und die Verteilung von Tochtergesellschaften im Ausland. Kleinere methodische Anpassungen an die jeweils zu betrachtende Branche erscheinen jedoch sinnvoll. Dies wurde bspw. in der vorliegenden Arbeit unternommen, indem etwa bei der Berechnung des Network Spread Indexes nicht die Gesamtzahl der Direktinvestitionen empfangenden Länder betrachtet wurde, sondern nur die im Kontext der Braubranche relevanten. Bei zukünftigen Untersuchungen der Brauindustrie scheint auch eine Gewichtung der Teilmärkte nach dem jeweiligen Absatzvolumen sinnvoll.

Im Zusammenhang mit den untersuchten bilanziellen Performance-Maßen wurde vor allem die besondere Eignung des RONA nachgewiesen, der im Untersuchungskontext internationaler Braukonzerne eine besondere Rolle spielt und für einen nachhaltigen Unternehmenserfolg von börsennotierten Braukonzernen von entscheidender Bedeutung ist. Da es sich bei der Brauwirtschaft um eine sehr anlagenintensive Industrie handelt, ist der RONA die zentrale Kennzahl, die sich aus der Relation des erwirtschafteten Ergebnisses des Konzerns zum eingesetzten Kapital, den Net Assets, ergibt. Da einerseits die Untersuchungsbefunde (siehe u. a. Kapitel III.6) der Bilanzanalyse ein hohes Bestimmtheitsmaß für die RONA-Variable ergaben und andererseits in Gesprächen mit Managern aus der Brauindustrie die Bedeutung des RONA für das operative Geschäft von Braukonzernen unterstrichen wurde, lässt sich schließen, dass es in künftigen Untersuchungen darauf ankommt, die jeweils im Branchenkontext wichtigen und geeigneten Performance-Indikatoren zu eruieren, um die nötige Validität und Reliabilität der Untersuchungsergebnisse zu gewährleisten.

Implikationen für zukünftige Kapitalmarkt orientierte Herangehensweisen an die Frage nach einer Erfolgswirkung von steigender Internationalisierung betreffen eine Fortführung bzw. Erweiterung der event study-Methodik auf verwandte Branchen. So böte sich bspw. ein Vergleich der untersuchten M\&A-Performance von Braukonzernen mit Transaktionen von börsennotierten Getränke-, Spirituosen- oder auch Molkereiunternehmen an. Auch erscheint es sinnvoll und fruchtbar, die Aktienkursreaktionen der übernommenen Unternehmen mit in eine weiter gehende Untersuchung einzubeziehen, was jedoch aufgrund der mangelnden Größe und fehlender Börsennotierung vieler Unternehmen erschwert werden könnte. 


\section{FAZIT DER ARBEIT}

Da multinational agierende Unternehmen sowohl auf eine ausreichend hohe Marktkapitalisierung als auch hohe Profitabilitätskennzahlen angewiesen sind, kommt einer umfassenden Betrachtungsweise eine besondere Bedeutung zu. Zumal im Zusammenhang mit den analysierten M\&A-Aktivitäten führender Braukonzerne spielen sowohl bilanzielle Kennzahlen als auch die Marktkapitalisierung eine gleichwertige Rolle, stellen doch beide Instrumente zur Finanzierung möglicher Übernahmen dar: mittels free cash flow und Fremdfinanzierung oder durch Kapitalerhöhung durch Ausgabe neuer Aktien. Dass letztere Möglichkeit zunehmend zur Finanzierung großer Akquisitionen verwandt wird, wurde bereits besprochen. Als methodisches Fazit der Arbeit lässt sich der Nachweis anführen, dass Kapitalmarkt orientierte und Bilanz orientierte Betrachtungsweisen der Erfolgsmessung von steigender internationaler Unternehmenstätigkeit nicht substitutiv, sondern vielmehr komplementär verwandt werden sollten, um ein umfassendes Bild unternehmerischen Erfolges und nachhaltiger finanzieller Performance erhalten zu können.

Neben den methodisch-empirischen Befunden sprechen die bilanziellen Zahlen eine deutliche Sprache. Unternehmen wie InBev, Heineken oder SABMilller haben in der vergangenen Dekade als Treiber zunehmender Internationalisierung die ehemals national und regional ausgerichtete Braubranche in eine global verflochtene Industrie transformiert und dabei teils außerordentliche Umsatz- und Profitabilitätswachstum generieren können. Die spannende Frage im Kontext der Weltbrauindustrie bleibt, ob es in naher Zukunft zu ersten ,Mega-Mergers' zwischen den führenden Brau- und Getränkekonzernen kommen wird. Nach Meinung des Autors wird das Konsolidierungs- und Konzentrationstempo der Brauunternehmen mit unverminderter Geschwindigkeit voranschreiten, damit diese langfristig im Wettbewerb mit den großen globalen Nahrungsmittel-, Getränke- und Spirituosenkonzernen bestehen können.

Die Quintessenz dieses Fazits - mit anderen Worten, der zusammenfassende Nukleus, auf den sich die vorliegende Arbeit verbal komprimieren lässt, soll mit folgendem abschließenden Satz verdeutlicht werden:

Mit der Globalisierung der Wirtschaft verhält es sich wie mit der Physik. Man muss sie nicht mögen, doch ist es hilfreich, ihre Gesetzmäßigkeiten zu verstehen. 


\section{ANHANG}

PUBLIKATIONEN UND KONFERENZTEILNAHMEN,

LEBENSLAUF 


\section{Publikationen und Konferenzteilnahmen}

\section{Beiträge mit Review-Prozess}

Ebneth, O. and Theuvsen, L. (2005): Large Mergers and Acquisitions of European Brewing Groups - Event Study Evidence on Value Creation. Submitted for: Agribusiness.

\section{Wissenschaftliche Publikationen}

Ebneth, O. and Theuvsen, L. (2005): Internationalization of Cooperatives in the Agribusiness - Concepts of Measurement and their Application, in: Strategies for Cooperatives, Theurl, Th. and Meyer, E. C. (Eds.), Aachen: 395-419.

Theuvsen, L. and Ebneth, O. (2004): Internationalization Strategies of European Dairy and Meat Cooperatives, in: Proceedings of the $2^{\text {nd }}$ HAED Conference: Management in Agribusiness, Josip Juracak (Ed.), Zagreb (Kroatia), $4^{\text {th }}-5^{\text {th }}$ of November 2004: 66-68.

\section{Konferenzbeiträge}

Ebneth, O. and Theuvsen, L. (2006): Internationalization and Financial Performance Brewing Groups, Paper submitted for the VIII ${ }^{\text {th }}$ IFSAM World Congress, $28^{\text {th }}-30^{\text {th }}$ of September 2006, Berlin.

Ebneth, O. and Theuvsen, L. (2006): Multinationality and Financial Performance - Findings from a Set of Listed Brewing Groups, Paper submitted for the $46^{\text {th }}$ GeWiSoLa Conference, $4^{\text {th }}-6^{\text {th }}$ of October 2006, Gießen.

Ebneth, O. and Theuvsen, L. (2006): Internationalization and Performance - Capital-MarketBased and Accounting-Based Measures in Comparison. Paper submitted for the USDA and AIEA2 International Meeting, $15^{\text {th }}-16^{\text {th }}$ of June 2006, Bologna.

Ebneth, O. and Theuvsen, L. (2006): Growth and Profit Opportunities - Analyzing the World Brewing Industry. Poster will be presented at the $16^{\text {th }}$ Annual World Food and Agribusiness Symposium and Forum, $10^{\text {th }}-13^{\text {th }}$ of June 2006, Buenos Aires.

Ebneth, O. and Theuvsen, L. (2006): The Relationship between Business Internationalization and Financial Performance of Stock-Listed Brewing Groups. Paper will be presented at the $16^{\text {th }}$ Annual World Food and Agribusiness Symposium and Forum, $10^{\text {th }}-13^{\text {th }}$ of June 2006, Buenos Aires.

Ebneth, O. and Theuvsen, L. (2005): Internationalisierung und Unternehmenserfolg - Ein Vergleich europäischer Molkereigenossenschaften, Paper presented at the GeWiSoLa Conference, $5^{\text {th }}-7^{\text {th }}$ of October 2005, Göttingen. 
Ebneth, O. and Theuvsen, L. (2005): Internationalization and Corporate Success - Empirical Evidence from the European Dairy Sector. Paper presented at the $\mathrm{XI}^{\text {th }}$ International Congress of the European Association of Agricultural Economists (EAAE) 2005, Copenhagen.

Ebneth, O. and Theuvsen, L. (2005): European Dairy Cooperatives. Impacts of Internationalization and Corporate Success. Paper presented at the $\mathrm{V}^{\text {th }}$ International PENSA Conference, $27^{\text {th }}-30^{\text {th }}$ of July 2005, Ribeirão Preto (Brazil).

Ebneth, O. and Theuvsen, L. (2005): Globalization of the Brewing Industry. Trends, Perspectives and Strategies. Paper presented at the $\mathrm{V}^{\text {th }}$ International PENSA Conference, $27^{\text {th }}-30^{\text {th }}$ of July 2005, Ribeirão Preto (Brazil).

Ebneth, O. and Theuvsen, L. (2005): Internationalization and Corporate Success- Event Study Evidence on M\&As of European Brewing Groups. Paper presented at the $15^{\text {th }}$ Annual World Food and Agribusiness Symposium and Forum, $25^{\text {th }}-28^{\text {th }}$ of June 2005, Chicago.

Ebneth, O. and Theuvsen, L. (2005): Internationalization and Financial Performance of Cooperatives - Empirical Evidence from the European Dairy Sector. Paper presented at the $15^{\text {th }}$ Annual World Food and Agribusiness Symposium and Forum, $25^{\text {th }}-28^{\text {th }}$ of June 2005, Chicago.

Ebneth, O. and Theuvsen, L. (2005): Does Internationalization Pay Off?- Accounting-Based Evaluation of M\&As of European Brewers. Poster presented at the $15^{\text {th }}$ Annual World Food and Agribusiness Symposium and Forum, $25^{\text {th }}-28^{\text {th }}$ of June 2005, Chicago.

Theuvsen, L. and Ebneth, O. (2004): Exploring Globalization in the Brewing Industry. Internationalization Strategies of World-wide Leading Brewers. Poster presented at the $14^{\text {th }}$ Annual World Food and Agribusiness Forum, $12^{\text {th }}-15^{\text {th }}$ of June 2005, Montreux.

\section{Artikel in Praxis orientierten Zeitschriften}

Ebneth, O. (2006): Overview about Northern American Beer Markets. Will be published in: Brewing and Beverage Industry International 6(2006).

Ebneth, O. (2006): Recent Developments in Southern American Beer Markets. Will be published in: Brewing and Beverage Industry International 5(2006).

Ebneth, O. (2006): Recent Developments in Southern American Beer Markets. Will be published in: Brewing and Beverage Industry Espagñol 3(2006).

Ebneth, O. (2006): Main West European Beer Markets at a Glance. Will be published in: Brewing and Beverage Industry International 4(2006).

Ebneth, O. (2006): Wachstum im Weltbiermarkt - Profit Pools in der Brauindustrie. Erscheint in: Brauindustrie 6(2006). 
Ebneth, O. (2006): Review of the Central \& Eastern European Beer Markets. Will be published in: Brewing and Beverage Industry International 3(2006).

Ebneth, O. (2006): The World's Largest Beer Market. Review of the Chinese Brewing Sector, in: Brewing and Beverage Industry International 2(2006): 10-13.

Ebneth, O. (2006): The World's Largest Beer Market. Review of the Chinese Brewing Sector. Will be published in: Brewing and Beverage Industry China 2(2006).

Ebneth, O. (2006): Oportunidades de Crecimiento en Aceleración, in: Brewing and Beverage Industry Espagñol 1(2006): 10-14.

Ebneth, O. (2006): Accelerating Growth Opportunities - Profit Pools in the World Brewing Industry, in: Brewing and Beverage Industry China 1(2006): 6-9.

Ebneth, O. (2006): Accelerating Growth Opportunities - Profit Pools in the World Brewing Industry, in: Brewing and Beverage Industry International 1(2006): 10-13.

Ebneth, O. (2005): Am Ausland führt kein Weg vorbei, in: DLZ Agrarmagazin 12(2005): 132-135.

Ebneth, O. et al. (2005): Wertschöpfungskette Milch muss an einem Strang ziehen, in: Land\&Forst 35(2005): 5-7.

Ebneth, O. (2005): Konzentrationstempo - Internationalisierung und Erfolg europäischer Braukonzerne, in: Brauindustrie 9(2005): 44-49.

Ebneth, O. (2005): Globalización de la Industria Cervecera. Tendencias, Perspectivas y Estrategias, in: Brewing and Beverage Industry Espagñol 2(2005): 32-37.

Ebneth, O. (2005): Globalization of the Brewing Industry. Trends, Perspectives and Strategies, in: Brewing and Beverage Industry International 2(2005): 10-15.

Ebneth, O. und Theuvsen, L. (2005): Genossenschaften im Wettbewerb. Internationalisierung genossenschaftlicher Molkerei- und Schlachtunternehmen, in: Neue Landwirtschaft 1(2005): 22-26.

Ebneth, O. und Theuvsen, L. (2004): Genossenschaften. Können sie mithalten?, in: DLGMitteilungen 9(2004): 70-71.

Ebneth, O. (2003): Mehrgefahrenversicherung. Dem Ausland nacheifern?, in: DLGMitteilungen 12(2003): 20-24. 


\section{LEBENSLAUF \\ OLIVER JOHANNES EBNETH}

Geboren am 10. Januar 1977 in Weiden in der Oberpfalz (Bayern)

\section{PRAKTISCHE ERFAHRUNGEN}

seit 04.2006

$03.2003-03.2006$

$08.2005-09.2005$

$10.2002-03.2003$

$03.2002-04.2002$

$07.2001-09.2001$
$11.2004-12.2004$

$05.2003-07.2003$

Brau Holding InTERnAtional GmbH \& Co. KGaA: München

Assistent des Vorstandsvorsitzenden, Herrn Dr. Friedrich Georg Hoepfner. Tätigkeitsschwerpunkte: Unternehmensplanung, externes Wachstum, Beteiligungsmanagement (M\&A).

\section{PROMOTION und wissenschaftlicher Mitarbeiter}

Lehrstuhl für BWL des Agribusiness, Universität Göttingen Forschungsschwerpunkte: empirische Kapitalmarktforschung, Bilanzanalyse, Unternehmensbewertung, Internationalisierungsstrategien europäischer Braukonzerne.

\section{HEINEKEN INTERNATIONAL: Headoffice Amsterdam}

Zweimalige Mitarbeit an einem Projekt zu Unternehmensakquisitionen in der Mergers\&Acquisitions-Abteilung (Corporate Business Development). Profitabilitätsanalyse weltweiter Biermärkte.

\section{KFW BANKENGRUPPE: KfW/DEG-Office Nairobi}

Assistenz des Büroleiters Oskar von Maltzan. Recherche-Arbeiten zum kenianischen Agrobusiness für die DEG.

\section{MÜNCHENER RÜCKVERSICHERUNGS-GESELLSCHAFT: München}

Stipendiat und Diplomand der Agro-Gruppe (CUGC 2.5) der Münchener Rück. Inhaltliche Betreuung meiner Masterarbeit: „Mehrgefahrenversicherungen als Risk-Management-Instrument für die deutsche Landwirtschaft“.

\section{MÜNCHENER RÜCKVERSICHERUNGS-GESELLSCHAFT: München}

Werkstudent in der Westeuropa-Abteilung (E 2/LA 2.1) der Münchener Rück.

FOOD AND Agriculture ORGANIZATION of the United Nations: Rom

Praktikum in der Commodity and Trade Group des Economic and Social Departments im FAO-Headquarter in Rom.

$03.2001-04.2001$

\section{EUROPÄISCHES PARLAMENT: Brüssel und Straßburg}

Praktikum im Abgeordnetenbüro von Frau Emilia Müller, MdEP. 
$10.1998-02.2003$

$10.1997-09.1998$

$09.1996-08.1997$

07.1996

\section{STUDIUM der Agrarökonomie}

TU München-Weihenstephan (Vordiplom), Institut National Agronomique Paris (Auslandsstudium als Erasmus-Student), Abschluss an der Universität Göttingen nach acht Fachsemestern als Master of Science (M.Sc.).

\section{STUDIUM der Germanistik und bayerischen Geschichte}

Ludwig-Maximilians-Universität München.

WEHRDIENST als Klarinettist im Heeresmusikkorps 4 in Regensburg.

ABITUR am Gymnasium Neustadt an der Waldnaab (Bayern).

\section{BERUFLICHE WEITERBILDUNG}

\section{Konferenzteilnahmen}

06.2006

06.2006

08.2005

07.2005

06.2005

09.2004

06.2004

\section{Managementseminar}

09.2002
VIII $^{\text {th }}$ USDA and AIEA2 International Meeting in Bologna.

$16^{\text {th }}$ Annual World Food and Agribusiness Forum in Buenos Aires.

$\mathrm{XI}^{\text {th }}$ International EAAE Conference in Kopenhagen.

$\mathrm{V}^{\text {th }}$ International PENSA Conference in São Paulo.

$15^{\text {th }}$ Annual World Food and Agribusiness Forum in Chicago.

International Cooperatives Forum in Münster.

$14^{\text {th }}$ Annual World Food and Agribusiness Forum in Montreux.

\section{SPRACH - UND EDV-KENNTNISSE}

Englisch (verhandlungssicher), französisch (gut), italienisch (Grundkenntnisse).

Textverarbeitung mit MS-Word, Tabellenkalkulation mit MS-Excel (beides sehr gut), Internet, Power Point.

\section{ENG A GEMENT UND HOBBYS}

Langjährige ehrenamtliche Mitarbeit als Vorstandsmitglied in einer politischen Jugendorganisation.

Ausbildertätigkeit (Klarinette) in der musikalischen Jugendarbeit.

Aktiver Musiker in einem Studentenorchester (Klarinettist).

Klassische Musik, Politik und Zeitgeschehen, Fußball und Skifahren, Schach,

zeitgenössische deutsche Literatur und französischer Film. 


\section{ERKLÄRUNGEN}

1. Hiermit erkläre ich, dass diese Arbeit weder in gleicher noch in ähnlicher Form bereits anderen Prüfungsbehörden vorgelegen hat.

Weiter erkläre ich, dass ich mich an keiner anderen Hochschule um einen Doktorgrad beworben habe.

Göttingen, den 24. Mai 2006

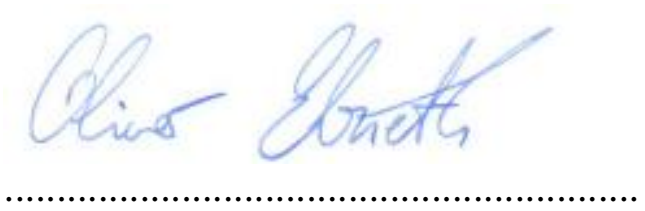

(Unterschrift)

2. Hiermit erkläre ich eidesstattlich, dass diese Dissertation selbständig und ohne unerlaubte Hilfe angefertigt wurde.

Göttingen, den 24. Mai 2006

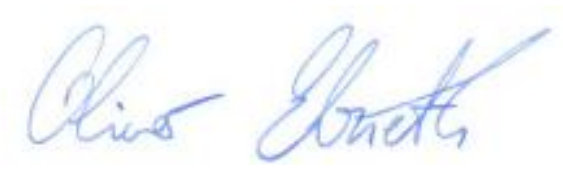

(Unterschrift) 